DOE/RW-0006, Rev. 12

December 1996

\title{
Integrated Data Base Report-1995: \\ U.S. Spent Nuclear Fuel and Radioactive Waste Inventories, Projections, and Characteristics
}

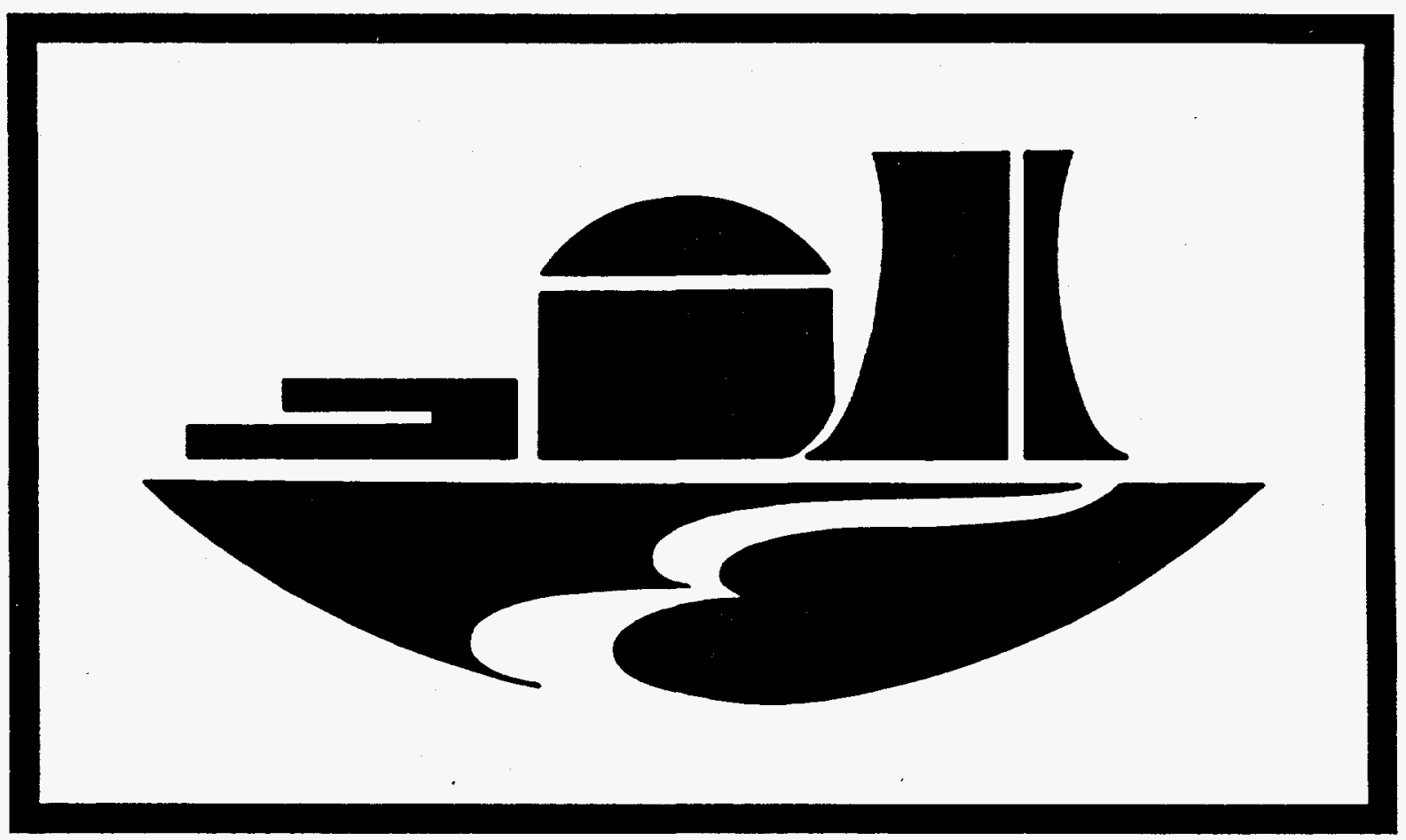

Prepared for

U.S. Department of Energy

Office of Environmental Management

Washington, D.C. 20585

Prepared by

Oak Ridge National Laboratory

Managed by Lockheed Martin Energy Research Corp. for the U.S. Department of Energy under contract DE-AC05-96OR22464 


\section{DISCLAMMER}

Portions of this document may be illegible in electronic image products. Images are produced from the best available original document. 


\section{DISCLAIMER}

This report was prepared as an account of work sponsored by an agency of the United States Government. Neither the United States Government nor any agency thereof, nor any of their employees, make any warranty, express or implied, or assumes any legal liability or responsibility for the accuracy, completeness, or usefulness of any information, apparatus, product, or process disclosed, or represents that its use would not infringe privately owned rights. Reference herein to any specific commercial product, process, or service by trade name, trademark, manufacturer, or otherwise does not necessarily constitute or imply its endorsement, recommendation, or favoring by the United States Government or any agency thereof. The views and opinions of authors expressed herein do not necessarily state or reflect those of the United States Government or any agency thereof. 


\section{PREFACE}

The information in this report summarizes the U.S. Department of Energy (DOE) data base for inventories, projections, and characteristics of domestic spent nuclear fuel and radioactive waste. This report is updated annually to keep abreast of continual waste inventory and projection changes in both the government and commercial sectors. Baseline information is provided for $\mathrm{DOE}$ program planning purposes and to support $\mathrm{DOE}$ program decisions. Although the primary purpose of this document is to provide background information for program planning within the DOE community, it has also been found useful by state and local governments, the academic community, and some private citizens. To sustain the objectives of this program in providing accurate and complete data in this field of operation, comments and suggestions to improve the quality and coverage are encouraged. Such comments and any general inquiries should be directed to DOE's Office of Environmental Management at either of the following:

Office of Waste Management

Route Symbol EM-35

Trevion II Building

19901 Germantown Road

Germantown, MD 20874-1290
Office of Environmental Restoration

Route Symbol EM-43

Cloverleaf Building

20400 Century Boulevard

Germantown, MD 20874

This report was prepared by the Integrated Data Base Program, which is sponsored by the DOE Office of Environmental Management. Suggestions, questions, and requests for information may be directed to any of the following:

$$
\begin{gathered}
\text { M. J. Zenkowich, DOE/EM-35, Germantown, MD 20874-1290 } \\
\text { Telephone: (301) 903-7126 } \\
\text { E-mail: Mathew.Zenkowich@em.doe.gov } \\
\text { M. D. Tolbert-Smith, DOE/EM-43, Germantown, MD } 20874 \\
\text { Telephone: (301) 903-8121 } \\
\text { E-mail: Marilyn. Tolbert-Smith@em.doe.gov } \\
\text { J. A. Klein, ORNL, P.O. Box 2008, Oak Ridge, TN 37831-6111 } \\
\text { Telephone: (423) 574-6823 } \\
\text { E-mail: jak@oml.gov }
\end{gathered}
$$

An important part of the Integrated Data Base Program is the Steering Committee, whose members provide both generic guidance and technical input. The membership of this committee, shown on the following page, represents all of the major DOE sites and programs for spent nuclear fuel and radioactive waste management. Each support committee member is assisted by a technical liaison as needed. The participation and assistance of these individuals are acknowledged with appreciation.

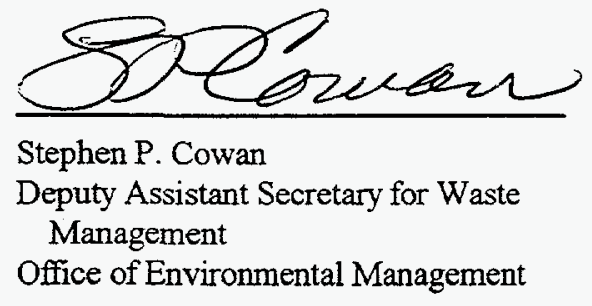

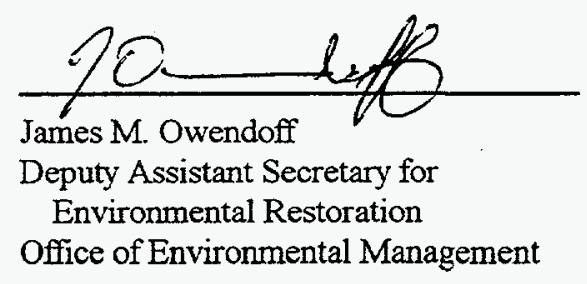




\section{Integrated Data Base Program Steering Committee}

\section{Executive Committee}

\section{Voting members}

M. D. Tolbert-Smith, DOE/EM

M. J. Zenkowich, DOE/EM
Advisorvmember

M. L. Payton, DOE/RW

J. A. Klein, ORNL

\section{Support Committee}

Chapter title/support area

Spent nuclear fuel

Commercial

DOE

High-level waste

Transuranic waste

Low-level waste

DOE

Commercial

Uranium mill tailings

Environmental restoration wastes

Mixed low-level waste

Waste minimization

\section{Committee member}

M. L. Payton, DOE/RW

J. F. Baker, DOE/EM

R. L. Sweeney, DOE/EM

M. L. Matthews, DOE/CAO

G. J. Duggan, DOE/EM

T. L. Plummer, DOE/EM

J. G. Snook, DOE/ID

T. Chung, DOE/EIA

M. D. Tolbert-Smith, DOE/EM

H. L. Belencan, DOE/EM

G. T. McBrien, DOE/EM $\underline{\text { Technical liaison }}$

R. R. MacDonald, CRWMS$\mathrm{M} \& O / J A I$

A. R. Bringhurst, LMIT

A. Sidpara, DOE/RL

P. E. Drez, DEA

J. Hwang, ATL

R. L. Fuchs, LMIT

T. Chung, DOE/ELA

J. M. Peterson, ANL-E M. M. MacDonell, ANL-E

T. D. Kirkpatrick, MACTEC

G. T. McBrien, DOE/EM 


\section{Chapter topic}

Overview

Spent nuclear fuel

High-level waste

Transuranic waste

Low-level waste

Uranium mill tailings

Environmental restoration wastes

Naturally occurring and acceleratorproduced radioactive material

Mixed low-level waste

Waste source terms

\section{Direct support service}

Clerical/IDB report

Clerical/IDB Program

Computer support (data base setup and analysis)

Computer graphics

Editing

Distribution
Author(s)

J. A. Klein and S. N. Storch

S. N. Storch ${ }^{\mathrm{a}}$

S. L. Loghry

S. Chakraborti (SAIC) ${ }^{\text {b }}$ and P. E. Drez (WTAC) ${ }^{c}$

A. S. Icenhour, S. L. Loghry, and M. L. Tharp

T. Chung (DOE/EIA)

M. Tolbert-Smith (DOE/EM-40), J. M. Peterson (ANL-E), and M. M. MacDonell (ANL-E)

S. N. Storch

T. D. Kirpatrick (MACTEC) and S. N. Storch

A. S. Icenhour and S. L. Loghry

Contributor(s)

D. T. Brooksbank

S. L. McDaniel

M. L. Tharp and S. L. Loghry

S. L. McDaniel

R. W. Sharpe

S. L. McDaniel, D. T. Brooksbank, and S. N. Storch

\footnotetext{
aWith support from DOE/EIA and DOE Office of Spent Fuel Management.

b Science Applications International Corporation (SAIC).

${ }^{c}$ Waste Isolation Pilot Plant Technical Assistance Contractor (WTAC). The WTAC provided the transuranic waste chapter for this report.

¿With support from DOE LLW Management Program (ATL International) and National Low-Level Waste Management Program.
} 
Major DOE data sources supporting the Integrated Data Base Program report

\begin{tabular}{|c|c|c|c|}
\hline Name of data source & Waste type information provided & Supporting DOE program office & $\begin{array}{l}\text { Principal coniractor and } \\
\text { mailing address } \\
\text { (Phone number) }\end{array}$ \\
\hline $\begin{array}{l}\text { DOE Office of Civilian Radioactive } \\
\text { Waste Management }\end{array}$ & $\begin{array}{l}\text { Commercial spent nuclear fuel } \\
\text { inventories }\end{array}$ & $\begin{array}{l}\text { DOE/RW Office of Storage and } \\
\text { Transportation }(\mathrm{RW}-40)\end{array}$ & $\begin{array}{l}\text { DOE/RW Waste Acceptance Division } \\
\text { (DOE/RW-44) } \\
\text { Forrestal Building } \\
1000 \text { Independence Avenue, S.W. } \\
\text { Washington, D.C. 20585-0001 } \\
\text { (202/586-9867) }\end{array}$ \\
\hline $\begin{array}{l}\text { Integrated Spent Nuclear Fuel Database } \\
\text { System }\end{array}$ & DOE spent nuclear fuel & $\begin{array}{l}\text { Office of Spent Fuel Management } \\
\text { (EM-67) }\end{array}$ & $\begin{array}{l}\text { Lockheed Martin Idaho Technologies } \\
\text { National Spent Fuel Program } \\
\text { P.O. Box } 1625 \\
\text { Idaho Falls, W } 83415 \\
\text { (208/526-8909) }\end{array}$ \\
\hline $\begin{array}{l}\text { Environmental Restoration Core } \\
\text { Database }\end{array}$ & $\begin{array}{l}\text { DOE/EM-40 contaminated } \\
\text { media/waste }\end{array}$ & $\begin{array}{l}\text { Office of Environmental Restoration } \\
(\mathrm{EM}-40)\end{array}$ & $\begin{array}{l}\text { DOE/EM Office of Program Integration } \\
\text { (DOE/EM-43) } \\
\text { Cloverleaf Building } \\
\text { U.S. Department of Energy } \\
\text { 20400 Century Boulevard } \\
\text { Germantown, MD } 20874 \\
\text { (301/903-8121) }\end{array}$ \\
\hline $\begin{array}{l}\text { International Nuclear Model, Personal } \\
\text { Computer Version (PCINM) }\end{array}$ & $\begin{array}{l}\text { Commercial spent nuclear fuel } \\
\text { projections }\end{array}$ & $\begin{array}{l}\text { Energy Information Administration } \\
\text { (DOE/EIA) }\end{array}$ & $\begin{array}{l}\text { DOE/EIA Analysis and Systems } \\
\quad \text { Division } \\
\text { Nuclear Fuel Cycle Branch } \\
\text { Route Symbol EI-531 } \\
950 \text { L'Enfant Plaza } \\
\text { Washington, D.C. } 20024 \\
\text { (202/426-1129) }\end{array}$ \\
\hline $\begin{array}{l}\text { Manifest Information Management } \\
\text { System (MIMS) }\end{array}$ & Commercial low-level waste & Office of Waste Management (EM-30) & $\begin{array}{l}\text { Lockheed Martin Idaho Technologies } \\
\text { National Low-Level Waste } \\
\quad \text { Management Program } \\
\text { P.O. Box } 1625 \\
\text { Idaho Falls, ID } 83415 \\
\text { (208/526-9717) }\end{array}$ \\
\hline
\end{tabular}


Major DOE data sources supporting the Integrated Data Base Program report (continued)

\begin{tabular}{|c|c|c|c|}
\hline Name of data source & Waste type information provided & Supporting DOE program office & $\begin{array}{l}\text { Principal contractor and } \\
\text { mailing address } \\
\text { (Phone number) }\end{array}$ \\
\hline Uranium Reserve Assessment Program & Commercial uranium mill tailings & $\begin{array}{l}\text { Energy Information Administration } \\
\text { (DOE/EIA) }\end{array}$ & $\begin{array}{l}\text { DOE/EIA Analysis and Systems } \\
\quad \text { Division } \\
\text { Nuclear Fuel Cycle Branch } \\
\text { Route Symbol EI-531 } \\
\text { 950 L'Enfant Plaza } \\
\text { Washington, D.C. } 20024 \\
\text { (202/426-1128) }\end{array}$ \\
\hline $\begin{array}{l}\text { Transuranic Waste Baseline Inventory } \\
\text { Database and Report (TWBID and } \\
\text { TWBIR) }\end{array}$ & DOE transuranic waste & $\begin{array}{l}\text { DOE Carlsbad Area Office } \\
\text { National Transuranic Program Office } \\
\text { (NTPO) }\end{array}$ & $\begin{array}{l}\text { Drez Environmental Associates } \\
8816 \text { Cherry Hills Road, N.E. } \\
\text { Albuquerque, NM } 87111 \\
\text { (505/828-9857) }\end{array}$ \\
\hline $\begin{array}{l}\text { DOE Site Treatment Plan Database/ } \\
1995 \text { MWIR Database }\end{array}$ & RCRA-regulated DOE mixed wastes & Office of Waste Management (EM-30) & $\begin{array}{l}\text { DOE/EM Office of Waste Management } \\
\text { Office of Planning and Analysis } \\
\text { Route Symbol EM-35 } \\
\text { Trevion II Building } \\
\text { U.S. Department of Energy } \\
\text { 19901 Germantown Road } \\
\text { Germantown, MD 20874-1290 } \\
(301 / 903-7126)\end{array}$ \\
\hline
\end{tabular}





\section{CONTENTS}

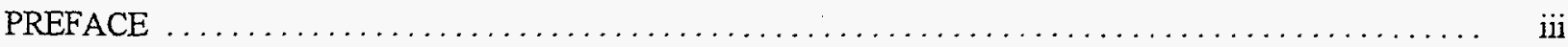

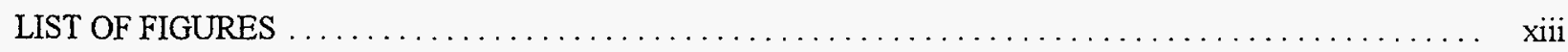

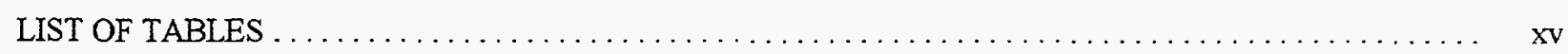

GLOSSARY OF ABBREVIATIONS, ACRONYMS, AND INITIALISMS $\ldots \ldots \ldots \ldots \ldots \ldots \ldots \ldots \ldots$ xxiii

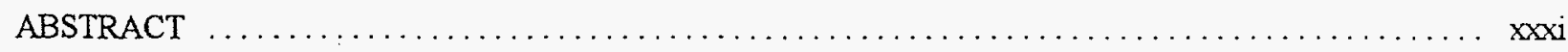

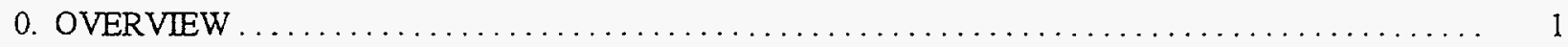

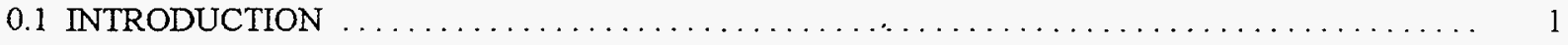

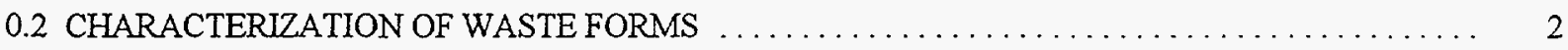

0.3 METHODS AND ASSUMPTIONS USED IN REPORT PREPARATION $\ldots \ldots \ldots \ldots \ldots \ldots \ldots \ldots$

0.4 WASTE CHARACTERISTICS AND UNITS REPORTED $\ldots \ldots \ldots \ldots \ldots \ldots \ldots \ldots \ldots \ldots \ldots \ldots$

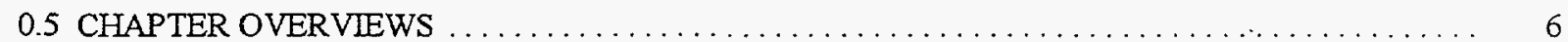

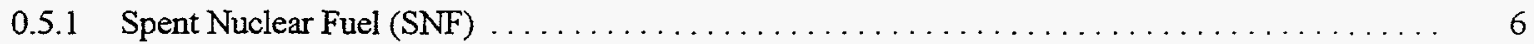

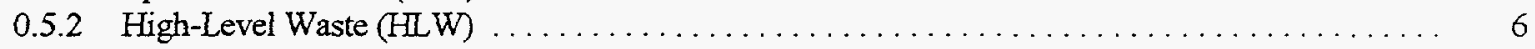

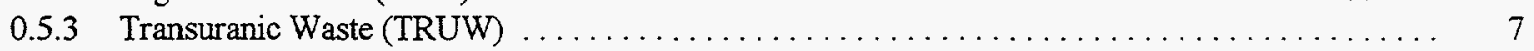

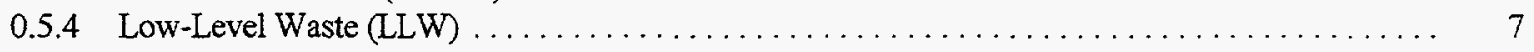

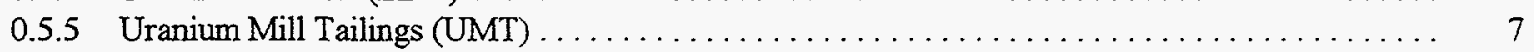

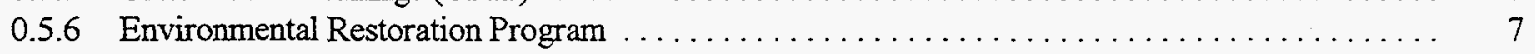

0.5.7 Naturally Occurring and Accelerator-Produced Radioactive Material (NARM) $\ldots \ldots \ldots \ldots \ldots . \quad 8$

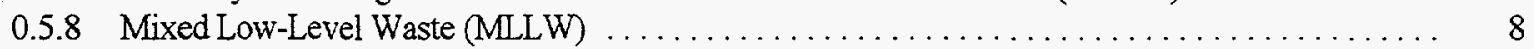

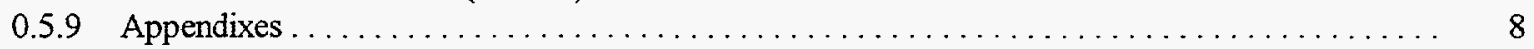

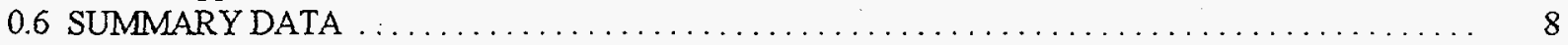

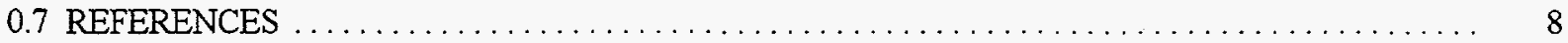

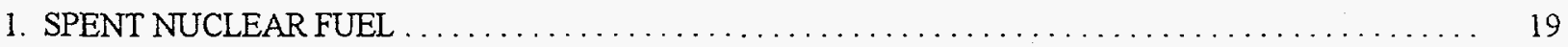

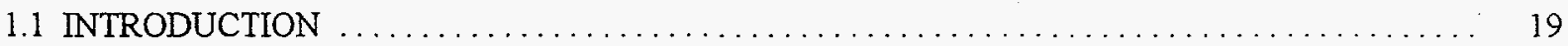

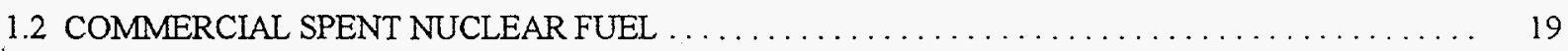

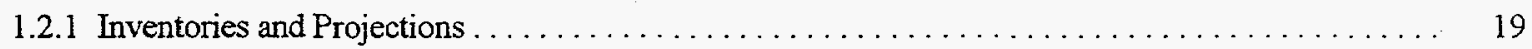

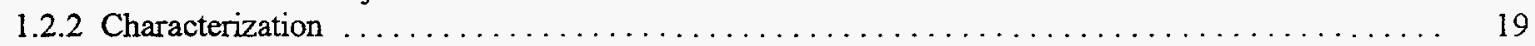

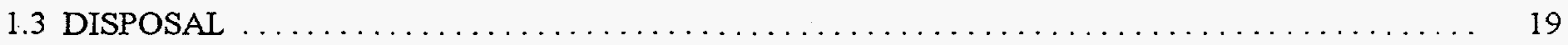

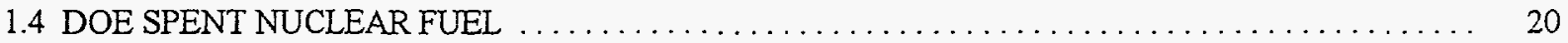

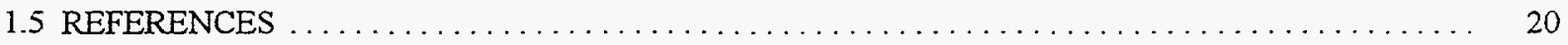

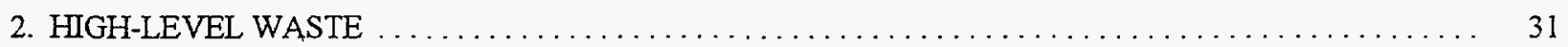

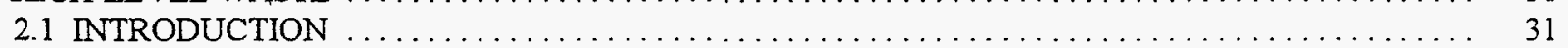

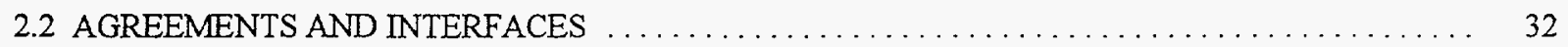

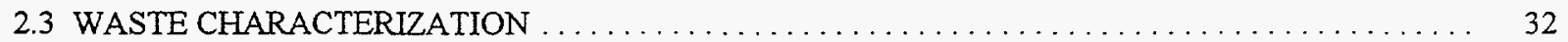

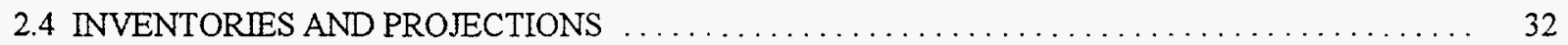

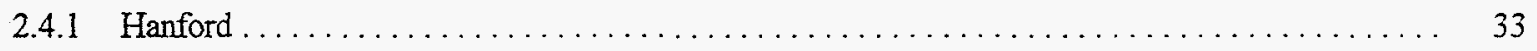

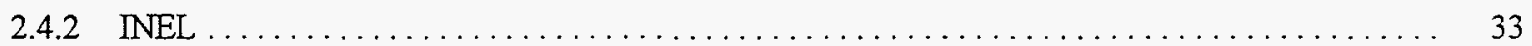




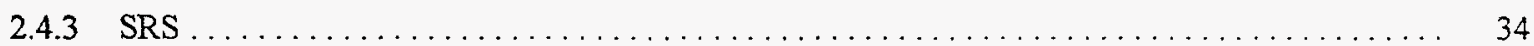

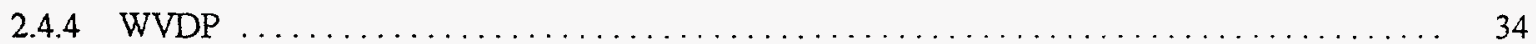

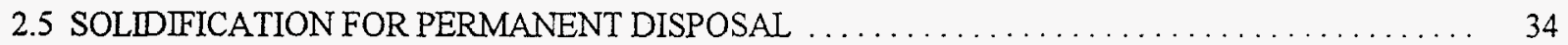

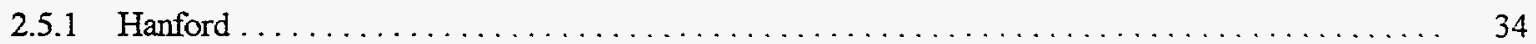

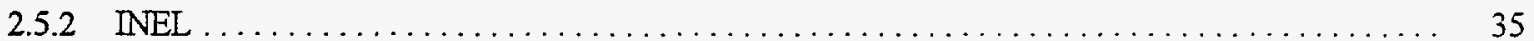

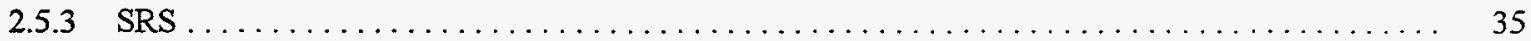

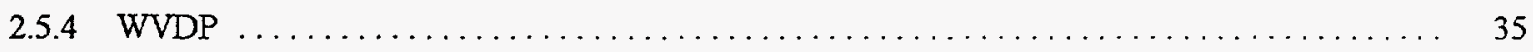

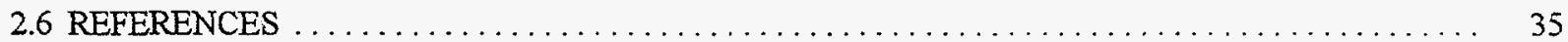

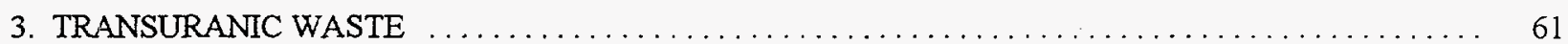

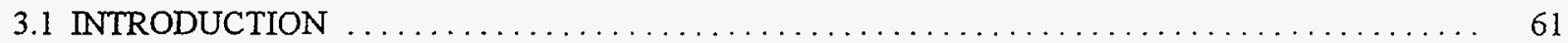

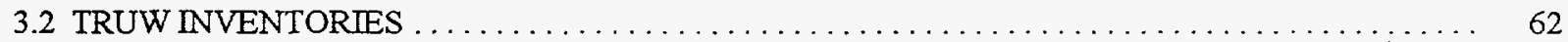

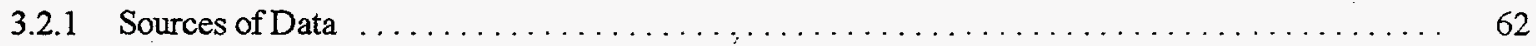

3.2.2 Site Locations—Summarized Volumes and Radioactivity $\ldots \ldots \ldots \ldots \ldots \ldots \ldots \ldots \ldots \ldots \ldots$

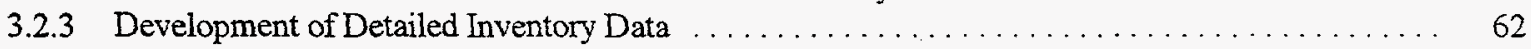

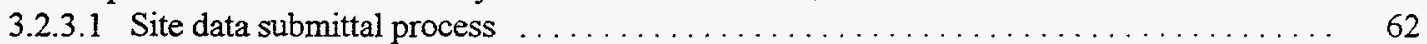

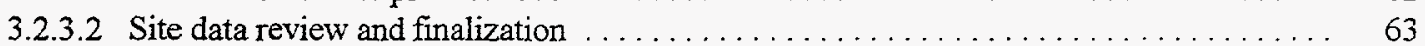

3.2.4 Volumes and Radioactivities of TRUW at DOE Sites $\ldots \ldots \ldots \ldots \ldots \ldots \ldots \ldots \ldots \ldots \ldots$

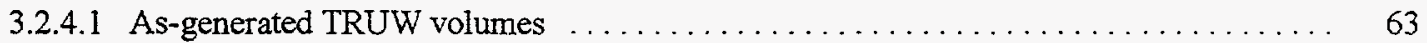

3.2.4.2 Final-form TRUW volumes ............................... 63

3.2.4.3 Decayed radioactivities for retrievably stored TRUW $\ldots \ldots \ldots \ldots \ldots \ldots \ldots \ldots \ldots$

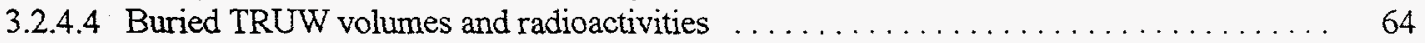

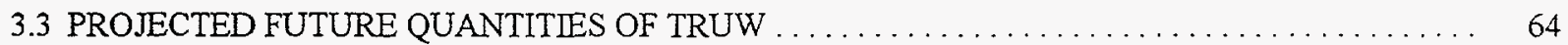

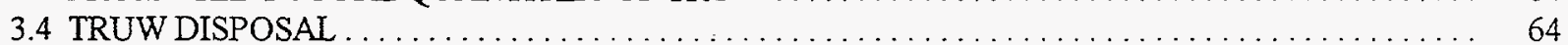

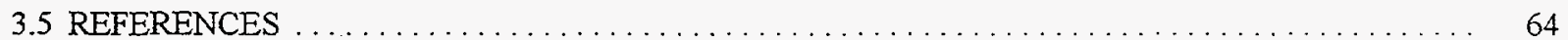

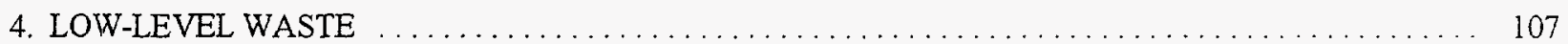

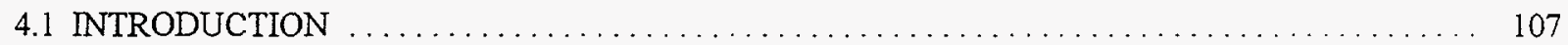

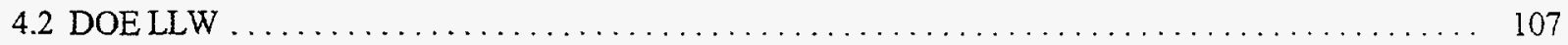

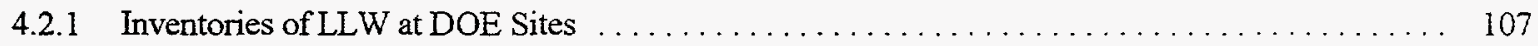

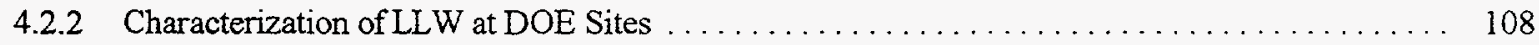

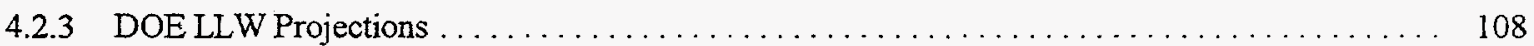

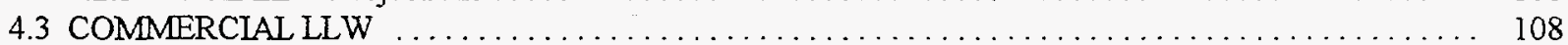

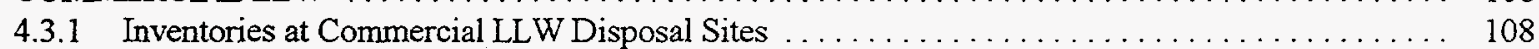

4.3.2 Characterization of $L L W$ at Commercial Disposal Sites . . . . . . . . . . . . . . . . . . . . 109

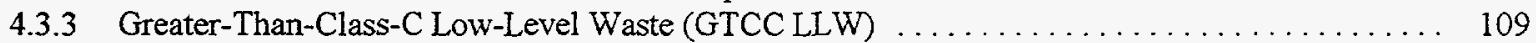

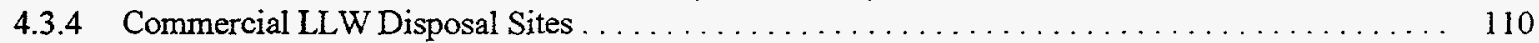

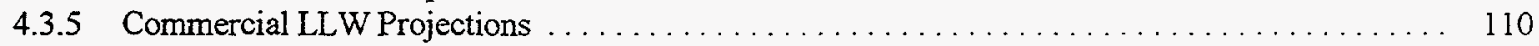

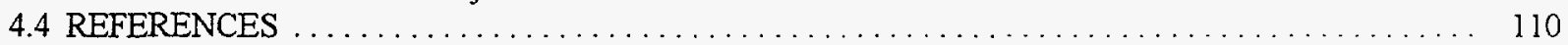

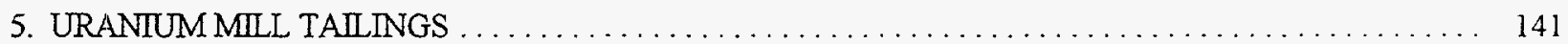

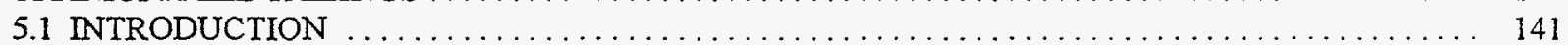

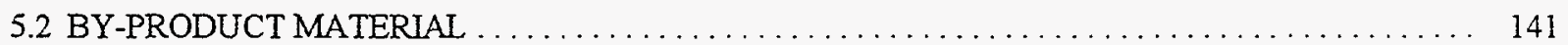

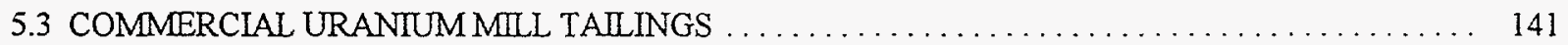

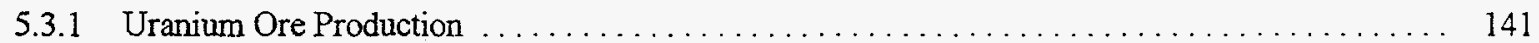

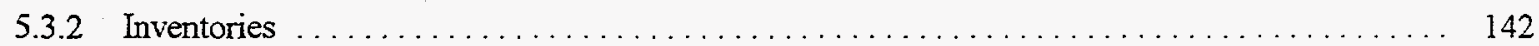

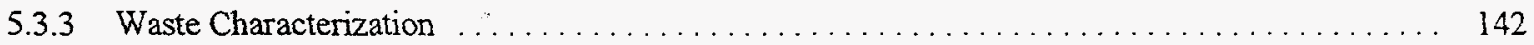

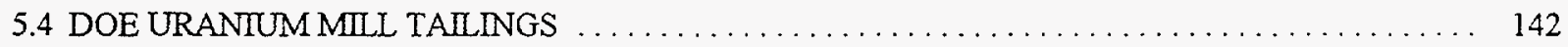

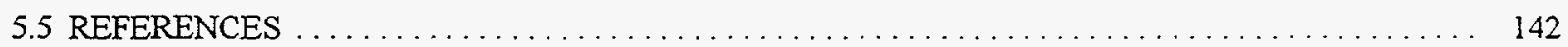

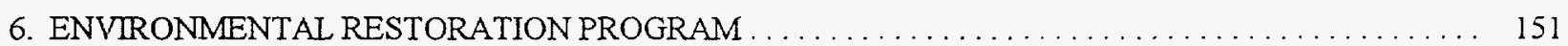

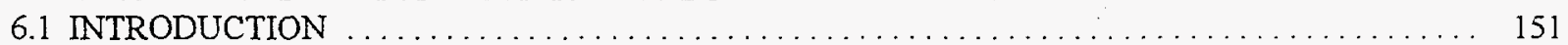




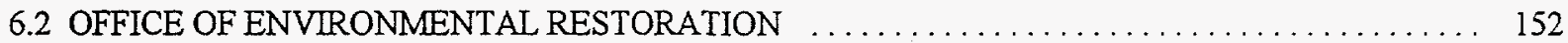

6.3 ENVIRONMENTAL RESTORATION WASTE CHARACTERISTICS $\ldots \ldots \ldots \ldots \ldots \ldots \ldots \ldots \ldots$

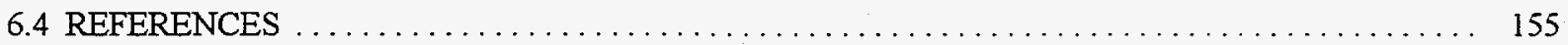

7. NATURALLY OCCURRING AND ACCELERATOR-PRODUCED RADIOACTIVE MATERIAL $\ldots \ldots .169$

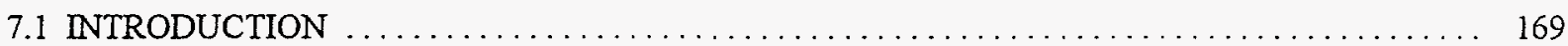

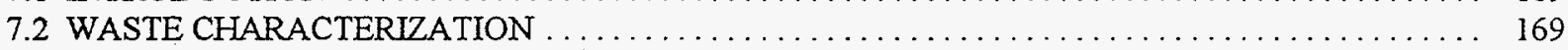

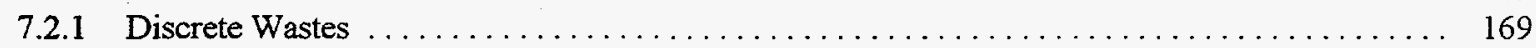

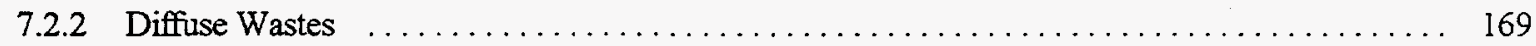

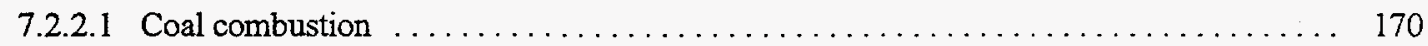

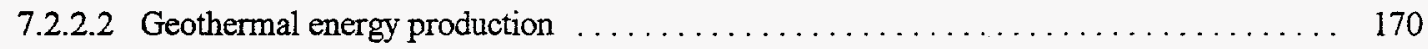

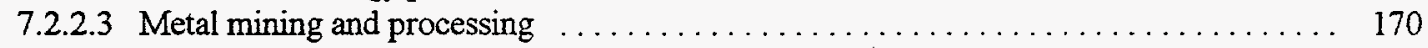

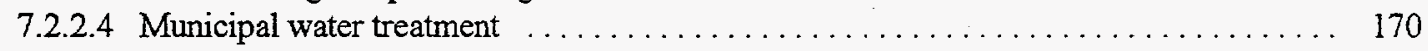

7.2.2.5 Oil and natural gas production and processing $\ldots \ldots \ldots \ldots \ldots \ldots \ldots \ldots \ldots \ldots \ldots$

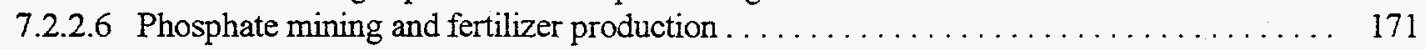

7.3 GENERATION, INVENTORIES AND PROJECTIONS $\ldots \ldots \ldots \ldots \ldots \ldots \ldots \ldots \ldots \ldots \ldots \ldots \ldots \ldots$

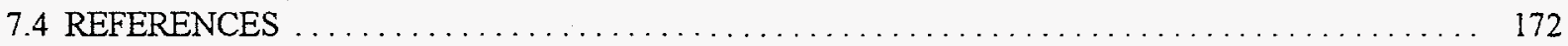

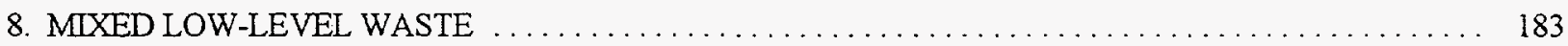

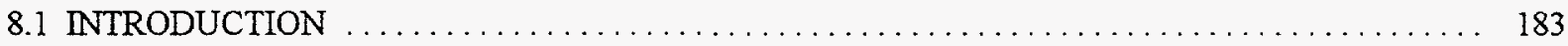

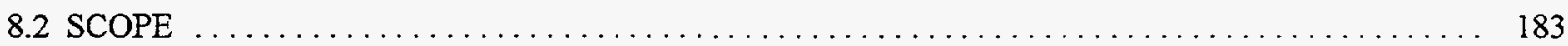

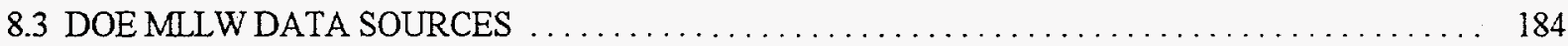

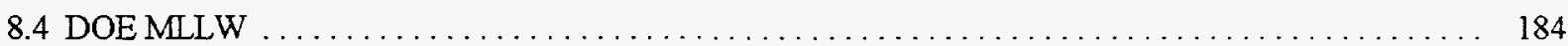

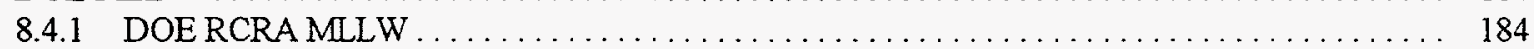

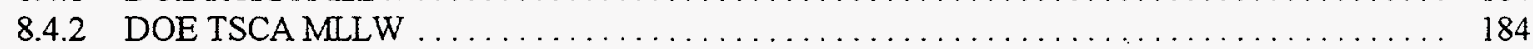

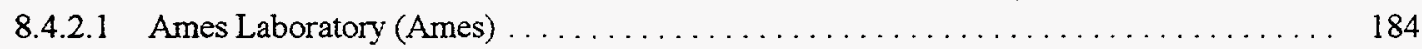

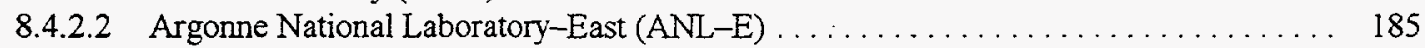

8.4.2.3 Argonne National Laboratory-West (ANL-W) $\ldots \ldots \ldots \ldots \ldots \ldots \ldots \ldots \ldots \ldots \ldots$

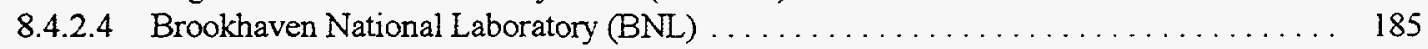

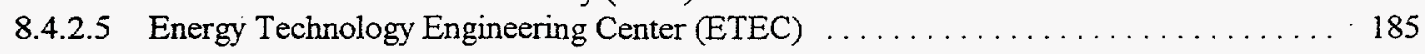

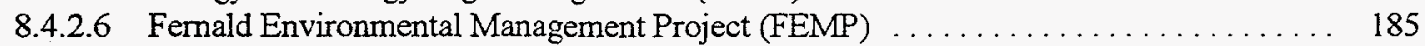

8.4.2.7 Fermi National Accelerator Laboratory (FNAI) . . . . . . . . . . . . . . . . . . . 185

8.4.2.8 Hanford Site (Hanford) . . . . . . . . . . . . . . . . . . . . . . . 185

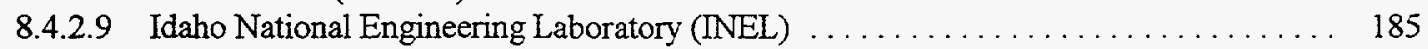

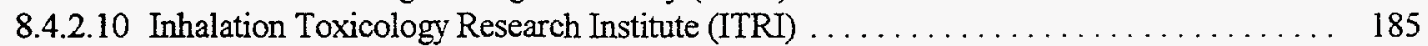

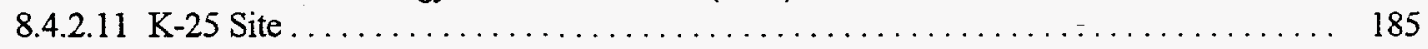

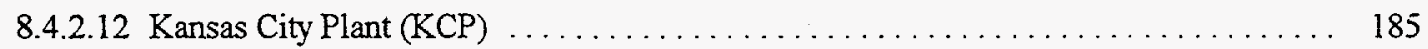

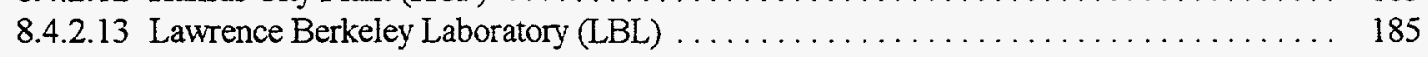

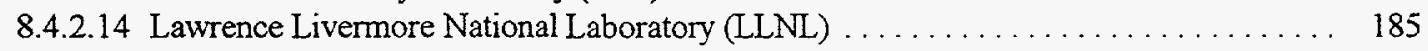

8.4.2.15 Los Alamos National Laboratory (LANL) . . . . . . . . . . . . . . . . . . . . . . . . 185

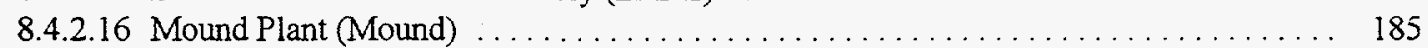

8.4.2.17 Naval Reactor (NR) Sites . . . . . . . . . . . . . . . . . . . . . . . . . . . . . . . . 186

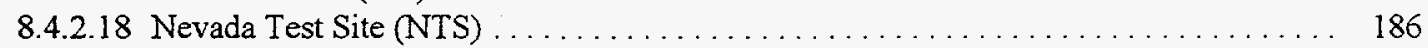

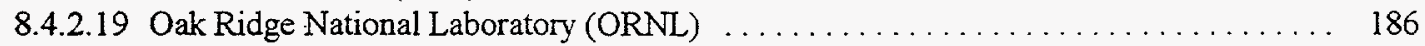

8.4.2.20 Oak Ridge Institute of Science and Education (ORISE) $\ldots \ldots \ldots \ldots \ldots \ldots \ldots \ldots$

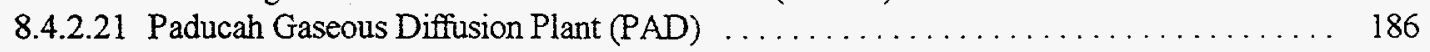

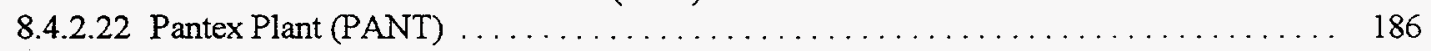

8.4.2.23 Pinellas Plant (Pinellas) . . . . . . . . . . . . . . . . . . . . . . . . . . 186

8.4.2.24 Portsmouth Gaseous Diffusion Plant (PORTS) . . . . . . . . . . . . . . . . . . . . 186

8.4.2.25 Princeton Plasma Physics Laboratory (PPPL) . . . . . . . . . . . . . . . . . . . 186

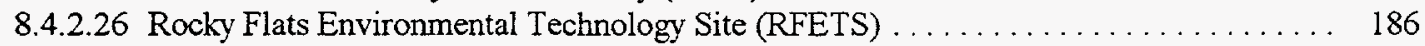

8.4.2.27 Stanford Linear Accelerator Center (SLAC) . . . . . . . . . . . . . . . . . . . . . . . 186

8.4.2.28 Sandia National Laboratory/California (SNL/CA) $\ldots \ldots \ldots \ldots \ldots \ldots \ldots \ldots \ldots$ 
8.4.2.29 Sandia National Laboratory/New Mexico (SNL/NM) $\ldots \ldots \ldots \ldots \ldots \ldots \ldots \ldots$

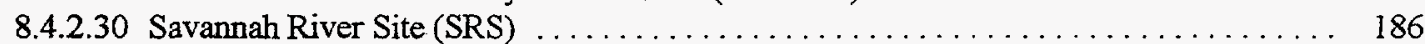

8.4.2.31 West Valley Demonstration Project (WVDP) $\ldots \ldots \ldots \ldots \ldots \ldots \ldots \ldots \ldots \ldots \ldots \ldots$

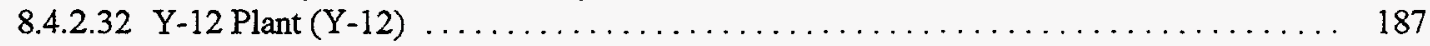

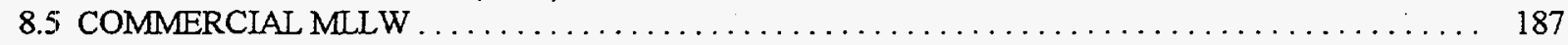

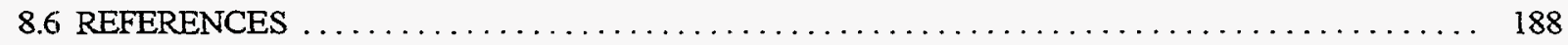

APPENDIX A. RADIOACTIVE WASTE SOURCE TERMS AND CHARACTERISTICS $\ldots \ldots \ldots \ldots \ldots \ldots 215$

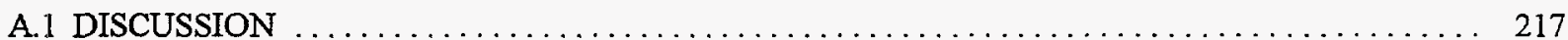

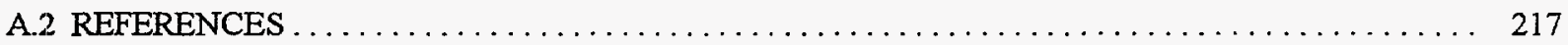

APPENDIX B. CHARACTERISTICS OF IMPORTANT RADIONUCLIDES $\ldots \ldots \ldots \ldots \ldots \ldots \ldots \ldots \ldots$

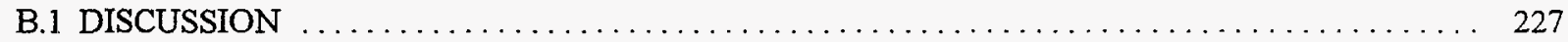

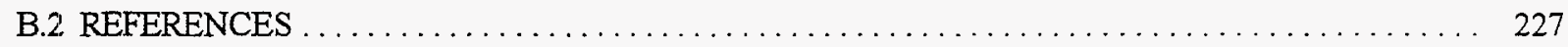

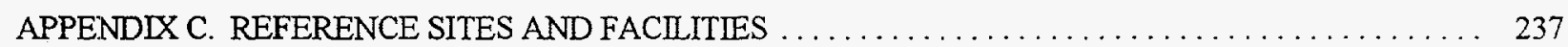

APPENDIX D. INTEGRATED DATA BASE DOCUMENT ACCESS ON THE INTERNET $\ldots \ldots \ldots \ldots \ldots 253$

APPENDIX E. INTEGRATED DATA BASE READER COMMENT FORM $\ldots \ldots \ldots \ldots \ldots \ldots \ldots \ldots \ldots$

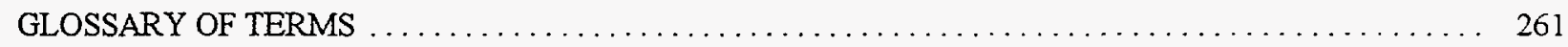

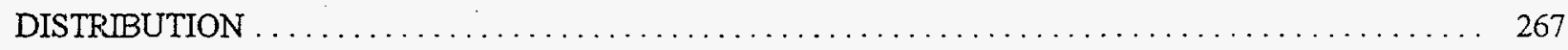




\section{LIST OF FIGURES}

\section{OVERVIEW}

0.1 Total volumes of DOE and commercial radioactive wastes and SNF through 1995

0.2 Total decayed radioactivities of DOE and commercial radioactive wastes and SNF through 1995

\section{SPENT NUCLEAR FUEL}

1.1 Locations of commercial nuclear power reactors as of December 31,1995 .

1.2 Historical and projected mass (MTIHM) of cumulative commercial SNF discharged for the DOE/EIA Low Case

\section{HIGH-LEVEL WASTE}

$2.1 \quad$ Locations and total volumes of $\mathrm{HLW}$ through $1995 \ldots \ldots \ldots \ldots \ldots$

2.2 Total volume and radioactivity of solid and liquid $\mathrm{HLW}$ through $1995 \ldots \ldots 37$

Historical and projected cumulative volumes of untreated $\mathrm{HI} W$ in storage $\ldots \ldots \ldots$

$2.4 \quad$ Projected cumulative number of $\mathrm{HLW}$ canisters $\ldots \ldots \ldots \ldots \ldots \ldots \ldots$

2.5 Treatment methods for tank waste and capsules at Hanford $\ldots \ldots \ldots \ldots$

2.6 Treatment methods for $\mathrm{HLW}$ in tanks and bins at $\mathrm{NEL} \ldots \ldots \ldots \ldots$

$2.7 \quad$ Treatment methods for $\mathrm{HLW}$ in tanks and canisters at $\mathrm{SRS} \ldots \ldots \ldots \ldots$

$2.8 \quad$ Treatment methods for HLW in tanks and canisters at WVDP $\ldots \ldots \ldots \ldots$

\section{TRANSURANIC WASTE}

3.1 U.S. Department of Energy transuranic waste generator/storage sites

\section{LOW-LEVEL WASTE}

4.1 Comparison of volume and radioactivity of LLW disposed at commercial and DOE facilities during 1995 
4.2 Total volume of $L L W$ disposed through $1995 \ldots \ldots \ldots \ldots \ldots \ldots \ldots \ldots \ldots \ldots \ldots \ldots$

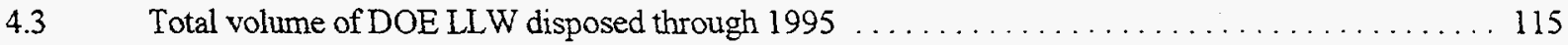

4.4 Locations and total volumes of DOE LLW disposed through $1995 \ldots \ldots \ldots \ldots \ldots \ldots \ldots \ldots$

4.5 Total volume of commercial LLW disposed through $1995 \ldots \ldots \ldots \ldots \ldots \ldots \ldots \ldots \ldots$

4.6 Locations and total volumes of commercial LLW disposed through $1995 \ldots \ldots \ldots \ldots \ldots \ldots$

\section{URANIUM MILL TAILINGS}

5.1 Location and status of uranium mills and plants at the end of 1995

\section{ENVIRONMENTAL RESTORATION PROGRAM}

6.1 Locations of field offices that direct the DOE environmental restoration program $\ldots \ldots \ldots \ldots \ldots 156$

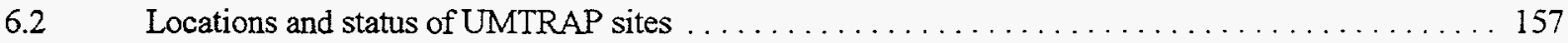

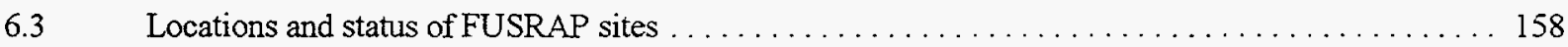

MIXED LOW-LEVEL WASTE

8.1 Estimated volume inventory of DOE MLLW (RCRA and TSCA) at the end of $1995 \ldots \ldots \ldots 191$

8.2 Estimated volume generation of DOE MLLW (RCRA and TSCA) during $1995 \ldots \ldots \ldots \ldots \ldots 1$ 


\section{LIST OF TABLES}

\section{OVERVIEW}

0.1 Types of radioactive wastes managed at DOE sites referenced in this report $\ldots \ldots \ldots 11$

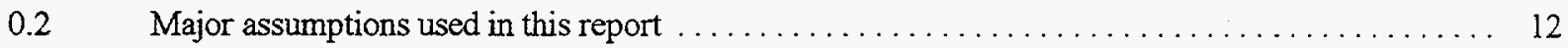

0.3 Cumulative SNF and radioactive waste inventories as of December $31,1995 \ldots 13$

$0.4 \quad$ Current and projected cumulative quantities of radioactive waste and $\mathrm{SNF} \ldots \ldots \ldots \ldots$

$0.5 \quad$ Volume $\left(\mathrm{m}^{3}\right)$ of DOE site wastes generated during $\mathrm{CY} 1995 \ldots \ldots \ldots \ldots$

0.6 Volume $\left(\mathrm{m}^{3}\right)$ inventory of stored DOE site SNF and radioactive wastes as of December 31, $1995 \ldots 16$

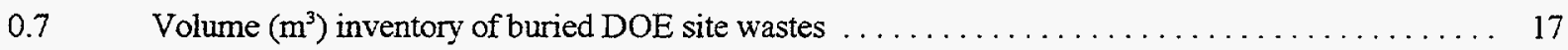

0.8 Volume $\left(\mathrm{m}^{3}\right)$ summary of radioactive wastes disposed of at the Envirocare Facility near

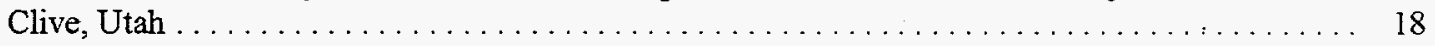

\section{SPENT NUCLEAR FUEL}

1.1 Historical and projected installed LWR electric power generating capacity for the DOE/EIA Low Case

1.2 Historical mass (MTIHM) of permanently discharged commercial SNF by reactor type $\ldots \ldots \ldots 25$

1.3 Current and projected mass (MTIHM) of permanently discharged commercial LWR SNF

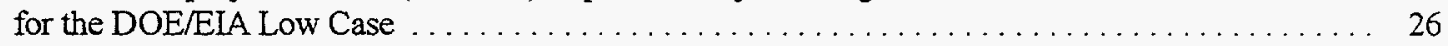

$1.4 \quad$ IDB reference characteristics of $L W R$ nuclear fuel assemblies $\ldots \ldots \ldots 27$

1.5 Summary inventory of DOE SNE for years 1996 and $2035 \ldots \ldots \ldots \ldots$

\section{HIGH-LEVEL WASTE}

2.1 Historical and projected cumulative volume of HLW stored in tanks, bins, and capsules, by site . . . 4l

2.2 Historical and projected cumulative decayed radioactivity of HLW stored in tanks, bins, and

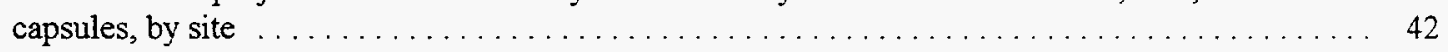

2.3 Historical and projected cumulative decayed thermal power of $H L W$ stored in tanks, bins, and

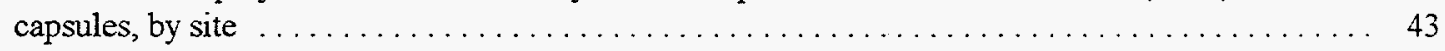

2.4 Projected annual and cumulative volume $\left(10^{3} \mathrm{~m}^{3}\right)$ of HLW glass stored in canisters, by site $\ldots \ldots 44$ 
2.5 Projected annual and cumulative decayed radioactivity $\left(10^{6} \mathrm{Ci}\right)$ of $\mathrm{HLW}$ glass stored in

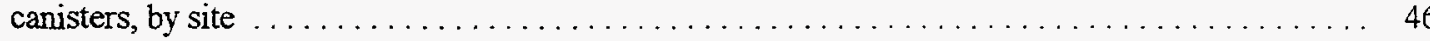

2.6 Projected annual and cumulative decayed thermal power $\left(10^{3} \mathrm{~W}\right)$ of $\mathrm{HLW}$ glass stored in

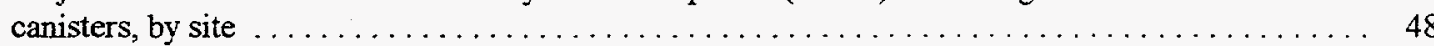

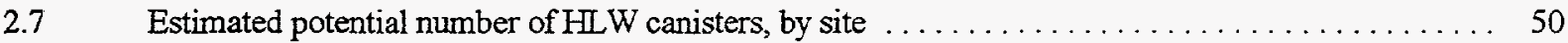

$2.8 \quad$ Current volume of $\mathrm{HLW}$ in storage by site through $1995 \ldots \ldots \ldots \ldots \ldots \ldots \ldots \ldots \ldots \ldots \ldots \ldots \ldots \ldots \ldots$

$2.9 \quad$ Current radioactivity of HLW in storage by site through $1995 \ldots \ldots \ldots \ldots \ldots \ldots \ldots \ldots \ldots \ldots$

$2.10 \quad$ Current thermal power of $H L W$ in storage by site through $1995 \ldots \ldots \ldots \ldots \ldots \ldots \ldots \ldots \ldots$

2.11 Major radionuclides comprising HLW and associated wastes at Hanford $\ldots \ldots \ldots \ldots \ldots \ldots$

$2.12 \quad$ Major radionuclides comprising $\mathrm{HLW}$ and associated wastes at INEL $\ldots \ldots \ldots \ldots \ldots \ldots \ldots$

$2.13 \quad$ Major radionuclides comprising $\mathrm{HLW}$ and associated wastes at $\mathrm{SRS} \ldots \ldots \ldots \ldots \ldots \ldots \ldots$

$2.14 \quad$ Major radionuclides comprising $\mathrm{HL} W$ and associated wastes at WVDP $\ldots \ldots \ldots \ldots \ldots \ldots$

2.15 Significant revisions and changes in the current values for HLW compared to the values in

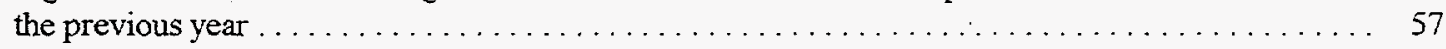

2.16 Proposed representative chemical composition of future HLW glass to be generated at Hanford . . . 58

2.17 Proposed representative chemical composition of future HLW glass to be generated at INEL . . . . 58

2.18 Proposed representative chemical composition of future HLW glass to be generated at SRS ..... 59

2.19 Proposed representative chemical composition of future $\mathrm{HLW}$ glass to be generated at WVDP .... 59

\section{TRANSURANIC WASTE}

3.1 Summary of as-generated waste-form volumes $\left(\mathrm{m}^{3}\right)$ of retrievably stored and projected

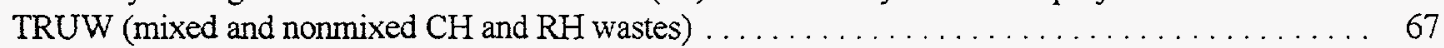

3.2 Summary of as-generated waste-form volumes $\left(\mathrm{m}^{3}\right)$ of retrievably stored and projected mixed and nonmixed CH TRUW

3.3 As-generated waste-form volumes $\left(\mathrm{m}^{3}\right)$ of retrievably stored and projected mixed $\mathrm{CH}$

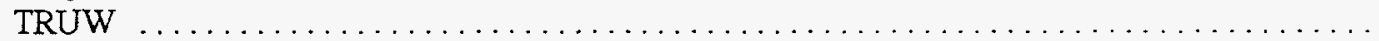

3.4 As-generated waste-form volumes $\left(\mathrm{m}^{3}\right)$ of retrievably stored and projected nonmixed $\mathrm{CH}$ TRUW

3.5 Summary of as-generated waste-form volumes $\left(\mathrm{m}^{3}\right)$ of retrievably stored and projected mixed and nonmixed RH TRUW

3.6 As-generated waste-form volumes $\left(\mathrm{m}^{3}\right)$ of retrievably stored and projected mixed $\mathrm{RH}$ TRUW 
3.7 As-generated waste-form volumes $\left(\mathrm{m}^{3}\right)$ of retrievably stored and projected nonmixed $\mathrm{RH}$ TRUW

3.8 Summary of final waste-form volumes $\left(\mathrm{m}^{3}\right)$ of retrievably stored and projected TRUW (mixed and nonmixed $\mathrm{CH}$ and $\mathrm{RH}$ wastes)

3.9 Summary of final waste-form volumes $\left(\mathrm{m}^{3}\right)$ of retrievably stored and projected mixed and

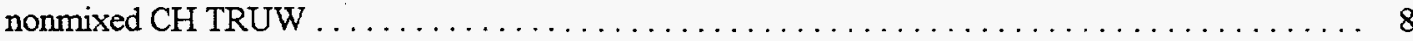

3.10 Final waste-form volumes $\left(\mathrm{m}^{3}\right)$ of retrievably stored and projected mixed $\mathrm{CH}$ TRUW $\ldots \ldots \ldots \ldots 8$

3.11 Final waste-form volumes $\left(\mathrm{m}^{3}\right)$ of retrievably stored and projected nonmixed $\mathrm{CH}$ TRUW $\ldots \ldots \ldots \quad 87$

3.12 Summary of final waste-form volumes $\left(\mathrm{m}^{3}\right)$ of retrievably stored and projected mixed and

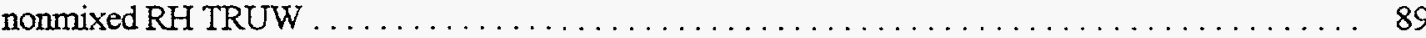

3.13 Final waste-form volumes $\left(\mathrm{m}^{3}\right)$ of retrievably stored and projected mixed RH TRUW . . . . . . 91

3.14 Final waste-form volumes $\left(\mathrm{m}^{3}\right)$ of retrievably stored and projected nonmixed $\mathrm{RH}$ TRUW $\ldots \ldots \ldots 93$

3.15 Estimated final-form volume $\left(\mathrm{m}^{3}\right)$ composition of retrievably stored and projected TRUW

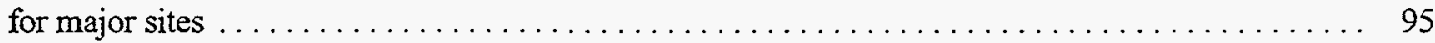

3.16 Estimated final-form volume $\left(\mathrm{m}^{3}\right)$ composition of retrievably stored and projected TRUW for small-quantity and commercial sites . . . . . . . . . . . . . . . . . . . . . . . 97

3.17 Summary of decayed radioactivity (Ci) isotopic content of retrievably stored $\mathrm{CH}$ TRUW $\ldots \ldots \ldots 99$

3.18 Summary of decayed mass $(\mathrm{g})$ isotopic content of retrievably stored $\mathrm{CH}$ TRUW $\ldots \ldots \ldots \ldots 101$

3.19 Summary of decayed radioactivity (Ci) isotopic content of retrievably stored RH TRUW $\ldots \ldots \ldots 103$

3.20 Summary of decayed mass $(\mathrm{g})$ isotopic content of retrievably stored RH TRUW $\ldots \ldots \ldots \ldots 105$

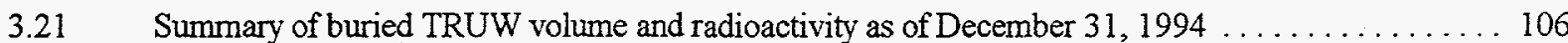

\section{LOW-LEVEL WASTE}

4.1 Summary of characteristics for disposed LLW as of December $31,1995 \ldots \ldots \ldots \ldots \ldots \ldots 117$

4.2 Historical and projected volume, radioactivity, and thermal power of disposed DOE LLW $\ldots \ldots \ldots 118$

4.3 Historical and projected volume, radioactivity, and thermal power of commercial LLW

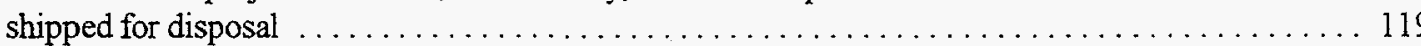

4.4 Historical annual additions and total volume of $L L W$ disposed at DOE sites $\ldots \ldots \ldots \ldots \ldots \ldots$

4.5 Description of physical forms used to characterize solid LLW from DOE activities $\ldots \ldots \ldots \ldots .121$

4.6 Actual 1995 generation and projected average annual generation of $L L W$ at DOE sites ........ 123

4.7 Actual 1995 cumulative storage inventory and projected average annual storage additions

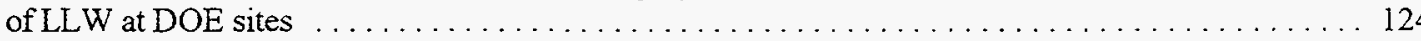


4.9 Breakdown by physical form of volumes of $L L W$ generated during 1995 at DOE sites $\ldots \ldots \ldots 126$

4.10 Breakdown by physical form of radioactivity of LLW generated during 1995 at DOE sites . . . . 127

4.11 Breakdown by physical form of cumulative volumes of $L L W$ stored at DOE sites $\ldots \ldots \ldots \ldots 128$

4.12 Breakdown by physical form of total gross radioactivity of $L L W$ stored at DOE sites $\ldots \ldots \ldots \ldots 129$

4.13 Breakdown by physical form of volumes of LLW disposed during 1995 at $D O E$ sites $\ldots \ldots \ldots 130$

4.14 Breakdown by physical form of radioactivity of LLW disposed during 1995 at DOE sites . ..... 131

4.15 DOE LLW disposed by methods other than shallow-land burial $\ldots \ldots \ldots \ldots \ldots \ldots \ldots \ldots 132$

4.16 Significant revisions and changes in the current values for LLW compared to the values in

the previous year.

4.17 Historical and projected volume, radioactivity, and thermal power characteristics of disposed DOE LLW, except SRS saltstone

4.18 Projected volume, radioactivity, and thermal power characteristics of DOE LLW saltstone

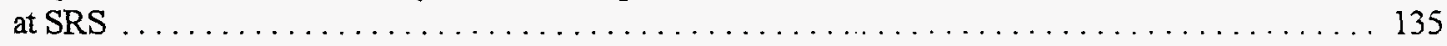

4.19 Historical annual additions and total volume of $L L W$ at commercial disposal sites $\ldots \ldots \ldots \ldots 136$

4.20 Historical annual additions and total undecayed radioactivity of LLW at commercial disposal sites

4.21 Distribution of total volume and radioactivity, by state, of LLW shipped to commercial disposal sites in 1995

4.22 Historical and projected cumulative volume and radioactivity summary of commercial GTCCLLW

4.23 Breakdown of 1995 low-level radioactive waste by type, volume, and radioactivity received by commercial disposal sites

\section{URANIUM MILL TAILINGS}

5.1 Uranium ore processed, $\mathrm{U}_{3} \mathrm{O}_{8}$ recovery rate, and tailings generated through 1995

5.2 Status of conventional uranium mill sites at the end of 1995

5.3 Typical characteristics of uranium mill tailings

\section{ENVIRONMENTAL RESTORATION PROGRAM}

6.1 List of sites in the DOE Environmental Restoration Program

6.2 Projected disposition of radioactively contaminated solid materials classified as LLW

xviii 
6.3 Projected disposition of radioactively contaminated solid materials classified as MLLW

6.4 Projected disposition of radioactively contaminated solid materials classified as TRUW 164

6.5 Projected disposition of radioactively contaminated solid materials classified as 1 le(2) by-product

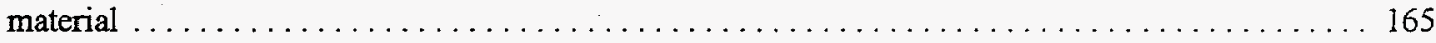

$6.6 \quad$ TSCA mixed waste associated with EM-40 activities $\ldots \ldots \ldots \ldots \ldots \ldots \ldots \ldots \ldots \ldots \ldots \ldots \ldots \ldots \ldots$

$6.7 \quad$ Volumes $\left(\mathrm{m}^{3}\right)$ of solid radioactive wastes in storage at EM-40 facilities $\ldots \ldots \ldots \ldots \ldots \ldots$

\section{NATURALLY OCCURRING AND ACCELERATOR-PRODUCED RADIOACTIVE MATERIAL}

7.1 Radionuclides found in accelerator-produced radioactive materials

7.2 Domestic processes that generate NORM waste

7.3 Major radionuclides found in diffuse NORM wastes

7.4 Radionuclide concentrations in coal combustion wastes

7.5 Radionuclide concentrations in geothermal energy production waste

7.6 Radionuclide concentrations in metal mining and processing wastes

7.7 Radionuclide concentrations in municipal water treatment wastes

7.8 Radionuclide concentrations in oil and natural gas production and processing wastes

7.9 Radionuclide concentrations in phosphate wastes

7.10 Quantities of radium disposed of by New York State generators during 1987-91

7.11 Estimates of domestic diffuse NORM waste generation, material density, cumulative inventory, and radioactive concentration

\section{MIXED LOW-LEVEL WASTE}

8.1 Thirty-nine states and territories with EPA mixed waste authorization as of the end of 1995

8.2 Summary of estimated total MLLW inventories and annual generation as of 1995

8.4 Treatability group matrix parameter categories used to characterize DOE RCRA MLLW

8.5 Estimated volume $\left(\mathrm{m}^{3}\right)$ inventories and five-year projections of DOE RCRA MLLW as of 1995, by site and physical form

8.6 Estimated volume $\left(\mathrm{m}^{3}\right)$ inventories and five-year projections of DOE RCRA MLLW as of 1995 , by physical form 
8.7 Significant revisions and changes in historical volume $\left(\mathrm{m}^{3}\right)$ inventory data for DOE RCRA $M L L W$ compared to volume $\left(\mathrm{m}^{3}\right)$ inventory data reported previously .

8.8 Mass $(\mathrm{kg})$ inventories through 1995, by hazard category, of TSCA-regulated MLLW from

DOE site activities

8.9 Volume $\left(\mathrm{m}^{3}\right)$ inventories through 1995 , by hazard category, of TSCA-regulated MLLW

from DOE site activities.

8.10 1995 mass $(\mathrm{kg})$ generation, by hazard category, of TSCA-regulated MLLW from DOE site activities

8.11 1995 volume $\left(\mathrm{m}^{3}\right)$ generation, by hazard category, of TSCA-regulated MLLW from DOE

site activities

8.12 Historical and projected annual mass $(\mathrm{kg})$ generation rates for TSCA-regulated MLLW

from DOE site activities

8.13 Historical and projected annual volume $\left(\mathrm{m}^{3}\right)$ generation rates for TSCA-regulated MLLW

from DOE site activities

8.14 Significant revisions and changes in historical inventory data for DOE TSCA MLLW compared

to inventory data reported previously

8.15 National commercially generated MLLW profile volume summary, by facility category

8.16 National commercially generated MLLW profile volume summary, by hazardous waste stream

\section{APPENDIX A. WASTE FLOWSHEETS, SOURCE TERMS, AND CHARACTERISTICS}

A.1 Estimated representative unit activity and thermal power characteristics of various types of radioactive materials and wastes

A.2 Representative DOE LLW radionuclide composition by percent activity

A.3 Average concentrations for representative radionuclides in $L L W$ at commercial disposal sites

A.4 Estimated sources and characteristics of commercial Greater-Than-Class-C LLW

A.5 Historical number and volume of drums and classes of LLW incorporated in cement generated in the WVDP Low-Level Radwaste Treatment System

\section{APPENDIX B. CHARACTERISTICS OF IMPORTANT RADIONUCLIDES}

B.1 Characteristics of important radionuclides 


\section{APPENDIX C. REFERENCE SITES AND FACILITIES}

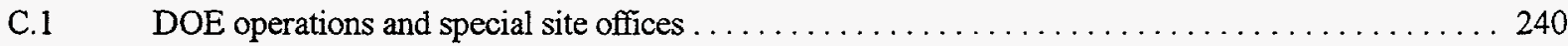

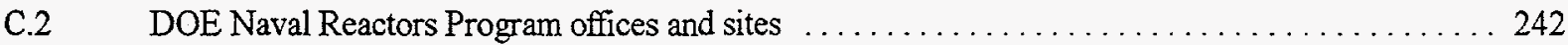

C.3 Major DOE sites and facilities referred to in this report $\ldots \ldots \ldots \ldots \ldots \ldots \ldots \ldots \ldots \ldots \ldots \ldots$

C.4 Major commercial radioactive waste disposal sites included in this report $\ldots \ldots \ldots \ldots \ldots \ldots 250$ 



\section{GLOSSARY OF ABBREVIATIONS, ACRONYMS, AND INITIALISMS}

In this report, all abbreviations, acronyms, and initialisms are defined in text before they are used. In some cases, they are redefined in text for the reader's convenience.

$\begin{array}{ll}\text { ACRR } & \text { Annular Core Research Reactor (at SNL/NM) } \\ \text { AEA } & \text { Atomic Energy Act of 1954 } \\ \text { AEC } & \text { Atomic Energy Commission } \\ \text { Ames } & \text { Ames Laboratory, Ames, Iowa } \\ \text { ANC } & \text { American Nuclear Corporation } \\ \text { ANL-E } & \text { Argonne National Laboratory-East, Argonne, Illinois } \\ \text { ANL-W } & \text { Argonne National Laboratory-West, INEL, Idaho } \\ \text { ARCO } & \text { Atlantic Richfield (Medical Products) Company, Reading, Pennsylvania } \\ \text { ARRR } & \text { Aerotest Radiography and Research Reactor, San Ramon, California } \\ \text { ASI } & \text { Advanced Sciences, Inc. } \\ \text { ATL } & \text { Advanced Technologies and Laboratories International, Inc. } \\ \text { ATL 1 } & \text { Atlantic Site 1 (38'30'N, 72'06'W) } \\ \text { ATL 2 } & \text { Atlantic Site 2 (37'50'N, 70'35'W) } \\ \text { ATR } & \text { Advanced Test Reactor (at INEL ICPP) } \\ & \\ \text { BAPL } & \text { Bettis Atomic Power Laboratory, West Mifflin, Pennsylvania } \\ \text { BARN } & \text { Barnwell, South Carolina (commercial waste site) } \\ \text { BCL } & \text { Battelle Columbus Laboratories, Columbus, Ohio } \\ \text { BCLDP } & \text { Battelle Columbus Laboratories Decommissioning Project, Columbus, Ohio } \\ \text { BDM } & \text { BDM Federal, Inc., Germantown, Maryland } \\ \text { BEMR } & \text { Baseline Environmental Management Report } \\ \text { BETY } & \text { Beatty, Nevada (commercial waste site) } \\ \text { BMI } & \text { Battelle Memorial Institute, Columbus, Ohio } \\ \text { BMRR } & \text { Brookhaven Medical Research Reactor (at BNL) } \\ \text { BNI } & \text { Bechtel National, Inc., Oak Ridge, Tennessee } \\ \text { BNL } & \text { Brookhaven National Laboratory, Upton, New York } \\ \text { BR } & \text { Belgium reactor } \\ \text { BSR } & \text { Bulk Shielding Reactor (at ORNL) } \\ \text { B\&W/LTC } & \text { Babcock and Wilcox/Lynchburg Technology Center, Lynchburg, Virginia } \\ \text { BWR } & \text { Boiling-water reactor } \\ & \\ \text { CADMUS } & \text { The Cadmus Group, Inc. } \\ \text { CANDU } & \text { Canadian Deuterium Reactor } \\ \text { CCMV } & \text { Converse County Mining Venture, Converse County, Wyoming } \\ \text { CE } & \text { Combustion Engineering Site (FUSRAP site), Windsor, Connecticut } \\ \text { CERCLA } & \text { Comprehensive Environmental Response, Compensation, and Liability Act of 1980 } \\ \text { CEU } & \text { Consolidated Edison Uranium (at ORNL) } \\ \text { CFC } & \text { Chlorinated fluorocarbon } \\ \text { CH } & \text { Contact-handled (transuranic waste) } \\ & \end{array}$


CISS

CRWMS-M\&O

CY

DAW

DOD

DOE

DOE/AL

DOE/CAO

DOE/CH

DOE/DP

DOENEIA

DOE/EM

DOE/FN

DOE/HQ

DOE/ID

DOENNV

DOE/OAK

$\mathrm{DOE} / \mathrm{OH}$

DOE/OR

DOE/OSTI

DOE/RF

DOE/RL

DOERW

DOE/SR

DOE/WIPP

DOE/WVAO

DOT

DRCT

DWCS

DWMP

DWPF

$D \& D$

EA

EBR

EC

ECF

EG\&GID

EIA

EIS

EMAD

ENVR

EOY

EPA

ERR

ETEC

FEMP

FFCA

FFTF
Colonie Interim Storage Site, Colonie, New York

Civilian Radioactive Waste Management System-Management and Operating (contractor)

Calendar year

Dry active waste

Department of Defense, U.S.

Department of Energy, U.S.

DOE Albuquerque Operations Office, Albuquerque, New Mexico

DOE Carlsbad Area Office, Carlsbad, New Mexico

DOE Chicago Operations Office, Argonne, Illinois

DOE Office of Defense Programs (Headquarters), Germantown, Maryland

DOE Energy Information Administration, Washington, D.C.

DOE Office of Environmental Management (Headquarters), Germantown, Maryland

DOE Fernald Area Office, Cincinnati, Ohio

DOE Headquarters, Washington, D.C. and Germantown, Maryland

DOE Idaho Operations Office, Idaho Falls, Idaho

DOE Nevada Operations Office, Las Vegas, Nevada

DOE Oakland Operations Office, Oakland, California

DOE Ohio Field Office, Miamisburg, Ohio

DOE Oak Ridge Operations Office, Oak Ridge, Tennessee

DOE Office of Scientific and Technical Information, Oak Ridge, Tennessee

DOE Rocky Flats Office, Golden, Colorado

DOE Richland Operations Office, Richland, Washington

DOE Office of Civilian Radioactive Waste Management (Headquarters), Washington, D.C.

DOE Savannah River Operations Office, Aiken, South Carolina

DOE WIPP Project Office, Carlsbad, New Mexico

DOE West Valley Area Office, West Valley, New York

Department of Transportation, U.S.

Dry rod consolidation technology

Defueling water cleanup system

Defense Waste Management Plan

Defense Waste Processing Facility

Decontamination and decommissioning

Environmental assessment

Experimental Breeder Reactor (at ANL-E)

Electron capture

Extended Core Facility (at INEL NRF)

EG\&G Idaho, Inc., Idaho Falls, Idaho (former management and operating contractor for Idaho

National Engineering Laboratory)

Energy Information Administration (DOE)

Environmental impact statement

Engine maintenance assembly and disassembly (at NTS)

Envirocare facility, Clive, Utah

End of (calendar) year

Environmental Protection Agency, U.S.

Elk River Reactor, Elk River, Minnesota

Energy Technology Engineering Center, Canoga Park, California [also referred to as the Santa

Susana Field Laboratory (SSFL)]

Fernald Environmental Management Project, Fernald, Ohio

Federal Facility Compliance Act of 1992

Fast Flux Test Facility, Hanford, Washington 
FIS

FNAL

FRR

FSVR

FUSRAP

FY

GA

GAP CON

GDP

GE

GETR

GJPO

GJRAP

GPU

GTCC

Hanford

HAZWRAP

HCF

HEDL

HEN

HFBR

HFEF

$\mathrm{HIC}$

HLW

HTGR

HWVP

ICPP

IDB

IE

IFSF

II

INEL

ISA

ISFSI

ISL

IT

ITRI

JAI

JO

K-25

KAPL

KCP

KESS

LANL

LBL

LDR
Farallon Islands (Pacific Ocean off Central California)

Fermi National Accelerator Laboratory, Batavia, Illinois

Foreign Research Reactor

Fort St. Vrain Reactor, Platteville, Colorado

Formerly Utilized Sites Remedial Action Program

Fiscal year

General Atomics, San Diego, California

Gap conductance

Gaseous diffusion plant

General Electric

General Electric Test Reactor, Pleasanton, Califormia

(DOE) Grand Junction Projects Office, Grand Junction, Colorado

Grand Junction Remedial Action Project

General Public Utilities Corporation, Parsippany, New Jersey

Greater-than-Class-C (low-level waste)

Hanford Site, Richland, Washington

Hazardous Waste Remedial Actions Program

Hot Cell Facility (at SNL/NM)

Hanford Engineering Development Laboratory (at Hanford)

Cape Henry (Atlantic Ocean off Virginia)

High-Flux Beam Reactor (at BNL)

Hot Fuel Examination Facility (at ANL-W)

High-integrity container

High-level waste

High-temperature, gas-cooled reactor

Hanford Waste Vitrification Plant (at Hanford)

Idaho Chemical Processing Plant, Idaho National Engineering Laboratory

Integrated Data Base (Program)

Irradiation effects

Irradiated Fuel Storage Facility (at INEL)

Industrial and institutional (waste)

Idaho National Engineering Laboratory, Idaho Falls, Idaho

Interim Storage Area (at Hanford)

Independent Spent Fuel Storage Installation

In situ leaching

Isomeric transition

Inhalation Toxicology Research Institute, Kirtland Air Force Base, Albuquerque, New Mexico

(E.R.) Johnson Associates, Inc., Oakton, Virginia

Joint Integration Office, Albuquerque, New Mexico

Oak Ridge K-25 Site, Oak Ridge, Tennessee (formerly called the Oak Ridge Gaseous Diffusion Plant)

Knolls Atomic Power Laboratory, Schenectady, New York

Kansas City Plant, Kansas City, Missouri

Kesselring Site (at KAPL)

Los Alamos National Laboratory, Los Alamos, New Mexico

Lawrence Berkeley Laboratory, Berkeley, Califomia

Land disposal restriction 


\begin{tabular}{|c|c|}
\hline LEHR & Laboratory for Energy-Related Health Research, Davis, California \\
\hline LEU & Low-enriched uranium \\
\hline LFRSB & Loose fuel-rod shipping basket \\
\hline LGR & Light-water cooled, graphite-moderated reactor \\
\hline LLNL. & Lawrence Livermore National Laboratory, Livermore, California \\
\hline LLR & LOFT lead rod \\
\hline LLRWPA & Low-Level Radioactive Waste Policy Act of 1980 \\
\hline LLRWPAA & Low-Level Radioactive Waste Policy Amendments Act of 1985 \\
\hline LLW & Low-level waste \\
\hline LLWMP & Low-Level Waste Management Program \\
\hline LMER & Lockheed Martin Energy Research (Corporation), Oak Ridge, Tennessee \\
\hline LMES & Lockheed Martin Energy Systems, Inc., Oak Ridge, Tennessee \\
\hline LMFBR & Liquid Metal Fast Breeder Reactor \\
\hline LMIT & Lockheed Martin Idaho Technologies, Idaho Falls, Idaho \\
\hline LOC & Loss of coolant \\
\hline LOFT & Loss-of-fluid test \\
\hline LSA & Low specific activity \\
\hline LWBR & Light-water breeder reactor \\
\hline LWR & Light-water reactor \\
\hline MACTEC & MAC Technical Services Company \\
\hline MAPI & Mitsubishi Atomic Power Industries \\
\hline MASS & Massachusetts Bay \\
\hline MED & Manhattan Engineer District (Manhattan Project) \\
\hline MFKY & Maxey Flats, Kentucky (commercial waste site) \\
\hline MFRP & Midwest Fuel Recovery Plant, Morris, Illinois (commercial spent fuel storage site) \\
\hline MMMS & Manifest Information Management System \\
\hline MLLW & Mixed low-level waste \\
\hline Mound & Mound Plant, Miamisburg, Ohio \\
\hline MRM & Miscellaneous radioactive material \\
\hline MSR & Molten Salt Reactor (at ORNL) \\
\hline MSRE & Molten Salt Reactor Experiment \\
\hline MTIHM & Metric tons initial heavy metal \\
\hline MTR & Materials Test Reactor (at INEL) \\
\hline MTRUW & Mixed transuranic waste \\
\hline MTU & Metric tons uranium \\
\hline MURR & Missouri (University of) Research Reactor, Columbia, Missouri \\
\hline MWIR & Mixed Waste Inventory Report \\
\hline NA & Not applicable \\
\hline NARM & Naturally occurring and accelerator-produced radioactive material \\
\hline NBSR & National Bureau of Standards Reactor, Gaithersburg, Maryland \\
\hline NEPA & National Environmental Policy Act of 1969 \\
\hline NFS & Nuclear Fuel Services, Erwin, Tennessee \\
\hline NORM & Naturally occurring radioactive material \\
\hline NPL & National priorities list \\
\hline NR & Naval reactors \\
\hline NRC & Nuclear Regulatory Commission \\
\hline NRF & Naval Reactors Facility (at INEL) \\
\hline NTIS & National Technical Information Service, Springfield, Virginia \\
\hline NTPO & National Transuranic Waste Program Office \\
\hline NTS & Nevada Test Site, Mercury, Nevada \\
\hline NUMEC & Nuclear Uranium Materials and Equipment Corporation \\
\hline
\end{tabular}




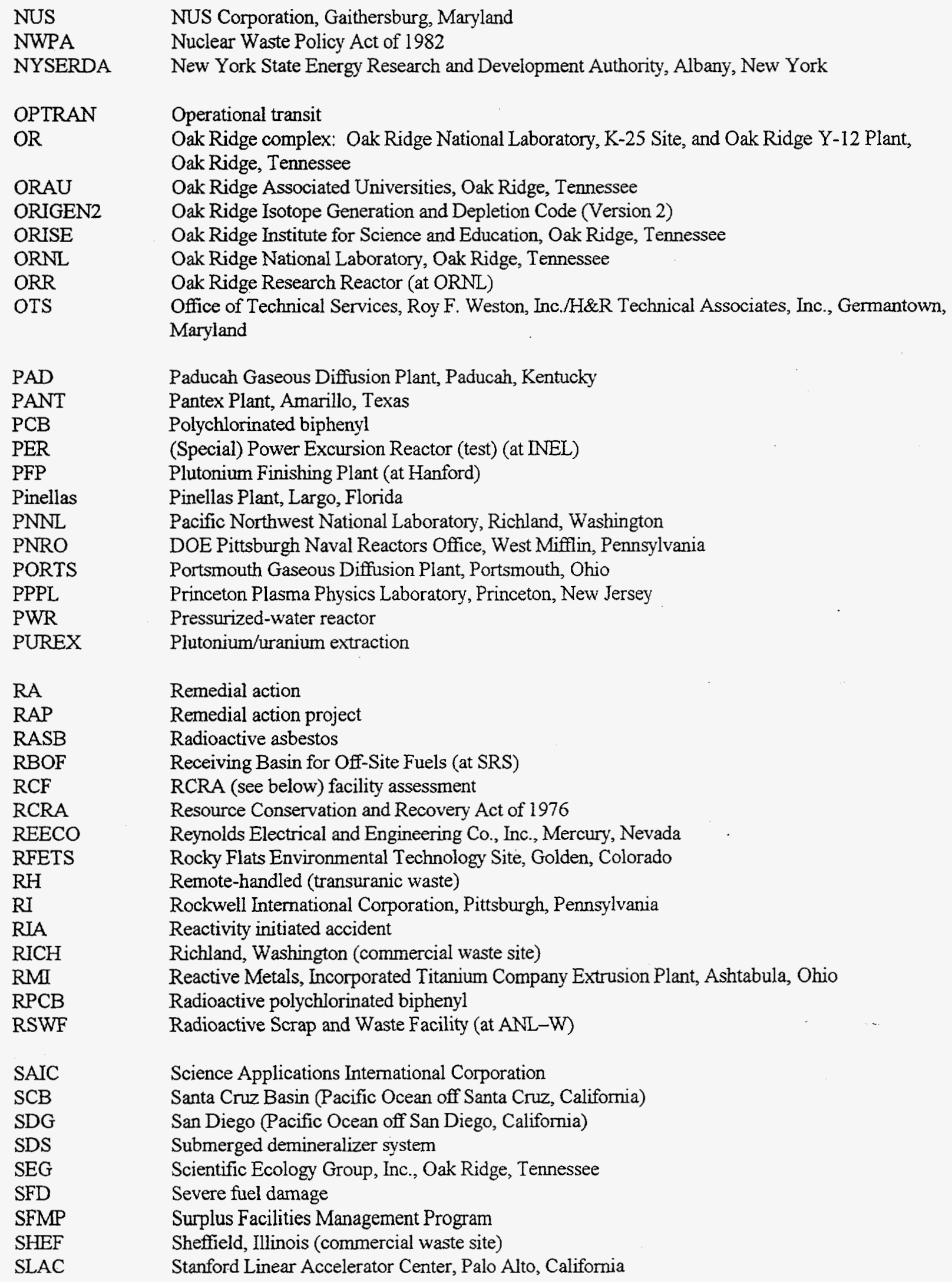




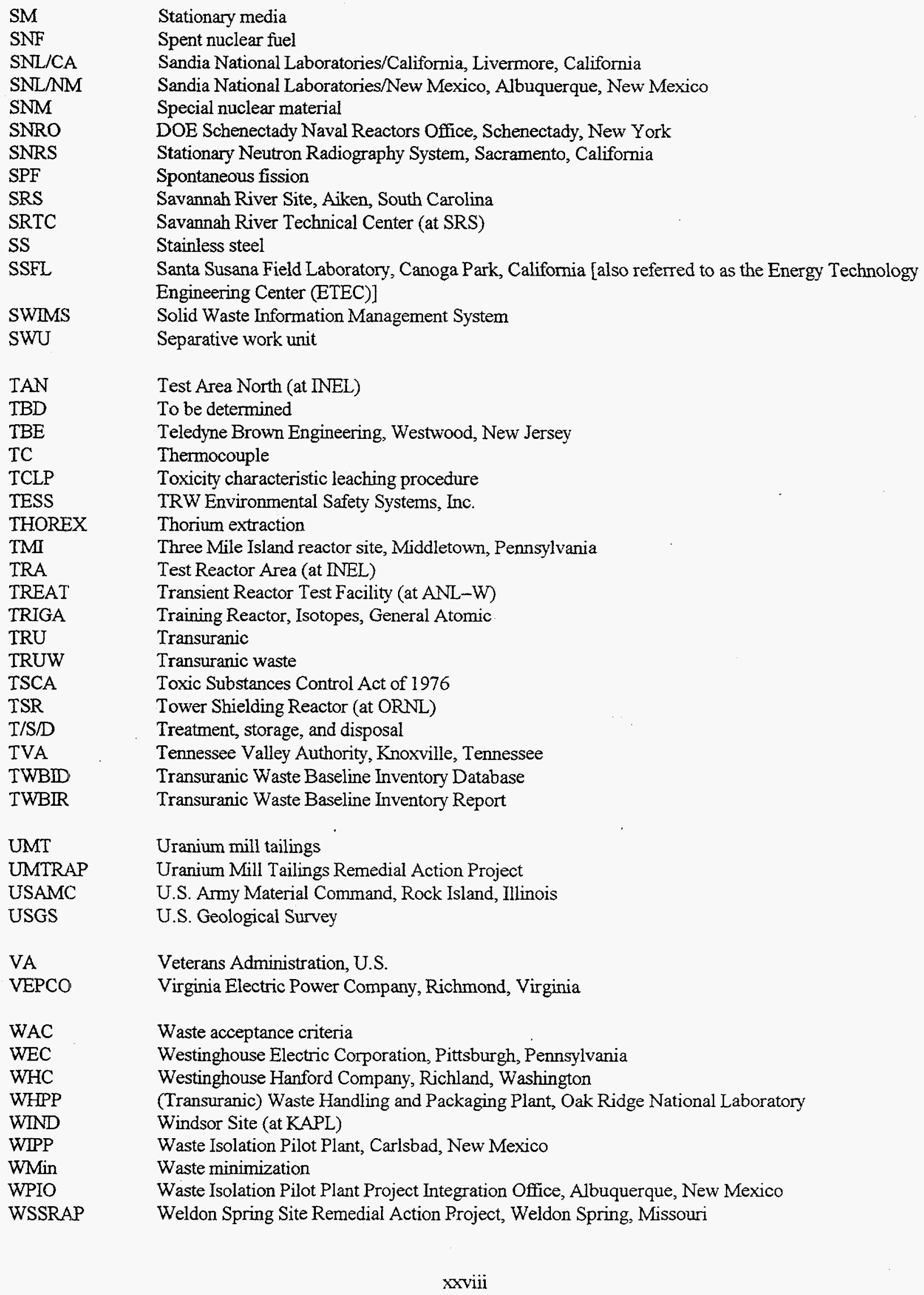


WTAC

WTWBIR

WVDP

WVNS

WVNY

W/WIPP

Y-12

$11 \mathrm{e}(2)$

M1 le(2)
Waste Isolation Pilot Plant Technical Assistance Contractor

Waste Isolation Pilot Plant Transuranic Waste Baseline Inventory Report

West Valley Demonstration Project, West Valley, New York

West Valley Nuclear Services Company, Inc., West Valley, New York

West Valley, New York (commercial waste site from 1963-1981)

Westinghouse/WIPP Project, Carlsbad, New Mexico

Oak Ridge Y-12 Plant, Oak Ridge, Tennessee

By-product material as defined in Sect. 11 e(2) of the Atomic Energy Act of 1954

Mixed 11 e(2) by-product material 



\title{
INTEGRATED DATA BASE REPORT-1995: U.S. SPENT NUCLEAR FUEL AND RADIOACTIVE WASTE INVENTORIES, PROJECTIONS, AND CHARACTERISTICS
}

\begin{abstract}
The Integrated Data Base Program has compiled historic data on inventories and characteristics of both commercial and U.S. Department of Energy (DOE) spent nuclear fuel and commercial and U.S. government-owned radioactive wastes. Except for transuranic wastes, inventories of these materials are reported as of December 31, 1995. Transuranic waste inventories are reported as of December 31,1994. All spent nuclear fuel and radioactive waste data reported are based on the most reliable information available from government sources, the open literature, technical reports, and direct contacts. The information forecasted is consistent with the latest DOE/Energy Information Administration (EIA) projections of U.S. commercial nuclear power growth and the expected DOE-related and private industrial and institutional activities.

The radioactive materials considered, on a chapter-by-chapter basis, are spent nuclear fuel, high-level waste, transuranic waste, low-level waste, uranium mill tailings, DOE Environmental Restoration Program contaminated environmental media, naturally occurring and accelerator-produced radioactive material, and mixed (hazardous and radioactive) low-level waste. For most of these categories, current and projected inventories are given through the calendaryear 2030, and the radioactivity and thermal power are calculated based on reported or estimated isotopic compositions.
\end{abstract}




\section{OVERVIEW}

\section{O.1 INTRODUCTION}

This report is an update of the previous document ${ }^{1}$ on spent nuclear fuel (SNF) and radioactive waste inventories and projections that was prepared for use in the planning and analysis of various SNF and waste management functions. Quantities of SNF and radioactive wastes produced from both commercial and U.S. Department of Energy (DOE)-sponsored activities are reported. Except for transuranic wastes (TRUW), whose inventories are reported as of December 31, 1994, historical SNF and radioactive waste inventories are reported as of December 31, 1995. Projections of future SNF and radioactive wastes are generally reported for the calendaryear (CY) period 1996-2030. Such projections may change in future revisions of this report as waste minimization (WMin), environmental restoration, and decontamination and decommissioning (D\&D) programs and activities at various government and commercial sites are defined and become operative. The tables of this document report historical inventories and projection data on a CY basis. These tables use a horizontal line to mark the point in time when past history ends and future projections begin. Because historical SNF and radioactive waste inventories are generally reported as of December 31, 1995, the line is drawn between the data entries for 1995 and 1996. Data reported for 1996 in this document are regarded as projected information.

This document contains information that has been assembled as a part of the Integrated Data Base (IDB) Program at Oak Ridge National Laboratory (ORNL), which has the lead responsibility for maintaining and reporting summary files of pertinent data on current and projected inventories and characteristics of permanently discharged domestic SNF and radioactive wastes. While this report documents inventories and projections of radioactive wastes from commercial and DOE site activities, estimates for certain other waste categories are not fully reported because of the current unavailability of data. These categories include naturally occurring and accelerator-produced radioactive material (NARM), discussed in Sect. 0.2, and wastes from U.S. Department of Defense (DOD) activities, particularly those resulting from the cleanup of military sites contaminated with significant amounts of radioactive waste. A study ${ }^{2}$ by the U.S. General Accounting Office indicates that as many as 420 DOD locations could be contaminated with radioactive wastes. Future updates of this report will work toward reporting information and data on NARM and DOD site radioactive wastes as they become available.

This report also does not report inventories of nonradioactive materials. These include hazardous wastes, sanitary wastes, and materials not categorized as waste (MNCAW), such as uncontaminated scrap metal. Preliminary estimates of inventories of MNCAW are reported in ref. 3.

Except for some NARM, most radioactive waste originates from five major sources: (1) the commercial nuclear fuel cycle; (2) DOE-related activities; (3) institutions such as hospitals, universities, and research foundations; (4) industrial uses of radioisotopes; and (5) mining and milling of uranium ore. The waste is broadly categorized as SNF, high-level waste (HLW), TRUW, low-level waste (LLW), and uranium mill tailings (UMT). Large quantities of radioactive waste also result from DOE site environmental restoration activities and the $D \& D$ programs of $D O E$ and commercial nuclear facilities. This report also documents inventories and projections of mixed low-level waste (MLLW), which is both hazardous and radioactive.

The primary purpose of this document is to report U.S. SNF and radioactive waste inventories, projections, and characteristics. The data presented were obtained through the cooperation and assistance of the offices and programs that were established by DOE to oversee the management of the various radioactive wastes and SNFs. In addition, the recent literature was reviewed to aid in selecting the data that are presented herein and to help establish a basis for many of the calculated radioactivity levels and heatgeneration rates that are included. In this report, SNF and radioactive wastes are characterized from the standpoint of their volumes (or masses) and their nuclear, physical, and 
chemical properties. The data reported are selected from more extensive information; that information is available upon request.

This annual inventory report contains summarized data of types found to be useful for programmatic planning purposes within the DOE community. The data are intended to provide a common basis for both $\mathrm{DOE}$ management-level planning and for more detailed analyses of the waste management system that are conducted by $\mathrm{DOE}$ contractors and field offices. However, this report is not intended to present the detailed types of information required as input to such analyses. The best sources of such information are the appropriate DOE operations offices, waste sites, or relevant documents previously issued, some of which may be referenced in this report.

This report does not address the programmatic implications of the data presented, such as the possible future need for interim SNF storage facilities. Discussion of the data is minimized to explain mainly what the data represent. Major $D O E$ data sources providing information and data for this report are identified in a table following the preface. Likewise, discussions of packaging details, shielding and transportation requirements, health and environmental effects, and costs are purposely avoided. Questions regarding the information and data presented may be addressed to the $\mathrm{DB}$ Program.

The DOE waste information and data contained in this report are furnished by the DOE contractor sites listed in Table 0.1. This table indicates also the types of radioactive waste managed at each site. The DOE site data (waste inventories, projections, and characteristics) are used by DOE-Headquarters (DOE-HQ), operations offices, and operating contractors for the management and strategic planning of various waste programs. The objective of this report is to provide waste information that is consistent, reflects current inventories and projections, and includes the types of basic data best suited to meet DOE waste program planning needs.

Information for this report is provided by a variety of sources. The DOE site waste data reported were received from $D O E$ contractors through $\mathrm{DOE}$ operations offices. DOE-HQ assigns to selected organizations major responsibilities for providing information on particular topics involving $\mathrm{SNF}$ and radioactive waste management. Further detailed information is generally available from data bases maintained at the specific DOE and commercial sites. Additional information on the reference sites and facilities referred to in this report is provided in Appendix C.

\subsection{CHARACTERIZATION OF WASTE FORMS}

The major characteristics of radioactive materials and wastes are described in the following:

\section{- Spent Nuclear Fuel (SNF)}

SNF consists of irradiated fuel discharged from a nuclear reactor. Unless otherwise identified, all SNFs discussed in this report are assumed to be permanently discharged and eligible for repository disposal. Three categories of permanently discharged SNF are considered: (1) fuel from commercial light-water reactors (LWRs); (2) fuel from non-LWR commercial reactors [e.g., the Fort St. Vrain high-temperature, gas-cooled reactor (HTGR)]; and (3) special fuels associated with government-sponsored research and demonstration programs, universities, and private industries. This report does not track the inventories of government production reactor SNFs that have been reprocessed in the manufacture of nuclear weapons for national defense. However, the inventories of $\mathrm{HLW}$ resulting from the reprocessing of these fuels are reported in Chapter 2. Also, Chapter 1 reports quantities of DOE SNF.

Currently, most LWR SNF assemblies are stored in pools at the reactor sites. The bulk of the remainder is in storage at the West Valley Demonstration Project (WVDP) site at West Valley, New York; the Idaho National Engineering Laboratory (INEL) at Idaho Falls, Idaho; and the Midwest Fuel Recovery Plant (MFRP) at Morris, Illinois. The WVDP facility is currently being decommissioned. All utility-owned SNF assemblies previously stored there have been returned to the utilities, and the fuel remaining is DOE-owned material.

SNFs discharged from a variety of reactors are currently stored at the Hanford Site (Hanford) and INEL. Hanford contains inventories of fuel from the $\mathrm{N}$ Reactor, the Fast Flux Test Facility (FFTF), and pressurized-water reactor (PWR)-Core II fuel from Shippingport. Fuel from the damaged Three Mile Island (TMI)-Unit 2 reactor, as well as some of the SNF from the Fort St. Vrain HTGR, are stored at INEL. Some special SNFs are stored at the Savannah River Site (SRS) and at INEL. These special fuels are government owned and are not scheduled for reprocessing in support of DOE activities. 


\section{- High-Level Waste (HLW)}

For this report, HLW means the highly radioactive material resulting from the reprocessing of SNF. This material includes mainly the liquid wastes remaining from the recovery of uranium and plutonium in a fuel reprocessing plant. This HLW may also be in the form of sludge, calcine, or other products into which such liquid wastes are converted to facilitate their handling and storage. Such waste contains fission products that result in the release of considerable decay energy. ${ }^{4}$ For this reason, heavy shielding is required to absorb penetrating radiation, and provisions (e.g., cooling systems) are needed to dissipate decay heat from $\mathrm{HLW}$.

\section{- Transuranic Waste (TRUW)}

TRUW refers to radioactive waste that contains more than $100 \mathrm{nCi} / \mathrm{g}$ of alpha-emitting isotopes with atomic numbers greater than 92 and half-lives greater than 20 years. $^{5,6}$ Such waste results primarily from fuel reprocessing and from the fabrication of plutonium weapons and plutonium-bearing reactor fuel. Generally, little or no shielding is required ["contact-handled" (CH) TRUW], but energetic gamma and neutron emissions from certain transuranic (TRU) nuclides and fission-product contaminants may require shielding or remote handling ["remote-handled" (RH) TRUW].

\section{- Low-Level Waste (LLW)}

Several statutes (refs. 4,6, and 7) define LLW not by what it is, but by what it is not. In general, LLW is radioactive waste not classified as SNF, HLW, TRUW, nor by-product materials such as UMT or thorium mill tailings. However, there are slight differences between the specific regulatory definitions of DOE-generated LLW and commercial LLW.

The definition of DOE LLW is based on DOE Order 5820.2A, ${ }^{6}$ which specifies DOE's policy for radioactive waste management. According to this order, LLW includes all radioactive waste not classified as either HLW, TRUW, SNF, or the bulk of the by-product tailings containing uranium or thorium and their decay products from processed ore. The DOE policy, as stated in Order 5820.2A, allows small volumes of fissionable material to be irradiated for research and development (R\&D) only-but not for the production of power or plutonium-and small concentrations of TRU $(<100 \mathrm{nCi} / \mathrm{g})$ radionuclides to be managed as LLW. The same DOE policy allows small volumes of $\mathrm{DOE}$ waste containing by-product material [specified in Sect. 11e(2) of the Atomic Energy Act of 1954 (AEA)] $]^{8}$ or NARM to be managed as LLW. Any LLW that also contains hazardous chemicals covered by either the Resource Conservation Recovery Act (RCRA) ${ }^{9}$ or the Toxic Substances Control Act $(\mathrm{TSCA})^{10}$ requires management as a "mixed waste."

The definition of commercial LLW is based on two statutes, the Nuclear Waste Policy Act (NWPA) ${ }^{4}$ and the Low-Level Radioactive Waste Policy Amendments Act (LLRWPAA). ${ }^{7}$ According to both the NWPA and the LLRWPAA, commercial LLW is radioactive material that (a) is not HLW, SNF, TRUW, or byproduct material as defined in Sect. 1 le(2) of the $\mathrm{AEA}$; and (b) consistent with existing law, is classified by the U.S. Nuclear Regulatory Commission (NRC) as LLW.

The radiation level from LLW waste may sometimes be high enough to require shielding for handling and transport. For commercial LLWs, the NRC has defined, in ref. 11, four disposal categories of LLW that require differing degrees of confinement and/or monitoring: classes A, B, C, and Greater-ThanClass-C (GTCC). The NRC excludes NARM from the LLW category. DOE LLWs are classified by groupings of disposal categories that are site specific, yet similar to the NRC categories. This report documents inventories of solid LLW either destined for disposal or awaiting treatment prior to disposal. It includes no liquid or gas waste in storage.

\section{- Uranium Mill Tailings (UMT)}

UMT are the earthen residues that remain after the extraction of uranium from ores. Tailings are generated in very large volumes and contain low concentrations of naturally occurring radioactive materials. These materials comprise a potential health hazard; the isotopes of major concern are ${ }^{226} \mathrm{Ra}$ and its daughter, ${ }^{222} \mathrm{Rn}$.

- Naturally Occurring and Accelerator-Produced Radioactive Material (NARM)

NARM wastes include both accelerator wastes (LLW) and naturally occurring radioactive material (NORM) that contain radionuclides (e.g., ${ }^{226} \mathrm{Ra},{ }^{222} \mathrm{Rn},{ }^{232} \mathrm{Th}$, ${ }^{238} \mathrm{U}$ ) existing throughout the earth's crust. Accelerator wastes include accelerator targets, wastes from accelerator maintenance or $D \& D$, and wastes from radiopharmaceutical manufacture. NORM 
wastes are classified according to their specific activity as either discrete or diffuse. Discrete NORM wastes have a relatively small volume but large radioactivity and include industrial gauges, old radium watch and industrial dials, radium needles in medical equipment, and resins (filters) that remove radioactive radium and some radiopharmaceutical waste from contaminated public drinking water. Diffuse NORM wastes are characterized by a relatively large volume with small radioactivity. These materials result from industrial processes and include:

- coal ash and slag from utility electrical generation;

- solid wastes from geothermal energy production;

- slag, leachate, and tailings from the mining and processing of metals other than uranium or thorium (e.g., copper);

- sludge from drinking water treatment;

- scale, shudge, produced water, and equipment from oil and natural-gas production containing NORM; and

- wastes (phosphogypsum and slag) from mining phosphate ores for fertilizer (ammonium phosphate) production. ${ }^{12,13}$

Current inventories of total domestic NARM wastes are not known. Future updates of this document will include additional information on NARM waste inventories, projections, and characteristics as they become available.

\section{- Mixed Low-Level Waste (MLLW)}

MLLW contains concentrations of both low-level radioactive materials and hazardous chemicals. The hazardous component of mixed waste has characteristics identified by any or all of the following statutes: the RCRA, as amended; ${ }^{9}$ the TSCA; ${ }^{10}$ and state regulations. Typically, MLLW from activities supporting DOE programs includes a variety of contaminated materials, such as air filters, cleaning solutions, engine oils and grease, paint residues, soils, construction and building materials, water-treatment chemicals, and decommissioned plant equipment. This report documents inventories and generation rates of various types of mixed wastes stored at DOE sites based on updated information and data from the DOE Site Treatment Plan Data Base ${ }^{14}$ and TSCA waste information and data reported to the $\mathrm{DB}$ Program by $\mathrm{DOE}$ sites.

\section{- Generated, Treated, Stored, and Disposed Wastes}

It should be emphasized that all of the types of radioactive materials and wastes discussed in this report can exist either as material generated, treated, stored, or disposed. The distinctions among these various waste conditions or "states" are as follows:

- Generated waste. A material recently discharged from a facility production process or operation that can be regarded as a waste because it has no economic value. In this report, quantities of generated waste are measured in units of volume [cubic meters $\left(\mathrm{m}^{3}\right)$ ] or mass $(\mathrm{kg})$ produced during a calendar year.

- Treated waste. A waste that, following generation, has been altered chemically or physically to reduce its toxicity or prepare it for storage or disposal on- or off-site. Waste treatment can include volume-reduction activities, such as incineration or compaction, which may be done prior to either storage or disposal or both (discussed next).

- Stored waste. A waste that, following generation (and usually some treatment), is being (temporarily) retained and monitored in a retrievable manner pending disposal. In this report, inventories and projections of stored radioactive materials or wastes are reported in volume $\left(\mathrm{m}^{3}\right)$ or mass $(\mathrm{kg})$ units or both.

- Disposed waste. A waste that has been put in final emplacement to ensure its isolation from the biosphere, with no intention of retrieval. Deliberate action is required to regain access to the waste. Disposed waste includes materials placed in a geologic repository, buried underground in shallow pits, dumped at sea, or discarded by hydrofracture injection. The latter two techniques were past practices and are no longer performed.

Throughout this report, the reader is urged to note the distinctions among these waste conditions. Such conditions have a great impact on the regulatory status of the waste materials considered in this report.

\subsection{METHODS AND ASSUMPTIONS USED IN REPORT PREPARATION}

This report consolidates a large amount of information from many sources. Some of these data are historical in nature, some are current, and some are projected; some have been calculated or estimated, and some have been measured. Over the years, waste regulations have been revised, waste category definitions have changed, 
measurement instruments and calibration methods have been improved, and record-keeping has been upgraded at all waste-generating and -receiving sites. In preparing this report, a major effort has been made to integrate waste data from many sources and to strive for a consistent and technically rational approach for the entire scope of coverage. Our primary sources of data are referenced, and, for calculated values (e.g., decayed radioactivity and thermal power), the bases for the calculations, are identified. To achieve adequate integration of data, numerous factors had to be considered; these are cited in footnotes that generally accompany the tables and figures of this report. In some cases, a more thorough explanation is provided in the text.

Each chapter details the assumptions on which its waste inventories and projections are based. The broader assumptions are mentioned here and are listed in Table 0.2. These include the projected time frame and specific assumptions used for estimating commercial and government (DOE) waste projections. For the commercial fuel cycle, the SNF and waste projections depend upon the nuclear power growth scenario. The commercial fuel cycle waste projections reported in this document assume a reference projection of nuclear power growth and no SNF reprocessing. The reference nuclear power electrical growth projection (and associated discharged SNF schedule) used throughout this report is the 1996 DOE/Energy Information Administration (EIA) Low Case (No New Orders). ${ }^{15}$ Throughout this report, this case will be referred to as the Low Case. The Low Case SNF and power-capacity projection cases are based on a set of assumptions involving nuclear electricity generation growth, reactor fuel burnup levels, reactor construction schedules, and reactor operating lifetimes and capacity factors. These assumptions are documented by DOE/EIA in ref. 15. In particular, the Low Case assumes that no new advanced LWRs will become operational before the year 2015 and that all current nuclear units are retired on the dates when their initial license-terms expire.

Detailed information about reactors already built, being built, or planned in the United States for domestic use or export as of December 31, 1995, is provided in report DOE/OSTI-8200-R59 (ref. 16), which contains a comprehensive listing of all domestic reactors categorized by primary function or purpose: civilian, production, military, export, and critical assembly.

The data for total waste inventories (which comprise historical data) are obviously less accurate than the values recorded for recent waste additions. The number of digits used in reporting these values is generally greater than justified in terms of numerical significance, but this proves useful and necessary for bookkeeping purposes. In some cases, the values cited are significantly different from those previously reported. This is generally a result of improved estimates, new measurements, or redefinition of terms. Explanations are given in such cases. Many of the comments received during the final review stage of this report deal with changes that have occurred after December 31, 1995-some as recently as November 1996. These changes are generally cited in footnotes.

For the sake of brevity, many of the figures and tables of this report use the exponential (E) notation. As examples of this notation, the constant $1.234 \mathrm{E}+2$ means $1.234 \times 10^{2}$, or 123.4 ; and $1.234 \mathrm{E}-4$ means $1.234 \times 10^{-4}$, which is 0.0001234 .

\subsection{WASTE CHARACTERISTICS AND UNTTS REPORTED}

Principal characteristics reported for most radioactive wastes discussed in this report include volume, radioactivity, and thermal power. All characteristics are reported in metric units and, depending on the waste form, can be significant considerations in meeting the requirements for waste treatment, storage, and disposal. Waste volume is reported in cubic meters $\left(\mathrm{m}^{3}\right)$ and generally reflects the amount of space occupied by the waste and its container. Radioactivity represents the rate of spontaneous disintegration of the radionuclides comprising the waste. In this report, radioactivity is measured by a unit called a curie $(\mathrm{Ci})$, which is $3.7 \times 10^{10}$ nuclear disintegrations per second. Over time, radionuclides decay to nonradioactive, stable isotopes. As an example, the short-lived radionuclides found in SNF rapidly decay during the first few years after the fuel is removed from a reactor.

It should be noted that while waste volumes accumulate with time by conventional addition, total radioactivity does not. Because of radioactive decay, cumulative activity cannot be based on reported annual additions; rather it must be estimated from knowledge of the waste composition, which includes the radionuclides comprising the waste, their concentrations, and decay attributes (e.g., half-lives and decay schemes). In this report, decayed radioactivity is generally estimated for some wastes by an abridged version of the ORIGEN2 code (ref. 17). Annual levels of radioactivity (Ci) reported in this document include contributions from both parent and daughter decay products.

Thermal power is a measure of the rate of heat-energy emission resulting from the decay of radionuclides in a waste. Like radioactivity, thermal power is not cumulative by conventional addition because of radioactive decay. Information on thermal power is needed in the design of shipping casks, storage facilities, and repositories where temperature rise, especially with regard to SNF and HLW, is an important concern. Thermal energy generation rates 
are highest for SNF, HLW, and RH TRUW. They may also be important for certain types of LLW. The unit of thermal power used in this report is the watt (W), which represents 1 joule $(J)$ of thermal energy emitted per second. Estimates of thermal power are based on radionuclide composition as well as total activity. While levels of thermal power may not be significant for certain waste forms (particularly some types of LLW), they are nevertheless reported for the major radioactive waste categories referenced in this report to provide a standard for comparison.

For SNF and TRUW, mass is reported to provide better assurances of accountability. SNF is reported in units of metric tons of initial heavy metal (MTIHM) to avoid difficulties and confusion arising from the need to estimate ranges of varied heavy-metal content (MTHM) that result from different levels of enrichment and reactor fuel burnup. Mass is reported in kilograms ( $\mathrm{kg}$ ) for the TRU radionuclides comprising TRUWs.

In this report, quantities of generated wastes are expressed in terms of either the amount of mass $(\mathrm{kg})$ or volume $\left(\mathrm{m}^{3}\right)$ produced in a given calendar year. Thus, generation rates for wastes are expressed in either kilograms per year $(\mathrm{kg} / \mathrm{year})$ or cubic meters per year ( $\mathrm{m}^{3} /$ year), depending on the availability of site information. Annual generation rates are reported in this document for SNF, TRUW, LLW, and MLLW. No HLW from SNF reprocessing was generated during 1995. In previous issues of this document, annual generation rates have not been reported for HLW in part because there are problems in accurately estimating $\mathrm{HLW}$ generation levels. One major difficulty is accounting for net waste-quantity changes due to the combined effects of various modes of site waste management operations such as evaporation and calcination.

Quantities of wastes can also be reported in terms of the number and types of waste containers. LWR SNF inventories and projections can be expressed in terms of the number of permanently discharged boiling-water reactor (BWR) and PWR fuel assemblies. HLW will be immobilized in either borosilicate glass or a glass/ceramic matrix solidified in stainless steel canisters. Quantities of LLW and stored TRUW can be based on the number and types of drums, boxes, or containers used or scheduled for use.

Waste characteristics are also identified by waste composition. Throughout this report, waste composition is expressed in terms of the following:

- radioactivity (Ci) or specific-activity $\left(\mathrm{Ci} / \mathrm{m}^{3}\right)$ breakdown by radionuclide (with accompanying daughter products) and

- physical form (solid, liquid, gas, or sludge) or chemical content (by chemical component), expressed in terms of either volume $\left(\mathrm{m}^{3}\right)$ or mass $(\mathrm{kg})$ or as a percentage of total weight (wt \%), volume (vol \%), or activity (act \%).

\subsection{CHAPTER OVERVIEWS}

A brief summary of each chapter in this report is presented in the following paragraphs.

\subsubsection{Spent Nuclear Fuel (SNF)}

Chapter 1 of this report presents national data on the quantities of permanently discharged SNF from commercial nuclear power reactors. Historical data on commercial SNF inventories are reported along with 1996 DOE/EIA projections for the Low Case. ${ }^{15}$ The Low Case is the baseline commercial scenario used throughout this report to make waste projections. For the projection period considered in this report (CYs 1996-2030), the Low Case assumes that no new reactors will be ordered.

DOE SNF inventories are also reported in Chapter 1. These include various types of research reactor SNFs which are stored at the SRS and the INEL.

In this report, the mass of discharged SNF is generally measured in MTIHM. The term "initial heavy metal" refers to the original mass of the actinide elements of the fuel, most of which is uranium. (Elements of the actinide group are those with atomic numbers greater than 89 .)

\subsubsection{High-Level Waste (HLW)}

The inventories of $\mathrm{HLW}$ in storage at the end of CY 1995 and projected through CY 2030 are given in Chapter 2. The waste forms include liquid, sludge, salt cake, slurry, calcine, precipitate, zeolite, glass, and capsules of separated strontium and cesium. Vitrified defense $\mathrm{HLW}$ is projected after the startup of the Defense Waste Processing Facility (DWPF) at SRS in 1996, and projections of vitrified $\mathrm{HLW}$ from commercial reprocessing activities are given for the WVDP. Projections recently made of the number of canisters containing the final immobilized form for the DOE HLW at Hanford and the INEL are also reported. In addition, Chapter 2 gives the locations, volumes, and radioactivities of HLW.

In 1992, DOE decided to phase out the reprocessing of its production reactor SNFs. Until then, the reprocessing activities recovered enriched uranium and plutonium which were used to support nuclear weapons production. As a consequence of ceasing to reprocess reactor SNFs, little additional HLW is expected to be generated at DOE sites in the future. 


\subsubsection{Transuranic Waste (TRUW)}

The locations, inventories, and projections of TRUW buried and stored at DOE sites are presented in Chapter 3. Inventories of TRUW are reported as of December 31, 1994, and are virtually all derived from government operations. The inventories documented in this report are based on data provided by the sites and include waste volumes and the masses and radioactivities of contained radionuclides. Projected future TRUW volumes through CY 2022 were also requested from the sites, but the sites were not able to make such estimates in all cases. Projections are reported through CY 2022 for those sites that provided estimates.

In 1984, DOE (with input from other federal agencies) revised the minimum radioactivity concentration level for defining TRUW from greater than $10 \mathrm{nCi} / g$ to greater than $100 \mathrm{nCi} / \mathrm{g}^{18}$ Consequently, some waste currently in the inventory may contain wastes stored under both criteria. This redefinition, as well as the development of instrumentation to detect these low levels of radioactivity, may reduce the volume of TRUW. As the waste is assayed, that portion of it which is greater than $10 \mathrm{nCj} / \mathrm{g}$ and less than $100 \mathrm{nCi} / \mathrm{g}$ will be reclassified to other waste categories.

\subsubsection{Low-Level Waste (LLW)}

Data for LLW from commercial and government activities are given in Chapter 4 and Appendix A. Commercial fuel-cycle LLW is generated from the conversion of yellowcake to uranium hexafluoride $\left(\mathrm{UF}_{6}\right)$, enrichment, fuel fabrication, and reactor operation. LLW also results from commercial operations by private organizations that are licensed to use radioactive materials. These include institutions and industries engaged in research and various medical and industrial activities. DOE LLW is similar in nature to the commercial industrial and institutional (I/) waste and the commercial fuel cycle LLW.

A wide variety of radionuclides are found in LLW. Uranium isotopes and their daughters dominate in the conversion, enrichment, and fuel-fabrication steps of the nuclear fuel cycle. Reactor operations produce LLW containing mostly activation products and fission products. A significant fraction of institutional LLW that is shipped to disposal sites is contaminated with small quantities of ${ }^{3} \mathrm{H}$ and ${ }^{14} \mathrm{C}$.

By the end of 1995 , approximately $66 \%$ of the total cumulative volume of disposed LLW resulted from various DOE activities. The remaining $34 \%$ resulted from domestic commercial activities. About $29 \%$ of the volume of LLW disposed during 1995 resulted from commercial activities.

\subsubsection{Uranium Mill Tailings (UMT)}

Current inventories and projections of tailings from commercial uranium mill operations are summarized in Chapter 5. Twenty-six licensed uranium mills have accumulated tailings from their operations. Half of these mills have accumulated both commercial and government tailings. By the end of 1995, only one NRC-licensed mill was operational. To date, almost all domestic uranium has been produced by conventional mining and milling methods from which these tailings derive. A small portion has been obtained via in situ leaching, recovery from mine water, recovery from copper/vanadium dump leach liquor, and recovery from wet-process phosphoric acid effluents. Tailings from the now inactive mills that produced uranium only for government operations are being stabilized under DOE's Environmental Restoration Program (see Chapter 6).

\subsubsection{Environmental Restoration Program}

The overall mission of the DOE Office of Environmental Restoration (EM-40) is to protect human health and the environment from the risks posed by inactive, surplus structures and contaminated environmental media. An overview of the EM-40 program is given below. Further details are provided in Chapter 6 . The scope of Chapter 6 is limited to radioactive and mixed (radioactive and chemically hazardous) wastes that could be generated by environmental restoration activities. Nonradioactive hazardous and sanitary wastes are outside the scope of this report.

The EM-40 program includes a bias for action to expedite actual cleanup wherever and whenever possible. Activities are prioritized based on factors such as the need to eliminate risks at sites not controlled by the federal government, the goal of reducing risks at all sites, and compliance with various laws, regulations, and agreements. Most activities are designed to either remove or contain contamination in the environment or to decommission contaminated structures. Related activities include treatment of contaminated materials and wastes, transportation of these materials and wastes to storage and disposal facilities, and disposal of wastes in permitted facilities.

The total volume of solid radioactively contaminated material being addressed by the EM-40 program is approximately 88 million $\mathrm{m}^{3}$. About one-half of this volume is expected to be managed in-situ using remedies such as capping, monitoring, and retention of land-use controls. The total volume of radioactive waste resulting from ex-situ remedies is approximately 44 million $\mathrm{m}^{3 ;}$ the largest source of waste is associated with the Uranium Mill Tailings Remedial Action Program ( 30 million $\mathrm{m}^{3}$ ) which 
is scheduled to be completed in 1997. Ninety percent of the waste from this program ( 27 million $\left.\mathrm{m}^{3}\right)$ is already in disposal cells. Most of the remaining waste volume is $\mathrm{LLW}\left(12\right.$ million $\left.\mathrm{m}^{3}\right)$. Strategies for managing the radioactive wastes associated with the EM- 40 program are presented in Chapter 6

\subsubsection{Naturally Occurring and Accelerator- Produced Radioactive Material (NARM)}

Chapter 7 describes the characteristics of NARM (and NORM). Some inventories and projections of these materials, based on information currently available, are also reported.

\subsubsection{Mixed Low-Level Waste (MLLW)}

Current inventories and generation rates of MLLW from both DOE and commercial sources are summarized in Chapter 8. These wastes are contaminated with both low-level radioactivity and chemically hazardous substances. The radioactive components are defined by the $\mathrm{AEA}^{8}{ }^{8}$ while the hazardous components are defined by the $\mathrm{RCRA}^{9}$ the TSCA, ${ }^{\mathrm{to}}$ and pertinent state regulations. As of the end of 1995, inventories of MLLW at DOE sites totaled about $131,400 \mathrm{~m}^{3}$.

\subsubsection{Appendixes}

Several appendixes are included in this report. Appendix $A$ is a compilation of source terms and characteristics used for waste projections. Source terms include both quantitative and descriptive characteristics used to describe radioactive wastes. As developed and used in the IDB Program, the source term for a particular waste is comprised of two components unique to that waste: (1) the number of curies of radioactivity, expressed either per unit of facility production or per unit of waste volume or mass, and (2) a listing of the relative contributions of component radioisotopes per curie of radioactivity of the waste. A tabulation of the properties of important radionuclides is given in Appendix B. Appendix $\mathrm{C}$ lists the sites and facilities referred to in this report. Appendix D describes how to get computer access to this document via the Internet, and Appendix $\mathrm{E}$ describes a reader comment form, which is provided in the front of this report.

\section{O.6 SUMMARY DATA}

A few graphical presentations and summary tables are included in this chapter to provide a broad overview. Figures 0.1 and 0.2 , respectively, show the volumes and decayed radioactivities of commercial and DOE wastes and SNF accumulated through 1995.

Summaries of SNF and radioactive waste inventories and projections are provided in Tables 0.3 and 0.4 . In general, material to be sent to R\&D facilities or to the proposed national geologic repository for SNF and HLW is still listed in each individual site's inventory.

DOE waste volume summaries for specific sites are given in Tables 0.5-0.7. Waste generation for 1995 is presented in Table 0.5, and cumulative 1995 inventories for stored and buried wastes are provided in Tables 0.6 and 0.7 , respectively.

In recent years, various types of DOE and commercial wastes have been disposed of at a facility operated by Envirocare of Utah, Inc., near Clive, Utah. A summary of 1995 disposed waste volume inventories at the Envirocare Facility is given in Table 0.8 (data from ref. 19).

\subsection{REFERENCES}

1. U.S. Department of Energy, Integrated Data Base Report-1994: U.S. Spent Nuclear Fuel and Radioactive Waste Inventories, Projections, and Characteristics, DOE/RW-0006, Rev. 11, Oak Ridge National Laboratory, Oak Ridge, Tennessee (September 1995).

2. U.S. General Accounting Office, Environmental Cleanup-Better Data Needed for Radioactively Contaminated Defense Sites, GAO/NSLAD-94-168, Washington, D.C. (August 1994).

3. Coreen Casey and Beth A. Heath, EG\&G Idaho, Inc., Radioactive Waste Technical Support Program, Material Not Categorized as Waste (MNCAW) Data Report, DOE/LLW-93, predecisional draft, Idaho National Engineering Laboratory, Idaho Falls, Idaho (November 1993).

4. U.S. Congress, Nuclear Waste Policy Act of 1982, Pub. L. 97-425, Jan. 7, 1983, as amended by the Budget Reconciliation Act for Fiscal Year 1988, Title V-Energy and Environment Programs, Pub. L. 100-203, Dec. 22, 1987. 
5. U.S. Environmental Protection Agency, "Environmental Radiation Protection Standards for Management and Disposal of Spent Nuclear Fuel, High-Level and Transuranic Radioactive Wastes," Code of Federal Regulations, 40 CFR Part 191 (July 1, 1996).

6. U.S. Department of Energy, DOE Order 5820.2A, Radioactive Waste Management, Washington, D.C., Sept. 26, 1988.

7. U.S. Congress, The Low-Level Radioactive Waste Policy Amendments Act of 1985, Pub. L. 99-240, Jan. 15, 1986.

8. U.S. Congress, Atomic Energy Act of 1954, Pub. L. 83-703, Aug. 15, 1954.

9. U.S. Congress, Resource Conservation and Recovery Act of 1976, Pub. L. 94-580, Oct. 21, 1976, as amended by the Hazardous and Solid Waste Amendments Acts of 1984, Pub. L. 98-616, Nov. 9, 1984.

10. U.S. Congress, Toxic Substances Control Act of 1976, Pub. L. 94-469, Oct. 11, 1976.

11. U.S. Nuclear Regulatory Commission, "Licensing Requirements for Land Disposal of Radioactive Waste-Waste Classification," Code of Federal Regulations, 10 CFR Part 61, Sect. 61.55 (Jan. 1, 1996).

12. The League of Women Voters Education Fund, The Nuclear Waste Primer, Lyons \& Burford, New York, 1993.

13. K. P. Smith, An Overview of Naturally Occurring Radioactive Materials (NORM) in the Petroleum Industry, ANL/EAIS-7, Argonne National Laboratory, Argonne, Illinois (December 1992).

14. U.S. Department of Energy, Office of Environmental Restoration, Office of Waste Management, Site Treatment Plan Data Base, updated through Oct. 31, 1996.

15. U.S. Department of Energy, Energy Information Administration, Nuclear Power Generation and Fuel Cycle Report 1996, DOE/ELA-0436(96), Washington, D.C. (October 1996).

16. U.S. Department of Energy, Office of Scientific and Technical Information, Nuclear Reactors Built, Being Built, or Planned: 1995, DOE/OSTI-8200-R59, Oak Ridge, Tennessee (August 1996).

17. A. G. Croff, ORIGEN2-A Revised and Updated Version of the Oak Ridge Isotope Generation and Depletion Code, ORNL-5621, Oak Ridge National Laboratory, Oak Ridge, Tennessee (July 1980).

18. U.S. Department of Energy, DOE Order 5820.2, Radioactive Waste Management, Washington, D.C., Feb. 6, 1984 [updated by DOE Order 5820.2A (ref. 6)].

19. Al Rafati, Envirocare of Utah, Inc., Salt Lake City, Utah, correspondence to Stephen N. Storch, IDB Program, ORNL, Oak Ridge, Tennessee, dated Aug. 8, 1996. 


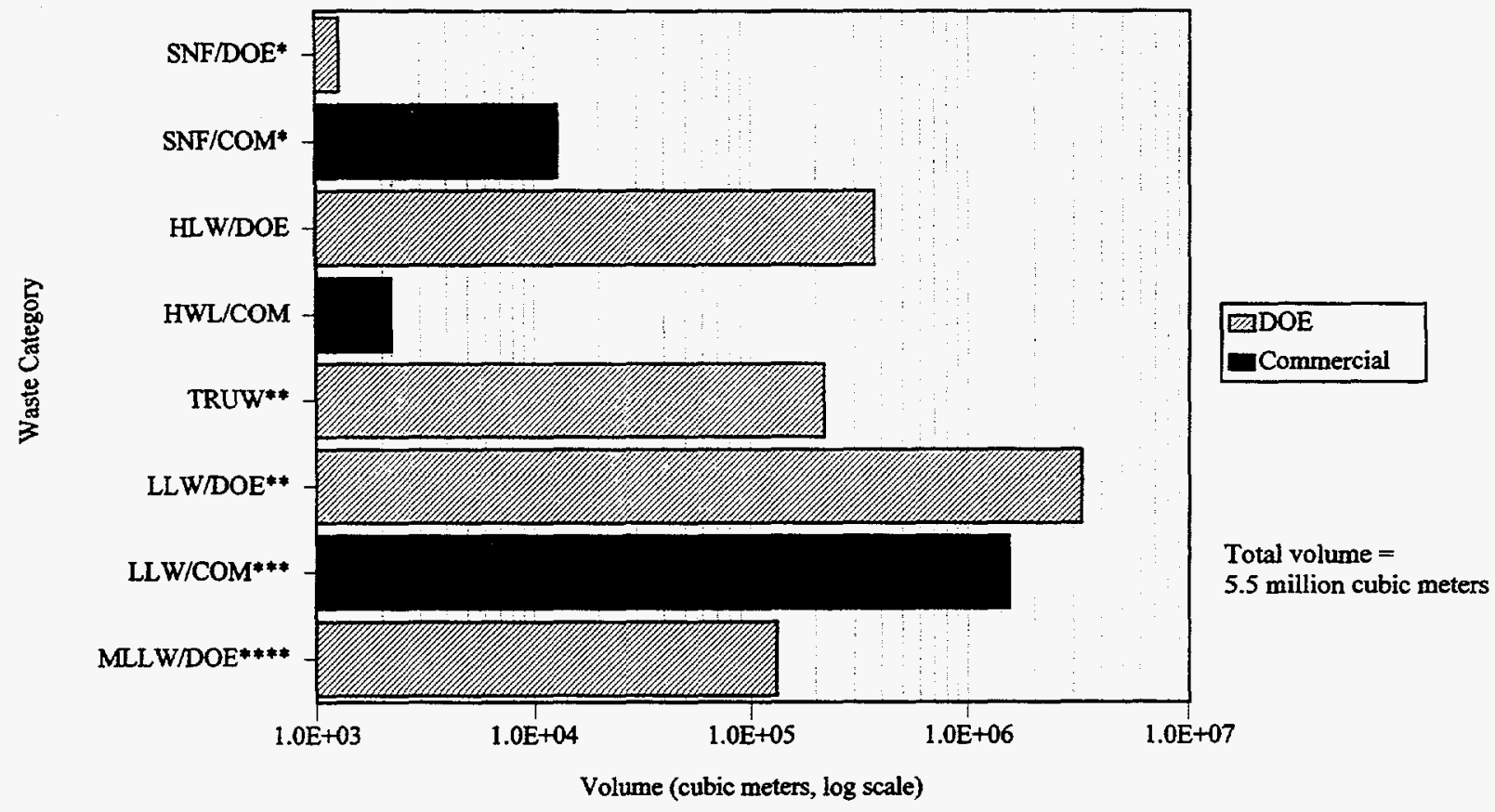

* Permanently discharged reactor fuel. Includes spacing between fuel assembly rods.

**DOE wastes include both retrievably stored and buried materials. Excludes wastes disposed of at the Envirocare Facility.

***Includes contributions from disposed wastes only. Excludes LLW disposed of at Envirocare.

**** Includes retrievably stored RCRA and TSCA materials only. Excludes MLLW disposed of at Envirocare.

Fig. 0.1. Total volumes of DOE and commercial radioactive wastes and SNF through 1995.

ORNL DWG 95-8850R2

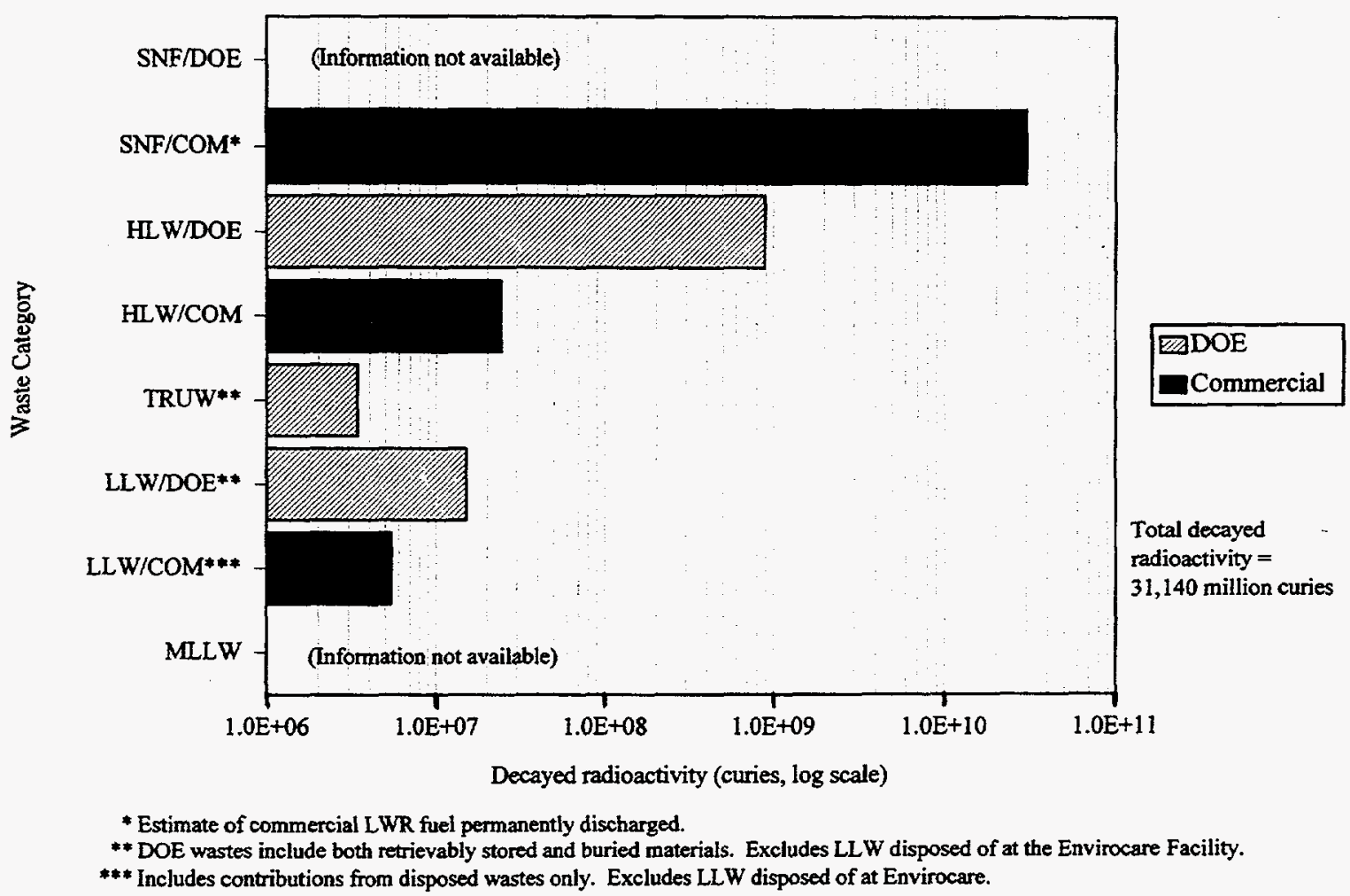

Fig. 0.2. Total decayed radioactivities of DOE and commercial radioactive wastes and SNF through 1995. 
Table 0.1. Types of radioactive wastes managed at DOE sites referenced in this report ${ }^{\mathrm{a}}$

\begin{tabular}{|c|c|c|c|c|c|c|c|}
\hline Site(s) & Symbol/label & SNF & HLW & TRUW & LLW & MLLW & UMT \\
\hline Ames Laboratory & Ames & & & $\mathrm{x}$ & $\mathrm{x}$ & $\mathrm{X}$ & \\
\hline Argonne National Laboratory-East & ANL-E & $\mathrm{X}^{\mathrm{a}}$ & & $\mathrm{x}$ & $\mathrm{x}$ & $\mathrm{X}$ & \\
\hline Argonne National Laboratory-West & ANL-W & $\mathrm{x}$ & & $\mathrm{x}$ & $\mathrm{x}$ & $\mathrm{X}$ & \\
\hline Atlantic Richfield (Medical Products) Company & ARCO & & & $\mathrm{x}$ & & & \\
\hline Battelle Columbus Laboratories & $\mathrm{BCL}$ & & & $\mathrm{X}$ & $\mathrm{x}$ & & \\
\hline Brookhaven National Laboratory & BNL & $\mathrm{x}$ & & & $\mathrm{X}$ & $\mathrm{x}$ & \\
\hline Energy Technology Engineering Center & ETEC & & & $\mathrm{x}$ & $\mathrm{X}$ & $\mathrm{X}$ & \\
\hline Fermi National Accelerator Laboratory & FNAL & & & & $\mathrm{x}$ & & \\
\hline Fernald Environmental Management Project & FEMP & & & & $\mathrm{x}$ & $\mathrm{x}$ & \\
\hline Hanford Site ${ }^{b}$ & Hanford & $\mathrm{x}$ & $\mathrm{x}$ & $\mathrm{X}$ & $\mathrm{x}$ & $\mathrm{x}$ & \\
\hline Idaho National Engineering Laboratory ${ }^{\mathcal{C}}$ & INEL & $\mathrm{X}$ & $\mathrm{x}$ & $\mathrm{X}$ & $\mathrm{x}$ & $\mathrm{x}$ & \\
\hline Inhalation Toxicology Research Institute & ITRI & & & & $\mathrm{X}$ & $\mathrm{x}$ & \\
\hline K-25 Site (Oak Ridge) & $\mathrm{K}-25$ & & & & $\mathrm{x}$ & $\mathrm{x}$ & \\
\hline Kansas City Plant & $\mathrm{KCP}$ & & & & $\mathrm{X}$ & $\mathrm{X}$ & \\
\hline Lawrence Berkeley Laboratory & LBL & & & $\mathrm{X}$ & $\mathrm{X}$ & $\mathrm{x}$ & \\
\hline Lawrence Livermore National Laboratory & LLNL & & & $\mathrm{X}$ & $\mathrm{X}$ & $\mathrm{X}$ & \\
\hline Los Alamos National Laboratory & LANL & $\mathrm{x}$ & & $\mathrm{x}$ & $\mathrm{X}$ & $\mathrm{x}$ & \\
\hline Missouri (Univ. of) Research Reactor & MURR & & & $\mathrm{x}$ & & & \\
\hline Mound Plant & Mound & & & $\mathrm{X}$ & $\mathrm{X}$ & $\mathrm{x}$ & \\
\hline Naval Reactors Facilities and Shipyards $d$ & NR Sites & & & $\mathrm{X}$ & $\mathrm{x}$ & $\mathrm{x}$ & \\
\hline Nevada Test Site & NTS & & & $\mathrm{X}$ & $\mathrm{X}$ & $\mathrm{x}$ & \\
\hline Oak Ridge Institute of Science and Education & ORISE & & & & $\mathrm{X}$ & & \\
\hline Oak Ridge National Laboratory & ORNL & $\mathrm{x}$ & & $\mathrm{x}$ & $\mathrm{X}$ & $\mathrm{x}$ & \\
\hline Paducah Gaseous Diffusion Plant & $\mathrm{PAD}$ & & & $\mathrm{x}$ & $\mathrm{x}$ & $\mathrm{x}$ & \\
\hline Pantex Plant & PANT & & & $\mathrm{X}$ & $\mathrm{x}$ & $\mathrm{x}$ & \\
\hline Pinellas Plant & Pinellas & & & & $\mathrm{X}$ & $\mathrm{x}$ & \\
\hline Portsmouth Gaseous Diffusion Plant & PORTS & & & & $\mathrm{X}$ & $\mathrm{x}$ & \\
\hline Princeton Plasma Physics Laboratory & PPPL & & & & $\mathrm{X}$ & $\mathrm{x}$ & \\
\hline Reactive Metals, Inc., Extrusion Plant & RMI & & & & $\mathrm{x}$ & $\mathrm{x}$ & \\
\hline Remedial Action Program Sites ${ }^{\mathrm{e}}$ & RAP Sites & & & $\mathrm{x}$ & $\mathrm{X}$ & $\mathrm{X}$ & $\mathrm{X}$ \\
\hline Rocky Flats Environmental Technology Site & RFETS & & & $X$ & $\mathrm{X}$ & $\mathrm{X}$ & \\
\hline Sandia National Laboratory/California & SNL/CA & & & & $\mathrm{x}$ & $\mathrm{x}$ & \\
\hline Sandia National Laboratory/New Mexico & SNL/NM & $\mathrm{x}$ & & $\mathrm{x}$ & $\mathrm{x}$ & $\mathrm{x}$ & \\
\hline Savannah River Site & SRS & $\mathrm{X}$ & $\mathrm{x}$ & $\mathrm{X}$ & $\mathrm{X}$ & $\mathrm{X}$ & \\
\hline Stanford Linear Accelerator Center & SLAC & & & & $\mathrm{X}$ & & \\
\hline Teledyne Brown Engineering & TBE & & & $\mathrm{x}$ & & & \\
\hline U.S. Army Material Command & USAMC & & & $\mathrm{x}$ & & & \\
\hline West Valley Demonstration Project & WVDP & $\mathrm{x}$ & $\mathrm{x}$ & $\mathrm{X}$ & $\mathrm{x}$ & $\mathrm{x}$ & \\
\hline Y-12 Plant (Oak Ridge) & $\mathrm{Y}-12$ & & & & $\mathrm{x}$ & $\mathrm{x}$ & \\
\hline
\end{tabular}

aAn " $\mathrm{X}$ " entry in this table indicates that the listed site manages waste of the category indicated. General site information is given in Appendix C.

Includes Pacific Northwest National Laboratory (PNNL).

Includes the Idaho Chemical Processing Plant (ICPP); excludes ANL-W.

$\mathrm{d}$ These sites are listed in Table C. 2 of Appendix C.

These sites are listed in Chapter 6. 
Table 0.2. Major assumptions used in this report

\section{Inventory/projection basis}

- Inventories are reported as of December 31, 1995

- Projections are generally reported for the CYs 1996-2030

\section{HLW solidification activities}

- For WVDP, HLW solidification (glass production) starts in 1996 and is completed in 1998

- For SRS, HLW solidification (glass production) at the Defense Waste Processing Facility (DWPF) starts in 1996 and continues through 2021

- For INEL, HLW solidification (immobilization) starts in 2019 and continues through 2035

- For Hanford, HWW solidification (borosilicate glass production) starts in 2002 and concludes in 2028

\section{Commercial activities}

- DOE/EIA projections of installed net LWR electrical capacity for the Low Case ${ }^{\mathrm{a}}$ of ref. 15 :

\section{Low Case}

$\begin{array}{crrrrrrrr}C Y & 1996 & 2000 & 2005 & 2010 & 2015 & 2020 & 2025 & 2030 \\ \text { GW(e) } & 101 & 101 & 101 & 94 & 64 & 49 & 22 & 2\end{array}$

- DOE/EIA assumptions for LWR fuel enrichment and design burnup:

\begin{tabular}{lccc}
\hline LWR fuel & $\begin{array}{c}\text { CY fuel is } \\
\text { loaded }\end{array}$ & $\begin{array}{c}\text { Fuel enrichment } \\
\left(\%{ }^{23} \mathrm{U}\right)\end{array}$ & $\begin{array}{c}\text { Design bumup } \\
\text { (MWd/MTIHM) }\end{array}$ \\
\hline BWR & 1993 & 3.17 & 36,000 \\
& 1998 & 3.34 & 40,000 \\
& 2006 & 3.50 & 43,000 \\
PWR & 2018 & 3.67 & 46,000 \\
& 1993 & 3.84 & 42,000 \\
& 1998 & 4.07 & 46,000 \\
& 2004 & 4.39 & 50,000 \\
& 2008 & 4.73 & 55,000 \\
& 2018 & 5.04 & 60,000 \\
\hline
\end{tabular}

- SNF from commercial reactors is not reprocessed. Thus, a fuel cycle without reprocessing is assumed for all commercial projections

${ }^{a}$ This case assumes that each reactor will be retired when the expiration date specified in its operating license is reached. Previously, this case was referred to as the No New Orders Case. 
Table 0.3. Cumulative SNF and radioactive waste inventories as of December 31, 1995

\begin{tabular}{|c|c|c|c|c|c|}
\hline Waste category & $\begin{array}{c}\text { TRU } \\
\text { isotopes } \\
\text { (kg) }\end{array}$ & $\begin{array}{c}\text { Mass } \\
\text { (MTIHM) }\end{array}$ & $\begin{array}{l}\text { Volume } \\
\left(\mathrm{m}^{3}\right)\end{array}$ & $\begin{array}{l}\text { Radioactivity } \\
\qquad\left(10^{6} \mathrm{Ci}\right)\end{array}$ & $\begin{array}{l}\text { Thermal } \\
\text { power } \\
\left(10^{3} \mathrm{~W}\right)\end{array}$ \\
\hline \multicolumn{6}{|l|}{ SNF } \\
\hline \multicolumn{6}{|l|}{ Commercial } \\
\hline BWRs & & 11,415 & $4,602^{b}$ & $\mathrm{c}$ & $\mathrm{c}$ \\
\hline PWRs & & 20,538 & $8,279^{b}$ & c & c \\
\hline DOE & & 2,647 & 1,282 & $\mathrm{c}$ & c \\
\hline \multicolumn{6}{|l|}{ HLW } \\
\hline Savannah River (DOE) & & & 126,500 & 502.2 & $1,406.0$ \\
\hline Idaho (DOE) & & & 11,170 & 49.3 & 142.8 \\
\hline Hanford (DOE) ${ }^{d}$ & & & 233,500 & 339.9 & 976.7 \\
\hline West Valley (commercial) & & & 2,200 & 24.1 & 69.7 \\
\hline \multicolumn{6}{|l|}{ TRUW (DOE) ${ }^{\mathbf{e}}$} \\
\hline Buried & c & & 141,000 & 0.75 & c \\
\hline Stored (site operations) ${ }^{f}$ & c & & 75,600 & 2.63 & c \\
\hline $\begin{array}{l}\text { Stored (environmental restoration } \\
\text { activities) }\end{array}$ & c & & 43 & c & c \\
\hline \multicolumn{6}{|l|}{ LLW } \\
\hline \multicolumn{6}{|l|}{ DOE sites } \\
\hline Generated & & & 29,199 & 0.4 & $\mathrm{c}$ \\
\hline Stored (site operations) & & & 65,775 & 2.5 & c \\
\hline $\begin{array}{l}\text { Stored (environmental restoration } \\
\text { activities) }\end{array}$ & & & 180,000 & $\mathrm{c}$ & $\mathrm{c}$ \\
\hline Disposed & & & $3,011,459$ & 12.5 & 22.2 \\
\hline \multicolumn{6}{|l|}{ Commercial sites } \\
\hline Disposed & & & $1,538,416$ & 5.4 & 20.8 \\
\hline Envirocare disposal facility & & & 155,694 & c & c \\
\hline \multicolumn{6}{|l|}{ Uranium mill tailings (commercial } \\
\hline licensed mill sites)g & & & $118,700,000$ & c & c \\
\hline $\begin{array}{l}\text { 11e(2) by-product material (from DOE } \\
\text { environmental restoration) }\end{array}$ & & c & $27,000,000$ & $\mathrm{c}$ & c \\
\hline \multicolumn{6}{|l|}{ MLLW (stored) } \\
\hline DOE $E^{i}$ & & c & 131,379 & c & c \\
\hline Commercial & & c & c & c & c \\
\hline Envirocare disposal facility & & c & 20,143 & c & c \\
\hline
\end{tabular}

a'Except for transuranic wastes, radioactivity data are calculated decayed values as of December 31, 1995.

Bincludes volume of spacing between the fuel tods of each assembly.

Information not available.

${ }^{d}$ Hanford tank wastes consist of HLW, TRUW, and LLW. However, in the interim storage mode, the tank wastes are managed as if they contain $\mathrm{HLW}$ and, therefore, are included in the $\mathrm{HLW}$ inventory.

Eata as of December 31, 1994.

$\mathrm{f}_{\text {As-generated wastes, mixed and nonmixed. }}$

gIncludes contributions from 26 NRC-licensed mills.

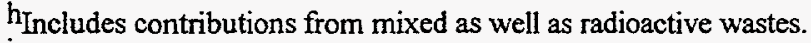

iIncludes contributions from environmental restoration activities. 
Table 0.4. Current and projected cumulative quantities of radioactive waste and SNF [Quantities are expressed as volume $\left(10^{3} \mathrm{~m}^{3}\right)$ unless otherwise indicated]

\begin{tabular}{|c|c|c|c|c|c|}
\hline \multirow{2}{*}{ Source and type of material } & \multicolumn{5}{|c|}{ End of calendar year } \\
\hline & 1995 & 2000 & 2010 & 2020 & 2030 \\
\hline \multicolumn{6}{|l|}{ DOE sites } \\
\hline SNF, mass, MTIHM ${ }^{a}$ & 2,647 & $\mathrm{~b}$ & $b$ & b & $\mathrm{b}$ \\
\hline \multicolumn{6}{|l|}{$\mathrm{HLW}$} \\
\hline Interim storage & 373.4 & 337 & 284 & 129 & 4 \\
\hline Glass or glass/ceramic ${ }^{c}$ & 0 & 0.7 & 2.6 & 10.7 & 18.8 \\
\hline \multicolumn{6}{|l|}{ TRUW } \\
\hline Buried & $141^{\mathrm{d}}$ & 141 & 141 & 141 & 141 \\
\hline Stored (as generated) & $77.0^{\mathrm{d}}$ & b & b & b & b \\
\hline \multicolumn{6}{|l|}{ LLW } \\
\hline Buriede & 3,011 & 3,427 & 4,124 & 4,813 & 5,503 \\
\hline Stored & 246 & b & b & $\mathrm{b}$ & b \\
\hline MLLW & 131.4 & b & b & $\mathrm{b}$ & b \\
\hline \multicolumn{6}{|l|}{ Commercial sites } \\
\hline \multicolumn{6}{|l|}{$\begin{array}{l}\text { LWR SNF, mass, MTLHM } \\
\text { (no reprocessing) }\end{array}$} \\
\hline Low Case & 32,022 & 43,100 & 63,600 & 80,000 & 87,900 \\
\hline \multicolumn{6}{|l|}{ HLW (WVDP) } \\
\hline Interim storage & 2.180 & 0.0 & 0.0 & 0.0 & 0.0 \\
\hline Glass & 0.0 & 0.24 & 0.24 & 0.24 & 0.24 \\
\hline LLW (no reprocessing) & 1,538 & 1,636 & $\mathrm{~b}$ & b & b \\
\hline Mill tailings (no reprocessing) & 118,700 & $\mathbf{b}$ & $b$ & b & $b$ \\
\hline MLLW & $\mathrm{b}$ & $b$ & $\mathrm{~b}$ & $b$ & $\mathrm{~b}$ \\
\hline \multicolumn{6}{|l|}{ Envirocare disposal facilityg } \\
\hline LLW & 155.7 & $\mathrm{~b}$ & $\mathrm{~b}$ & $b$ & b \\
\hline MLLW & 20.1 & $b$ & $b$ & $b$ & b \\
\hline NARM & 277.6 & $\mathrm{~b}$ & $\mathrm{~b}$ & $b$ & b \\
\hline 11 e(2) by-product & 127.4 & $\mathrm{~b}$ & $b$ & $\mathrm{~b}$ & b \\
\hline
\end{tabular}

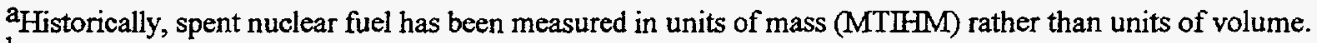

binformation not available.

Tncludes projections for glass at SRS and glass/ceramic at INEL.

dProjected inventory level for end of CY 1995. Includes mixed and nonmixed wastes.

Projections include contributions from SRS saltstone.

$f_{\text {The }} 1995$ discharged spent nuclear fuel mass is a BWR and PWR mass sum rounded to the nearest metric ton. Such rounding may result in slight differences between the spent nuclear fuel inventories and projections reported in this document and those reported by DOE/EIA.

gIncludes wastes from DOE-, commercial-, DOD-, and EPA-sponsored activities. 
Table 0.5. Volume $\left(\mathrm{m}^{3}\right)$ of DOE site wastes generated during CY 1995

\begin{tabular}{|c|c|c|c|c|c|c|c|}
\hline \multirow{2}{*}{ Site(s) } & \multirow{2}{*}{$\mathrm{HLW}$} & \multicolumn{2}{|c|}{ TRUW } & \multirow{2}{*}{$\operatorname{LLW}^{\mathrm{b}}$} & \multicolumn{2}{|c|}{ MLLW } & \multirow{2}{*}{ Total (site) } \\
\hline & & $\mathrm{CH}^{\mathrm{a}}$ & $\mathrm{RH}^{\mathrm{a}}$ & & $\mathrm{RCRA}^{\mathrm{c}}$ & TSCA & \\
\hline Ames & & & & 10 & $\mathrm{~d}$ & e & 10 \\
\hline ANL -E & & & & 253 & 22 & & 275 \\
\hline ANL-W & & & & & 74 & $\mathrm{e}$ & 74 \\
\hline $\mathrm{BNL}$ & & & & 480 & $<1$ & $<1$ & 482 \\
\hline ETEC & & & & 528 & $<1$ & 19 & 548 \\
\hline FEMP & & & & & 31 & $\mathrm{~d}$ & 31 \\
\hline FNAL & & & & 34 & $\mathrm{~d}$ & & 34 \\
\hline Hanford & & 450 & 72 & 3,902 & 840 & 7 & 5,271 \\
\hline INEL & & & & 2,624 & 180 & $<1$ & 2,805 \\
\hline ITRI & & & & 33 & $\mathrm{~d}$ & & 33 \\
\hline $\mathrm{K}-25$ & & & & 2,922 & 269 & 322 & 3,513 \\
\hline $\mathrm{KCP}$ & & & & $<<1$ & $\mathrm{~d}$ & & $\ll 1$ \\
\hline LANL & & 262 & 1 & 1,585 & 21 & 69 & 1,938 \\
\hline LBL & & & & 24 & 1 & $<1$ & 26 \\
\hline LLNL & & 27 & & 124 & 353 & & 504 \\
\hline Mound & & & & 687 & 1 & 7 & 695 \\
\hline NR sites & & $<<1$ & 1 & 1,818 & 31 & 22 & 1,872 \\
\hline NTS & & & & 3 & 530 & & 533 \\
\hline ORISE & & & & 10 & d & & 10 \\
\hline ORNL & & 9 & 12 & 1,668 & 113 & 20 & 1,822 \\
\hline $\mathrm{PAD}$ & & & & & & 1,061 & 1,061 \\
\hline PANT & & & & 139 & 184 & 358 & 681 \\
\hline Pinellas & & & & 201 & $\mathrm{~d}$ & & 201 \\
\hline PORTS & & & & & 709 & 260 & 969 \\
\hline PPPL & & & & 22 & d & & 22 \\
\hline RFETS & & 108 & & 549 & 801 & d & 1,458 \\
\hline SLAC & & & & 15 & d & & 15 \\
\hline SNL/CA & & & & 171 & d & & 171 \\
\hline SNL/NM & & d & & 77 & 68 & & 145 \\
\hline SRS & & 427 & 4 & 10,043 & 1,135 & & 11,605 \\
\hline WVDP & & 5 & & 686 & 8 & 36 & 735 \\
\hline$Y-12$ & & & & 591 & 346 & 52 & 989 \\
\hline Others $\mathrm{f}$ & & $<<1$ & & & 7 & & 7 \\
\hline Total & $0 g$ & 1,288 & 90 & 29,199 & 5,725 & 2,234 & 38,536 \\
\hline
\end{tabular}

aprojected annual addition to as-generated waste stored inventory during 1995. Includes contributions from both mixed and nonmixed wastes.

Excludes wastes from DOE environmental restoration programs.

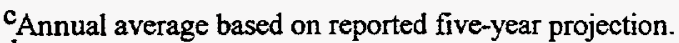

Information unavailable or unknown.

eTrace amount.

$\mathrm{f}_{\text {Includes contributions from } 14 \text { sites. }}$

gFrom SNF reprocessing. (No SNF was reprocessed during CY 1995.) 
Table 0.6. Volume $\left(\mathrm{m}^{3}\right)$ inventory of stored DOE site SNF and radioactive wastes as of December 31, 1995

\begin{tabular}{|c|c|c|c|c|c|c|c|c|}
\hline \multirow{2}{*}{ Site(s) } & \multirow{2}{*}{ SNF } & \multirow{2}{*}{ HLW } & \multicolumn{2}{|c|}{ TRUW } & \multirow{2}{*}{ LLW } & \multicolumn{2}{|c|}{ MLLW } & \multirow{2}{*}{ Total (site) } \\
\hline & & & $\mathrm{CH}$ & $\mathrm{RH}$ & & RCRA & TSCA & \\
\hline Ames & & & & & 18 & & 1 & 19 \\
\hline$A N L-E$ & 0.1 & & 25 & & 564 & 34 & 57 & 680 \\
\hline ANL-W & 9 & & 3 & 22 & & 412 & 13 & 459 \\
\hline $\mathrm{BNL}$ & 9 & & & & 297 & 24 & 3 & 333 \\
\hline ETEC & & & 2 & 6 & 425 & 1 & & 434 \\
\hline FEMP & & & & & 140,000 & 2,390 & & 142,390 \\
\hline FNAL & & & & & 91 & $b$ & & 91 \\
\hline Hanford & 378 & 233,500 & 11,450 & 273 & & 8,170 & 103 & 253,874 \\
\hline INEL & 493 & 11,170 & 39,300 & 200 & 18,634 & 25,734 & 2 & 95,533 \\
\hline ITRI & & & & & 50 & & & 50 \\
\hline $\mathrm{K}-25$ & & & & & 13,638 & 23,076 & 8,159 & 44,873 \\
\hline $\mathrm{KCP}$ & & & & & $<<1$ & & & $<<1$ \\
\hline LANL & $<1$ & & 11,262 & 94 & & 609 & 6,192 & 18,158 \\
\hline LBL & & & $<I$ & & 35 & 6 & $<1$ & 43 \\
\hline ILNL & & & 257 & & 644 & 454 & $<1$ & 1,356 \\
\hline Mound & & & 274 & & 3,392 & 97 & 45 & 3,808 \\
\hline NR sites & 88 & & & $<1$ & 3 & 77 & 18 & 187 \\
\hline NTS & & & 616 & & 301 & 306 & $\ll<1$ & 1,223 \\
\hline ORISE & & & & & $\ll 1$ & $b$ & & b \\
\hline ORNL & 17 & & 1,339 & 1,842 & 1,842 & 2,841 & 16 & 7,897 \\
\hline $\mathrm{PAD}$ & & & 2 & & 5,200 & 1,032 & 3,930 & 10,164 \\
\hline PANT & & & $<1$ & & 208 & 132 & 3 & 344 \\
\hline Pinellas & & & & & 124 & & & 124 \\
\hline PORTS & & & & & 9,000 & 8,011 & 3,600 & 20,611 \\
\hline \multicolumn{9}{|l|}{ PPPL } \\
\hline RFETS & & & 1,978 & & 5,463 & 13,558 & 191 & 21,190 \\
\hline SLAC & & & & & 174 & $\mathrm{c}$ & & 174 \\
\hline SNL/CA & & & & & 26 & & & 26 \\
\hline SNL/NM & 6 & & 7 & & 360 & 70 & & 443 \\
\hline SRS & 130 & 126,500 & 6,977 & & 1,616 & 6,940 & 110 & 142,273 \\
\hline WVDP & 11 & 2,200 & 43 & 529 & 14,936 & 148 & 82 & 17,949 \\
\hline$Y-12$ & & & & & 2,934 & 13,730 & 941 & 17,605 \\
\hline Others & 140 & & & 581 & 25,800 & 59 & & 26,580 \\
\hline Total & 1,282 & 373,370 & 73,537 & 3,548 & 245,775 & 107,911 & 23,468 & 828,891 \\
\hline
\end{tabular}

aProjected for the end of CY 1995. Includes both mixed and nonmixed as-generated wastes. $b_{\text {Information unavailable or unknown. }}$ 
Table 0.7. Volume $\left(m^{3}\right)$ inventory of buried DOE site wastes ${ }^{a, b, c, d}$

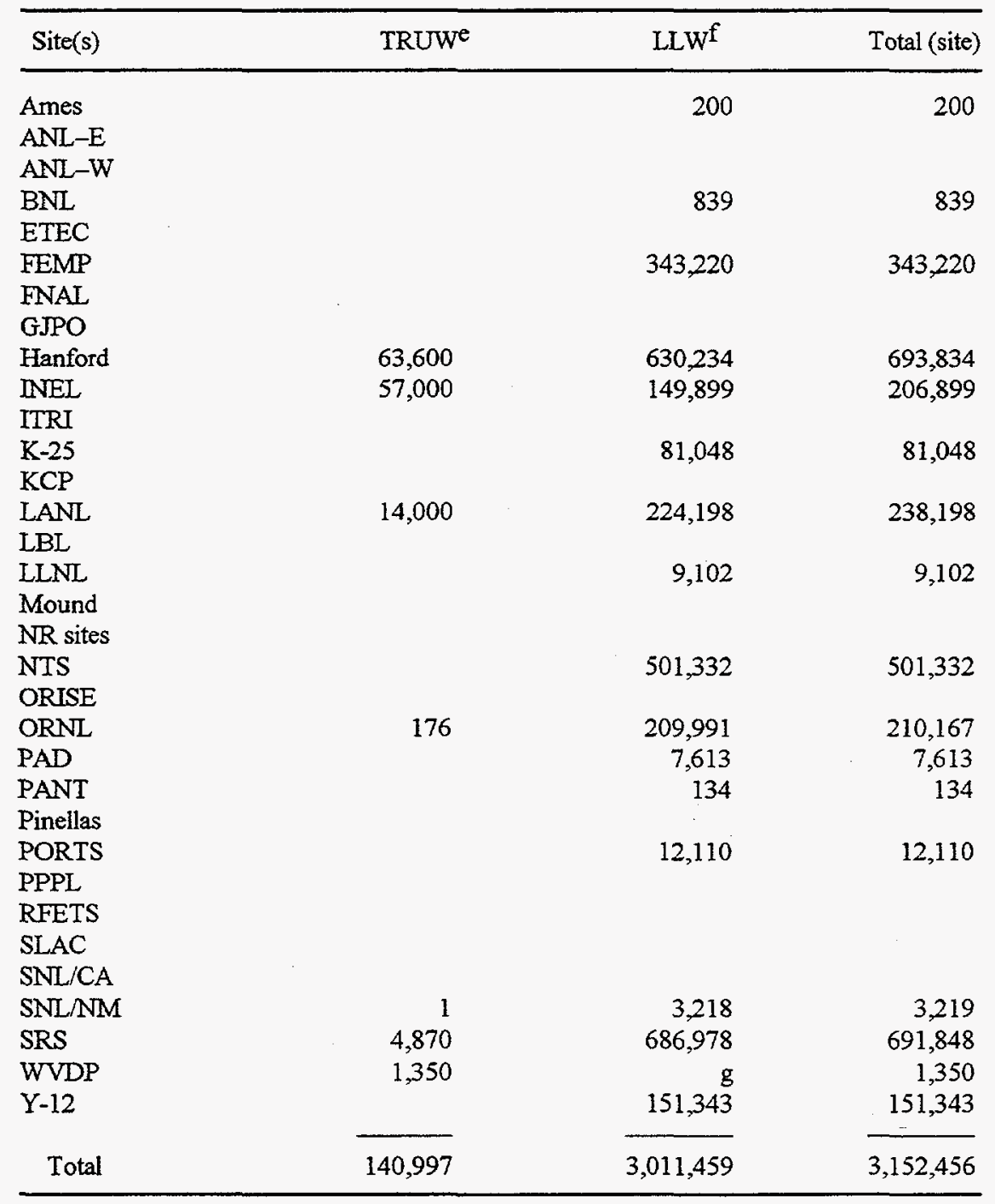

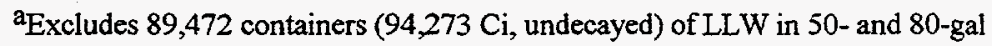
drums disposed of by sea dumping.

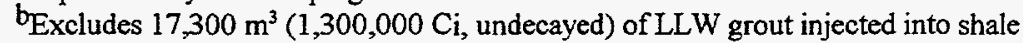
underlying the ORNL site.

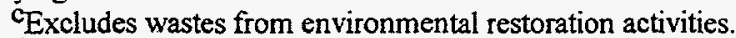

dExcludes contributions from DOE wastes disposed of at the Envirocare Facility as of the end of 1995 . See Table 0.8 .

eAs of December 31, 1994.

$f_{A s}$ of December 31,1995 . The data listed for LLW represent disposed inventories and include materials that are not buried.

gonly commercial LLW is buried at WVDP. 
Table 0.8. Volume $\left(\mathrm{m}^{3}\right)$ summary of radioactive wastes disposed of at the Envirocare Facility near Clive, Utah ${ }^{\mathrm{a}}$

\begin{tabular}{|c|c|c|c|c|c|c|c|c|}
\hline \multirow{2}{*}{ Waste type } & \multicolumn{2}{|c|}{ DOE wastes } & \multicolumn{2}{|c|}{ Commercial wastes } & \multicolumn{2}{|c|}{ Other wastes ${ }^{b}$} & \multicolumn{2}{|c|}{ Total radioactive wastes } \\
\hline & 1995 & Cumulative & 1995 & Cumulative & 1995 & Cumulative & 1995 & Cumulative \\
\hline$L L W^{c}$ & 11,698 & 14,118 & 12,860 & 133,290 & 483 & 8,286 & 25,041 & 155,694 \\
\hline MLLW & 4,398 & 5,667 & 2,821 & 6,713 & 3,676 & 7,763 & 10,895 & 20,143 \\
\hline NARM & 0 & 0 & 591 & 12,524 & 37,053 & 265,110 & 37,644 & 277,634 \\
\hline $11 e(2)^{d}$ & 21,098 & 24,922 & 62,343 & 102,510 & 0 & 0 & 83,441 & 127,432 \\
\hline Total & 37,194 & 44,707 & 78,615 & 255,037 & 41,212 & 281,159 & 157,021 & 580,903 \\
\hline
\end{tabular}

aBased on ref. 19.

bWastes from activities supported by DOD and EPA.

${ }^{\mathrm{c}} \mathrm{LLW}$ received from nonroutine operations, such as cleanups.

By-product material as defined in Sect. 1 le(2) of the Atomic Energy Act of 1954 (Pub. L. 83-703). 


\section{SPENT NUCLEAR FUEL}

\subsection{INTRODUCTION}

This chapter reports the quantities and characteristics of spent nuclear fuel (SNF) that has been permanently discharged from commercial LWRs and one-of-a-kind reactors. In addition, this chapter contains a mass summary report of DOE SNE (see Sect. 1.4). Though currently in storage at numerous commercial and DOE sites, this fuel will ultimately require geologic disposal.

Some commercial SNF in inventory will be reinserted into reactors for further irradiation. However, this amount is relatively small, and the schedules for reinsertions are not always predictable. Therefore, for the purposes of this report all SNF is considered permanently discharged from the reactors.

Historical inventories of commercial LWR SNF have been updated through December 31, 1995. ${ }^{1}$ The data reported in this chapter include the inventories of SNF stored at the West Valley Demonstration Project (WVDP), the Midwest Fuel Recovery Plant (MFRP), and the Idaho National Engineering Laboratory (INEL) sites in addition to those stored at the various reactor sites. Figure 1.1 shows the locations of existing and planned power reactor sites and commercial LWR SNF storage facilities. A list of commercial reactors is given also in report DOE/ OSTI-8200-R58 (ref. 2).

Projections of nuclear capacity and SNF discharges are given for the calendar years (CY) 1996-2030 for the DOE/EIA Low Case (No New Orders) forecast schedule reported in ref. 3. Starting with an estimate for 1996 of $100.6 \mathrm{GW}(\mathrm{e})$, the Low Case (No New Orders) forecast, hereafter referred to simply as the Low Case, projects installed commercial nuclear electric capacity to start declining in the year 2000 , ultimately decreasing to $2.3 \mathrm{GW}(\mathrm{e})$ by 2030 .

The Low Case reference scenario considered for projecting accumulated SNF assumes a fuel cycle with no reprocessing. SNF discharge projections, in terms of cumulative mass discharged, are graphically illustrated for the DOE/EIA Low Case in Fig. 1.2.

DOE/EIA projections for the Low Case assume that current average equilibrium burnup levels of discharged
SNF will be 33,000 and 41,000 MWd/MTIHM for BWR and PWR fuel, respectively. ${ }^{3}$

\subsection{COMMERCIAL SPENT NUCLEAR FUEL}

\subsubsection{Inventories and projections}

The total inventory of commercial LWR SNF in storage at the WVDP site, the MFRP, INEL, and the reactor sites as of December 31, 1995, amounted to 32,022 MTIHM. Of this total amount, 26 MTIHM are in storage at the WVDP site, ${ }^{4} 674$ MTIHM are in storage at the MFRP, ${ }^{1}$ and 42 MTIHM are in storage at INEL. ${ }^{1}$ The remainder is stored at the reactor sites. These inventories do not include the SNF reprocessed at the WVDP site when the facility was operated as a fuel reprocessing plant. The WVDP SNF inventories include 125 LWR fuel assemblies (representing 26 MTIHM), which are owned by $D O E$.

A BWRPWR breakdown of the electric power generating capacity for the Low Case forecast, along with historical reactor capacity data, is given in Table 1.1. The buildups of permanently discharged LWR SNF mass are given on a historical basis in Table 1.2 and for the projected DOE/EIA Low Case in Table 1.3.

\subsubsection{Characterization}

Reference characteristics of BWR and PWR fuel assemblies, obtained from refs. 5 and 6 , are summarized in Table 1.4. More detailed information on SNF characteristics may be found in ref. 7 .

\subsection{DISPOSAL}

Through the first half of 1995, the Office of Civilian Radioactive Waste Management (OCRWM) met regulatory milestones by completing the first Technical Basis Report on surface processes and by submitting the 
Annotated Outline for the License Application. Extensive surface-based and underground investigation is being conducted. This investigation is essential to evaluate the suitability of the Yucca Mountain site before a license application can be submitted to the Nuclear Regulatory Commission (NRC). This work is well underway and will lead to a determination of the suitability of the Yucca Mountain site in 1998.

Tumnel excavation is proceeding well despite ground conditions that have been more difficult than anticipated. The assembly of the 25-ft-diam Tunnel Boring Machine (TBM) was completed, and the TBM was moved into the Starter Tunnel in early August 1994. The TBM has excavated over 3.5 miles of the 5-mile exploratory studies facility tumnel. A revised program plan was issued in June 1996. Primary emphasis remains on evaluating the suitability of the Yucca Mountain site.

The DOE Office of Civilian Radioactive Waste Management (OCRWM) is developing an approach for the performance of its waste acceptance, storage, and transportation responsibilities as set forth in the Nuclear Waste Policy Act (NWPA), as amended, and in the Standard Contract for disposal of SNF and HLW (see Glossary of Terms). A presolicitation conference was held on July 9,1996 , to discuss technical and contractual issues related to the potential acquisition of transportation services. At the presolicitation conference, OCRWM made available a draft Statement of Work and a draft Concept of Operations for the waste acceptance and transportation services acquisition. The Department anticipates that a draft request for proposals (RFP) should be issued for public review and comment in December 1996 , followed by another presolicitation conference in February or March 1997.

OCRWM is pursuing an acquisition process that is market-driven, relying on private industry (contractors) to provide all necessary services and equipment to fulfill OCRWM's mission at competitive fixed prices and fixed rates. The contractors would accept SNF from its owners and generators (purchasers) and supply casks and equipment for transporting SNF to (and possibly storage at) a designated federal facility. Contractors would work with purchasers to determine the best way to service a site and would recommend to the $\mathrm{NRC}$ preferred transportation routes to a federal facility.

OCRWM may award up to four contracts, covering four regions of the United States. The intent is to develop a market infrastructure to foster competition and innovative approaches to waste acceptance and transportation. The phased approach to contract implementation would allow flexibility and would include sequential development of business/servicing plans that would describe each contractor's general approach, how it might acquire casks and equipment, and its approach to transportation operations.

\subsection{DOE SPENT NUCLEAR FUEL}

Summary inventory characteristics of DOE SNF as of June 1996 are given in Table 1.5 (based on ref. 4). Projected site inventories for CY 2035 are also included.

For purposes of clarification, the quantities of SNF reported in Table 1.5 include contributions from other nuclear fuels besides those permanently discharged from production reactors. SNFs reported in this table also include DOE-owned nuclear fuel that has been withdrawn from or resides for storage in a nuclear reactor following irradiation, the constituent elements of which have not been separated by processing. In addition to intact fuel, reactor-irradiated fuel materials requiring special handling (e.g., defective fuel and special fuel forms) are also considered SNF and are eligible for inclusion in Table 1.5. This table also lists some commercially generated nuclear fuels and nuclear fuels from foreign reactors and university research reactors which are stored at DOE sites. More detailed information on these special nuclear fuels will be included in future updates of this report.

\subsection{REFERENCES}

1. U.S. Department of Energy, Energy Information Administration, Nuclear Fuel Data Form RW-859, Washington, D.C. (data as of December 31, 1995).

2. U.S. Department of Energy, Office of Scientific and Technical Information, Nuclear Reactors Built, Being Built, or Planned: 1995, DOE/OSTI-8200-R59, Oak Ridge, Tennessee (August 1996).

3. U.S. Department of Energy, Energy Information Administration, Nuclear Power Generation and Fuel Cycle Report 1996, DOE/ELA-0436(96), Washington, D.C. (October 1996). 
4. Elwood P. Stroupe, National Spent Nuclear Fuel Program, Idaho National Engineering Laboratory, Idaho Falls, Idaho, correspondence to Jerry A. Klein, IDB Program, Oak Ridge National Laboratory, Oak Ridge, Tennessee, "Spent Nuclear Fuel Data for the Integrated Data Base," EPS-1 17-96, dated Nov. 19, 1996.

5. General Electric Company, General Electric Standard Safety Analysis Report, BWR/6, Docket STN 50-447, San Jose, California (1973).

6. Westinghouse Nuclear Energy Systems, Reference Safety Analysis Report, RESAR-3, Docket STN 50-480, Pittsburgh, Pennsylvania (1972).

7. U.S. Department of Energy, Characteristics of Potential Repository Wastes, Vols. 1-4, DOE/RW-0184-R1, Oak Ridge National Laboratory, Oak Ridge, Tennessee (July 1992). 


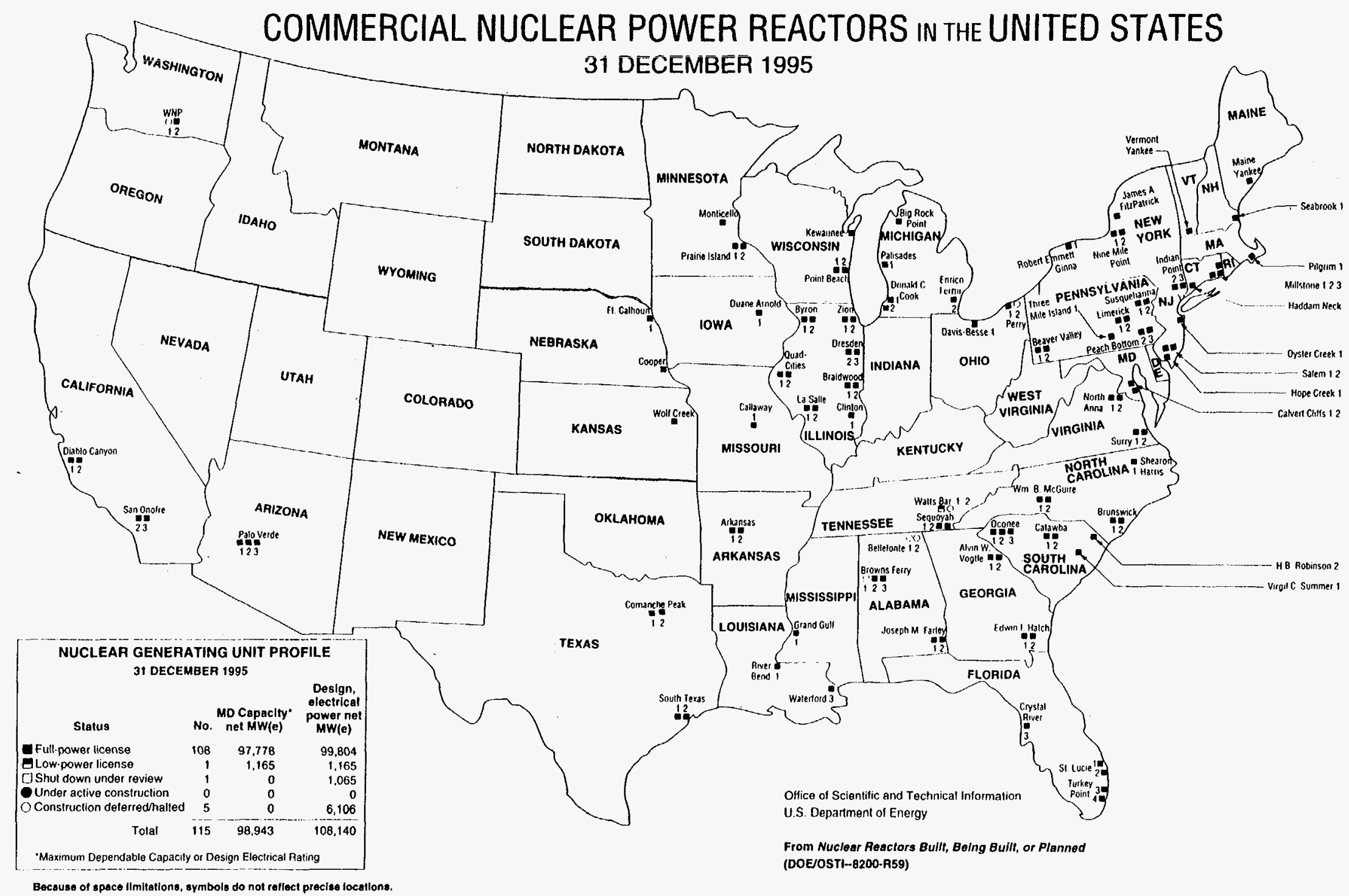

Fig. 1.1. Locations of commercial nuclear power reactors as of December 31, 1995. Courtesy of U.S. Department of Energy, Office of Scientific and Technical Information, Oak Ridge, Tennessee. 
ORNL DWG 96-3532

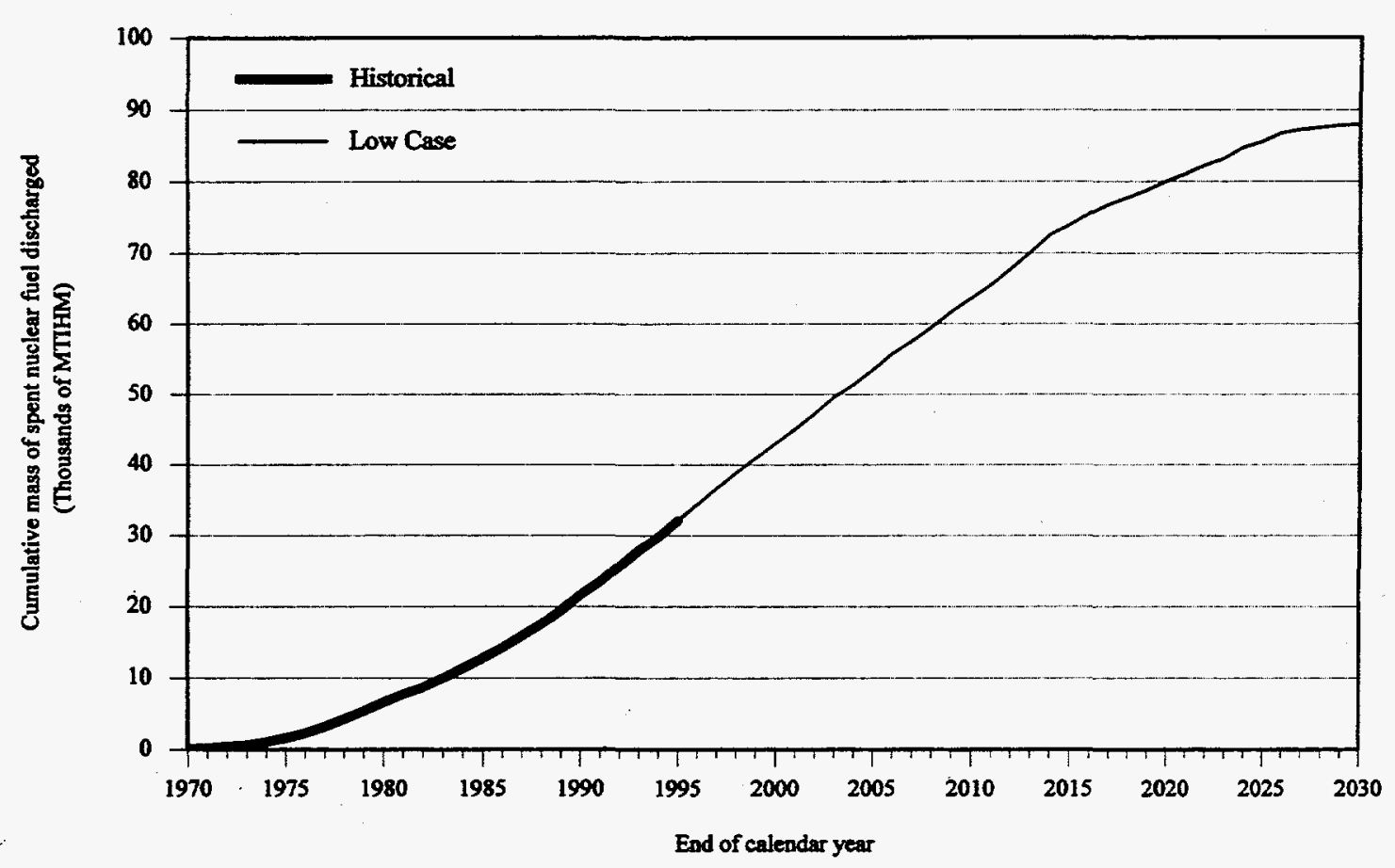

Fig. 1.2. Historical and projected mass (MTIHM) of cumulative commercial spent nuclear fuel discharged for the DOE/EIA Low Case. 
Table 1.1. Historical and projected installed LWR electric power generating capacity for the DOE/EIA Low Case

\begin{tabular}{|c|c|c|c|c|c|c|c|}
\hline \multirow{2}{*}{$\begin{array}{l}\text { End of } \\
\text { calendar } \\
\text { year }\end{array}$} & \multicolumn{3}{|c|}{$\begin{array}{l}\text { Historical capacity }{ }^{a} \\
{[G W(e)]}\end{array}$} & \multirow{2}{*}{$\begin{array}{c}\text { End of } \\
\text { calendar } \\
\text { year }\end{array}$} & \multicolumn{3}{|c|}{$\begin{array}{l}\text { Low Case projected capacity } \\
\qquad[\mathrm{GW}(\mathrm{e})]\end{array}$} \\
\hline & BWR & PWR & Total & & BWR & PWR & Total \\
\hline 1960 & 0.1 & 0.2 & 0.3 & 1996 & 33.5 & 67.1 & 100.6 \\
\hline 1961 & 0.1 & 0.2 & 0.3 & 1997 & 33.5 & 67.1 & 100.6 \\
\hline 1962 & 0.1 & 0.2 & 0.4 & 1998 & 33.5 & 67.1 & 100.6 \\
\hline 1963 & 0.1 & 0.2 & 0.4 & 1999 & 33.5 & 67.1 & 100.6 \\
\hline 1964 & 0.1 & 0.2 & 0.4 & 2000 & 33.5 & 67.0 & 100.5 \\
\hline 1965 & 0.1 & 0.2 & 0.4 & 2001 & 33.5 & 67.0 & 100.5 \\
\hline 1966 & 0.1 & 0.2 & 0.4 & 2002 & 33.5 & 67.0 & 100.5 \\
\hline 1967 & 0.1 & 1.3 & 1.4 & 2003 & 33.5 & 67.0 & 100.5 \\
\hline 1968 & 0.2 & 1.2 & 1.4 & 2004 & 33.5 & 67.0 & 100.5 \\
\hline 1969 & 0.8 & 1.7 & 2.6 & 2005 & 33.5 & 67.0 & 100.5 \\
\hline 1970 & 2.9 & 2.9 & 5.8 & 2006 & 33.2 & 66.5 & 99.7 \\
\hline 1971 & 4.3 & 3.7 & 8.0 & 2007 & 32.8 & 65.6 & 98.4 \\
\hline 1972 & 7.0 & 6.5 & 13.5 & 2008 & 32.5 & 65.0 & 97.5 \\
\hline 1973 & 8.1 & 14.1 & 22.1 & 2009 & 31.9 & 63.9 & 95.8 \\
\hline 1974 & 13.3 & 19.4 & 32.7 & 2010 & 31.2 & 62.3 & 93.5 \\
\hline 1975 & 15.0 & 23.3 & 38.3 & 2011 & 30.9 & 61.8 & 92.7 \\
\hline 1976 & 16.8 & 27.9 & 44.7 & 2012 & 29.5 & 59.1 & 88.6 \\
\hline 1977 & 16.8 & 30.4 & 47.2 & 2013 & 25.4 & 50.7 & 76.1 \\
\hline 1978 & 17.6 & 32.2 & 49.8 & 2014 & 21.9 & 43.7 & 65.6 \\
\hline 1979 & 17.6 & 32.2 & 49.8 & 2015 & 21.2 & 42.5 & 63.7 \\
\hline 1980 & 17.6 & 34.3 & 51.9 & 2016 & 19.2 & 38.3 & 57.5 \\
\hline 1981 & 17.6 & 38.6 & 56.2 & 2017 & 18.2 & 36.5 & 54.7 \\
\hline 1982 & 18.7 & 40.5 & 59.2 & 2018 & 17.4 & 34.8 & 52.2 \\
\hline 1983 & 19.7 & 43.6 & 63.3 & 2019 & 17.4 & 34.8 & 52.2 \\
\hline 1984 & 24.2 & 45.8 & 70.0 & 2020 & 16.4 & 32.7 & 49.1 \\
\hline 1985 & 26.8 & 51.7 & 78.5 & 2021 & 15.3 & 30.7 & 46.0 \\
\hline 1986 & 28.9 & 55.2 & 84.1 & 2022 & 13.9 & 27.9 & 41.8 \\
\hline 1987 & 31.8 & 60.8 & 92.6 & 2023 & 12.6 & 25.1 & 37.7 \\
\hline 1988 & 31.8 & 63.1 & 94.9 & 2024 & 9.6 & 19.2 & 28.8 \\
\hline 1989 & 33.8 & 64.1 & 97.9 & 2025 & 7.4 & 14.7 & 22.1 \\
\hline 1990 & 32.9 & 66.7 & 99.6 & 2026 & 4.2 & 8.4 & 12.6 \\
\hline 1991 & 32.0 & 67.7 & 99.6 & 2027 & 2.3 & 4.7 & 7.0 \\
\hline 1992 & 31.8 & 67.1 & 98.9 & 2028 & 1.1 & 4.6 & 5.7 \\
\hline 1993 & 31.8 & 67.2 & 99.0 & 2029 & 0.0 & 3.5 & 3.5 \\
\hline 1994 & 31.9 & 67.2 & 99.1 & 2030 & 0.0 & 2.3 & 2.3 \\
\hline 1995 & 32.2 & 67.2 & 99.4 & & & & \\
\hline
\end{tabular}

aBased on ref. 1.

bata from ref. 3. Assumes that no new reactors will be ordered. Projections assume that one-third of the total capacity will be provided by BWRs and the remainder by PWRs. 
Table 1.2. Historical mass (MTHHM) of permanently discharged commercial SNF by reactor type ${ }^{a}$

\begin{tabular}{|c|c|c|c|c|c|c|}
\hline \multirow{2}{*}{$\begin{array}{l}\text { End of } \\
\text { calendar } \\
\text { year }\end{array}$} & \multicolumn{2}{|c|}{$\mathrm{BWR}^{\mathbf{b}}$} & \multicolumn{2}{|c|}{$\mathrm{PWR}^{\mathrm{c}}$} & \multicolumn{2}{|c|}{ Total LWR ${ }^{d}$} \\
\hline & Annual & Cumulative & Annual & Cumulative & Annual & Cumulative \\
\hline $1968-1970$ & & 16 & & 39 & & 55 \\
\hline 1971 & 65 & 81 & 44 & 83 & 109 & 164 \\
\hline 1972 & 146 & 226 & 100 & 183 & 246 & 410 \\
\hline 1973 & 94 & 320 & 67 & 250 & 161 & 570 \\
\hline 1974 & 242 & 562 & 208 & 458 & 449 & 1,020 \\
\hline 1975 & 226 & 787 & 322 & 780 & 548 & 1,567 \\
\hline 1976 & 298 & 1,085 & 401 & 1,181 & 699 & 2,266 \\
\hline 1977 & 383 & 1,469 & 467 & 1,648 & 850 & 3,116 \\
\hline 1978 & 384 & 1,852 & 699 & 2,346 & 1,082 & 4,199 \\
\hline 1979 & 400 & 2,252 & 721 & 3,068 & 1,121 & 5,320 \\
\hline 1980 & 620 & 2,872 & 618 & 3,686 & 1,238 & 6,558 \\
\hline 1981 & 459 & 3,331 & 676 & 4,362 & 1,135 & 7,692 \\
\hline 1982 & 357 & 3,688 & 640 & 5,002 & 998 & 8,690 \\
\hline 1983 & 491 & 4,179 & 771 & 5,773 & 1,263 & 9,952 \\
\hline 1984 & 498 & 4,677 & 841 & 6,614 & 1,339 & 11,291 \\
\hline 1985 & 532 & 5,209 & 861 & 7,475 & 1,393 & 12,684 \\
\hline 1986 & 458 & 5,667 & 996 & 8,472 & 1,454 & 14,139 \\
\hline 1987 & 597 & 6,264 & 1,109 & 9,581 & 1,706 & 15,844 \\
\hline 1988 & 536 & 6,799 & 1,117 & 10,697 & 1,652 & 17,497 \\
\hline 1989 & 698 & 7,497 & 1,215 & 11,913 & 1,913 & 19,410 \\
\hline 1990 & 633 & 8,130 & 1,504 & 13,417 & 2,137 & 21,547 \\
\hline 1991 & 588 & 8,718 & 1,271 & 14,688 & 1,859 & 23,406 \\
\hline 1992 & 695 & 9,413 & 1,596 & 16,284 & 2,291 & 25,697 \\
\hline 1993 & 700 & 10,113 & 1,532 & 17,816 & 2,232 & 27,929 \\
\hline 1994 & 675 & 10,788 & 1,207 & 19,024 & 1,882 & 29,811 \\
\hline 1995 & 627 & 11,415 & 1,514 & 20,538 & 2,141 & $31,952^{\mathrm{e}}$ \\
\hline
\end{tabular}

${ }^{a}$ Based on refs. 1 and 3.

$\mathrm{b}_{\mathrm{BWR}}=$ boiling-water reactor.

CPWR $=$ pressurized-water reactor.

$\mathrm{d}_{\mathrm{LWR}}=$ light-water reactor.

Excludes $70 \mathrm{MTHH}$ of discharged fuel assemblies that are expected to be reinserted. 
Table 1.3. Current and projected mass (MTIHM) of permanently discharged commercial LWR

SNF for the DOE/EIA Low Case ${ }^{a}$

\begin{tabular}{|c|c|c|}
\hline $\begin{array}{l}\text { End of } \\
\text { calendar } \\
\text { year }\end{array}$ & Annual & Cumulative \\
\hline $1995^{\mathrm{b}}$ & 2,141 & $32,022^{\mathrm{c}}$ \\
\hline 1996 & 2,300 & 34,300 \\
\hline 1997 & 2,400 & 36,700 \\
\hline 1998 & 2,200 & 38,900 \\
\hline 1999 & 1,900 & 40,900 \\
\hline 2000 & 2,200 & 43,100 \\
\hline 2001 & 2,000 & 45,100 \\
\hline 2002 & 2,300 & 47,300 \\
\hline 2003 & 2,300 & 49,600 \\
\hline 2004 & 1,800 & 51,400 \\
\hline 2005 & 2,000 & 53,500 \\
\hline 2006 & 2,200 & 55,700 \\
\hline 2007 & 1,800 & 57,500 \\
\hline 2008 & 2,000 & 59,500 \\
\hline 2009 & 2,200 & 61,700 \\
\hline 2010 & 1,900 & 63,600 \\
\hline 2011 & 2,000 & 65,500 \\
\hline 2012 & 2,100 & 67,700 \\
\hline 2013 & 2,500 & 70,100 \\
\hline 2014 & 2,400 & 72,500 \\
\hline 2015 & 1,400 & 73,900 \\
\hline 2016 & 1,600 & 75,500 \\
\hline 2017 & 1,300 & 76,800 \\
\hline 2018 & 1,000 & 77,800 \\
\hline 2019 & 1,000 & 78,800 \\
\hline 2020 & 1,100 & 80,000 \\
\hline 2021 & 1,000 & 81,000 \\
\hline 2022 & 1,300 & 82,200 \\
\hline 2023 & 1,000 & 83,200 \\
\hline 2024 & 1,400 & 84,700 \\
\hline 2025 & 800 & 85,500 \\
\hline 2026 & 1,200 & 86,700 \\
\hline 2027 & 600 & 87,200 \\
\hline 2028 & 200 & 87,500 \\
\hline 2029 & 300 & 87,800 \\
\hline 2030 & 100 & 87,900 \\
\hline
\end{tabular}

${ }^{a}$ Assumes no future fuel reprocessing. Note that cumulative levels reported may not equal sum of annual additions because of independent rounding.

b Actual data.

TIncludes 70 MTIHM of discharged fuel assemblies that are expected to be reinserted. 
Table 1.4. IDB reference characteristics of $L W R$ nuclear fuel assemblies

\begin{tabular}{lll}
\hline \multicolumn{1}{c}{ Characteristics } & \multicolumn{1}{c}{ BWR $^{\mathrm{a}}$} & PWR $^{\mathrm{b}}$ \\
\hline Overall assembly length, $\mathrm{m}$ & 4.470 & 4.059 \\
Cross section, cm & $13.9 \times 13.9$ & $21.4 \times 21.4$ \\
Fuel rod length, $\mathrm{m}$ & 4.064 & 3.851 \\
Active fuel height, $\mathrm{m}$ & 3.759 & 3.658 \\
Fuel rod outer diameter, cm & 1.252 & 0.950 \\
Fuel rod array & $8 \times 8$ & $17 \times 17$ \\
Fuel rods per assembly & 63 & 264 \\
Assembly total weight, $\mathrm{kg}$ & 319.9 & 657.9 \\
Uranium/assembly, $\mathrm{kg}$ & 183.3 & 461.4 \\
UO $/$ assembly, $\mathrm{kg}$ & 208.0 & 523.4 \\
Zircaloy/assembly, $\mathrm{kg}$ & $103.3^{\mathrm{c}}$ & $108.4^{\mathrm{d}}$ \\
Hardware/assembly, $\mathrm{kg}$ & $8.6^{\mathrm{e}}$ & $26.1^{\mathrm{f}}$ \\
Total metalassembly, $\mathrm{kg}$ & 111.9 & $134.5^{\mathrm{g}}$ \\
Nominal volume/assembly, $\mathrm{m}^{3}$ & $0.0864 \mathrm{~g}$ & $0.186 \mathrm{~g}$ \\
\hline
\end{tabular}

aRef. 5.

bef. 6.

Includes Zircaloy fuel-rod spacers and fuel channel.

dincludes Zircaloy control-rod guide thimbles.

Includes stainless steel tie-plates, Inconel springs, and plenum springs.

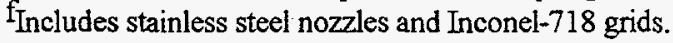

SBased on overall outside dimension. Includes spacing between the stacked fuel rods of an assembly. 
Table 1.5. Summary inventory of DOE SNF for years 1996 and $2035^{a}$

\begin{tabular}{|c|c|c|c|c|c|}
\hline \multirow{2}{*}{ Site/category } & \multirow{2}{*}{ Facility ${ }^{b}$} & \multirow{2}{*}{$\begin{array}{c}1996 \\
\text { total mass } \\
(\mathrm{kg})\end{array}$} & \multirow{2}{*}{$\begin{array}{c}1996 \\
\text { volume } \\
\left(\mathrm{m}^{3}\right)\end{array}$} & \multicolumn{2}{|c|}{$\begin{array}{l}\text { Discharged heavy metal } \\
\text { (MTHM) }\end{array}$} \\
\hline & & & & 1996 & 2035 \\
\hline \multirow[t]{2}{*}{$\mathrm{ANL} \sim \mathrm{E}$} & $\begin{array}{l}\text { Alpha-gamma hot cell } \\
\text { Chicago Pile-5 }\end{array}$ & $\begin{array}{r}257.10 \\
12.80\end{array}$ & $\begin{array}{l}0.10 \\
0.01\end{array}$ & $\begin{array}{l}0.08 \\
0.00\end{array}$ & $\begin{array}{l}0.08 \\
0.00\end{array}$ \\
\hline & Subtotal & 269.90 & 0.12 & 0.08 & 0.08 \\
\hline \multirow[t]{5}{*}{ ANL-W } & EBR-II & $10,242.05$ & 0.84 & 8.72 & 8.72 \\
\hline & HFEF & $5,172.90$ & 0.95 & 0.61 & 0.61 \\
\hline & RSWF & $24,878.44$ & 2.34 & 18.83 & 18.83 \\
\hline & TREAT & 206.81 & 4.95 & 0.02 & 0.02 \\
\hline & Subtotal & $40,500.20$ & 9.08 & 28.18 & 28.18 \\
\hline \multirow[t]{3}{*}{$\mathrm{BNL}$} & BMRR & 165.46 & 0.13 & 0.00 & 0.01 \\
\hline & HFBR & $4 ; 263.69$ & 8.46 & 0.25 & 1.34 \\
\hline & Subtotal & $4,429.15$ & 8.59 & 0.26 & 1.34 \\
\hline FRR & FRR & - & - & - & 16.37 \\
\hline FSVR & ISFSI & $187,392.00$ & 130.27 & 14.73 & 14.73 \\
\hline \multirow[t]{12}{*}{ Hanford } & Area 200 (burial) & 801.95 & 143.79 & 0.32 & 0.32 \\
\hline & Area 400 (ISA) & 317.60 & 0.08 & 0.02 & 0.02 \\
\hline & Area 618 (burial) & 0.00 & 0.00 & 0.00 & 0.00 \\
\hline & Bldg. 324 & $4,077.50$ & 1.19 & 2.28 & 2.28 \\
\hline & Bldg. 325 & 50.00 & 0.00 & 0.01 & 0.01 \\
\hline & Bldg. 327 & 39.00 & 0.01 & 0.02 & 0.02 \\
\hline & FFTF & $56,083.89$ & 18.28 & 11.02 & 11.02 \\
\hline & PFP & $1,023.40$ & 0.02 & 0.01 & 0.01 \\
\hline & T-plant & $38,500.00$ & 9.45 & 15.82 & 15.82 \\
\hline & 105-K East Basin & $1,723,772.00$ & 99.94 & $1,146.43$ & $1,146.43$ \\
\hline & 105-K West Basin & $1,804,017.00$ & 104.91 & 956.05 & 956.05 \\
\hline & Subtotal & $3,628,682.34$ & 377.67 & $2,131.98$ & $2,131.98$ \\
\hline \multirow[t]{11}{*}{ INEL } & ICPP-603 & $20,548.68$ & 9.38 & 2.03 & 2.03 \\
\hline & ICPP-666 & $461,754.34$ & 148.54 & 14.56 & 14.56 \\
\hline & ICPP-749 & $257,781.88$ & 96.55 & 78.60 & 78.60 \\
\hline & ICPP-IFSF & $131,390.79$ & 86.11 & 10.02 & 10.02 \\
\hline & MTR canal & $1,990.70$ & 0.75 & 0.26 & 0.26 \\
\hline & PER-620 & $5,580.50$ & 0.84 & 0.56 & 0.56 \\
\hline & TAN-607 & $333,480.56$ & 131.85 & 85.29 & 85.29 \\
\hline & TAN-790 & $56,375.01$ & 11.78 & 38.37 & 38.37 \\
\hline & TRA-660 & 577.62 & 0.46 & 0.23 & 0.23 \\
\hline & TRA-670 & $6,578.04$ & 6.74 & 0.69 & 2.53 \\
\hline & Subtotal & $1,276,058.12$ & 493.00 & 230.59 & 232.44 \\
\hline INEL/NRF & ECF & $381,784.69$ & 88.34 & 6.01 & 6.01 \\
\hline LANL & CMR Building & 421.40 & 0.60 & 0.01 & 0.01 \\
\hline
\end{tabular}


Table 1.5 (continued)

\begin{tabular}{|c|c|c|c|c|c|}
\hline \multirow[t]{2}{*}{ Site/category } & \multirow[t]{2}{*}{ Facility ${ }^{b}$} & \multirow{2}{*}{$\begin{array}{c}1996 \\
\text { total mass } \\
(\mathrm{kg})\end{array}$} & \multirow{2}{*}{$\begin{array}{c}1996 \\
\text { volume } \\
\left(\mathrm{m}^{3}\right)\end{array}$} & \multicolumn{2}{|c|}{$\begin{array}{l}\text { Discharged heavy metal } \\
\text { (MTHM) }\end{array}$} \\
\hline & & & & 1996 & 2035 \\
\hline \multirow[t]{8}{*}{ ORNL } & Bldg. 7827 & $1,978.90$ & 4.01 & 0.15 & 0.15 \\
\hline & Bldg. 7829 & 782.40 & 0.48 & 0.03 & 0.03 \\
\hline & Bldg. 7920 & 204.30 & 0.10 & 0.00 & 0.00 \\
\hline & Bldg. 7900 pools & $9,233.40$ & 7.86 & 0.47 & 1.15 \\
\hline & BSR & 327.04 & 0.40 & 0.06 & 0.06 \\
\hline & MSR & $8,940.00$ & 3.88 & 0.04 & 0.04 \\
\hline & TSR & 182.00 & 0.10 & 0.01 & 0.01 \\
\hline & Subtotal & $21,648.04$ & 16.82 & 0.75 & 1.43 \\
\hline \multirow[t]{5}{*}{ SNL/NM } & ACRR & 100.00 & 0.09 & 0.00 & 2.36 \\
\hline & Dense pack & 900.00 & 1.12 & 0.10 & 0.10 \\
\hline & HCF & 15.00 & 0.00 & 0.00 & 0.00 \\
\hline & Manzano storage & $12,800.00$ & 5.20 & 0.00 & 0.00 \\
\hline & Subtotal & $13,815.00$ & 6.41 & 0.10 & 2.46 \\
\hline \multirow[t]{9}{*}{ SRS } & F-Canyon & $23,268.24$ & 5.20 & 22.61 & 22.61 \\
\hline & H-Canyon & 430.00 & 0.42 & 0.07 & 0.07 \\
\hline & K-Basin & $20,400.00$ & 29.14 & 3.24 & 3.24 \\
\hline & K/L/P-Basin & $2,478.40$ & 3.36 & 0.06 & 0.06 \\
\hline & L-Basin & $135,986.47$ & 43.22 & 117.96 & 117.96 \\
\hline & P-Basin & $10,700.00$ & 14.51 & 1.39 & 1.39 \\
\hline & RBOF & $96,527.97$ & 33.65 & 60.82 & 60.82 \\
\hline & SRTC & 5.28 & 0.00 & 0.00 & 0.00 \\
\hline & Subtotal & $289,796.35$ & 129.50 & 206.14 & 206.14 \\
\hline U.S. Navy & U.S. Navy Shipyards & - & - & - & 50.15 \\
\hline WVDP & Fuel Receiving and Storage Facility & $42,780.00$ & 11.34 & 26.31 & 26.31 \\
\hline \multirow[t]{16}{*}{ Other } & Non-DOE-owned domestic reactors & & & & \\
\hline & $\begin{array}{l}\text { Armed Forces Radiobiological } \\
\text { Facility }\end{array}$ & 323.00 & 0.08 & 0.02 & 0.02 \\
\hline & ARRR & 263.90 & 0.07 & 0.01 & 0.01 \\
\hline & DOW TRIGA Reactor & 265.20 & 0.06 & 0.01 & 0.01 \\
\hline & GA TRIGA Reactor Facility & 884.60 & 0.22 & 0.06 & 0.06 \\
\hline & GE Pleasanton & 80.00 & 0.05 & 0.01 & 0.01 \\
\hline & Hot cell facility (GA) & 28.90 & 0.08 & 0.01 & 0.01 \\
\hline & Lynchburg Technology Center & 77.05 & 0.03 & 0.04 & 0.04 \\
\hline & NBSR & 830.98 & 2.16 & 0.02 & 0.11 \\
\hline & Omaha VA Medical Center & 162.40 & 0.04 & 0.01 & 0.01 \\
\hline & SNRS & 306.00 & 0.07 & 0.02 & 0.02 \\
\hline & USGS Facility of Denver, Colorado & 547.40 & 0.13 & 0.03 & 0.04 \\
\hline & Subtotal & $3,769.43$ & 3.00 & 0.24 & 0.34 \\
\hline & University & & & & \\
\hline & Cornell University & 418.20 & 0.10 & 0.02 & 0.03 \\
\hline & Georgia Institute of Technology & 0.00 & 0.00 & 0.00 & 0.15 \\
\hline
\end{tabular}


Table 1.5 (continued)

\begin{tabular}{|c|c|c|c|c|c|}
\hline \multirow{2}{*}{ Site/category } & \multirow{2}{*}{ Facility ${ }^{b}$} & \multirow{2}{*}{$\begin{array}{c}1996 \\
\text { total mass } \\
(\mathrm{kg})\end{array}$} & \multirow{2}{*}{$\begin{array}{c}1996 \\
\text { volume } \\
\left(\mathrm{m}^{3}\right)\end{array}$} & \multicolumn{2}{|c|}{$\begin{array}{l}\text { Discharged heavy metal } \\
\text { (MTHM) }\end{array}$} \\
\hline & & & & 1996 & 2035 \\
\hline \multirow[t]{29}{*}{ Other (contd.) } & University (contd.) & & & & \\
\hline & Iowa State University Reactor & 38.55 & 0.19 & 0.02 & 0.02 \\
\hline & Kansas State University & 377.40 & 0.09 & 0.02 & 0.03 \\
\hline & $\begin{array}{l}\text { Massachusetts Institute of } \\
\text { Technology }\end{array}$ & 292.00 & 0.14 & 0.03 & 0.12 \\
\hline & North Carolina State University & 880.00 & 0.24 & 0.53 & 0.53 \\
\hline & Ohio State University & 200.00 & 2.14 & 0.02 & 0.02 \\
\hline & Oregon State University & 326.40 & 0.08 & 0.02 & 0.04 \\
\hline & Pennsylvania State University & 615.40 & 0.15 & 0.04 & 0.04 \\
\hline & Purdue University & 3.72 & 0.01 & 0.00 & 0.03 \\
\hline & Reed Reactor Facility & 227.80 & 0.06 & 0.01 & 0.01 \\
\hline & $\begin{array}{l}\text { Rhode Island Nuclear Science } \\
\text { Center }\end{array}$ & 99.00 & 0.10 & 0.02 & 0.17 \\
\hline & $\begin{array}{l}\text { State University of New York- } \\
\text { Buffalo }\end{array}$ & $1,000.00$ & 0.13 & 0.66 & 0.66 \\
\hline & Texas A\&M & 632.40 & 0.15 & 0.03 & 0.08 \\
\hline & University of California-Irvine & 384.20 & 0.09 & 0.02 & 0.02 \\
\hline & University of Massachusetts-Lowell & 230.00 & 0.15 & 0.00 & 0.05 \\
\hline & University of Texas-Austin & 523.60 & 0.13 & 0.03 & 0.03 \\
\hline & University of Arizona & 333.20 & 0.08 & 0.02 & 0.02 \\
\hline & University of Florida & 313.00 & 0.66 & 0.01 & 0.03 \\
\hline & University of mlinois & 662.00 & 0.16 & 0.04 & 0.05 \\
\hline & University of Maryland & 316.20 & 0.08 & 0.02 & 0.03 \\
\hline & University of Michigan & 598.00 & 0.71 & 0.10 & 0.47 \\
\hline & University of Missouri & 793.60 & 0.93 & 0.08 & 0.59 \\
\hline & University of Utah & 403.10 & 0.11 & 0.03 & 0.03 \\
\hline & University of Virginia & 191.40 & 0.19 & 0.04 & 0.09 \\
\hline & University of Wisconsin & 775.20 & 0.19 & 0.04 & 0.09 \\
\hline & Washington State University & 731.00 & 0.18 & 0.04 & 0.09 \\
\hline & Worcester Institute of Technology & 150.80 & 0.16 & 0.02 & 0.02 \\
\hline & Subtotal & $11,516.17$ & 7.41 & 1.91 & 3.57 \\
\hline & Grand total & $5,902,862.79$ & $1,282.13$ & $2,647.32$ & $2,721.56$ \\
\hline
\end{tabular}

Based on ref. 8. Inventories for 1996 are reported as of June 1996.

${ }^{b}$ Abbreviations used in this table: ACRR-Annular Core Research Reactor, ARRR-Aerotest Radiography and Research Reactor, BMRR-Brookhaven Medical Research Reactor, BSR - Bulk Shielding Reactor, CMR-Chemistry and Metallurgical Research, EBR-Experimental Breeder Reactor, ECF_-Extended Core Facility, FFTF_-Fast Flux Test Facility, FRR-Foreign Research Reactor, FSVR-Fort St. Vrain Reactor, GA-General Atomics, GE-General Electric, HCF-Hot Cell Facility, HFBR-(Brookhaven) High Flux Beam (Research) Reactor, HFEF-Hot Fuel Examination Facility, ICPP-Idaho Chemical Processing Plant, IFSF-Irradiated Fuel Storage Facility, ISA-Interim Storage Area, ISFSI-Independent Spent Fuel Storage Installation, MSR - Molten Salt Reactor, MTR-Materials Test Reactor, NBSR - National Bureau of Standards Reactor, PER-(Special) Power Excursion Reactor (Test), PFP--plutonium finishing plant, RBOF-Receiving Basin for Off-Site Fuels, RSWF-Radioactive Scrap and Waste Facility, SNRS-Stationary Neutron Radiography System, SRTC-Savannah River Technical Center, TAN-Test Area North, TRA-Test Reactor Area, TREAT-Transient Reactor Test Facility, TRIGA-Training Reactor, Isotopes, General Atomic, TSR-Tower Shielding Reactor, USGS-U.S. Geological Survey, and VA-Veterans Administration. 


\section{HIGH-LEVEL WASTE}

\subsection{INTRODUCTION}

High-level waste (HLW) is generated by the chemical reprocessing of spent research and production reactor fuel, irradiated targets, and naval propulsion fuel. HLW generally contains more than $99 \%$ of the nonvolatile fission products produced during reactor operation. HLW from a facility that recovers both uranium and plutonium contains a residual amount of about $0.5 \%$ of those elements, while $\mathrm{HLW}$ from a facility that recovers only uranium contains a residual $0.5 \%$ of the uranium and essentially all of the plutonium Most fission products have short half-lives and therefore quickly decay. $\mathrm{HLW}$ older than 1 year contains primarily the fission radionuclides ${ }^{137} \mathrm{Cs}$ and ${ }^{90} \mathrm{Sr}$ and very small amounts of transuranic (TRU) nuclides.

When generated, HLW is a highly radioactive, acidic liquid which generates heat and must be handled remotely behind heavy shielding in corrosion-resistant vessels, usually made of stainless steel. At the Hanford Site (Hanford), because stainless steel was in short supply, HLW was neutralized with caustic soda (sodium hydroxide), and sodium nitrite was then added for corrosion control so that the HLW could be stored safely in carbon-steel tanks. This practice continued at Hanford, the Savannah River Site (SRS), and the West Valley Demonstration Project (WVDP), even when stainless steel became more readily available. Neutralization with caustic soda forms sodium nitrate (which remains in solution) and hydrated oxides of certain radionuclides and nonradioactive chemicals (which precipitate and collect as a sludge on the floor of the tank). In addition, the ${ }^{137} \mathrm{Cs}$ remains largely in solution. At the Idaho National Engineering Laboratory (INEL), however, the waste has always been stored as an acidic liquid in stainless steel tanks and then converted later into a granular solid (calcine) by thermal processing, which drives off water and decomposes nitrate salts to oxides.

The supernatant liquid resulting from neutralization may become concentrated by evaporation-either by selfboiling or in evaporators. If enough water is removed from the waste, sodium nitrate and sodium nitrite will crystallize from the solution. The crystals then will settle to the bottom of the tank liquid and on top of the sludge. If there are many crystals, a salt cake will form.

To reduce heat generation in tanks, large quantities of the radionuclides ${ }^{137} \mathrm{Cs}$ and ${ }^{90} \mathrm{Sr}$ were removed from some Hanford HLW and encapsulated in concentrated form as halide salts. Some of these capsules were subsequently leased to non-DOE organizations for beneficial use. All of the leased capsules have now been returned to Hanford.

In summary, HLW exists in a variety of physical or chemical forms (alkaline or acidic, supernatant liquid, sludge, salt cake, calcine solid, etc.), all of which must be stored behind heavy shielding and usually in underground tanks or bins.

Most of the current U.S. inventory of HLW has resulted from DOE activities. $\mathrm{HLW}$ is stored at SRS (Aiken, South Carolina), INEL (Idaho Falls, Idaho), and Hanford (Richland, Washington). A small amount of HLW was generated by commercial operations at the Nuclear Fuel Services (NFS) plant, near West Valley, New York, between 1966 and 1972, at a site owned by the New York State Energy Research and Development Authority (NYSERDA). After 1972, fuel reprocessing operations at this plant were discontinued. In 1980, Congress passed the West Valley Demonstration Project Act (Pub. L. 96-368), which authorizes DOE to conduct, jointly with NYSERDA ( $90 \%$ DOE, 10\% NYSERDA), a demonstration of solidification of HLW for disposal and the decontamination and decommissioning (D\&D) of facilities used in the demonstration. The HLW data presented in this chapter are based on separate submittals provided by Hanford, INEL, SRS, and WVDP in ref. 1 .

In 1992, DOE decided to phase out the domestic reprocessing of irradiated nuclear fuel for the recovery of enriched uranium or plutonium in support of defense activities; thus, no new HLW is expected to be generated by this source for the immediate future. However, some future D\&D activities may generate waste with radioactivity levels high enough such that the waste will require management in ways similar to $\mathrm{HLW}$. An example of such a future activity is the flushing of residual wastes found in reprocessing facilities. 


\subsection{AGREEMENTS AND INTERFACES}

HLW is considered to be a mixed waste (i.e., waste containing both radioactivity and hazardous substances) unless demonstrated to the contrary. The hazardous substances of $\mathrm{HLW}$ are defined by the Resource Conservation and Recovery Act (RCRA). ${ }^{2}$ Liquid HLW is charasteristic mixed waste (i.e., as stored, it shows the characteristic of corrosivity because of its acidity or alkalinity). Some HLW may also be listed mixed waste (i.e., it: contains substances on any one of several lists regulated under $R C R A$ ). Mixed wastes must be managed according to RCRA ${ }^{2}$ and Atomic Energy Act $(\mathrm{AEA})^{3}$ requirements.

The Federal Facility Compliance Act (FFCA) ${ }^{4}$ of 1992 amends the Solid Waste Disposal Act to require (among other things) that DOE prepare a Site Treatment Plan (STP) for each site which generates, stores, or treats mixed waste. In effect, these STPs constitute a legally enforceable agreement between DOE and the host state that DOE must comply with certain requirements for mixed waste management. STPs must be approved by the host state for the site.

Two such prior agreements exist: both involve triparty arrangements. One (for Hanford) is among DOE, the U.S. Environmental Protection Agency (EPA), and the state of Washington. This tri-party agreement ${ }^{5}$ serves as an STP, is legally enforceable, and requires DOE to undertake specifici actions at Hanford on a prescriptive timetable.

The other tri-party agreement (for SRS) involves DOE, EPA, and the state of South Carolina. However, this agreement applies only to those waste storage tanks that do not meet current DOE and regulatory criteria for secondary containment and leak detection. Consequently, an STP is being prepared for SRS.

Concerning INEL, an amended Notice of Noncompliance Consent Order was issued to DOE in March 1994 by the Idaho Department of Health and Welfare. The Order includes provisions for proposing waste treatment technologies and deadlines for removing liquid waste from specific tanks. Because no prior agreements with WVDP specifically address mixed waste treatment, a proposed STP has been submitted to the state regulator for WVDP to address such treatment.

\subsection{WASTE CHARACTERIZATION}

Generic characterization of HLW at some sites has been hampered over the years by the use of several different flowsheets for the processes that generated the waste or prepared the wastes for storage (e.g., nuclide separation, precipitation, and evaporation). In some instances, wastes have been blended. Information for all sites is based on historic records of reprocessing feeds and, for Hanford, SRS, and WVDP, extensive sampling of stored HLW.

Prior to Revision 11 of this report, HLW data were presented by physical form in some detail (e.g., liquid, sludge, slurry, salt cake, and precipitate). Starting with Revision 11 and continuing in this year's revision, the data are more simply categorized as solid, liquid, or processgenerated (canistered) material. Each of these three waste categories requires different storage and processing methods. As HLW pretreatment and vitrification processes proceed, inventories of liquid and solid waste will generally decrease, and canistered material will increase.

Chemical and radionuclide compositions and inventories are given for the current and projected HLW at Hanford (Tables 2.11 and 2.16), INEL (Tables 2.12 and 2.17), SRS (Tables 2.13 and 2.18); and WVDP (Tables 2.14 and 2.19).

\subsection{INVENTORIES AND PROJECTIONS}

Tables 2.1-2.3, respectively, present historical and projected volumes, radioactivity, and thermal power inventories of HLW currently in storage. The radioactivity and resultant thermal power of $\mathrm{HLW}$ decay, over time, in a manner characteristic of the constituent radionuclides, but, as previously mentioned, the volume depends significantly on the specific treatment history of the waste. When one takes into account all radionuclides in HLW, total radioactivity and thermal power each decrease about 2 to $4 \%$ per year within storage units to which no new waste has been added.

Locations of the four HLW sites and the relative volumes of HLW are represented in Fig. 2.1. The total volume and radioactivity for the HLW (solid and liquid) stored at the four sites is shown graphically in Fig. 2.2. Historical and projected cumulative volumes of HLW stored or produced at each site are graphically illustrated in Fig. 2.3. The number of waste canisters projected to be produced by each site are depicted in Fig. 2.4.

Current DOE plans are to immobilize and package HLW for disposal in a licensed, underground geologic repository. Figures $2.5-2.8$ show, for each of the four sites, the general treatment processes by which the HLW will be immobilized to a form acceptable for disposal in a repository. Tables $2.4-2.6$, respectively, give the projected volume, radioactivity, and thermal power for $\mathrm{HLW}$ immobilized as borosilicate glass. Table 2.7 gives estimates, year by year and by site, of the number of HLW canisters to be produced based on reference flowsheets. 
Canister estimates for SRS [Defense Waste Processing Facility (DWPF)] and WVDP are fairly well established (both projects began radioactive operations in FY 1996), while canister estimates for INEL and Hanford are less certain because pretreatment and waste minimization processes have not yet been finalized. Tables 2.8-2.10 give the volume, radioactivity, and thermal power, respectively, of stored HLW by site and by physical form. Currently available summary information about the radionuclide distribution for stored $\mathrm{HLW}$ and projected associated wastes for each site has been given in Tables 2.11-2.14. Significant changes in any of these tables from the previous IDB report (Rev. 11) ${ }^{6}$ are presented in Table 2.15. It should be noted that the radioactivity reported in Tables $2.2,2.5$, and 2.9 include contributions from both parent and daughter products.

Projected inventories (volume, radioactivity, and thermal power) for $H L W$ presented in Tables 2.1-2.6 have been generated by each site based on certain assumptions and therefore should be considered only as current best estimates. It is possible that new treatment methods or waste forms may be developed and may supersede current baseline projections for Hanford or INEL. All sites are moving toward shutdown; consequently, very little additional $\mathrm{HLW}$ will be generated. Major $\mathrm{HLW}$ activities will be (a) continued safe storage, (b) preparation for solidification, (c) solidification, and (d) interim storage pending shipment to a national repository. Thus, the inventory of liquid HLW in storage generally will decrease, and the inventory of solidified $\mathrm{HLW}$ in interim storage, pending shipment to a national repository, will increase. The Waste Acceptance System Requirements Document $t^{7}$ for the national repository states that receipt of DOE HLW will begin no sooner than 6 years after initial receipt of utility spent nuclear fuel (SNF). Thus, the canisters of. solidified HLW will remain in interim storage, most likely at the production site. The current projected number of HLW canisters (Table 2.7) for Hanford, INEL, and SRS is significantly different from the value reported in the previous IDB report. For INEL, the new projections reflect a state of Idaho, Department of the Navy, and DOE Settlement Agreements completed in 1995.

Summary flowsheets of the reference solidification processes are given for Hanford (Fig. 2.5), INEL (Fig. 2.6), SRS (Fig. 2.7), and WVDP (Fig. 2.8). Overall, these flowsheets are very similar; process differences reflect differences among sites in waste characteristics.

\subsubsection{Hanford}

Hanford $\mathrm{HLW}$ is stored in underground carbon-steel tanks. The $\mathrm{HLW}$ inventory (as of December 31, 1995) consists of $143,700 \mathrm{~m}^{3}$ considered to be "solid" HLW (salt cake and sludge in single- and double-shell tanks) and $89,800 \mathrm{~m}^{3}$ of "liquid" HLW (supernatant in single- and double-shell tanks and drainable interstitial liquid in single-shell tanks), for a total of $233,500 \mathrm{~m}^{3}$. At Hanford, waste in single- and double-shell tanks consists of HLW, TRUW, and several LLWs. However, in the interim storage mode, the tanks are managed as if they contain only HLW. Thus, their contents are included in the HLW inventory.

A total of 2,217 capsules have been manufactured at Hanford, some of which have been leased off-site for beneficial purposes. Of the 1,577 cesium and 640 strontium capsules, a total of 249 cesium capsules and 35 strontium capsules have been dismantled. The inventory of capsules that have been dismantled is not expected to be returned to Hanford for interim storage and future processing. This leaves 1,328 cesium capsules and 605 strontium capsules to be processed (overpacked) and disposed of as HLW.

The HLW projections for Hanford are based on the assumptions that (1) fuel reprocessing is not resumed, (2) pumpable liquids are transferred from single-shell tanks to double-shell tanks, (3) double-shell tanks will continue to receive D\&D-generated waste, and (4) volume reduction of stored wastes through evaporation will continue.

\subsubsection{INEL}

INEL HLW is currently being stored as both acidic liquid and calcined solids (calcine). Underground, highintegrity, stainless-steel tanks contain about $7,400 \mathrm{~m}^{3}$ of acidic liquid waste. [Of this waste, only $1,100 \mathrm{~m}^{3}$ is actual $\mathrm{HLW}$; the rest is sodium-bearing waste (SBW), which is managed the same way as is HLW because of site practice.] Underground stainless-steel bins currently store about $3,800 \mathrm{~m}^{3}$ of calcine, an interim waste form. More than $90 \%$ of the total radioactivity is in the calcine.

For INEL, HLW projections are based on the recommended option from a new systems engineering approach to integrate all DOE Office of Environmental Management waste streams at the INEL. The HLW projections include streams associated "with the intermediate calcining of liquid waste, followed by separation of HLW and LLW fractions in the remaining liquid waste and redissolved calcine. No new HLW from reprocessing activities was produced after FY 1992; SNF reprocessing facilities are being placed into cold standby pending $D \& D$. The current reference waste form at INEL is a glass. According to the October 17,1995, Settlement Agreement, INEL is to calcine all of the liquid waste currently stored in the tanks by December 31,2012 . All of the HLW must be treated to be converted to the final 
waste form and be "road ready" by December 31, 2035. It is assumed that INEL will begin radioactive operations and thus produce canisters in 2020 and continue this operation through 2035 (see Fig. 2.6). The projections reported in Tables 2.1-2.7 reflect this assumption.

\subsubsection{SRS}

SRS HLW is stored as alkaline liquid, sludge, salt cake, and precipitate. The current inventory of about $126,500 \mathrm{~m}^{3}$ is stored in underground, single- and doubleshelled carbon-steel tanks. Although reprocessing operations are being phased out, the HLW tank farms are continuing to receive $\mathrm{HLW}$ from the canyons as part of cleanout operations. The preparation of some HLW for vitrification during the original in-tank precipitation demonstration generated cesium precipitate, and on-going HLW siludge-washing in extended sludge processing (ESP) has generated partially washed sludge. Pretreatment of $\mathrm{HLW}$ is performed in the In-Tank Precipitation Facility. Characterization data for SRS HLW are based on sampling and process knowledge. Allowable facility design variability of feed composition is limited; therefore, the data reported in Tables 2.1-2.6 assume a uniform feed rate and composition. The current budget projections have both (a) limited the waste-blending capability to feed a uniform composition and (b) reduced the feed rate. The radiochemical streams are assumed to be processed at a uniform rate with adjustments made for radioactive decay.

\subsubsection{WVDP}

Reprocessing at the West Valley NFS plant was terminated in 1972, after which no additional HLW has been generated. HLW at WVDP is stored in underground tanks. The current $\mathrm{HLW}$ inventory of $2,200 \mathrm{~m}^{3}$ consists of liquid alkaline waste and solid waste (composed of both alkaline sludge and inorganic zeolite ion-exchange material contaminated with radio-cesium). The waste is stored in an underground carbon-steel tank. The cesium-loaded zeolite was transferred and blended with the sludge and alkaline waste in 1995. The small amount of acidic waste remaining from reprocessing of a thorium fuel was also blended into the alkaline waste in 1995.

\subsection{SOLIDIFICATION FOR PERMANENT DISPOSAL}

All HLW will be processed and solidified to a form acceptable for permanent disposal in a geologic repository. ${ }^{8-12}$ Borosilicate glass has been selected as the reference waste form for all sites. ${ }^{13}$ Projections are based on current funding guidance provided to the sites by $D O E$.

\subsubsection{Hanford}

The current technical baseline for Hanford is to retrieve and process all ( $>99 \mathrm{vol} \%$ ) of the tank wastes using a two-phase approach which will depend on private contractors to design, construct, operate, and finance most of the required processing capability. The demonstration phase (Phase I) facilities for supernatant (liquid) pretreatment and LLW and HLW immobilization are scheduled to begin operation in June 2002 and will process waste through 2011. HLW sludges will be pretreated in-tank using water washing and caustic leaching as appropriate. Up to $13 \mathrm{vol} \%$ of the supernatant and 6 vol $\%$ of the sludges will be processed during Phase I. Full-scale production facilities, including out-of-tank sludge pretreatment, are scheduled to begin operating in 2012. These facilities will be sized to complete immobilization of LLW by 2024 and HLW by 2028 in order to meet current Tri-Party Agreement milestones.

The pretreatment processes separate the majority of the radioactivity contained in the tank waste for HLW vitrification and the bulk of the tank waste volume for LLW immobilization. The current technical baseline uses settle/decant to separate solids from the liquids, ion exchange to reduce the radioactivity in the supernatants, and caustic leaching to reduce the volume of $H L W$ sludges requiring vitrification. Other pretreatment processes that are evaluated in the Tank Waste Remediation System Program Environmental Impact Statement ${ }^{14}$ are acid dissolution, advanced separations, and organic destruction.

Hanford's reference canister for HLW disposal has a diameter of $61 \mathrm{~cm}$ and a length of $4.5 \mathrm{~m}$. The nominal glass volume is estimated at $1.1 \mathrm{~m}^{3}$ with a minimum waste oxide loading of $25 \mathrm{vol} \%$ (excluding sodium and silicon). Processing of the cesium and strontium capsules will be performed in the $\mathrm{HLW}$ vitrification facility concurrent with the $\mathrm{HLW}$ tank waste vitrification. Because the projected volume, radioactivity, and thermal power of the HLW final form have not been finalized, no data on the final LLW form are presented in Table 2.11.

An interim storage facility will be built at Hanford with sufficient capacity to store the entire $\mathrm{HLW}$ volume of glass produced by the HLW vitrification facility. Storage will continue until the HLW canisters are shipped to a geologic repository. It is assumed for planning purposes that shipment to the repository will commence no sooner than 2035. Thus values for glass volume, curies, watts and number of canisters given in Tables 2.4 through 2.7, respectively, represent the total accumulation of Hanford's HLW canisters. 


\subsubsection{INEL}

In the baseline plan, ${ }^{15}$ the New Waste Calcining Facility is assumed to operate through 2012 and to complete calcining the SBW inventory as required by the Settlement Agreement. A new separations-vitrification facility is planned to be on line by 2020 . Newly generated liquid waste and calcine will be processed to separate the high-activity radionuclides from the low-activity waste. In addition, the required land disposal restriction (LDR) treatments for the RCRA constituents in the waste will then be made as required. The high-activity waste will be vitrified in a new facility and stored until final disposition after 2035. The separated low-activity waste will be grouted and disposed. All HLW is projected to be processed by December 31, 2035, to meet the Settlement Agreement.

\subsubsection{SRS}

The plan to process SRS HLW into glass is detailed in High Level Waste System Plan Revision $5(U)^{16}$ (i.e., Rev. 5 baseline case, similar to Rev. 4), which was transmitted to DOE April 27, $1995 . \quad$ Briefly, Rev. 5 depicts three funding cases, and the data included in this report represent the baseline case with completion of the immobilization of the current inventory of HLW in FY 2021.

For SRS, canyon cleanout operations are scheduled to be completed by FY 2002. Additional HLW from canyon cleanout activities until then will represent a maximum increase of about $14.5 \%$ of current inventory. Pretreatment (sludge-washing) of liquid HLW has been started, and the DWPF began producing canisters of solidified HLW in FY 1996. The HLW glass waste forms will be stored at SRS until a national repository is ready to accept them (see Fig. 2.7).

\subsubsection{WVDP}

Pretreatment at the WVDP is complete. In May 1988, the processing of liquid HLW was initiated. This alkaline liquid was decontaminated to LLW in the WVDP Supernatant Treatment System (STS) in preparation for the incorporation of all HLW at the WVDP into a glass. In the STS, an ion-exchange process that is operated in a batch mode is used to remove cesium from the alkaline liquid waste (see Fig. 2.8). The ion-exchange columns are located in the underground carbon-steel tank, which was originally installed as a backup tank for the storage of alkaline HLW. The sludge in the bottom of the tank has been mixed with the residual supernatant and an alkaline solution. Both shudge-wash processing cycles were completed in 1994. The wash solutions are also treated in the STS before they are incorporated in cement. The washed sludge, the acidic waste, and the loaded zeolite will be combined and incorporated into a glass. The primary vitrification campaign will begin in FY 1996 and be completed by FY 1998. Tank heels and residual material will then continue to be vitrified through mid-FY 2001. The glass will be stored on-site until it is transferred to a federal repository.

\subsection{REFERENCES}

1. DOE site HLW data submittal attachments, submitted to the DB Program during September-October 1996. The following HLW submittals were received and reviewed by the IDB Program before analysis and integration. Preceding each submittal is the site (in parentheses) to which it refers.

a. (Hanford) William J. Taylor, DOE Richland Operations Office, Richland, Washington, correspondence to Robin L. Sweeney, High-Level Waste Program Manager, DOE-HQ, copy to Steve Loghry, IDB Program, ORNL, Oak Ridge, Tennessee, "High-Level Waste (HLW) Information Request for the 1996 Integrated Data Base Report," 96-WDD-098, dated July 15, 1996.

b. (INEL) D. A. Knecht, Lockheed Martin Idaho Technologies Company, Idaho Falls, Idaho, correspondence to P. J. Dirkmaat, DOE-ID, Idaho Falls, Idaho, copy to S. Loghry, IDB Program, ORNL, Oak Ridge, Tennessee, "INEL HLW 1996 Integrated Data Base Submittal for 12/31/95 Waste Quantities," DAK-07-95, dated July 29, 1996.

c. (SRS) J. R. Hester, Westinghouse Savannah River Company, Aiken, South Carolina, correspondence to Steve Loghry, IDB Program, ORNL, Oak Ridge, Tennessee, "1996 DOE Integrated Data Base Input," dated June 17, 1996. 
d. (WVDP) P. E. Walters, West Valley Nuclear Services Company, Inc., West Valley, New York, correspondence to Steve Loghry, IDB Program, ORNL, Oak Ridge, Tennessee, "Submittal of High-Level Waste Information for the 1996 Integrated Data Base Report," WZ:96:0070, dated Aug. 1, 1996.

2. U.S. Congress, Resource Conservation and Recovery Act of 1976, Pub. L. 94-580, 1976, as amended.

3. U.S. Congress, Atomic Energy Act of 1954, Pub. L. 83-703, Aug. 15, 1994.

4. U.S. Congress, The Federal Facility Compliance Act of 1992, Pub. L. 102-386, Oct. 6, 1992.

5. Washington State Department of Ecology, U.S. Environmental Protection Agency, and U.S. Department of Energy, Hanford Federal Facility Agreement and Consent Order, EPA Docket Number 1089-03-040120, Ecology Docket Number 89-54, Richland, Washington (May 1989).

6. U.S. Department of Energy, Integrated Data Base Report-1994: U.S. Spent Nuclear Fuel and Radioactive Waste Inventories, Projections, and Characteristics, DOE/RW-0006, Rev. 11, Oak Ridge National Laboratory, Oak Ridge, Tennessee (September 1995).

7. U.S. Department of Energy, Office of Civilian Radioactive Waste Management, Waste Acceptance System Requirements Document, DOE/RW-0351P, Rev. 1, Washington, D.C., (March 1994).

8. U.S. Department of Energy, Final Environmental Impact Statement, Disposal of Hanford Defense High-Level, Transuranic, and Tank Waste, Hanford Site, Richland, Washington, DOE/EIS-0113, DOE Richland Operations Office, Richland, Washington (December 1987).

9. U.S. Congress, The Nuclear Waste Policy Act of 1982, Pub. L. 97-425, Sect. 8, Jan. 7, 1983, as amended.

10. Ronald Reagan, President of the United States, Washington, D.C., letter to John S. Herrington, Secretary of Energy, "Disposal of Defense Waste in a Commercial Repository," dated Apr. 30, 1985.

11. U.S. Department of Energy, "Civilian Radioactive Waste Management: Calculating Nuclear Waste Fund Disposal Fees for Department of Energy Defense Program Waste; Notice," Fed. Regis. 56(161), 31508 (Aug. 20, 1987).

12. U.S. Congress, The Nuclear Waste Policy Amendments Act of 1987, Pub. L. 100-203, Title V, Subtitle A, Dec. 22, 1987.

13. U.S. Department of Energy, Office of Defense Waste and Transportation Management, Defense Waste and Transportation Management Program Implementation Plan, DOE/DP-0059, Washington, D.C. (August 1988).

14. U.S. Department of Energy and Washington State Department of Ecology, Tank Waste Remediation System, Hanford Site, Richland, Washington-Final Environmental Impact Statement," DOE/EIS-0189, DOE Richland Operations Office, Richland, Washington (August 1996).

15. U.S. Department of Energy, Integration of EM Activities at the INEL, Idaho National Engineering Laboratory, Idaho Falls, Idaho (March 31, 1995).

16. U.S. Department of Energy, High-Level Waste System Plan Revision $5(U)$, HLW-OVP-95-0031, Savannah River Site, Aiken, South Carolina (April 26, 1995). 


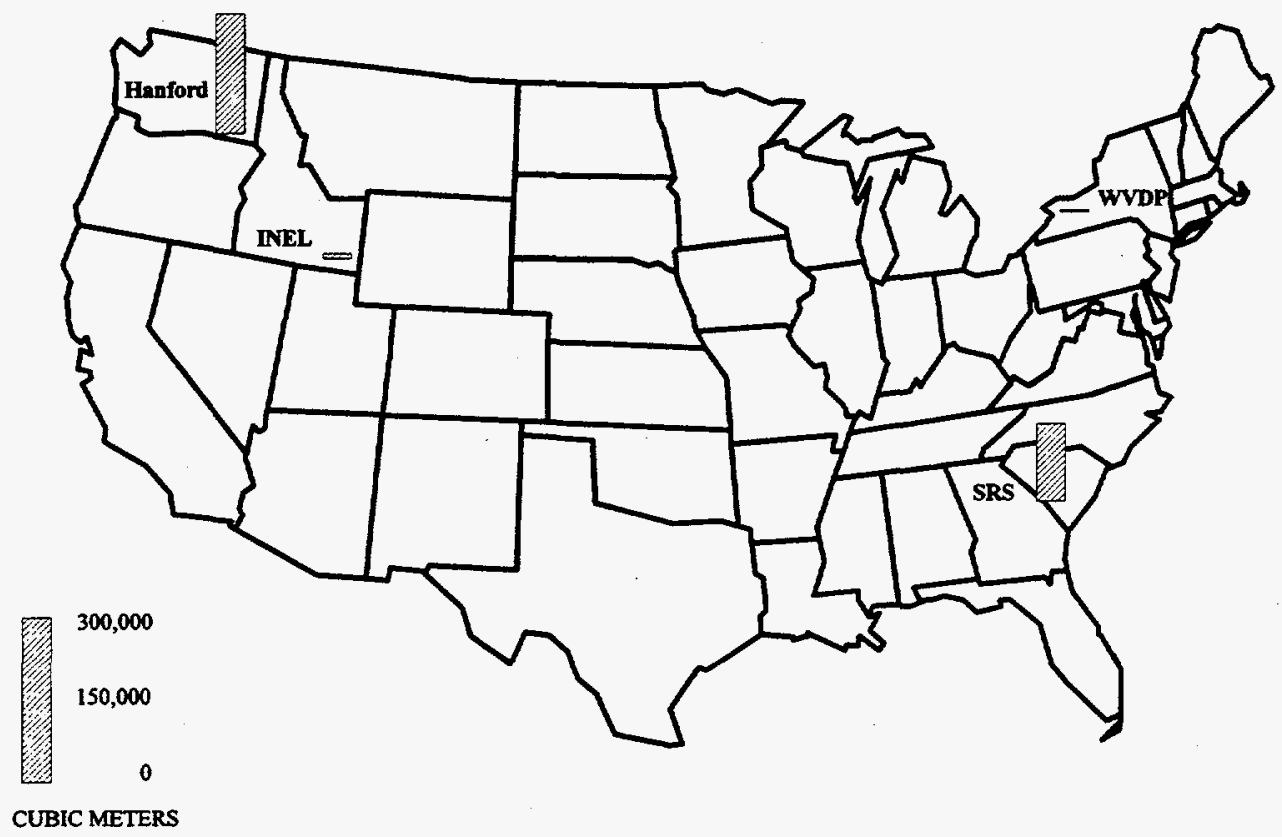

Fig. 2.1. Locations and total volumes of HLW through 1995.

ORNL DWG 95-8223R
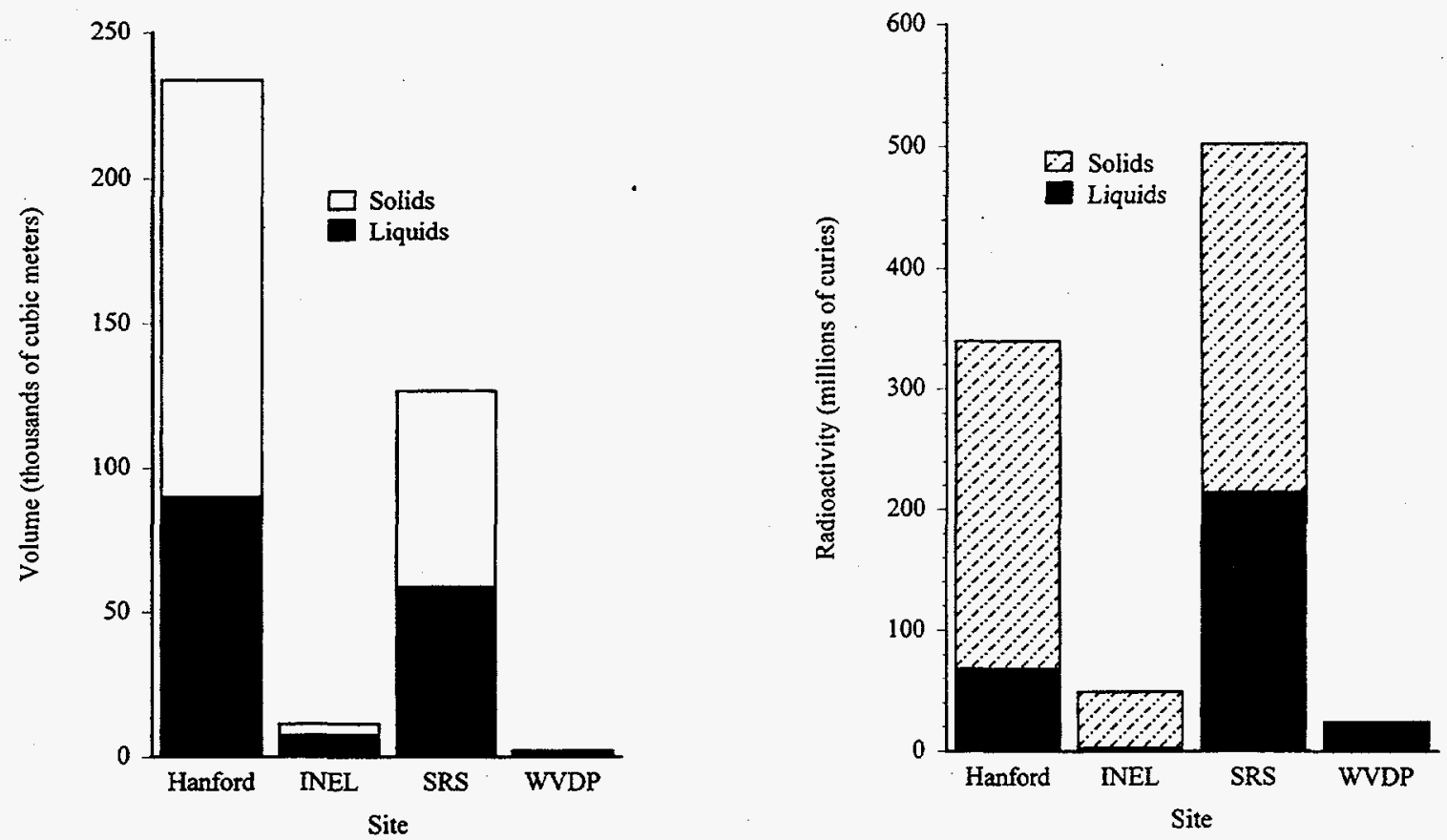

Fig. 2.2. Total volume and radioactivity of solid and liquid HLW through 1995. 


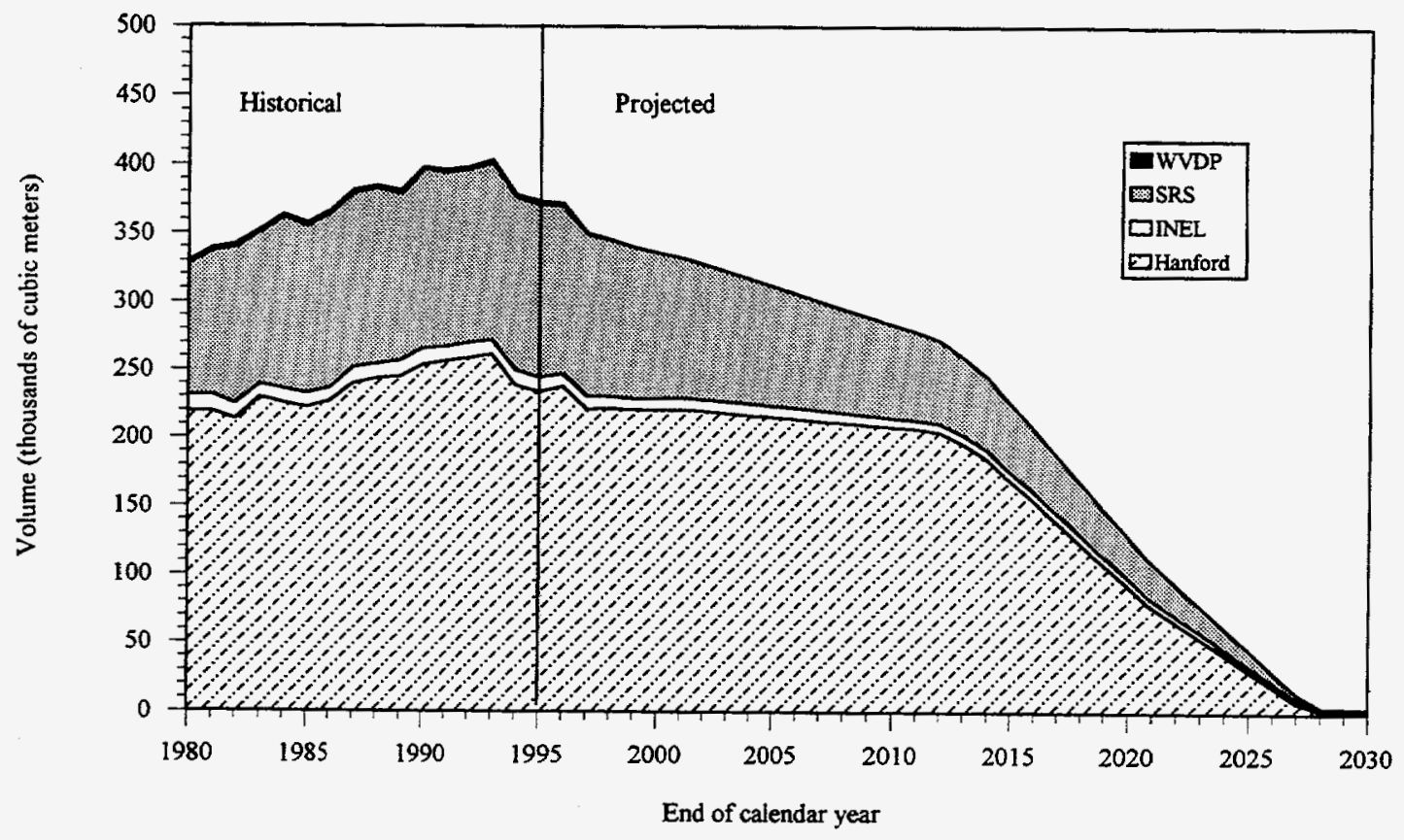

Fig. 2.3. Historical and projected cumulative volumes of untreated HLW in storage.

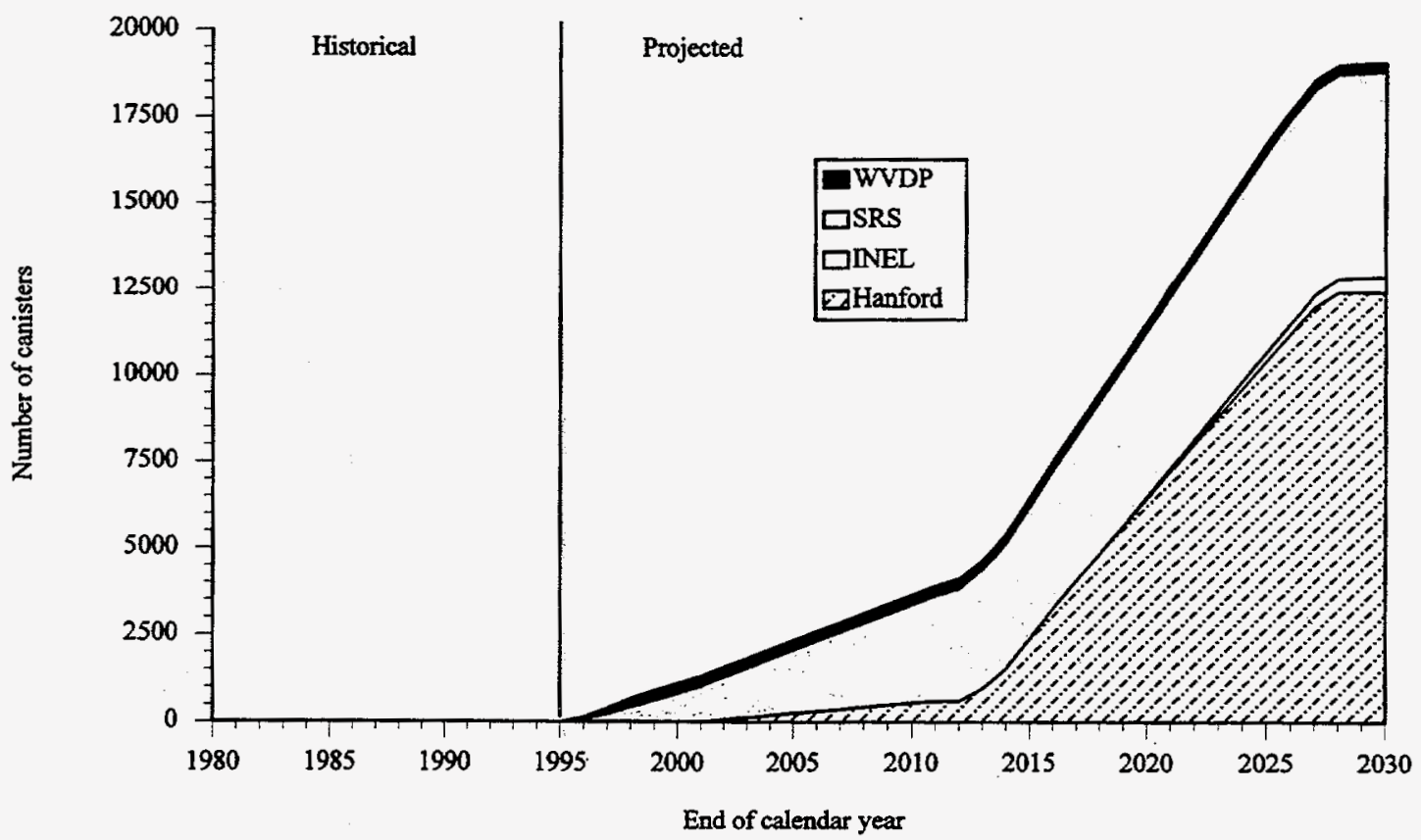

Fig. 2.4. Projected cumulative number of HLW canisters. 


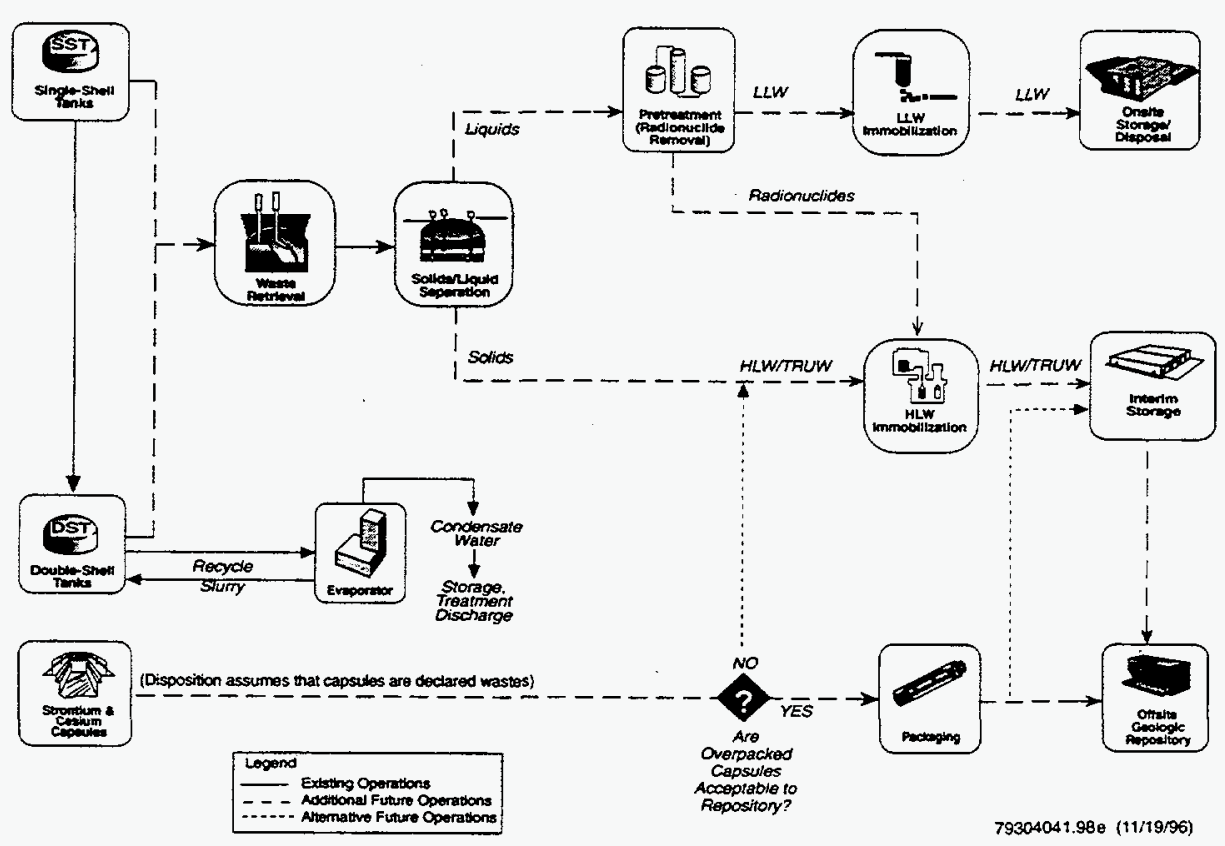

Fig. 2.5. Treatment methods for tank waste and capsules at Hanford.

ORNL DWG 96-7943

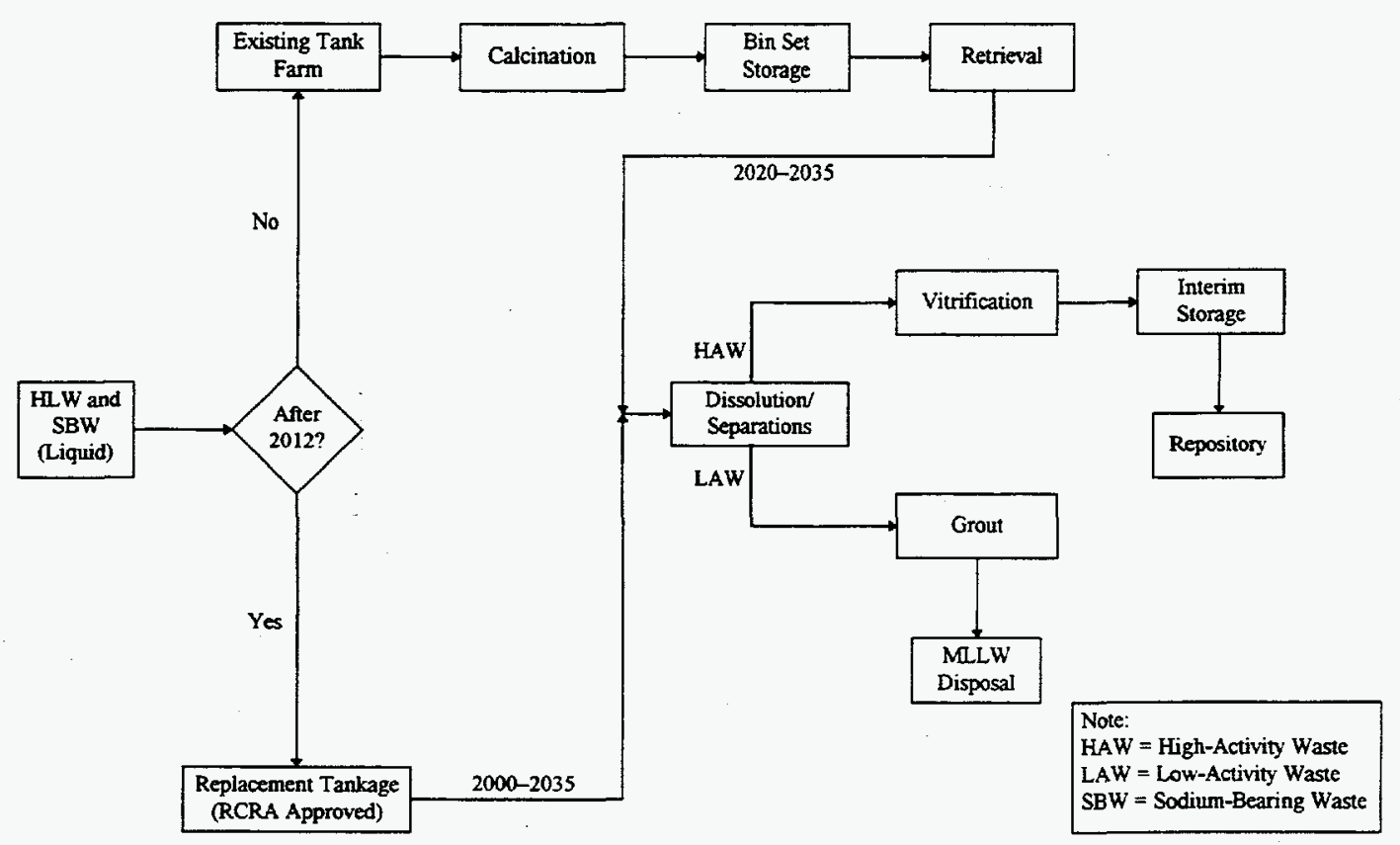

Fig. 2.6. Treatment methods for HLW in tanks and bins at INEL. 


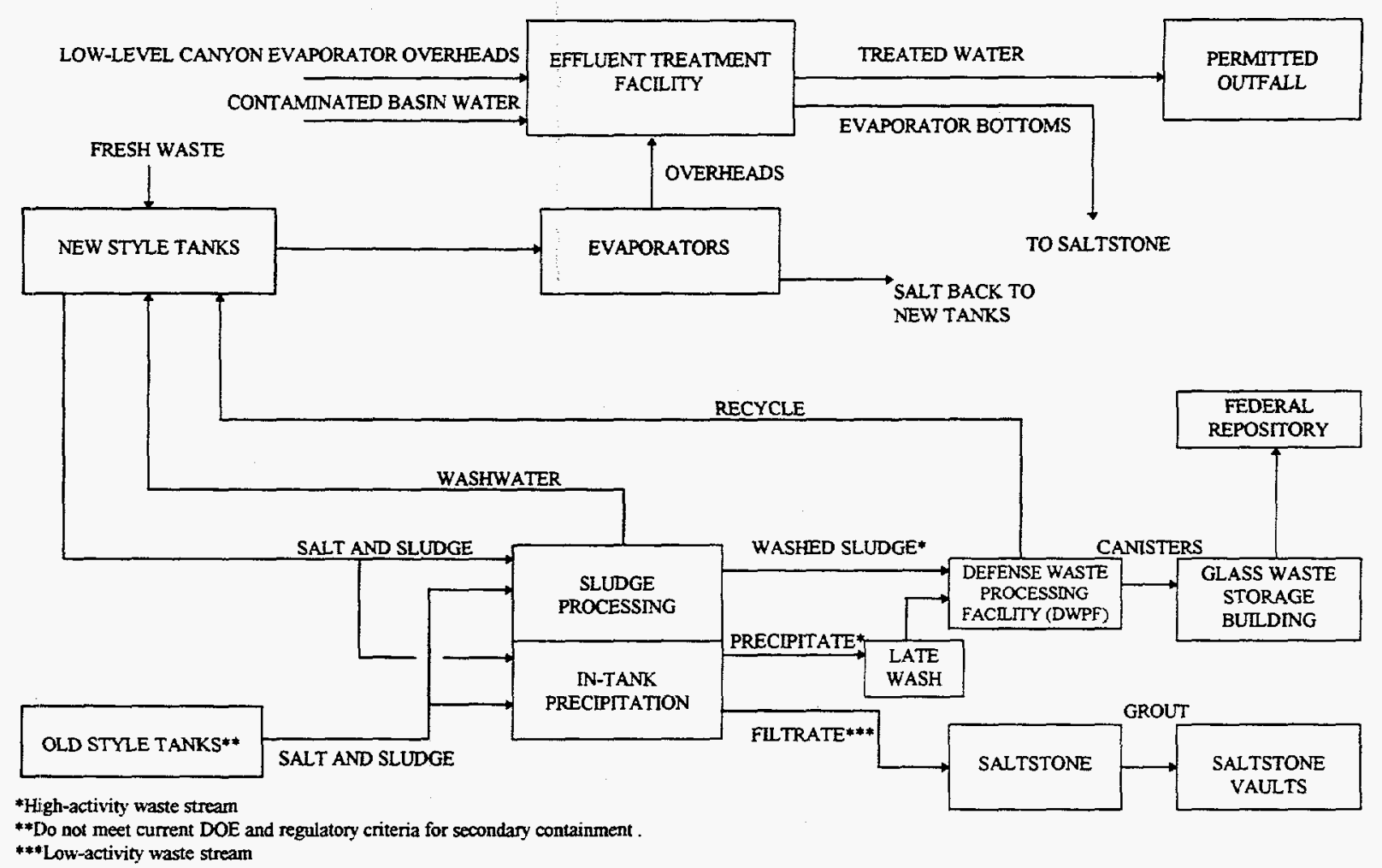

Fig. 2.7. Treatment methods for HLW in tanks and canisters at SRS.

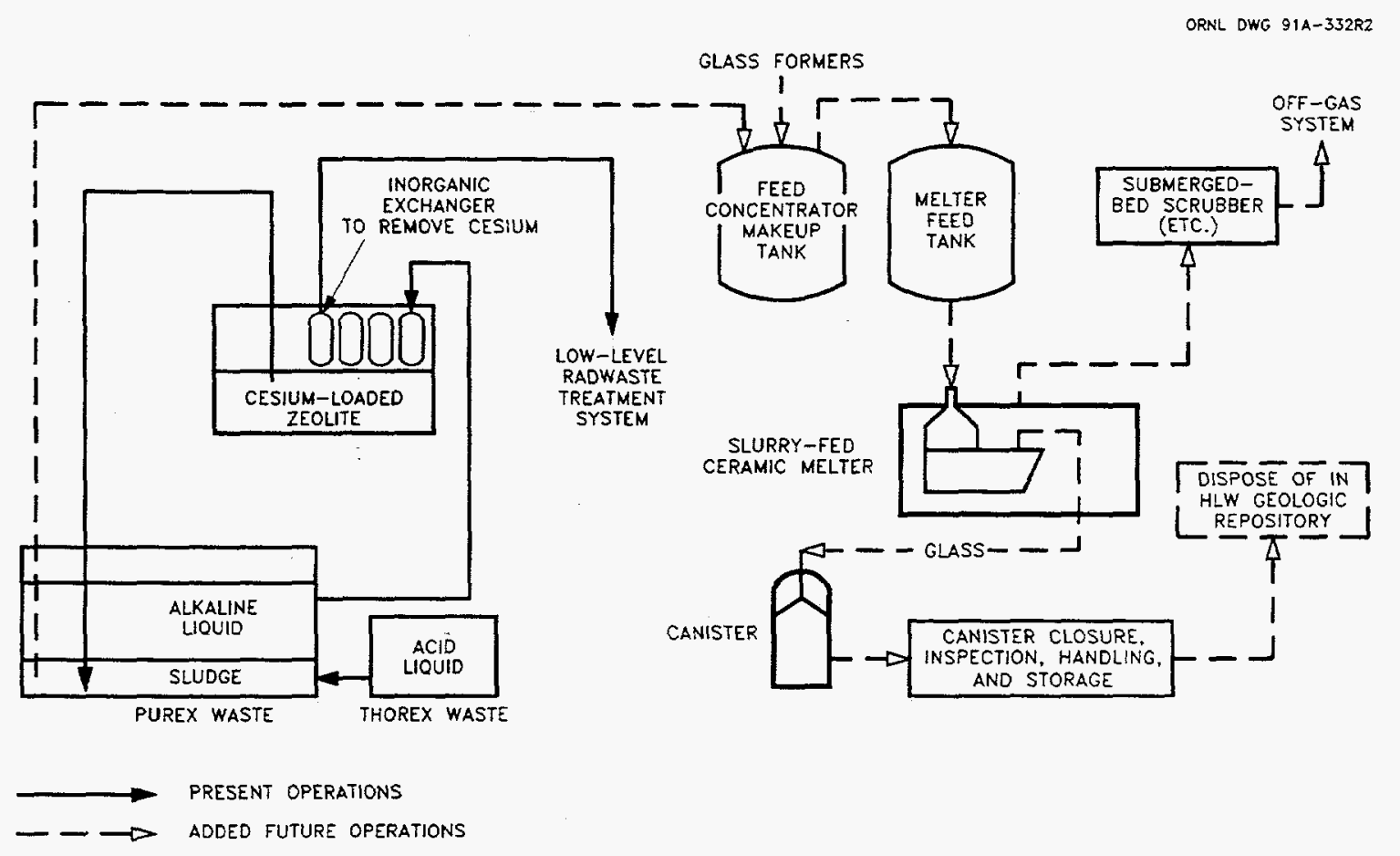

Fig. 2.8. Treatment methods for HLW in tanks and canisters at WVDP. 
Table 2.1. Historical and projected cumulative volume of HLW stored in tanks, bins, and capsules, by site ${ }^{a, b}$

\begin{tabular}{|c|c|c|c|c|c|}
\hline \multirow{2}{*}{$\begin{array}{l}\text { End of } \\
\text { calendar } \\
\text { year }\end{array}$} & \multicolumn{5}{|c|}{ Volume, $10^{3} \mathrm{~m}^{3}$} \\
\hline & Hanford & INEL & SRS & WVDP & Total \\
\hline 1990 & 253.6 & 12.0 & 131.7 & 1.2 & 398.5 \\
\hline 1991 & 256.4 & 10.4 & 127.9 & 1.7 & 396.5 \\
\hline 1992 & 258.7 & 11.2 & 126.9 & 1.6 & 398.3 \\
\hline 1993 & 261.7 & 10.5 & 129.3 & 2.0 & 403.5 \\
\hline 1994 & 238.9 & 11.0 & 126.3 & 2.2 & 378.4 \\
\hline 1995 & 233.5 & 11.2 & 126.5 & 2.2 & 373.4 \\
\hline 1996 & 237.8 & 9.6 & 122.7 & 1.8 & 371.9 \\
\hline 1997 & 221.0 & 9.4 & 118.9 & 0.8 & 350.0 \\
\hline 1998 & 221.8 & 8.9 & 115.0 & 0.2 & 345.9 \\
\hline 1999 & 220.9 & 8.2 & 111.2 & & 340.2 \\
\hline 2000 & 220.9 & 8.2 & 107.4 & & 336.5 \\
\hline 2001 & 221.3 & 8.3 & 103.5 & & 333.1 \\
\hline 2002 & 219.9 & 8.4 & 99.7 & & 328.0 \\
\hline 2003 & 218.4 & 8.5 & 95.9 & & 322.8 \\
\hline 2004 & 217.0 & 8.5 & 92.0 & & 317.6 \\
\hline 2005 & 215.6 & 8.3 & 88.2 & & 312.1 \\
\hline 2006 & 214.2 & 8.1 & 84.4 & & 306.6 \\
\hline 2007 & 212.8 & 7.9 & 80.5 & & 301.2 \\
\hline 2008 & 211.4 & 7.3 & 76.7 & & 295.3 \\
\hline 2009 & 209.9 & 7.2 & 72.8 & & 289.9 \\
\hline 2010 & 208.5 & 6.6 & 69.0 & & 284.2 \\
\hline 2011 & 207.1 & 6.5 & 65.2 & & 278.7 \\
\hline 2012 & 204.5 & 6.4 & 61.3 & & 272.3 \\
\hline 2013 & 196.3 & 6.5 & 57.5 & & 260.3 \\
\hline 2014 & 185.2 & 6.5 & 53.7 & & 245.4 \\
\hline 2015 & 169.8 & 6.6 & 49.8 & & 226.2 \\
\hline 2016 & 154.4 & 6.6 & 46.0 & & 207.0 \\
\hline 2017 & 138.9 & 6.7 & 42.2 & & 187.8 \\
\hline 2018 & 123.5 & 6.7 & 38.3 & & 168.6 \\
\hline 2019 & 108.1 & 6.2 & 34.5 & & 148.8 \\
\hline 2020 & 92.7 & 5.7 & 30.7 & & 129.1 \\
\hline 2021 & 77.2 & 5.3 & 26.8 & & 109.4 \\
\hline 2022 & 65.7 & 4.8 & 23.0 & & -93.6 \\
\hline 2023 & 54.2 & 4.4 & 19.2 & & 77.8 \\
\hline 2024 & 42.6 & 4.0 & 15.3 & & 61.9 \\
\hline 2025 & 31.1 & 3.5 & 11.5 & & 46.1 \\
\hline 2026 & 19.6 & 3.1 & 7.7 & & 30.3 \\
\hline 2027 & 8.0 & 2.7 & 3.8 & & 14.5 \\
\hline 2028 & $2.2^{\mathrm{c}}$ & 2.3 & 0.0 & & 4.5 \\
\hline 2029 & $2.2^{\mathrm{c}}$ & 1.9 & & & 4.1 \\
\hline 2030 & $2.2^{\mathrm{c}}$ & 1.5 & & & 3.7 \\
\hline 2031 & $2.2^{\mathrm{c}}$ & 1.1 & & & 3.3 \\
\hline 2032 & $2.2^{\mathrm{c}}$ & 0.7 & & & 2.9 \\
\hline 2033 & $2.2^{\mathrm{c}}$ & 0.5 & & & 2.7 \\
\hline 2034 & $2.2^{\mathrm{c}}$ & 0.2 & & & 2.4 \\
\hline 2035 & $2.2^{c}$ & 0.0 & & & 2.2 \\
\hline
\end{tabular}

a Historical inventories for HLW volume are taken from the previous edition of this report [i.e., DOE/RW-0006, Rev. 10 (December 1994)]. The inventories for 1995 and the projections through 2030 are taken from ref. 1.

b Numbers shown as 0.0 are less than $50 \mathrm{~m}^{3}$. Values of 0.0 or blank do not imply tank cleanout will be $100 \%$.

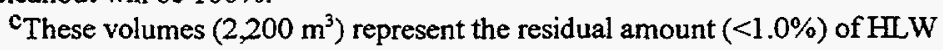
which will remain in tanks until 2035 or later, as per agreement between DOE, the Washington State Department of Ecology, and the EPA (see ref. 5). 
Table 2.2. Historical and projected cumulative decayed radioactivity of HLW stored in tanks, bins, and capsules, by site ${ }^{a, b}$

\begin{tabular}{|c|c|c|c|c|c|}
\hline \multirow{2}{*}{$\begin{array}{l}\text { End of } \\
\text { calendar } \\
\text { year }\end{array}$} & \multicolumn{5}{|c|}{ Radioactivity, $10^{6} \mathrm{Ci}$} \\
\hline & Hanford & INEL & SRS & WVDP & Total \\
\hline 1990 & 399.3 & 63.2 & 561.6 & 26.7 & $1,050.8$ \\
\hline 1991 & 384.2 & 59.4 & 537.6 & 26.2 & $1,007.4$ \\
\hline 1992 & 372.1 & 50.8 & 632.4 & 25.9 & $1,081.2$ \\
\hline 1993 & 361.4 & 52.5 & 606.0 & 25.3 & $1,045.3$ \\
\hline 1994 & 348.0 & 51.6 & 534.5 & 24.7 & 958.8 \\
\hline 1995 & 339.9 & 49.3 & 502.2 & 24.1 & 915.4 \\
\hline 1996 & 332.1 & 48.1 & 465.4 & 19.2 & 864.8 \\
\hline 1997 & 324.4 & 45.9 & 440.2 & 8.5 & 819.0 \\
\hline 1998 & 316.9 & 44.4 & 416.0 & 2.1 & 779.4 \\
\hline 1999 & 309.6 & 43.3 & 392.8 & & 745.7 \\
\hline 2000 & 302.4 & 42.2 & 370.6 & & 715.2 \\
\hline 2001 & 295.4 & 41.2 & 349.2 & & 685.8 \\
\hline 2002 & 287.8 & 40.3 & 328.6 & & 656.6 \\
\hline 2003 & 280.4 & 39.3 & 308.8 & & 628.4 \\
\hline 2004 & 273.1 & 38.3 & 289.7 & & 601.2 \\
\hline 2005 & 266.0 & 37.5 & 271.4 & & 575.0 \\
\hline 2006 & 259.2 & 36.5 & 253.8 & & 549.5 \\
\hline 2007 & 252.5 & 35.7 & 236.8 & & 525.0 \\
\hline 2008 & 245.9 & 34.8 & 220.5 & & 501.3 \\
\hline 2009 & 239.6 & 34.0 & 204.8 & & 478.4 \\
\hline 2010 & 233.4 & 33.1 & 189.7 & & 456.2 \\
\hline 2011 & 227.3 & 32.3 & 175.2 & & 434.8 \\
\hline 2012 & 222.1 & 31.4 & 161.3 & & 414.8 \\
\hline 2013 & 189.8 & 30.7 & 147.9 & & 368.4 \\
\hline 2014 & 156.9 & 30.0 & 135.0 & & 321.9 \\
\hline 2015 & 122.4 & 29.3 & 122.6 & & 274.3 \\
\hline 2016 & 89.4 & 28.6 & 110.7 & & 228.7 \\
\hline 2017 & 79.7 & 27.9 & 99.3 & & 206.9 \\
\hline 2018 & 70.5 & 27.3 & 88.3 & & 186.1 \\
\hline 2019 & 61.6 & 26.7 & 77.7 & & 166.0 \\
\hline 2020 & 53.1 & 21.8 & 67.6 & & 142.5 \\
\hline 2021 & 45.0 & 18.4 & 57.9 & & 121.3 \\
\hline 2022 & 37.2 & 15.1 & 48.6 & & 100.9 \\
\hline 2023 & 29.7 & 12.2 & 39.7 & & 81.6 \\
\hline 2024 & 22.6 & 9.5 & 31.1 & & 63.1 \\
\hline 2025 & 15.8 & 6.9 & 22.9 & & 45.5 \\
\hline 2026 & 9.2 & 4.6 & 15.0 & & 28.8 \\
\hline 2027 & 3.0 & 2.9 & 7.5 & & 13.4 \\
\hline 2028 & 0.0 & 1.3 & 0.2 & & 1.6 \\
\hline 2029 & 0.0 & 0.7 & & & 0.7 \\
\hline 2030 & 0.0 & 0.5 & & & 0.5 \\
\hline 2031 & 0.0 & 0.2 & & & 0.2 \\
\hline 2032 & 0.0 & 0.0 & & & 0.0 \\
\hline 2033 & 0.0 & 0.0 & & & 0.0 \\
\hline 2034 & 0.0 & 0.0 & & & 0.0 \\
\hline 2035 & 0.0 & 0.0 & & & 0.0 \\
\hline
\end{tabular}

a Historical inventories for HLW radioactivity are taken from the previous edition of this report [i.e., DOE/RW-0006, Rev. 10 (December 1994)]. The inventories for 1995 and the projections through 2030 are taken from ref. 1 .

b Numbers shown as 0.0 are less than $5,000 \mathrm{Ci}$. Values of 0.0 or blank do not imply tank cleanout will be $100 \%$. 
Table 2.3. Historical and projected cumulative decayed thermal power of HLW stored in tanks, bins, and capsules, by site ${ }^{a, b}$

\begin{tabular}{|c|c|c|c|c|c|}
\hline \multirow{2}{*}{$\begin{array}{l}\text { End of } \\
\text { calendar } \\
\text { year }\end{array}$} & \multicolumn{5}{|c|}{ Thermal power, $10^{3} \mathrm{~W}$} \\
\hline & Hanford & NNEL & SRS & WVDP & Total \\
\hline 1990 & $1,150.3$ & 184.4 & $1,566.7$ & 76.9 & $2,978.3$ \\
\hline 1991 & $1,106.5$ & 172.0 & $1,509.3$ & 75.9 & $2,863.7$ \\
\hline 1992 & $1,073.1$ & 147.3 & $1,724.3$ & 79.1 & $3,023.8$ \\
\hline 1993 & $1,043.1$ & 153.7 & $1,615.3$ & 74.1 & $2,886.3$ \\
\hline 1994 & 999.8 & 150.8 & $1,497.3$ & 78.1 & $2,726.0$ \\
\hline 1995 & 976.7 & 142.8 & 1.406 .0 & 69.7 & 2.595 .2 \\
\hline 1996 & 954.1 & 139.6 & $1,311.3$ & 55.7 & $2,460.7$ \\
\hline 1997 & 932.1 & 132.7 & $1,248.5$ & 24.8 & $2,337.9$ \\
\hline 1998 & 910.5 & 128.2 & $1,187.6$ & 6.2 & $2,232.5$ \\
\hline 1999 & 889.5 & 125.0 & $1,128.0$ & & $2,142.5$ \\
\hline 2000 & 868.9 & 121.8 & $1,069.5$ & & $2,060.2$ \\
\hline 2001 & 848.9 & 118.7 & $1,012.2$ & & $1,979.7$ \\
\hline 2002 & 826.8 & 116.7 & 956.1 & & $1,899.6$ \\
\hline 2003 & 805.4 & 113.6 & 901.5 & & $1,820.5$ \\
\hline 2004 & 784.4 & 110.6 & 848.4 & & $1,743.4$ \\
\hline 2005 & 764.1 & 108.4 & 796.9 & & $1,669.4$ \\
\hline 2006 & 744.2 & 105.3 & 746.9 & & $1,596.4$ \\
\hline 2007 & 724.9 & 103.2 & 698.5 & & $1,526.6$ \\
\hline 2008 & 706.0 & 100.5 & 651.8 & & $1,458.3$ \\
\hline 2009 & 687.7 & 98.2 & 606.6 & & $1,392.4$ \\
\hline 2010 & 669.8 & 95.7 & 562.9 & & $1,328.5$ \\
\hline 2011 & 652.4 & 93.0 & 520.8 & & $1,266.3$ \\
\hline 2012 & 637.3 & 90.8 & 480.2 & & $1,208.4$ \\
\hline 2013 & 548.3 & 88.7 & 441.0 & & $1,078.1$ \\
\hline 2014 & 456.9 & 86.7 & 403.3 & & 946.9 \\
\hline 2015 & 360.5 & 84.7 & 366.9 & & 812.1 \\
\hline 2016 & 268.3 & 82.7 & 331.8 & & 682.9 \\
\hline 2017 & 239.4 & 80.8 & 298.0 & & 618.2 \\
\hline 2018 & 211.6 & 78.9 & 265.5 & & 556.0 \\
\hline 2019 & 185.0 & 77.0 & 234.2 & & 496.2 \\
\hline 2020 & 159.4 & 62.9 & 204.1 & & 426.4 \\
\hline 2021 & 135.0 & 53.1 & 175.1 & & 363.2 \\
\hline 2022 & 111.6 & 43.7 & 147.2 & & 302.5 \\
\hline 2023 & 89.2 & 35.3 & 120.3 & & 244.9 \\
\hline 2024 & 67.8 & 27.4 & 94.5 & & 189.7 \\
\hline 2025 & 47.3 & 19.8 & 69.7 & & 136.8 \\
\hline 2026 & 27.8 & 13.3 & 45.8 & & 86.8 \\
\hline 2027 & 9.1 & 8.5 & 22.8 & & 40.4 \\
\hline 2028 & 0.0 & 3.9 & 0.8 & & 4.7 \\
\hline 2029 & 0.0 & 2.0 & & & 2.1 \\
\hline 2030 & 0.0 & 1.3 & & & 1.4 \\
\hline 2031 & 0.0 & 0.7 & & & 0.7 \\
\hline 2032 & 0.0 & 0.0 & & & 0.1 \\
\hline 2033 & 0.0 & 0.0 & & & 0.1 \\
\hline 2034 & 0.0 & 0.0 & & & 0.1 \\
\hline 2035 & 0.0 & 0.0 & & & 0.0 \\
\hline
\end{tabular}

${ }^{a}$ Historical inventories for $\mathrm{HLW}$ thermal power are taken from the previous edition of this report [i.e., DOE/RW-0006, Rev. 10 (December 1994)]. The inventories for 1995 and the projections through 2030 are taken from ref. 1 .

bumbers shown as 0.0 are less than $50 \mathrm{~W}$. Values of 0.0 or blank do not imply tank cleanout will be $100 \%$. 
Table 2.4. Projected annual and cumulative volume $\left(10^{3} \mathrm{~m}^{3}\right)$ of $\mathrm{HLW}$ glass stored in canisters, by site $\mathrm{a}^{\mathrm{a}, \mathrm{b}}$

\begin{tabular}{|c|c|c|c|c|c|c|c|c|c|c|}
\hline \multirow{2}{*}{$\begin{array}{l}\text { End of } \\
\text { calendar } \\
\text { year }\end{array}$} & \multicolumn{2}{|c|}{ Hanford ${ }^{c}$} & \multicolumn{2}{|c|}{ INEL $^{\mathrm{d}}$} & \multicolumn{2}{|c|}{ SRS } & \multicolumn{2}{|c|}{ WVDPf } & \multicolumn{2}{|c|}{ Total } \\
\hline & Annual & Cumulative & Annual & Cumulative & Annual & Cumulative & Annual & Cumulative & Annual & Cumulative \\
\hline 1996 & & & & & 0.044 & 0.044 & 0.050 & 0.050 & 0.094 & 0.094 \\
\hline 1997 & & & & & 0.094 & 0.138 & 0.100 & 0.150 & 0.194 & 0.288 \\
\hline 1998 & & & & & 0.125 & 0.263 & 0.060 & 0.210 & 0.185 & 0.473 \\
\hline 1999 & & & & & 0.125 & 0.388 & 0.020 & 0.230 & 0.145 & 0.618 \\
\hline 2000 & & & & & 0.125 & 0.513 & & 0.230 & 0.125 & 0.743 \\
\hline 2001 & & & & & 0.125 & 0.638 & & 0.230 & 0.125 & 0.868 \\
\hline 2002 & 0.069 & 0.069 & & & 0.125 & 0.764 & & 0.230 & 0.194 & 1.062 \\
\hline 2003 & 0.069 & 0.138 & & & 0.125 & 0.889 & & 0.230 & 0.194 & 1.256 \\
\hline 2004 & 0.069 & 0.207 & & & 0.125 & 1.014 & & 0.230 & 0.194 & 1.450 \\
\hline 2005 & 0.069 & 0.275 & & & 0.125 & 1.139 & & 0.230 & 0.194 & 1.644 \\
\hline 2006 & 0.069 & 0.344 & & & 0.125 & 1.264 & & 0.230 & 0.194 & 1.839 \\
\hline 2007 & 0.069 & 0.413 & & & 0.125 & 1.389 & & 0.230 & 0.194 & 2.033 \\
\hline 2008 & 0.069 & 0.482 & & & 0.125 & 1.515 & & 0.230 & 0.194 & 2.227 \\
\hline 2009 & 0.069 & 0.551 & & & 0.125 & 1.640 & & 0.230 & 0.194 & 2.421 \\
\hline 2010 & 0.069 & 0.620 & & & 0.125 & 1.765 & & 0.230 & 0.194 & 2.615 \\
\hline 2011 & 0.069 & 0.689 & & & 0.125 & 1.890 & & 0.230 & 0.194 & 2.809 \\
\hline 2012 & & 0.689 & & & 0.125 & 2.015 & & 0.230 & 0.125 & 2.934 \\
\hline 2013 & 0.414 & 1.102 & & & 0.125 & 2.140 & & 0.230 & 0.539 & 3.473 \\
\hline 2014 & 0.643 & 1.745 & & & 0.125 & 2.266 & & 0.230 & 0.768 & 4.241 \\
\hline 2015 & 0.987 & 2.733 & & & 0.125 & 2.391 & . & 0.230 & 1.113 & 5.354 \\
\hline 2016 & 0.987 & 3.720 & & & 0.125 & 2.516 & & 0.230 & 1.113 & 6.466 \\
\hline 2017 & 0.918 & 4.638 & & & 0.125 & 2.641 & & 0.230 & 1.043 & 7.509 \\
\hline 2018 & 0.918 & 5.556 & & & 0.125 & 2.766 & & 0.230 & 1.043 & 8.553 \\
\hline 2019 & 0.918 & 6.474 & 0.005 & 0.005 & 0.125 & 2.892 & & 0.230 & 1.048 & 9.601 \\
\hline 2020 & 0.918 & 7.392 & 0.051 & 0.056 & 0.125 & 3.017 & & 0.230 & 1.094 & 10.695 \\
\hline 2021 & 0.918 & 8.310 & 0.051 & 0.107 & 0.125 & 3.142 & & 0.230 & 1.094 & 11.789 \\
\hline 2022 & 0.918 & 9.228 & 0.051 & 0.158 & 0.125 & 3.267 & & 0.230 & 1.094 & 12.883 \\
\hline 2023 & 0.918 & 10.146 & 0.051 & 0.208 & 0.125 & 3.392 & & 0.230 & 1.094 & 13.977 \\
\hline 2024 & 0.918 & 11.064 & 0.051 & 0.259 & 0.125 & 3.517 & & 0.230 & 1.094 & 15.071 \\
\hline 2025 & 0.918 & 11.982 & 0.051 & 0.309 & 0.125 & 3.643 & & 0.230 & 1.094 & 16.164 \\
\hline 2026 & 0.918 & 12.900 & 0.050 & 0.359 & 0.078 & 3.720 & & 0.230 & 1.045 & 17.210 \\
\hline 2027 & 0.918 & 13.818 & 0.049 & 0.409 & & 3.720 & & 0.230 & 0.967 & 18.177 \\
\hline 2028 & 0.459 & 14.277 & 0.050 & 0.458 & & 3.720 & & 0.230 & 0.509 & 18.686 \\
\hline 2029 & & 14.277 & 0.046 & 0.505 & & 3.720 & & 0.230 & 0.046 & 18.732 \\
\hline 2030 & & 14.277 & 0.045 & 0.550 & & 3.720 & & 0.230 & 0.045 & 18.777 \\
\hline 2031 & & 14.277 & 0.045 & 0.595 & & 3.720 & & 0.230 & 0.045 & 18.822 \\
\hline
\end{tabular}


Table 2.4 (continued)

\begin{tabular}{|c|c|c|c|c|c|c|c|c|c|c|}
\hline \multirow{2}{*}{$\begin{array}{l}\text { End of } \\
\text { calendar } \\
\text { year }\end{array}$} & \multicolumn{2}{|c|}{ Hanford } & \multicolumn{2}{|c|}{$\mathbb{I N E L}^{\mathrm{d}}$} & \multicolumn{2}{|c|}{ SRS $^{e}$} & \multicolumn{2}{|c|}{ WVDpf } & \multicolumn{2}{|c|}{ Total } \\
\hline & Annual & Cumulative & Annual & Cumulative & Annual & Cumulative & Annual & Cumulative & Annual & Cumulative \\
\hline 2032 & & 14.277 & 0.045 & 0.640 & & 3.720 & & 0.230 & 0.045 & 18.868 \\
\hline 2033 & & 14.277 & 0.027 & 0.667 & & 3.720 & & 0.230 & 0.027 & 18.895 \\
\hline 2034 & & 14.277 & 0.027 & 0.694 & & 3.720 & & 0.230 & 0.027 & 18.922 \\
\hline 2035 & & 14.277 & 0.026 & 0.720 & & 3.720 & & 0.230 & 0.026 & 18.948 \\
\hline
\end{tabular}

aTaken from data given in ref. 1 . Glass may be in storage at the site, in transit to a repository, or in a repository.

bSee Table 2.7 for the projected number of canisters.

cHanford's reference canister has a diameter of $61 \mathrm{~cm}$ and is $450 \mathrm{~cm}$ long (about $2 \mathrm{ft}$ in diam by about $15 \mathrm{ft}$ in length). The nominal glass volume is expected to be $1.1 \mathrm{~m}^{3}$ with a minimum waste oxide loading of $25 \mathrm{vol} \%$ (excluding sodium and silicon). Hanford values include HLW glass plus overpacked cesium and strontium capsule canisters, assuming capsules to be declared waste and treated as HLW.

dINEL's canister projections assume the use of a canister containing $1.26 \mathrm{~m}^{3}$ of glass.

eAt SRS, the DWPF canisters are $0.6 \mathrm{~m}$ in diam by $3 \mathrm{~m}$ in length (about $2 \mathrm{ft}$ in diam by about $10 \mathrm{ft}$ in length). Each canister is assumed to contain $0.625 \mathrm{~m}^{3}$ of glass [i.e., $85 \%$ of the usable capacity $\left(0.735 \mathrm{~m}^{3}\right)$ ] made with HLW from the reprocessing of SNF at SRS. The glass incorporates $36 \mathrm{wt} \%$ oxides from waste ( $28 \mathrm{wt} \%$ from SNF and $8 \mathrm{wt} \%$ from processing chemicals) and $64 \mathrm{wt} \%$ oxides from nonradioactive glass frit. Volumes reported are for the glass waste form and not the canisters.

For WVDP, it is assumed that 276 canisters $0.6 \mathrm{~m}$ in diam by $3 \mathrm{~m}$ in length ( $2 \mathrm{ft}$ in diam by $10 \mathrm{ft}$ in length) are filled with waste glass during 1996-1999 and that each canister contains $0.8 \mathrm{~m}^{3}$ of glass at the filling temperature. Tank heels and residual materials will continue to be vitrified through mid-FY 2001 
Table 2.5. Projected annual and cumulative decayed radioactivity $\left(10^{6} \mathrm{Ci}\right)$ of $\mathrm{HLW}$ glass stored in canisters, by site ${ }^{\mathrm{a}, \mathrm{b}}$

\begin{tabular}{|c|c|c|c|c|c|c|c|c|c|c|}
\hline \multirow{2}{*}{$\begin{array}{l}\text { End of } \\
\text { calendar } \\
\text { year }\end{array}$} & \multicolumn{2}{|c|}{ Hanford ${ }^{c}$} & \multicolumn{2}{|c|}{ INEL } & \multicolumn{2}{|c|}{ SRS } & \multicolumn{2}{|c|}{ WVDP } & \multicolumn{2}{|c|}{ Total } \\
\hline & Annual & Cumulative & Annual & Cumulative & Annual & Cumulative & Annual & Cumulative & Annual & Cumulative \\
\hline 1996 & & & & & 15.31 & 15.31 & 4.89 & 4.89 & 20.20 & 20.20 \\
\hline 1997 & & & & & 14.96 & 29.90 & 9.56 & 14.45 & 24.52 & 44.35 \\
\hline 1998 & & & & & 14.61 & 43.80 & 5.60 & 20.05 & 20.21 & 63.85 \\
\hline 1999 & & & & & 14.28 & 57.05 & 1.83 & 21.88 & 16.11 & 78.93 \\
\hline 2000 & & & & & 13.95 & 69.67 & & 21.37 & 13.95 & 91.04 \\
\hline 2001 & & & & & 13.64 & 81.69 & & 20.87 & 13.64 & 102.56 \\
\hline 2002 & 0.81 & 0.81 & & & 13.33 & 93.14 & & 20.38 & 14.14 & 114.33 \\
\hline 2003 & 0.79 & 1.58 & & & 13.03 & 104.03 & & 19.91 & 13.82 & 125.52 \\
\hline 2004 & 0.77 & 2.32 & & & 12.74 & 114.38 & & 19.44 & 13.51 & 136.14 \\
\hline 2005 & 0.75 & 3.02 & & & 12.46 & 124.22 & & 18.99 & 13.21 & 146.23 \\
\hline 2006 & 0.74 & 3.68 & & & 12.18 & 133.56 & & 18.55 & 12.92 & 155.79 \\
\hline 2007 & 0.72 & 4.32 & & & 11.91 & 142.43 & & 18.12 & 12.63 & 164.86 \\
\hline 2008 & 0.70 & 4.92 & & & 11.64 & 150.84 & & 17.69 & 12.35 & 173.45 \\
\hline 2009 & 0.69 & 5.49 & & & 11.39 & 158.80 & & 17.28 & 12.07 & 181.57 \\
\hline 2010 & 0.67 & 6.04 & & & 11.13 & 166.34 & & 16.88 & 11.81 & 189.25 \\
\hline 2011 & 0.66 & 6.55 & & & 10.89 & 173.46 & & 16.48 & 11.54 & 196.50 \\
\hline 2012 & & 6.40 & & & 10.65 & 180.20 & & 16.10 & 10.65 & 202.70 \\
\hline 2013 & 27.13 & 33.39 & & & 10.42 & 186.55 & & 15.72 & 37.55 & 235.66 \\
\hline 2014 & 28.54 & 61.16 & & & 10.19 & 192.53 & & 15.36 & 38.73 & 269.05 \\
\hline 2015 & 30.87 & 90.62 & & & 9.96 & 198.17 & & 15.00 & 40.83 & 303.79 \\
\hline 2016 & 30.16 & 118.69 & & & 9.75 & 203.47 & & 14.65 & 39.91 & 336.81 \\
\hline 2017 & 7.59 & 123.55 & & & 9.54 & 208.44 & & 14.31 & 17.13 & 346.29 \\
\hline 2018 & 7.42 & 128.11 & & & 9.33 & 213.10 & & 13.97 & 16.75 & 355.19 \\
\hline 2019 & 7.25 & 132.41 & 0.04 & 0.04 & 9.13 & 217.46 & & 13.65 & 16.41 & 363.56 \\
\hline 2020 & 7.08 & 136.43 & 4.32 & 4.31 & 8.93 & 221.54 & & 13.33 & 20.33 & 375.61 \\
\hline 2021 & 6.91 & 140.20 & 2.95 & 7.12 & 8.74 & 225.33 & & 13.02 & 18.60 & 385.67 \\
\hline 2022 & 6.76 & 143.72 & 2.88 & 9.81 & 8.55 & 228.87 & & 12.72 & 18.19 & 395.11 \\
\hline 2023 & 6.60 & 147.00 & 2.57 & 12.10 & 8.37 & 232.14 & & 12.42 & 17.54 & 403.67 \\
\hline 2024 & 6.45 & 150.06 & 2.50 & 14.30 & 8.19 & 235.18 & & 12.13 & 17.14 & 411.67 \\
\hline 2025 & 6.30 & 152.90 & 2.43 & 16.40 & 8.02 & 237.98 & & 11.85 & 16.74 & 419.12 \\
\hline 2026 & 6.15 & 155.52 & 2.14 & 18.10 & 7.85 & 240.55 & & 11.57 & 16.14 & 425.75 \\
\hline 2027 & 6.01 & 157.95 & 1.57 & 19.30 & & 235.23 & & 11.30 & 7.58 & 423.78 \\
\hline 2028 & 2.94 & 157.24 & 1.55 & 20.30 & & 230.04 & & 11.04 & 4.49 & 418.62 \\
\hline 2029 & & 153.61 & 0.62 & 20.50 & & 224.97 & & 10.78 & 0.62 & 409.86 \\
\hline 2030 & & 150.07 & 0.22 & 20.20 & & 220.01 & & 10.53 & 0.22 & 400.81 \\
\hline 2031 & & d & 0.22 & 20.00 & & d & & $\mathrm{d}$ & & d \\
\hline 2032 & & d & 0.21 & 19.70 & & d & & d & & d \\
\hline
\end{tabular}


Table 2.5 (continued)

\begin{tabular}{|c|c|c|c|c|c|c|c|c|c|c|}
\hline \multirow{2}{*}{$\begin{array}{l}\text { End of } \\
\text { calendar } \\
\text { year }\end{array}$} & \multicolumn{2}{|c|}{ Hanford ${ }^{\mathfrak{c}}$} & \multicolumn{2}{|c|}{ INEL } & \multicolumn{2}{|c|}{ SRS } & \multicolumn{2}{|c|}{ WVDP } & \multicolumn{2}{|c|}{ Total } \\
\hline & Annual & Cumulative & Annual & Cumulative & Annual & Cumulative & Annual & Cumulative & Annual & Cumulative \\
\hline 2033 & & d & 0.01 & 19.30 & & $d$ & & $d$ & & d \\
\hline 2034 & & d & 0.01 & 18.80 & & d & & $d$ & & d \\
\hline 2035 & & $d$ & 0.01 & 18.40 & & $\mathrm{~d}$ & & d & & d \\
\hline
\end{tabular}

aTaken from data given in ref. 1 .

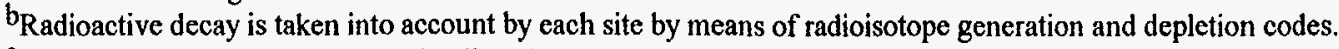

The significant increase in annual radioactivity for the years 2013-2016 reflects the accelerated processing schedule for the strontium and cesium capsules at Hanford (see Sect. 2.5.1). Hanford values include HLW glass plus overpacked cesium and strontium capsule canisters, assuming capsules to be declared waste and treated as HLW.

$\mathrm{d}_{\text {Not available. }}$ 
Table 2.6. Projected annual and cumulative decayed thermal power $\left(10^{3} \mathrm{~W}\right)$ of HLW glass stored in canisters, by site ${ }^{a}, \mathrm{~b}$

\begin{tabular}{|c|c|c|c|c|c|c|c|c|c|c|}
\hline \multirow{2}{*}{$\begin{array}{c}\text { End of } \\
\text { calendar } \\
\text { year }\end{array}$} & \multicolumn{2}{|c|}{ Hanford ${ }^{c}$} & \multicolumn{2}{|c|}{ INEL } & \multicolumn{2}{|c|}{ SRS } & \multicolumn{2}{|c|}{ WVDP } & \multicolumn{2}{|c|}{ Total } \\
\hline & Annual & Cumulative & Annual & Cumulative & Annual & Cumulative & Annual & Cumulative & Annual & Cumulative \\
\hline 1996 & & & & & 43.15 & 43.15 & 14.17 & 14.17 & 57.32 & 57.32 \\
\hline 1997 & & & & & 42.42 & 84.81 & 27.71 & 41.88 & 70.13 & 126.69 \\
\hline 1998 & & & & & 41.71 & 125.05 & 16.23 & 58.10 & 57.94 & 183.15 \\
\hline 1999 & & & & & 41.00 & 163.82 & 5.30 & 63.40 & 46.30 & 227.22 \\
\hline 2000 & & & & & 40.27 & 201.08 & & 61.91 & 40.27 & 262.99 \\
\hline 2001 & & & & & 39.54 & 236.82 & & 60.46 & 39.54 & 297.28 \\
\hline 2002 & 2.43 & 2.43 & & & 38.79 & 271.02 & & 59.05 & 41.22 & 332.49 \\
\hline 2003 & 2.37 & 4.74 & & & 38.05 & 303.72 & & 57.66 & 40.42 & 366.12 \\
\hline 2004 & 2.31 & 6.94 & & & 37.31 & 334.93 & & 56.31 & 39.62 & 398.19 \\
\hline 2005 & 2.26 & 9.05 & & & 36.57 & 364.70 & & 54.99 & 38.83 & 428.74 \\
\hline 2006 & 2.21 & 11.05 & & & 35.84 & 393.08 & & 53.71 & 38.05 & 457.83 \\
\hline 2007 & 2.16 & 12.95 & & & 35.12 & 420.10 & & 52.45 & 37.28 & 485.49 \\
\hline 2008 & 2.11 & 14.76 & & & 34.42 & 445.81 & & 51.22 & 36.52 & 511.79 \\
\hline 2009 & 2.06 & 16.48 & & & 33.72 & 470.26 & & 50.02 & 35.78 & 536.76 \\
\hline 2010 & 2.01 & 18.11 & & & 33.03 & 493.51 & & 48.85 & 35.05 & 560.46 \\
\hline 2011 & 1.97 & 19.66 & & & 32.36 & 515.58 & & 47.71 & 34.33 & 582.94 \\
\hline 2012 & & 19.20 & & & 31.71 & 536.53 & & 46.59 & 31.71 & 602.32 \\
\hline 2013 & 74.34 & 93.10 & & & 31.06 & 556.40 & & 45.50 & 105.41 & 695.00 \\
\hline 2014 & 78.74 & 169.69 & & & 30.44 & 575.23 & & 44.43 & 109.17 & 789.36 \\
\hline 2015 & 85.87 & 251.65 & & & 29.82 & 593.07 & & 43.39 & 115.69 & 888.11 \\
\hline 2016 & 83.89 & 329.73 & & & 29.22 & 609.95 & & 42.37 & 113.11 & 982.05 \\
\hline 2017 & 22.79 & 344.91 & & & 28.63 & 625.90 & & 41.38 & 51.42 & $1,012.20$ \\
\hline 2018 & 22.27 & 359.22 & & & 28.06 & 640.97 & & 40.41 & 50.32 & $1,040.61$ \\
\hline 2019 & 21.75 & 372.69 & 0.12 & 0.12 & 27.50 & 655.19 & & 39.47 & 49.37 & $1,067.47$ \\
\hline 2020 & 21.25 & 385.35 & 12.40 & 12.40 & 26.95 & 668.59 & & 38.54 & 60.60 & $1,104.89$ \\
\hline 2021 & 20.76 & 397.23 & 8.49 & 20.50 & 26.41 & 681.21 & & 37.64 & 55.67 & $1,136.59$ \\
\hline 2022 & 20.29 & 408.37 & 8.29 & 28.20 & 25.89 & 693.08 & & 36.76 & 54.47 & $1,166.40$ \\
\hline 2023 & 19.82 & 418.78 & 7.41 & 34.90 & 25.38 & 704.22 & & 35.90 & 52.61 & $1,193.80$ \\
\hline 2024 & 19.36 & 428.50 & 7.22 & 41.20 & 24.89 & 714.67 & & 35.06 & 51.47 & $1,219.43$ \\
\hline 2025 & 18.92 & 437.55 & 7.01 & 47.20 & 24.40 & 724.46 & & 34.24 & 50.33 & $1,243.45$ \\
\hline 2026 & 18.49 & 445.97 & 6.19 & 52.20 & 23.93 & 733.61 & & 33.43 & 48.60 & $1,265.20$ \\
\hline 2027 & 18.06 & 453.76 & 4.54 & 55.50 & & 718.68 & & 32.65 & 22.60 & $1,260.59$ \\
\hline 2028 & 8.82 & $452: 15$ & 4.46 & 58.60 & & 704.09 & & 31.89 & 13.28 & $1,246.73$ \\
\hline 2029 & & 441.75 & 1.79 & 59.00 & & 689.84 & & 31.14 & 1.79 & $1,221.73$ \\
\hline 2030 & & 431.59 & 0.65 & 58.30 & & 675.91 & & 30.41 & 0.65 & $1,196.21$ \\
\hline 2031 & & $\mathrm{~d}$ & 0.63 & 57.60 & & d & & d & 0.63 & d \\
\hline 2032 & & $\mathrm{~d}$ & 0.62 & 56.90 & & $d$ & & $\mathrm{~d}$ & 0.62 & $d$ \\
\hline
\end{tabular}


Table 2.6 (continued)

\begin{tabular}{|c|c|c|c|c|c|c|c|c|c|c|}
\hline \multirow{2}{*}{$\begin{array}{l}\text { End of } \\
\text { calendar } \\
\text { year }\end{array}$} & \multicolumn{2}{|c|}{ Hanford ${ }^{\mathrm{c}}$} & \multicolumn{2}{|c|}{ INEL } & \multicolumn{2}{|c|}{ SRS } & \multicolumn{2}{|c|}{ WVDP } & \multicolumn{2}{|c|}{ Total } \\
\hline & Annual & Cumulative & Annual & Cumulative & Annual & Cumulative & Annual & Cumulative & Annual & Cumulative \\
\hline 2033 & & d & 0.02 & 55.60 & & d & & d & 0.02 & $\mathrm{~d}$ \\
\hline 2034 & & $\mathrm{~d}$ & 0.02 & 54.30 & & d & & d & 0.02 & d \\
\hline 2035 & & d & 0.01 & 53.00 & & d & & d & 0.01 & $d$ \\
\hline
\end{tabular}

a Taken from data given in ref. 1.

${ }^{b}$ Thermal power is taken into account by each site by means of radioisotope generation and depletion codes.

The significant increase in annual thermal power for the ycars 2013-2016 reflects the accelerated processing schedule for the strontium and cesium capsules at Hanford (see Sect. 2.5.1). Hanford values include HLW glass plus overpacked cesium and strontium capsule canisters, assuming capsules to be declared waste and treated as HLW.

$\mathrm{d}_{\text {Not available. }}$ 
Table 2.7. Estimated potential number of HLW canisters, by site ${ }^{a}$

\begin{tabular}{|c|c|c|c|c|c|c|c|c|c|c|}
\hline \multirow{2}{*}{$\begin{array}{l}\text { End of } \\
\text { calendar } \\
\text { year }\end{array}$} & \multicolumn{2}{|c|}{ Hanford $b$} & \multicolumn{2}{|c|}{ INEL $^{c}$} & \multicolumn{2}{|c|}{ SRS $^{\mathrm{d}}$} & \multicolumn{2}{|c|}{ WVDPe } & \multicolumn{2}{|c|}{ Total } \\
\hline & Annual & Cumulative & Annual & Cumulative & Annual & Cumulative & Annual & Cumulative & Annual & Cumulative \\
\hline 1996 & & & & & 70 & 70 & 55 & 55 & 125 & 125 \\
\hline 1997 & & & & & 150 & 220 & 118 & 173 & 268 & 393 \\
\hline 1998 & & & & & 200 & 420 & 76 & 249 & 276 & 669 \\
\hline 1999 & & & & & 200 & 620 & 27 & 276 & 227 & 896 \\
\hline 2000 & & & & & 200 & 820 & & 276 & 200 & 1,096 \\
\hline 2001 & & & & & 200 & 1,020 & & 276 & 200 & 1,296 \\
\hline 2002 & 60 & 60 & & & 200 & 1,220 & & 276 & 260 & 1,556 \\
\hline 2003 & 60 & 120 & & & 200 & 1,420 & & 276 & 260 & 1,816 \\
\hline 2004 & 60 & 180 & & & 200 & 1,620 & & 276 & 260 & 2,076 \\
\hline 2005 & 60 & 240 & & & 200 & 1,820 & & 276 & 260 & 2,336 \\
\hline 2006 & 60 & 300 & & & 200 & 2,020 & & 276 & 260 & 2,596 \\
\hline 2007 & 60 & 360 & & & 200 & 2,220 & & 276 & 260 & 2,856 \\
\hline 2008 & 60 & 420 & & & 200 & 2,420 & & 276 & 260 & 3,116 \\
\hline 2009 & 60 & 480 & & & 200 & 2,620 & & 276 & 260 & 3,376 \\
\hline 2010 & 60 & 540 & & & 200 & 2,820 & & 276 & 260 & 3,636 \\
\hline 2011 & 60 & 600 & & & 200 & 3,020 & & 276 & 260 & 3,896 \\
\hline 2012 & & 600 & & & 200 & 3,220 & & 276 & 200 & 4,096 \\
\hline 2013 & 361 & 961 & & & 200 & 3,420 & & 276 & 561 & 4,657 \\
\hline 2014 & 561 & 1,521 & & & 200 & 3,620 & & 276 & 761 & 5,417 \\
\hline 2015 & 861 & 2,382 & & & 200 & 3,820 & & 276 & 1,061 & 6,478 \\
\hline 2016 & 861 & 3,242 & & & 200 & 4,020 & & 276 & 1,061 & 7,538 \\
\hline 2017 & 800 & 4,042 & & & 200 & 4,220 & & 276 & 1,000 & 8,538 \\
\hline 2018 & 800 & 4,842 & & & 200 & 4,420 & & 276 & 1,000 & 9,538 \\
\hline 2019 & 800 & 5,642 & 4 & 4 & 200 & 4,620 & & 276 & 1,004 & 10,542 \\
\hline 2020 & 800 & 6,442 & 40 & 45 & 200 & 4,820 & & 276 & 1,040 & 11,583 \\
\hline 2021 & 800 & 7,242 & 40 & 85 & 200 & 5,020 & & 276 & 1,040 & 12,623 \\
\hline 2022 & 800 & 8,042 & 40 & 125 & 200 & 5,220 & & 276 & 1,040 & 13,663 \\
\hline 2023 & 800 & 8,842 & 40 & 165 & 200 & 5,420 & & 276 & 1,040 & 14,703 \\
\hline 2024 & 800 & 9,642 & 40 & 206 & 200 & 5,620 & & 276 & 1,040 & 15,744 \\
\hline 2025 & 800 & 10,442 & 40 & 246 & 200 & 5,820 & & 276 & 1,040 & 16,784 \\
\hline 2026 & 800 & 11,242 & 40 & 285 & 124 & 5,944 & & 276 & 964 & 17,747 \\
\hline 2027 & 800 & 12,042 & 39 & 324 & & 5,944 & & 276 & 839 & 18,586 \\
\hline 2028 & 400 & 12,442 & 39 & 364 & & 5,944 & & 276 & 439 & 19,026 \\
\hline 2029 & & 12,442 & 37 & 400 & & 5,944 & & 276 & 37 & 19,062 \\
\hline 2030 & & 12,442 & 36 & 436 & & 5,944 & & 276 & 36 & 19,098 \\
\hline 2031 & & 12,442 & 36 & 472 & & 5,944 & & 276 & 36 & 19,134 \\
\hline
\end{tabular}


Table 2.7 (continued)

\begin{tabular}{|c|c|c|c|c|c|c|c|c|c|c|}
\hline \multirow{2}{*}{$\begin{array}{l}\text { End of } \\
\text { calendar } \\
\text { year }\end{array}$} & \multicolumn{2}{|c|}{ Hanford ${ }^{b}$} & \multicolumn{2}{|c|}{ INELC } & \multicolumn{2}{|c|}{ SRS $^{\mathrm{d}}$} & \multicolumn{2}{|c|}{ WVDpe } & \multicolumn{2}{|c|}{ Total } \\
\hline & Annual & Cumulative & Annual & Cumulative & Annual & Cumulative & Annual & Cumulative & Annual & Cumulative \\
\hline 2032 & & 12,442 & 36 & 508 & & 5,944 & & 276 & 36 & 19,170 \\
\hline 2033 & & 12,442 & 21 & 530 & & 5,944 & & 276 & 21 & 19,192 \\
\hline 2034 & & 12,442 & 21 & 551 & & 5,944 & & 276 & 21 & 19,213 \\
\hline 2035 & & 12,442 & 21 & 572 & & 5,944 & & 276 & 21 & 19,234 \\
\hline
\end{tabular}

${ }^{a}$ Taken from ref. 1. The projected waste volume, radioactivity, and thermal power values (Tables 2.4-2.6) are consistent with the number of canisters reported. Canister projections may not be calculated by the site in whole numbers, as presented here. Due to round-off, numbers may not add exactly. The projections reported for Hanford and INEL reflect major changes in the HLW solidification schedule. These changes are mainly caused by current DOE funding guidance.

b Hanford's reference canister has a diameter of $61 \mathrm{~cm}$ and is $450 \mathrm{~cm}$ long (about $2 \mathrm{ft}$ in diam by about $15 \mathrm{ft}$ in length). The nominal glass volume is expected to be $1.1 \mathrm{~m}^{3}$ with a minimum waste oxide loading of 25 vol \% (excluding sodium and silicon). Cesium and strontium capsules will also be disposed of in these canisters.

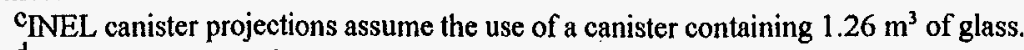

${ }^{d}$ Canisters are $0.6 \mathrm{~m}$ in diam by $3 \mathrm{~m}$ in length (about $2 \mathrm{ft}$ in diam by about $10 \mathrm{ft}$ in length). Each canister is assumed to contain $0.625 \mathrm{~m}^{3}$ of glass made with $\mathrm{HLW}$ from the reprocessing of SNF at SRS. The glass incorporates $36 \mathrm{wt} \%$ oxides from waste $(28 \mathrm{wt} \%$ from SNF and $8 \mathrm{wt} \%$ from processing chemicals) and $64 \mathrm{wt} \%$ oxides from nonradioactive glass frit.

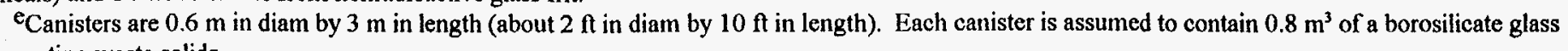
incorporating waste solids. 
Table 2.8. Current volume of HLW in storage by site through $1995^{\mathfrak{a}}$

\begin{tabular}{|c|c|c|c|c|c|c|}
\hline \multirow{3}{*}{ Site } & \multicolumn{6}{|c|}{ Volume, $10^{3} \mathrm{~m}^{3}$} \\
\hline & \multicolumn{2}{|c|}{ Tank waste } & \multicolumn{2}{|c|}{ Capsules } & \multirow{2}{*}{$\begin{array}{l}\text { Canister } \\
\text { material }\end{array}$} & \multirow{2}{*}{ Total } \\
\hline & Liquid $^{b}$ & Solid $^{\mathrm{C}}$ & $\mathrm{Sr}$ & Cs & & \\
\hline Hanford & 89.839 & 143.668 & 0.0011 & 0.0024 & & 233.5 \\
\hline INEL & 7.35 & 3.83 & & & & 11.2 \\
\hline SRS & 58.7 & 67.8 & & & & 126.5 \\
\hline WVDPd & 2.2 & & & & & 2.2 \\
\hline Total & 158.1 & 215.3 & 0.0011 & 0.0024 & 0 & 373.4 \\
\hline
\end{tabular}

${ }^{a}$ Taken from ref. 1.

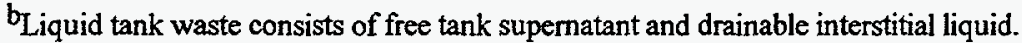

'Solid tank waste consists of sludge, salt cake, zeolite, calcine, and precipitate.

$d_{W V D P}$ liquid waste includes sludge and zeolite.

Table 2.9. Current radioactivity of HLW in storage by site through $1995^{a}$

\begin{tabular}{|c|c|c|c|c|c|c|}
\hline \multirow{3}{*}{ Site } & \multicolumn{6}{|c|}{ Radioactivity, $10^{6} \mathrm{Ci}$} \\
\hline & \multicolumn{2}{|c|}{ Tank waste } & \multicolumn{2}{|c|}{ Capsules } & \multirow{2}{*}{$\begin{array}{l}\text { Canister } \\
\text { material }\end{array}$} & \multirow{2}{*}{ Total } \\
\hline & Liquid $^{b}$ & Solid $\mathrm{c}^{\mathrm{C}}$ & $\mathrm{Sr}$ & Cs & & \\
\hline Hanford & 68.5 & 125.3 & 44.9 & 101.2 & & 339.9 \\
\hline INEL & 2.7 & 46.6 & & & & 49.3 \\
\hline SRS & 214.6 & 287.6 & & & & 502.2 \\
\hline WVDP $^{d}$ & 24.05 & & & & & 24.1 \\
\hline Total & 309.8 & 459.5 & 44.9 & 101.2 & 0 & 915.4 \\
\hline
\end{tabular}

${ }^{\text {a Taken from ref. } 1 .}$

biquid tank waste consists of free tank supernatant and drainable interstitial liquid.

'Solid tank waste consists of sludge, salt cake, zeolite, calcine, and precipitate.

dWVDP liquid waste includes sludge and zeolite. 
Table 2.10. Current thermal power of HLW in storage by site through $1995^{\mathrm{a}}$

\begin{tabular}{|c|c|c|c|c|c|c|}
\hline \multirow{3}{*}{ Site } & \multicolumn{6}{|c|}{ Thermal power, $10^{3} \mathrm{~W}$} \\
\hline & \multicolumn{2}{|c|}{ Tank waste } & \multicolumn{2}{|c|}{ Capsules } & \multirow{2}{*}{$\begin{array}{l}\text { Canister } \\
\text { material }\end{array}$} & \multirow{2}{*}{ Total } \\
\hline & Liquid $^{b}$ & Solid ${ }^{c}$ & $\mathrm{Sr}$ & Cs & & \\
\hline Hanford & 166.7 & 414.24 & 150.3 & 245.4 & & 976.7 \\
\hline INEL & 7.8 & 135.0 & & & & 142.8 \\
\hline SRS & 476.2 & 929.84 & & & & $1,406.0$ \\
\hline WVDPd & 69.7 & & & & & 69.7 \\
\hline Total & 720.4 & $1,479.1$ & 150.3 & 245.4 & 0 & $2,595.2$ \\
\hline
\end{tabular}

aTaken from ref. 1.

$\mathrm{b}_{\text {Liquid tank waste consists of free tank supernatant and drainable interstitial liquid. }}$

'Solid tank waste consists of sludge, salt cake, zeolite, calcine, and precipitate.

WVDP liquid waste includes sludge and zeolite.

Table 2.11. Major radionuclides comprising $H L W$ and associated wastes at $H a n$ ford $^{\mathrm{a}}$

\begin{tabular}{|c|c|c|c|c|c|c|}
\hline \multirow{3}{*}{ Radionuclides } & \multicolumn{6}{|c|}{ Radioactivity ( $\mathrm{Ci}$ ) by waste category } \\
\hline & \multicolumn{2}{|c|}{ Interim forms ${ }^{b}$} & \multicolumn{4}{|c|}{ Final forms ${ }^{c}$} \\
\hline & Tank waste & $\begin{array}{c}\text { Other } \\
\text { (capsules) }\end{array}$ & $\begin{array}{l}\text { HLW glass } \\
\text { canisters }\end{array}$ & $\begin{array}{l}\text { LLW form } \\
\text { (glass) }^{d}\end{array}$ & $\begin{array}{l}\text { Cumulative } \\
\text { out of system }\end{array}$ & $\begin{array}{c}\text { Other } \\
\text { (capsule canisters) }\end{array}$ \\
\hline${ }^{3} \mathrm{H}$ & e & e & & & & \\
\hline${ }^{14} \mathrm{C}$ & $4.574 \mathrm{E}+03$ & & $4.555 \mathrm{E}+03$ & & & \\
\hline${ }^{90} \mathrm{Sr}$ & $5.952 E+07$ & $2.246 \mathrm{E} \div 07$ & $2.713 E+07$ & & & $1.024 E+07$ \\
\hline${ }^{90} \mathrm{Y}$ & $5.952 \mathrm{E}+07$ & $2.246 \mathrm{E}+07$ & $2.713 E+07$ & & & $1.024 \mathrm{E}+07$ \\
\hline${ }^{99} \mathrm{Tc}$ & $3.210 \mathrm{E}+04$ & & $3.209 E+04$ & & & \\
\hline${ }^{129} \mathrm{~T}$ & $2.980 \mathrm{E}-01$ & & $2.979 E-01$ & & & \\
\hline${ }^{137} \mathrm{Cs}$ & $3.772 \mathrm{E}+07$ & $5.196 \mathrm{E}+07$ & $1.760 E+07$ & & & $2.425 \mathrm{E}+07$ \\
\hline${ }^{137 \mathrm{~m}} \mathrm{Ba}$ & $3.572 \mathrm{E}+07$ & $4.921 E+07$ & $1.667 E+07$ & & & $2.296 \mathrm{E}+07$ \\
\hline${ }^{151} \mathrm{Sm}$ & $1.058 \mathrm{E}+06$ & & $8.205 E+05$ & & & \\
\hline${ }^{238} \mathrm{Pu}$ & $1.415 E+03$ & & $1.090 E+03$ & & & \\
\hline${ }^{239} \mathrm{Pu}$ & $2.635 \mathrm{E}+04$ & & $2.632 E+04$ & & & \\
\hline${ }^{240} \mathrm{Pu}$ & $6.692 \mathrm{E}+03$ & & $6.667 E+03$ & & & \\
\hline${ }^{241} \mathrm{Pu}$ & $9.316 \mathrm{E}+04$ & & $1.903 E+04$ & & & \\
\hline${ }^{242} \mathrm{Pu}$ & $2.802 E-01$ & & $2.801 E-01$ & & & \\
\hline${ }^{241} \mathrm{Am}$ & $1.037 \mathrm{E}+05$ & & $1.007 E+05$ & & & \\
\hline${ }^{242} \mathrm{Am}$ & $6.247 \mathrm{E}+01$ & & $5.373 \mathrm{E}+01$ & & & \\
\hline Total & $1.938 \mathrm{E}+08$ & $1.461 E+08$ & $8.955 E+07$ & & & $6.769 E+07$ \\
\hline
\end{tabular}

a Data taken from ref. 1(a).

${ }_{\text {As of December } 31,1995 .}$

$c_{\text {As of December } 31,2028 .}$

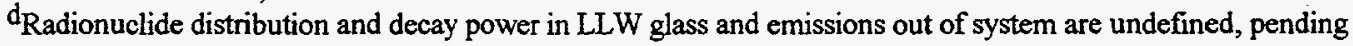
flowsheet development and regulatory decisions.

Estimate of December 31,1995 , inventory for ${ }^{3} \mathrm{H}$ is currently unavailable. 
Table 2.12. Major radionuclides comprising $H L W$ and associated wastes at NEL $^{a}$

\begin{tabular}{|c|c|c|c|c|}
\hline \multirow{3}{*}{ Radionuclides } & \multicolumn{4}{|c|}{ Radioactivity ( $\mathrm{Ci}$ ) by waste category } \\
\hline & \multicolumn{2}{|c|}{ Interim forms ${ }^{b}$} & \multicolumn{2}{|c|}{ Final forms $\mathrm{c}^{\mathrm{c}}$} \\
\hline & Liquid & Calcine & Glass & Grout \\
\hline${ }^{90} \mathrm{Sr}$ & $6.23 E+05$ & $1.10 \mathrm{E}+07$ & $4.28 \mathrm{E}+06$ & $4.28 \mathrm{E}+02$ \\
\hline${ }^{90} \mathrm{Y}$ & $6.23 E+05$ & $1.10 \mathrm{E}+07$ & $4.28 E+06$ & $4.28 E+02$ \\
\hline${ }^{106} \mathrm{Ru}$ & $3.03 E+02$ & $1.87 E+03$ & $8.90 E-10$ & $8.90 E-10$ \\
\hline${ }^{106} \mathrm{Rh}$ & $3.03 \mathrm{E}+02$ & $1.87 \mathrm{E}+03$ & $8.90 E-10$ & $8.90 E-10$ \\
\hline${ }^{134} \mathrm{Cs}$ & $4.48 E+03$ & $3.79 E+04$ & $5.95 E-02$ & $5.95 E-06$ \\
\hline${ }^{137} \mathrm{Cs}$ & $7.34 \mathrm{E}+05$ & $1.26 \mathrm{E}+07$ & $5.05 \mathrm{E}+06$ & $5.05 \mathrm{E}+02$ \\
\hline${ }^{137 m} \mathrm{Ba}$ & $6.96 \mathrm{E}+05$ & $1.19 E+07$ & $4.79 \mathrm{E}+06$ & $4.79 \mathrm{E}+02$ \\
\hline${ }^{144} \mathrm{Ce}$ & $1.59 E+03$ & $7.17 \mathrm{E}+03$ & $2.84 \mathrm{E}-12$ & $2.84 \mathrm{E}-16$ \\
\hline${ }^{144} \mathrm{Pr}$ & $1.59 E+03$ & $7.17 E+03$ & $2.84 E-12$ & $2.84 E-16$ \\
\hline${ }^{147} \mathrm{Pm}$ & & $6.15 E+04$ & $1.71 E+00$ & $1.71 \mathrm{E}-04$ \\
\hline${ }^{154} \mathrm{Eu}$ & $4.16 \mathrm{E}+03$ & $4.76 \mathrm{E}+04$ & $1.92 E+03$ & $1.92 \mathrm{E}-01$ \\
\hline Total & $2.69 E+06$ & $4.66 \mathrm{E}+07$ & $1.84 \mathrm{E}+07$ & $1.84 E+03$ \\
\hline
\end{tabular}

a Data taken from ref. $1(b)$

${ }^{b}$ As of December 31, 1995.

$\mathrm{c}_{\text {As of December } 31,2035 .}$ 
Table 2.13. Major radionuclides comprising $H L W$ and associated wastes at SRS ${ }^{a}$

\begin{tabular}{|c|c|c|c|c|}
\hline \multirow{3}{*}{ Radionuclides } & \multicolumn{4}{|c|}{ Radioactivity ( $\mathrm{Ci}$ ) by waste category } \\
\hline & \multirow{2}{*}{$\frac{\text { Interim form }{ }^{b}}{\text { Tank waste }}$} & \multicolumn{3}{|c|}{ Final forms $s^{c}$} \\
\hline & & $\begin{array}{l}\text { Canister } \\
\text { material }\end{array}$ & $\begin{array}{l}\text { Saltstone } \\
\text { (LLW) }\end{array}$ & Outfal \\
\hline${ }^{3} \mathrm{H}$ & $8.06 E+04$ & $2.41 E+03$ & $7.82 E-01$ & d \\
\hline${ }^{14} \mathrm{C}$ & $2.00 \mathrm{E}+01$ & $5.99 \mathrm{E}-01$ & $1.94 E-04$ & $d$ \\
\hline${ }^{90} \mathrm{Sr}$ & $1.19 \mathrm{E}+08$ & $3.57 \mathrm{E}+06$ & $1.16 \mathrm{E}+03$ & $\mathrm{~d}$ \\
\hline${ }^{90} \mathrm{Y}$ & $1.19 E+08$ & $3.57 E+06$ & $1.16 E+03$ & $d$ \\
\hline${ }^{99} \mathrm{Tc}$ & $2.28 \mathrm{E}+04$ & $6.82 E+02$ & $2.21 E-01$ & d \\
\hline${ }^{129} \mathrm{I}$ & $3.68 \mathrm{E}+01$ & $1.10 \mathrm{E}+00$ & $3.57 \mathrm{E}-04$ & $\mathrm{~d}$ \\
\hline${ }^{137} \mathrm{Cs}$ & $1.16 E+08$ & $3.47 \mathrm{E}+06$ & $1.12 E+03$ & d \\
\hline${ }^{137 \mathrm{~m}} \mathrm{Ba}$ & $1.10 \mathrm{E}+08$ & $3.28 \mathrm{E}+06$ & $1.06 \mathrm{E}+03$ & d \\
\hline${ }^{238} \mathrm{Pu}$ & $1.71 E+06$ & $5.13 E+04$ & $1.66 \mathrm{E}+01$ & d \\
\hline${ }^{239} \mathrm{Pu}$ & $3.64 \mathrm{E}+04$ & $1.09 E+03$ & $3.54 \mathrm{E}-01$ & d \\
\hline${ }^{240} \mathrm{Pu}$ & $1.66 \mathrm{E}+04$ & $4.96 E+02$ & $1.61 \mathrm{E}-01$ & d \\
\hline${ }^{241} \mathrm{Pu}$ & $7.53 E+05$ & $2.26 E+04$ & $7.31 \mathrm{E}+00$ & d \\
\hline${ }^{242} \mathrm{Pu}$ & $2.84 \mathrm{E}+01$ & $8.51 \mathrm{E}-01$ & $2.76 \mathrm{E}-04$ & $d$ \\
\hline${ }^{241} \mathrm{Am}$ & $6.06 \mathrm{E}+04$ & $1.82 \mathrm{E}+03$ & $5.88 E-01$ & d \\
\hline${ }^{242 \mathrm{~m}} \mathrm{Am}$ & $6.38 E+01$ & $1.91 E+00$ & $6: 20 E-04$ & d \\
\hline${ }^{232} \mathrm{Th}$ & $1.47 \mathrm{E}+00$ & $4.41 E-02$ & $1.43 E-05$ & $\mathrm{~d}$ \\
\hline${ }^{233} \mathrm{U}$ & $1.08 \mathrm{E}+02$ & $3.25 \mathrm{E}+00$ & $1.05 \mathrm{E}-03$ & d \\
\hline${ }^{234} \mathrm{U}$ & $3.01 E+01$ & $9.00 \mathrm{E}-01$ & $2.92 \mathrm{E}-04$ & $\mathrm{~d}$ \\
\hline${ }^{237} \mathrm{~Np}$ & $7.04 \mathrm{E}+01$ & $2.11 \mathrm{E}+00$ & $6.83 E-04$ & d \\
\hline${ }^{244} \mathrm{Cm}$ & $2.50 \mathrm{E}+03$ & $7.50 \mathrm{E}+01$ & $2.43 E-02$ & $\mathrm{~d}$ \\
\hline Total & $4.67 \mathrm{E}+08$ & $1.40 \mathrm{E}+07$ & $4.53 E+03$ & d \\
\hline
\end{tabular}

a Data taken from ref. 1(c).

${ }^{b}$ As of December 31, 1995.

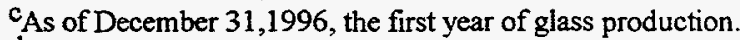

degligible contribution. 
Table 2.14. Major radionuclides comprising HLW and associated wastes at WVDPa

\begin{tabular}{|c|c|c|c|c|}
\hline \multirow{3}{*}{ Radionuclides } & \multicolumn{4}{|c|}{ Radioactivity, $\mathrm{Ci}$, by waste category } \\
\hline & \multirow{2}{*}{$\frac{\text { Interim form }}{\text { Tank waste }}$} & \multicolumn{3}{|c|}{ Final forms ${ }^{c}$} \\
\hline & & Canister material & LLW form & Emissions \\
\hline${ }^{3} \mathrm{H}$ & $1.23 E+00$ & & & \\
\hline${ }^{14} \mathrm{C}$ & $5.49 \mathrm{E}-01$ & & & \\
\hline${ }^{90} \mathrm{Sr}$ & $5.81 E+06$ & & & \\
\hline${ }^{90} \mathrm{Y}$ & $5.81 E+06$ & & & \\
\hline${ }^{99} \mathrm{Tc}$ & $1.09 E+02$ & & & \\
\hline${ }^{106} \mathrm{Ru}$ & $2.30 E-01$ & & & \\
\hline${ }^{106} \mathrm{Rh}$ & $2.30 \mathrm{E}-01$ & & & \\
\hline${ }^{129} \mathrm{I}$ & $1.81 E-01$ & & & \\
\hline${ }^{134} \mathrm{Cs}$ & $6.87 E+02$ & & & \\
\hline${ }^{135} \mathrm{Cs}$ & $1.61 E+02$ & & & \\
\hline${ }^{137} \mathrm{Cs}$ & $6.28 \mathrm{E}+06$ & & & \\
\hline${ }^{137} \mathrm{~m} \mathrm{Ba}$ & $5.94 E+06$ & & & \\
\hline${ }^{147} \mathrm{Pm}$ & $1.79 E+04$ & & & \\
\hline${ }^{238} \mathrm{Pu}$ & $7.92 \mathrm{E}+03$ & & & \\
\hline${ }^{239} \mathrm{Pu}$ & $1.63 \mathrm{E}+03$ & & & \\
\hline${ }^{240} \mathrm{Pu}$ & $1.19 E+03$ & & & \\
\hline${ }^{241} \mathrm{Pu}$ & $6.04 \mathrm{E}+04$ & & & \\
\hline${ }^{242} \mathrm{Pu}$ & $1.62 \mathrm{E}+00$ & & & \\
\hline${ }^{241} \mathrm{Am}$ & $5.36 E+04$ & & & \\
\hline${ }^{242} \mathrm{Am}$ & $2.87 \mathrm{E}+02$ & & & \\
\hline${ }^{244} \mathrm{Cm}$ & $6.07 E+03$ & & & \\
\hline Total & $2.40 \mathrm{E}+07$ & & & - \\
\hline
\end{tabular}

a Data taken from ref. 1 (d).

${ }^{b}$ As of December 31, 1995.

${ }$ Radionuclide information for the final forms generated from HLW treatment is currently unavailable. 
Table 2.15. Significant revisions and changes in the current values for HLW compared to the values in the previous year ${ }^{a, b}$

\begin{tabular}{|c|c|c|c|c|}
\hline Waste characteristics & $\begin{array}{l}\text { Previous report } \\
\text { values }^{\mathrm{a}}\end{array}$ & $\begin{array}{l}\text { Significant revisions } \\
\text { and changes }\end{array}$ & Updated values $^{b}$ & Explanation \\
\hline \multicolumn{5}{|c|}{ Hanford Site } \\
\hline Number of canisters & See Table 2.7 & $\begin{array}{l}\text { Canister production } \\
\text { schedule updated }\end{array}$ & See Table 2.7 & \\
\hline \multicolumn{5}{|c|}{ Idaho National Engineering Laboratory } \\
\hline Number of canisters & See Table 2.7 & $\begin{array}{l}\text { Canister production } \\
\text { schedule updated }\end{array}$ & See Table 2.7 & $\begin{array}{l}\text { Based on current funding guidance from DOE } \\
\text { and the INEL Full Treatment Alternative } \\
\text { (see ref. 15) }\end{array}$ \\
\hline \multicolumn{5}{|c|}{ Savannah River Site } \\
\hline Number of canisters & See Table 2.7 & $\begin{array}{l}\text { Canister production } \\
\text { schedule updated }\end{array}$ & See Table 2.7 & $\begin{array}{l}\text { Based on current funding guidance from DOE } \\
\text { and the SRS High-Level Waste System Plan } \\
\text { Revision } 6(U) \text { (see ref. 16) }\end{array}$ \\
\hline \multicolumn{5}{|c|}{ West Valley Demonstration Project } \\
\hline $\begin{array}{l}\text { Volume, radioactivity, and } \\
\text { thermal power }\end{array}$ & See Tables 2.8-2.10 & $\begin{array}{l}\text { Values reported are } \\
\text { for liquid, sludge, } \\
\text { and zeolite }\end{array}$ & See Tables 2.8-2.10 & Wastes have been blended prior to vitrification \\
\hline
\end{tabular}

aData are for December 31, 1994. See tables and text cited in Chapter 2 of ref. 6 (DOE/RW-0006, Rev. 11).

${ }^{b}$ Data are for December 31,1995 , as reported in this document (DOE/RW-0006, Rev. 12). 
Table 2.16. Proposed representative chemical composition of future HLW glass to be generated at Hanford ${ }^{2}$

\begin{tabular}{ll||lr}
\hline Component & Wt $\%$ & Component & Wt \% \\
\hline $\mathrm{Al}_{2} \mathrm{O}_{3}$ & 9.31 & $\mathrm{Na}_{2} \mathrm{O}$ & 11.79 \\
$\mathrm{~B}_{2} \mathrm{O}_{3}$ & 7.02 & $\mathrm{Na}_{2} \mathrm{SO}_{4}$ & 0.10 \\
$\mathrm{Bi}_{2} \mathrm{O}_{3}$ & 1.15 & $\mathrm{NiO}$ & 1.08 \\
$\mathrm{CaO}$ & 0.83 & $\mathrm{P}_{2} \mathrm{O}_{5}$ & 1.56 \\
$\mathrm{Ce}_{2} \mathrm{O}_{3}$ & 1.13 & $\mathrm{PbO}_{2}$ & 0.14 \\
$\mathrm{Cr}_{2} \mathrm{O}_{3}$ & 0.36 & $\mathrm{SiO}_{2}$ & 46.11 \\
$\mathrm{Fe}_{2} \mathrm{O}_{3}$ & 4.49 & $\mathrm{SrO}$ & 0.18 \\
$\mathrm{~K}_{2} \mathrm{O}$ & 0.17 & $\mathrm{ThO}_{2}$ & 0.01 \\
$\mathrm{La}_{2} \mathrm{O}_{3}$ & 0.11 & $\mathrm{UO}_{3}$ & 6.69 \\
$\mathrm{Li}_{2} \mathrm{O}$ & 2.01 & $\mathrm{ZrO}_{2}$ & 3.79 \\
$\mathrm{MnO}_{2}$ & 1.17 & Other & 0.17 \\
$\mathrm{NaF}$ & 0.63 & & \\
\hline
\end{tabular}

aData taken from ref. 1(a).

Table 2.17. Proposed representative chemical composition of future HLW glass to be produced at INEL ${ }^{a}$

\begin{tabular}{|c|c|c|c|}
\hline \multirow{2}{*}{$\begin{array}{l}\text { Chemical } \\
\text { compound }\end{array}$} & \multicolumn{3}{|c|}{ Glass, wt $\%$, formed from high-activity fraction from } \\
\hline & $\begin{array}{l}\text { Dissolved } \\
\mathrm{Zr} \text { calcine }\end{array}$ & $\begin{array}{l}\text { Dissolved } \\
\text { Al calcine }\end{array}$ & Na-bearing-waste \\
\hline $\mathrm{Al}_{2} \mathrm{O}_{3}$ & 0.5 & 12.7 & 17.2 \\
\hline$A M P^{b}$ & 0.8 & 6.6 & . \\
\hline $\mathrm{B}_{2} \mathrm{O}_{3}$ & 12.2 & 8.1 & 11.3 \\
\hline $\mathrm{CaF}_{2}$ & 14.5 & & \\
\hline $\mathrm{CaO}$ & 0.4 & & \\
\hline $\mathrm{Cs}_{2} \mathrm{O}$ & & 0.1 & \\
\hline $\mathrm{Fe}_{2} \mathrm{O}_{3}$ & & 0.1 & 0.1 \\
\hline $\mathrm{Na}_{2} \mathrm{O}$ & 12.9 & 18.0 & 13.8 \\
\hline $\mathrm{P}_{2} \mathrm{O}_{5}$ & 0.1 & & \\
\hline $\mathrm{SiO}_{2}$ & 56.8 & 54.4 & 57.6 \\
\hline $\mathrm{ZrO}_{2}$ & 1.8 & & \\
\hline Total & 100.0 & 100.0 & 100.0 \\
\hline
\end{tabular}

aData taken from ref. 1(b); flowsheet estimate, not verified by laboratory tests.

$\mathrm{b}_{\text {Ammoniummolybdophosphate. }}$ 
Table 2.18. Proposed representative chemical composition of future HLW glass to be generated at SRS ${ }^{a}$

\begin{tabular}{lc||cr}
\hline Component & Wt \% & Component & Wt \% \\
\hline $\mathrm{Al}_{2} \mathrm{O}_{3}$ & 3.9 & $\mathrm{MgO}$ & 2.0 \\
$\mathrm{~B}_{2} \mathrm{O}_{3}$ & 7.3 & $\mathrm{MnO}$ & 1.2 \\
$\mathrm{CaO}$ & 0.6 & $\mathrm{Na}$ & $\mathrm{O}$ \\
$\left.\mathrm{Ca}_{3} \mathrm{PO}_{4}\right)_{2}$ & 1.1 & $\mathrm{NiO}$ & 0.7 \\
$\mathrm{Cr}_{2} \mathrm{O}_{3}$ & 0.2 & $\mathrm{SiO}_{2}$ & 53.4 \\
$\mathrm{CuO}$ & 0.4 & $\mathrm{TiO}_{2}$ & 0.3 \\
$\mathrm{FeO}_{\mathrm{Fe}}$ & 1.1 & $\mathrm{U}_{3} \mathrm{O}_{8}$ & 0.9 \\
$\mathrm{Fe}_{2} \mathrm{O}_{3}$ & 11.1 & $\mathrm{ZnO}_{2}$ & 0.1 \\
$\mathrm{~K}_{2} \mathrm{O}$ & 2.4 & Other & 0.4 \\
$\mathrm{Li}_{2} \mathrm{O}$ & 4.8 & & \\
& & Total & 100.0 \\
\hline
\end{tabular}

aData taken from ref. 1(c).

Table 2.19. Proposed representative chemical composition of future HLW glass to be generated at WVDP ${ }^{a}$

\begin{tabular}{|c|c|c|c|}
\hline Component & Wt \% & Composition & Wt \% \\
\hline $\mathrm{Al}_{2} \mathrm{O}_{3}$ & 6.00 & $\mathrm{Nd}_{2} \mathrm{O}_{3}$ & 0.14 \\
\hline $\mathrm{B}_{2} \mathrm{O}_{3}$ & 12.89 & $\mathrm{NiO}$ & 0.25 \\
\hline $\mathrm{BaO}$ & 0.16 & $\mathrm{P}_{2} \mathrm{O}_{5}$ & 1.20 \\
\hline $\mathrm{CaO}$ & 0.48 & $\mathrm{PdO}$ & 0.03 \\
\hline $\mathrm{Ce}_{2} \mathrm{O}_{3}$ & 0.31 & $\mathrm{Pr}_{6} \mathrm{O}_{11}$ & 0.04 \\
\hline $\mathrm{CoO}$ & 0.02 & $\mathrm{Rh}_{2} \mathrm{O}_{3}$ & 0.02 \\
\hline $\mathrm{Cr}_{2} \mathrm{O}_{3}$ & 0.14 & $\mathrm{RuO}_{2}$ & 0.08 \\
\hline $\mathrm{Cs}_{2} \mathrm{O}$ & 0.08 & $\mathrm{SO}_{3}$ & 0.23 \\
\hline $\mathrm{CuO}$ & 0.03 & $\mathrm{SiO}_{2}$ & 40.98 \\
\hline $\mathrm{Fe}_{2} \mathrm{O}_{3}$ & 12.02 & $\mathrm{Sm}_{2} \mathrm{O}_{3}$ & 0.03 \\
\hline $\mathrm{K}_{2} \mathrm{O}$ & 5.00 & $\mathrm{SrO}$ & 0.02 \\
\hline $\mathrm{La}_{2} \mathrm{O}_{3}$ & 0.04 & $\mathrm{ThO}_{2}$ & 3.56 \\
\hline $\mathrm{Li}_{2} \mathrm{O}$ & 3.71 & $\mathrm{TiO}_{2}$ & 0.80 \\
\hline $\mathrm{MgO}$ & 0.89 & $\mathrm{UO}_{3}$ & 0.63 \\
\hline $\mathrm{MnO}$ & 0.82 & $\mathrm{Y}_{2} \mathrm{O}_{3}$ & 0.02 \\
\hline $\mathrm{MoO}_{3}$ & 0.04 & $\mathrm{ZnO}$ & 0.02 \\
\hline \multirow[t]{2}{*}{$\mathrm{Na}_{2} \mathrm{O}$} & 8.00 & $\mathrm{ZrO}_{2}$ & 1.32 \\
\hline & & Total & 100.00 \\
\hline
\end{tabular}

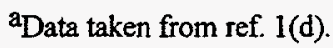





\section{TRANSURANIC WASTE}

\subsection{INTRODUCTION}

This chapter presents information on the inventories and characteristics of transuranic waste (TRUW) at various DOE transuranic (TRU) waste sites in the United States. TRUW is a waste category specific to DOE; it does not apply to wastes regulated by the U.S. Nuclear Regulatory Commission. DOE Order 5820.2A defines TRUW as waste that (1) is contaminated with alphaemitting transuranic (i. e., atomic numbers greater than 92) radionuclides with half-lives greater than 20 years and (2) contains a total concentration of such radionuclides in excess of $100 \mathrm{nCi} / \mathrm{g}$ of waste at the time of assay. ${ }^{1}$

DOE Order 5820.2A also states that heads of field elements can determine that other alpha-contaminated waste at the site(s) may be managed as TRUW. ${ }^{1}$ As a consequence of this provision, wastes containing radionuclides such as ${ }^{233} \mathrm{U}$ and ${ }^{244} \mathrm{Cm}$, which do not meet the strict definition of TRU radionuclides because of their respective atomic numbers or half-lives, may be managed as TRUW at some sites.

Most TRUW exists in solid form (e.g., items such as protective clothing, paper, rags, glass, miscellaneous tools, and equipment that have become contaminated with TRU radionuclides). ${ }^{2}$ Some TRUW is in the form of sludges or liquids resulting from chemical processing for recovery of plutonium or other TRU elements. ${ }^{2}$ Some of the liquids have been solidified, and some sludges have been dewatered. All sludge and liquid wastes scheduled for disposal in the Waste Isolation Pilot Plant (WIPP) will be solidified prior to their shipment to meet the current WIPP waste acceptance criteria (WAC). ${ }^{3}$

Before 1970, TRUW was disposed of on-site in shallow, landfill-type, DOE-owned burial sites. TRUW disposed of in this manner is referred to as "buried" TRUW. In 1970, DOE's predecessor agency, the U.S. Atomic Energy Commission, concluded that waste containing long-lived alpha-emitting radionuclides should have greater confinement from the environment. Thus, TRUW generated since the early 1970 s has been segregated from other waste types and placed in retrievable storage pending shipment and final disposal in a permanent geologic repository. ${ }^{4}$ This waste is referred to as "retrievably stored" TRUW.

Retrievably stored waste is contained in a variety of packagings (e.g., metal drums and wooden and metal boxes) and is stored in various facilities such as earthmounded berms, concrete culverts, buildings, and on outdoor pads. Many of these facilities have been upgraded and are now in compliance with applicable hazardous waste storage regulations.

TRUW packages are classified as either "contact handled" $(\mathrm{CH})$ or "remote handled" $(\mathrm{RH})$, depending on the radiation level at the surface of the package at the time of packaging. If this level equals or exceeds $200 \mathrm{mrem} / \mathrm{h}$, the package is classified as RH TRUW.

CH TRUW contains relatively small quantities of fission and activation products that produce highly penetrating radiation. Typically, its emissions consists mostly of alpha particles, some neutrons and primarily low-energy gamma and X-rays. RH TRUW typically contains a greater proportion of fission and activation products that produce highly penetrating radiation and produce a higher level of radiation at the surface of the package.

Mixed TRUW is defined as TRUW which contains, in addition to radioactive constituents, hazardous constituents defined and regulated in accordance with the Resource Conservation and Recovery Act (RCRA). Examples of mixed TRUW are radionuclide-contaminated sludges from plutonium recovery, discarded materials contaminated with both solvents and radioactive materials, scintillation fluids, and discarded, contaminated lead shielding. Mixed TRUW must be managed to comply with both the applicable hazardous waste regulations (e.g., RCRA) and the regulations applying only to radioactive TRUW.

Some TRUW may also be contaminated with hazardous materials defined by regulations other than RCRA (e.g., Toxic Substances Control Act and state regulations). Once identified, DOE continues to manage these wastes appropriately to meet all other hazardous waste regulations in addition to RCRA (if applicable). 
Under existing arrangements, retrievably stored TRUW is the responsibility of the DOE Office of Waste Management (EM-30). It is planned that the retrievably stored TRUW and newly generated TRUW from routine site operational activities will be shipped to WIPP for disposal; whereas, buried TRUW and TRUW generated from site remediation activities and decontamination and decommissioning (D\&D) activities are the responsibility of the Office of Environmental Restoration (EM-40). (See Chapter 6 of this document for a discussion of environmental restoration wastes.)

\subsection{TRUW INVENTORIES}

\subsubsection{Sources of Data}

The vast majority of the quantitative information contained in this chapter is either derived or summarized from end of the calendar year data furnished by the DOE sites and published in the Transuranic Waste Baseline Inventory Report (Revision 3) (TWBIR). ${ }^{2}$ In some cases, when no new data were made available by the sites (e.g., for TRU-contaminated soil volumes), the information presented in this chapter is the same as that presented in the previous revision of the $\mathrm{IDB} .^{5}$ As programs and plans evolve or are changed, modifications or additions will be made to the data and other information in this chapter. It is expected that the quality and accuracy of the data will improve with each annual revision of this document, thus improving the usefulness of the data for program planning and decision purposes.

Early TRUW inventory practices were not as stringent as current ones with regard to requirements for waste identification, categorization, and segregation. Consequently, early inventory data are based largely on process knowledge and on various studies and summaries related to site-specific practices. ${ }^{6}$ As these efforts continue and TRUWW is further characterized, there may be revisions in the estimated overall quantities of TRUW.

\subsubsection{Site Locations-Summarized Volumes and Radioactivity}

TRUW management activities (generation, retrievable storage, etc.) are performed at 10 major sites and 15 smallquantity sites (SQS). Figure 3.1 shows the locations of the DOE's TRU waste sites. Tables 3.1-3.21, which are discussed later in this chapter, summarize the distribution of TRUW and their radioactivities at various DOE TRU waste sites. The volumes reported in this document reflect two waste conditions: as-generated and final form. For stored waste, the as-generated volumes reflect the waste volumes currently in storage at the site, whereas for projected waste the as-generated volumes reflect the expected waste volumes at the time of generation. The final-form volumes reflect the expected volume of the waste following site processing, treatment, and repackaging of the as-generated waste for disposal at WIPP.

\subsubsection{Development of Detailed Inventory Data}

To optimize its resources and to enhance data quality, DOE integrated the TRUW data needs of the IDB report with those of the TWBIR and issued a joint data call to the sites in 1995. The information contained in this chapter is primarily a summary of the detailed data gathered through this integrated data-call process, supplemented by additional information received from some DOE sites after the original data submittals. The data reported in this document are based on both as-generated and final-form volumes of TRUW, and are derived from Rev. 3 of the TWBIR, which was published in June 1996 to document the TRUW inventory data used by Sandia National Laboratories for performance assessment calculations for the WIPP repository.

To meet the needs of the TWBIR, site data were collected at the waste-stream level in both their asgenerated and final forms. The data were grouped by similar physical and chemical properties and aggregated to produce estimated total volumes of the waste.

The data reported in the tables in this chapter include both the as-generated and final-form volumes at the end of CY 1994, and the projected increase in these respective volumes from 1995 through 2022.

\subsubsection{Site data submittal process}

As stated previously, the vast majority of the quantitative TRUW information presented in this chapter is derived or summarized from Rev. 3 of the TWBIR, published in June 1996. The sites provided waste stream profiles for each waste stream in their respective current and projected inventories through 2022. The waste stream profiles included such information as waste stream description, as-generated and final-form volumes, specific container data, and estimates of the amount of various materials present in the waste (e.g., iron-based metals and alloys, cellulosics, plastics, etc.). In addition, the sites were provided with a list of acceptable final waste forms for disposal at WIPP and asked to assign the appropriate final waste form to each waste stream that is eligible for disposal at WIPP. The assignment of final waste form helps to identify the general physical and chemical properties of the final form for each waste stream.

The sites provided radionuclide compositions and curies of each radionuclide added in each year of TRUW 
accumulation from 1970 through the end of CY 1994. The annual radioactivities reported for the currently retrievable stored waste in the site submittals are on an as-stored basis; that is, they represent the curies of each radionuclide added each year at the end of the year in which the currently retrievable waste was placed in storage. Only the cumulative site radioactivities decayed to the end of 1995 have been included in this chapter.

\subsubsection{Site data review and finalization}

The site data submittals for TRUW were reviewed to make certain, to the greatest extent possible, that the data supplied met the requirements of completeness and consistency. The data review process included modifying the formats of the data so that they could be easily converted to input data files.

In support of the TWBIR, waste streams at each TRUW site that were assigned the same final waste form were grouped together to form site-specific waste profiles. These profiles provide a condensed summary of the volume and waste material information found in the waste stream profiles for each site. Site-specific waste profiles with the same final waste form were then combined for all the TRU waste sites to produce WIPP waste profiles. The summary tables and figures presented in this chapter for final form volumes are based on aggregation of detailed waste stream profile data reported in Rev. 3 of the TWBIR.

\subsubsection{Volumes and Radioactivities of TRUW at DOE Sites}

\subsubsection{As-generated TRUW volumes}

Table 3.1 shows the total estimated as-generated TRUW volumes in storage at the end of 1994 and projected through 2022 for each DOE site, and Tables 3.2-3.7 show the breakup of this total as-generated inventory into total $\mathrm{CH}$ TRUW (Table 3.2), mixed $\mathrm{CH}$ TRUW (Table 3.3), nonmixed CH TRUW (Table 3.4), total RH TRUW (Table 3.5), mixed RH TRUW (Table 3.6), and nonmixed RH TRUW (Table 3.7).

\subsubsection{Final-form TRUW volumes}

Table 3.8 shows the expected final-form volumes of the stored and projected TRUW reported earlier in Table 3.1 after the waste is processed to its final form. Tables 3.9-3.14 show the breakup of this expected final form volume into total $\mathrm{CH}$ TRUW (Table 3.9), mixed $\mathrm{CH}$ TRUW (Table 3.10), nonmixed CH TRUW (Table 3.11), total RH TRUW (Table 3.12), mixed RH TRUW (Table 3.13), and nonmixed RH TRUW (Table 3.14).
Tables 3.15 and 3.16 show the expected total finalform volumes of TRUW at each site distributed among each final waste form (e.g., combustible waste). Table 3.15 shows the final waste form distribution for the major sites, whereas Table 3.16 presents the same for the SQS.

\subsubsection{Decayed radioactivities for retrievably stored TRUW}

The yearly undecayed activity reported for each radionuclide by each TRU waste site for CH TRUW and RH TRUW generated from 1970 through 1994 was decayed to the end of CY 1995 using the ORNL computer code ORIGEN2. ${ }^{\top}$ This code converts the annual as-stored activities to annual decayed activities and accumulates these quantities to produce tables showing cumulative decayed activity for each radionuclide at each site. Once the decayed activities were calculated, the decayed mass was estimated using standard values of the specific activity of each radionuclide as reported in Appendix B of Rev. 11 of the IDB. Waste volumes are assumed to be unaffected by radioactive decay.

Tables 3.17-3.20 show the distribution of the primary TRUW radionuclides for all the DOE sites (decayed to the of CY 1995) for retrievably stored CH TRUW and RH TRUW both by radioactivity and by mass. As shown in these tables, ${ }^{241} \mathrm{Pu},{ }^{238} \mathrm{Pu},{ }^{239} \mathrm{Pu},{ }^{241} \mathrm{Am}$, and ${ }^{240} \mathrm{Pu}$ cumulatively contribute more than $99 \%$ of the total activity for retrievably stored CH TRUW (Table 3.17), whereas ${ }^{238} \mathrm{Pu},{ }^{239} \mathrm{Pu},{ }^{232} \mathrm{Th},{ }^{235} \mathrm{U}$, and ${ }^{240} \mathrm{Pu}$ contribute more than $98 \%$ of the total radionuclide mass for retrievably stored CH TRUW (Table 3.18). Similar information on RH TRUW and on the relative distribution of both the radioactivity and mass of the primary radionuclides for the various DOE sites can be derived from Tables 3.19 and 3.20 .

For the purposes of this report, primary radionuclides are those that cumulatively contribute $98 \%$ of either the total radionuclide activity or mass. As compared to Rev. 11 of the IDB, the increase in the total activity of each of the primary radionuclides for CH TRUW is due to the inclusion of the residues from the Rocky Flats Environmental Technology Site that have been declared as TRUW and are planned to be disposed of in the WIPP repository.

Similarly, for RH TRUW, there has been an increase in the amount of ${ }^{238} \mathrm{U}$ and a corresponding decrease in ${ }^{235} \mathrm{U}$ activities and mass based on the results of RH TRUW sludge sampling data from ORNL. The new sludge sampling data indicated that the majority of the activity in the RH TRUW sludges at ORNL was due to the presence of ${ }^{238} U$ instead of ${ }^{235} U$, as was originally reported. The new 
data corrected the previously high estimates of ${ }^{235} \mathrm{U}$ in the RH TRUW sludges at ORNL.

\subsubsection{Buried TRUW volumes and radioactivities}

Table 3.21 summarizes the buried TRUW volumes and associated radioactivity, both as-stored and asdecayed. Buried TRUW volumes and radioactivities shown in Table 3.21 are based on data provided in the site submittals. The form of the site-submitted data for buried waste is identical to that of the retrievably stored waste-except that no distinction is made between $\mathrm{CH}$ and RH buried wastes. Table 3.21 shows both cumulative asstored and as-decayed radioactivities for all nuclides as well as for TRU nuclides only.

\subsection{PROJECTED FUTURE QUANTITIES OF TRUW}

Tables 3.1-3.16 show the data submitted by the sites for estimated future volumes of TRUW generation. Tables 3.1-3.7 give projections for as-generated waste forms, and Tables 3.8-3.16 give projections for final waste forms. The sites were not requested to estimate the radioactivities or isotopic compositions of these wastes because it was believed that there would be little basis for such estimates. The estimated volumes are given in terms of average annual rates ( $\mathrm{m}^{3} / \mathrm{year}$ ) for three time periods from 1995 to 1997 and for three cumulative time periods thereafter (1998-2002, 2003-2012, and 2013-2022).

\subsection{TRUW DISPOSAL}

The goals of the DOE TRUW Program are to terminate interim storage and achieve permanent disposal of DOE TRUW. ${ }^{8}$ As stated in Pub. L. 96-164, ${ }^{9}$ WIPP was to be constructed ".. as a defense activity of the DOE for the purpose of providing a research and development facility to demonstrate the safe disposal of radioactive waste resulting from defense activities and programs of the United States." Construction of the facility is essentially complete, and WIPP is now the only facility specifically designed for isolation of TRUW. It is designed to contain about $175,000 \mathrm{~m}^{3}\left(6.2 \text { million } \mathrm{ft}^{3}\right)^{10}$ of TRUW $655 \mathrm{~m}$ $(2,150 \mathrm{ft})$ below ground in a mined salt formation.

Waste received at WIPP must meet the WIPP WAC and associated quality assurance requirements specified in the WIPP WAC. ${ }^{3}$ A number of other approvals remain to be completed before $D O E$ can begin disposal operations at the facility. DOE is committed to demonstrating compliance with all applicable regulations before the permanent disposal of TRUW in the WIPP repository. These regulations are the environmental standards for management and disposal of TRU wastes, as mandated in 40 CFR Part 191 (ref. 11) and Part 194 (ref. 12), and the RCRA regulations. Compliance will be demonstrated through performance assessment calculations based on the inventory of existing and currently projected waste streams as reported by the DOE TRU waste sites in the TWBIR, Rev. 3. ${ }^{2}$

In 1992 the WIPP Legislative Land Withdrawal Act ${ }^{10}$ was passed, confirming congressional intent to have $\mathrm{DOE}$ continue with development and permitting of the facility. Since then, DOE has stated its intent to accelerate processes leading to the start of waste disposal operations at the WIPP.

\subsection{REFERENCES}

1. U.S. Department of Energy, Radioactive Waste Management, DOE Order 5820.2A, Washington, D.C. (Sept. 26, 1988).

2. U.S. Department of Energy, Transuranic Waste Baseline Inventory Report (Revision 3), CAO-95-1121, Carlsbad, New Mexico (June 1996).

3. U.S. Department of Energy, Waste Acceptance Criteria for the Waste Isolation Pilot Plant, DOE/WIPP-069, Rev. 5, Carlsbad, New Mexico (April 1996).

4. K S. Hollingsworth, Policy Statement Regarding Solid Waste Burial, AEC Directive IAD No. 0511-21, Washington, D.C. (Mar. 20, 1970)

5. U.S. Department of Energy, Integrated Data Base Report-1994: U.S. Spent Nuclear Fuel and Radioactive Waste Inventories, Projections, and Characteristics, DOE/RW-0006, Rev. 11, Oak Ridge National Laboratory, Oak Ridge, Tennessee (September 1995). 
6. U.S. Department of Energy, Defense Waste Management Plan for Buried Transuranic-Contaminated Waste, Transuranic-Contaminated Soil, and Difficult-to-Certify Transuranic Waste, DOE/DP-0044, Washington, D.C. (June 1987).

7. A G. Croff, "ORIGEN2: A Versatile Computer Code for Calculating the Nuclide Compositions and Characteristics of Nuclear Materials," Nuclear Technology, 62, 335-352 (November 1983).

8. U.S. Department of Energy, Long Range Master Plan for Defense Transuranic Waste Program, DOE/WIPP 88-028, Carlsbad, New Mexico (December 1988).

9. U.S. Congress, Department of Energy National Security and Military Application of Nuclear Energy Authorization Act of 1980, Pub. L. 96-164 (1980).

10. U.S. Congress, Waste Isolation Pilot Plant Land Withdrawal Act, Pub. L. 102-579 (1992).

11. U.S. Environmental Protection Agency, Environmental Radiation Protection Standards for the Management and Disposal of Spent Nuclear Fuel, High-Level and Transuranic Radioactive Wastes, 40 CFR Part 191, final rule, Fed. Regist. 58, 66398 (Dec. 20, 1993).

12. U.S. Environmental Protection Agency, Criteria for Certification and Recertification of the Waste Isolation Pilot Plant's Compliance with 40 CFR 191 Disposal Regulations, Final Rule, 40 CFR Part 194, Fed. Regist., (Feb. 9, 1996). 
AE Argonne Natlonal Laboratony-East AL. Amos Laboratory

AM ARCO Medical Products Company

AW Argonne National Laboratory-Wes!

BC Ballelle Columbus Leboralony

Bi. Baboock \& Whoox-t ynctiburg

BT Bells Alomic Power Leboralon

ET Energy Tectrotogy Englneerting Center

GE Generaj Elocarlc Vallecllas Nuclear Center

iN Idaho Nallonal Enghoortho Loboralon

KA Knolls Alomic Power Leboralory - Sctronectady

iA Los Alamos National Laboratory - Sctrenectady

IB Lawrence Benkeley Laboratory

UL Lawrence L Nemore Natlonal Laboratory
MC U.S. Asmy Malental Command

MD Mound Plant

MU Untverslyy of Missount Research Reactor

NT Nevadu Test SHe

OA Oak Ritge Nallonal Leboratory

PA Paducah Gasoous Difusion Plam

PX Pantex Plant

RF Pocky Flals Enmironmental Tectmology SHe

FL Harrond (Plictiance) SHo

SA Sande Nalloned Laboratorios

Sllo

WV Wosl Valloy Demonstration Propect

Fig. 3.1. U.S. Department of Energy transuranic waste generator/storage sites.

- Indicates Small Quantity Sito $\star$ Indicalos Major Sito 


\section{AS-GENERATED WASTE FORM}

Table 3.1. Summary of as-generated waste-form volumes $\left(\mathrm{m}^{3}\right)$ of retrievably stored and projected TRUW (mixed and nonmixed $\mathrm{CH}$ and $\mathrm{RH}$ wastes)

\begin{tabular}{|c|c|c|c|c|c|c|c|c|}
\hline \multirow{2}{*}{ Site } & \multirow{2}{*}{$\begin{array}{l}\text { End of } \\
\text { CY } 1994 \\
\text { total }\end{array}$} & \multicolumn{3}{|c|}{ Annual increase } & \multicolumn{3}{|c|}{ Cumulative increase } & \multirow{2}{*}{$\begin{array}{l}\text { End of } \\
\text { CY } 2022 \\
\text { total }\end{array}$} \\
\hline & & 1995 & 1996 & 1997 & 1998-2002 & 2003-2012 & $2013-2022$ & \\
\hline \multicolumn{9}{|l|}{ Major sites } \\
\hline ANL,-E & $2.48 \mathrm{E}+01$ & $0.00 \mathrm{E}+00$ & $4.20 \mathrm{E}+01$ & $3.33 \mathrm{E}+00$ & $1.79 \mathrm{E}+01$ & $3.33 \mathrm{E}+01$ & $3.33 E+01$ & $1.55 \mathrm{E}+02$ \\
\hline Hanford & $1.12 \mathrm{E}+04$ & $5.22 \mathrm{E}+02$ & $2.01 \mathrm{E}+02$ & $1.79 \mathrm{E}+02$ & $2.56 \mathrm{E}+03$ & $1.66 \mathrm{E}+04$ & $2.86 \mathrm{E}+04$ & $6.00 \mathrm{E}+04$ \\
\hline INEL & $3.95 E+04$ & $0.00 \mathrm{E}+00$ & $0.00 \mathrm{E}+00$ & $0.00 \mathrm{E}+00$ & $0.00 \mathrm{E}+00$ & $0.00 \mathrm{E}+00$ & $0.00 \mathrm{E}+00$ & $3.95 E+04$ \\
\hline LANL & $1.10 \mathrm{E}+04$ & $2.63 \mathrm{E}+02$ & $2.63 E+02$ & $2.63 E+02$ & $1.32 \mathrm{E}+03$ & $2.63 E+03$ & $2.64 \mathrm{E}+03$ & $1.84 \mathrm{E}+04$ \\
\hline LLNL & $2.30 \mathrm{E}+02$ & $2.66 \mathrm{E}+01$ & $1.32 E+01$ & $1.51 \mathrm{E}+01$ & $6.99 \mathrm{E}+01$ & $1.42 \mathrm{E}+02$ & $4.44 E+02$ & $9.41 \mathrm{E}+02$ \\
\hline Mound & $2.74 \mathrm{E}+02$ & $0.00 E+00$ & $0.00 \mathrm{E}+00$ & $0.00 \mathrm{E}+00$ & $0.00 \mathrm{E}+00$ & $0.00 \mathrm{E}+00$ & $0.00 \mathrm{E}+00$ & $2.74 \mathrm{E}+02$ \\
\hline NTS & $6.16 \mathrm{E}+02$ & $0.00 E+00$ & $0.00 \mathrm{E}+00$ & $0.00 \mathrm{E}+00$ & $0.00 \mathrm{E}+00$ & $2.75 \mathrm{E}+00$ & $2.77 \mathrm{E}+00$ & $6.21 E+02$ \\
\hline ORNL & $3.16 \mathrm{E}+03$ & $2.14 \mathrm{E}+01$ & $2.14 \mathrm{E}+01$ & $2.14 \mathrm{E}+01$ & $1.07 \mathrm{E}+02$ & $2.14 \mathrm{E}+02$ & $2.14 \mathrm{E}+02$ & $3.76 \mathrm{E}+03$ \\
\hline RFETS & $1.87 \mathrm{E}+03$ & $1.08 \mathrm{E}+02$ & $9.37 \mathrm{E}+01$ & $3.46 \mathrm{E}+01$ & $1.73 \mathrm{E}+02$ & $1.42 \mathrm{E}+03$ & $1.35 \mathrm{E}+03$ & $5.05 E+03$ \\
\hline SRS & $6.55 E+03$ & $4.27 \mathrm{E}+02$ & $8.84 \mathrm{E}+02$ & $6.05 E+02$ & $3.48 \mathrm{E}+03$ & $2.09 \mathrm{E}+03$ & $1.46 \mathrm{E}+03$ & $1.55 E+04$ \\
\hline Subtotal & $7.45 \mathrm{E}+04$ & $1.37 \mathrm{E}+03$ & $1.52 \mathrm{E}+03$ & $1.12 E+03$ & $7.73 E+03$ & $2.32 E+04$ & $3.48 \mathrm{E}+04$ & $1.44 \mathrm{E}+05$ \\
\hline \multicolumn{9}{|c|}{ Small-quantity sites } \\
\hline Ames & $0.00 \mathrm{E}+00$ & $0.00 \mathrm{E}+00$ & $0.00 \mathrm{E}+00$ & $0.00 \mathrm{E}+00$ & $0.00 \mathrm{E}+00$ & $4.16 \mathrm{E}-01$ & $0.00 \mathrm{E}+00$ & $4.16 \mathrm{E}-01$ \\
\hline $\mathrm{ANL}, \mathrm{W}$ & $2.51 \mathrm{E}+01$ & $0.00 \mathrm{E}+00$ & $0.00 \mathrm{E}+00$ & $0.00 E+00$ & $8.33 \mathrm{E}+01$ & $1.12 E+02$ & $9.16 \mathrm{E}+01$ & $3.12 \mathrm{E}+02$ \\
\hline $\mathrm{ARCO}$ & $4.00 \mathrm{E}-02$ & $2.40 E-03$ & $2.40 \mathrm{E}-03$ & $2.40 \mathrm{E}-03$ & $1.20 \mathrm{E}-02$ & $2.40 \mathrm{E}-02$ & $2.08 \mathrm{E}-02$ & $1.04 \mathrm{E}-01$ \\
\hline BAPL & $0.00 \mathrm{E}+00$ & $7.28 \mathrm{E}-01$ & $2.67 \mathrm{E}+01$ & $1.73 E+01$ & $7.62 \mathrm{E}+01$ & $3.88 \mathrm{E}+00$ & $0.00 \mathrm{E}+00$ & $1.25 \mathrm{E}+02$ \\
\hline $\mathrm{BCL}$ & $5.81 \mathrm{E}+02$ & $0.00 \mathrm{E}+00$ & $0.00 \mathrm{E}+00$ & $0.00 \mathrm{E}+00$ & $0.00 \mathrm{E}+00$ & $0.00 \mathrm{E}+00$ & $0.00 \mathrm{E}+00$ & $5.81 \mathrm{E}+02$ \\
\hline ETEC & $7.35 \mathrm{E}+00$ & $0.00 \mathrm{E}+00$ & $4.20 \mathrm{E}-01$ & $4.20 \mathrm{E}-01$ & $0.00 \mathrm{E}+00$ & $0.00 \mathrm{E}+00$ & $0.00 \mathrm{E}+00$ & $8.19 E+00$ \\
\hline KAPL & $2.40 \mathrm{E}-01$ & $2.00 \mathrm{E}-01$ & $2.00 \mathrm{E}-01$ & $2.00 \mathrm{E}-01$ & $1.00 \mathrm{E}+00$ & $2.00 \mathrm{E}+00$ & $2.00 \mathrm{E}+00$ & $5.84 \mathrm{E}+00$ \\
\hline LBL & $6.24 \mathrm{E}-01$ & $0.00 \mathrm{E}+00$ & $0.00 \mathrm{E}+00$ & $0.00 \mathrm{E}+00$ & $2.08 \mathrm{E}-01$ & $4.16 E-01$ & $4.16 \mathrm{E}-01$ & $1.66 \mathrm{E}+00$ \\
\hline MURR & $2.08 \mathrm{E}-01$ & $2.08 \mathrm{E}-01$ & $2.08 \mathrm{E}-01$ & $2.08 \mathrm{E}-01$ & $2.08 \mathrm{E}-01$ & $0.00 \mathrm{E}+00$ & $0.00 \mathrm{E}+00$ & $1.04 \mathrm{E}+00$ \\
\hline PAD & $2.10 \mathrm{E}+00$ & $0.00 \mathrm{E}+00$ & $0.00 \mathrm{E}+00$ & $0.00 \mathrm{E}+00$ & $0.00 \mathrm{E}+00$ & $0.00 \mathrm{E}+00$ & $0.00 \hat{E}+00$ & $2.10 \mathrm{E}+00$ \\
\hline PANT & $6.24 \mathrm{E}-01$ & $0.00 \mathrm{E}+00$ & $0.00 \mathrm{E}+00$ & $0.00 \mathrm{E}+00$ & $0.00 \mathrm{E}+00$ & $0.00 \mathrm{E}+00$ & $0.00 \mathrm{E}+00$ & $6.24 \mathrm{E}-01$ \\
\hline SNL/NM & $6.66 \mathrm{E}+00$ & $2.08 \mathrm{E}-01$ & $1.87 \mathrm{E}+00$ & $2.08 \mathrm{E}-01$ & $1.04 \mathrm{E}+00$ & $2.08 \mathrm{E}+00$ & $2.08 \mathrm{E}+00$ & $1.41 \mathrm{E}+01$ \\
\hline TBE & $2.08 \mathrm{E}-01$ & $0.00 \mathrm{E}+00$ & $0.00 \mathrm{E}+00$ & $0.00 \mathrm{E}+00$ & $0.00 \mathrm{E}+00$ & $0.00 \mathrm{E}+00$ & $0.00 \mathrm{E}+00$ & $2.08 \mathrm{E}-01$ \\
\hline USAMC & $0.00 \mathrm{E}+00$ & $0.00 E+00$ & $0.00 \mathrm{E}+00$ & $0.00 \mathrm{E}+00$ & $0.00 \mathrm{E}+00$ & $0.00 \mathrm{E}+00$ & $0.00 \mathrm{E}+00$ & $0.00 \mathrm{E}+00$ \\
\hline Subtotal & $6.24 \mathrm{E}+02$ & $1.35 E+00$ & $2.94 \mathrm{E}+01$ & $1.84 \mathrm{E}+01$ & $1.62 \mathrm{E}+02$ & $1.21 \mathrm{E}+02$ & $9.62 \mathrm{E}+01$ & $1.05 E+03$ \\
\hline
\end{tabular}




\section{AS-GENERATED WASTE FORM}

Table 3.1 (continued)

\begin{tabular}{|c|c|c|c|c|c|c|c|c|}
\hline \multirow{2}{*}{ Site } & \multirow{2}{*}{$\begin{array}{l}\text { End of } \\
\text { CY } 1994 \\
\text { total }\end{array}$} & \multicolumn{3}{|c|}{ Annual increase } & \multicolumn{3}{|c|}{ Cumulative increase } & \multirow{2}{*}{$\begin{array}{l}\text { End of } \\
\text { CY } 2022 \\
\text { total }\end{array}$} \\
\hline & & 1995 & 1996 & 1997 & 1998-2002 & $2003-2012$ & 2013-2022 & \\
\hline \multicolumn{9}{|l|}{ Commercial site } \\
\hline WVDP & $5.22 \mathrm{E}+02$ & $4.98 \mathrm{E}+01$ & $5.34 \mathrm{E}+00$ & $5.34 \mathrm{E}+00$ & $7.48 \mathrm{E}+01$ & $5.34 \mathrm{E}+01$ & $5.34 \mathrm{E}+01$ & $5.34 \mathrm{E}+01$ \\
\hline $\begin{array}{l}\text { Total } \\
\text { (all sites) }\end{array}$ & $7.56 \mathrm{E}+04$ & $1.42 \mathrm{E}+03$ & $1.55 \mathrm{E}+03$ & $1.15 \mathrm{E}+03$ & $7.97 \mathrm{E}+03$ & $2.34 \mathrm{E}+04$ & $3.49 \mathrm{E}+04$ & $1.46 \mathrm{E}+05$ \\
\hline
\end{tabular}




\section{AS-GENERATED WASTE FORM}

Table 3.2. Summary of as-generated waste-form volumes $\left(\mathrm{m}^{3}\right)$ of retrievably stored and projected mixed and nonmixed CH TRUW

\begin{tabular}{|c|c|c|c|c|c|c|c|c|}
\hline \multirow{2}{*}{ Site } & \multirow{2}{*}{$\begin{array}{l}\text { End of } \\
\text { CY } 1994 \\
\text { total }\end{array}$} & \multicolumn{3}{|c|}{ Annual increase } & \multicolumn{3}{|c|}{ Cumulative increase } & \multirow{2}{*}{$\begin{array}{l}\text { End of } \\
\text { CY } 2022 \\
\text { total }\end{array}$} \\
\hline & & 1995 & 1996 & 1997 & 1998-2002 & $2003-2012$ & $2013-2022$ & \\
\hline \multicolumn{9}{|l|}{ Major sites } \\
\hline ANL-E & $2.48 \mathrm{E}+01$ & $0.00 \mathrm{E}+00$ & $4.20 \mathrm{E}+01$ & $3.33 \mathrm{E}+00$ & $1.79 \mathrm{E}+01$ & $3.33 \mathrm{E}+01$ & $3.33 \mathrm{E}+01$ & $1.55 \mathrm{E}+02$ \\
\hline Hanford & $1.10 \mathrm{E}+04$ & $4.50 \mathrm{E}+02$ & $1.97 \mathrm{E}+02$ & $1.68 E+02$ & $2.28 \mathrm{E}+03$ & $1.22 \mathrm{E}+04$ & $1.19 \mathrm{E}+04$ & $3.82 \mathrm{E}+04$ \\
\hline INEL & $3.93 E+04$ & $0.00 \mathrm{E}+00$ & $0.00 \mathrm{E}+00$ & $0.00 \mathrm{E}+00$ & $0.00 \mathrm{E}+00$ & $0.00 \mathrm{E}+00$ & $0.00 \mathrm{E}+00$ & $3.93 E+04$ \\
\hline LANL & $1.10 \mathrm{E}+04$ & $2.62 \mathrm{E}+02$ & $2.62 \mathrm{E}+02$ & $2.62 E+02$ & $1.31 \mathrm{E}+03$ & $2.62 \mathrm{E}+03$ & $2.63 E+03$ & $1.83 \mathrm{E}+04$ \\
\hline LLNL & $2.30 \mathrm{E}+02$ & $2.66 \mathrm{E}+01$ & $1.32 \mathrm{E}+01$ & $1.51 \mathrm{E}+01$ & $6.99 \mathrm{E}+01$ & $1.42 \mathrm{E}+02$ & $4.44 E+02$ & $9.41 \mathrm{E}+02$ \\
\hline Mound & $2.74 \mathrm{E}+02$ & $0.00 \mathrm{E}+00$ & $0.00 \mathrm{E}+00$ & $0.00 \mathrm{E}+00$ & $0.00 \mathrm{E}+00$ & $0.00 E+00$ & $0.00 \mathrm{E}+00$ & $2.74 \mathrm{E}+02$ \\
\hline NTS & $6.16 \mathrm{E}+02$ & $0.00 \mathrm{E}+00$ & $0.00 \mathrm{E}+00$ & $0.00 \mathrm{E}+00$ & $0.00 \mathrm{E}+00$ & $2.75 \mathrm{E}+00$ & $2.77 \mathrm{E}+00$ & $6.21 E+02$ \\
\hline ORNL & $1.33 \mathrm{E}+03$ & $9.15 \mathrm{E}+00$ & $9.15 \mathrm{E}+00$ & $9.15 \mathrm{E}+00$ & $4.58 \mathrm{E}+01$ & $9.15 \mathrm{E}+01$ & $9.15 \mathrm{E}+01$ & $1.58 \mathrm{E}+03$ \\
\hline RFETS & $1.87 \mathrm{E}+03$ & $1.08 \mathrm{E}+02$ & $9.37 \mathrm{E}+01$ & $3.46 \mathrm{E}+01$ & $1.73 E+02$ & $1.42 \mathrm{E}+03$ & $1.35 \mathrm{E}+03$ & $5.05 E+03$ \\
\hline SRS & $6.55 \mathrm{E}+03$ & $4.27 E+02$ & $8.84 \mathrm{E}+02$ & $6.05 E+02$ & $3.48 E+03$ & $2.09 \mathrm{E}+03$ & $1.46 \mathrm{E}+03$ & $1.55 E+04$ \\
\hline Subtotal & $7.21 \mathrm{E}+04$ & $1.28 \mathrm{E}+03$ & $1.50 \mathrm{E}+03$ & $1.10 \mathrm{E}+03$ & $7.38 \mathrm{E}+03$ & $1.86 \mathrm{E}+04$ & $1.79 \mathrm{E}+04$ & $1.20 \mathrm{E}+05$ \\
\hline \multicolumn{9}{|c|}{ Small-quantity sites } \\
\hline Ames & $0.00 \mathrm{E}+00$ & $0.00 \mathrm{E}+00$ & $0.00 \mathrm{E}+00$ & $0.00 \mathrm{E}+00$ & $0.00 \mathrm{E}+00$ & $4.16 \mathrm{E}-01$ & $0.00 \mathrm{E}+00$ & $4.16 E-01$ \\
\hline ANL-W & $3.47 \mathrm{E}+00$ & $0.00 \mathrm{E}+00$ & $0.00 E+00$ & $0.00 \mathrm{E}+00$ & $7.65 \mathrm{E}+01$ & $9.98 \mathrm{E}+01$ & $8.51 E+01$ & $2.65 \mathrm{E}+02$ \\
\hline ARCO & $4.00 \mathrm{E}-02$ & $2.40 \mathrm{E}-03$ & $2.40 \mathrm{E}-03$ & $2.40 \mathrm{E}-03$ & $1.20 \mathrm{E}-02$ & $2.40 \mathrm{E}-02$ & $2.08 \mathrm{E}-02$ & $1.04 \mathrm{E}-01$ \\
\hline BAPL & $0.00 \mathrm{E}+00$ & $4.16 \mathrm{E}-01$ & $2.65 E+01$ & $1.70 \mathrm{E}+01$ & $7.56 \mathrm{E}+01$ & $3.78 \mathrm{E}+00$ & $0.00 \mathrm{E}+00$ & $1.23 \mathrm{E}+02$ \\
\hline $\mathrm{BCL}$ & $0.00 \mathrm{E}+00$ & $0.00 \mathrm{E}+00$ & $0.00 \mathrm{E}+00$ & $0.00 \mathrm{E}+00$ & $0.00 \mathrm{E}+00$ & $0.00 \mathrm{E}+00$ & $0.00 \mathrm{E}+00$ & $0.00 \mathrm{E}+00$ \\
\hline ETEC & $1.68 \mathrm{E}+00$ & $0.00 \mathrm{E}+00$ & $0.00 \mathrm{E}+00$ & $0.00 \mathrm{E}+00$ & $0.00 \mathrm{E}+00$ & $0.00 \mathrm{E}+00$ & $0.00 \mathrm{E}+00$ & $1.68 \mathrm{E}+00$ \\
\hline KAPL & $0.00 \mathrm{E}+00$ & $0.00 \mathrm{E}+00$ & $0.00 \mathrm{E}+00$ & $0.00 \mathrm{E}+00$ & $0.00 \mathrm{E}+00$ & $0.00 \mathrm{E}+00$ & $0.00 \mathrm{E}+00$ & $0.00 \mathrm{E}+00$ \\
\hline LBL & $6.24 \mathrm{E}-01$ & $0.00 \mathrm{E}+00$ & $0.00 \mathrm{E}+00$ & $0.00 \mathrm{E}+00$ & $2.08 \mathrm{E}-01$ & $4.16 \mathrm{E}-01$ & $4.16 \mathrm{E}-01$ & $1.66 \mathrm{E}+00$ \\
\hline MURR & $2.08 \mathrm{E}-01$ & $2.08 E-01$ & $2.08 E-01$ & $2.08 \mathrm{E}-01$ & $2.08 \mathrm{E}-01$ & $0.00 \mathrm{E}+00$ & $0.00 \mathrm{E}+00$ & $1.04 \mathrm{E}+00$ \\
\hline $\mathrm{PAD}$ & $2.10 \mathrm{E}+00$ & $0.00 \mathrm{E}+00$ & $0.00 E+00$ & $0.00 \mathrm{E}+00$ & $0.00 \mathrm{E}+00$ & $0.00 \mathrm{E}+00$ & $0.00 \mathrm{E}+00$ & $2.10 \mathrm{E}+00$ \\
\hline PANT & $6.24 \mathrm{E}-01$ & $0.00 \mathrm{E}+00$ & $0.00 \mathrm{E}+00$ & $0.00 \mathrm{E}+00$ & $0.00 \mathrm{E}+00$ & $0.00 \mathrm{E}+00$ & $0.00 \mathrm{E}+00$ & $6.24 \mathrm{E}-01$ \\
\hline SNL/NM & $6.66 \mathrm{E}+00$ & $2.08 \mathrm{E}-01$ & $1.87 \mathrm{E}+00$ & $2.08 \mathrm{E}-01$ & $1.04 \mathrm{E}+00$ & $2.08 \mathrm{E}+00$ & $2.08 \mathrm{E}+00$ & $1.41 \mathrm{E}+01$ \\
\hline TBE & $2.08 \mathrm{E}-01$ & $0.00 \mathrm{E}+00$ & $0.00 \mathrm{E}+00$ & $0.00 \mathrm{E}+00$ & $0.00 \mathrm{E}+00$ & $0.00 \mathrm{E}+00$ & $0.00 \mathrm{E}+00$ & $2.08 \mathrm{E}-01$ \\
\hline USAMC & $0.00 \mathrm{E}+00$ & $0.00 \mathrm{E}+00$ & $0.00 \mathrm{E}+00$ & $0.00 \mathrm{E}+00$ & $0.00 \mathrm{E}+00$ & $0.00 \mathrm{E}+00$ & $0.00 \mathrm{E}+00$ & $0.00 \mathrm{E}+00$ \\
\hline Subtotal & $1.56 \mathrm{E}+01$ & $8.34 \mathrm{E}-01$ & $2.85 \mathrm{E}+01$ & $1.74 \mathrm{E}+01$ & $1.54 \mathrm{E}+02$ & $1.07 \mathrm{E}+02$ & $8.76 \mathrm{E}+01$ & $4.10 \mathrm{E}+02$ \\
\hline
\end{tabular}




\begin{tabular}{|c|c|c|c|c|c|c|c|c|}
\hline & & & AS-GENER & TED WAS & FORM & & & \\
\hline & & & Tab & 3.2 (continu & & & & \\
\hline & End of & & Innual increas & & & Imulative incre & & End of \\
\hline & CY 1994 & 1995 & 1996 & 1997 & 1998-2002 & $2003-2012$ & 2013-2022 & total \\
\hline $\begin{array}{l}\text { Commercial site } \\
\text { WVDP }\end{array}$ & $3.79 \mathrm{E}+01$ & $5.34 \mathrm{E}+00$ & $5.34 \mathrm{E}+00$ & $5.34 \mathrm{E}+00$ & $2.67 E+01$ & $5.34 \mathrm{E}+01$ & $5.34 E+01$ & $1.87 \mathrm{E}+02$ \\
\hline $\begin{array}{l}\text { Total } \\
\text { (all sites) }\end{array}$ & $7.22 \mathrm{E}+04$ & $1.29 \mathrm{E}+03$ & $1.54 \mathrm{E}+03$ & $1.12 \mathrm{E}+03$ & $7.56 \mathrm{E}+03$ & $1.88 \mathrm{E}+04$ & $1.81 \mathrm{E}+04$ & $1.21 \mathrm{E}+05$ \\
\hline
\end{tabular}




\section{AS-GENERATED WASTE FORM}

Table 3.3. As-generated waste-form volumes $\left(\mathrm{m}^{3}\right)$ of retrievably stored and projected mixed CH TRUW

\begin{tabular}{|c|c|c|c|c|c|c|c|c|}
\hline \multirow{2}{*}{ Site } & \multirow{2}{*}{$\begin{array}{l}\text { End of } \\
\text { CY } 1994 \\
\text { total }\end{array}$} & \multicolumn{3}{|c|}{ Annual increase } & \multicolumn{3}{|c|}{ Cumulative increase } & \multirow{2}{*}{$\begin{array}{c}\text { End of } \\
\text { CY } 2022 \\
\text { total }\end{array}$} \\
\hline & & 1995 & 1996 & 1997 & $1998-2002$ & 2003-2012 & 2013-2022 & \\
\hline \multicolumn{9}{|l|}{ Major sites } \\
\hline ANL-E & $6.48 E+00$ & $0.00 \mathrm{E}+00$ & $0.00 \mathrm{E}+00$ & $0.00 \mathrm{E}+00$ & $1.29 \mathrm{E}+00$ & $0.00 \mathrm{E}+00$ & $0.00 \mathrm{E}+00$ & $7.77 \mathrm{E}+00$ \\
\hline Hanford & $2.77 \mathrm{E}+02$ & $6.94 \mathrm{E}+01$ & $6.88 \mathrm{E}+01$ & $7.35 \mathrm{E}+01$ & $3.95 E+02$ & $6.34 E+03$ & $7.17 \mathrm{E}+03$ & $1.44 \mathrm{E}+04$ \\
\hline INEL & $3.02 \mathrm{E}+04$ & $0.00 \mathrm{E}+00$ & $0.00 \mathrm{E}+00$ & $0.00 \mathrm{E}+00$ & $0.00 \mathrm{E}+00$ & $0.00 \mathrm{E}+00$ & $0.00 \mathrm{E}+00$ & $3.02 \mathrm{E}+04$ \\
\hline LANL & $7.67 \mathrm{E}+03$ & $1.35 \mathrm{E}+02$ & $1.35 \mathrm{E}+02$ & $1.35 E+02$ & $6.73 E+02$ & $1.35 \mathrm{E}+03$ & $1.35 \mathrm{E}+03$ & $1.14 \mathrm{E}+04$ \\
\hline LLNL & $8.35 E+00$ & $2.52 \mathrm{E}+00$ & $6.24 \mathrm{E}-01$ & $2.52 \mathrm{E}+00$ & $6.92 \mathrm{E}+00$ & $1.57 \mathrm{E}+01$ & $2.14 E+01$ & $5.80 \mathrm{E}+01$ \\
\hline Mound & $3.54 \mathrm{E}+00$ & $0.00 \mathrm{E}+00$ & $0.00 \mathrm{E}+00$ & $0.00 \mathrm{E}+00$ & $0.00 \mathrm{E}+00$ & $0.00 \mathrm{E}+00$ & $0.00 \mathrm{E}+00$ & $3.54 \mathrm{E}+00$ \\
\hline NTS & $6.15 E+02$ & $0.00 \mathrm{E}+00$ & $0.00 \mathrm{E}+00$ & $0.00 \mathrm{E}+00$ & $0.00 \mathrm{E}+00$ & $2.75 \mathrm{E}+00$ & $2.77 \mathrm{E}+00$ & $6.20 \mathrm{E}+02$ \\
\hline ORNL & $6.97 \mathrm{E}+02$ & $9.15 E+00$ & $9.15 \mathrm{E}+00$ & $9.15 E+00$ & $4.58 \mathrm{E}+01$ & $9.15 E+01$ & $9.15 \mathrm{E}+01$ & $9.53 \mathrm{E}+02$ \\
\hline RFETS & $9.49 E+02$ & $5.87 \mathrm{E}+01$ & $4.91 \mathrm{E}+01$ & $2.19 \mathrm{E}+01$ & $1.14 \mathrm{E}+02$ & $1.06 \mathrm{E}+03$ & $1.01 E+03$ & $3.26 \mathrm{E}+03$ \\
\hline SRS & $3.14 \mathrm{E}+03$ & $6.18 \mathrm{E}+01$ & $1.20 \mathrm{E}+02$ & $8.05 \mathrm{E}+01$ & $4.59 \mathrm{E}+02$ & $3.09 \mathrm{E}+02$ & $2.01 E+02$ & $4.37 \mathrm{E}+03$ \\
\hline Subtotal & $4.35 \mathrm{E}+04$ & $3.36 \mathrm{E}+02$ & $3.82 \mathrm{E}+02$ & $3.22 \mathrm{E}+02$ & $1.69 \mathrm{E}+03$ & $9.16 \mathrm{E}+03$ & $9.84 \mathrm{E}+03$ & $6.53 \mathrm{E}+04$ \\
\hline \multicolumn{9}{|c|}{ Small-quantity sites } \\
\hline Ames & $0.00 \mathrm{E}+00$ & $0.00 \mathrm{E}+00$ & $0.00 \mathrm{E}+00$ & $0.00 \mathrm{E}+00$ & $0.00 \mathrm{E}+00$ & $4.16 \mathrm{E}-01$ & $0.00 \mathrm{E}+00$ & $4.16 \mathrm{E}-01$ \\
\hline ANL_W & $1.18 \mathrm{E}+00$ & $0.00 \mathrm{E}+00$ & $0.00 \mathrm{E}+00$ & $0.00 \mathrm{E}+00$ & $2.04 \mathrm{E}+00$ & $0.00 \mathrm{E}+00$ & $0.00 \mathrm{E}+00$ & $3.22 \mathrm{E}+00$ \\
\hline $\mathrm{ARCO}$ & $0.00 \mathrm{E}+00$ & $0.00 \mathrm{E}+00$ & $0.00 \mathrm{E}+00$ & $0.00 \mathrm{E}+00$ & $0.00 \mathrm{E}+00$ & $0.00 \mathrm{E}+00$ & $0.00 \mathrm{E}+00$ & $0.00 \mathrm{E}+00$ \\
\hline BAPL & $0.00 \mathrm{E}+00$ & $0.00 \mathrm{E}+00$ & $0.00 \mathrm{E}+00$ & $0.00 \mathrm{E}+00$ & $0.00 \mathrm{E}+00$ & $0.00 \mathrm{E}+00$ & $0.00 \mathrm{E}+00$ & $0.00 \mathrm{E}+00$ \\
\hline $\mathrm{BCL}$ & $0.00 \mathrm{E}+00$ & $0.00 \mathrm{E}+00$ & $0.00 \mathrm{E}+00$ & $0.00 \mathrm{E}+00$ & $0.00 \mathrm{E}+00$ & $0.00 \mathrm{E}+00$ & $0.00 \mathrm{E}+00$ & $0.00 \mathrm{E}+00$ \\
\hline ETEC & $0.00 \mathrm{E}+00$ & $0.00 \mathrm{E}+00$ & $0.00 \mathrm{E}+00$ & $0.00 \mathrm{E}+00$ & $0.00 \mathrm{E}+00$ & $0.00 \mathrm{E}+00$ & $0.00 \mathrm{E}+00$ & $0.00 \mathrm{E}+00$ \\
\hline KAPL & $0.00 \mathrm{E}+00$ & $0.00 \mathrm{E}+00$ & $0.00 \mathrm{E}+00$ & $0.00 \mathrm{E}+00$ & $0.00 \mathrm{E}+00$ & $0.00 \mathrm{E}+00$ & $0.00 \mathrm{E}+00$ & $0.00 \mathrm{E}+00$ \\
\hline LBL & $0.00 \mathrm{E}+00$ & $0.00 \mathrm{E}+00$ & $0.00 \mathrm{E}+00$ & $0.00 \mathrm{E}+00$ & $0.00 \mathrm{E}+00$ & $0.00 \mathrm{E}+00$ & $0.00 \mathrm{E}+00$ & $0.00 \mathrm{E}+00$ \\
\hline MURR & $2.08 \mathrm{E}-01$ & $2.08 \mathrm{E}-01$ & $2.08 \mathrm{E}-01$ & $2.08 \mathrm{E}-01$ & $2.08 \mathrm{E}-01$ & $0.00 \mathrm{E}+00$ & $0.00 \mathrm{E}+00$ & $1.04 \mathrm{E}+00$ \\
\hline PAD & $2.10 \mathrm{E}+00$ & $0.00 \mathrm{E}+00$ & $0.00 \mathrm{E}+00$ & $0.00 \mathrm{E}+00$ & $0.00 \mathrm{E}+00$ & $0.00 \mathrm{E}+00$ & $0.00 \mathrm{E}+00$ & $2.10 \mathrm{E}+00$ \\
\hline PANT & $0.00 \mathrm{E}+00$ & $0.00 \mathrm{E}+00$ & $0.00 \mathrm{E}+00$ & $0.00 \mathrm{E}+00$ & $0.00 \mathrm{E}+00$ & $0.00 \mathrm{E}+00$ & $0.00 \mathrm{E}+00$ & $0.00 \mathrm{E}+00$ \\
\hline SNL/NM & $0.00 \mathrm{E}+00$ & $0.00 \mathrm{E}+00$ & $0.00 \mathrm{E}+00$ & $0.00 \mathrm{E}+00$ & $0.00 \mathrm{E}+00$ & $0.00 \mathrm{E}+00$ & $0.00 \mathrm{E}+00$ & $0.00 \mathrm{E}+00$ \\
\hline TBE & $0.00 \mathrm{E}+00$ & $0.00 \mathrm{E}+00$ & $0.00 \mathrm{E}+00$ & $0.00 \mathrm{E}+00$ & $0.00 \mathrm{E}+00$ & $0.00 \mathrm{E}+00$ & $0.00 \mathrm{E}+00$ & $0.00 \mathrm{E}+00$ \\
\hline USAMC & $0.00 \mathrm{E}+00$ & $0.00 \mathrm{E}+00$ & $0.00 \mathrm{E}+00$ & $0.00 \mathrm{E}+00$ & $0.00 \mathrm{E}+00$ & $0.00 \mathrm{E}+00$ & $0.00 \mathrm{E}+00$ & $0.00 \mathrm{E}+00$ \\
\hline Subtotal & $3.49 \mathrm{E}+00$ & $2.08 \mathrm{E}-01$ & $2.08 \mathrm{E}-01$ & $2.08 \mathrm{E}-01$ & $2.25 \mathrm{E}+00$ & $4.16 \mathrm{E}-01$ & $0.00 \mathrm{E}+00$ & $6.78 \mathrm{E}+00$ \\
\hline
\end{tabular}


AS-GENERATED WASTE FORM

Table 3.3 (continued)

\begin{tabular}{|c|c|c|c|c|c|c|c|c|}
\hline \multirow{2}{*}{ Site } & \multirow{2}{*}{$\begin{array}{l}\text { End of } \\
\text { CY } 1994 \\
\text { total }\end{array}$} & \multicolumn{3}{|c|}{ Annual increase } & \multicolumn{3}{|c|}{ Cumulative increase } & \multirow{2}{*}{$\begin{array}{l}\text { End of } \\
\text { CY } 2022 \\
\text { total }\end{array}$} \\
\hline & & 1995 & 1996 & 1997 & $1998-2002$ & 2003-2012 & 2013-2022 & \\
\hline \multicolumn{8}{|c|}{ Commercial site } & $1.42 \mathrm{E}+01$ \\
\hline Total & $4.36 \mathrm{E}+04$ & $3.36 \mathrm{E}+02$ & $3.83 \mathrm{E}+02$ & $3.22 \mathrm{E}+02$ & $1.70 \mathrm{E}+03$ & $9.16 \mathrm{E}+03$ & $9.84 \mathrm{E}+03$ & $6.53 \mathrm{E}+04$ \\
\hline
\end{tabular}




\section{AS-GENERATED WASTE FORM}

Table 3.4. As-generated waste-form volumes $\left(\mathrm{m}^{3}\right)$ of retrievably stored and projected nonmixed $\mathrm{CH}$ TRUW

\begin{tabular}{|c|c|c|c|c|c|c|c|c|}
\hline \multirow{2}{*}{ Site } & \multirow{2}{*}{$\begin{array}{l}\text { End of } \\
\text { CY } 1994 \\
\text { total }\end{array}$} & \multicolumn{3}{|c|}{ Annual increase } & \multicolumn{3}{|c|}{ Cumulative increase } & \multirow{2}{*}{$\begin{array}{c}\text { End of } \\
\text { CY } 2022 \\
\text { total }\end{array}$} \\
\hline & & 1995 & 1996 & 1997 & $1998-2002$ & 2003-2012 & 2013-2022 & \\
\hline \multicolumn{9}{|l|}{ Major sites } \\
\hline ANL-E & $1.83 \mathrm{E}+01$ & $0.00 E+00$ & $4.20 \mathrm{E}+01$ & $3.33 E+00$ & $1.66 \mathrm{E}+01$ & $3.33 E+01$ & $3.33 E+01$ & $1.47 \mathrm{E}+02$ \\
\hline Hanford & $1.08 \mathrm{E}+04$ & $3.81 E+02$ & $1.28 \mathrm{E}+02$ & $9.47 \mathrm{E}+01$ & $1.89 \mathrm{E}+03$ & $5.86 E+03$ & $4.75 E+03$ & $2.39 \mathrm{E}+04$ \\
\hline INEL & $9.08 E+03$ & $0.00 \mathrm{E}+00$ & $0.00 \mathrm{E}+00$ & $0.00 \mathrm{E}+00$ & $0.00 \mathrm{E}+00$ & $0.00 \mathrm{E}+00$ & $0.00 \mathrm{E}+00$ & $9.08 \mathrm{E}+03$ \\
\hline LANL & $3.28 \mathrm{E}+03$ & $1.28 \mathrm{E}+02$ & $1.28 \mathrm{E}+02$ & $1.28 \mathrm{E}+02$ & $6.40 \mathrm{E}+02$ & $1.28 \mathrm{E}+03$ & $1.28 \mathrm{E}+03$ & $6.86 E+03$ \\
\hline LLNL & $2.22 \mathrm{E}+02$ & $2.40 \mathrm{E}+01$ & $1.26 \mathrm{E}+01$ & $1.26 \mathrm{E}+01$ & $6.30 \mathrm{E}+01$ & $1.26 \mathrm{E}+02$ & $4.23 E+02$ & $8.83 E+02$ \\
\hline Mound & $2.70 \mathrm{E}+02$ & $0.00 \mathrm{E}+00$ & $0.00 \mathrm{E}+00$ & $0.00 \mathrm{E}+00$ & $0.00 \mathrm{E}+00$ & $0.00 \mathrm{E}+00$ & $0.00 \mathrm{E}+00$ & $2.70 \mathrm{E}+02$ \\
\hline NTS & $9.00 \mathrm{E}-01$ & $0.00 \mathrm{E}+00$ & $0.00 \mathrm{E}+00$ & $0.00 \mathrm{E}+00$ & $0.00 \mathrm{E}+00$ & $0,00 \mathrm{E}+00$ & $0.00 \mathrm{E}+00$ & $9.00 \mathrm{E}-01$ \\
\hline ORNL & $6.29 \mathrm{E}+02$ & $0.00 \mathrm{E}+00$ & $0.00 \mathrm{E}+00$ & $0.00 \mathrm{E}+00$ & $0.00 \mathrm{E}+00$ & $0.00 \mathrm{E}+00$ & $0.00 \mathrm{E}+00$ & $6.29 E+02$ \\
\hline RFETS & $9.19 \mathrm{E}+02$ & $4.95 E+01$ & $4.46 \mathrm{E}+01$ & $1.27 \mathrm{E}+01$ & $5.94 \mathrm{E}+01$ & $3.61 \mathrm{E}+02$ & $3.43 \mathrm{E}+02$ & $1.79 \mathrm{E}+03$ \\
\hline SRS & $3.42 \mathrm{E}+03$ & $3.65 E+02$ & $\cdot 7.64 \mathrm{E}+02$ & $5.24 \mathrm{E}+02$ & $3.02 \mathrm{E}+03$ & $1.79 E+03$ & $1.26 E+03$ & $1.11 E+04$ \\
\hline Subtotal & $2.86 \mathrm{E}+04$ & $9.47 \mathrm{E}+02$ & $1.12 \mathrm{E}+03$ & $7.76 \mathrm{E}+02$ & $5.69 \mathrm{E}+03$ & $9.45 \mathrm{E}+03$ & $8.08 E+03$ & $5.46 E+04$ \\
\hline \multicolumn{9}{|c|}{ Small-quantity sites } \\
\hline Ames & $0.00 \mathrm{E}+00$ & $0.00 \mathrm{E}+00$ & $0.00 \mathrm{E}+00$ & $0.00 \mathrm{E}+00$ & $0.00 \mathrm{E}+00$ & $0.00 \mathrm{E}+00$ & $0.00 \mathrm{E}+00$ & $0.00 \mathrm{E}+00$ \\
\hline ANL-W & $2.29 \mathrm{E}+00$ & $0.00 \mathrm{E}+00$ & $0.00 \mathrm{E}+00$ & $0.00 \mathrm{E}+00$ & $7.45 \mathrm{E}+01$ & $9.98 \mathrm{E}+01$ & $8.51 \mathrm{E}+01$ & $2.62 \mathrm{E}+02$ \\
\hline ARCO & $4.00 \mathrm{E}-02$ & $2.40 E-03$ & $2.40 \mathrm{E}-03$ & $2.40 \mathrm{E}-03$ & $1.20 \mathrm{E}-02$ & $2.40 E-02$ & $2.08 \mathrm{E}-02$ & $1.04 \mathrm{E}-01$ \\
\hline BAPL & $0.00 \mathrm{E}+00$ & $4.16 \mathrm{E}-01$ & $2.65 \mathrm{E}+01$ & $1.70 \mathrm{E}+01$ & $7.56 \mathrm{E}+01$ & $3.78 \mathrm{E}+00$ & $0.00 \mathrm{E}+00$ & $1.23 E+02$ \\
\hline $\mathrm{BCL}$ & $0.00 \mathrm{E}+00$ & $0.00 \mathrm{E}+00$ & $0.00 \mathrm{E}+00$ & $0.00 \mathrm{E}+00$ & $0.00 \mathrm{E}+00$ & $0.00 \mathrm{E}+00$ & $0.00 \mathrm{E}+00$ & $0.00 \mathrm{E}+00$ \\
\hline ETEC & $1.68 \mathrm{E}+00$ & $0.00 \mathrm{E}+00$ & $0.00 \mathrm{E}+00$ & $0.00 \mathrm{E}+00$ & $0.00 \mathrm{E}+00$ & $0.00 E+00$ & $0.00 \mathrm{E}+00$ & $1.68 \mathrm{E}+00$ \\
\hline KAPL, & $0.00 \mathrm{E}+00$ & $0.00 \mathrm{E}+00$ & $0.00 \mathrm{E}+00$ & $0.00 \mathrm{E}+00$ & $0.00 \mathrm{E}+00$ & $0.00 \mathrm{E}+00$ & $0.00 \mathrm{E}+00$ & $0.00 \mathrm{E}+00$ \\
\hline LBL & $6.24 \mathrm{E}-01$ & $0.00 \mathrm{E}+00$ & $0.00 \mathrm{E}+00$ & $0.00 \mathrm{E}+00$ & $2.08 \mathrm{E}-01$ & $4.16 \mathrm{E}-01$ & $4.16 \mathrm{E}-01$ & $1.66 E+00$ \\
\hline MURR & $0.00 \mathrm{E}+00$ & $0.00 \mathrm{E}+00$ & $0.00 E+00$ & $0.00 E+00$ & $0.00 \mathrm{E}+00$ & $0.00 \mathrm{E}+00$ & $0.00 E+00$ & $0.00 \mathrm{E}+00$ \\
\hline PAD & $0.00 \mathrm{E}+00$ & $0.00 \mathrm{E}+00$ & $0.00 \mathrm{E}+00$ & $0.00 \mathrm{E}+00$ & $0.00 \mathrm{E}+00$ & $0.00 \mathrm{E}+00$ & $0.00 \mathrm{E}+00$ & $0.00 \mathrm{E}+00$ \\
\hline PANT & $6.24 \mathrm{E}-01$ & $0.00 \mathrm{E}+00$ & $0.00 \mathrm{E}+00$ & $0.00 \mathrm{E}+00$ & $0.00 \mathrm{E}+00$ & $0.00 \mathrm{E}+00$ & $0.00 \mathrm{E}+00$ & $6.24 \mathrm{E}-01$ \\
\hline SNL/NM & $6.66 \mathrm{E}+00$ & $2.08 \mathrm{E}-01$ & $1.87 \mathrm{E}+00$ & $2.08 \mathrm{E}-01$ & $1.04 \mathrm{E}+00$ & $2.08 \mathrm{E}+00$ & $2.08 \mathrm{E}+00$ & $1.41 \mathrm{E}+01$ \\
\hline TBE & $2.08 \mathrm{E}-01$ & $0.00 \mathrm{E}+00$ & $0.00 \mathrm{E}+00$ & $0.00 \mathrm{E}+00$ & $0.00 \mathrm{E}+00$ & $0.00 \mathrm{E}+00$ & $0.00 \mathrm{E}+00$ & $2.08 \mathrm{E}-01$ \\
\hline USAMC & $0.00 \mathrm{E}+00$ & $0.00 \mathrm{E}+00$ & $0.00 \mathrm{E}+00$ & $0.00 \mathrm{E}+00$ & $0.00 \mathrm{E}+00$ & $0.00 \mathrm{E}+00$ & $0.00 \mathrm{E}+00$ & $0.00 \mathrm{E}+00$ \\
\hline Subtotal & $1.21 \mathrm{E}+01$ & $6.26 \mathrm{E}-01$ & $2.83 \mathrm{E}+01$ & $1.72 E+01$ & $1.51 \mathrm{E}+02$ & $1.06 \mathrm{E}+02$ & $8.76 \mathrm{E}+01$ & $4.03 \mathrm{E}+02$ \\
\hline
\end{tabular}




\section{AS-GENERATED WASTE FORM}

Table 3.4 (continued)

\begin{tabular}{|c|c|c|c|c|c|c|c|c|}
\hline \multirow{2}{*}{ Site } & \multirow{2}{*}{$\begin{array}{l}\text { End of } \\
\text { CY } 1994 \\
\text { total }\end{array}$} & \multicolumn{3}{|c|}{ Annual increase } & \multicolumn{3}{|c|}{ Cumulative increase } & \multirow{2}{*}{$\begin{array}{l}\text { End of } \\
\text { CY } 2022 \\
\text { total }\end{array}$} \\
\hline & & 1995 & 1996 & 1997 & $1998-2002$ & 2003--2012 & $2013-2022$ & \\
\hline \multicolumn{9}{|l|}{ Commercial site } \\
\hline WVDP & $2.38 E+01$ & $5.34 \mathrm{E}+00$ & $5.34 \mathrm{E}+00$ & $5.34 \mathrm{E}+00$ & $2.67 E+01$ & $5.34 \mathrm{E}+01$ & $5.34 \mathrm{E}+01$ & $1.73 \mathrm{E}+02$ \\
\hline $\begin{array}{l}\text { Total } \\
\text { (all sites) }\end{array}$ & $2.86 \mathrm{E}+04$ & $9.53 \mathrm{E}+02$ & $1.15 \mathrm{E}+03$ & $7.98 \mathrm{E}+02$ & $5.86 \mathrm{E}+03$ & $9.61 E+03$ & $8.22 \mathrm{E}+03$ & $5.52 \mathrm{E}+04$ \\
\hline
\end{tabular}




\section{AS-GENERATED WASTE FORM}

Table 3.5. Summary of as-generated waste-form volumes $\left(\mathrm{m}^{3}\right)$ of retrievably stored and projected mixed and nonmixed RH TRUW

\begin{tabular}{|c|c|c|c|c|c|c|c|c|}
\hline \multirow{2}{*}{ Site } & \multirow{2}{*}{$\begin{array}{c}\text { End of } \\
\text { CY } 1994 \\
\text { total }\end{array}$} & \multicolumn{3}{|c|}{ Annual increase } & \multicolumn{3}{|c|}{ Cumulative increase } & \multirow{2}{*}{$\begin{array}{l}\text { End of } \\
\text { CY } 2022 \\
\text { total }\end{array}$} \\
\hline & & 1995 & 1996 & 1997 & $1998-2002$ & 2003-2012 & $2013-2022$ & \\
\hline \multicolumn{9}{|l|}{ Major sites } \\
\hline ANL-E & $0.00 \mathrm{E}+00$ & $0.00 \mathrm{E}+00$ & $0.00 \mathrm{E}+00$ & $0.00 \mathrm{E}+00$ & $0.00 \mathrm{E}+00$ & $0.00 \mathrm{E}+00$ & $0.00 \mathrm{E}+00$ & $0.00 \mathrm{E}+00$ \\
\hline Hanford & $2.01 \mathrm{E}+02$ & $7.17 \mathrm{E}+01$ & $4.05 \mathrm{E}+00$ & $1.12 \mathrm{E}+01$ & $2.82 E+02$ & $4.44 \mathrm{E}+03$ & $1.67 \mathrm{E}+04$ & $2.17 \mathrm{E}+04$ \\
\hline INEL & $2.00 \mathrm{E}+02$ & $0.00 \mathrm{E}+00$ & $0.00 \mathrm{E}+00$ & $0.00 \mathrm{E}+00$ & $0.00 \mathrm{E}+00$ & $0.00 \mathrm{E}+00$ & $0.00 \mathrm{E}+00$ & $2.00 \mathrm{E}+02$ \\
\hline LANL & $9.32 \mathrm{E}+01$ & $8.90 \mathrm{E}-01$ & $8.90 \mathrm{E}-01$ & $8.90 \mathrm{E}-01$ & $4.45 \mathrm{E}+00$ & $8.90 \mathrm{E}+00$ & $1.78 \mathrm{E}+01$ & $1.27 \mathrm{E}+02$ \\
\hline LLNL & $0.00 \mathrm{E}+00$ & $0.00 \mathrm{E}+00$ & $0.00 \mathrm{E}+00$ & $0.00 \mathrm{E}+00$ & $0.00 E+00$ & $0.00 \mathrm{E}+00$ & $0.00 \mathrm{E}+00$ & $0.00 \mathrm{E}+00$ \\
\hline Mound & $0.00 E+00$ & $0.00 \mathrm{E}+00$ & $0.00 \mathrm{E}+00$ & $0.00 \mathrm{E}+00$ & $0.00 \mathrm{E}+00$ & $0.00 \mathrm{E}+00$ & $0.00 \mathrm{E}+00$ & $0.00 \mathrm{E}+00$ \\
\hline NTS & $0.00 \mathrm{E}+00$ & $0.00 \mathrm{E}+00$ & $0.00 \mathrm{E}+00$ & $0.00 \mathrm{E}+00$ & $0.00 \mathrm{E}+00$ & $0.00 \mathrm{E}+00$ & $0.00 \mathrm{E}+00$ & $0.00 \mathrm{E}+00$ \\
\hline ORNL & $1.83 \mathrm{E}+03$ & $1.23 \mathrm{E}+01$ & $1.23 \mathrm{E}+01$ & $1.23 \mathrm{E}+01$ & $6.14 \mathrm{E}+01$ & $1.23 E+02$ & $1.23 E+02$ & $2.18 \mathrm{E}+03$ \\
\hline RFETS & $0.00 \mathrm{E}+00$ & $0.00 \mathrm{E}+00$ & $0.00 \mathrm{E}+00$ & $0.00 \mathrm{E}+00$ & $0.00 \mathrm{E}+00$ & $0.00 \mathrm{E}+00$ & $0.00 \mathrm{E}+00$ & $0.00 \mathrm{E}+00$ \\
\hline SRS & $0.00 \mathrm{E}+00$ & $0.00 \mathrm{E}+00$ & $0.00 \mathrm{E}+00$ & $0.00 \mathrm{E}+00$ & $0.00 \mathrm{E}+00$ & $0.00 \mathrm{E}+00$ & $0.00 \mathrm{E}+00$ & $0.00 \mathrm{E}+00$ \\
\hline Subtotal & $2.33 E+03$ & $8.49 \mathrm{E}+01$ & $1.72 \mathrm{E}+01$ & $2.43 \mathrm{E}+01$ & $3.48 \mathrm{E}+02$ & $4.57 \mathrm{E}+03$ & $1.69 E+04$ & $2.42 \mathrm{E}+04$ \\
\hline \multicolumn{9}{|c|}{ Small-quantity sites } \\
\hline Ames & $0.00 \mathrm{E}+00$ & $0.00 \mathrm{E}+00$ & $0.00 \mathrm{E}+00$ & $0.00 \mathrm{E}+00$ & $0.00 \mathrm{E}+00$ & $0.00 \mathrm{E}+00$ & $0.00 \mathrm{E}+00$ & $0.00 \mathrm{E}+00$ \\
\hline ANL-W & $2.17 \mathrm{E}+01$ & $0.00 \mathrm{E}+00$ & $0.00 \mathrm{E}+00$ & $0.00 \mathrm{E}+00$ & $6.82 \mathrm{E}+00$ & $1.24 \mathrm{E}+01$ & $6.57 \mathrm{E}+00$ & $4.74 \mathrm{E}+01$ \\
\hline $\mathrm{ARCO}$ & $0.00 \mathrm{E}+00$ & $0.00 \mathrm{E}+00$ & $0.00 \mathrm{E}+00$ & $0.00 \mathrm{E}+00$ & $0.00 \mathrm{E}+00$ & $0.00 \mathrm{E}+00$ & $0.00 \mathrm{E}+00$ & $0.00 \mathrm{E}+00$ \\
\hline BAPL & $0.00 \mathrm{E}+00$ & $3.12 \mathrm{E}-01$ & $2.08 \mathrm{E}-01$ & $3.12 \mathrm{E}-01$ & $6.24 \mathrm{E}-01$ & $1.04 \mathrm{E}-01$ & $0.00 \mathrm{E}+00$ & $1.56 \mathrm{E}+00$ \\
\hline $\mathrm{BCL}$ & $5.81 \mathrm{E}+02$ & $0.00 \mathrm{E}+00$ & $0.00 \mathrm{E}+00$ & $0.00 \mathrm{E}+00$ & $0.00 \mathrm{E}+00$ & $0.00 \mathrm{E}+00$ & $0.00 \mathrm{E}+00$ & $5.81 \mathrm{E}+02$ \\
\hline ETEC & $5.67 \mathrm{E}+00$ & $0.00 \mathrm{E}+00$ & $4.20 \mathrm{E}-01$ & $4.20 \mathrm{E}-01$ & $0.00 \mathrm{E}+00$ & $0.00 \mathrm{E}+00$ & $0.00 \mathrm{E}+00$ & $6.51 \mathrm{E}+00$ \\
\hline KAPL & $2.40 E-01$ & $2.00 \mathrm{E}-01$ & $2.00 E-01$ & $2.00 \mathrm{E}-01$ & $1.00 \mathrm{E}+00$ & $2.00 \mathrm{E}+00$ & $2.00 \mathrm{E}+00$ & $5.84 \mathrm{E}+00$ \\
\hline LBL & $0.00 \mathrm{E}+00$ & $0.00 \mathrm{E}+00$ & $0.00 \mathrm{E}+00$ & $0.00 \mathrm{E}+00$ & $0.00 \mathrm{E}+00$ & $0.00 \mathrm{E}+00$ & $0.00 \mathrm{E}+00$ & $0.00 \mathrm{E}+00$ \\
\hline MURR & $0.00 E+00$ & $0.00 \mathrm{E}+00$ & $0.00 \mathrm{E}+00$ & $0.00 \mathrm{E}+00$ & $0.00 \mathrm{E}+00$ & $0.00 \mathrm{E}+00$ & $0.00 \mathrm{E}+00$ & $0.00 \mathrm{E}+00$ \\
\hline $\mathrm{PAD}$ & $0.00 \mathrm{E}+00$ & $0.00 \mathrm{E}+00$ & $0.00 \mathrm{E}+00$ & $0.00 \mathrm{E}+00$ & $0.00 \mathrm{E}+00$ & $0.00 \mathrm{E}+00$ & $0.00 \mathrm{E}+00$ & $0.00 \mathrm{E}+00$ \\
\hline PANT & $0.00 \mathrm{E}+00$ & $0.00 \mathrm{E}+00$ & $0.00 \mathrm{E}+00$ & $0.00 \mathrm{E}+00$ & $0.00 \mathrm{E}+00$ & $0.00 \mathrm{E}+00$ & $0.00 \mathrm{E}+00$ & $0.00 \mathrm{E}+00$ \\
\hline SNL/NM & $0.00 \mathrm{E}+00$ & $0.00 \mathrm{E}+00$ & $0.00 \mathrm{E}+00$ & $0.00 \mathrm{E}+00$ & $0.00 \mathrm{E}+00$ & $0.00 \mathrm{E}+00$ & $0.00 \mathrm{E}+00$ & $0.00 \mathrm{E}+00$ \\
\hline TBE & $0.00 \mathrm{E}+00$ & $0.00 \mathrm{E}+00$ & $0.00 \mathrm{E}+00$ & $0.00 \mathrm{E}+00$ & $0.00 \mathrm{E}+00$ & $0.00 \mathrm{E}+00$ & $0.00 \mathrm{E}+00$ & $0.00 \mathrm{E}+00$ \\
\hline USAMC & $0.00 \mathrm{E}+00$ & $0.00 \mathrm{E}+00$ & $0.00 \mathrm{E}+00$ & $0.00 \mathrm{E}+00$ & $0.00 \mathrm{E}+00$ & $0.00 \mathrm{E}+00$ & $0.00 \mathrm{E}+00$ & $0.00 \mathrm{E}+00$ \\
\hline Subtotal & $6.08 \mathrm{E}+02$ & $5.12 \mathrm{E}-01$ & $8.28 \mathrm{E}-01$ & $9.32 E-01$ & $8.44 \mathrm{E}+00$ & $1.45 \mathrm{E}+01$ & $8.57 \mathrm{E}+00$ & $6.42 \mathrm{E}+02$ \\
\hline
\end{tabular}




\section{AS-GENERATED WASTE FORM}

Table 3.5 (continued)

\begin{tabular}{|c|c|c|c|c|c|c|c|c|}
\hline \multirow{2}{*}{ Site } & \multirow{2}{*}{$\begin{array}{l}\text { End of } \\
\text { CY } 1994 \\
\text { total }\end{array}$} & \multicolumn{3}{|c|}{ Annual increase } & \multicolumn{3}{|c|}{ Cumulative increase } & \multirow{2}{*}{$\begin{array}{l}\text { End of } \\
\text { CY } 2022 \\
\text { total }\end{array}$} \\
\hline & & 1995 & 1996 & 1997 & $1998-2002$ & 2003-2012 & $2013-2022$ & \\
\hline \multicolumn{9}{|l|}{ Commercial site } \\
\hline WVDP & $4.84 \mathrm{E}+02$ & $4.45 \mathrm{E}+01$ & $0.00 \mathrm{E}+00$ & $0.00 E+00$ & $4.81 \mathrm{E}+01$ & $0.00 \mathrm{E}+00$ & $0.00 \mathrm{E}+00$ & $5.76 \mathrm{E}+02$ \\
\hline $\begin{array}{l}\text { Total } \\
\text { (all sites) }\end{array}$ & $3.42 \mathrm{E}+03$ & $1.30 \mathrm{E}+02$ & $1.80 \mathrm{E}+01$ & $2.53 \mathrm{E}+01$ & $4.04 \mathrm{E}+02$ & $4.58 \mathrm{E}+03$ & $1.69 \mathrm{E}+04$ & $2.54 \mathrm{E}+04$ \\
\hline
\end{tabular}




\section{AS-GENERATED WASTE FORM}

Table 3.6. As-generated waste-form volumes $\left(\mathrm{m}^{3}\right)$ of retrievably stored and projected mixed RH TRUW

\begin{tabular}{|c|c|c|c|c|c|c|c|c|}
\hline \multirow{2}{*}{ Site } & \multirow{2}{*}{$\begin{array}{l}\text { End of } \\
\text { CX } 1994 \\
\text { total }\end{array}$} & \multicolumn{3}{|c|}{ Annual increase } & \multicolumn{3}{|c|}{ Cumulative increase } & \multirow{2}{*}{$\begin{array}{l}\text { End of } \\
\text { CY } 2022 \\
\text { total }\end{array}$} \\
\hline & & 1995 & 1996 & 1997 & 1998-2002 & 2003-2012 & 2013-2022 & \\
\hline \multicolumn{9}{|l|}{ Major sites } \\
\hline ANL-E & $0.00 \mathrm{E}+00$ & $0.00 \mathrm{E}+00$ & $0.00 \mathrm{E}+00$ & $0.00 \mathrm{E}+00$ & $0.00 \mathrm{E}+00$ & $0.00 \mathrm{E}+00$ & $0.00 \mathrm{E}+00$ & $0.00 \mathrm{E}+00$ \\
\hline Hanford & $2.06 \mathrm{E}+00$ & $4.05 E+00$ & $4.05 \mathrm{E}+00$ & $1.12 \mathrm{E}+01$ & $2.82 \mathrm{E}+02$ & $4.08 \mathrm{E}+03$ & $1.09 \mathrm{E}+04$ & $1.53 E+04$ \\
\hline INEL & $1.52 \mathrm{E}+02$ & $0.00 \mathrm{E}+00$ & $0.00 \mathrm{E}+00$ & $0.00 \mathrm{E}+00$ & $0.00 \mathrm{E}+00$ & $0.00 \mathrm{E}+00$ & $0.00 \mathrm{E}+00$ & $1.52 \mathrm{E}+02$ \\
\hline LANL & $1.64 \mathrm{E}+01$ & $8.90 \mathrm{E}-01$ & $8.90 \mathrm{E}-01$ & $8.90 \mathrm{E}-01$ & $4.45 \mathrm{E}+00$ & $8.90 \mathrm{E}+00$ & $1.78 \mathrm{E}+01$ & $5.02 \mathrm{E}+01$ \\
\hline LLNL & $0.00 \mathrm{E}+00$ & $0.00 \mathrm{E}+00$ & $0.00 \mathrm{E}+00$ & $0.00 \mathrm{E}+00$ & $0.00 \mathrm{E}+00$ & $0.00 \mathrm{E}+00$ & $0.00 \mathrm{E}+00$ & $0.00 \mathrm{E}+00$ \\
\hline Mound & $0.00 \mathrm{E}+00$ & $0.00 \mathrm{E}+00$ & $0.00 E+00$ & $0.00 \mathrm{E}+00$ & $0.00 \mathrm{E}+00$ & $0.00 \mathrm{E}+00$ & $0.00 \mathrm{E}+00$ & $0.00 \mathrm{E}+00$ \\
\hline NTS & $0.00 \mathrm{E}+00$ & $0.00 \mathrm{E}+00$ & $0.00 \mathrm{E}+00$ & $0.00 \mathrm{E}+00$ & $0.00 \mathrm{E}+00$ & $0.00 \mathrm{E}+00$ & $0.00 \mathrm{E}+00$ & $0.00 \mathrm{E}+00$ \\
\hline ORNL & $1.77 \mathrm{E}+03$ & $1.23 E+01$ & $1.23 E+01$ & $1.23 \mathrm{E}+01$ & $6.14 E+01$ & $1.23 \mathrm{E}+02$ & $1.23 \mathrm{E}+02$ & $2.12 E+03$ \\
\hline RFETS & $0.00 \mathrm{E}+00$ & $0.00 \mathrm{E}+00$ & $0.00 \mathrm{E}+00$ & $0.00 \mathrm{E}+00$ & $0.00 \mathrm{E}+00$ & $0.00 \mathrm{E}+00$ & $0.00 \mathrm{E}+00$ & $0.00 \mathrm{E}+00$ \\
\hline SRS & $0.00 \mathrm{E}+00$ & $0.00 \mathrm{E}+00$ & $0.00 \mathrm{E}+00$ & $0.00 \mathrm{E}+00$ & $0.00 \mathrm{E}+00$ & $0.00 \mathrm{E}+00$ & $0.00 \mathrm{E}+00$ & $0.00 \mathrm{E}+00$ \\
\hline Subtotal & $1.94 \mathrm{E}+03$ & $1.72 \mathrm{E}+01$ & $1.72 \mathrm{E}+01$ & $2.43 \mathrm{E}+01$ & $3.48 \mathrm{E}+02$ & $4.21 \mathrm{E}+03$ & $1.10 \mathrm{E}+04$ & $1.76 \mathrm{E}+04$ \\
\hline \multicolumn{9}{|c|}{ Small-quantity sites } \\
\hline Ames & $0.00 \mathrm{E}+00$ & $0.00 \mathrm{E}+00$ & $0.00 \mathrm{E}+00$ & $0.00 \mathrm{E}+00$ & $0.00 \mathrm{E}+00$ & $0.00 \mathrm{E}+00$ & $0.00 \mathrm{E}+00$ & $0.00 \mathrm{E}+00$ \\
\hline ANL-W & $1.54 \mathrm{E}+01$ & $0.00 \mathrm{E}+00$ & $0.00 \mathrm{E}+00$ & $0.00 \mathrm{E}+00$ & $5.82 \mathrm{E}+00$ & $1.04 \mathrm{E}+01$ & $4.77 \mathrm{E}+00$ & $3.64 \mathrm{E}+01$ \\
\hline ARCO & $0.00 \mathrm{E}+00$ & $0.00 \mathrm{E}+00$ & $0.00 \mathrm{E}+00$ & $0.00 \mathrm{E}+00$ & $0.00 \mathrm{E}+00$ & $0.00 \mathrm{E}+00$ & $0.00 \mathrm{E}+00$ & $0.00 \mathrm{E}+00$ \\
\hline BAPL & $0.00 \mathrm{E}+00$ & $0.00 \mathrm{E}+00$ & $0.00 E+00$ & $0.00 \mathrm{E}+00$ & $0.00 E+00$ & $0.00 \mathrm{E}+00$ & $0.00 \mathrm{E}+00$ & $0.00 \bar{E}+00$ \\
\hline $\mathrm{BCL}$ & $0.00 \mathrm{E}+00$ & $0.00 \mathrm{E}+00$ & $0.00 \mathrm{E}+00$ & $0.00 \mathrm{E}+00$ & $0.00 \mathrm{E}+00$ & $0.00 \mathrm{E}+00$ & $0.00 \mathrm{E}+00$ & $0.00 \mathrm{E}+00$ \\
\hline ETEC & $5.67 \mathrm{E}+00$ & $0.00 \mathrm{E}+00$ & $4.20 \mathrm{E}-01$ & $4.20 \mathrm{E}-01$ & $0.00 \mathrm{E}+00$ & $0.00 \mathrm{E}+00$ & $0.00 \mathrm{E}+00$ & $6.51 \mathrm{E}+00$ \\
\hline KAPL & $0.00 \mathrm{E}+00$ & $4.00 \mathrm{E}-02$ & $4.00 \mathrm{E}-02$ & $4.00 \mathrm{E}-02$ & $1.40 \mathrm{E}-01$ & $2.00 \mathrm{E}-01$ & $2.00 \mathrm{E}-01$ & $6.60 \mathrm{E}-01$ \\
\hline LBL & $0.00 \mathrm{E}+00$ & $0.00 \mathrm{E}+00$ & $0.00 \mathrm{E}+00$ & $0.00 \mathrm{E}+00$ & $0.00 \mathrm{E}+00$ & $0.00 \mathrm{E}+00$ & $0.00 \mathrm{E}+00$ & $0.00 \mathrm{E}+00$ \\
\hline MURR & $0.00 \mathrm{E}+00$ & $0.00 \mathrm{E}+00$ & $0.00 \mathrm{E}+00$ & $0.00 \mathrm{E}+00$ & $0.00 \mathrm{E}+00$ & $0.00 \mathrm{E}+00$ & $0.00 \mathrm{E}+00$ & $0.00 \mathrm{E}+00$ \\
\hline PAD & $0.00 \mathrm{E}+00$ & $0.00 \mathrm{E}+00$ & $0.00 \mathrm{E}+00$ & $0.00 \mathrm{E}+00$ & $0.00 \mathrm{E}+00$ & $0.00 \mathrm{E}+00$ & $0.00 \mathrm{E}+00$ & $0.00 \mathrm{E}+00$ \\
\hline PANT & $0.00 \mathrm{E}+00$ & $0.00 \mathrm{E}+00$ & $0.00 \mathrm{E}+00$ & $0.00 \mathrm{E}+00$ & $0.00 \mathrm{E}+00$ & $0.00 \mathrm{E}+00$ & $0.00 \mathrm{E}+00$ & $0.00 \mathrm{E}+00$ \\
\hline SNL/NM & $0.00 \mathrm{E}+00$ & $0.00 \mathrm{E}+00$ & $0.00 \mathrm{E}+00$ & $0.00 \mathrm{E}+00$ & $0.00 \mathrm{E}+00$ & $0.00 \mathrm{E}+00$ & $0.00 \mathrm{E}+00$ & $0.00 \mathrm{E}+00$ \\
\hline TBE & $0.00 \mathrm{E}+00$ & $0.00 \mathrm{E}+00$ & $0.00 \mathrm{E}+00$ & $0.00 \mathrm{E}+00$ & $0.00 \mathrm{E}+00$ & $0.00 \mathrm{E}+00$ & $0.00 \mathrm{E}+00$ & $0.00 \mathrm{E}+00$ \\
\hline USAMC & $0.00 \mathrm{E}+00$ & $0.00 \mathrm{E}+00$ & $0.00 \mathrm{E}+00$ & $0.00 \mathrm{E}+00$ & $0.00 \mathrm{E}+00$ & $0.00 \mathrm{E}+00$ & $0.00 \mathrm{E}+00$ & $0.00 \mathrm{E}+00$ \\
\hline Subtotal & $2.11 \mathrm{E}+01$ & $4.00 \mathrm{E}-02$ & $4.60 \mathrm{E}-01$ & $4.60 \mathrm{E}-01$ & $5.96 \mathrm{E}+00$ & $1.06 \mathrm{E}+01$ & $4.97 \mathrm{E}+00$ & $4.36 \mathrm{E}+01$ \\
\hline
\end{tabular}




\section{AS-GENERATED WASTE FORM}

Table 3.6 (continued)

\begin{tabular}{|c|c|c|c|c|c|c|c|c|}
\hline \multirow{2}{*}{ Site } & \multirow{2}{*}{$\begin{array}{l}\text { End of } \\
\text { CY } 1994 \\
\text { total }\end{array}$} & \multicolumn{3}{|c|}{ Annual increase } & \multicolumn{3}{|c|}{ Cumulative increase } & \multirow{2}{*}{$\begin{array}{l}\text { End of } \\
\text { CY } 2022 \\
\text { total }\end{array}$} \\
\hline & & 1995 & 1996 & 1997 & $1998-2002$ & $2003-2012$ & $2013-2022$ & \\
\hline \multicolumn{9}{|l|}{ Commercial site } \\
\hline WVDP & $0.00 \mathrm{E}+00$ & $0.00 \mathrm{E}+00$ & $0.00 \mathrm{E}+00$ & $0.00 \mathrm{E}+00$ & $0.00 \mathrm{E}+00$ & $0.00 \mathrm{E}+00$ & $0.00 \mathrm{E}+00$ & $0.00 \mathrm{E}+00$ \\
\hline $\begin{array}{l}\text { Total } \\
\quad \text { (all sites) }\end{array}$ & $1.96 \mathrm{E}+03$ & $1.73 \mathrm{E}+01$ & $1.77 E+01$ & $2.48 \mathrm{E}+01$ & $3.54 \mathrm{E}+02$ & $4.22 \mathrm{E}+03$ & $1.10 \mathrm{E}+04$ & $1.76 \mathrm{E}+04$ \\
\hline
\end{tabular}




\section{AS-GENERATED WASTE FORM}

Table 3.7. As-generated waste-form volumes $\left(\mathrm{m}^{3}\right)$ of retrievably stored and projected nonmixed RH TRUW

\begin{tabular}{|c|c|c|c|c|c|c|c|c|}
\hline \multirow{2}{*}{ Site } & \multirow{2}{*}{$\begin{array}{l}\text { End of } \\
\text { CY } 1994 \\
\text { total }\end{array}$} & \multicolumn{3}{|c|}{ Annual increase } & \multicolumn{3}{|c|}{ Cumulative increase } & \multirow{2}{*}{$\begin{array}{c}\text { End of } \\
\text { CY } 2022 \\
\text { total }\end{array}$} \\
\hline & & 1995 & 1996 & 1997 & $1998-2002$ & 2003-2012 & $2013-2022$ & \\
\hline \multicolumn{9}{|l|}{ Major sites } \\
\hline ANL-E & $0.00 \mathrm{E}+00$ & $0.00 \mathrm{E}+00$ & $0.00 \mathrm{E}+00$ & $0.00 \mathrm{E}+00$ & $0.00 \mathrm{E}+00$ & $0.00 \mathrm{E}+00$ & $0.00 \mathrm{E}+00$ & $0.00 \mathrm{E}+00$ \\
\hline Hanford & $1.99 \mathrm{E}+02$ & $6.76 E+01$ & $0.00 \mathrm{E}+00$ & $0.00 \mathrm{E}+00$ & $0.00 \mathrm{E}+00$ & $3.56 \mathrm{E}+02$ & $5.83 E+03$ & $6.45 E+03$ \\
\hline INEL & $4.87 \mathrm{E}+01$ & $0.00 \mathrm{E}+00$ & $0.00 \mathrm{E}+00$ & $0.00 \mathrm{E}+00$ & $0.00 \mathrm{E}+00$ & $0.00 \mathrm{E}+00$ & $0.00 E+00$ & $4.87 \mathrm{E}+01$ \\
\hline LANL & $7.68 \mathrm{E}+01$ & $0.00 \mathrm{E}+00$ & $0.00 \mathrm{E}+00$ & $0.00 \mathrm{E}+00$ & $0.00 \mathrm{E}+00$ & $0.00 \mathrm{E}+00$ & $0.00 \mathrm{E}+00$ & $7.68 \mathrm{E}+01$ \\
\hline LLNL & $0.00 \mathrm{E}+00$ & $0.00 \mathrm{E}+00$ & $0.00 \mathrm{E}+00$ & $0.00 \mathrm{E}+00$ & $0.00 \mathrm{E}+00$ & $0.00 \mathrm{E}+00$ & $0.00 \mathrm{E}+00$ & $0.00 \mathrm{E}+00$ \\
\hline Mound & $0.00 \mathrm{E}+00$ & $0.00 \mathrm{E}+00$ & $0.00 \mathrm{E}+00$ & $0.00 \mathrm{E}+00$ & $0.00 \mathrm{E}+00$ & $0.00 \mathrm{E}+00$ & $0.00 \mathrm{E}+00^{\circ}$ & $0.00 \mathrm{E}+00$ \\
\hline NTS & $0.00 \mathrm{E}+00$ & $0.00 \mathrm{E}+00$ & $0.00 \mathrm{E}+00$ & $0.00 \mathrm{E}+00$ & $0.00 \mathrm{E}+00$ & $0.00 \mathrm{E}+00$ & $0.00 \mathrm{E}+00$ & $0.00 \mathrm{E}+00$ \\
\hline ORNL & $5.94 E+01$ & $0.00 \mathrm{E}+00$ & $0.00 \mathrm{E}+00$ & $0.00 E+00$ & $0.00 \mathrm{E}+00$ & $0.00 \mathrm{E}+00$ & $0.00 \mathrm{E}+00$ & $5.94 \mathrm{E}+01$ \\
\hline RFETS & $0.00 \mathrm{E}+00$ & $0.00 \mathrm{E}+00$ & $0.00 \mathrm{E}+00$ & $0.00 \mathrm{E}+00$ & $0.00 \mathrm{E}+00$ & $0.00 \mathrm{E}+00$ & $0.00 \mathrm{E}+00$ & $0.00 \mathrm{E}+00$ \\
\hline SRS & $0.00 \mathrm{E}+00$ & $0.00 \mathrm{E}+00$ & $0.00 \mathrm{E}+00$ & $0.00 \mathrm{E}+00$ & $0.00 \mathrm{E}+00$ & $0.00 \mathrm{E}+00$ & $0.00 \mathrm{E}+00$ & $0.00 \mathrm{E}+00$ \\
\hline Subtotal & $3.84 \mathrm{E}+02$ & $6.76 E+01$ & $0.00 \mathrm{E}+00$ & $0.00 \mathrm{E}+00$ & $0.00 \mathrm{E}+00$ & $3.56 \mathrm{E}+02$ & $5.83 E+03$ & $6.64 \mathrm{E}+03$ \\
\hline \multicolumn{9}{|c|}{ Small-quantity sites } \\
\hline Ames & $0.00 \mathrm{E}+00$ & $0.00 \mathrm{E}+00$ & $0.00 E+00$ & $0.00 \mathrm{E}+00$ & $0.00 \mathrm{E}+00$ & $0.00 \mathrm{E}+00$ & $0.00 \mathrm{E}+00$ & $0.00 \mathrm{E}+00$ \\
\hline ANL-W & $6.21 \mathrm{E}+00$ & $0.00 \mathrm{E}+00$ & $0.00 \mathrm{E}+00$ & $0.00 \mathrm{E}+00$ & $1.00 \mathrm{E}+00$ & $2.00 \mathrm{E}+00$ & $1.80 \mathrm{E}+00$ & $1.10 \mathrm{E}+01$ \\
\hline ARCO & $0.00 \mathrm{E}+00$ & $0.00 \mathrm{E}+00$ & $0.00 E+00$ & $0.00 \mathrm{E}+00$ & $0.00 \mathrm{E}+00$ & $0.00 \mathrm{E}+00$ & $0.00 \mathrm{E}+00$ & $0.00 \mathrm{E}+00$ \\
\hline BAPL & $0.00 \mathrm{E}+00$ & $3.12 \mathrm{E}-01$ & $2.08 \mathrm{E}-01$ & $3.12 \mathrm{E}-01$ & $6.24 \mathrm{E}-01$ & $1.04 \mathrm{E}-01$ & $0.00 \mathrm{E}+00$ & $1.56 \mathrm{E}+00$ \\
\hline $\mathrm{BCL}$ & $5.81 \mathrm{E}+02$ & $0.00 \mathrm{E}+00$ & $0.00 \mathrm{E}+00$ & $0.00 \mathrm{E}+00$ & $0.00 \mathrm{E}+00$ & $0.00 \mathrm{E}+00$ & $0.00 \mathrm{E}+00$ & $5.81 \mathrm{E}+02$ \\
\hline ETEC & $0.00 \mathrm{E}+00$ & $0.00 \mathrm{E}+00$ & $0.00 \mathrm{E}+00$ & $0.00 \mathrm{E}+00$ & $0.00 \mathrm{E}+00$ & $0.00 \mathrm{E}+00$ & $0.00 \mathrm{E}+00$ & $0.00 \mathrm{E}+00$ \\
\hline KAPL & $2.40 \mathrm{E}-01$ & $1.60 \mathrm{E}-01$ & $1.60 \mathrm{E}-01$ & $1.60 \mathrm{E}-01$ & $8.60 \mathrm{E}-01$ & $1.80 \mathrm{E}+00$ & $1.80 \mathrm{E}+00$ & $5.18 \mathrm{E}+00$ \\
\hline LBL & $0.00 \mathrm{E}+00$ & $0.00 \mathrm{E}+00$ & $0.00 \mathrm{E}+00$ & $0.00 \mathrm{E}+00$ & $0.00 \mathrm{E}+00$ & $0.00 \mathrm{E}+00$ & $0.00 \mathrm{E}+00$ & $0.00 \mathrm{E}+00$ \\
\hline MURR & $0.00 \mathrm{E}+00$ & $0.00 \mathrm{E}+00$ & $0.00 \mathrm{E}+00$ & $0.00 \mathrm{E}+00$ & $0.00 \mathrm{E}+00$ & $0.00 \mathrm{E}+00$ & $0.00 \mathrm{E}+00$ & $0.00 \mathrm{E}+00$ \\
\hline PAD & $0.00 E+00$ & $0.00 \mathrm{E}+00$ & $0.00 \mathrm{E}+00$ & $0.00 \mathrm{E}+00$ & $0.00 \mathrm{E}+00$ & $0.00 \mathrm{E}+00$ & $0.00 \mathrm{E}+00$ & $0.00 \mathrm{E}+00$ \\
\hline PANT & $0.00 \mathrm{E}+00$ & $0.00 \mathrm{E}+00$ & $0.00 E+00$ & $0.00 \mathrm{E}+00$ & $0.00 \mathrm{E}+00$ & $0.00 \mathrm{E}+00$ & $0.00 \mathrm{E}+00$ & $0.00 \mathrm{E}+00$ \\
\hline SNL/NM & $0.00 \mathrm{E}+00$ & $0.00 \mathrm{E}+00$ & $0.00 \mathrm{E}+00$ & $0.00 \mathrm{E}+00$ & $0.00 \mathrm{E}+00$ & $0.00 \mathrm{E}+00$ & $0.00 \mathrm{E}+00$ & $0.00 \mathrm{E}+00$ \\
\hline TBE & $0.00 \mathrm{E}+00$ & $0.00 \mathrm{E}+00$ & $0.00 \mathrm{E}+00$ & $0.00 \mathrm{E}+00$ & $0.00 \mathrm{E}+00$ & $0.00 \mathrm{E}+00$ & $0.00 \mathrm{E}+00$ & $0.00 \mathrm{E}+00$ \\
\hline USAMC & $0.00 \mathrm{E}+00$ & $0.00 \mathrm{E}+00$ & $0.00 \mathrm{E}+00$ & $0.00 \mathrm{E}+00$ & $0.00 \mathrm{E}+00$ & $0.00 \mathrm{E}+00$ & $0.00 \mathrm{E}+00$ & $0.00 \mathrm{E}+00$ \\
\hline Subtotal & $5.87 \mathrm{E}+02$ & $4.72 \mathrm{E}-01$ & $3.68 \mathrm{E}-01$ & $4.72 \mathrm{E}-01$ & $2.48 \mathrm{E}+00$ & $3.90 \mathrm{E}+00$ & $3.60 \mathrm{E}+00$ & $5.98 \mathrm{E}+02$ \\
\hline
\end{tabular}




\begin{tabular}{|c|c|c|c|c|c|c|c|c|}
\hline & & & AS-GENEI & TED WAS & FORM & & & \\
\hline & & & Tab & 3.7 (continu & & & & \\
\hline Si & End of & & Annual increa & & & Imulative incre & & End of \\
\hline & total & 1995 & 1996 & 1997 & $1998-2002$ & $2003-2012$ & $2013-2022$ & total \\
\hline $\begin{array}{l}\text { Commercial site } \\
\text { WVDP }\end{array}$ & $4.84 \mathrm{E}+02$ & $4.45 \mathrm{E}+01$ & $0.00 \mathrm{E}+00$ & $0.00 \mathrm{E}+00$ & $4.81 \mathrm{E}+01$ & $0.00 \mathrm{E}+00$ & $0.00 \mathrm{E}+00$ & $5.76 \mathrm{E}+02$ \\
\hline $\begin{array}{l}\text { Total } \\
\text { (all sites) }\end{array}$ & $1.45 E+03$ & $1.13 E+02$ & $3.68 \mathrm{E}-01$ & $4.72 \mathrm{E}-01$ & $5.06 \mathrm{E}+01$ & $3.60 \mathrm{E}+02$ & $5.84 \mathrm{E}+03$ & $7.81 \mathrm{E}+03$ \\
\hline
\end{tabular}


FINAL WASTE FORM

Table 3.8. Summary of final waste-form volumes $\left(\mathrm{m}^{3}\right)$ of retrievably stored and projected TRUW (mixed and nonmixed $\mathrm{CH}$ and $\mathrm{RH}$ wastes)

\begin{tabular}{|c|c|c|c|c|c|c|c|c|}
\hline \multirow{2}{*}{ Site } & \multirow{2}{*}{$\begin{array}{l}\text { End of } \\
\text { CY } 1994 \\
\text { total }\end{array}$} & \multicolumn{3}{|c|}{ Annual increase } & \multicolumn{3}{|c|}{ Cumulative increase } & \multirow{2}{*}{$\begin{array}{c}\text { End of } \\
\text { CY } 2022 \\
\text { total }\end{array}$} \\
\hline & & 1995 & 1996 & 1997 & $1998-2002$ & $2003-2012$ & 2013-2022 & \\
\hline \multicolumn{9}{|l|}{ Major sites } \\
\hline ANL-E & $2.48 \mathrm{E}+01$ & $0.00 \mathrm{E}+00$ & $4.20 \mathrm{E}+01$ & $3.33 \mathrm{E}+00$ & $1.79 \mathrm{E}+01$ & $3.33 E+01$ & $3.33 \mathrm{E}+01$ & $1.55 \mathrm{E}+02$ \\
\hline Hanford & $1.26 \mathrm{E}+04$ & $4.57 \mathrm{E}+02$ & $1.19 \mathrm{E}+02$ & $1.31 \mathrm{E}+02$ & $2.32 \mathrm{E}+03$ & $1.96 \mathrm{E}+04$ & $3.23 E+04$ & $6.75 \mathrm{E}+04$ \\
\hline INEL & $2.88 \mathrm{E}+04$ & $0.00 \mathrm{E}+00$ & $0.00 \mathrm{E}+00$ & $0.00 \mathrm{E}+00$ & $0.00 \mathrm{E}+00$ & $0.00 \mathrm{E}+00$ & $0.00 \mathrm{E}+00$ & $2.88 \mathrm{E}+04$ \\
\hline LANL & $1.11 \mathrm{E}+04$ & $2.63 E+02$ & $2.65 \mathrm{E}+02$ & $2.65 E+02$ & $1.33 E+03$ & $2.66 \mathrm{E}+03$ & $2.67 \mathrm{E}+03$ & $1.86 \mathrm{E}+04$ \\
\hline LLNL & $2.30 \mathrm{E}+02$ & $3.74 \mathrm{E}+01$ & $2.40 \mathrm{E}+01$ & $2.59 \mathrm{E}+01$ & $1.24 \mathrm{E}+02$ & $2.50 \mathrm{E}+02$ & $2.50 \mathrm{E}+02$ & $9.41 \mathrm{E}+02$ \\
\hline Mound & $3.20 \mathrm{E}+02$ & $0.00 \mathrm{E}+00$ & $0.00 \mathrm{E}+00$ & $0.00 \mathrm{E}+00$ & $0.00 \mathrm{E}+00$ & $0.00 \mathrm{E}+00$ & $0.00 \mathrm{E}+00$ & $3.20 \mathrm{E}+02$ \\
\hline NTS & $6.19 \mathrm{E}+02$ & $0.00 \mathrm{E}+00$ & $0.00 \mathrm{E}+00$ & $0.00 \mathrm{E}+00$ & $0.00 E+00$ & $6.21 \mathrm{E}+00$ & $2.77 \mathrm{E}+00$ & $6.28 E+02$ \\
\hline ORNL & $3.80 \mathrm{E}+03$ & $1.65 \mathrm{E}+01$ & $2.54 \mathrm{E}+01$ & $2.54 \mathrm{E}+01$ & $1.27 E+02$ & $2.54 \mathrm{E}+02$ & $2.54 \mathrm{E}+02$ & $4.50 \mathrm{E}+03$ \\
\hline RFETS & $7.08 \mathrm{E}+02$ & $1.05 E+02$ & $8.96 E+01$ & $3.24 \mathrm{E}+01$ & $1.61 E+02$ & $1.75 E+03$ & $1.77 \mathrm{E}+03$ & $4.61 E+03$ \\
\hline SRS & $2.88 \mathrm{E}+03$ & $2.50 \mathrm{E}-01$ & $2.50 \mathrm{E}-01$ & $2.50 \mathrm{E}-01$ & $1.25 E+00$ & $2.50 \mathrm{E}+00$ & $6.76 \mathrm{E}+03$ & $9.65 E+03$ \\
\hline Subtotal & $6.11 \mathrm{E}+04$ & $8.79 E+02$ & $5.66 \mathrm{E}+02$ & $4.84 \mathrm{E}+02$ & $4.08 \mathrm{E}+03$ & $2.46 \mathrm{E}+04$ & $4.41 E+04$ & $1.36 E+05$ \\
\hline \multicolumn{9}{|c|}{ Small-quantity sites } \\
\hline Ames & $0.00 \mathrm{E}+00$ & $0.00 \mathrm{E}+00$ & $0.00 \mathrm{E}+00$ & $0.00 \mathrm{E}+00$ & $0.00 \mathrm{E}+00$ & $4.16 \mathrm{E}-01$ & $0.00 \mathrm{E}+00$ & $4.16 \mathrm{E}-01$ \\
\hline ANL-W & $2.58 \mathrm{E}+01$ & $0.00 \mathrm{E}+00$ & $0.00 \mathrm{E}+00$ & $0.00 \mathrm{E}+00$ & $5.47 \mathrm{E}+02$ & $7.91 \mathrm{E}+02$ & $6.68 \mathrm{E}+02$ & $2.03 E+03$ \\
\hline $\mathrm{ARCO}$ & $0.00 \mathrm{E}+00$ & $0.00 \mathrm{E}+00$ & $0.00 \mathrm{E}+00$ & $0.00 \mathrm{E}+00$ & $4.16 \mathrm{E}-01$ & $0.00 \mathrm{E}+00$ & $0.00 \mathrm{E}+00$ & $4.16 E-01$ \\
\hline BAPL & $0.00 \mathrm{E}+00$ & $1.75 \mathrm{E}+00$ & $2.73 E+01$ & $1.83 \mathrm{E}+01$ & $7.83 \mathrm{E}+01$ & $4.22 \mathrm{E}+00$ & $0.00 \mathrm{E}+00$ & $1.30 \mathrm{E}+02$ \\
\hline BCL & $5.81 \mathrm{E}+02$ & $0.00 \mathrm{E}+00$ & $0.00 \mathrm{E}+00$ & $0.00 \mathrm{E}+00$ & $0.00 \mathrm{E}+00$ & $0.00 \mathrm{E}+00$ & $0.00 \mathrm{E}+00$ & $5.81 \mathrm{E}+02$ \\
\hline ETEC & $7.98 \mathrm{E}+00$ & $0.00 \mathrm{E}+00$ & $4.16 \mathrm{E}-01$ & $4.16 \mathrm{E}-01$ & $0.00 \mathrm{E}+00$ & $0.00 \mathrm{E}+00$ & $0.00 \mathrm{E}+00$ & $8.81 E+00$ \\
\hline KAPL & $2.50 \mathrm{E}+00$ & $2.08 \mathrm{E}+00$ & $2.08 \mathrm{E}+00$ & $2.08 \mathrm{E}+00$ & $1.04 \mathrm{E}+01$ & $2.08 \mathrm{E}+01$ & $2.08 E+01$ & $6.07 E+01$ \\
\hline LBL & $6.24 \mathrm{E}-01$ & $0.00 \mathrm{E}+00$ & $0.00 \mathrm{E}+00$ & $0.00 E+00$ & $2.08 \mathrm{E}-01$ & $4.16 \mathrm{E}-01$ & $4.16 \mathrm{E}-01$ & $1.66 \mathrm{E}+00$ \\
\hline MURR & $2.08 \mathrm{E}-01$ & $2.08 \mathrm{E}-01$ & $2.08 \mathrm{E}-01$ & $2.08 \mathrm{E}-01$ & $2.08 \mathrm{E}-01$ & $0.00 \mathrm{E}+00$ & $0.00 \mathrm{E}+00$ & $1.04 \mathrm{E}+00$ \\
\hline PAD & $0.00 \mathrm{E}+00$ & $0.00 \mathrm{E}+00$ & $0.00 E+00$ & $0.00 \mathrm{E}+00$ & $0.00 \mathrm{E}+00$ & $0.00 \mathrm{E}+00$ & $3.78 \mathrm{E}+00$ & $3.78 \mathrm{E}+00$ \\
\hline PANT & $6.24 \mathrm{E}-01$ & $0.00 \mathrm{E}+00$ & $0.00 \mathrm{E}+00$ & $0.00 \mathrm{E}+00$ & $0.00 \mathrm{E}+00$ & $0.00 \mathrm{E}+00$ & $0.00 \mathrm{E}+00$ & $6.24 \mathrm{E}-01$ \\
\hline SNL/NM & $6.66 \mathrm{E}+00$ & $2.08 \mathrm{E}-01$ & $1.87 \mathrm{E}+00$ & $2.08 \mathrm{E}-01$ & $1.04 \mathrm{E}+00$ & $2.08 \mathrm{E}+00$ & $2.08 E+00$ & $1.41 \mathrm{E}+01$ \\
\hline TBE & $2.08 \mathrm{E}-01$ & $0.00 \mathrm{E}+00$ & $0.00 \mathrm{E}+00$ & $0.00 \mathrm{E}+00$ & $0.00 \mathrm{E}+00$ & $0.00 \mathrm{E}+00$ & $0.00 E+00$ & $2.08 \mathrm{E}-01$ \\
\hline USAMC & $2.50 \mathrm{E}+00$ & $0.00 \mathrm{E}+00$ & $0.00 \mathrm{E}+00$ & $0.00 \mathrm{E}+00$ & $0.00 \mathrm{E}+00$ & $0.00 \mathrm{E}+00$ & $0.00 \mathrm{E}+00$ & $2.50 \mathrm{E}+00$ \\
\hline Subtotal & $6.28 \mathrm{E}+02$ & $4.25 \mathrm{E}+00$ & $3.19 \mathrm{E}+01$ & $2.13 \mathrm{E}+01$ & $6.37 \mathrm{E}+02$ & $8.19 \mathrm{E}+02$ & $6.95 \mathrm{E}+02$ & $2.84 \mathrm{E}+03$ \\
\hline
\end{tabular}




\section{FINAL WASTE FORM}

Table 3.8 (continued)

\begin{tabular}{|c|c|c|c|c|c|c|c|c|}
\hline \multirow{2}{*}{ Site } & \multirow{2}{*}{$\begin{array}{l}\text { End of } \\
\text { CY } 1994 \\
\text { total }\end{array}$} & \multicolumn{3}{|c|}{ Annual increase } & \multicolumn{3}{|c|}{ Cumulative increase } & \multirow{2}{*}{$\begin{array}{c}\text { End of } \\
\text { CY } 2022 \\
\text { total }\end{array}$} \\
\hline & & 1995 & 1996 & 1997 & 1998--2002 & 2003-2012 & $2013-2022$ & \\
\hline \multicolumn{9}{|l|}{ Commercial site } \\
\hline WVDP & $3.42 \mathrm{E}+02$ & $1.78 E+01$ & $4.18 \mathrm{E}+00$ & $4.18 \mathrm{E}+00$ & $5.38 \mathrm{E}+01$ & $4.18 \mathrm{E}+01$ & $4.18 \mathrm{E}+01$ & $5.06 \mathrm{E}+02$ \\
\hline $\begin{array}{l}\text { Total } \\
\text { (all sites) }\end{array}$ & $6.21 E+04$ & $9.01 E+02$ & $6.02 \mathrm{E}+02$ & $5.09 \mathrm{E}+02$ & $4.77 \mathrm{E}+03$ & $2.54 \mathrm{E}+04$ & $4.48 \mathrm{E}+04$ & $1.39 \mathrm{E}+05$ \\
\hline
\end{tabular}


FINAL WASTE FORM

Table 3.9. Summary of final waste-form volumes $\left(\mathrm{m}^{3}\right)$ of retrievably stored and projected mixed and nonmixed CH TRUW

\begin{tabular}{|c|c|c|c|c|c|c|c|c|}
\hline \multirow{2}{*}{ Site } & \multirow{2}{*}{$\begin{array}{c}\text { End of } \\
\text { CY } 1994 \\
\text { total }\end{array}$} & \multicolumn{3}{|c|}{ Annual increase } & \multicolumn{3}{|c|}{ Cumulative increase } & \multirow{2}{*}{$\begin{array}{l}\text { End of } \\
\text { CY } 2022 \\
\text { total }\end{array}$} \\
\hline & & 1995 & 1996 & 1997 & $1998-2002$ & 2003-2012 & $2013-2022$ & \\
\hline \multicolumn{9}{|l|}{ Major sites } \\
\hline ANL-E & $2.48 \mathrm{E}+01$ & $0.00 \mathrm{E}+00$ & $4.20 \mathrm{E}+01$ & $3.33 \mathrm{E}+00$ & $1.79 \mathrm{E}+01$ & $3.33 \mathrm{E}+01$ & $3.33 E+01$ & $1.55 \mathrm{E}+02$ \\
\hline Hanford & $1.24 \mathrm{E}+04$ & $3.85 \mathrm{E}+02$ & $1.15 \mathrm{E}+02$ & $1.20 \mathrm{E}+02$ & $2.04 \mathrm{E}+03$ & $1.52 \mathrm{E}+04$ & $1.56 \mathrm{E}+04$ & $4.58 \mathrm{E}+04$ \\
\hline INEL & $2.86 \mathrm{E}+04$ & $0.00 \mathrm{E}+00$ & $0.00 \mathrm{E}+00$ & $0.00 \mathrm{E}+00$ & $0.00 \mathrm{E}+00$ & $0.00 \mathrm{E}+00$ & $0.00 \mathrm{E}+00$ & $2.86 \mathrm{E}+04$ \\
\hline LANL & $1.11 \mathrm{E}+04$ & $2.62 \mathrm{E}+02$ & $2.62 \mathrm{E}+02$ & $2.62 \mathrm{E}+02$ & $1.31 \mathrm{E}+03$ & $2.62 \mathrm{E}+03$ & $2.63 \mathrm{E}+03$ & $1.84 \mathrm{E}+04$ \\
\hline LLNL & $2.30 \mathrm{E}+02$ & $3.74 \mathrm{E}+01$ & $2.40 \mathrm{E}+01$ & $2.59 \mathrm{E}+01$ & $1.24 \mathrm{E}+02$ & $2.50 \mathrm{E}+02$ & $2.50 \mathrm{E}+02$ & $9.41 \mathrm{E}+02$ \\
\hline Mound & $3.20 \mathrm{E}+02$ & $0.00 \mathrm{E}+00$ & $0.00 \mathrm{E}+00$ & $0.00 \mathrm{E}+00$ & $0.00 \mathrm{E}+00$ & $0.00 \mathrm{E}+00$ & $0.00 \mathrm{E}+00$ & $3.20 \mathrm{E}+02$ \\
\hline NTS & $6.19 \mathrm{E}+02$ & $0.00 \mathrm{E}+00$ & $0.00 \mathrm{E}+00$ & $0.00 \mathrm{E}+00$ & $0.00 \mathrm{E}+00$ & $6.21 E+00$ & $2.77 \mathrm{E}+00$ & $6.28 \mathrm{E}+02$ \\
\hline ORNL & $1.33 E+03$ & $9.15 E+00$ & $9.15 \mathrm{E}+00$ & $9.15 \mathrm{E}+00$ & $4.58 \mathrm{E}+01$ & $9.15 \mathrm{E}+01$ & $9.15 \mathrm{E}+01$ & $1.58 \mathrm{E}+03$ \\
\hline RFETS & $7.08 \mathrm{E}+02$ & $1.05 \mathrm{E}+02$ & $8.96 \mathrm{E}+01$ & $3.24 \mathrm{E}+01$ & $1.61 \mathrm{E}+02$ & $1.75 E+03$ & $1.77 E+03$ & $4.61 \mathrm{E}+03$ \\
\hline SRS & $2.88 \mathrm{E}+03$ & $2.50 \mathrm{E}-01$ & $2.50 \mathrm{E}-01$ & $2.50 \mathrm{E}-01$ & $1.25 \mathrm{E}+00$ & $2.50 \mathrm{E}+00$ & $6.76 \mathrm{E}+03$ & $9.65 \mathrm{E}+03$ \\
\hline Subtotal & $5.81 E+04$ & $7.99 \mathrm{E}+02$ & $5.42 \mathrm{E}+02$ & $4.53 \mathrm{E}+02$ & $3.70 \mathrm{E}+03$ & $1.99 \mathrm{E}+04$ & $2.71 E+04$ & $1.11 E+05$ \\
\hline \multicolumn{9}{|c|}{ Small-quantity sites } \\
\hline Ames & $0.00 \mathrm{E}+00$ & $0.00 \mathrm{E}+00$ & $0.00 \mathrm{E}+00$ & $0.00 \mathrm{E}+00$ & $0.00 \mathrm{E}+00$ & $4.16 \mathrm{E}-01$ & $0.00 \mathrm{E}+00$ & $4.16 E-01$ \\
\hline ANL-W & $6.54 \mathrm{E}+00$ & $0.00 \mathrm{E}+00$ & $0.00 \mathrm{E}+00$ & $0.00 \mathrm{E}+00$ & $2.14 \mathrm{E}+02$ & $2.85 \mathrm{E}+02$ & $2.42 \mathrm{E}+02$ & $7.47 \mathrm{E}+02$ \\
\hline $\mathrm{ARCO}$ & $0.00 \mathrm{E}+00$ & $0.00 \mathrm{E}+00$ & $0.00 \mathrm{E}+00$ & $0.00 \mathrm{E}+00$ & $4.16 \mathrm{E}-01$ & $0.00 \mathrm{E}+00$ & $0.00 \mathrm{E}+00$ & $4.16 \mathrm{E}-01$ \\
\hline BAPL & $0.00 \mathrm{E}+00$ & $4.16 \mathrm{E}-01$ & $2.65 E+01$ & $1.70 \mathrm{E}+01$ & $7.56 \mathrm{E}+01$ & $3.78 \mathrm{E}+00$ & $0.00 \mathrm{E}+00$ & $1.23 \mathrm{E}+02$ \\
\hline $\mathrm{BCL}$ & $0.00 \mathrm{E}+00$ & $0.00 \mathrm{E}+00$ & $0.00 \mathrm{E}+00$ & $0.00 \mathrm{E}+00$ & $0.00 \mathrm{E}+00$ & $0.00 \mathrm{E}+00$ & $0.00 \mathrm{E}+00$ & $0.00 \mathrm{E}+00$ \\
\hline ETEC & $1.68 \mathrm{E}+00$ & $0.00 \mathrm{E}+00$ & $0.00 \mathrm{E}+00$ & $0.00 \mathrm{E}+00$ & $0.00 \mathrm{E}+00$ & $0.00 \mathrm{E}+00$ & $0.00 \mathrm{E}+00$ & $1.68 \mathrm{E}+00$ \\
\hline KAPL & $0.00 \mathrm{E}+00$ & $0.00 \mathrm{E}+00$ & $0.00 \mathrm{E}+00$ & $0.00 \mathrm{E}+00$ & $0.00 \mathrm{E}+00$ & $0.00 \mathrm{E}+00$ & $0.00 \mathrm{E}+00$ & $0.00 \mathrm{E}+00$ \\
\hline LBL & $6.24 \mathrm{E}-01$ & $0.00 \mathrm{E}+00$ & $0.00 \mathrm{E}+00$ & $0.00 \mathrm{E}+00$ & $2.08 \mathrm{E}-01$ & $4.16 E-01$ & $4.16 \mathrm{E}-01$ & $1.66 \mathrm{E}+00$ \\
\hline MURR & $2.08 \mathrm{E}-01$ & $2.08 \mathrm{E}-01$ & $2.08 \mathrm{E}-01$ & $2.08 \mathrm{E}-01$ & $2.08 \mathrm{E}-01$ & $0.00 \mathrm{E}+00$ & $0.00 \mathrm{E}+00$ & $1.04 \mathrm{E}+00$ \\
\hline PAD & $0.00 \mathrm{E}+00$ & $0.00 \mathrm{E}+00$ & $0.00 \mathrm{E}+00$ & $0.00 \mathrm{E}+00$ & $0.00 \mathrm{E}+00$ & $0.00 \mathrm{E}+00$ & $3.78 E+00$ & $3.78 \mathrm{E}+00$ \\
\hline PANT & $6.24 \mathrm{E}-01$ & $0.00 \mathrm{E}+00$ & $0.00 \mathrm{E}+00$ & $0.00 \mathrm{E}+00$ & $0.00 \mathrm{E}+00$ & $0.00 \mathrm{E}+00$ & $0.00 \mathrm{E}+00$ & $6.24 \mathrm{E}-01$ \\
\hline SNL/NM & $6.66 \mathrm{E}+00$ & $2.08 \mathrm{E}-01$ & $1.87 \mathrm{E}+00$ & $2.08 \mathrm{E}-01$ & $1.04 \mathrm{E}+00$ & $2.08 \mathrm{E}+00$ & $2.08 \mathrm{E}+00$ & $1.41 E+01$ \\
\hline TBE & $2.08 \mathrm{E}-01$ & $0.00 \mathrm{E}+00$ & $0.00 \mathrm{E}+00$ & $0.00 \mathrm{E}+00$ & $0.00 \mathrm{E}+00$ & $0.00 \mathrm{E}+00$ & $0.00 \mathrm{E}+00$ & $2.08 \mathrm{E}-01$ \\
\hline USAMC & $2.50 \mathrm{E}+00$ & $0.00 \mathrm{E}+00$ & $0.00 \mathrm{E}+00$ & $0.00 \mathrm{E}+00$ & $0.00 \mathrm{E}+00$ & $0.00 \mathrm{E}+00$ & $0.00 \mathrm{E}+00$ & $2.50 \mathrm{E}+00$ \\
\hline Subtotal & $1.90 \mathrm{E}+01$ & $8.32 E-01$ & $2.85 \mathrm{E}+01$ & $1.74 \mathrm{E}+01$ & $2.91 E+02$ & $2.91 \mathrm{E}+02$ & $2.49 \mathrm{E}+02$ & $8.97 \mathrm{E}+02$ \\
\hline
\end{tabular}




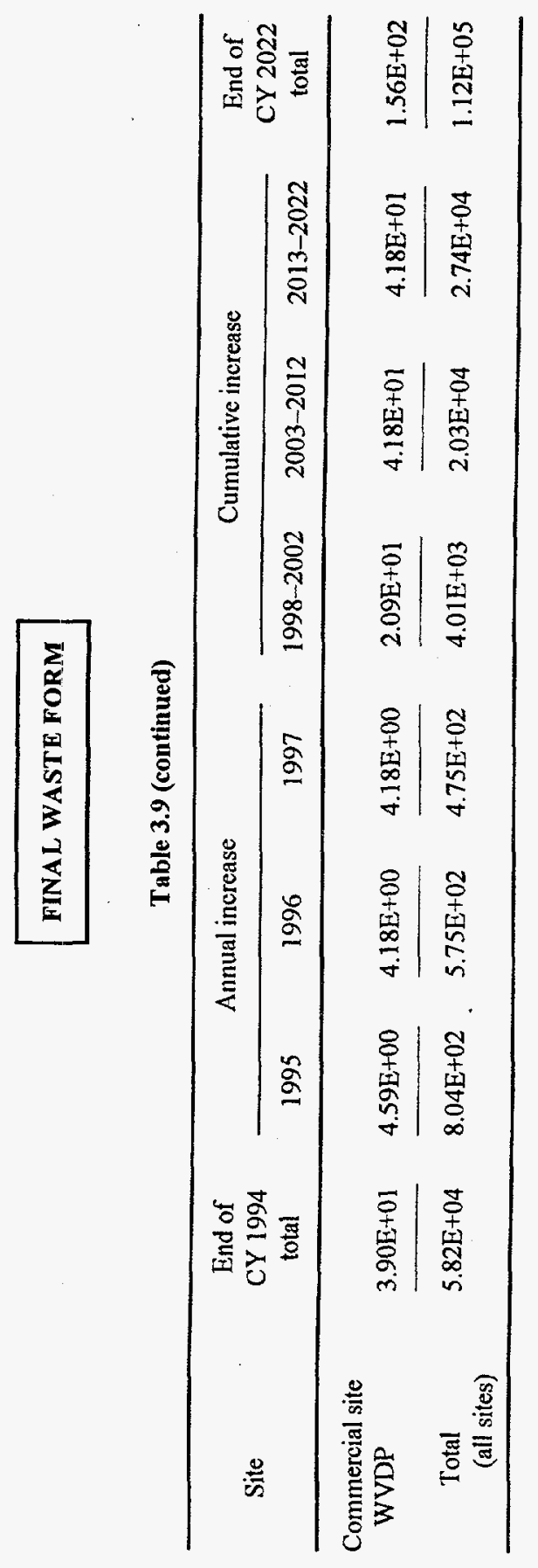


FINAL WASTE FORM

Table 3.10. Final waste-form volumes $\left(\mathrm{m}^{3}\right)$ of retrievably stored and projected mixed $\mathrm{CH}$ TRUW

\begin{tabular}{|c|c|c|c|c|c|c|c|c|}
\hline \multirow{2}{*}{ Site } & \multirow{2}{*}{$\begin{array}{l}\text { End of } \\
\text { CY } 1994 \\
\text { total }\end{array}$} & \multicolumn{3}{|c|}{ Annual increase } & \multicolumn{3}{|c|}{ Cumulative increase } & \multirow{2}{*}{$\begin{array}{c}\text { End of } \\
\text { CY } 2022 \\
\text { total }\end{array}$} \\
\hline & & 1995 & 1996 & 1997 & $1998-2002$ & 2003--2012 & $2013-2022$ & \\
\hline \multicolumn{9}{|l|}{ Major sites } \\
\hline ANL-E & $6.48 \mathrm{E}+00$ & $0.00 \mathrm{E}+00$ & $0.00 \mathrm{E}+00$ & $0.00 \mathrm{E}+00$ & $1.29 \mathrm{E}+00$ & $0.00 \mathrm{E}+00$ & $0.00 \mathrm{E}+00$ & $7.77 \mathrm{E}+00$ \\
\hline Hanford & $2.78 \mathrm{E}+02$ & $2.60 \mathrm{E}+01$ & $2.53 \mathrm{E}+01$ & $3.18 \mathrm{E}+01$ & $1.84 \mathrm{E}+02$ & $6.19 \mathrm{E}+03$ & $7.09 \mathrm{E}+03$ & $1.38 \mathrm{E}+04$ \\
\hline INEL & $2.33 \mathrm{E}+04$ & $0.00 \mathrm{E}+00$ & $0.00 \mathrm{E}+00$ & $0.00 \mathrm{E}+00$ & $0.00 \mathrm{E}+00$ & $0.00 \mathrm{E}+00$ & $0.00 \mathrm{E}+00$ & $2.33 E+04$ \\
\hline LANL & $7.71 \mathrm{E}+03$ & $1.35 E+02$ & $1.35 E+02$ & $1.35 E+02$ & $6.73 E+02$ & $1.35 \mathrm{E}+03$ & $1.35 \mathrm{E}+03$ & $1.15 \mathrm{E}+04$ \\
\hline LLNL & $8.34 \mathrm{E}+00$ & $2.72 \mathrm{E}+00$ & $8.32 \mathrm{E}-01$ & $2.72 \mathrm{E}+00$ & $7.94 \mathrm{E}+00$ & $1.78 \mathrm{E}+01$ & $1.76 \mathrm{E}+01$ & $5.79 \mathrm{E}+01$ \\
\hline Mound & $3.54 \mathrm{E}+00$ & $0.00 \mathrm{E}+00$ & $0.00 \mathrm{E}+00$ & $0.00 \mathrm{E}+00$ & $0.00 \mathrm{E}+00$ & $0.00 \mathrm{E}+00$ & $0.00 \mathrm{E}+00$ & $3.54 \mathrm{E}+00$ \\
\hline NTS & $6.13 E+02$ & $0.00 \mathrm{E}+00$ & $0.00 \mathrm{E}+00$ & $0.00 \mathrm{E}+00$ & $0.00 \mathrm{E}+00$ & $6.21 \mathrm{E}+00$ & $2.77 \mathrm{E}+00$ & $6.22 \mathrm{E}+02$ \\
\hline ORNL & $6.99 \mathrm{E}+02$ & $9.15 \mathrm{E}+00$ & $9.15 \mathrm{E}+00$ & $9.15 E+00$ & $4.58 \mathrm{E}+01$ & $9.15 \mathrm{E}+01$ & $9.15 \mathrm{E}+01$ & $9.55 \mathrm{E}+02$ \\
\hline RFETS & $4.37 \mathrm{E}+02$ & $6.41 \mathrm{E}+01$ & $5.37 \mathrm{E}+01$ & $2.37 \mathrm{E}+01$ & $1.23 E+02$ & $1.32 \mathrm{E}+03$ & $1.31 \mathrm{E}+03$ & $3.34 \mathrm{E}+03$ \\
\hline SRS & $1.48 \mathrm{E}+03$ & $0.00 \mathrm{E}+00$ & $0.00 \mathrm{E}+00$ & $0.00 \mathrm{E}+00$ & $0.00 \mathrm{E}+00$ & $0.00 \mathrm{E}+00$ & $2.87 \mathrm{E}+03$ & $4.35 E+03$ \\
\hline Subtotal & $3.45 \mathrm{E}+04$ & $2.37 \mathrm{E}+02$ & $2.24 \mathrm{E}+02$ & $2.02 \mathrm{E}+02$ & $1.03 \mathrm{E}+03$ & $8.98 \mathrm{E}+03$ & $1.27 \mathrm{E}+04$ & $5.79 \mathrm{E}+04$ \\
\hline \multicolumn{9}{|c|}{ Small-quantity sites } \\
\hline Ames & $0.00 \mathrm{E}+00$ & $0.00 \mathrm{E}+00$ & $0.00 \mathrm{E}+00$ & $0.00 \mathrm{E}+00$ & $0.00 \mathrm{E}+00$ & $4.16 \mathrm{E}-01$ & $0.00 \mathrm{E}+00$ & $4.16 \mathrm{E}-01$ \\
\hline ANL $-W$ & $4.25 \mathrm{E}+00$ & $0.00 \mathrm{E}+00$ & $0.00 \mathrm{E}+00$ & $0.00 \mathrm{E}+00$ & $2.04 \mathrm{E}+00$ & $0.00 \mathrm{E}+00$ & $0.00 \mathrm{E}+00$ & $6.29 \mathrm{E}+00$ \\
\hline $\mathrm{ARCO}$ & $0.00 \mathrm{E}+00$ & $0.00 \mathrm{E}+00$ & $0.00 \mathrm{E}+00$ & $0.00 \mathrm{E}+00$ & $0.00 \mathrm{E}+00$ & $0.00 \mathrm{E}+00$ & $0.00 \mathrm{E}+00$ & $0.00 \mathrm{E}+00$ \\
\hline BAPL & $0.00 \mathrm{E}+00$ & $0.00 \mathrm{E}+00$ & $0.00 \mathrm{E}+00$ & $0.00 \mathrm{E}+00$ & $0.00 \mathrm{E}+00$ & $0.00 \mathrm{E}+00$ & $0.00 \mathrm{E}+00$ & $0.00 \mathrm{E}+00$ \\
\hline $\mathrm{BCL}$ & $0.00 \mathrm{E}+00$ & $0.00 \mathrm{E}+00$ & $0.00 \mathrm{E}+00$ & $0.00 \mathrm{E}+00$ & $0.00 \mathrm{E}+00$ & $0.00 \mathrm{E}+00$ & $0.00 \mathrm{E}+00$ & $0.00 \mathrm{E}+00$ \\
\hline ETEC & $0.00 \mathrm{E}+00$ & $0.00 \mathrm{E}+00$ & $0.00 \mathrm{E}+00$ & $0.00 \mathrm{E}+00$ & $0.00 \mathrm{E}+00$ & $0.00 \mathrm{E}+00$ & $0.00 \mathrm{E}+00$ & $0.00 \mathrm{E}+00$ \\
\hline KAPL & $0.00 \mathrm{E}+00$ & $0.00 \mathrm{E}+00$ & $0.00 \mathrm{E}+00$ & $0.00 \mathrm{E}+00$ & $0.00 \mathrm{E}+00$ & $0.00 \mathrm{E}+00$ & $0.00 \mathrm{E}+00$ & $0.00 \mathrm{E}+00$ \\
\hline LBL & $0.00 \mathrm{E}+00$ & $0.00 \mathrm{E}+00$ & $0.00 \mathrm{E}+00$ & $0.00 \mathrm{E}+00$ & $0.00 \mathrm{E}+00$ & $0.00 \mathrm{E}+00$ & $0.00 \mathrm{E}+00$ & $0.00 \mathrm{E}+00$ \\
\hline MURR & $2.08 \mathrm{E}-01$ & $2.08 \mathrm{E}-01$ & $2.08 \mathrm{E}-01$ & $2.08 E-01$ & $2.08 \mathrm{E}-01$ & $0.00 E+00$ & $0.00 \mathrm{E}+00$ & $1.04 \mathrm{E}+00$ \\
\hline $\mathrm{PAD}$ & $0.00 \mathrm{E}+00$ & $0.00 \mathrm{E}+00$ & $0.00 \mathrm{E}+00$ & $0.00 \mathrm{E}+00$ & $0.00 \mathrm{E}+00$ & $0.00 \mathrm{E}+00$ & $3.78 E+00$ & $3.78 \mathrm{E}+00$ \\
\hline PANT & $0.00 \mathrm{E}+00$ & $0.00 \mathrm{E}+00$ & $0.00 \mathrm{E}+00$ & $0.00 \mathrm{E}+00$ & $0.00 \mathrm{E}+00$ & $0.00 \mathrm{E}+00$ & $0.00 \mathrm{E}+00$ & $0.00 \mathrm{E}+00$ \\
\hline SNL/NM & $0.00 \mathrm{E}+00$ & $0.00 \mathrm{E}+00$ & $0.00 \mathrm{E}+00$ & $0.00 \mathrm{E}+00$ & $0.00 \mathrm{E}+00$ & $0.00 E+00$ & $0.00 \mathrm{E}+00$ & $0.00 \mathrm{E}+00$ \\
\hline TBE & $0.00 \mathrm{E}+00$ & $0.00 \mathrm{E}+00$ & $0.00 \mathrm{E}+00$ & $0.00 \mathrm{E}+00$ & $0.00 \mathrm{E}+00$ & $0.00 \mathrm{E}+00$ & $0.00 \mathrm{E}+00$ & $0.00 \mathrm{E}+00$ \\
\hline USAMC & $0.00 \mathrm{E}+00$ & $0.00 \mathrm{E}+00$ & $0.00 \mathrm{E}+00$ & $0.00 \mathrm{E}+00$ & $0.00 \mathrm{E}+00$ & $0.00 \mathrm{E}+00$ & $0.00 E+00$ & $0.00 \mathrm{E}+00$ \\
\hline Subtotal & $4.46 \mathrm{E}+00$ & $2.08 \mathrm{E}-01$ & $2.08 E-01$ & $2.08 \mathrm{E}-01$ & $2.25 \mathrm{E}+00$ & $4.16 \mathrm{E}-01$ & $3.78 \mathrm{E}+00$ & $1.15 \mathrm{E}+01$ \\
\hline
\end{tabular}




\section{FINAL WASTE FORM}

Table 3.10 (continued)

\begin{tabular}{|c|c|c|c|c|c|c|c|c|}
\hline \multirow{2}{*}{ Site } & \multirow{2}{*}{$\begin{array}{l}\text { End of } \\
\text { CY } 1994 \\
\text { total }\end{array}$} & \multicolumn{3}{|c|}{ Annual increase } & \multicolumn{3}{|c|}{ Cumulative increase } & \multirow{2}{*}{$\begin{array}{c}\text { End of } \\
\text { CY } 2022 \\
\text { total }\end{array}$} \\
\hline & & 1995 & 1996 & 1997 & $1998-2002$ & $2003-2012$ & 2013-2022 & \\
\hline \multicolumn{9}{|l|}{ Commercial site } \\
\hline WVDP & $1.39 E+01$ & $0.00 \mathrm{E}+00$ & $0.00 \mathrm{E}+00$ & $0.00 \mathrm{E}+00$ & $0.00 \mathrm{E}+00$ & $0.00 \mathrm{E}+00$ & $0.00 \mathrm{E}+00$ & $1.39 \mathrm{E}+01$ \\
\hline $\begin{array}{l}\text { Total } \\
\text { (all sites) }\end{array}$ & $3.45 \mathrm{E}+04$ & $2.37 \mathrm{E}+02$ & $2.24 \mathrm{E}+02$ & $2.02 \mathrm{E}+02$ & $1.04 \mathrm{E}+03$ & $8.98 \mathrm{E}+03$ & $1.27 \mathrm{E}+04$ & $5.79 \mathrm{E}+04$ \\
\hline
\end{tabular}


FINAL WASTE FORM

Table 3.11. Final waste-form volumes $\left(\mathrm{m}^{3}\right)$ of retrievably stored and projected nonmixed CH TRUW

\begin{tabular}{|c|c|c|c|c|c|c|c|c|}
\hline \multirow{2}{*}{ Site } & \multirow{2}{*}{$\begin{array}{l}\text { End of } \\
\text { CY } 1994 \\
\text { total }\end{array}$} & \multicolumn{3}{|c|}{ Annual increase } & \multicolumn{3}{|c|}{ Cumulative increase } & \multirow{2}{*}{$\begin{array}{c}\text { End of } \\
\text { CY } 2022 \\
\text { total }\end{array}$} \\
\hline & & 1995 & 1996 & 1997 & $1998-2002$ & $2003-2012$ & $2013-2022$ & \\
\hline \multicolumn{9}{|l|}{ Major sites } \\
\hline ANL-E & $1.83 \mathrm{E}+01$ & $0.00 \mathrm{E}+00$ & $4.20 \mathrm{E}+01$ & $3.33 E+00$ & $1.66 \mathrm{E}+01$ & $3.33 E+01$ & $3.33 \mathrm{E}+01$ & $1.47 \mathrm{E}+02$ \\
\hline Hanford & $1.21 \mathrm{E}+04$ & $3.59 \mathrm{E}+02$ & $8.95 \mathrm{E}+01$ & $8.78 \mathrm{E}+01$ & $1.85 \mathrm{E}+03$ & $8.97 E+03$ & $8.51 \mathrm{E}+03$ & $3.20 \mathrm{E}+04$ \\
\hline INEL & $5.33 E+03$ & $0.00 \mathrm{E}+00$ & $0.00 \mathrm{E}+00$ & $0.00 \mathrm{E}+00$ & $0.00 \mathrm{E}+00$ & $0.00 \mathrm{E}+00$ & $0.00 \mathrm{E}+00$ & $5.33 \mathrm{E}+03$ \\
\hline LANL & $3.34 \mathrm{E}+03$ & $1.28 \mathrm{E}+02$ & $1.28 \mathrm{E}+02$ & $1.28 \mathrm{E}+02$ & $6.40 E+02$ & $1.28 \mathrm{E}+03$ & $1.28 \mathrm{E}+03$ & $6.92 \mathrm{E}+03$ \\
\hline LLNL & $2.22 \mathrm{E}+02$ & $3.46 \mathrm{E}+01$ & $2.32 \mathrm{E}+01$ & $2.32 \mathrm{E}+01$ & $1.16 \mathrm{E}+02$ & $2.32 \mathrm{E}+02$ & $2.32 \mathrm{E}+02$ & $8.83 \mathrm{E}+02$ \\
\hline Mound & $3.17 \mathrm{E}+02$ & $0.00 \mathrm{E}+00$ & $0.00 \mathrm{E}+00$ & $0.00 \mathrm{E}+00$ & $0.00 \mathrm{E}+00$ & $0.00 \mathrm{E}+00$ & $0.00 \mathrm{E}+00$ & $3.17 \mathrm{E}+02$ \\
\hline NTS & $5.67 \mathrm{E}+00$ & $0.00 \mathrm{E}+00$ & $0.00 \mathrm{E}+00$ & $0.00 \mathrm{E}+00$ & $0.00 \mathrm{E}+00$ & $0.00 \mathrm{E}+00$ & $0.00 \mathrm{E}+00$ & $5.67 \mathrm{E}+00$ \\
\hline ORNL & $6.29 E+02$ & $0.00 \mathrm{E}+00$ & $0.00 \mathrm{E}+00$ & $0.00 \mathrm{E}+00$ & $0.00 \mathrm{E}+00$ & $0.00 \mathrm{E}+00$ & $0.00 \mathrm{E}+00$ & $6.29 E+02$ \\
\hline RFETS & $2.71 \mathrm{E}+02$ & 4.10E+01 & $3.60 \mathrm{E}+01$ & $8.68 \mathrm{E}+00$ & $3.77 E+01$ & $4.28 \mathrm{E}+02$ & $4.55 \mathrm{E}+02$ & $1.28 \mathrm{E}+03$ \\
\hline SRS & $1.40 \mathrm{E}+03$ & $2.50 \mathrm{E}-01$ & $2.50 \mathrm{E}-01$ & $2.50 \mathrm{E}-01$ & $1.25 \mathrm{E}+00$ & $2.50 \mathrm{E}+00$ & $3.89 \mathrm{E}+03$ & $5.30 \mathrm{E}+03$ \\
\hline Subtotal & $2.36 \mathrm{E}+04$ & $5.62 \mathrm{E}+02$ & $3.19 \mathrm{E}+02$ & $2.51 \mathrm{E}+02$ & $2.66 E+03$ & $1.09 \mathrm{E}+04$ & $1.44 \mathrm{E}+04$ & $5.28 \mathrm{E}+04$ \\
\hline \multicolumn{9}{|c|}{ Small-quantity sites } \\
\hline Ames & $0.00 \mathrm{E}+00$ & $0.00 \mathrm{E}+00$ & $0.00 \mathrm{E}+00$ & $0.00 \mathrm{E}+00$ & $0.00 \mathrm{E}+00$ & $0.00 \mathrm{E}+00$ & $0.00 \mathrm{E}+00$ & $0.00 \mathrm{E}+00$ \\
\hline ANL-W & $2.29 \mathrm{E}+00$ & $0.00 \mathrm{E}+00$ & $0.00 \mathrm{E}+00$ & $0.00 \mathrm{E}+00$ & $2.12 \mathrm{E}+02$ & $2.85 \mathrm{E}+02$ & $2.42 \mathrm{E}+02$ & $7.41 \mathrm{E}+02$ \\
\hline $\mathrm{ARCO}$ & $0.00 \mathrm{E}+00$ & $0.00 \mathrm{E}+00$ & $0.00 \mathrm{E}+00$ & $0.00 \mathrm{E}+00$ & $4.16 \mathrm{E}-01$ & $0.00 \mathrm{E}+00$ & $0.00 \mathrm{E}+00$ & $4.16 \mathrm{E}-01$ \\
\hline BAPL & $0.00 \mathrm{E}+00$ & $4.16 \mathrm{E}-01$ & $2.65 \mathrm{E}+01$ & $1.70 \mathrm{E}+01$ & $7.56 \mathrm{E}+01$ & $3.78 \mathrm{E}+00$ & $0.00 \mathrm{E}+00$ & $1.23 \mathrm{E}+02$ \\
\hline $\mathrm{BCL}$ & $0.00 \mathrm{E}+00$ & $0.00 \mathrm{E}+00$ & $0.00 \mathrm{E}+00$ & $0.00 \mathrm{E}+00$ & $0.00 \mathrm{E}+00$ & $0.00 \mathrm{E}+00$ & $0.00 \mathrm{E}+00$ & $0.00 \mathrm{E}+00$ \\
\hline ETEC & $1.68 \mathrm{E}+00$ & $0.00 \mathrm{E}+00$ & $0.00 \mathrm{E}+00$ & $0.00 \mathrm{E}+00$ & $0.00 \mathrm{E}+00$ & $0.00 \mathrm{E}+00$ & $0.00 \mathrm{E}+00$ & $1.68 \mathrm{E}+00$ \\
\hline KAPL & $0.00 \mathrm{E}+00$ & $0.00 \mathrm{E}+00$ & $0.00 \mathrm{E}+00$ & $0.00 \mathrm{E}+00$ & $0.00 \mathrm{E}+00$ & $0.00 \mathrm{E}+00$ & $0.00 \mathrm{E}+00$ & $0.00 \mathrm{E}+00$ \\
\hline LBL & $6.24 \mathrm{E}-01$ & $0.00 \mathrm{E}+00$ & $0.00 \mathrm{E}+00$ & $0.00 \mathrm{E}+00$ & $2.08 \mathrm{E}-01$ & $4.16 \mathrm{E}-01$ & $4.16 \mathrm{E}-01$ & $1.66 \mathrm{E}+00$ \\
\hline MURR & $0.00 \mathrm{E}+00$ & $0.00 \mathrm{E}+00$ & $0.00 \mathrm{E}+00$ & $0.00 \mathrm{E}+00$ & $0.00 \mathrm{E}+00$ & $0.00 \mathrm{E}+00$ & $0.00 \mathrm{E}+00$ & $0.00 \mathrm{E}+00$ \\
\hline PAD & $0.00 \mathrm{E}+00$ & $0.00 \mathrm{E}+00$ & $0.00 \mathrm{E}+00$ & $0.00 \mathrm{E}+00$ & $0.00 \mathrm{E}+00$ & $0.00 \mathrm{E}+00$ & $0.00 E+00$ & $0.00 \mathrm{E}+00$ \\
\hline PANT & $6.24 \mathrm{E}-01$ & $0.00 \mathrm{E}+00$ & $0.00 \mathrm{E}+00$ & $0.00 \mathrm{E}+00$ & $0.00 E+00$ & $0.00 \mathrm{E}+00$ & $0.00 \mathrm{E}+00$ & $6.24 \mathrm{E}-01$ \\
\hline SNL/NM & $6.66 \mathrm{E}+00$ & $2.08 \mathrm{E}-01$ & $1.87 \mathrm{E}+00$ & $2.08 E-01$ & $1.04 \mathrm{E}+00$ & $2.08 \mathrm{E}+00$ & $2.08 \mathrm{E}+00$ & $1.41 \mathrm{E}+01$ \\
\hline TBE & $2.08 \mathrm{E}-01$ & $0.00 \mathrm{E}+00$ & $0.00 \mathrm{E}+00$ & $0.00 \mathrm{E}+00$ & $0.00 \mathrm{E}+00$ & $0.00 \mathrm{E}+00$ & $0.00 \mathrm{E}+00$ & $2.08 \mathrm{E}-01$ \\
\hline USAMC & $2.50 \mathrm{E}+00$ & $0.00 \mathrm{E}+00$ & $0.00 \mathrm{E}+00$ & $0.00 \mathrm{E}+00$ & $0.00 \mathrm{E}+00$ & $0.00 \mathrm{E}+00$ & $0.00 \mathrm{E}+00$ & $2.50 \mathrm{E}+00$ \\
\hline Subtotal & $1.46 \mathrm{E}+01$ & $6.24 \mathrm{E}-01$ & $2.83 \mathrm{E}+01$ & $1.72 \mathrm{E}+01$ & $2.89 \mathrm{E}+02$ & $2.91 \mathrm{E}+02$ & $2.45 \mathrm{E}+02$ & $8.86 \mathrm{E}+02$ \\
\hline
\end{tabular}




\begin{tabular}{|c|c|c|c|c|c|c|c|c|}
\hline & & & FINA & WASTE FC & & & & \\
\hline & & & Tabl & 11 (contim & & & & \\
\hline & End of & & nnual increa & & & mulative incre & & End of \\
\hline & total & 1995 & 1996 & 1997 & $1998-2002$ & $2003-2012$ & $2013-2022$ & total \\
\hline $\begin{array}{l}\text { Commercial site } \\
\text { WVDP }\end{array}$ & $2.50 \mathrm{E}+01$ & $4.59 \mathrm{E}+00$ & $4.18 \mathrm{E}+00$ & $4.18 \mathrm{E}+00$ & $2.09 \mathrm{E}+01$ & $4.18 \mathrm{E}+01$ & $4.18 \mathrm{E}+01$ & $1.42 \mathrm{E}+02$ \\
\hline $\begin{array}{l}\text { Total } \\
\text { (all sites) }\end{array}$ & $2.37 E+04$ & $5.68 \mathrm{E}+02$ & $3.51 \mathrm{E}+02$ & $2.73 E+02$ & $2.97 \mathrm{E}+03$ & $1.13 \mathrm{E}+04$ & $1.47 \mathrm{E}+04$ & $5.38 \mathrm{E}+04$ \\
\hline
\end{tabular}


FINAL WASTE FORM

Table 3.12. Summary of final waste-form volumes $\left(\mathrm{m}^{3}\right)$ of retrievably stored and projected mixed and nonmixed RH TRUW

\begin{tabular}{|c|c|c|c|c|c|c|c|c|}
\hline \multirow{2}{*}{ Site } & \multirow{2}{*}{$\begin{array}{l}\text { End of } \\
\text { CY } 1994 \\
\text { total }\end{array}$} & \multicolumn{3}{|c|}{ Annual increase } & \multicolumn{3}{|c|}{ Cumulative increase } & \multirow{2}{*}{$\begin{array}{l}\text { End of } \\
\text { CY } 2022 \\
\text { total }\end{array}$} \\
\hline & & 1995 & 1996 & 1997 & 1998-2002 & 2003-2012 & 2013-2022 & \\
\hline \multicolumn{9}{|l|}{ Major sites } \\
\hline ANL-E & $0.00 \mathrm{E}+00$ & $0.00 \mathrm{E}+00$ & $0.00 \mathrm{E}+00$ & $0.00 \mathrm{E}+00$ & $0.00 \mathrm{E}+00$ & $0.00 \mathrm{E}+00$ & $0.00 \mathrm{E}+00$ & $0.00 \mathrm{E}+00$ \\
\hline Hanford & $2.02 \mathrm{E}+02$ & $7.21 \mathrm{E}+01$ & $4.45 \mathrm{E}+00$ & $1.16 \mathrm{E}+01$ & $2.84 \mathrm{E}+02$ & $4.44 \mathrm{E}+03$ & $1.67 \mathrm{E}+04$ & $2.17 \mathrm{E}+04$ \\
\hline INEL & $2.21 \mathrm{E}+02$ & $0.00 \mathrm{E}+00$ & $0.00 \mathrm{E}+00$ & $0.00 \mathrm{E}+00$ & $0.00 \mathrm{E}+00$ & $0.00 \mathrm{E}+00$ & $0.00 \mathrm{E}+00$ & $2.21 E+02$ \\
\hline LANL & $9.43 \mathrm{E}+01$ & $8.90 \mathrm{E}-01$ & $2.67 \mathrm{E}+00$ & $2.67 \mathrm{E}+00$ & $1.33 \mathrm{E}+01$ & $3.38 \mathrm{E}+01$ & $4.54 \mathrm{E}+01$ & $1.93 \mathrm{E}+02$ \\
\hline LLNL & $0.00 \mathrm{E}+00$ & $0.00 \mathrm{E}+00$ & $0.00 \mathrm{E}+00$ & $0.00 \mathrm{E}+00$ & $0.00 \mathrm{E}+00$ & $0.00 \mathrm{E}+00$ & $0.00 \mathrm{E}+00$ & $0.00 \mathrm{E}+00$ \\
\hline Mound & $0.00 \mathrm{E}+00$ & $0.00 \mathrm{E}+00$ & $0.00 \mathrm{E}+00$ & $0.00 \mathrm{E}+00$ & $0.00 \mathrm{E}+00$ & $0.00 \mathrm{E}+00$ & $0.00 \mathrm{E}+00$ & $0.00 \mathrm{E}+00$ \\
\hline NTS & $0.00 \mathrm{E}+00$ & $0.00 E+00$ & $0.00 \mathrm{E}+00$ & $0.00 \mathrm{E}+00$ & $0.00 \mathrm{E}+00$ & $0.00 \mathrm{E}+00$ & $0.00 \mathrm{E}+00$ & $0.00 \mathrm{E}+00$ \\
\hline ORNL & $2.47 \mathrm{E}+03$ & $7.39 \mathrm{E}+00$ & $1.63 \mathrm{E}+01$ & $1.63 E+01$ & $8.14 \mathrm{E}+01$ & $1.63 E+02$ & $1.63 E+02$ & $2.92 \mathrm{E}+03$ \\
\hline RFETS & $0.00 \mathrm{E}+00$ & $0.00 \mathrm{E}+00$ & $0.00 \mathrm{E}+00$ & $0.00 \mathrm{E}+00$ & $0.00 \mathrm{E}+00$ & $0.00 \mathrm{E}+00$ & $0.00 \mathrm{E}+00$ & $0.00 \mathrm{E}+00$ \\
\hline SRS & $0.00 \mathrm{E}+00$ & $0.00 \mathrm{E}+00$ & $0.00 \mathrm{E}+00$ & $0.00 \mathrm{E}+00$ & $0.00 \mathrm{E}+00$ & $0.00 \mathrm{E}+00$ & $0.00 \mathrm{E}+00$ & $0.00 \mathrm{E}+00$ \\
\hline Subtotal & $2.99 \mathrm{E}+03$ & $8.04 E+01$ & $2.34 \mathrm{E}+01$ & $3.05 \mathrm{E}+01$ & $3.79 \mathrm{E}+02$ & $4.64 \mathrm{E}+03$ & $1.69 \mathrm{E}+04$ & $2.51 E+04$ \\
\hline \multicolumn{9}{|c|}{ Small-quantity sites } \\
\hline Ames & $0.00 \mathrm{E}+00$ & $0.00 \mathrm{E}+00$ & $0.00 \mathrm{E}+00$ & $0.00 \mathrm{E}+00$ & $0.00 \mathrm{E}+00$ & $0.00 \mathrm{E}+00$ & $0.00 \mathrm{E}+00$ & $0.00 \mathrm{E}+00$ \\
\hline ANL, W & $1.93 E+01$ & $0.00 \mathrm{E}+00$ & $0.00 \mathrm{E}+00$ & $0.00 \mathrm{E}+00$ & $3.33 \mathrm{E}+02$ & $5.06 \mathrm{E}+02$ & $4.25 \mathrm{E}+02$ & $1.28 \mathrm{E}+03$ \\
\hline ARCO & $0.00 \mathrm{E}+00$ & $0.00 \mathrm{E}+00$ & $0.00 \mathrm{E}+00$ & $0.00 \mathrm{E}+00$ & $0.00 \mathrm{E}+00$ & $0.00 \mathrm{E}+00$ & $0.00 \mathrm{E}+00$ & $0.00 \mathrm{E}+00$ \\
\hline BAPL & $0.00 \mathrm{E}+00$ & $1.33 \mathrm{E}+00$ & $8.90 \mathrm{E}-01$ & $1.33 \mathrm{E}+00$ & $2.67 \mathrm{E}+00$ & $4.45 \mathrm{E}-01$ & $0.00 \mathrm{E}+00$ & $6.67 \mathrm{E}+00$ \\
\hline $\mathrm{BCL}$ & $5.81 \mathrm{E}+02$ & $0.00 \mathrm{E}+00$ & $0.00 E+00$ & $0.00 \mathrm{E}+00$ & $0.00 \mathrm{E}+00$ & $0.00 \mathrm{E}+00$ & $0.00 \mathrm{E}+00$ & $5.81 E+02$ \\
\hline ETEC & $6.30 \mathrm{E}+00$ & $0.00 \mathrm{E}+00$ & $4.16 \mathrm{E}-01$ & $4.16 \mathrm{E}-01$ & $0.00 \mathrm{E}+00$ & $0.00 \mathrm{E}+00$ & $0.00 \mathrm{E}+00$ & $7.13 \mathrm{E}+00$ \\
\hline KAPL & $2.50 \mathrm{E}+00$ & $2.08 \mathrm{E}+00$ & $2.08 \mathrm{E}+00$ & $2.08 \mathrm{E}+00$ & $1.04 \mathrm{E}+01$ & $2.08 \mathrm{E}+01$ & $2.08 \mathrm{E}+01$ & $6.07 \mathrm{E}+01$ \\
\hline LBL & $0.00 \mathrm{E}+00$ & $0.00 \mathrm{E}+00$ & $0.00 \mathrm{E}+00$ & $0.00 \mathrm{E}+00$ & $0.00 \mathrm{E}+00$ & $0.00 \mathrm{E}+00$ & $0.00 \mathrm{E}+00$ & $0.00 \mathrm{E}+00$ \\
\hline MURR & $0.00 \mathrm{E}+00$ & $0.00 \mathrm{E}+00$ & $0.00 \mathrm{E}+00$ & $0.00 \mathrm{E}+00$ & $0.00 \mathrm{E}+00$ & $0.00 \mathrm{E}+00$ & $0.00 \mathrm{E}+00$ & $0.00 \mathrm{E}+00$ \\
\hline PAD & $0.00 \mathrm{E}+00$ & $0.00 \mathrm{E}+00$ & $0.00 \mathrm{E}+00$ & $0.00 \mathrm{E}+00$ & $0.00 \mathrm{E}+00$ & $0.00 \mathrm{E}+00$ & $0.00 \mathrm{E}+00$ & $0.00 \mathrm{E}+00$ \\
\hline PANT & $0.00 \mathrm{E}+00$ & $0.00 \mathrm{E}+00$ & $0.00 \mathrm{E}+00$ & $0.00 \mathrm{E}+00$ & $0.00 \mathrm{E}+00$ & $0.00 \mathrm{E}+00$ & $0.00 \mathrm{E}+00$ & $0.00 \mathrm{E}+00$ \\
\hline SNL/NM & $0.00 \mathrm{E}+00$ & $0.00 \mathrm{E}+00$ & $0.00 \mathrm{E}+00$ & $0.00 \mathrm{E}+00$ & $0.00 \mathrm{E}+00$ & $0.00 \mathrm{E}+00$ & $0.00 \mathrm{E}+00$ & $0.00 \mathrm{E}+00$ \\
\hline TBE & $0.00 \mathrm{E}+00$ & $0.00 \mathrm{E}+00$ & $0.00 \mathrm{E}+00$ & $0.00 \mathrm{E}+00$ & $0.00 \mathrm{E}+00$ & $0.00 \mathrm{E}+00$ & $0.00 \mathrm{E}+00$ & $0.00 \mathrm{E}+00$ \\
\hline USAMC & $0.00 \mathrm{E}+00$ & $0.00 \mathrm{E}+00$ & $0.00 \mathrm{E}+00$ & $0.00 \mathrm{E}+00$ & $0.00 \mathrm{E}+00$ & $0.00 \mathrm{E}+00$ & $0.00 \mathrm{E}+00$ & $0.00 \mathrm{E}+00$ \\
\hline Subtotal & $6.09 \mathrm{E}+02$ & $3.42 \mathrm{E}+00$ & 3.39E+00 & $3.83 \mathrm{E}+00$ & $3.46 \mathrm{E}+02$ & $5.28 \mathrm{E}+02$ & $4.46 \mathrm{E}+02$ & $1.94 E+03$ \\
\hline
\end{tabular}




\begin{tabular}{|c|c|c|c|c|c|c|c|c|}
\hline \multicolumn{9}{|c|}{ FINAL WASTE FORM } \\
\hline \multirow{3}{*}{ Site } & \multirow{3}{*}{$\begin{array}{l}\text { End of } \\
\text { CY } 1994 \\
\text { total }\end{array}$} & \multicolumn{6}{|c|}{ Table 3.12 (continued) } & \multirow{3}{*}{$\begin{array}{c}\text { End of } \\
\text { CY } 2022 \\
\text { total }\end{array}$} \\
\hline & & \multicolumn{3}{|c|}{ Annual increase } & \multicolumn{3}{|c|}{ Cumulative increase } & \\
\hline & & 1995 & 1996 & 1997 & $1998-2002$ & 2003-2012 & $2013-2022$ & \\
\hline $\begin{array}{l}\text { Commercial site } \\
\text { WVDP }\end{array}$ & $3.03 E+02$ & $1.32 \mathrm{E}+01$ & $0.00 \mathrm{E}+00$ & $0.00 \mathrm{E}+00$ & $3.29 \mathrm{E}+01$ & $0.00 \mathrm{E}+00$ & $0.00 \mathrm{E}+00$ & $3.50 \mathrm{E}+02$ \\
\hline $\begin{array}{l}\text { Total } \\
\text { (all sites) }\end{array}$ & $3.90 \mathrm{E}+03$ & $9.70 \mathrm{E}+01$ & $2.68 \mathrm{E}+01$ & $3.44 \mathrm{E}+01$ & $7.58 \mathrm{E}+02$ & $5.16 \mathrm{E}+03$ & $1.74 \mathrm{E}+04$ & $2.73 \mathrm{E}+04$ \\
\hline
\end{tabular}


FINAL WASTE FORM

Table 3.13. Fingl waste-form volumes $\left(\mathrm{m}^{3}\right)$ of retrievably stored and projected mixed RH TRUW

\begin{tabular}{|c|c|c|c|c|c|c|c|c|}
\hline \multirow{2}{*}{ Site } & \multirow{2}{*}{$\begin{array}{l}\text { End of } \\
\text { CY } 1994 \\
\text { total }\end{array}$} & \multicolumn{3}{|c|}{ Annual increase } & \multicolumn{3}{|c|}{ Cumulative increase } & \multirow{2}{*}{$\begin{array}{c}\text { End of } \\
\text { CY } 2022 \\
\text { total }\end{array}$} \\
\hline & & 1995 & 1996 & 1997 & $1998-2002$ & $2003-2012$ & $2013-2022$ & \\
\hline \multicolumn{9}{|l|}{ Major sites } \\
\hline ANL,-E & $0.00 \mathrm{E}+00$ & $0.00 \mathrm{E}+00$ & $0.00 \mathrm{E}+00$ & $0.00 \mathrm{E}+00$ & $0.00 \mathrm{E}+00$ & $0.00 \mathrm{E}+00$ & $0.00 \mathrm{E}+00$ & $0.00 \mathrm{E}+00$ \\
\hline Hanford & $2.67 \mathrm{E}+00$ & $4.45 \mathrm{E}+00$ & $4.45 \mathrm{E}+00$ & $1.16 \mathrm{E}+01$ & $2.84 \mathrm{E}+02$ & $4.08 \mathrm{E}+03$ & $1.09 E+04$ & $1.53 E+04$ \\
\hline INEL & $1.95 \mathrm{E}+02$ & $0.00 \mathrm{E}+00$ & $0.00 \mathrm{E}+00$ & $0.00 \mathrm{E}+00$ & $0.00 \mathrm{E}+00$ & $0.00 \mathrm{E}+00$ & $0.00 \mathrm{E}+00$ & $1.95 \mathrm{E}+02$ \\
\hline LANL & $1.69 E+01$ & $8.90 \mathrm{E}-01$ & $8.90 E-01$ & $8.90 \mathrm{E}-01$ & $4.45 E+00$ & $8.90 \mathrm{E}+00$ & $1.78 \mathrm{E}+01$ & $5.07 \mathrm{E}+01$ \\
\hline LLNL & $0.00 \mathrm{E}+00$ & $0.00 \mathrm{E}+00$ & $0.00 \mathrm{E}+00$ & $0.00 \mathrm{E}+00$ & $0.00 E+00$ & $0.00 \mathrm{E}+00$ & $0.00 \mathrm{E}+00$ & $0.00 \mathrm{E}+00$ \\
\hline Mound & $0.00 \mathrm{E}+00$ & $0.00 E+00$ & $0.00 \mathrm{E}+00$ & $0.00 \mathrm{E}+00$ & $0.00 \mathrm{E}+00$ & $0.00 \mathrm{E}+00$ & $0.00 \mathrm{E}+00$ & $0.00 \mathrm{E}+00$ \\
\hline NTS & $0.00 \mathrm{E}+00$ & $0.00 \mathrm{E}+00$ & $0.00 \mathrm{E}+00$ & $0.00 \mathrm{E}+00$ & $0.00 \mathrm{E}+00$ & $0.00 \mathrm{E}+00$ & $0.00 E+00$ & $0.00 \mathrm{E}+00$ \\
\hline ORNL & $2.38 E+03$ & $7.39 \mathrm{E}+00$ & $1.63 \mathrm{E}+01$ & $1.63 \mathrm{E}+01$ & $8.14 \mathrm{E}+01$ & $1.63 \mathrm{E}+02$ & $1.63 \mathrm{E}+02$ & $2.83 \mathrm{E}+03$ \\
\hline RFETS & $0.00 \mathrm{E}+00$ & $0.00 \mathrm{E}+00$ & $0.00 \mathrm{E}+00$ & $0.00 \mathrm{E}+00$ & $0.00 \mathrm{E}+00$ & $0.00 \mathrm{E}+00$ & $0.00 \mathrm{E}+00$ & $0.00 \mathrm{E}+00$ \\
\hline SRS & $0.00 \mathrm{E}+00$ & $0.00 \mathrm{E}+00$ & $0.00 \mathrm{E}+00$ & $0.00 \mathrm{E}+00$ & $0.00 \mathrm{E}+00$ & $0.00 \mathrm{E}+00$ & $0.00 \mathrm{E}+00$ & $0.00 \mathrm{E}+00$ \\
\hline Subtotal & $2.60 \mathrm{E}+03$ & $1.27 \mathrm{E}+01$ & $2.16 E+01$ & $2.87 \mathrm{E}+01$ & $3.70 \mathrm{E}+02$ & $4.26 \mathrm{E}+03$ & $1.11 E+04$ & $1.84 \mathrm{E}+04$ \\
\hline \multicolumn{9}{|c|}{ Small-quantity sites } \\
\hline Ames & $0.00 \mathrm{E}+00$ & $0.00 \mathrm{E}+00$ & $0.00 \mathrm{E}+00$ & $0.00 \mathrm{E}+00$ & $0.00 \mathrm{E}+00$ & $0.00 \mathrm{E}+00$ & $0.00 \mathrm{E}+00$ & $0.00 \mathrm{E}+00$ \\
\hline ANL-W & $1.78 \mathrm{E}+00$ & $0.00 \mathrm{E}+00$ & $0.00 \mathrm{E}+00$ & $0.00 \mathrm{E}+00$ & $8.90 \mathrm{E}+00$ & $1.69 \mathrm{E}+01$ & $8.90 \mathrm{E}+00$ & $3.65 \mathrm{E}+01$ \\
\hline $\mathrm{ARCO}$ & $0.00 \mathrm{E}+00$ & $0.00 \mathrm{E}+00$ & $0.00 \mathrm{E}+00$ & $0.00 \mathrm{E}+00$ & $0.00 \mathrm{E}+00$ & $0.00 \mathrm{E}+00$ & $0.00 \mathrm{E}+00$ & $0.00 \mathrm{E}+00$ \\
\hline BAPL & $0.00 \mathrm{E}+00$ & $0.00 \mathrm{E}+00$ & $0.00 \mathrm{E}+00$ & $0.00 \mathrm{E}+00$ & $0.00 \mathrm{E}+00$ & $0.00 \mathrm{E}+00$ & $0.00 \mathrm{E}+00$ & $0.00 \mathrm{E}+00$ \\
\hline $\mathrm{BCL}$ & $0.00 \mathrm{E}+00$ & $0.00 \mathrm{E}+00$ & $0.00 \mathrm{E}+00$ & $0.00 \mathrm{E}+00$ & $0.00 \mathrm{E}+00$ & $0.00 \mathrm{E}+00$ & $0.00 \mathrm{E}+00$ & $0.00 \mathrm{E}+00$ \\
\hline ETEC & $6.30 \mathrm{E}+00$ & $0.00 \mathrm{E}+00$ & $4.16 \mathrm{E}-01$ & $4.16 \mathrm{E}-01$ & $0.00 \mathrm{E}+00$ & $0.00 \mathrm{E}+00$ & $0.00 \mathrm{E}+00$ & $7.13 E+00$ \\
\hline KAPL & $0.00 \mathrm{E}+00$ & $4.16 \mathrm{E}-01$ & $4.16 \mathrm{E}-01$ & $4.16 \mathrm{E}-01$ & $1.46 \mathrm{E}+00$ & $2.08 \mathrm{E}+00$ & $2.08 \mathrm{E}+00$ & $6.86 \mathrm{E}+00$ \\
\hline LBL & $0.00 \mathrm{E}+00$ & $0.00 \mathrm{E}+00$ & $0.00 \mathrm{E}+00$ & $0.00 \mathrm{E}+00$ & $0.00 \mathrm{E}+00$ & $0.00 \mathrm{E}+00$ & $0.00 \mathrm{E}+00$ & $0.00 \mathrm{E}+00$ \\
\hline MURR & $0.00 \mathrm{E}+00$ & $0.00 \mathrm{E}+00$ & $0.00 \mathrm{E}+00$ & $0.00 \mathrm{E}+00$ & $0.00 \mathrm{E}+00$ & $0.00 \mathrm{E}+00$ & $0.00 \mathrm{E}+00$ & $0.00 E+00$ \\
\hline PAD & $0.00 \mathrm{E}+00$ & $0.00 \mathrm{E}+00$ & $0.00 \mathrm{E}+00$ & $0.00 \mathrm{E}+00$ & $0.00 \mathrm{E}+00$ & $0.00 \mathrm{E}+00$ & $0.00 E+00$ & $0.00 \mathrm{E}+00$ \\
\hline PANT & $0.00 \mathrm{E}+00$ & $0.00 \mathrm{E}+00$ & $0.00 \mathrm{E}+00$ & $0.00 \mathrm{E}+00$ & $0.00 \mathrm{E}+00$ & $0.00 \mathrm{E}+00$ & $0.00 \mathrm{E}+00$ & $0.00 \mathrm{E}+00$ \\
\hline SNL/NM & $0.00 \mathrm{E}+00$ & $0.00 \mathrm{E}+00$ & $0.00 \mathrm{E}+00$ & $0.00 \mathrm{E}+00$ & $0.00 \mathrm{E}+00$ & $0.00 \mathrm{E}+00$ & $0.00 \mathrm{E}+00$ & $0.00 \mathrm{E}+00$ \\
\hline TBE & $0.00 \mathrm{E}+00$ & $0.00 E+00$ & $0.00 E+00$ & $0.00 \mathrm{E}+00$ & $0.00 \mathrm{E}+00$ & $0.00 \mathrm{E}+00$ & $0.00 \mathrm{E}+00$ & $0.00 \mathrm{E}+00$ \\
\hline USAMC & $0.00 \mathrm{E}+00$ & $0.00 \mathrm{E}+00$ & $0.00 \mathrm{E}+00$ & $0.00 \mathrm{E}+00$ & $0.00 \mathrm{E}+00$ & $0.00 \mathrm{E}+00$ & $0.00 \mathrm{E}+00$ & $0.00 \mathrm{E}+00$ \\
\hline Subtotal & $8.08 \mathrm{E}+00$ & $4.16 \mathrm{E}-01$ & $8.32 \mathrm{E}-01$ & $8.32 \mathrm{E}-01$ & $1.04 \mathrm{E}+01$ & $1.90 \mathrm{E}+01$ & $1.10 \mathrm{E}+01$ & $5.05 \mathrm{E}+01$ \\
\hline
\end{tabular}




\section{FINAL WASTE FORM}

Table 3.13 (continued)

\begin{tabular}{|c|c|c|c|c|c|c|c|c|}
\hline \multirow{2}{*}{ Site } & \multirow{2}{*}{$\begin{array}{c}\text { End of } \\
\text { CY } 1994 \\
\text { total }\end{array}$} & \multicolumn{3}{|c|}{ Annual increase } & \multicolumn{3}{|c|}{ Cumulative increase } & \multirow{2}{*}{$\begin{array}{l}\text { End of } \\
\text { CY } 2022 \\
\text { total }\end{array}$} \\
\hline & & 1995 & 1996 & 1997 & $1998-2002$ & 2003-2012 & $2013-2022$ & \\
\hline \multicolumn{9}{|l|}{ Commercial site } \\
\hline WVDP & $0.00 \mathrm{E}+00$ & $0.00 \mathrm{E}+00$ & $0.00 \mathrm{E}+00$ & $0.00 \mathrm{E}+00$ & $0.00 \mathrm{E}+00$ & $0.00 \mathrm{E}+00$ & $0.00 \mathrm{E}+00$ & $0.00 \mathrm{E}+00$ \\
\hline $\begin{array}{l}\text { Total } \\
\text { (all sites) }\end{array}$ & $2.61 \mathrm{E}+03$ & $1.31 E+01$ & $2.25 \mathrm{E}+01$ & $2.96 \mathrm{E}+01$ & $3.80 \mathrm{E}+02$ & $4.27 \mathrm{E}+03$ & $1.11 E+04$ & $1.84 E+04$ \\
\hline
\end{tabular}




\section{FINAL WASTE FORM}

Table 3.14. Final waste-form volumes $\left(\mathrm{m}^{3}\right)$ of retrievably stored and projected nonmixed RH TRUW

\begin{tabular}{|c|c|c|c|c|c|c|c|c|}
\hline \multirow{2}{*}{ Site } & \multirow{2}{*}{$\begin{array}{l}\text { End of } \\
\text { CY } 1994 \\
\text { total }\end{array}$} & \multicolumn{3}{|c|}{ Annual increase } & \multicolumn{3}{|c|}{ Cumulative increase } & \multirow{2}{*}{$\begin{array}{c}\text { End of } \\
\text { CY } 2022 \\
\text { total }\end{array}$} \\
\hline & & 1995 & 1996 & 1997 & $1998-2002$ & 2003-2012 & 2013-2022 & \\
\hline \multicolumn{9}{|l|}{ Major sites } \\
\hline ANL-E & $0.00 \mathrm{E}+00$ & $0.00 \mathrm{E}+00$ & $0.00 \mathrm{E}+00$ & $0.00 \mathrm{E}+00$ & $0.00 \mathrm{E}+00$ & $0.00 \mathrm{E}+00$ & $0.00 \mathrm{E}+00$ & $0.00 \mathrm{E}+00$ \\
\hline Hanford & $1.99 \mathrm{E}+02$ & $6.76 E+01$ & $0.00 \mathrm{E}+00$ & $0.00 \mathrm{E}+00$ & $0.00 \mathrm{E}+00$ & $3.56 \mathrm{E}+02$ & $5.83 E+03$ & $6.46 \mathrm{E}+03$ \\
\hline INEL & $2.61 \mathrm{E}+01$ & $0.00 \mathrm{E}+00$ & $0.00 \mathrm{E}+00$ & $0.00 \mathrm{E}+00$ & $0.00 \mathrm{E}+00$ & $0.00 \mathrm{E}+00$ & $0.00 \mathrm{E}+00$ & $2.61 \mathrm{E}+01$ \\
\hline LANL & $7.74 \mathrm{E}+01$ & $0.00 \mathrm{E}+00$ & $1.78 \mathrm{E}+00$ & $1.78 \mathrm{E}+00$ & $8.90 \mathrm{E}+00$ & $2.49 \mathrm{E}+01$ & $2.76 \mathrm{E}+01$ & $1.42 \mathrm{E}+02$ \\
\hline LLNL & $0.00 \mathrm{E}+00$ & $0.00 \mathrm{E}+00$ & $0.00 \mathrm{E}+00$ & $0.00 \mathrm{E}+00$ & $0.00 \mathrm{E}+00$ & $0.00 \mathrm{E}+00$ & $0.00 \mathrm{E}+00$ & $0.00 \mathrm{E}+00$ \\
\hline Mound & $0.00 \mathrm{E}+00$ & $0.00 \mathrm{E}+00$ & $0.00 \mathrm{E}+00$ & $0.00 \mathrm{E}+00$ & $0.00 \mathrm{E}+00$ & $0.00 \mathrm{E}+00$ & $0.00 E+00$ & $0.00 \mathrm{E}+00$ \\
\hline NTS & $0.00 \mathrm{E}+00$ & $0.00 \mathrm{E}+00$ & $0.00 \mathrm{E}+00$ & $0.00 \mathrm{E}+00$ & $0.00 \mathrm{E}+00$ & $0.00 \mathrm{E}+00$ & $0.00 \mathrm{E}+00$ & $0.00 \mathrm{E}+00$ \\
\hline ORNL & $8.54 E+01$ & $0.00 \mathrm{E}+00$ & $0.00 \mathrm{E}+00$ & $0.00 \mathrm{E}+00$ & $0.00 \mathrm{E}+00$ & $0.00 \mathrm{E}+00$ & $0.00 \mathrm{E}+00$ & $8.54 \mathrm{E}+01$ \\
\hline RFETS & $0.00 \mathrm{E}+00$ & $0.00 \mathrm{E}+00$ & $0.00 \mathrm{E}+00$ & $0.00 \mathrm{E}+00$ & $0.00 \mathrm{E}+00$ & $0.00 \mathrm{E}+00$ & $0.00 \mathrm{E}+00$ & $0.00 \mathrm{E}+00$ \\
\hline SRS & $0.00 \mathrm{E}+00$ & $0.00 \mathrm{E}+00$ & $0.00 \mathrm{E}+00$ & $0.00 \mathrm{E}+00$ & $0.00 \mathrm{E}+00$ & $0.00 \mathrm{E}+00$ & $0.00 \mathrm{E}+00$ & $0.00 \mathrm{E}+00$ \\
\hline Subtotal & $3.88 \mathrm{E}+02$ & $6.76 \mathrm{E}+01$ & $1.78 \mathrm{E}+00$ & $1.78 E+00$ & $8.90 \mathrm{E}+00$ & $3.81 \mathrm{E}+02$ & $5.86 \mathrm{E}+03$ & $6.71 E+03$ \\
\hline \multicolumn{9}{|c|}{ Small-quantity sites } \\
\hline Ames & $0.00 \mathrm{E}+00$ & $0.00 \mathrm{E}+00$ & $0.00 \mathrm{E}+00$ & $0.00 \mathrm{E}+00$ & $0.00 \mathrm{E}+00$ & $0.00 \mathrm{E}+00$ & $0.00 \mathrm{E}+00$ & $0.00 \mathrm{E}+00$ \\
\hline ANL-W & $1.75 E+01$ & $0.00 \mathrm{E}+00$ & $0.00 \mathrm{E}+00$ & $0.00 \mathrm{E}+00$ & $3.24 E+02$ & $4.89 \mathrm{E}+02$ & $4.17 \mathrm{E}+02$ & $1.25 \mathrm{E}+03$ \\
\hline ARCO & $0.00 \mathrm{E}+00$ & $0.00 \mathrm{E}+00$ & $0.00 \mathrm{E}+00$ & $0.00 \mathrm{E}+00$ & $0.00 \mathrm{E}+00$ & $0.00 \mathrm{E}+00$ & $0.00 \mathrm{E}+00$ & $0.00 \mathrm{E}+00$ \\
\hline BAPL & $0.00 \mathrm{E}+00$ & $1.33 \mathrm{E}+00$ & $8.90 E-01$ & $1.33 \mathrm{E}+00$ & $2.67 \mathrm{E}+00$ & $4.45 \mathrm{E}-01$ & $0.00 \mathrm{E}+00$ & $6.67 \mathrm{E}+00$ \\
\hline $\mathrm{BCL}$ & $5.81 \mathrm{E}+02$ & $0.00 \mathrm{E}+00$ & $0.00 \mathrm{E}+00$ & $0.00 \mathrm{E}+00$ & $0.00 \mathrm{E}+00$ & $0.00 \mathrm{E}+00$ & $0.00 \mathrm{E}+00$ & $5.81 \mathrm{E}+02$ \\
\hline ETEC & $0.00 \mathrm{E}+00$ & $0.00 \mathrm{E}+00$ & $0.00 \mathrm{E}+00$ & $0.00 \mathrm{E}+00$ & $0.00 \mathrm{E}+00$ & $0.00 \mathrm{E}+00$ & $0.00 \mathrm{E}+00$ & $0.00 \mathrm{E}+00$ \\
\hline KAPL & $2.50 \mathrm{E}+00$ & $1.66 \mathrm{E}+00$ & $1.66 \mathrm{E}+00$ & $1.66 \mathrm{E}+00$ & $8.94 \mathrm{E}+00$ & $1.87 \mathrm{E}+01$ & $1.87 \mathrm{E}+01$ & $5.39 E+01$ \\
\hline LBL & $0.00 \mathrm{E}+00$ & $0.00 \mathrm{E}+00$ & $0.00 \mathrm{E}+00$ & $0.00 \mathrm{E}+00$ & $0.00 \mathrm{E}+00$ & $0.00 \mathrm{E}+00$ & $0.00 \mathrm{E}+00$ & $0.00 \mathrm{E}+00$ \\
\hline MURR & $0.00 \mathrm{E}+00$ & $0.00 \mathrm{E}+00$ & $0.00 E+00$ & $0.00 \mathrm{E}+00$ & $0.00 \mathrm{E}+00$ & $0.00 \mathrm{E}+00$ & $0.00 \mathrm{E}+00$ & $0.00 \mathrm{E}+00$ \\
\hline PAD & $0.00 \mathrm{E}+00$ & $0.00 \mathrm{E}+00$ & $0.00 \mathrm{E}+00$ & $0.00 \mathrm{E}+00$ & $0.00 \mathrm{E}+00$ & $0.00 \mathrm{E}+00$ & $0.00 \mathrm{E}+00$ & $0.00 \mathrm{E}+00$ \\
\hline PANT & $0.00 \mathrm{E}+00$ & $0.00 E+00$ & $0.00 \mathrm{E}+00$ & $0.00 \mathrm{E}+00$ & $0.00 \mathrm{E}+00$ & $0.00 \mathrm{E}+00$ & $0.00 \mathrm{E}+00$ & $0.00 \mathrm{E}+00$ \\
\hline SNL/NM & $0.00 \mathrm{E}+00$ & $0.00 \mathrm{E}+00$ & $0.00 \mathrm{E}+00$ & $0.00 \mathrm{E}+00$ & $0.00 \mathrm{E}+00$ & $0.00 \mathrm{E}+00$ & $0.00 \mathrm{E}+00$ & $0.00 \mathrm{E}+00$ \\
\hline TBE & $0.00 \mathrm{E}+00$ & $0.00 \mathrm{E}+00$ & $0.00 \mathrm{E}+00$ & $0.00 \mathrm{E}+00$ & $0.00 \mathrm{E}+00$ & $0.00 \mathrm{E}+00$ & $0.00 \mathrm{E}+00$ & $0.00 \mathrm{E}+00$ \\
\hline USAMC & $0.00 \mathrm{E}+00$ & $0.00 \mathrm{E}+00$ & $0.00 \mathrm{E}+00$ & $0.00 \mathrm{E}+00$ & $0.00 \mathrm{E}+00$ & $0.00 \mathrm{E}+00$ & $0.00 \mathrm{E}+00$ & $0.00 \mathrm{E}+00$ \\
\hline Subtotal & $6.01 \mathrm{E}+02$ & $3.00 \mathrm{E}+00$ & $2.55 \mathrm{E}+00$ & $3.00 \mathrm{E}+00$ & $3.36 \mathrm{E}+02$ & $5.09 \mathrm{E}+02$ & $4.35 \mathrm{E}+02$ & $1.89 \mathrm{E}+03$ \\
\hline
\end{tabular}




\section{FINAL WASTE FORM}

Table 3.14 (continued)

\begin{tabular}{|c|c|c|c|c|c|c|c|c|}
\hline \multirow{2}{*}{ Site } & \multirow{2}{*}{$\begin{array}{l}\text { End of } \\
\text { CY } 1994 \\
\text { total }\end{array}$} & \multicolumn{3}{|c|}{ Annual increase } & \multicolumn{3}{|c|}{ Cumulative increase } & \multirow{2}{*}{$\begin{array}{l}\text { End of } \\
\text { CY } 2022 \\
\text { total }\end{array}$} \\
\hline & & 1995 & 1996 & 1997 & 1998-2002 & $2003-2012$ & $2013-2022$ & \\
\hline \multicolumn{9}{|l|}{ Commercial site } \\
\hline WVDP & $3.03 \mathrm{E}+02$ & $1.32 \mathrm{E}+01$ & $0.00 \mathrm{E}+00$ & $0.00 \mathrm{E}+00$ & $3.29 \mathrm{E}+01$ & $0.00 \mathrm{E}+00$ & $0.00 \mathrm{E}+00$ & $3.50 \mathrm{E}+02$ \\
\hline $\begin{array}{l}\text { Total } \\
\text { (all sites) }\end{array}$ & $1.29 \mathrm{E}+03$ & $8.39 E+01$ & $4.33 \mathrm{E}+00$ & $4.78 E+00$ & $3.77 \mathrm{E}+02$ & $8.90 \mathrm{E}+02$ & $6.29 \mathrm{E}+03$ & $8.95 E+03$ \\
\hline
\end{tabular}




\section{FINAL WASTE FORM}

Table 3.15. Estimated final-form volume $\left(\mathrm{m}^{3}\right)$ composition of retrievably stored and projected TRUW for major sites ${ }^{\mathrm{a}}$

\begin{tabular}{|c|c|c|c|c|c|c|c|}
\hline \multirow{2}{*}{$\begin{array}{l}\text { Major } \\
\text { sites }\end{array}$} & \multirow{2}{*}{ Category } & \multicolumn{3}{|c|}{ Contact-handled } & \multicolumn{3}{|c|}{ Remote-handled } \\
\hline & & Stored & Projected & Total & Stored & Projected & Total \\
\hline \multirow[t]{5}{*}{$\mathrm{ANL}-\mathrm{E}^{\mathrm{a}}$} & Lead/cadmium metal waste & 1.07 & 1.29 & 2.36 & 0.00 & 0.00 & 0.00 \\
\hline & Solidified inorganics & 5.20 & 0.00 & 5.20 & 0.00 & 0.00 & 0.00 \\
\hline & Solidified organics & 0.21 & 0.00 & 0.21 & 0.00 & 0.00 & 0.00 \\
\hline & Uncategorized metal & 4.99 & 128.54 & 133.53 & 0.00 & 0.00 & 0.00 \\
\hline & Subtotal & 11.47 & 129.83 & 141.30 & 0.00 & 0.00 & 0.00 \\
\hline \multirow[t]{8}{*}{ Hanford ${ }^{c}$} & Combustible & 455.73 & $1,247.26$ & $1,702.99$ & 0.00 & 0.00 & 0.00 \\
\hline & Heterogeneous & $11,190.75$ & $6,271.29$ & $17,462.04$ & 199.36 & $4,066.41$ & $3,006.87$ \\
\hline & Lead/cadmium metal waste & 14.17 & 34.53 & 48.69 & 2.67 & 60.52 & 0.00 \\
\hline & Soils & 119.48 & $5,961.70$ & $6,081.17$ & 0.00 & 0.00 & 0.00 \\
\hline & Solidified inorganics & 12.94 & 7.07 & 20.02 & 0.00 & 0.00 & 0.00 \\
\hline & Solidified organics & 7.36 & 9.36 & 16.72 & 0.00 & 0.00 & 0.00 \\
\hline & Uncategorized metal & 444.86 & $19,635.15$ & $20,080.01$ & 0.00 & $17,400.39$ & $17,400.39$ \\
\hline & Subtotal & $12,280.03$ & $33,235.41$ & $45,515.44$ & 202.03 & $21,527.32$ & $21,729.35$ \\
\hline \multirow[t]{11}{*}{ INEL } & Combustible & $3,304.94$ & 0.00 & $3,304.94$ & 21.36 & 0.00 & 21.36 \\
\hline & Filter & 131.05 & 0.00 & 131.05 & 0.00 & 0.00 & 0.00 \\
\hline & Graphite & 498.19 & 0.00 & 498.19 & 0.00 & 0.00 & 0.00 \\
\hline & Heterogeneous & $10,608.50$ & 0.00 & $10,608.50$ & 49.84 & 0.00 & 49.84 \\
\hline & Inorganic nonmetal & $3,028.24$ & 0.00 & $3,028.24$ & 46.28 & 0.00 & 46.28 \\
\hline & Lead/cadmium metal waste & 14.37 & 0.00 & 14.37 & 3.56 & 0.00 & 3.56 \\
\hline & Salt waste & 20.52 & 0.00 & 20.52 & 0.00 & 0.00 & 0.00 \\
\hline & Solidified inorganics & $4,344.44$ & 0.00 & $4,344.44$ & 65.27 & 0.00 & 65.27 \\
\hline & Solidified organics & 789.67 & 0.00 & 789.67 & 3.56 & 0.00 & 3.56 \\
\hline & Uncategorized metal & $5,866.82$ & 0.00 & $5,866.82$ & 30.85 & 0.00 & 30.85 \\
\hline & Subtotal & $28,606.72$ & 0.00 & $28,606.72$ & 220.72 & 0.00 & 220.72 \\
\hline \multirow[t]{8}{*}{ LANL } & Combustible & $1,821.45$ & $2,464.60$ & $4,197.64$ & 15.13 & 48.95 & 64.08 \\
\hline & Heterogeneous & 16.02 & 29.12 & 45.14 & 11.57 & 0.00 & 11.57 \\
\hline & Lead/cadmium metal waste & 1.89 & 0.00 & 1.89 & 0.00 & 0.00 & 0.00 \\
\hline & Soils & 110.57 & 29.12 & 139.69 & 0.00 & 0.00 & 0.00 \\
\hline & Solidified inorganics & $4,888.20$ & $2,033.82$ & $6,922.02$ & 0.00 & 0.00 & 0.00 \\
\hline & Solidified organics & 1.46 & 29.12 & 30.58 & 0.00 & 0.00 & 0.00 \\
\hline & Uncategorized metals & $4,214.43$ & $2,853.76$ & $7,068.19$ & 67.64 & 49.84 & 117.48 \\
\hline & Subtotal & $11,054.01$ & $7,351.14$ & $18,405.15$ & 94.34 & 98.79 & 193.13 \\
\hline \multirow[t]{6}{*}{ LLNL } & Filter & 15.54 & 32.28 & 47.82 & 0.00 & 0.00 & 0.00 \\
\hline & Heterogeneous & 198.85 & 663.78 & 862.63 & 0.00 & 0.00 & 0.00 \\
\hline & Salt waste & 0.62 & 3.02 & 3.64 & 0.00 & 0.00 & 0.00 \\
\hline & Solidified inorganics & 14.35 & 5.82 & 20.18 & 0.00 & 0.00 & 0.00 \\
\hline & Solidified organics & 1.04 & 5.82 & 6.86 & 0.00 & 0.00 & 0.00 \\
\hline & Subtotal & 230.40 & 710.73 & 941.13 & 0.00 & 0.00 & 0.00 \\
\hline
\end{tabular}


FINAL WASTE FORM

Table 3.15 (continued)

\begin{tabular}{|c|c|c|c|c|c|c|c|}
\hline \multirow{2}{*}{$\begin{array}{l}\text { Major } \\
\text { sites }\end{array}$} & \multirow{2}{*}{ Category } & \multicolumn{3}{|c|}{ Contact-handled } & \multicolumn{3}{|c|}{ Remote-handled } \\
\hline & & Stored & Projected & Total & Stored & Projected & Total \\
\hline \multirow[t]{7}{*}{ Mound ${ }^{d}$} & Combustible & 7.07 & 0.00 & 7.07 & 0.00 & 0.00 & 0.00 \\
\hline & Filter & 0.83 & 0.00 & 0.83 & 0.00 & 0.00 & 0.00 \\
\hline & Heterogeneous & 0.62 & 0.00 & 0.62 & 0.00 & 0.00 & 0.00 \\
\hline & Soils & 177.18 & 0.00 & 177.18 & 0.00 & 0.00 & 0.00 \\
\hline & Solidified inorganics & 6.03 & 0.00 & 6.03 & 0.00 & 0.00 & 0.00 \\
\hline & Uncategorized metal & 82.48 & 0.00 & 82.48 & 0.00 & 0.00 & 0.00 \\
\hline & Subtotal & 274.22 & 0.00 & 272.22 & 0.00 & 0.00 & 0.00 \\
\hline \multirow[t]{3}{*}{ NTS } & Heterogeneous & 613.26 & 8.98 & 622.24 & 0.00 & 0.00 & 0.00 \\
\hline & Solidified inorganics & 5.67 & 0.00 & 5.67 & 0.00 & 0.00 & 0.00 \\
\hline & Subtotal & 618.93 & 8.98 & 627.91 & 0.00 & 0.00 & 0.00 \\
\hline \multirow[t]{3}{*}{ ORNLe } & Heterogeneous & $1,304.16$ & 256.26 & $1,560.42$ & $1,432.01$ & 240.30 & $1,672.31$ \\
\hline & Solidified inorganics & 0.00 & 0.00 & 0.00 & $1,036.85$ & 206.48 & $1,243.33$ \\
\hline & Subtotal & $1,304.16$ & 256.26 & $1,560.42$ & $2,468.86$ & 446.78 & $2,915.64$ \\
\hline \multirow[t]{11}{*}{ RFETS ${ }^{f}$} & Combustible & 185.54 & 861.08 & $1,046.62$ & 0.00 & 0.00 & 0.00 \\
\hline & Filter & 72.09 & 477.51 & 549.60 & 0.00 & 0.00 & 0.00 \\
\hline & Graphite & 13.73 & 47.57 & 61.30 & 0.00 & 0.00 & 0.00 \\
\hline & Heterogeneous & 3.86 & 0.00 & 3.86 & 0.00 & 0.00 & 0.00 \\
\hline & Inorganic nonmetal & 58.29 & 866.74 & 925.03 & 0.00 & 0.00 & 0.00 \\
\hline & Lead/cadmium metal waste & 3.95 & 298.27 & 302.22 & 0.00 & 0.00 & 0.00 \\
\hline & Salt waste & 0.00 & 325.92 & 325.92 & 0.00 & 0.00 & 0.00 \\
\hline & Solidified inorganics & 165,36 & $1,257.64$ & $1,423.01$ & 0.00 & 0.00 & 0.00 \\
\hline & Solidified organics & 109.82 & 31.11 & 140.93 & 0.00 & 0.00 & 0.00 \\
\hline & Uncategorized metal & 93.31 & 236.12 & 329.43 & 0.00 & 0.00 & 0.00 \\
\hline & Subtotal & $1,133.13$ & $5,901.46$ & $7,034.59$ & 0.00 & 0.00 & 0.00 \\
\hline \multirow[t]{5}{*}{ SRS } & Heterogeneous & $2,611.61$ & $5,475.85$ & $8,087.46$ & 0.00 & 0.00 & 0.00 \\
\hline & Solidified inorganics & 200.19 & $1,169.61$ & $1,369.80$ & 0.00 & 0.00 & 0.00 \\
\hline & Uncategorized metal & 70.35 & 120.54 & 190.89 & 0.00 & 0.00 & 0.00 \\
\hline & Subtotal & $2,882.14$ & $6,766.01$ & $9,648.15$ & 0.00 & 0.00 & 0.00 \\
\hline & Total (major sites) & $57,968.04$ & $52,860.31$ & $110,828.35$ & $2,985.95$ & $22,072.89$ & $25,058.84$ \\
\hline
\end{tabular}

aThe totals for ANL-E do not include $13.31 \mathrm{~m}^{3}$ of "as-generated" stored CH-TRU waste because final waste-form volumes have not been estimated at this time.

J_ead/cadmium metal wastes include materials that contain either lead or cadmium or both.

cThe totals for Hanford do not include $94.82 \mathrm{~m}^{3}$ of "as-generated" stored CH-TRU waste and $459.49 \mathrm{~m}^{3}$ of "as-generated" projected $\mathrm{CH}$-TRU waste because final waste-form volumes have not been estimated at this time.

dThe totals for Mound do not include $30.57 \mathrm{~m}^{3}$ of "as-generated" stored CH-TRU waste because final waste-form volumes have not been estimated at this time.

eThe totals for ORNL do not include $23 \mathrm{~m}^{3}$ of "as-generated" stored CH-TRU waste and $0.03 \mathrm{~m}^{3}$ of "as-generated" stored RH-TRU waste because final waste-form volumes have not been estimated at this time.

The totals for RFETS do not include $0.83 \mathrm{~m}^{3}$ of "as-génerated" stored CH-TRU waste because final waste-form volumes have not been estimated at this time. 
FINAL WASTE FORM

Table 3.16. Estimated final-form volume $\left(\mathrm{m}^{3}\right)$ composition of retrievably stored and projected TRUW for small-quantity and commercial sites ${ }^{a}, b$

\begin{tabular}{|c|c|c|c|c|c|c|c|}
\hline \multirow{2}{*}{$\begin{array}{l}\text { Major } \\
\text { sites }\end{array}$} & \multirow{2}{*}{ Category } & \multicolumn{3}{|c|}{ Contact-handled } & \multicolumn{3}{|c|}{ Remote-handled } \\
\hline & & Stored & Projected & Total & Stored & Projected & Total \\
\hline \multicolumn{8}{|c|}{ Small-quantity sites (SQS) } \\
\hline Ames & Solidified inorganics & 0.00 & 0.42 & 0.42 & 0.00 & 0.00 & 0.00 \\
\hline \multirow[t]{7}{*}{ ANL_-Wa } & Combustible & 0.00 & 101.64 & 101.64 & 0.00 & 0.00 & 0.00 \\
\hline & Heterogeneous & 6.54 & 345.35 & 351.89 & 0.00 & $1,208.62$ & $1,208.62$ \\
\hline & Inorganic nonmetal & 0.00 & 0.00 & 0.00 & 0.00 & 21.36 & 21.36 \\
\hline & Lead/cadmium metal waste ${ }^{\mathrm{b}}$ & 0.00 & 0.00 & 0.00 & 0.00 & 6.23 & 6.23 \\
\hline & Solidified inorganics & 0.00 & 0.00 & 0.00 & 1.78 & 28.48 & 30.26 \\
\hline & Uncategorized metal & 0.00 & 293.76 & 293.76 & 17.51 & 0.00 & 17.51 \\
\hline & Subtotal & 6.54 & 740.76 & 747.30 & 19.29 & $1,264.69$ & $1,283.98$ \\
\hline $\mathrm{ARCO}$ & Heterogeneous & $0.04^{\mathrm{c}}$ & 0.42 & 0.46 & 0.00 & 0.00 & 0.00 \\
\hline $\mathrm{BCL}$ & Heterogeneous & 0.00 & 0.00 & 0.00 & 580.50 & 0.00 & 580.50 \\
\hline BAPL & Heterogeneous & 0.00 & 123.27 & 123.27 & 0.00 & 6.67 & 6.67 \\
\hline \multirow[t]{3}{*}{ ETEC $^{d}$} & Heterogeneous & 1.68 & 0.00 & 1.68 & 0.00 & 0.00 & 0.00 \\
\hline & Lead/cadmium metal waste & 0.00 & 0.00 & 0.00 & 0.89 & 0.00 & 0.89 \\
\hline & Subtotal & 1.68 & 0.00 & 1.68 & 0.89 & 0.00 & 0.89 \\
\hline KAPL & Heterogeneous & 0.00 & 0.00 & 0.00 & 2.50 & 58.24 & 60.74 \\
\hline LBL & Heterogeneous & 0.62 & 1.04 & 1.66 & 0.00 & 0.00 & 0.00 \\
\hline MURR & Heterogeneous & 0.21 & 0.83 & 1.04 & 0.00 & 0.00 & 0.00 \\
\hline PAD & Inorganic nonmetal & 0.00 & 1.89 & 1.89 & 0.00 & 0.00 & 0.00 \\
\hline PANT & Heterogeneous & 0.62 & 0.00 & 0.62 & 0.00 & 0.00 & 0.00 \\
\hline SNL/NM & Heterogeneous & 6.66 & 7.49 & 14.14 & 0.00 & 0.00 & 0.00 \\
\hline TBE & Inorganic nonmetal & 0.21 & 0.00 & 0.21 & 0.00 & 0.00 & 0.00 \\
\hline USAMC & Heterogeneous & 2.50 & 0.00 & 2.50 & 0.00 & 0.00 & 0.00 \\
\hline & SQS subtotal & 19.08 & 876.12 & 895.20 & 603.18 & $1,329.60$ & $1,932.78$ \\
\hline
\end{tabular}




\section{FINAL WASTE FORM}

Table 3.16 (continued)

\begin{tabular}{|c|c|c|c|c|c|c|c|}
\hline \multirow{2}{*}{$\begin{array}{l}\text { Major } \\
\text { sites }\end{array}$} & \multirow{2}{*}{ Category } & \multicolumn{3}{|c|}{ Contact-handled } & \multicolumn{3}{|c|}{ Remote-handled } \\
\hline & & Stored & Projected & Total & Stored & Projected & Total \\
\hline \multicolumn{8}{|c|}{ Commercial site } \\
\hline \multirow[t]{7}{*}{ WVDPf } & Filter & 0.00 & 0.00 & 0.00 & 48.95 & 46.15 & 95.10 \\
\hline & Heterogeneous & 19.36 & 117.40 & 136.76 & 17.80 & 0.00 & 17.80 \\
\hline & Lead/cadmium metal waste & 2.08 & 0.00 & 2.08 & 0.00 & 0.00 & 0.00 \\
\hline & Solidified inorganics & 6.69 & 0.00 & 6.69 & 0.00 & 0.00 & 0.00 \\
\hline & Uncategorized metal & 0.42 & 0.00 & 0.42 & 236.74 & 0.00 & 236.74 \\
\hline & $\begin{array}{l}\text { Commercial site } \\
\text { subtotal }\end{array}$ & 28.55 & 117.40 & 145.95 & 303.49 & 46.15 & 349.64 \\
\hline & Total & 47.63 & 993.52 & $1,041.15$ & 906.67 & $1,375.75$ & $2,282.42$ \\
\hline
\end{tabular}

aThe totals for ANL $-\mathrm{W}$ do not include $14.76 \mathrm{~m}^{3}$ of "as-generated" stored RH-TRU waste because the final wasteform volumes have not been estimated at this time.

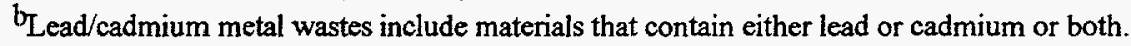

"The stored CH-TRU waste volume reported in this table is in "as-generated" form because final waste-form volumes have not been estimated at this time.

"The totals for ETEC do not include $5.46 \mathrm{~m}^{3}$ of "as-generated" stored RH-TRU waste and $0.84 \mathrm{~m}^{3}$ of "asgenerated" projected RH-TRU waste because the final waste-form is unknown at this time.

"The totals for PANT do not include $0.90 \mathrm{~m}^{3}$ of "as-generated" stored CH-TRU waste and $3.78 \mathrm{~m}^{3}$ of "final form" projected $\mathrm{CH}$-TRU waste because the final waste-form is unknown at this time.

The totals for WVDP do not include $10.50 \mathrm{~m}^{3}$ of "as-generated" stored CH-TRU waste because the final wasteform volumes have not been estimated at this time. 
Table 3.17. Summary of decayed radioactivity (Ci) isotopic content of retrievably stored CH TRUWa

\begin{tabular}{|c|c|c|c|c|c|c|c|}
\hline Site & ${ }^{241} \mathrm{Pu}$ & ${ }^{238} \mathrm{Pu}$ & ${ }^{239} \mathrm{Pu}$ & ${ }^{241} \mathrm{Am}$ & ${ }^{240} \mathrm{Pu}$ & $\begin{array}{l}\text { Total } \\
\text { curies }\end{array}$ & $\begin{array}{c}\text { Curies } \\
(\%)\end{array}$ \\
\hline \multicolumn{8}{|c|}{ Major sites } \\
\hline ANL-E & $5.98 \mathrm{E}+01$ & $2.14 \mathrm{E}+00$ & $3.28 \mathrm{E}+01$ & $5.73 \mathrm{E}+00$ & $9.42 \mathrm{E}+00$ & $1.10 \mathrm{E}+02$ & 0.00 \\
\hline Hanford & $3.78 \mathrm{E}+04$ & $8.05 E+04$ & $2.63 E+04$ & $4.73 E+03$ & $6.15 E+03$ & $1.55 \mathrm{E}+05$ & 6.19 \\
\hline INEL & $1.50 \mathrm{E}+05$ & $5.98 \mathrm{E}+04$ & $4.01 \mathrm{E}+04$ & $9.01 \mathrm{E}+04$ & $9.82 \mathrm{E}+03$ & $3.50 \mathrm{E}+05$ & 13.93 \\
\hline LANL & $1.62 \mathrm{E}+03$ & $1.15 E+05$ & $7.69 \mathrm{E}+04$ & $9.11 \mathrm{E}+03$ & $1.00 \mathrm{E}+02$ & $2.02 \mathrm{E}+05$ & 8.06 \\
\hline LLNL & $1.63 \mathrm{E}+03$ & $7.65 \mathrm{E}+01$ & $1.58 \mathrm{E}+02$ & $1.44 \mathrm{E}+02$ & $6.44 \mathrm{E}+01$ & $2.07 \mathrm{E}+03$ & 0.08 \\
\hline Mound & $0.00 \mathrm{E}+00$ & $1.53 E+03$ & $2.98 \mathrm{E}+01$ & $0.00 \mathrm{E}+00$ & $0.00 \mathrm{E}+00$ & $1.56 \mathrm{E}+03$ & 0.06 \\
\hline NTS & $2.40 \mathrm{E}+02$ & $1.95 \mathrm{E}+02$ & $2.76 \mathrm{E}+03$ & $2.84 \mathrm{E}+02$ & $1.88 \mathrm{E}+01$ & $3.50 \mathrm{E}+03$ & 0.14 \\
\hline ORNL & $4.79 \mathrm{E}+04$ & $3.50 \mathrm{E}+03$ & $1.01 \mathrm{E}+03$ & $1.61 \mathrm{E}+03$ & $9.48 \mathrm{E}+02$ & $5.50 \mathrm{E}+04$ & 2.19 \\
\hline RFETS & $7.74 \mathrm{E}+05$ & $8.43 E+03$ & $1.94 \mathrm{E}+05$ & $1.30 \mathrm{E}+05$ & $4.94 E+04$ & $1.16 \mathrm{E}+06$ & 46.06 \\
\hline SRS & $6.20 \mathrm{E}+04$ & $4.87 \mathrm{E}+05$ & $9.29 E+03$ & $3.70 \mathrm{E}+03$ & $2.29 \mathrm{E}+03$ & $5.64 \mathrm{E}+05$ & 22.48 \\
\hline Subtotal & $1.07 \mathrm{E}+06$ & $7.55 \mathrm{E}+05$ & $3.51 E+05$ & $2.40 \mathrm{E}+05$ & $6.88 \mathrm{E}+04$ & $2.49 \mathrm{E}+06$ & 99.21 \\
\hline \multicolumn{8}{|c|}{ Small-quantity sites (SQS) } \\
\hline Ames & $0.00 \mathrm{E}+00$ & $0.00 E+00$ & $0.00 E+00$ & $0.00 \mathrm{E}+00$ & $0.00 \mathrm{E}+00$ & $0.00 \mathrm{E}+00$ & 0.00 \\
\hline ANL-W & $0.00 \mathrm{E}+00$ & $0.00 \mathrm{E}+00$ & $0.00 \mathrm{E}+00$ & $0.00 \mathrm{E}+00$ & $0.00 \mathrm{E}+00$ & $0.00 \mathrm{E}+00$ & 0.00 \\
\hline $\mathrm{ARCO}$ & $0.00 \mathrm{E}+00$ & $3.70 \mathrm{E}+02$ & $0.00 \mathrm{E}+00$ & $0.00 \mathrm{E}+00$ & $0.00 \mathrm{E}+00$ & $3.70 \mathrm{E}+02$ & 0.01 \\
\hline $\mathrm{BCL}$ & $0.00 \mathrm{E}+00$ & $0.00 \mathrm{E}+00$ & $0.00 \mathrm{E}+00$ & $0.00 \mathrm{E}+00$ & $0.00 \mathrm{E}+00$ & $0.00 \mathrm{E}+00$ & 0.00 \\
\hline BAPL & $0.00 \mathrm{E}+00$ & $0.00 \mathrm{E}+00$ & $0.00 \mathrm{E}+00$ & $0.00 \mathrm{E}+00$ & $0.00 \mathrm{E}+00$ & $0.00 \mathrm{E}+00$ & 0.00 \\
\hline ETEC & $6.22 \mathrm{E}+00$ & $1.11 \mathrm{E}-01$ & $1.79 \mathrm{E}+00$ & $5.19 \mathrm{E}-01$ & $6.12 \mathrm{E}-01$ & $9.25 \mathrm{E}+00$ & 0.00 \\
\hline KAPL & $0.00 \mathrm{E}+00$ & $0.00 \mathrm{E}+00$ & $0.00 \mathrm{E}+00$ & $0.00 \mathrm{E}+00$ & $0.00 \mathrm{E}+00$ & $0.00 \mathrm{E}+00$ & 0.00 \\
\hline LBL & $4.48 \mathrm{E}-07$ & $2.32 \mathrm{E}-04$ & $8.45 E-06$ & $9.17 \mathrm{E}-02$ & $5.14 \mathrm{E}-03$ & $9.71 \mathrm{E}-02$ & 0.00 \\
\hline MURR & $6.32 E-03$ & $0.00 \mathrm{E}+00$ & $2.46 \mathrm{E}-02$ & $3.24 \mathrm{E}-01$ & $0.00 \mathrm{E}+00$ & $3.55 \mathrm{E}-01$ & 0.00 \\
\hline PAD & $0.00 \mathrm{E}+00$ & $0.00 E+00$ & $5.57 \mathrm{E}+01$ & $0.00 \mathrm{E}+00$ & $0.00 \mathrm{E}+00$ & $5.57 E+01$ & 0.00 \\
\hline PANT & $0.00 \mathrm{E}+00$ & $0.00 \mathrm{E}+00$ & $5.55 \mathrm{E}-02$ & $0.00 \mathrm{E}+00$ & $0.00 \mathrm{E}+00$ & $5.55 \mathrm{E}-02$ & 0.00 \\
\hline SNL/NM & $0.00 \mathrm{E}+00$ & $0.00 \mathrm{E}+00$ & $0.00 \mathrm{E}+00$ & $0.00 \mathrm{E}+00$ & $0.00 \mathrm{E}+00$ & $0.00 \mathrm{E}+00$ & 0.00 \\
\hline TBE & $0.00 \mathrm{E}+00$ & $0.00 \mathrm{E}+00$ & $0.00 \mathrm{E}+00$ & $0.00 \mathrm{E}+00$ & $0.00 \mathrm{E}+00$ & $0.00 \mathrm{E}+00$ & 0.00 \\
\hline USAMC & $0.00 \mathrm{E}+00$ & $0.00 \mathrm{E}+00$ & $1.80 \mathrm{E}+01$ & $0.00 \mathrm{E}+00$ & $0.00 \mathrm{E}+00$ & $1.80 \mathrm{E}+01$ & 0.00 \\
\hline Subtotal & $6.22 \mathrm{E}+00$ & $3.70 \mathrm{E}+02$ & $7.56 \mathrm{E}+01$ & $9.34 \mathrm{E}-01$ & $6.17 \mathrm{E}-01$ & $4.54 \mathrm{E}+02$ & 0.02 \\
\hline
\end{tabular}


Table 3.17 (continued)

\begin{tabular}{|c|c|c|c|c|c|c|c|}
\hline Site & ${ }^{241} \mathrm{Pu}$ & ${ }^{238} \mathrm{Pu}$ & ${ }^{239} \mathrm{Pu}$ & ${ }^{241} \mathrm{Am}$ & ${ }^{240} \mathrm{Pu}$ & $\begin{array}{l}\text { Total } \\
\text { curies }\end{array}$ & $\begin{array}{l}\text { Curies } \\
(\%)\end{array}$ \\
\hline \multicolumn{8}{|c|}{ Commercial site } \\
\hline WVDP & $3.43 \mathrm{E}+01$ & $0.00 \mathrm{E}+00$ & $0.00 \mathrm{E}+00$ & $2.15 \mathrm{E}+00$ & $0.00 \mathrm{E}+00$ & $3.65 \mathrm{E}+01$ & 0.00 \\
\hline Total (all sites) & $1.07 \mathrm{E}+06$ & $7.56 \mathrm{E}+05$ & $3.51 E+05$ & $2.40 \mathrm{E}+05$ & $6.88 \mathrm{E}+04$ & $2.49 \mathrm{E}+06$ & 99.23 \\
\hline Total curies, $\%$ & 42.83 & 30.12 & 13.99 & 9.55 & 2.74 & & \\
\hline Cumulative, $\%$ & 42.83 & 72.96 & 86.94 & 96.49 & 99.23 & & \\
\hline
\end{tabular}

aDecayed to the end of CY 1995. 
Table 3.18. Summary of decayed mass (g) isotopic content of retrievably stored CH TRUW

\begin{tabular}{|c|c|c|c|c|c|c|c|}
\hline Site & ${ }^{238} \mathrm{U}$ & ${ }^{239} \mathrm{Pu}$ & ${ }^{232} \mathrm{Th}$ & ${ }^{235} \mathrm{U}$ & ${ }^{240} \mathrm{Pu}$ & $\begin{array}{l}\text { Total } \\
\text { grams }\end{array}$ & $\begin{array}{c}\text { Grams } \\
(\%)\end{array}$ \\
\hline \multicolumn{8}{|c|}{ Major sites } \\
\hline ANL-E & $0.00 \mathrm{E}+00$ & $5.29 E+02$ & $0.00 \mathrm{E}+00$ & $0.00 \mathrm{E}+00$ & $4.15 \mathrm{E}+0 \mathrm{I}$ & $5.70 E+02$ & 0.00 \\
\hline Hanford & $1.74 \mathrm{E}+07$ & $4.24 E+05$ & $6.12 \mathrm{E}+05$ & $7.91 \mathrm{E}+05$ & $2.71 E+04$ & $1.93 \mathrm{E}+07$ & 65.49 \\
\hline INEL & $3.44 \mathrm{E}+05$ & $6.47 \mathrm{E}+05$ & $3.01 \mathrm{E}+06$ & $2.86 \mathrm{E}+04$ & $4.33 E+04$ & $4.07 \mathrm{E}+06$ & 13.84 \\
\hline LANL & $7.03 \mathrm{E}+04$ & $1.24 \mathrm{E}+06$ & $2.09 \mathrm{E}+04$ & $2.44 \mathrm{E}+05$ & $4.42 \mathrm{E}+02$ & $1.57 \mathrm{E}+06$ & 5.35 \\
\hline LLNL & $9.00 \mathrm{E}+04$ & $2.55 \mathrm{E}+03$ & $8.54 \mathrm{E}-09$ & $2.74 \mathrm{E}+02$ & $2.84 \mathrm{E}+02$ & $9.31 \mathrm{E}+04$ & 0.32 \\
\hline Mound & $0.00 \mathrm{E}+00$ & $4.81 \mathrm{E}+02$ & $0.00 \mathrm{E}+00$ & $1.24 \mathrm{E}-01$ & $0.00 \mathrm{E}+00$ & $4.81 \mathrm{E}+02$ & 0.00 \\
\hline NTS & $4.88 \mathrm{E}+02$ & $4.45 \mathrm{E}+04$ & $7.46 \mathrm{E}-09$ & $2.84 \mathrm{E}+01$ & $8.27 \mathrm{E}+01$ & $4.51 \mathrm{E}+04$ & 0.15 \\
\hline ORNL & $1.27 \mathrm{E}+05$ & $1.62 E+04$ & $7.82 \mathrm{E}+03$ & $3.77 \mathrm{E}+03$ & $4.18 \mathrm{E}+03$ & $1.59 E+05$ & 0.54 \\
\hline RFETS & $2.11 \mathrm{E}-02$ & $3.13 E+06$ & $2.41 E-05$ & $7.43 E+02$ & $2.18 \mathrm{E}+05$ & $3.35 \mathrm{E}+06$ & 11.39 \\
\hline SRS & $1.70 \mathrm{E}+04$ & $1.50 \mathrm{E}+05$ & $1.94 \mathrm{E}+05$ & $2.70 \mathrm{E}+03$ & $1.01 E+04$ & $3.74 \mathrm{E}+05$ & 1.27 \\
\hline Subtotal & $1.81 E+07$ & $5.66 \mathrm{E}+06$ & $3.85 E+06$ & $1.07 E+06$ & $3.03 E+05$ & $2.89 \mathrm{E}+07$ & 98.34 \\
\hline
\end{tabular}

Small-quantity sites (SQS)

\begin{tabular}{llllllll} 
Ames & $0.00 \mathrm{E}+00$ & $0.00 \mathrm{E}+00$ & $0.00 \mathrm{E}+00$ & $0.00 \mathrm{E}+00$ & $0.00 \mathrm{E}+00$ & $0.00 \mathrm{E}+00$ & 0.00 \\
ANL-W & $0.00 \mathrm{E}+00$ & $0.00 \mathrm{E}+00$ & $0.00 \mathrm{E}+00$ & $0.00 \mathrm{E}+00$ & $0.00 \mathrm{E}+00$ & $0.00 \mathrm{E}+00$ & 0.00 \\
ARCO & $0.00 \mathrm{E}+00$ & $0.00 \mathrm{E}+00$ & $0.00 \mathrm{E}+00$ & $0.00 \mathrm{E}+00$ & $0.00 \mathrm{E}+00$ & $0.00 \mathrm{E}+00$ & 0.00 \\
BCL & $0.00 \mathrm{E}+00$ & $0.00 \mathrm{E}+00$ & $0.00 \mathrm{E}+00$ & $0.00 \mathrm{E}+00$ & $0.00 \mathrm{E}+00$ & $0.00 \mathrm{E}+00$ \\
BAPL & $0.00 \mathrm{E}+00$ & $0.00 \mathrm{E}+00$ & $0.00 \mathrm{E}+00$ & $0.00 \mathrm{E}+00$ & $0.00 \mathrm{E}+00$ & $0.00 \mathrm{E}+00$ \\
ETEC & $1.39 \mathrm{E}-07$ & $2.89 \mathrm{E}+01$ & $1.47 \mathrm{E}-10$ & $4.89 \mathrm{E}-03$ & $2.70 \mathrm{E}+00$ & $3.16 \mathrm{E}+01$ & 0.00 \\
KAPL & $0.00 \mathrm{E}+00$ & $0.00 \mathrm{E}+00$ & $0.00 \mathrm{E}+00$ & $0.00 \mathrm{E}+00$ & $0.00 \mathrm{E}+00$ & $0.00 \mathrm{E}+00$ & 0.00 \\
LBL & $5.48 \mathrm{E}-05$ & $1.36 \mathrm{E}-04$ & $4.86 \mathrm{E}-12$ & $1.54 \mathrm{E}-08$ & $2.26 \mathrm{E}-02$ & $2.28 \mathrm{E}-02$ & 0.00 \\
MURR & $3.46 \mathrm{E}-01$ & $3.97 \mathrm{E}-01$ & $0.00 \mathrm{E}+00$ & $2.06 \mathrm{E}-05$ & $0.00 \mathrm{E}+00$ & $7.43 \mathrm{E}-01$ & 0.00 \\
PAD & $0.00 \mathrm{E}+00$ & $8.98 \mathrm{E}+02$ & $0.00 \mathrm{E}+00$ & $1.52 \mathrm{E}-01$ & $0.00 \mathrm{E}+00$ & $8.98 \mathrm{E}+02$ & 0.00 \\
PANT & $0.00 \mathrm{E}+00$ & $8.95 \mathrm{E}-01$ & $0.00 \mathrm{E}+00$ & $5.06 \mathrm{E}-05$ & $0.00 \mathrm{E}+00$ & $8.95 \mathrm{E}-01$ & 0.00 \\
SNL/NM & $0.00 \mathrm{E}+00$ & $0.00 \mathrm{E}+00$ & $0.00 \mathrm{E}+00$ & $0.00 \mathrm{E}+00$ & $0.00 \mathrm{E}+00$ & $0.00 \mathrm{E}+00$ & 0.00 \\
TBE & $0.00 \mathrm{E}+00$ & $0.00 \mathrm{E}+00$ & $0.00 \mathrm{E}+00$ & $0.00 \mathrm{E}+00$ & $0.00 \mathrm{E}+00$ & $0.00 \mathrm{E}+00$ & 0.00 \\
USAMC & $0.00 \mathrm{E}+00$ & $2.90 \mathrm{E}+02$ & $0.00 \mathrm{E}+00$ & $8.21 \mathrm{E}-03$ & $0.00 \mathrm{E}+00$ & $2.90 \mathrm{E}+02$ & 0.00 \\
\multicolumn{1}{c}{ Subtotal } & $3.46 \mathrm{E}-01$ & $1.22 \mathrm{E}+03$ & $1.52 \mathrm{E}-10$ & $1.66 \mathrm{E}-01$ & $2.72 \mathrm{E}+00$ & $1.22 \mathrm{E}+03$ & 0.00
\end{tabular}


Table 3.18 (continued)

\begin{tabular}{|c|c|c|c|c|c|c|c|}
\hline Site & ${ }^{238} \mathrm{U}$ & ${ }^{239} \mathrm{Pu}$ & ${ }^{232} \mathrm{Th}$ & ${ }^{235} \mathrm{U}$ & ${ }^{240} \mathrm{Pu}$ & $\begin{array}{l}\text { Total } \\
\text { grams }\end{array}$ & $\begin{array}{c}\text { Grams } \\
(\%)\end{array}$ \\
\hline \multicolumn{8}{|c|}{ Commercial site } \\
\hline WVDP & $0.00 E+00$ & $0.00 \mathrm{E}+00$ & $0.00 \mathrm{E}+00$ & $0.00 \mathrm{E}+00$ & $0.00 \mathrm{E}+00$ & $0.00 \mathrm{E}+00$ & 0.00 \\
\hline Total (all sites) & $1.81 \mathrm{E}+07$ & $5.66 \mathrm{E}+06$ & $3.85 \mathrm{E}+06$ & $1.07 \mathrm{E}+06$ & $3.03 \mathrm{E}+05$ & $2.89 E+07$ & 98.35 \\
\hline Total grams, \% & 61.39 & 19.22 & 13.07 & 3.64 & 1.03 & & \\
\hline Cumulative, \% & 61.39 & 80.62 & 93.68 & 97.32 & 98.35 & & \\
\hline
\end{tabular}

a Decayed to the end of CY 1995. 
Table 3.19. Summary of decayed radioactivity (Ci) isotopic content of retrievably stored RH TRUWa

\begin{tabular}{|c|c|c|c|c|c|c|c|c|c|c|c|}
\hline Site & ${ }^{90} \mathrm{Sr}$ & ${ }^{90} \mathrm{Y}$ & ${ }^{137} \mathrm{Cs}$ & ${ }^{137 / m} \mathrm{Ba}$ & ${ }^{241} \mathrm{Pu}$ & ${ }^{152} \mathrm{Eu}$ & ${ }^{154} \mathrm{Eu}$ & ${ }^{244} \mathrm{Cm}$ & ${ }^{60} \mathrm{Co}$ & $\begin{array}{l}\text { Total } \\
\text { curies }\end{array}$ & $\begin{array}{c}\text { Curies } \\
(\%)\end{array}$ \\
\hline \multicolumn{12}{|c|}{ Major sites } \\
\hline ANL-E & $0.00 \mathrm{E}+00$ & $0.00 \mathrm{E}+00$ & $0.00 \mathrm{E}+00$ & $0.00 \mathrm{E}+00$ & $0.00 \mathrm{E}+00$ & $0.00 \mathrm{E}+00$ & $0.00 \mathrm{E}+00$ & $0.00 \mathrm{E}+00$ & $0.00 \mathrm{E}+00$ & $0.00 E+00$ & 0.00 \\
\hline Hanford & $6.46 \mathrm{E}+03$ & $6.46 \mathrm{E}+03$ & $6.98 \mathrm{E}+03$ & $6.61 \mathrm{E}+03$ & $4.67 \mathrm{E}+03$ & $0.00 \mathrm{E}+00$ & $0.00 \mathrm{E}+00$ & $0.00 \mathrm{E}+00$ & $3.36 \mathrm{E}+02$ & $3.15 E+04$ & 22.68 \\
\hline INEL & $1.70 \mathrm{E}+03$ & $1.70 \mathrm{E}+03$ & $1.90 \mathrm{E}+03$ & $1.80 \mathrm{E}+03$ & $4.82 \mathrm{E}+01$ & $1.14 \mathrm{E}-01$ & $7.92 \mathrm{E}-01$ & $9.63 \mathrm{E}-02$ & $1.31 \mathrm{E}+01$ & $7.15 \mathrm{E}+03$ & 5.14 \\
\hline LANL & $1.24 \mathrm{E}+02$ & $1.24 \mathrm{E}+02$ & $1.35 \mathrm{E}+02$ & $1.28 \mathrm{E}+02$ & $0.00 \mathrm{E}+00$ & $5.09 \mathrm{E}-04$ & $3.50 \mathrm{E}-02$ & $0.00 \mathrm{E}+00$ & 4.17E+00 & $5.16 \mathrm{E}+02$ & 0.37 \\
\hline LLNL & $0.00 \mathrm{E}+00$ & $0.00 \mathrm{E}+00$ & $0.00 \mathrm{E}+00$ & $0.00 \mathrm{E}+00$ & $0.00 \mathrm{E}+00$ & $0.00 \mathrm{E}+00$ & $0.00 \mathrm{E}+00$ & $0.00 \mathrm{E}+00$ & $0.00 \mathrm{E}+00$ & $0.00 \mathrm{E}+00$ & 0.00 \\
\hline Mound & $0.00 \mathrm{E}+00$ & $0.00 \mathrm{E}+00$ & $0.00 \mathrm{E}+00$ & $0.00 \mathrm{E}+00$ & $0.00 \mathrm{E}+00$ & $0.00 \mathrm{E}+00$ & $0.00 \mathrm{E}+00$ & $0.00 \mathrm{E}+00$ & $0.00 \mathrm{E}+00$ & $0.00 \mathrm{E}+00$ & 0.00 \\
\hline NTS & $0.00 \mathrm{E}+00$ & $0.00 \mathrm{E}+00$ & $0.00 \mathrm{E}+00$ & $0.00 \mathrm{E}+00$ & $6.60 \mathrm{E}-05$ & $0.00 \mathrm{E}+00$ & $0.00 \mathrm{E}+00$ & $1.55 \mathrm{E}+02$ & $0.00 \mathrm{E}+00$ & $1.55 \mathrm{E}+02$ & 0.11 \\
\hline ORNL & $3.53 \mathrm{E}+04$ & $3.53 \mathrm{E}+04$ & $9.78 \mathrm{E}+03$ & $9.25 \mathrm{E}+03$ & $3.98 \mathrm{E}-07$ & $3.66 \mathrm{E}+03$ & $1.77 E+03$ & $9.44 \mathrm{E}+02$ & $6.17 \mathrm{E}+02$ & $9.65 \mathrm{E}+04$ & 69.47 \\
\hline RFETS & $0.00 \mathrm{E}+00$ & $0.00 \mathrm{E}+00$ & $0.00 \mathrm{E}+00$ & $0.00 \mathrm{E}+00$ & $0.00 \mathrm{E}+00$ & $0.00 \mathrm{E}+00$ & $0.00 \mathrm{E}+00$ & $0.00 \mathrm{E}+00$ & $0.00 \mathrm{E}+00$ & $0.00 \mathrm{E}+00$ & 0.00 \\
\hline SRS & $6.85 \mathrm{E}+00$ & $6.85 \mathrm{E}+00$ & $6.86 \mathrm{E}+00$ & $6.49 \mathrm{E}+00$ & $0.00 \mathrm{E}+00$ & $0.00 \mathrm{E}+00$ & $0.00 \mathrm{E}+00$ & $4.68 \mathrm{E}+00$ & $0.00 \mathrm{E}+00$ & $3.17 \mathrm{E}+01$ & 0.02 \\
\hline Subtotal & $4.35 E+04$ & $4.36 E+04$ & $1.88 \mathrm{E}+04$ & $1.78 \mathrm{E}+04$ & $4.71 \mathrm{E}+03$ & $3.66 \mathrm{E}+03$ & $1.77 \mathrm{E}+03$ & $1.10 \mathrm{E}+03$ & $9.71 \mathrm{E}+02$ & $1.36 E+05$ & 97.79 \\
\hline \multicolumn{12}{|c|}{ Small-quantity sites (SQS) } \\
\hline Ames & $0.00 \mathrm{E}+00$ & $0.00 \mathrm{E}+00$ & $0.00 \mathrm{E}+00$ & $0.00 \mathrm{E}+00$ & $0.00 \mathrm{E}+00$ & $0.00 \mathrm{E}+00$ & $0.00 \mathrm{E}+00$ & $0.00 \mathrm{E}+00$ & $0.00 \mathrm{E}+00$ & $0.00 \mathrm{E}+00$ & 0.00 \\
\hline ANL-W & $0.00 \mathrm{E}+00$ & $0.00 \mathrm{E}+00$ & $0.00 \mathrm{E}+00$ & $0.00 \mathrm{E}+00$ & $0.00 \mathrm{E}+00$ & $0.00 \mathrm{E}+00$ & $0.00 \mathrm{E}+00$ & $0.00 \mathrm{E}+00$ & $0.00 \mathrm{E}+00$ & $0.00 \mathrm{E}+00$ & 0.00 \\
\hline ARCO & $0.00 \mathrm{E}+00$ & $0.00 \mathrm{E}+00$ & $0.00 \mathrm{E}+00$ & $0.00 \mathrm{E}+00$ & $0.00 \mathrm{E}+00$ & $0.00 \mathrm{E}+00$ & $0.00 \mathrm{E}+00$ & $0.00 \mathrm{E}+00$ & $0.00 \mathrm{E}+00$ & $0.00 \mathrm{E}+00$ & 0.00 \\
\hline BCL & $0.00 \mathrm{E}+00$ & $0.00 \mathrm{E}+00$ & $0.00 \mathrm{E}+00$ & $0.00 \mathrm{E}+00$ & $0.00 \mathrm{E}+00$ & $0.00 \mathrm{E}+00$ & $0.00 \mathrm{E}+00$ & $0.00 \mathrm{E}+00$ & $0.00 \mathrm{E}+00$ & $0.00 E+00$ & 0.00 \\
\hline BAPL & $0.00 \mathrm{E}+00$ & $0.00 \mathrm{E}+00$ & $0.00 \mathrm{E}+00$ & $0.00 \mathrm{E}+00$ & $0.00 \mathrm{E}+00$ & $0.00 \mathrm{E}+00$ & $0.00 \mathrm{E}+00$ & $0.00 \mathrm{E}+00$ & $0.00 \mathrm{E}+00$ & $0.00 \mathrm{E}+00$ & 0.00 \\
\hline ETEC & $2.62 \mathrm{E}+00$ & $2.62 \mathrm{E}+00$ & $2.62 \mathrm{E}+00$ & $2.48 \mathrm{E}+00$ & $0.00 \mathrm{E}+00$ & $0.00 \mathrm{E}+00$ & $0.00 \mathrm{E}+00$ & $0.00 \mathrm{E}+00$ & $2.30 \mathrm{E}+00$ & $1.26 \mathrm{E}+01$ & 0.01 \\
\hline KAPL & $5.70 E+01$ & $5.70 E+01$ & $5.71 E+01$ & $5.40 \mathrm{E}+01$ & $7.77 \mathrm{E}-01$ & $0.00 \mathrm{E}+00$ & $1.40 \mathrm{E}+00$ & $0.00 \mathrm{E}+00$ & $2.75 E-01$ & $2.28 \mathrm{E}+02$ & 0.16 \\
\hline LBL & $0.00 \mathrm{E}+00$ & $0.00 \mathrm{E}+00$ & $0.00 \mathrm{E}+00$ & $0.00 \mathrm{E}+00$ & $0.00 \mathrm{E}+00$ & $0.00 \mathrm{E}+00$ & $0.00 \mathrm{E}+00$ & $0.00 \mathrm{E}+00$ & $0.00 \mathrm{E}+00$ & $0.00 \mathrm{E}+00$ & 0.00 \\
\hline MURR & $0.00 \mathrm{E}+00$ & $0.00 \mathrm{E}+00$ & $0.00 \mathrm{E}+00$ & $0.00 \mathrm{E}+00$ & $0.00 \mathrm{E}+00$ & $0.00 \mathrm{E}+00$ & $0.00 \mathrm{E}+00$ & $0.00 \mathrm{E}+00$ & $0.00 \mathrm{E}+00$ & $0.00 \mathrm{E}+00$ & 0.00 \\
\hline $\mathrm{PAD}$ & $0.00 \mathrm{E}+00$ & $0.00 \mathrm{E}+00$ & $0.00 E+00$ & $0.00 \mathrm{E}+00$ & $0.00 \mathrm{E}+00$ & $0.00 \mathrm{E}+00$ & $0.00 E+00$ & $0.00 \mathrm{E}+00$ & $0.00 \mathrm{E}+00$ & $0.00 E+00$ & 0.00 \\
\hline PANT & $0.00 \mathrm{E}+00$ & $0.00 \mathrm{E}+00$ & $0.00 \mathrm{E}+00$ & $0.00 \mathrm{E}+00$ & $0.00 \mathrm{E}+00$ & $0.00 \mathrm{E}+00$ & $0.00 \mathrm{E}+00$ & $0.00 \mathrm{E}+00$ & $0.00 \mathrm{E}+00$ & $0.00 \mathrm{E}+00$ & 0.00 \\
\hline SNL/NM & $0.00 \mathrm{E}+00$ & $0.00 \mathrm{E}+00$ & $0.00 \mathrm{E}+00$ & $0.00 \mathrm{E}+00$ & $0.00 \mathrm{E}+00$ & $0.00 \mathrm{E}+00$ & $0.00 \mathrm{E}+00$ & $0.00 \mathrm{E}+00$ & $0.00 \mathrm{E}+00$ & $0.00 \mathrm{E}+00$ & 0.00 \\
\hline TBE & $0.00 E+00$ & $0.00 \mathrm{E}+00$ & $0.00 \mathrm{E}+00$ & $0.00 \mathrm{E}+00$ & $0.00 \mathrm{E}+00$ & $0.00 \mathrm{E}+00$ & $0.00 \mathrm{E}+00$ & $0.00 \mathrm{E}+00$ & $0.00 \mathrm{E}+00$ & $0.00 \mathrm{E}+00$ & 0.00 \\
\hline USAMC & $0.00 \mathrm{E}+00$ & $0.00 \mathrm{E}+00$ & $0.00 \mathrm{E}+00$ & $0.00 \mathrm{E}+00$ & $0.00 \mathrm{E}+00$ & $0.00 \mathrm{E}+00$ & $0.00 \mathrm{E}+00$ & $0.00 \mathrm{E}+00$ & $0.00 \mathrm{E}+00$ & $0.00 \mathrm{E}+00$ & 0.00 \\
\hline Subtotal & $5.96 \mathrm{E}+01$ & $5.96 \mathrm{E}+01$ & $5.97 \mathrm{E}+01$ & $5.65 E+01$ & $7.77 \mathrm{E}-01$ & $0.00 \mathrm{E}+00$ & $1.40 \mathrm{E}+00$ & $0.00 \mathrm{E}+00$ & $2.58 \mathrm{E}+00$ & $2.41 E+02$ & 0.17 \\
\hline
\end{tabular}


Table 3.19 (continued)

\begin{tabular}{|c|c|c|c|c|c|c|c|c|c|c|c|}
\hline Site & ${ }^{90} \mathrm{Sr}$ & ${ }^{90} \mathrm{Y}$ & ${ }^{137} \mathrm{Cs}$ & ${ }^{137} \mathrm{~m} \mathrm{Ba}$ & ${ }^{241} \mathrm{Pu}$ & ${ }^{152} \mathrm{Eu}$ & ${ }^{154} \mathrm{Eu}$ & ${ }^{214} \mathrm{Cm}$ & ${ }^{60} \mathrm{Co}$ & $\begin{array}{l}\text { Total } \\
\text { curies }\end{array}$ & $\begin{array}{c}\text { Curies } \\
(\%)\end{array}$ \\
\hline \multicolumn{12}{|c|}{ Commercial site } \\
\hline WVDP & $1.96 \mathrm{E}+01$ & $1.96 \mathrm{E}+01$ & $5.35 \mathrm{E}+01$ & $5.06 \mathrm{E}+01$ & $0.00 \mathrm{E}+00$ & $0.00 \mathrm{E}+00$ & $0.00 \mathrm{E}+00$ & $0.00 \mathrm{E}+00$ & $0.00 \mathrm{E}+00$ & $1.43 \mathrm{E}+02$ & 0.10 \\
\hline Total (all sites) & $4.36 E+04$ & $4.36 \mathrm{E}+04$ & $1.89 \mathrm{E}+04$ & $1.79 \mathrm{E}+04$ & $4.71 E+03$ & $3.66 \mathrm{E}+03$ & $1.77 \mathrm{E}+03$ & $1.10 \mathrm{E}+03$ & $9.73 \mathrm{E}+02$ & $1.36 \mathrm{E}+05$ & 98.07 \\
\hline Total curies, $\%$ & 31.39 & 31.40 & 13.61 & 12.87 & 3.39 & 2.64 & 1.28 & 0.79 & 0.70 & 98.07 & \\
\hline Cumulative, $\%$ & 31.39 & 62.79 & 76.40 & 89.27 & 92.66 & 95.30 & 96.57 & 97.37 & 98.07 & & \\
\hline
\end{tabular}

a Decayed to the end of CY 1995. 
Table 3.20. Summary of decayed mass ( $g$ ) isotopic content of retrievably stored RH TRUW

\begin{tabular}{lcr}
\hline Site & \multicolumn{2}{c}{$\begin{array}{c}\text { Grams } \\
(\%)\end{array}$} \\
\hline & Major sites & \\
& & \\
ANL-E & $0.00 \mathrm{E}+00$ & 0.00 \\
Hanford & $3.05 \mathrm{E}+04$ & 0.03 \\
INEL & $4.11 \mathrm{E}+03$ & 0.00 \\
LANL & $5.95 \mathrm{E}+01$ & 0.00 \\
LLNL & $0.00 \mathrm{E}+00$ & 0.00 \\
Mound & $0.00 \mathrm{E}+00$ & 0.00 \\
NTS & $7.57 \mathrm{E}-12$ & 0.00 \\
ORNL & $9.07 \mathrm{E}+07$ & 98.52 \\
RFETS & $0.00 \mathrm{E}+00$ & 0.00 \\
SRS & $0.00 \mathrm{E}+00$ & 0.00 \\
\multicolumn{1}{c}{ Subtotal } & - & -0.56
\end{tabular}

Small-quantity sites (SQS)

\begin{tabular}{lll} 
Ames & $0.00 \mathrm{E}+00$ & 0.00 \\
ANL-W & $0.00 \mathrm{E}+00$ & 0.00 \\
ARCO & $0.00 \mathrm{E}+00$ & 0.00 \\
BCL & $0.00 \mathrm{E}+00$ & 0.00 \\
BAPL & $0.00 \mathrm{E}+00$ & 0.00 \\
ETEC & $0.00 \mathrm{E}+00$ & 0.00 \\
KAPL & $1.02 \mathrm{E}-08$ & 0.00 \\
LBL & $0.00 \mathrm{E}+00$ & 0.00 \\
MURR & $0.00 \mathrm{E}+00$ & 0.00 \\
PAD & $0.00 \mathrm{E}+00$ & 0.00 \\
PANT & $0.00 \mathrm{E}+00$ & 0.00 \\
SNL $/ \mathrm{NM}$ & $0.00 \mathrm{E}+00$ & 0.00 \\
TBE & $0.00 \mathrm{E}+00$ & 0.00 \\
USAMC & $0.00 \mathrm{E}+00$ & 0.00 \\
\hline & & \\
Subtotal & $1.02 \mathrm{E}-08$ & 0.00
\end{tabular}

Commercial site

\begin{tabular}{lcc} 
WVP & $\frac{0.00 \mathrm{E}+00}{}$ & 0.00 \\
\cline { 2 - 2 } Total (all sites) & $9.07 \mathrm{E}+07$ & 98.56 \\
Total grams, \% & 98.56 & \\
Cumulative, \% & $9.86 \mathrm{E}-01$ & \\
\hline
\end{tabular}

a Decayed to the end of CY 1995. 
Table 3.21. Summary of buried TRUW volume and radioactivity as of December 31, 1994

\begin{tabular}{|c|c|c|c|c|c|}
\hline \multirow[b]{2}{*}{ Site } & \multirow{2}{*}{$\begin{array}{c}\text { Cumulative } \\
\text { volume } \\
\left(\mathrm{m}^{3}\right)\end{array}$} & \multicolumn{2}{|c|}{$\begin{array}{l}\text { Cumulative as-stored } \\
\text { radioactivity }\end{array}$} & \multicolumn{2}{|c|}{ Decayed radioactivity } \\
\hline & & $\begin{array}{c}\text { All } \\
\text { nuclides } \\
\left(10^{3} \mathrm{Ci}\right)\end{array}$ & $\begin{array}{c}\text { TRU } \\
\text { only } \\
\left(10^{3} \mathrm{Ci}\right)\end{array}$ & $\begin{array}{c}\text { All } \\
\text { nuclides } \\
\left(10^{3} \mathrm{Ci}\right)\end{array}$ & $\begin{array}{c}\text { TRU } \\
\text { only } \\
\left(10^{3} \mathrm{Ci}\right)\end{array}$ \\
\hline \multicolumn{6}{|c|}{ Major sites } \\
\hline ANL-E & $0.00 E+00$ & $0.00 \mathrm{E}+00$ & $0.00 \mathrm{E}+00$ & $0.00 \mathrm{E}+00$ & $0.00 E+00$ \\
\hline Hanford & $6.36 E+04$ & $6.02 \mathrm{E}+02$ & $1.14 \mathrm{E}+02$ & $1.73 \mathrm{E}+02$ & $9.38 \mathrm{E}+01$ \\
\hline NNEL & $5.70 \mathrm{E}+04$ & $2.49 E+02$ & b & b & b \\
\hline LANL & $1.40 E+04$ & c & d & c & c \\
\hline LINL & $0.00 \mathrm{E}+00$ & $0.00 \mathrm{E}+00$ & $0.00 \mathrm{E}+00$ & $0.00 \mathrm{E}+00$ & $0.00 \mathrm{E}+00$ \\
\hline Mound & $0.00 \mathrm{E}+00$ & $0.00 E+00$ & $0.00 \mathrm{E}+00$ & $0.00 \mathrm{E}+00$ & $0.00 E+00$ \\
\hline NTS & $0.00 \mathrm{E}+00$ & $0.00 E+00$ & $0.00 \mathrm{E}+00$ & $0.00 \mathrm{E}+00$ & $0.00 \mathrm{E}+00$ \\
\hline ORNL & $1.76 \mathrm{E}+02$ & $2.40 \mathrm{E}-01$ & $1.00 \mathrm{E}-01$ & $5.43 E+02$ & $2.00 \mathrm{E}-01$ \\
\hline RFETS & $0.00 E+00$ & $0.00 \mathrm{E}+00$ & $0.00 \mathrm{E}+00$ & $0.00 \mathrm{E}+00$ & $0.00 \mathrm{E}+00$ \\
\hline SRS & $4.87 \mathrm{E}+03$ & $3.37 \mathrm{E}+01$ & $3.37 \mathrm{E}+01$ & $3.05 E+01$ & $3.05 E+01$ \\
\hline Subtotal & $1.40 \mathrm{E}+05$ & $8.84 \mathrm{E}+02$ & $1.48 \mathrm{E}+02$ & $7.47 \mathrm{E}+02$ & $1.25 \mathrm{E}+02$ \\
\hline \multicolumn{6}{|c|}{ Small-quantity sites (SQS) } \\
\hline Ames & $0.00 E+00$ & $0.00 \mathrm{E}+00$ & $0.00 \mathrm{E}+00$ & $0.00 E+00$ & $0.00 \mathrm{E}+00$ \\
\hline ANL-W & $0.00 \mathrm{E}+00$ & $0.00 \mathrm{E}+00$ & $0.00 \mathrm{E}+00$ & $0.00 E+00$ & $0.00 E+00$ \\
\hline ARCO & $0.00 \mathrm{E}+00$ & $0.00 E+00$ & $0.00 \mathrm{E}+00$ & $0.00 \mathrm{E}+00$ & $0.00 \mathrm{E}+00$ \\
\hline $\mathrm{BCL}$ & $0.00 \mathrm{E}+00$ & $0.00 \mathrm{E}+00$ & $0.00 \mathrm{E}+00$ & $0.00 \mathrm{E}+00$ & $0.00 \mathrm{E}+00$ \\
\hline BAPL & $0.00 \mathrm{E}+00$ & $0.00 \mathrm{E}+00$ & $0.00 \mathrm{E}+00$ & $0.00 \mathrm{E}+00$ & $0.00 \mathrm{E}+00$ \\
\hline ETEC & $0.00 E+00$ & $0.00 \mathrm{E}+00$ & $0.00 \mathrm{E}+00$ & $0.00 E+00$ & $0.00 \mathrm{E}+00$ \\
\hline KAPL & $0.00 E+00$ & $0.00 E+00$ & $0.00 E+00$ & $0.00 E+00$ & $0.00 \mathrm{E}+00$ \\
\hline LBL & $0.00 E+00$ & $0.00 \mathrm{E}+00$ & $0.00 E+00$ & $0.00 E+00$ & $0.00 \mathrm{E}+00$ \\
\hline MURR & $0.00 \mathrm{E}+00$ & $0.00 \mathrm{E}+00$ & $0.00 \mathrm{E}+00$ & $0.00 E+00$ & $0.00 E+00$ \\
\hline PAD & $0.00 E+00$ & $0.00 \mathrm{E}+00$ & $0.00 \mathrm{E}+00$ & $0.00 \mathrm{E}+00$ & $0.00 \mathrm{E}+00$ \\
\hline PANT & $0.00 \mathrm{E}+00$ & $0.00 E+00$ & $0.00 E+00$ & $0.00 E+00$ & $0.00 E+00$ \\
\hline SNL/NM & $1.33 E+00$ & $0.00 E+00$ & $0.00 \mathrm{E}+00$ & $0.00 \mathrm{E}+00$ & $0.00 E+00$ \\
\hline TBE & $0.00 E+00$ & $0.00 \mathrm{E}+00$ & $0.00 \mathrm{E}+00$ & $0.00 E+00$ & $0.00 \mathrm{E}+00$ \\
\hline USAMC & $0.00 E+00$ & $0.00 E+00$ & $0.00 E+00$ & $0.00 \mathrm{E}+00$ & $0.00 E+00$ \\
\hline Subtotal & $1.33 \mathrm{E}+00$ & $0.00 \mathrm{E}+00$ & $0.00 \mathrm{E}+00$ & $0.00 \mathrm{E}+00$ & $0.00 \mathrm{E}+00$ \\
\hline \multicolumn{6}{|c|}{ Commercial site } \\
\hline WVDP & $1.35 \mathrm{E}+03$ & $6.52 E+02$ & $6.52 \mathrm{E}+02$ & $0.00 \mathrm{E}+00$ & $0.00 \mathrm{E}+00$ \\
\hline Total & $1.41 E+05$ & $1.54 E+03$ & $8.00 E+02$ & $7.47 E+02$ & $1.25 E+02$ \\
\hline
\end{tabular}

${ }^{a}$ Any small differences in volumes reported in this table and in TWBIR, Rev. 3 and in the numerical totals shown result from rounding.

bNEL data did not include any isotopic compositions, so no TRU isotope or decay calculations could be made.

Information not available.

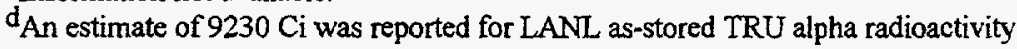
(as of the end of CY 1991) in Integrated Data Base for 1992: U.S. Spent Fuel and Radioactive Waste Inventories, Projections, and Characteristics, DOE/RW-0006, Rev. 8 (October 1992). 


\section{LOW-LEVEL WASTE}

\subsection{INTRODUCTION}

As used in this chapter, LLW has the same meaning as in The Low-Level Waste Policy Act (Pub. L. 95-573, Dec. 22, 1980). Namely, LLW is radioactive waste not classified as high-level radioactive waste, transuranic waste (TRUW), spent nuclear fuel (SNF), or by-product material specified as uranium or thorium tailings and waste. Tailings (viz., mill tailings) are considered in Chapters 5 and 6 . Another waste classification not delineated in this chapter is "mixed" low-level waste (MLLW), which contains both chemically hazardous and radioactive constituents (see Chapter 8 ). Specific definitions of these waste types (as defined by DOE Order $5820.2 \mathrm{~A}$ ) are given in the glossary of this report. DOE generates LLW through its defense activities, naval nuclear propulsion program, and various $R \& D$ activities. The data for $D O E$ sites represent a summary of information obtained from each site.'

Disposal of LLW at commercial sites accounted for about 21 vol \% of the LLW disposed during 1995. In this chapter, commercial sites exclude the Envirocare site which is treated separately because it has not only commercial and DOE wastes, but also wastes from activities sponsored by other (non-DOE) federal agencies. Commercially disposed LLW is generally divided into five categories: academic, government, industrial, medical, and utility. ${ }^{2}$ The academic category includes university hospitals and university medical and nonmedical research facilities. The government category includes state and non-DOE federal agencies. The industrial category is comprised of private entities such as R\&D companies, manufacturers, nondestructive-testing operations, mining works, fuel fabrication facilities, and radiopharmaceutical manufacturers. The medical category includes hospitals and clinics, research facilities, and private medical offices. The utility category includes commercial nuclear reactors. In earlier revisions of the IDB report, commercially disposed waste was reported by fuel cycle and industrial/institutional (//) type activities. However, to achieve more consistency with other reporting agencies, the five categories previously described were used, starting with Rev. 9 of the IDB report.
Some LLW is also disposed of at the Envirocare facility located in Clive, Utah. Envirocare is a commercially operated facility that disposes of $\mathrm{LLW}$, MLLW, NARM/NORM, and 11 e(2) by-product material for both federal and private customers (see Table 0.8). The volume of $L L W$ disposed of at Envirocare is presented in Table 4.1. Radioactivity data were not available. Envirocare accounted for about $27 \mathrm{vol} \%$ of the LLW disposed during 1995.

Some LLW is also generated by DOE environmental restoration programs (see Chapter 6). Other LLW will be generated in future years by nonroutine $\mathrm{D} \& \mathrm{D}$ operations. Waste from past commercial D\&D operations is included in the disposed commercial waste inventories reported in this chapter.

The categorization of LLW according to DOE and commercial activities permits a comparison of the radioactivity levels and volumes of waste arising from each of these major sources (Figs. 4.1 and 4.2). Envirocare is not included in the comparisons presented in Fig. 4.1 since radioactivity data were not available. Summary data on LLW disposal are given in Table 4.1. Historical and projected data by year for DOE LLW disposal are presented in Table 4.2. In Table 4.3, similar data are shown for commercial LLW disposal.

\subsection{DOE LLW}

\subsubsection{Inventories of LLW at DOE Sites}

An abridged picture of DOE LLW inventories, projections, and characteristics through 1995 is given in Figs. 4.1-4.4, as well as Tables 4.1, 4.2, and 4.4-4.18. The data in these tables are derived from responses to questionnaires submitted to DOE sites. ${ }^{l}$ As reflected in the tables, DOE LLW data can be grouped into three major areas: generation, storage, and disposal.

Summaries of DOE site-generated LLW volumes and associated radioactivity are presented in Tables 4.6, 4.9, and 4.10 . 
Tables $4.7,4.11$, and 4.12 provide summary volume and radioactivity data concerning LLW storage at DOE sites.

A summary of DOE LLW disposed volume and radioactivity is presented in Tables 4.1, 4.2, 4.4, 4.8, 4.13, 4.14, and 4.17. Before October 1979, some LLW generated by DOE contractors was shipped to commercial disposal sites. The volume and radioactivity data for DOE LLW that were shipped to commercial disposal sites are contained in the commercial LLW tables in this chapter. Currently, LLW generated by DOE activities is generally disposed of at DOE sites (see Figs. 4.3 and 4.4). It should be noted that the DOE Environmental Restoration Program (discussed in Chapter 6) is currently using, and will likely expand its use of, commercial LLW disposal facilities.

Simall quantities of DOE LIW have been disposed of by dumping the LLW into the sea or by hydrofracture. ${ }^{3}$ Table 4.15 shows the estimated quantity and radioactivity of $L L W$ disposed of by these methods. Dumping of LLW into the sea was halted by the United States in 1970, and hydrofracture was terminated in 1983.

\subsubsection{Characterization of LLW at DOE Sites}

Based on information reported in ref. 1, summaries of physical characteristics of DOE LLW are reported in Tables 4.6-4.14. Table 4.5 provides definitions of each of the physical forms used in the tables. For DOE site activities sponsored by the Office of Waste Management (EM-30), this set of physical forms provides a detailed description of the LLW that has been generated, stored, or disposed. However, the DOE Environmental Restoration Prograrn (EM-40) has large volumes of LLW currently in storage (such as unsolidified sludges and resins) which, except for the "other" category, do not fit into the categories described in Table 4.5. Actual 1995 and projected generation, storage, and disposal volume and radioactivity data are presented in Tables 4.6-4.8, respectively. A more detailed breakdown of this data, by DOE site, is provided in Tables 4.9-4.14.

In addition to shallow-land burial, DOE LLW has been disposed by dumping wastes into the sea and hydrofracture (Table 4.15). Most of the DOE wastes that were dumped into the sea were incorporated into cement matrix material and packaged in steel drums (55- or 80-gal capacity). Hydrofracture was developed at ORNL for the permanent disposal of locally generated, low-level (approximately $0.25 \mathrm{Ci} / \mathrm{L}$ ) liquid waste concentrates. ${ }^{4}$ Waste was mixed with a blend of cement and other additives, and the resulting grout was injected into shale at a depth of 200 to $300 \mathrm{~m}$. The injected grout hardened into thin, horizontal sheets several hundred meters wide.
Significant changes in DOE LLW inventory and characteristics data from that reported in Rev. 11 of this report are summarized in Table 4.16.

\subsubsection{DOE LLW Projections}

Low-level waste generation, storage, and disposal projections reported by DOE sites are presented in Tables 4.6-4.8, respectively. Projections are provided for three time periods: $1996,1997-2001$, and 2002-2030. The data presented in Tables 4.6-4.8 represent the average annual generation, addition to storage, or disposal, respectively, during each of the three periods.

The projected disposal data (Table 4.8), combined with actual 1995 (Tables 4.8, 4.13, and 4.14) and historical (ref. 5) disposal data, were used to calculate historical and projected volume, radioactivity, and thermal power of DOE disposed LLW. LLW data were collected by a different breakdown in previous revisions of the $\mathrm{IB}$ report (Revs. 1-10). Historical disposal data through 1993 were decayed from the year of addition through 2030 using the representative radionuclide compositions given in Table A.2 of Appendix A. ${ }^{6}$ Beginning with 1994, disposal data were decayed from the year of addition through 2030 using radionuclide compositions provided by each disposal site in the data call for Rev. 11 of this report.

Projected volume, radioactivity, and thermal power for disposed DOE LLW are presented in Tables 4.2, 4.17, and 4.18. Table 4.17 summarizes disposed DOE LLW excluding SRS saltstone. Table 4.18 summarizes projections of saltstone, an LLW by-product from the solidification of HLW at SRS. This saltstone (see Fig. 2.7 in Chapter 2) is to be stored in concrete vaults at SRS. Table 4.2 is the summation of Tables 4.17 and 4.18. Grout-immobilized LLW derived from processing doubleshell waste at Hanford (see Fig. 2.5 in Chapter 2) is excluded from the projections in Table 4.2 because the schedule and formulation for immobilization are not yet firmly defined.

\subsection{COMMERCIAL LLW}

\subsubsection{Inventories at Commercial LLW Disposal Sites}

There are six commercial shallow-land disposal sites for LIW (Figs. 4.2, 4.5, and 4.6), but only two are currently in operation: Barnwell, South Carolina; and Richland, Washington. Commercial operations at the Maxey Flats, Kentucky; West Valley, New York; Sheffield, Illinois; and Beatty, Nevada, sites have been 
halted. Until 1986, a second NRC-licensed burial ground at West Valley continued to receive wastes generated on-site from cleamup and water treatment operations. However, disposal operations at the WVDP have been suspended since 1986 pending the preparation of an environmental impact statement (EIS) report for the West Valley site closure. The historical data for annual additions and inventories of volume and radioactivity (undecayed) at each commercial disposal site through the end of 1995 are listed in Tables 4.19 and 4.20, respectively (compiled from refs. 5 and 7). The volumes are depicted in Figs. 4.2, 4.5, and 4.6. Sources of the historical reported data through 1984 are given in ref. 3, and those through 1994 are given in ref. 5. Quantities of LLW shipped to disposal sites during 1995 are listed in Table 4.21 on a state-by-state basis. ${ }^{7}$ Table 4.3 is a summary of historical and projected volumes, radioactivity (decayed), and thermal power for commercial LLW. Projections are made only through 2005 because of large uncertainties in commercial disposal facility operations. Not included in Table 4.3 are the drums of cemented LLW to be generated by the WVDP as a result of the vitrification of HLW. This LLW from the WVDP is described in Table A. 5 of Appendix A. Table 4.3 also does not include $25,041 \mathrm{~m}^{3}\left(11,698 \mathrm{~m}^{3}\right.$ from DOE generators, $12,860 \mathrm{~m}^{3}$ from commercial generators, and $483 \mathrm{~m}^{3}$ from other federal agency generators) of LLW disposed of at the Envirocare disposal facility near Clive, Utah. Additional information on Envirocare disposals can be found in Table 0.8 of Chapter 0 .

\subsubsection{Characterization of LLW at Commercial Disposal Sites}

All of the LLW accepted for commercial disposal is categorized as Class A, B, or C in compliance with NRC specifications. ${ }^{8}$ The LLW that exceeds these specifications is currently in storage at the generator site or at a DOE site which has accepted it for study (see Sect. 4.3.3). A calculated representative radionuclide composition for disposed commercial LLW is given in Table A.3 of Appendix A. This composition is periodically updated to reflect changes in waste management practices and in the regulations governing $L L W$ disposal.

\subsubsection{Greater-Than-Class-C Low-Level Waste (GTCC LLW)}

In 1980, federal law made each state responsible for providing the disposal capacity for LLW generated within its borders, except for certain waste generated by the federal government. ${ }^{9}$ In 10 CFR Part 61 (ref. 8), the NRC codifies disposal requirements for three classes of LLW, as mentioned above, generally suitable for near-surface disposal, namely, Class A, B, and C (with Class C waste requiring the most rigorous disposal specifications). Waste with concentrations above Class $\mathrm{C}$ limits for certain short- and long-lived radionuclides (i.e., GTCC LLW) was found not generally suitable for near-surface disposal, except on a case-by-case evaluation of the waste and the proposed disposal method by NRC or state licensing agency. The Low-Level Radioactive Waste Policy Amendments Act (LLRWPAA) ${ }^{10}$ made the states responsible for the disposal of Classes $A, B$, and CLLW and made the federal government (viz., DOE) responsible for disposal of GTCC LLW. The law also required that GTCC LLW generated by licensees of NRC be disposed of in a facility licensed by NRC. The projected amounts of GTCC LLW are uncertain, both because of regulatory uncertainties affecting the definition of HLW (i.e., a clearly defined all-inclusive list of wastes considered HLW may include more than those described in Chapter 2) and because of limited information on the sources, volumes, and characteristics of GTCC LLW."

In May 1989, NRC promulgated a rule that requires disposal of GTCC LLW in a deep geologic repository unless disposal elsewhere has been approved by NRC. The rule as amended states: "Waste that is not generally acceptable for near-surface disposal is waste for which form and disposal methods must be different and, in general, more stringent than those specified for Class $C$ waste. In the absence of specific requirements in this part, such waste must be disposed of in a geologic repository as defined in Part 60 of this chapter unless proposals for disposal of such waste in a disposal site licensed pursuant to this part are approved by the Commission."12 A disposal facility (other than a deep geologic repository) for GTCC LLW will probably not be available for several decades because of the complexities of siting and of NRC licensing. A generic description of estimated sources and forms of GTCC LLW is presented in Table A.4 of Appendix A.

DOE recently revised an earlier study to provide information about estimates of current and future GTCC LLW to the year 2035 (2055 in some instances). Information garnered during the study ${ }^{13}$ includes identification of generators, waste form characteristics, volumes, and radionuclide activities. The study categorizes GTCC LLW as (1) nuclear utilities waste, (2) sealed-sources waste, (3) DOE-held potential GTCC LLW, and (4) other generator waste. Various scenarios for data projection were used: (a) unpackaged volumes; (b) packaged volumes based on the application of packaging factors to the unpackaged volumes; and (c) concentration averaging, mixing, or blending of similar materials with different radionuclide concentration values 
applied to the packaged volumes. Each of the three scenarios is treated for three cases: low, base, and high.

Based on base-case projections of after-concentrationaveraged-packaged waste to the year 2035 , the study deternnined that the largest volume of GTCC wastes (approximately 53\%) is generated by nuclear power plants. The "other" generator waste category contributes approximately $31 \%$ of the total GTCC LLW volume projected to the year 2035 . Sealed sources are about $16 \%$ of the total projected volume of GTCC LLW. A summary of historical and projected cumulative volume and radioactivity for GTCC LLW is presented in Table 4.22.

\subsubsection{Commercial LLW Disposal Sites}

Of the six commercial LLW disposal sites, only two remain open: Barnwell and Richland. Historical information regarding commercial LLW facilities can be found in previous editions of the $\mathrm{IBB}$ report. Closure dates for the four closed facilities are presented in the footnotes of Tables 4.19 and 4.20 .

The Barnwell, South Carolina, disposal site is operated by Chem-Nuclear Systems, Inc. During 1995, Barnwell received about $70 \%$ of the total volume of commercial LLW shipped for disposal. Barnwell was scheduled to restrict access to Southeast compact members starting July 1, 1995; however, Barnwell withdrew from the Southeast compact. As a result, Barnwell is open for disposal to all states with the exception of North Carolina.
The Richland, Washington, disposal site is operated by U.S. Ecology, Inc. During 1995, Richland received about $30 \%$ of the total volume of commercial LLW shipped for disposal. Richland accepted wastes only from member states of the Northwest and Rocky Mountain compacts (Alaska, Colorado, Hawaii, Idaho, Montana, Nevada, New Mexico, Oregon, Washington, and Wyoming).

Table 4.23 provides a breakdown of waste received at Barnwell and Richland in 1995 by category (i.e., academic, government, industrial, medical, or utility LLW).

\subsubsection{Commercial LLW Projections}

This report presents summary information for disposed commercial waste. Historical volume, radioactivity, and thermal power data through 1979 are taken from an earlier version of this report (DOE/RW-0006, Rev. 8). After 1979, the source term for commercial LLW in Table A.4 of Appendix $A$ is used to decay the annual waste additions to the commercial sites.

Projections for disposed commercial LLW are made only through 2005 because of uncertainties in current facility operations and the availability of future sites. Neither Barnwell nor Richland have a published closure date. ${ }^{7}$ As a result, for projections, the disposal rates for 1996-2005 are assumed to be the same as the rates reported for 1995. Historical and projected volume, radioactivity, and thermal power for disposed commercial LLW are presented in Table 4.3.

\subsection{REFERENCES}

1. DOE site LLW data submittal attachments, submitted to the IDB Program during September-November 1996. The following LLW submittals were received and reviewed by the IDB Program before analysis and integration. Preceding each submittal is the site (in parentheses) to which it refers.

a. (Ames) Kay Lampe Hannasch, Ames Laboratory, Ames, Iowa, correspondence to Alan Icenhour or Steve Storch, IDB Program, ORNL, Oak Ridge, Tennessee, "Low-Level Waste Data Request for Integrated Data Base Report," dated Sept. 10, 1996.

b. (ANL-E) R.F. Coley, Argonne National Laboratory, Argonne, Illinois, correspondence to Timothy S. Crawford, DOE Argonne Group Manager, Argonne, Illinois, "Low-Level Waste Data Request for the Integrated Data Base Report," dated Sept. 19, 1996.

c. (ANL-W) See reference for INEL.

d. (BNL) Angela C. Harvey, DOE Brookhaven Area Office, Upton, New York, correspondence to Steven Storch, IDB Program, ORNL, Oak Ridge, Tennessee, "Low-Level Waste Data for Brookhaven National Laboratory," dated Sept. 26, 1996. 
e. (ETEC) M. Lee, Rockwell International Corporation, Rocketdyne Division, Canoga Park, California, correspondence to Alan Icenhour or Steve Storch, IDB Program, ORNL, "ETEC/Rocketdyne Low-Level Waste Data for Integrated Data Base (IDB) Report," dated Sept. 12, 1996.

f. (FNAL) Andrew E. Mravca, DOE Fermi Group, Batavia, Illinois, correspondence to Alan Icenhour, IDB Program, ORNL, Oak Ridge, Tennessee, "Low-Level Waste Data Request for Integrated Data Base Report," dated Sept. 13, 1996.

g. (Hanford) D. E. McKenney, Westinghouse Hanford Company, Richland, Washington, correspondence to T. K. Teynor, DOE Richland Operations Office, Richland, Washington, "Low-Level Waste Data Request for the Integrated Data Base Report," 9602190 R1, dated Sept. 20, 1996.

h. (INEL) Roger R. Piscitella, Idaho National Engineering Laboratory, Idaho Falls, Idaho, correspondence to Steve Storch, IDB Program, ORNL, Oak Ridge, Tennessee, "Idaho National Engineering Laboratory (INEL) Input to Integrated Data Base (IDB) Report (DOE/RW-0006, Rev. 12)," RRP-08-96, dated Sept. 20, 1996.

i. (ITRD) Stephen R Rohrer, Lovelace Biomedical and Environmental Research Institute, Inc., Albuquerque, New Mexico, correspondence to Alan Icenhour, IDB Program, ORNL, Oak Ridge, Tennessee, transmitting updated ITRI LLW information and data, dated Aug. 28, 1996.

j. (K-25) A. L. Rivera, Lockheed Martin Energy Systems, Inc., Oak Ridge, Tennessee, correspondence to S. N. Storch, DB Program, ORNL, Oak Ridge, Tennessee, "1996 Low-Level Waste (LLW) Management Data Call," dated Sept. 30, 1996.

k. (KCP) Margaret E. Stockdale, U.S. Department of Energy, Kansas City Area Office, Kansas City, Missouri, correspondence to Steve Storch, IDB Program, ORNL, Oak Ridge, Tennessee, "LLW Integrated Data Base," dated Nov. 14, 1996.

1. (LANL) I-Li Chen, Los Alamos National Laboratory, Los Alamos, New Mexico, correspondence to Alan Icenhour and Steve Storch, IDB Program, ORNL, Oak Ridge, Tennessee, "For 1996 Low-Level Waste," CST-14-96-139, Sept. 18, 1996.

m. (LBL) Robin Wendt, Lawrence Berkeley Laboratory, Berkeley, California, correspondence to Steve Storch, IDB Program, ORNL, Oak Ridge, Tennessee, "LLW Data Request for IDB," WM-96-256, dated Sept. 12, 1996.

n. (LLNL) Richard W. Crawford, Lawrence Liverinore National Laboratory, Livermore, California, correspondence to Alan Icenhour or Steve Storch, IDB Program, ORNL, Oak Ridge, Tennessee, transmitting updated LLNL LLW information and data, dated Sept. 20, 1996.

o. (MOUND) James J. Zahora, EG\&G Mound Applied Technologies, Miamisburg, Ohio, correspondence to Robert S. Rothman, DOE Miamisburg Area Office, Miamisburg, Ohio, "Low-level Waste Management Data Call," dated Oct. 16, 1996.

p. (NR sites) E. D. Shollenberger, Pittsburgh Naval Reactors Office, West Mifflin, Pennsylvania, correspondence to Alan Icenhour, IDB Program, ORNL, Oak Ridge, Tennessee, "Low-Level Waste Information for the 1996 Integrated Data Base Report," dated Oct. 24, 1996.

(NR sites) A. R. Seepo, Schenectady Naval Reactors Office, Schenectady, New York, correspondence to Alan Icenhour, IDB Program, ORNL, Oak Ridge, Tennessee, "Low-Level Waste Information for the 1996 Integrated Data Base Report," SBF \#96-38, dated Nov. 5, 1996. 
q. (NTS) L. S. Sygitowicz, Bechtel Nevada, Las Vegas, Nevada, correspondence to IDB Program, ORNL, Oak Ridge, Tennessee, "Low-Level Waste Data Request for Integrated Data Base Report," E100-WM-96-1550, dated Sept. 20, 1996.

r. (ORISE) Chuck Scott, Oak Ridge Institute for Science and Education, Oak Ridge, Tennessee, correspondence to Alan Icenhour, IBB Program, ORNL, Oak Ridge, Tennessee, transmitting updated ORISE LLW information and data, dated Oct. 21, 1996.

s. (ORNL) A. L. Rivera, Lockheed Martin Energy Systems, Inc., Oak Ridge, Tennessee, correspondence to S. N. Storch, IDB Program, ORNL, Oak Ridge, Tennessee, "1996 Low-Level Waste (LLW) Management Data Call," dated Sept. 30, 1996.

t. (PANT) W. A. Weinreich, Mason \& Hanger Corporation, Amarillo, Texas, correspondence to Alan Icenhour, IDB Program, ORNL, Oak Ridge, Tennessee, "Low-Level Waste Data Request for Integrated Database Report," GM96-03263-791, dated Sept. 18, 1996.

u. (Pinellas) Gary Schmidtke, U.S. Department of Energy, Pinellas Area Office, Largo, Florida, correspondence to Steve Storch, IDB Program, ORNL, Oak Ridge, Tennessee, "Pinellas Plant LLW Information for the IDB," dated Nov. 8, 1996.

v. (PPPL) Jerry W. Faul, DOE Princeton Group, correspondence to R. Lang, DOE Chicago Operations Office, Argonne, Illinois, "PPPL's Low-Level Waste Data for Integrated Data Base Report," TEC.960927.0002, dated Sept. 25, 1996.

w. (RFETS) Lisa O'Mary, DOE Rocky Flats Office, Golden, Colorado, correspondence to Steve Storch, IDB Program, ORNL, Oak Ridge National Laboratory, transmitting updated RFETS LLW information and data, dated Sept. 17, 1996.

x. (SLAC) Michael Scharfenstein, Stanford University, Stanford, California, correspondence to Alan Icenhour or Steve Storch, IDB Program, ORNL, Oak Ridge, Tennessee, transmitting updated SLAC LLW information and data, dated Sept. 9, 1996.

y. (SNL/CA) Sarah O'Connor, Lockheed Martin Sandia Corporation, Livermore, California, correspondence to Alan Icenhour and Steve Storch, IDB Program, ORNL, Oak Ridge, Tennessee, "Response to Low-Level Waste Data Request for Integrated Data Base Report," dated Sept. 20, 1996.

z. (SNL/NM) Scott A. Schrader, Sandia National Laboratories/New Mexico, Albuquerque, New Mexico, correspondence to Steve Storch, IDB Program, ORNL, Oak Ridge, Tennessee, "Integrated Data Base Low-Level Waste Data Request," dated Oct. 1, 1996.

aa. (SRS) Nathaniel S. Roddy, Westinghouse Savannah River Company, Aiken, South Carolina, correspondence to Steven Storch, IDB Program, ORNL, Oak Ridge, Tennessee, "Low-Level Waste Management Data Call for 1996 (U)," SWE-SWE-96-0411, dated Oct. 4, 1996.

ab. (WVDP) E. B. Martin, West Valley Nuclear Services Company, Inc., West Valley, New York, correspondence to Steven N. Storch, $\mathrm{DB}$ Program, ORNL, Oak Ridge, Tennessee, "Submittal of Low-Level Waste Data Call for the 1995 (sic) Integrated Data Base," WZ:96:0100, dated Sept. 26, 1996.

ac. (Y-12) A. L. Rivera, Lockheed Martin Energy Systems, Inc., Oak Ridge, Tennessee, correspondence to S. N. Storch, IDB Program, ORNL, Oak Ridge, Tennessee, "1996 Low-Level Waste (LLW) Management Data Call," dated Sept. 30, 1996. 
2. R L. Fuchs, “1995 State-by-State Assessment of Low-Level Radioactive Wastes Received at Commercial Disposal Sites," DOE/LLW-237, Idaho National Engineering Laboratory, Idaho Falls, Idaho (September 1996).

3. U.S. Department of Energy, Spent Fuel and Radioactive Waste Inventories, Projections, and Characteristics, DOE/RW-0006, Rev. 1, Oak Ridge National Laboratory, Oak Ridge, Tennessee (December 1985).

4. U.S. Energy Research and Development Administration, Environmental Statement, Radioactive Waste Facilities, WASH-1532 (Draft), Oak Ridge National Laboratory, Oak Ridge, Tennessee (January 1974).

5. U.S. Department of Energy, Integrated Data Base Report-1994: U.S. Spent Nuclear Fuel and Radioactive Waste Inventories, Projections, and Characteristics, DOE/RW-0006, Rev. 11, Oak Ridge National Laboratory, Oak Ridge, Tennessee (September 1995).

6. C. W. Forsberg, W. L. Carter, and A. H. Kibbey, Flowsheets and Source Terms for Radioactive Waste Projections, ORNL/TM-8462, Oak Ridge National Laboratory, Oak Ridge, Tennessee (March 1985).

7. R L. Fuchs, Lockheed Martin Idaho Technologies Company, National Low-Level Waste Management Program, Idaho Falls, Idaho, correspondence to A. S. Icenhour, Oak Ridge National Laboratory, Oak Ridge, Tennessee, "Transmittal of the 1995 Commercial Disposal Inventory Data," dated July 2, 1996.

8. U.S. Nuclear Regulatory Commission, "Licensing Requirements for Land Disposal of Radioactive Wastes," Code of Federal Regulations, 10 CFR Part 61, Jan. 1, 1994.

9. U.S. Congress, The Low-Level Radioactive Waste Policy Act, Pub. L. 95-573, Dec. 22, 1980.

10. U.S. Congress, The Low-Level Radioactive Waste Policy Amendments Act of 1985, Pub. L. 99-240, Jan. 15, 1986.

11. U.S. Department of Energy, Recommendations for Management of Greater-than-Class-C Low-Level Radioactive Waste, DOENE-0077, Washington, D.C. (February 1987).

12. U.S. Nuclear Regulatory Commission, amendments to 10 CFR Part 61, "Disposal of Radioactive Wastes," Final Rule, Fed. Regist. 54(100), 22578-83 (May 25, 1989).

13. Lockheed Idaho Technologies Company, Greater-Than-Class-C Low-Level Radioactive Waste Characterization: Estimated Volumes, Radionuclide Activities, and Other Characteristics, DOE/LLW-114, Rev. 1, Idaho Falls, Idaho (September 1994). 


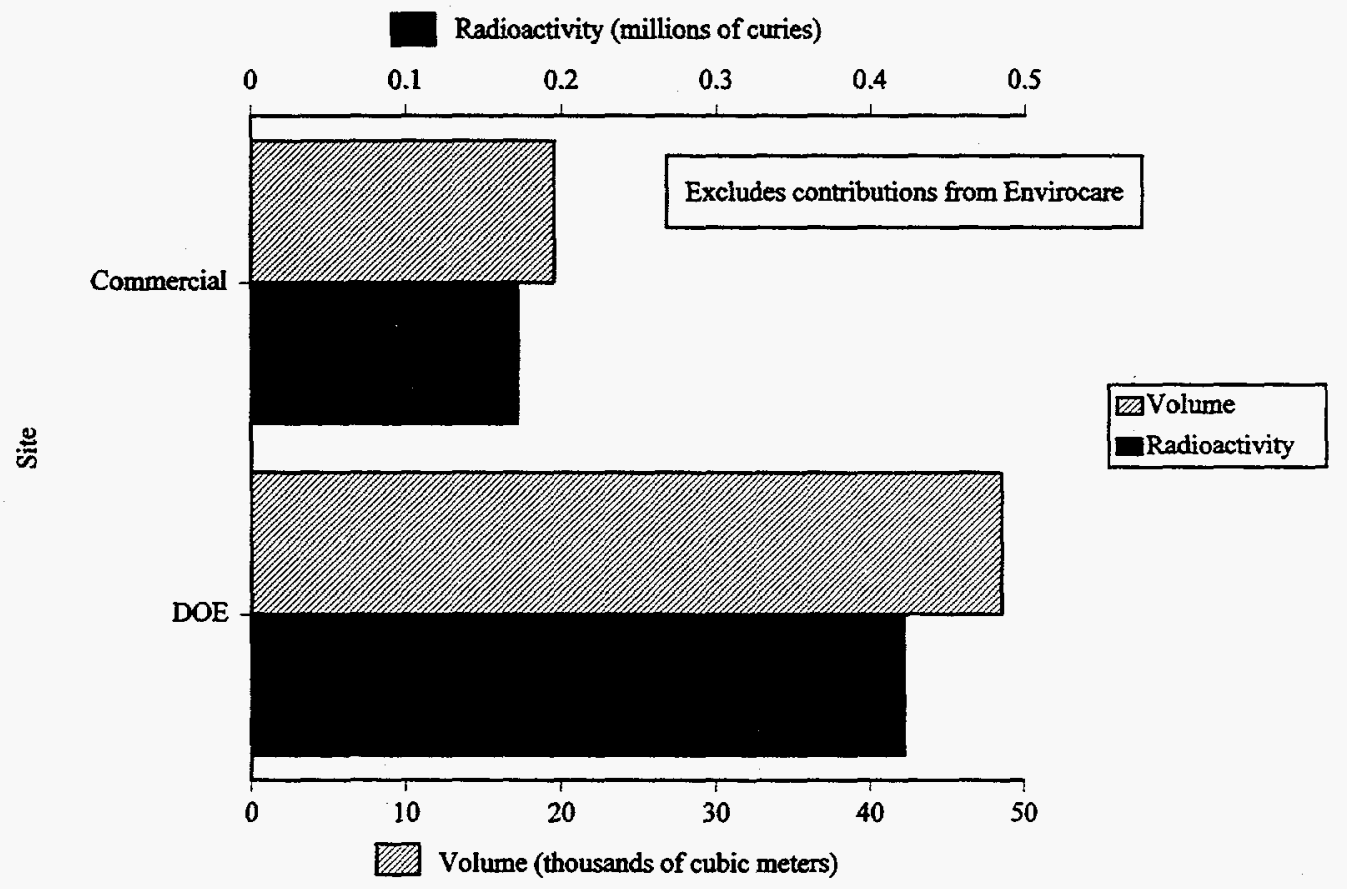

Fig. 4.1. Comparison of volume and radioactivity of LLW disposed at commercial and DOE facilities during 1995.

ORNL DWG 95-8839R

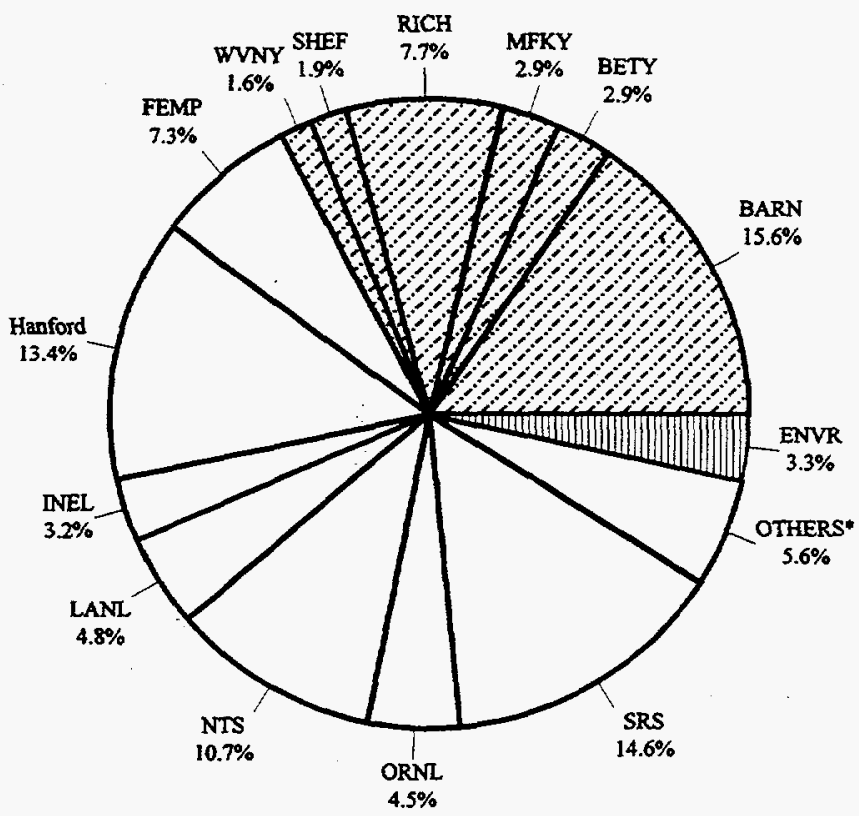

\begin{tabular}{lc} 
SITE & $\begin{array}{c}\text { CUBIC } \\
\text { METERS }\end{array}$ \\
\hline BARN & $7.359 E+05$ \\
BETY & $1.375 E+05$ \\
MFKY & $1.353 E+05$ \\
RICH & $3.644 E+05$ \\
SHEF & $8.833 E+04$ \\
WVNY & $7.707 E+04$ \\
FEMP & $3.432 E+05$ \\
Hanford & $6.302 E+05$ \\
INEL & $1.498 E+05$ \\
LANL & $2.242 E+05$ \\
NTS & $5.013 E+05$ \\
ORNL & $2.100 E+05$ \\
SRS & $6.870 E+05$ \\
OTHERS* & $2.655 E+05$ \\
ENVR & $1.560 E+05$ \\
\hline TOTAL & $4.705 E+06$ \\
- Includes contributions & \\
BNN, Krom Ames, \\
PORTS, SNLLNM, PAD, PANT, and Y-12.
\end{tabular}

Commercial

$\square$ DOE

[IIII] Envirocare

Fig. 4.2. Total volume of LLW disposed through 1995. 
ORNL DWG 95-8845R

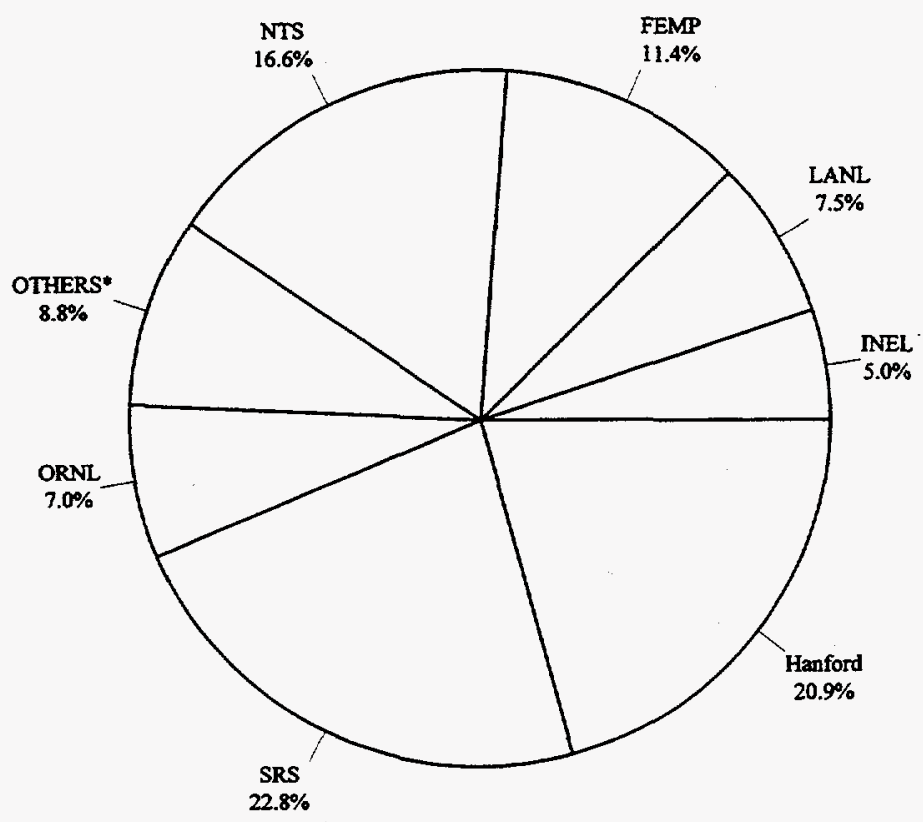

\begin{tabular}{lc}
\multicolumn{1}{c}{ SITE } & $\begin{array}{c}\text { CUBIC } \\
\text { METERS }\end{array}$ \\
\hline FEMP & $3.432 \mathrm{E}+05$ \\
Hanford & $6.302 \mathrm{E}+05$ \\
INEL & $1.498 \mathrm{E}+05$ \\
LANL & $2.242 \mathrm{E}+05$ \\
NTS & $5.013 \mathrm{E}+05$ \\
ORNL & $2.100 \mathrm{E}+05$ \\
SRS & $6.870 \mathrm{E}+05$ \\
OTHERS & $2.655 \mathrm{E}+05$ \\
\hline TOTAL & $3.011 \mathrm{E}+06$
\end{tabular}

-Includes contributions from Ames,

BNL, K-25, LLNL, PAD, PANT,

PORTS, SNLNM., and Y-12

Fig. 4.3. Total volume of DOE LLW disposed through 1995.

ORNL DWG 95-8846

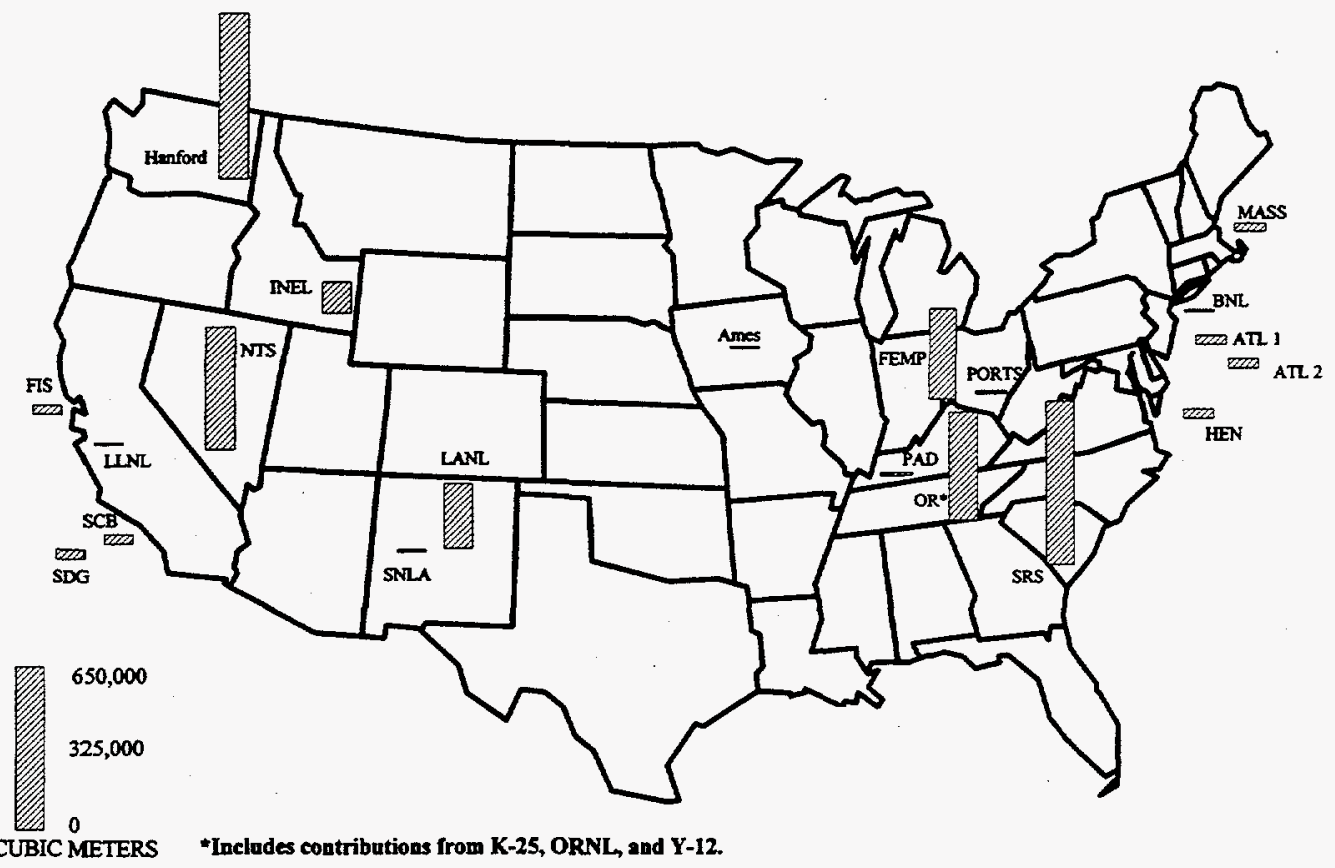

Fig. 4.4. Locations and total volumes of DOE LLW disposed through 1995. 
ORNL DWG 95-8129R

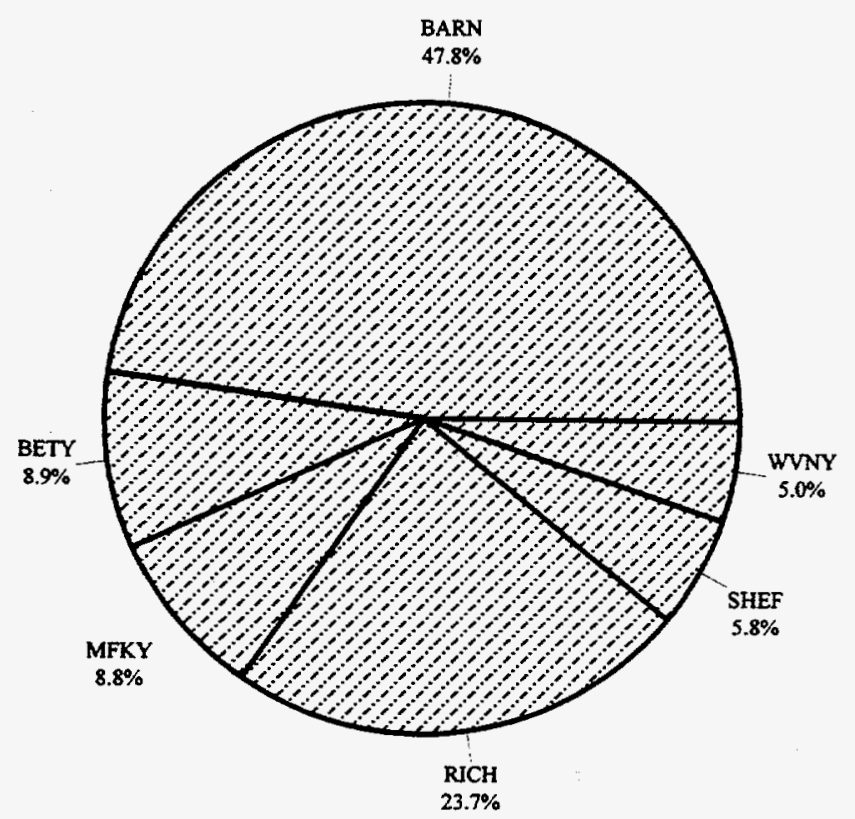

\begin{tabular}{lc} 
SITE & $\begin{array}{c}\text { CUBIC } \\
\text { METERS }\end{array}$ \\
\hline BARN & $7.359 E+05$ \\
BETY & $1.375 E+05$ \\
MFKY & $1.353 \mathrm{E}+05$ \\
RICH & $3.644 \mathrm{E}+05$ \\
SHEF & $8.833 \mathrm{E}+04$ \\
WVNY & $7.707 \mathrm{E}+04$ \\
\hline TOTAL & $1.538 \mathrm{E}+06$
\end{tabular}

Fig. 4.5. Total volume of commercial LLW disposed through 1995.

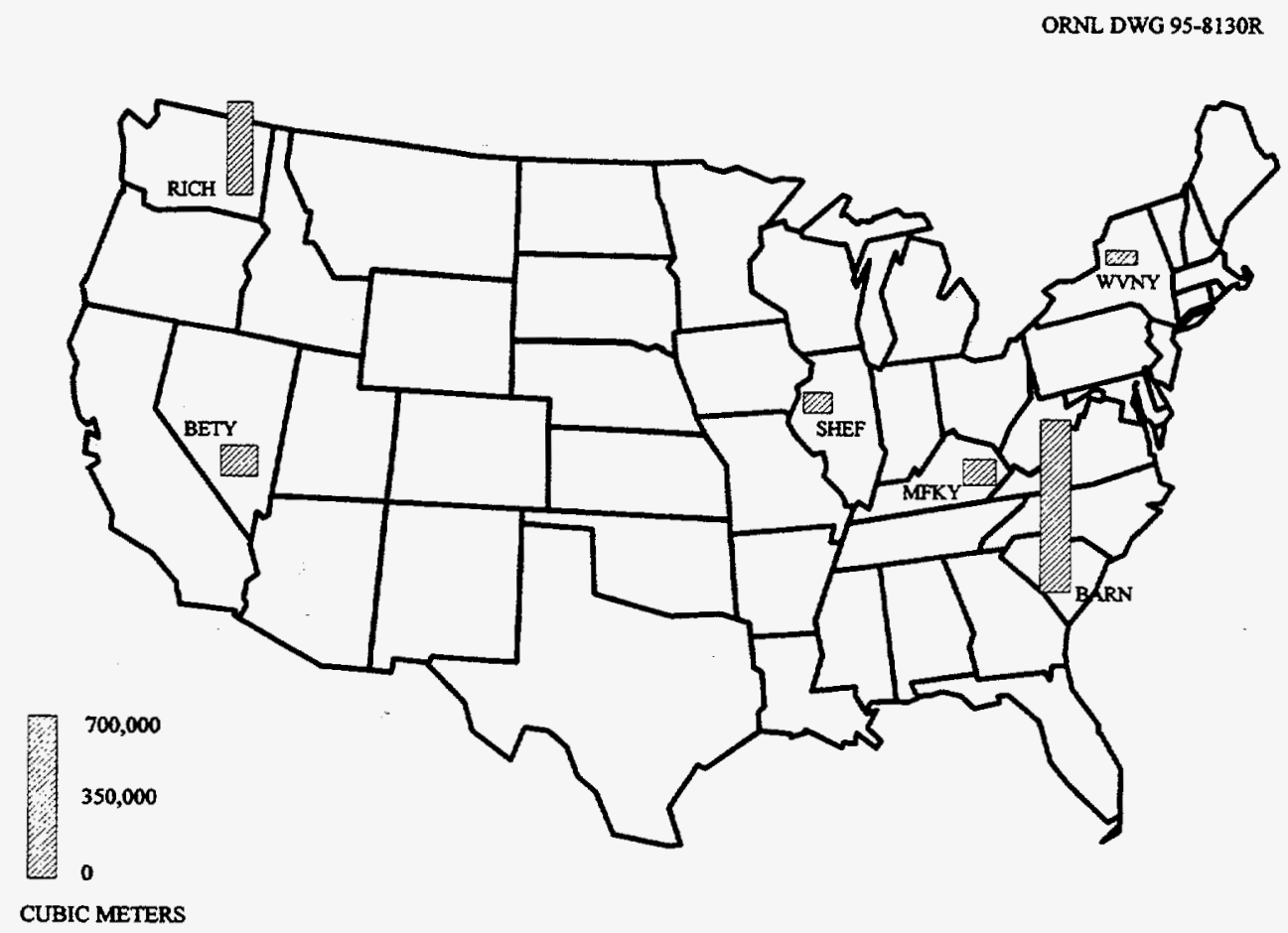

Fig. 4.6. Locations and total volumes of commercial LLW disposed through 1995. 
Table 4.1. Summary of characteristics for disposed LLW as of December 31, 1995

\begin{tabular}{|c|c|c|c|c|c|c|}
\hline \multirow{2}{*}{ Category } & \multicolumn{2}{|c|}{$\begin{array}{l}\text { Volume } \\
\left(10^{3} \mathrm{~m}^{3}\right)\end{array}$} & \multicolumn{2}{|c|}{$\begin{array}{l}\text { Radioactivity } \\
\qquad\left(10^{3} \mathrm{Ci}\right)\end{array}$} & \multicolumn{2}{|c|}{$\begin{array}{c}\text { Thermal power } \\
\text { (W) }\end{array}$} \\
\hline & Annual ${ }^{a}$ & Cumulative & Annual & Cumulative & Annual & Cumulative \\
\hline DOE sites & 48.5 & 3,011 & 422 & 12,550 & 2,378 & 22,195 \\
\hline Commercial sites $^{b}$ & 19.5 & 1,538 & 172 & 5,376 & 869 & 20,815 \\
\hline Envirocare ${ }^{c}$ & 25.0 & 156 & d & d & $d$ & $d$ \\
\hline Total & 93.0 & 4,705 & 594 & 17,926 & 3,247 & 43,010 \\
\hline
\end{tabular}

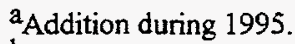

bIncludes contributions from Beatty, West Valley, Maxey Flats, Richland, Sheffield, and Barnwell.

CEnvirocare is a commercially operated facility that disposes of LLW, MLLW, NARM/NORM, and

11 (2) by-product material for both federal and private customers. See Table 0.8 .

UUnavailable. 
Table 4.2. Historical and projected volume, radioactivity, and thermal power of disposed DOE LLW

\begin{tabular}{|c|c|c|c|c|c|c|}
\hline \multirow{2}{*}{$\begin{array}{l}\text { End of } \\
\text { calendar } \\
\text { year }\end{array}$} & \multicolumn{2}{|c|}{$\begin{array}{l}\text { Volume } \\
\left(10^{3} \mathrm{~m}^{3}\right)\end{array}$} & \multicolumn{2}{|c|}{$\begin{array}{l}\text { Radioactivity } \\
\qquad\left(10^{3} \mathrm{Ci}\right)\end{array}$} & \multicolumn{2}{|c|}{$\begin{array}{l}\text { Thermal power } \\
\text { (W) }\end{array}$} \\
\hline & Annual & Cumulative & Annual & Cumulative $^{b}$ & Annual & Cumulative \\
\hline 1990 & 60.0 & 2,759 & 545 & 13,516 & 2,013 & 17,844 \\
\hline 1991 & 53.6 & 2,812 & 717 & 13,277 & 2,788 & 18,220 \\
\hline 1992 & 48.3 & 2,860 & 1078 & 13,401 & 4,947 & 20,741 \\
\hline 1993 & 50.5 & 2,911 & 894 & 13,147 & 3,263 & 20,398 \\
\hline 1994 & 52.1 & 2,963 & 621 & 12,858 & 3,463 & 21,534 \\
\hline 1995 & 48.5 & 3,011 & 422 & 12,550 & 2,378 & 22,195 \\
\hline 1996 & 82.5 & 3,121 & 797 & 12,700 & 4,665 & 25,356 \\
\hline 1997 & 76.5 & 3,197 & 744 & 12,786 & 4,383 & 27,911 \\
\hline 1998 & 76.5 & 3,274 & 744 & 12,861 & 4,382 & 30,186 \\
\hline 1999 & 76.5 & 3,350 & 745 & 12,922 & 4,381 & 32,186 \\
\hline 2000 & 76.5 & 3,427 & 745 & 12,973 & 4,379 & 33,928 \\
\hline 2001 & 76.5 & 3,503 & 745 & 13,015 & 4,375 & 35,435 \\
\hline 2002 & 68.9 & 3,572 & 382 & 12,686 & 2,211 & 34,577 \\
\hline 2003 & 68.9 & 3,641 & 382 & 12,399 & 2,209 & 33,831 \\
\hline 2004 & 68.9 & 3,710 & 382 & 12,142 & 2,207 & 33,162 \\
\hline 2005 & 68.9 & 3,779 & 382 & 11,910 & 2,206 & 32,561 \\
\hline 2006 & 68.9 & 3,848 & 382 & 11,701 & 2,206 & 32,017 \\
\hline 2007 & 68.9 & 3,917 & 382 & 11,511 & 2,206 & 31,524 \\
\hline 2008 & 68.9 & 3,986 & 382 & 11,338 & 2,206 & 31,074 \\
\hline 2009 & 68.9 & 4,055 & 382 & 11,180 & 2,206 & 30,661 \\
\hline 2010 & 68.9 & 4,124 & 382 & 11,036 & 2,206 & 30,283 \\
\hline 2011 & 68.9 & 4,193 & 382 & 10,905 & 2,206 & 29,936 \\
\hline 2012 & 68.9 & 4,262 & 382 & 10,785 & 2,207 & 29,614 \\
\hline 2013 & 68.9 & 4,331 & 382 & 10,675 & 2,207 & 29,316 \\
\hline 2014 & 68.9 & 4,400 & 382 & 10,574 & 2,207 & 29,039 \\
\hline 2015 & 68.9 & 4,469 & 382 & 10,483 & 2,207 & 28,782 \\
\hline 2016 & 68.9 & 4,538 & 382 & 10,399 & 2,207 & 28,541 \\
\hline 2017 & 68.9 & 4,607 & 382 & 10,324 & 2,208 & 28,317 \\
\hline 2018 & 68.9 & 4,675 & 382 & 10,255 & 2,208 & 28,107 \\
\hline 2019 & 68.9 & 4,744 & 381 & 10,193 & 2,208 & 27,909 \\
\hline 2020 & 68.9 & 4,813 & 381 & 10,137 & 2,208 & 27,723 \\
\hline 2021 & 68.9 & 4,882 & 381 & 10,087 & 2,208 & 27,548 \\
\hline 2022 & 68.9 & 4,951 & 381 & 10,042 & 2,208 & 27,383 \\
\hline 2023 & 68.9 & 5,020 & 381 & 10,003 & 2,209 & 27,226 \\
\hline 2024 & 68.9 & 5,089 & 381 & 9,968 & 2,209 & 27,078 \\
\hline 2025 & 68.9 & 5,158 & 381 & 9,938 & 2,209 & 26,939 \\
\hline 2026 & 68.9 & 5,227 & 381 & 9,913 & 2,209 & 26,805 \\
\hline 2027 & 68.9 & 5,296 & 381 & 9,891 & 2,210 & 26,679 \\
\hline 2028 & 68.9 & 5,365 & 381 & 9,874 & 2,210 & 26,559 \\
\hline 2029 & 68.9 & 5,434 & 381 & 9,859 & 2,210 & 26,445 \\
\hline 2030 & 68.9 & 5,503 & 381 & 9,848 & 2,210 & 26,336 \\
\hline
\end{tabular}

asummation of values in Tables 4.17 (buried DOE LLW, except SRS saitstone) and 4.18 (LLW saltstone at SRS)

The radioactivity added each year for each waste type is decayed as described in the footnotes of Tables 4.17 and 4.18 . 
Table 4.3. Historical and projected volume, radioactivity, and thermal power of commercial LLW shipped for disposal ${ }^{a}$

\begin{tabular}{|c|c|c|c|c|c|c|}
\hline \multirow{2}{*}{$\begin{array}{l}\text { End of } \\
\text { calendar } \\
\text { year }\end{array}$} & \multicolumn{2}{|c|}{$\begin{array}{l}\text { Volume } \\
\left(10^{3} \mathrm{~m}^{3}\right)\end{array}$} & \multicolumn{2}{|c|}{$\begin{array}{l}\text { Radioactivity } \\
\qquad\left(10^{3} \mathrm{Ci}\right)\end{array}$} & \multicolumn{2}{|c|}{$\begin{array}{l}\text { Thermal power } \\
\text { (W) }\end{array}$} \\
\hline & Annual & Cumulative & Annual & Cumulative $^{\mathrm{b}}$ & Annual & Cumulative \\
\hline 1990 & 32.4 & 1,384 & 549 & 4,979 & 2,774 & 16,457 \\
\hline 1991 & 38.8 & 1,423 & 800 & 5,272 & 4,044 & 18,424 \\
\hline 1992 & 49.4 & 1,472 & 1,000 & 5,708 & 5,057 & 21,117 \\
\hline 1993 & 22.4 & 1,495 & 643 & 5,709 & 3,252 & 21,627 \\
\hline 1994 & 24.3 & 1,519 & 751 & 5,841 & 3,799 & 22,746 \\
\hline 1995 & 19.5 & 1,538 & 172 & 5,376 & 869 & 20,815 \\
\hline $1996^{\mathrm{c}}$ & 19.5 & 1,558 & 172 & 5,020 & 869 & 19,330 \\
\hline 1997 & 19.5 & 1,577 & 172 & 4,726 & 869 & 18,093 \\
\hline 1998 & 19.5 & 1,597 & 172 & 4,479 & 869 & 17,041 \\
\hline 1999 & 19.5 & 1,617 & 172 & 4,268 & 869 & 16,140 \\
\hline 2000 & 19.5 & 1,636 & 172 & 4,086 & 869 & 15,365 \\
\hline 2001 & 19.5 & 1,656 & 172 & 3,928 & 869 & 14,697 \\
\hline 2002 & 19.5 & 1,675 & 172 & 3,788 & 869 & 14,122 \\
\hline 2003 & 19.5 & 1,695 & 172 & 3,665 & 869 & 13,626 \\
\hline 2004 & 19.5 & 1,714 & 172 & 3,556 & 869 & 13,200 \\
\hline 2005 & 19.5 & 1,734 & 172 & 3,459 & 869 & 12,834 \\
\hline
\end{tabular}

anciudes LLW disposed of at the following commercial sites: Beatty, Nevada; West Valley, New York; Maxey Flats, Kentucky; Richland, Washington; Sheffield, Illinois; and Barnwell, South Carolina.

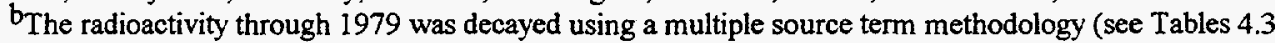
and $4.20-4.25$ of Rev: 8 of this report for a description of this method). After 1979, the radioactivity is decayed from the year of addition using the representative compositions given in Table A.4 of Appendix A.

CProjections were made based on disposal operations at Richland, Washington, and Barnwell, South Carolina, as described in Sect. 4.3.5. Projections were made only through 2005 because of large uncertainties in commercial disposal facility operations. 
Table 4.4. Historical annual additions and total volume of $L L W$ disposed at DOE sites $^{\mathrm{a}, b}$

\begin{tabular}{|c|c|c|c|c|c|c|c|c|c|c|c|}
\hline \multirow[b]{2}{*}{ Year } & \multicolumn{11}{|c|}{ Volume of waste disposed annually, $10^{3} \mathrm{~m}^{3}$} \\
\hline & FEMP & Hanford & INEL & LANL & NTS & ORNL & SRS & $Y-12^{c}$ & $\begin{array}{c}\text { All } \\
\text { others }\end{array}$ & $\begin{array}{c}\text { Total } \\
\text { annual } \\
\text { addition }\end{array}$ & $\begin{array}{c}\text { Total } \\
\text { cumulative } \\
\text { volume }\end{array}$ \\
\hline $1975^{\mathrm{e}}$ & 309.3 & 352.0 & 84.6 & 131.6 & 8.3 & 181.5 & 269.1 & 58.4 & 83.9 & $1,478.9$ & 1,479 \\
\hline 1976 & 14.4 & 4.1 & 6.2 & 8.8 & 0.0 & 3.8 & 8.1 & 2.7 & 0.9 & 49.0 & 1,528 \\
\hline 1977 & 2.8 & 10.7 & 6.6 & 3.6 & 0.5 & 2.4 & 14.7 & 1.5 & 1.1 & 43.9 & 1,572 \\
\hline 1978 & 1.9 & 9.8 & 5.9 & 7.5 & 10.0 & 2.0 & 15.5 & 1.4 & 3.2 & 57.2 & 1,629 \\
\hline 1979 & 1.6 & 17.5 & 5.3 & 4.9 & 15.8 & 2.1 & 18.2 & 1.1 & 1.1 & 67.6 & 1,697 \\
\hline 1980 & 1.3 & 10.4 & 5.1 & 4.8 & 13.3 & 2.0 & 19.6 & 1.4 & 0.7 & 58.6 & 1,755 \\
\hline 1981 & 1.5 & 12.8 & 3.1 & 5.5 & 21.1 & 1.4 & 20.1 & 1.2 & 1.6 & 68.3 & 1,824 \\
\hline 1982 & 2.8 & 11.7 & 3.2 & 4.5 & 57.0 & 1.3 & 22.4 & 2.2 & 2.0 & 107.1 & 1,931 \\
\hline 1983 & 3.4 & 17.9 & 5.5 & 3.2 & 12.1 & 1.8 & 26.7 & 3.4 & 1.7 & 75.7 & 2,006 \\
\hline 1984 & 3.5 & 18.8 & 3.9 & 5.4 & 36.0 & 2.2 & 26.1 & 7.2 & 10.6 & 113.7 & 2,120 \\
\hline 1985 & 0.7 & 17.0 & 3.1 & 6.7 & 41.7 & 2.2 & 30.5 & 18.7 & 2.1 & 122.7 & 2,243 \\
\hline 1986 & 0.0 & 21.1 & 3.4 & 4.5 & 27.9 & 1.8 & 30.1 & 15.0 & 1.0 & 104.8 & 2,348 \\
\hline 1987 & 0.0 & 20.3 & 3.0 & 3.7 & 81.1 & 0.5 & 28.2 & 16.2 & 1.0 & 154.0 & 2,501 \\
\hline 1988 & 0.0 & 16.8 & 2.0 & 4.3 & 39.1 & 0.6 & 30.2 & 10.6 & 1.0 & 104.6 & 2,606 \\
\hline 1989 & 0.0 & 13.7 & 1.3 & 6.4 & 35.0 & 1.3 & 26.8 & 5.7 & 2.3 & 92.5 & 2,699 \\
\hline 1990 & 0.0 & 13.4 & 1.8 & 4.5 & 9.1 & 0.3 & 26.6 & 4.4 & 0.0 & 60.1 & 2,759 \\
\hline 1991 & 0.0 & 10.6 & 1.3 & 5.8 & 11.6 & 0.2 & 23.8 & 0.3 & 0.0 & 53.6 & 2,812 \\
\hline 1992 & 0.0 & 10.9 & 0.8 & 2.3 & 20.1 & 1.1 & 13.0 & 0.0 & 0.0 & 48.2 & 2,860 \\
\hline 1993 & 0.0 & 12.1 & 0.9 & 2.7 & 18.6 & 0.8 & 15.3 & 0.1 & 0.0 & 50.5 & 2,911 \\
\hline 1994 & 0.0 & 13.7 & 1.9 & 1.9 & 22.9 & 0.3 & 11.4 & 0.0 & 0.0 & 52.1 & 2,963 \\
\hline 1995 & 0.0 & 14.9 & 0.9 & 1.6 & 20.0 & 0.4 & 10.6 & 0.0 & 0.0 & 48.5 & 3,011 \\
\hline Total & 343.2 & 630.2 & 149.8 & 224.2 & 501.3 & 210.0 & 687.0 & 151.3 & 114.2 & 3,011 & \\
\hline
\end{tabular}

${ }^{a}$ No TRUW included; data from refs. 1 and 5 . Slight differences in values shown and those actually reported result from round-off and truncation of numbers.

${ }^{b}$ See Tables 4.8 and 4.13 for breakdown of disposed volumes by physical form.

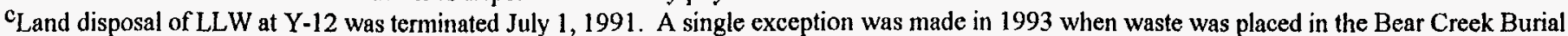
Ground walk-in pits.

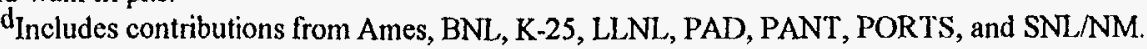

e Values for 1975 are cumulative volumes to this date (ref. 3 ). 
Table 4.5. Description of physical forms used to characterize solid LLW from DOE activities ${ }^{\text {a }}$

Physical form

Debris waste

Debris-combustible

Debris—noncombustible and compactible

Debris-noncombustible and combustible mixed

Activated metal/equipment/ hardware

Contaminated metal/equipment/ hardware

Reactor component/compartment

Elemental lead shielding

Filter media

Biological waste and carcasses

Asbestos-contaminated waste

Soil/sediment/rubble
Description

Waste that is at least $50 \%$ by volume solid materials that are intended for disposal and that are either manufactured objects, plant, or animal matter or that are natural geologic materials

Waste containing any material that will sustain combustion in air when exposed to an ignition source of $315^{\circ} \mathrm{C}\left(600^{\circ} \mathrm{F}\right)$ for a period of $5 \mathrm{~min}$

Waste composed of materials that are capable of undergoing volume reduction or of being compacted and that are not capable of being burned or rapidly oxidized

Waste composed of a mixture of materials that can and cannot sustain combustion

Waste materiais that are contaminated with radionuclides generated through neutron activation of metal, equipment, and/or hardware. Metal waste includes slag generated from removal of dross from molten metal, activated aluminum ingots, and metal generated by the decontamination and decommissioning (D\&D) of site structures and equipment. Other examples are activated reactor hardware, glove box parts, dehumidifiers, freezers, engines, pumps, and compressors

Waste materials that are externally contaminated with radioactive material. Metal waste includes slag generated from removal of dross from molten metal, nonreusable drums, and metal generated by the D\&D of site structures and equipment

Reactor components are those components which have been removed from a reactor pressure vessel or reactor compartment. A reactor compartment is the section that contains the reactor that is separated and removed from a submarine

Wastes containing at least $50 \%$ by volume elemental lead. Examples include lead bricks, sheets, and pipes

A category of solid waste that includes waste that is primarily (i.e., $\geq 50 \%$ by volume) filters constructed of one (or more than one) type of material. Examples of filter media waste are mechanical filters, cartridge-type filter media, and air filters

Waste that consists of any plant or animal material such as shrubs, trees, animal carcasses, animal bedding and litter wastes, including any absorbent material and chemical agents such as lime, animal tissues in vials, and cultures

Waste that contains about $80 \%$ by volume, or more, asbestos or asbestos-based material. The asbestos contained in the waste may be friable or nonfriable asbestos. Friable asbestos is a material that, when dry, may be crumbled, pulverized, or reduced to powder by hand pressure. Nonfriable asbestos is that which is completely encapsulated or fixed in a matrix material that prevents the asbestos fibers from being released upon handling

A category of solid waste estimated to contain about $50 \%$ by volume soil and other ground media including, but not limited to, sand, silt, grass, rock, and gravel. The category also includes waste containing sediment which constitutes material in drainage and natural ponds and rubble which comprises waste material from building or demolition processes and concrete chunks and blocks from D\&D activities 
Table 4.5 (continued)

Fhysical form, Description

Solidified liquids/chelates/oils

Soliditied sludge/resin

Sources

Paint waste

Salt waste

Incinerator ash

Activated carbon (charcoal)

Other inorganic particulates

Other
Wastes that contain liquids, any polydentate ligand that will solubilize metal ions, and oils that have been immobilized or stabilized with agents, such as cement, polymers, and bitumen, and properly cured into a solidified form. The waste should not contain free liquids in excess of $1 \%$ of the volume of the waste when the waste is in a disposal container or $0.5 \%$ of the volume of the waste processed to a stable form. The waste should be in a form that meets stability requirements

Sludge is any solid, semisolid, or liquid waste generated from any of a number of airor water-treatment processes. Waste with a total suspended/settled solids (TSS) content of $>30 \%$ is a sludge. The waste may be a mixture of sludge and stabilization agents cured into a solidified form

Resin waste includes waste that is primarily (i.e., $\geq 50 \%$ by volume) unused or spent inorganic or organic-based resins used in wastewater treatment or other applications. An example of this waste is inorganic/organic ion-exchange resins

Waste material composed of encapsulated radioactive material whose main purpose is to generate known amounts of radiation. Sealed sources are defined as a category of special-form radioactive material in 10 CFR Part 71.4. Special-form radioactive material is radioactive material which satisfies the following conditions: (1) it is either a single, solid piece or is contained in a sealed capsule that can be opened only by destroying the capsule; (2) the piece or capsule has at least one dimension not less than $5 \mathrm{~mm}$; and (3) it satisfies the test requirements of 10 CFR Part 71.75

Waste that is at least $50 \%$ by volume new, used, or removed paint. Examples of waste in this category are dried paint chips; containers filled with dried paint; and opened or unopened cans of heavy, viscous paint. This category does not include waste that is $50 \%$ by volume, or more, paint-related solids such as empty paint cans, depressurized spray paint cans, or other painting equipment (brushes, rollers, etc.)

Waste that is at least $50 \%$ by volume salts including interstitial liquids, if present. Chloride salts and sulfate salt wastes contain more than (i.e., $>1000 \mathrm{ppm}$ ) levels of chlorides or other halogens and of sulfur compounds, respectively. Nitrate salts are predominantly (i.e., $>50 \%$ by volume of salt) nitrates. Metal oxides/hydroxides waste is predominantly metal oxides or hydroxides

An inorganic particulate waste that is primarily (i.e., $\geq 50 \%$ by volume) bottom or fly ash resulting from incineration

An inorganic particulate waste that is primarily (i.e., $\geq 50 \%$ by volume) spent or unused activated carbon including any residual liquids. The activated carbon may be in powdered (typically 50 to $100 \mu \mathrm{m}$ ) or granular (typically 0.1 to $1 \mathrm{~mm}$ ) form

Waste that is at least $50 \%$ by volume inorganic particulates including any residual or absorbed liquids. Examples of other inorganic particulate waste are sandblasting media, inorganic particulate absorbent material (e.g., vermiculite), and ion-exchange media

This category includes solid wastes that are not characterized sufficiently or that do not meet the criteria for assignment into one of the above categories

\footnotetext{
apatrice M. Bubar, DOE Office of Planning and Analysis, Office of Waste Management, Environmental Management,
correspondence to DOE field offices and DOE site points of contact, "Low-Level Waste Data Request for Integrated Data Base Report," Attachment 1, dated Aug. 9, 1996.
} 
Table 4.6. Actual 1995 generation and projected average annual generation of LLW at DOE sites

\begin{tabular}{|c|c|c|c|c|c|c|c|c|}
\hline \multirow{3}{*}{ Physical form } & \multirow{2}{*}{\multicolumn{2}{|c|}{1995 generation }} & \multicolumn{6}{|c|}{ Projections } \\
\hline & & & \multicolumn{2}{|c|}{1996} & \multicolumn{2}{|c|}{ 1997-2001 } & \multicolumn{2}{|c|}{$2002-2030$} \\
\hline & $\begin{array}{l}\text { Volume } \\
\left(\mathrm{m}^{3}\right)\end{array}$ & $\begin{array}{l}\text { Activity } \\
\text { (Ci) }\end{array}$ & $\begin{array}{c}\text { Volume } \\
\left(\mathrm{m}^{3}\right)\end{array}$ & $\begin{array}{l}\text { Activity } \\
\text { (Ci) }\end{array}$ & $\begin{array}{c}\text { Volume } \\
\left(\mathrm{m}^{3} / \text { year }\right)\end{array}$ & $\begin{array}{c}\text { Activity } \\
\text { (Ci/year) }\end{array}$ & $\begin{array}{c}\text { Volume } \\
\left(\mathrm{m}^{3} / \text { year }\right)\end{array}$ & $\begin{array}{l}\text { Activity } \\
\text { (Ci/year) }\end{array}$ \\
\hline \multicolumn{9}{|l|}{ Debris } \\
\hline Combustible & 10,185 & 135 & 10,120 & 357 & 9,332 & 293 & 6,348 & 164 \\
\hline Noncombustible and compactible & 1,781 & 1,599 & 922 & 8,146 & 873 & 4 & 4,430 & 2 \\
\hline Noncombustible and combustible mixed & 7,577 & 23,925 & 8,512 & 7,294 & 7,778 & 137,796 & 5,084 & 136,698 \\
\hline Activated metal/equipment/hardware & 690 & 69,015 & 848 & 159,142 & 1,396 & 153,384 & 164 & 78,639 \\
\hline Contaminated metal/equipment/hardware & 3,546 & 36,537 & 4,813 & 11,263 & 3,679 & 11,718 & 2,107 & 5,319 \\
\hline Reactor component/compartment & 275 & 56,902 & 126 & 40 & 531 & 295,500 & 1,242 & 796,000 \\
\hline Elemental lead shielding & 5 & $<<1$ & 0 & 0 & 0 & 0 & 0 & 0 \\
\hline Filter media (as-is, solidified, dewatered) & 337 & 378 & 285 & 136 & 311 & 156 & 203 & 134 \\
\hline Biological waste and carcasses (as-is, solidified) & 33 & $\ll<1$ & 11 & 1 & 37 & 1 & 37 & 1 \\
\hline Asbestos-contaminated waste (as-is, solidified) & 443 & 2 & 394 & $<1$ & 255 & $<1$ & 191 & $<1$ \\
\hline Soil/sediment/rubble (as-is, solidified, dewatered) & 527 & 12 & 1,545 & 16 & 2,627 & 12 & 296 & 9 \\
\hline Solidified liquids/chelates/oils & 250 & 158,760 & 636 & 160,020 & 105 & 170,019 & 90 & 165,003 \\
\hline Solidified sludge/resin (dewatered, solidified) & 727 & 221 & 543 & 560 & 700 & 186 & 498 & 141 \\
\hline Sources (sealed sources, devices, and gauges) & $1^{a}$ & $1,036^{\mathrm{a}}$ & $<1^{b}$ & $124^{b}$ & $<\mathbf{l}^{\mathbf{c}}$ & $16^{\mathrm{c}}$ & $<1^{d}$ & $2^{\mathrm{d}}$ \\
\hline Paint waste & 5 & $<<1$ & 11 & $\ll 1$ & 11 & $<<1$ & 11 & $\ll 1$ \\
\hline Salt waste & 1,501 & 23 & 2,771 & 41 & 174 & $\ll<1$ & 145 & $<<1$ \\
\hline Incinerator ash (solidified, as-is) & 6 & $\ll<1$ & 4 & $\ll 1$ & 227 & $<<1$ & 245 & $\ll<1$ \\
\hline Activated carbon (charcoal) & 3 & 865 & 2 & 6 & 4 & 1 & 1 & 1 \\
\hline Other inorganic particulates & 160 & 245 & 592 & 20,777 & 671 & 15,912 & 458 & 12,129 \\
\hline Other & 1,148 & 22,932 & 97 & 22,034 & 330 & 52 & 151 & 40 \\
\hline Total & 29,199 & 372,586 & 32,232 & 389,956 & 29,040 & 785,051 & 21,701 & $1,194,283$ \\
\hline
\end{tabular}

${ }^{a}$ Two hundred fifty-six sources with no volume estimate are included in radioactivity value for sources.

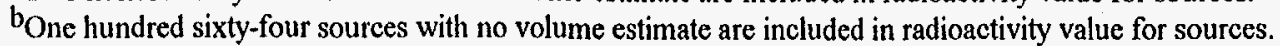

${ }^{c}$ Thirty-eight sources with no volume estimate are included in radioactivity value for sources.

dFifteen sources with no volume estimate are included in radioactivity value for sources. 
Table 4.7. Actual 1995 cumulative storage inventory and projected average annual storage additions of LLW at DOE sites

\begin{tabular}{|c|c|c|c|c|c|c|c|c|}
\hline \multirow{3}{*}{ Physical form } & \multirow{2}{*}{\multicolumn{2}{|c|}{ Cumulative $^{b}$}} & \multicolumn{6}{|c|}{ Projected annuai adjoition ${ }^{a}$} \\
\hline & & & \multicolumn{2}{|c|}{1996} & \multicolumn{2}{|c|}{$1997-2001$} & \multicolumn{2}{|c|}{$2002-2030$} \\
\hline & $\begin{array}{c}\text { Volume } \\
\left(\mathrm{m}^{3}\right)\end{array}$ & $\begin{array}{l}\text { Activity } \\
\text { (Ci) }\end{array}$ & $\begin{array}{c}\text { Volume } \\
\left(\mathrm{m}^{3}\right)\end{array}$ & $\begin{array}{l}\text { Activity } \\
\text { (Ci) }\end{array}$ & $\begin{array}{l}\text { Volume } \\
\left(\mathrm{m}^{3} / \text { year }\right)\end{array}$ & $\begin{array}{c}\text { Activity } \\
\text { (Ci/year) }\end{array}$ & $\begin{array}{c}\text { Volume } \\
\left(\mathrm{m}^{3} / \text { year }\right)\end{array}$ & $\begin{array}{l}\text { Activity } \\
\text { (Ci/year) }\end{array}$ \\
\hline \multicolumn{9}{|l|}{ Debris } \\
\hline Combustible & 9,656 & 43,044 & 2,625 & $-1,355$ & 1,899 & 89 & 400 & 96 \\
\hline Noncombustible and compactible & 4,443 & 31 & -629 & 7,934 & -446 & -665 & 44 & -159 \\
\hline Noncombustible and combustible mixed & 10,391 & 801,424 & 2,105 & $-11,096$ & 1,976 & 2,161 & 1,613 & 2,161 \\
\hline Activated metal/equipment/hardware & 1,873 & 457,511 & 321 & 38,619 & 101 & 50 & 111 & 55 \\
\hline Contaminated metal/equipment/hardware & 10,367 & 19,731 & 2,899 & 1,543 & 376 & 1,538 & 447 & 1,540 \\
\hline Reactor component/compartment & 10 & $<1$ & 0 & 0 & 0 & 0 & 0 & 0 \\
\hline Elemental lead shielding & 15 & $\ll 1$ & 0 & 0 & 0 & 0 & 0 & 0 \\
\hline Filter media (as-is, solidified, dewatered) & 780 & 4 & 341 & $<1$ & 42 & 2 & 36 & 2 \\
\hline Biological waste and carcasses (as-is, solidified) & 13 & $<1$ & 9 & 1 & 7 & 1 & 8 & 1 \\
\hline Asbestos-contaminated waste (as-is, solidified) & 1,129 & $\ll<1$ & 183 & $\ll<1$ & 154 & $<<1$ & 23 & $\ll<1$ \\
\hline Soil/sediment/rubble (as-is, solidified, dewatered) & 5,805 & 447 & -215 & -110 & 1,442 & -66 & 71 & $<1$ \\
\hline Solidified liquids/chelates/oils & 50 & $1,140,003$ & 9 & 160,004 & 51 & 115,004 & 51 & 115,004 \\
\hline Solidified sludge/resin (dewatered, solidified) & 8,291 & 220 & 6,296 & 305 & 140 & -42 & 140 & -9 \\
\hline Sources (sealed sources, devices, and gauges) & $10^{\mathrm{c}}$ & $216^{\mathrm{c}}$ & $2^{\mathrm{d}}$ & $142^{d}$ & $2^{\mathrm{e}}$ & $469^{\mathrm{e}}$ & $2^{f}$ & $468^{f}$ \\
\hline Paint waste & 11 & $\ll 1$ & 1 & $\ll 1$ & 1 & $<<1$ & 1 & $<<1$ \\
\hline Salt waste & 29 & g & 1 & $\mathrm{~g}$ & 1 & g & $<1$ & $\mathrm{~g}$ \\
\hline Incinerator ash (solidified, as-is) & 306 & $\ll i$ & 4 & $<<1$ & -1 & $\ll 1$ & 2 & $<<1$ \\
\hline Activated carbon (charcoal) & 65 & $\ll<1$ & 5 & $\ll 1$ & 3 & $\ll 1$ & 0 & 0 \\
\hline Other inorganic particulates & 462 & 26 & 124 & 5 & 35 & $\mathrm{~g}$ & 2 & $\mathrm{~g}$ \\
\hline \multicolumn{9}{|l|}{ Other } \\
\hline EM-30 program waste & 12,068 & 501 & 5,806 & 666 & -887 & -189 & $\ll<1$ & $\ll<1$ \\
\hline$E M-40$ program waste & $180,000^{\mathrm{h}, \mathrm{i}}$ & 8 & $\mathrm{~g}$ & $\mathrm{~g}$ & g & $\mathrm{g}$ & $\mathrm{g}$ & $\mathrm{g}$ \\
\hline Total & 245,775 & $2,463,158$ & 19,888 & 196,659 & 4,895 & 118,351 & 2,950 & 119,159 \\
\hline
\end{tabular}

aNegative values indicate a decrease in stored volume or radioactivity.

bCumulative storage as of December $31,1995$.

CIncluded in radioactivity value for sources are 118 sources with no volume estimate

dincluded in radioactivity value for sources are 91 sources with no volume estimate.

e Included in radioactivity value for sources are 31 sources with no volume estimate.

Included in radioactivity value for sources are 30 sources with no volume estimate.

gNot available.

hFor EM-40 program LLW, data available only for cumulative storage volume for physical form "other." See Table 6.7 in Chapter 6 . The physical form categories listed above do not apply to EM-40 program wastes.

iTotal shown is rounded to two significant figures. 
Table 4.8. Actual disposal during 1995 and projected average annual disposal of LLW at DOE sites

\begin{tabular}{|c|c|c|c|c|c|c|c|c|}
\hline \multirow{3}{*}{ Physical form } & & & \multicolumn{6}{|c|}{ Projections ${ }^{\mathrm{a}, \mathrm{b}}$} \\
\hline & \multicolumn{2}{|c|}{1995 disposal } & \multicolumn{2}{|c|}{1996} & \multicolumn{2}{|c|}{$1997-2001$} & \multicolumn{2}{|c|}{$2002-2030$} \\
\hline & $\begin{array}{c}\text { Volume } \\
\left(\mathrm{m}^{3}\right)\end{array}$ & $\begin{array}{c}\text { Activity } \\
\text { (Ci) }\end{array}$ & $\begin{array}{c}\text { Volume } \\
\left(\mathrm{m}^{3}\right)\end{array}$ & $\begin{array}{l}\text { Activity } \\
\text { (Ci) }\end{array}$ & $\begin{array}{l}\text { Volume } \\
\left(\mathrm{m}^{3} / \text { year }\right)\end{array}$ & $\begin{array}{l}\text { Activity } \\
\text { (Ci/year) }\end{array}$ & $\begin{array}{l}\text { Volume } \\
\left(\mathrm{m}^{3} / \text { year }\right)\end{array}$ & $\begin{array}{c}\text { Activity } \\
\text { (Ci/year) }\end{array}$ \\
\hline Debris-dry solids & 25,258 & 27,002 & 23,323 & 3,496 & 20,395 & 232 & 6,322 & 199 \\
\hline Activated metal/equipment/hardware & 568 & 51,495 & 300 & 136,600 & 567 & 90,350 & 89 & 38,790 \\
\hline Contaminated metal/equipment/hardware & 10,607 & 37,123 & 2,806 & 12,234 & 1,844 & 8,172 & 951 & 5,486 \\
\hline Reactor component/compartment & 9,047 & 256,100 & 8,120 & 484,200 & 7,654 & 520,500 & 3,529 & 240,317 \\
\hline Filter media (as-is, solidified, dewatered) & 246 & 3 & 33 & 13 & 25 & 8 & 19 & 4 \\
\hline Biological waste and carcasses (as-is, solidified) & 22 & $\ll 1$ & 16 & 56 & 18 & 33 & 17 & 18 \\
\hline Asbestos-contaminated waste (as-is, solidified) & 99 & $<1$ & 57 & $<1$ & 48 & $\ll 1$ & 45 & $<<1$ \\
\hline Soil/sediment/rubble (as-is, solidified, dewatered) & 494 & 11 & 1,703 & 19 & 335 & 11 & 140 & 6 \\
\hline Solidified liquids/chelates/oils & 252 & 31,270 & 700 & 19 & 102 & 17 & 20 & 15 \\
\hline Solidified sludge/resin (dewatered, solidified) & 144 & 237 & 88 & 286 & 66 & 169 & 51 & 94 \\
\hline Sources (sealed sources, devices, and gauges) & $\ll 1^{\mathrm{c}}$ & $1,033^{\mathrm{c}}$ & $<1^{d}$ & $133^{\mathrm{d}}$ & $<1$ & 12 & $<1$ & 10 \\
\hline Paint waste & 0 & 0 & 1 & e & 1 & e & 1 & e \\
\hline Salt waste & 1,500 & 23 & 0 & 0 & 172 & e & 144 & e \\
\hline Incinerator ash (solidified, as-is) & 6 & $\ll<1$ & 10 & $\ll<1$ & 6 & $\ll<1$ & 3 & $<<1$ \\
\hline Activated carbon (charcoal) & 0 & 0 & 0 & 0 & 0 & 0 & 0 & 0 \\
\hline Other inorganic particulates & 1 & $\ll 1$ & 22 & 81 & 32 & 49 & 11 & 27 \\
\hline Other & 259 & 17,622 & 323 & 24,840 & 230 & 14,620 & 102 & 8,128 \\
\hline Total & 48,503 & 421,919 & 37,500 & 661,977 & 31,495 & 634,172 & 11,445 & 293,095 \\
\hline
\end{tabular}

asome radioactivity projections unavailable from Hanford and SRS. Some volume and radioactivity projections unavailable from NTS.

bolume and radioactivity projections do not agree with those in Table 4.17. Projected values, shown in Table 4.17, were estimated for the unavailable data (from Hanford, SRS, and NTS).

cIncluded in radioactivity value for sources are 105 sources with no volume estimate.

$\mathrm{d}_{\text {Included in radioactivity value for sources are } 6 \text { sources with no volume estimate. }}$

eUnknown. 
Table 4.9. Breakdown by physical form of volumes of LLW generated during 1995 at DOE sites

\begin{tabular}{|c|c|c|c|c|c|c|c|c|c|c|}
\hline \multirow[b]{2}{*}{ Physical form } & \multicolumn{10}{|c|}{ Volume, $\mathrm{m}^{3}$} \\
\hline & Hanford & INEL & $\mathrm{K}-25$ & LANL & Mound & NR sites $^{a}$ & ORNL & SRS & $\begin{array}{l}\text { All } \\
\text { others } b\end{array}$ & Total \\
\hline \multicolumn{11}{|l|}{ Debris } \\
\hline Combustible & 1,111 & 1,357 & 2,115 & 558 & 47 & 223 & 0 & 4,070 & 704 & 10,185 \\
\hline Noncombustible and compactible & 1,479 & 0 & 13 & 107 & 0 & 101 & 0 & 0 & 81 & 1,781 \\
\hline $\begin{array}{l}\text { Noncombustible and combustible } \\
\text { mixed }\end{array}$ & 14 & 0 & 330 & 323 & 423 & 530 & 1,640 & 3,500 & 817 & 7,577 \\
\hline Activated metal/equipment/hardware & 313 & 5 & 0 & 0 & 0 & 28 & 0 & 227 & 117 & 690 \\
\hline Contaminated metal/equipment/hardware & 504 & 158 & 310 & 367 & 0 & 421 & 0 & 746 & 1,040 & 3,546 \\
\hline Reactor component/compartment & 0 & 0 & 0 & 0 & 0 & 270 & 0 & 0 & 5 & 275 \\
\hline Elemental lead shielding & 0 & 0 & 0 & 0 & 0 & 0 & 0 & 0 & 5 & 5 \\
\hline Filter media (as-is, solidified, dewatered) & 101 & 6 & 3 & 15 & 0 & 46 & 0 & 0 & 166 & 337 \\
\hline $\begin{array}{l}\text { Biological waste and carcasses } \\
\text { (as-is, solidified) }\end{array}$ & 9 & 0 & 0 & $<<1$ & 0 & 0 & 13 & 0 & 11 & 33 \\
\hline $\begin{array}{l}\text { Asbestos-contaminated waste } \\
\text { (as-is, solidified) }\end{array}$ & 18 & 1 & 129 & 40 & 7 & 182 & 12 & 0 & 55 & 443 \\
\hline $\begin{array}{l}\text { Soil/sediment/rubble (as-is, solidified, } \\
\text { dewatered) }\end{array}$ & 181. & 107 & 4 & 107 & 0 & 2 & 0 & 0 & 126 & 527 \\
\hline Solidified liquids/chelates/oils & 171 & 0 & 0 & 4 & 65 & 2 & 0 & 0 & 8 & 250 \\
\hline $\begin{array}{l}\text { Solidified sludge/resin (dewatered, } \\
\text { solidified) }\end{array}$ & 0 & 1 & 0 & 61 & 145 & 12 & 0 & 0 & 508 & 727 \\
\hline $\begin{array}{l}\text { Sources (sealed sources, devices, and } \\
\text { gauges) }\end{array}$ & 0 & $0^{\mathrm{c}}$ & 0 & 1 & 0 & $<<1^{d}$ & 0 & 0 & $0^{\mathbf{e}}$ & 1 \\
\hline Paint waste & 0 & 0 & 0 & 0 & 0 & 0 & 0 & 0 & 5 & 5 \\
\hline Salt waste & 0 & 0 & $<1$ & 0 & 0 & 0 & 0 & 1,500 & $<1$ & 1,501 \\
\hline Incinerator ash (solidified, as-is) & 1 & 0 & 0 & 0 & 0 & 0 & 0 & 0 & 5 & 6 \\
\hline Activated carbon (charcoal) & 0 & 0 & 1 & 0 & 0 & 1 & 0 & 0 & $<1$ & 3 \\
\hline Other inorganic particulates & 0 & 63 & 9 & 0 & 0 & 0 & 0 & 0 & 88 & 160 \\
\hline Other & 0 & 927 & 7 & 2 & 0 & 0 & 3 & 0 & 209 & 1,148 \\
\hline Total & 3,902 & 2,625 & 2,921 & 1,585 & 687 & 1,818 & 1,668 & 10,043 & 3,950 & 29,199 \\
\hline
\end{tabular}

a Naval Reactors (NR) sites include KAPL, BAPL, and NRF.

Includes Ames, ANL-E, BNL, ETEC, FNAL, ITRI, KCP, LBL, LLNL, NTS, PANT, Pinellas, PPPL, RFETS, SLAC, SNL/CA, SNL/NM, WVDP and $\mathrm{Y}-12$.

CINEL reported 63 sources for which a volume estimate was not available.

dNR sites reported 35 sources for which a volume estimate was not available.

Four sites reported 158 sources for which a volume estimate was not available (38 at Ames, 9 at BNL, 100 at ETEC, and 11 at SNL/NM). 
Table 4.10. Breakdown by physical form of radioactivity of LLW generated during 1995 at DOE sites

\begin{tabular}{|c|c|c|c|c|c|c|c|c|c|c|}
\hline \multirow[b]{2}{*}{ Physical form } & \multicolumn{10}{|c|}{ Radioactivity, $\mathrm{Ci}$} \\
\hline & Hanford & INEL & $\mathrm{K}-25$ & LANL & Mound & NR sites ${ }^{\mathrm{a}}$ & ORNL & SRS & $\begin{array}{c}\text { All } \\
\text { others } b\end{array}$ & Total \\
\hline \multicolumn{11}{|l|}{ Debris } \\
\hline Combustible & 22 & 5 & c & 24 & 24 & $<1$ & 0 & 44 & 16 & 135 \\
\hline Noncombustible and compactible & 1,595 & 0 & c & $<1$ & 0 & 1 & 0 & 0 & 3 & 1,599 \\
\hline $\begin{array}{l}\text { Noncombustible and combustible } \\
\text { mixed }\end{array}$ & 3,380 & 0 & c & 25 & 216 & 27 & 3,708 & 2,380 & 14,189 & 23,925 \\
\hline Activated metal/equipment/hardware & 21,577 & 8,018 & 0 & 0 & 0 & 17,500 & 0 & 21,900 & 20 & 69,015 \\
\hline Contaminated metal/equipment/hardware & 34,730 & 5 & c & 1,290 & 0 & 6 & 0 & c & 506 & 36,537 \\
\hline Reactor component/compartment & 0 & 0 & 0 & 0 & 0 & 56,902 & 0 & 0 . & $\ll<1$ & 56,902 \\
\hline Elemental lead shielding & 0 & 0 & 0 & 0 & 0 & 0 & 0 & 0 & $\ll<$ & $<<1$ \\
\hline Filter media (as-is, solidified, dewatered) & 3 & $<<1$ & $\mathrm{c}$ & $<<1$ & 0 & 372 & 0 & 0 & 3 & 378 \\
\hline $\begin{array}{l}\text { Biological waste and carcasses } \\
\text { (as-is, solidified) }\end{array}$ & $\ll<1$ & 0 & 0 & $\ll 1$ & 0 & 0 & $\ll 1$ & 0 & $\ll<1$ & $<<1$ \\
\hline $\begin{array}{l}\text { Asbestos-contaminated waste } \\
\text { (as-is, solidified) }\end{array}$ & $<<1$ & $<<1$ & $\mathrm{c}$ & $<1$ & $\ll 1$ & $<1$ & 0 & 0 & 1 & 2 \\
\hline $\begin{array}{l}\text { Soil/sediment/rubble (as-is, solidified, } \\
\text { dewatered) }\end{array}$ & $<1$ & 10 & c & $\ll 1$ & 0 & 1 & 0 & 0 & 1 & 12 \\
\hline Solidified liquids/chelates/oils & 1,760 & 0 & 0 & $\ll<1$ & 157,000 & $\ll<1$ & 0 & 0 & $\ll<1$ & 158,760 \\
\hline $\begin{array}{l}\text { Solidified sludge/resin (dewatered, } \\
\text { solidified) }\end{array}$ & 0 & $<1$ & 0 & 2 & $<1$ & $<1$ & 0 & 0 & 218 & 221 \\
\hline $\begin{array}{l}\text { Sources (sealed sources, devices, and } \\
\text { gauges) }\end{array}$ & 0 & 109 & 0 & 905 & 0 & $\ll 1$ & 0 & 0 & 22 & 1,036 \\
\hline Paint waste & 0 & 0 & 0 & 0 & 0 & 0 & 0 & 0 & $\ll 1$ & $<<1$ \\
\hline Salt waste & 0 & 0 & $\mathrm{c}$ & 0 & 0 & 0 & 0 & 22 & 1 & 23 \\
\hline Incinerator ash (solidified, as-is) & $<<1$ & 0 & 0 & 0 & 0 & 0 & 0 & 0 & $\ll 1$ & $<<1$ \\
\hline Activated carbon (charcoal) & 0 & 0 & $\mathrm{c}$ & 0 & 0 & $\ll<1$ & 0 & 0 & 865 & 865 \\
\hline Other inorganic particulates & 0 & 235 & c & 0 & 0 & 0 & 0 & 0 & 10 & 245 \\
\hline Other & 0 & 17,620 & c & 2 & 0 & 0 & $\ll<1$ & 0 & 5,310 & 22,932 \\
\hline Total & 63,067 & 26,002 & c & 2,248 & 157,240 & 74,810 & 3,708 & 24,346 & 21,165 & 372,586 \\
\hline
\end{tabular}

a Naval Reactors (NR) sites include KAPL, BAPL, and NRF

bIncludes Ames, ANL-E, BNL, ETEC, FNAL, ITRI, KCP, LBL, LLNL, NTS, PANT, Pinellas, PPPL, RFETS, SLAC, SNL/CA, SNL/NM, WVDP, and Y-12. 'Unknown. 
Table 4.11. Breakdown by physical form of cumulative volumes of LLW stored at DOE sites ${ }^{\mathrm{a}}$

\begin{tabular}{|c|c|c|c|c|c|c|c|c|c|c|c|}
\hline \multirow[b]{2}{*}{ Physical form } & \multicolumn{11}{|c|}{ Volume, $\mathrm{m}^{3}$} \\
\hline & FEMP & INEL & $\mathrm{K}-25$ & Mound & ORNL & RFETS & SRS & WVDP & $\mathrm{Y}-12$ & $\begin{array}{c}\text { All } \\
\text { others } b\end{array}$ & Total \\
\hline \multicolumn{12}{|l|}{ Debris } \\
\hline Combustible & 0 & 4,102 & 1,777 & 282 & 0 & 2,380 & 0 & 121 & 869 & 125 & 9,656 \\
\hline Noncombustible and compactible & 0 & 3,637 & 432 & 0 & 0 & 62 & 0 & 117 & 131 & 64 & 4,443 \\
\hline $\begin{array}{l}\text { Noncombustible and combustible } \\
\text { mixed }\end{array}$ & 0 & 0 & 3,072 & 2,590 & 1,842 & 558 & 376 & 113 & 1,019 & 821 & 10,391 \\
\hline Activated metal/equipment/hardware & 0 & 0 & 0 & 0 & 0 & 0 & 1,240 & 0 & 0 & 633 & 1,873 \\
\hline Contaminated metal/equipment/hardware & 0 & 2,250 & 4,800 & 0 & 0 & 987 & 0 & 1,542 & 343 & 445 & 10,367 \\
\hline Reactor component/compartment & 0 & 0 & 0 & 0 & 0 & 0 & 0 & 0 & 0 & 10 & 10 \\
\hline Elemental lead shielding & 0 & 0 & 5 & 0 & 0 & 0 & 0 & 0 & 0 & 10 & 15 \\
\hline Filter media (as-is, solidified, dewatered) & 0 & 0 & 76 & 0 & 0 & 504 & 0 & 5 & 51 & 144 & 780 \\
\hline $\begin{array}{l}\text { Biological waste and carcasses } \\
\text { (as-is, solidified) }\end{array}$ & 0 & 0 & 0 & 0 & 0 & 6 & 0 & 1 & 0 & 6 & 13 \\
\hline $\begin{array}{l}\text { Asbestos-contaminated waste } \\
\text { (as-is, solidified) }\end{array}$ & 0 & 0 & 856 & 42 & 0 & 0 & 0 & 30 & 192 & 9 & 1,129 \\
\hline $\begin{array}{l}\text { Soil/sediment/rubble (as-is, solidified, } \\
\text { dewatered) }\end{array}$ & 0 & 4,016 & 816 & 0 & 0 & 100 & 0 & 455 & 234 & 184 & 5,805 \\
\hline Solidified liquids/chelates/oils & 0 & 0 & 0 & 33 & 0 & $<1$ & 0 & 3 & 0 & 14 & 50 \\
\hline $\begin{array}{l}\text { Solidified sludge/resin (dewatered, } \\
\text { solidified) }\end{array}$ & 0 & 0 & 895 & 445 & 0 & 723 & 0 & 6,188 & 26 & 14 & 8,291 \\
\hline $\begin{array}{l}\text { Sources (sealed sources, devices, and } \\
\text { gauges) }\end{array}$ & 0 & 0 & 0 & 0 & 0 & $<1$ & 0 & 0 & 6 & $4^{\mathrm{c}}$ & 10 \\
\hline Paint waste & 0 & 0 & 0 & 0 & 0 & 0 & 0 & 1 & 2 & 8 & 11 \\
\hline Salt waste & 0 & 0 & 27 & 0 & 0 & $<1$ & 0 & 0 & 1 & 1 & 29 \\
\hline Incinerator ash (solidified, as-is) & 0 & 0 & 291 & 0 & 0 & 1 & 0 & $<1$ & 0 & 14 & 306 \\
\hline Activated carbon (charcoal) & 0 & 0 & 49 & 0 & 0 & 0 & 0 & 5 & 0 & 11 & 65 \\
\hline Other inorganic particulates & 0 & 0 & 223 & 0 & 0 & 116 & 0 & 57 & 10 & 56 & 462 \\
\hline \multicolumn{12}{|l|}{ Other d } \\
\hline EM-30 program wastc & 0 & 4,629 & 319 & 0 & 0 & 24 & 0 & 6,298 & 50 & 748 & 12,068 \\
\hline EM-40 program waste & $140,000^{\mathrm{e}}$ & 0 & 0 & 0 & 0 & 0 & 0 & 0 & 0 & $40,000^{\mathrm{e}}$ & $180,000^{\circ}$ \\
\hline Total & 140,000 & 18,634 & 13,638 & 3,392 & 1,842 & 5,463 & 1,616 & 14,936 & 2,934 & 43,321 & 245,775 \\
\hline
\end{tabular}

ac Cumulative storage as of December 31, 1995.

bIncludes Ames, ANL-E, BNL, ETEC, FNAL, FUSRAP, GA, ITRI, KCP, LBL, LLNL, NRF, NTS, ORISE, PAD, PANT, Pinellas, RMI, SLAC, SNL/CA, and SNL/NM.

cThree sites reported 118 sources for which a volume estimate was not available (13 at BNL, 102 at ETEC, and 3 at ORISE).

dFor EM-40 program LLW, data available only for curnulative storage volume for physical form "other." The physical form categories listed above do not apply to EM-40 program wastes.

ESee Table 6.7 in Chapter 6. Reported volume rounded to two significant figures. 
Table 4.12. Breakdown by physical form of total gross radioactivity of LLW stored at DOE sites ${ }^{\text {a }}$

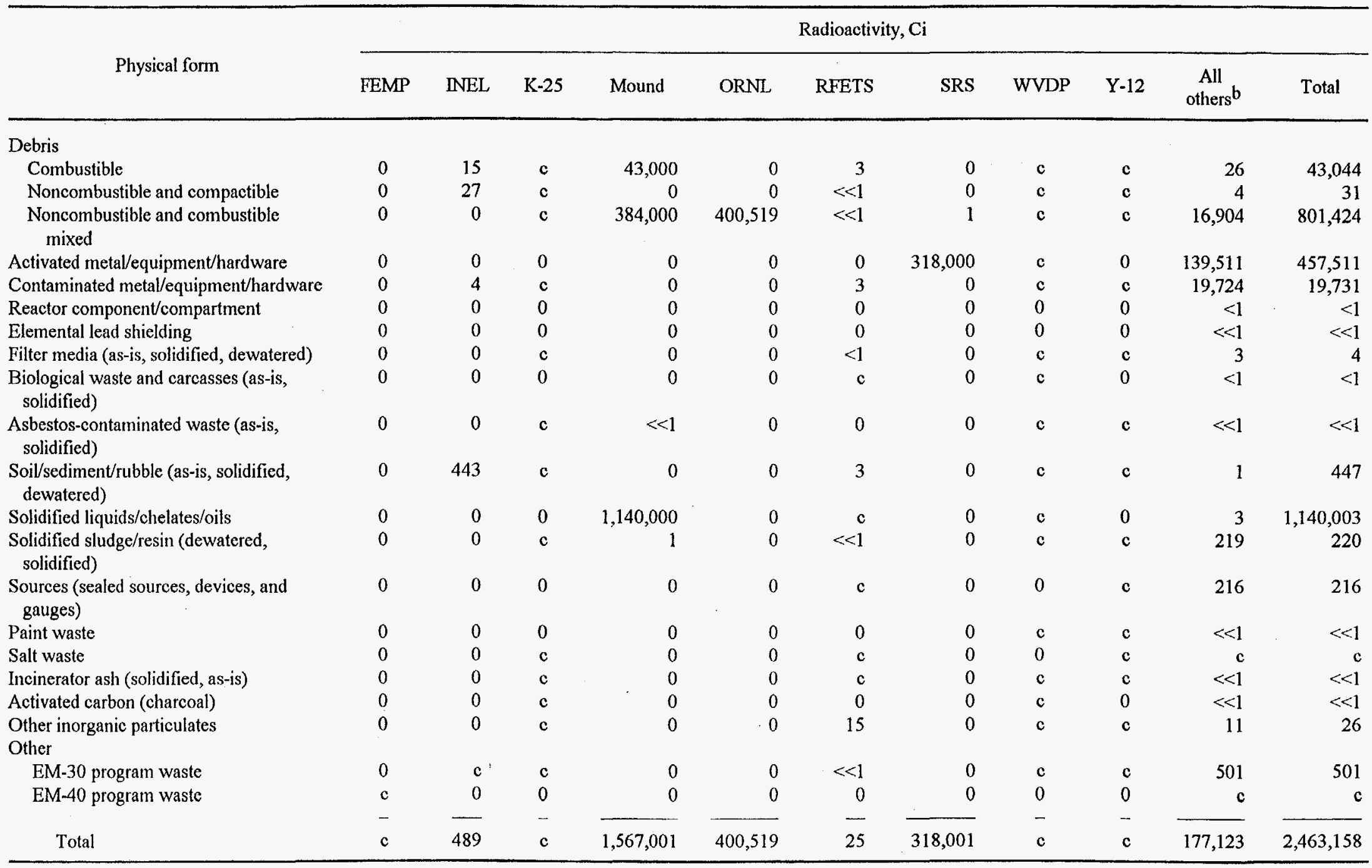

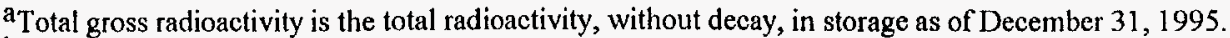

bIncludes Ames, ANL--E, BNL, ETEC, FNAL, ITRI, KCP, LBL, LLNL, NRF, NTS, ORISE, PANT, Pinellas, SLAC, SNL/CA, and SNL/NM. Radioactivity data not reported for EM-40 sites.

$\mathrm{c}_{\text {Not available. }}$ 
Table 4.13. Breakdown by physical form of volumes of LLW disposed during 1995 at DOE sites

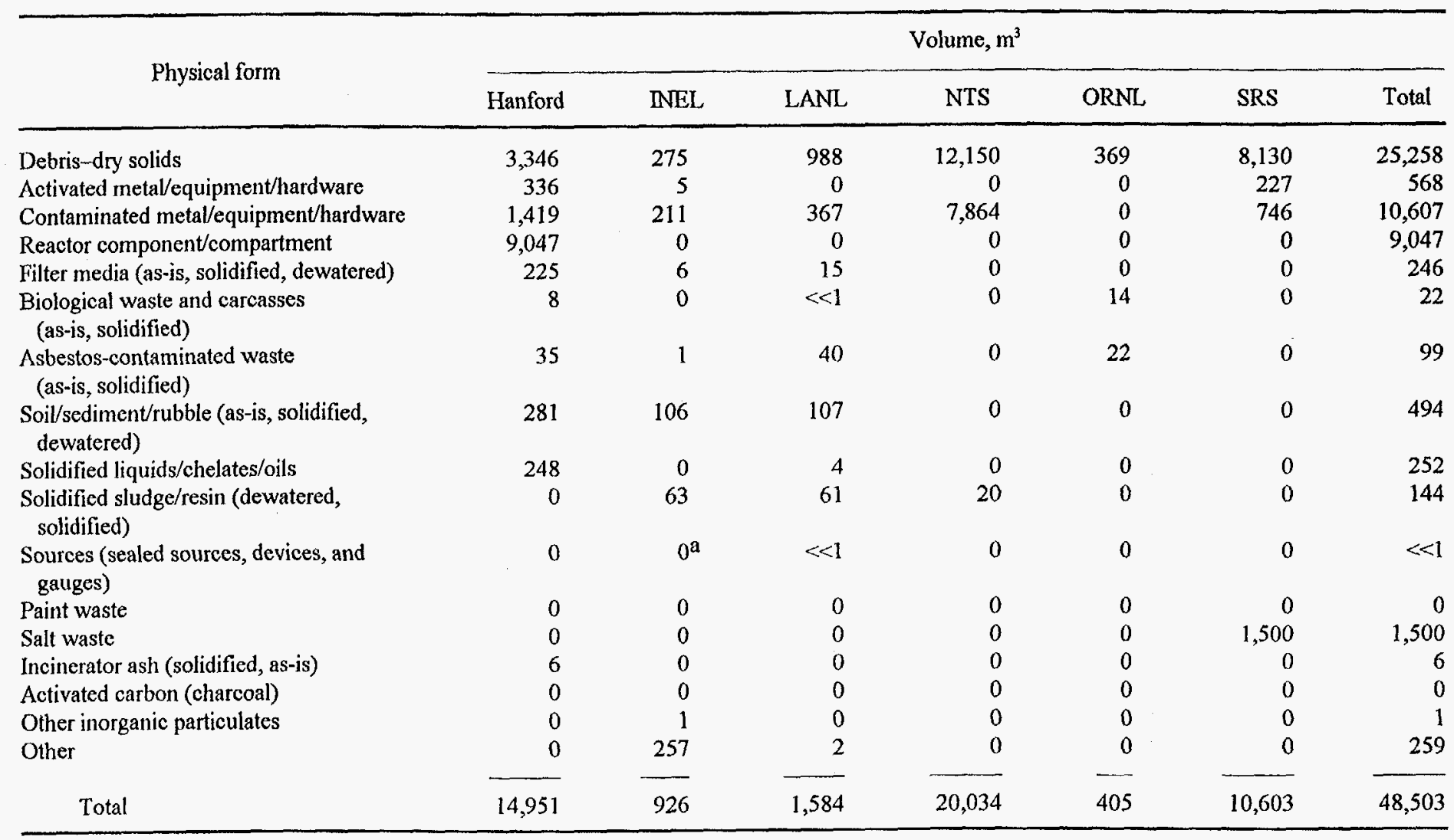

aINEL, reported 105 sources for which volume estimates were not available. 
Table 4.14. Breakdown by physical form of radioactivity of LLW disposed during 1995 at DOE sites

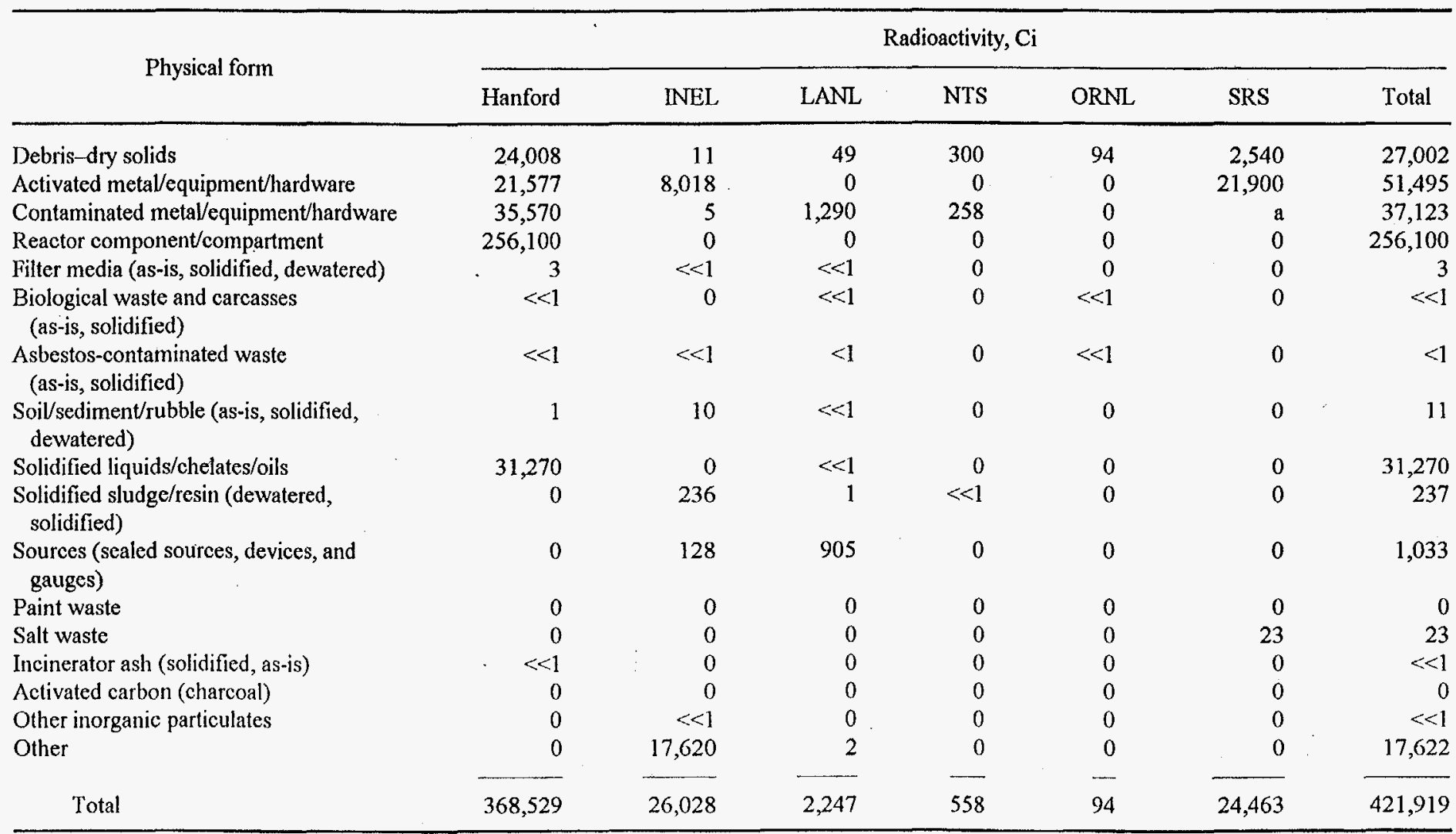

aunnown. 
Table 4.15. DOE LLW disposed by methods other than shallow-land burial ${ }^{a}$

\begin{tabular}{|c|c|c|c|c|}
\hline Site & Location & $\begin{array}{l}\text { Site use } \\
\text { (year) }\end{array}$ & $\begin{array}{l}\text { Waste } \\
\text { containers } \\
\text { buried }^{b}\end{array}$ & $\begin{array}{l}\text { Undecayed } \\
\text { radioactive } \\
\text { content } \\
\text { (Ci) }\end{array}$ \\
\hline \multicolumn{5}{|c|}{ Atlantic Ocean } \\
\hline Atlantic & $\begin{array}{l}38^{\circ} 30 \mathrm{~N} \\
72^{\circ} 06^{\circ} \mathrm{W}\end{array}$ & $\begin{array}{l}\text { 1951-1956; } \\
1959-1962\end{array}$ & 14,300 & $74,400^{\mathrm{c}}$ \\
\hline Atlantic & $\begin{array}{l}37^{\circ} 50 \mathrm{~N} \\
70^{\circ} 35^{\prime} \mathrm{W}\end{array}$ & $1957-1959$ & 14,500 & 2,100 \\
\hline Massachusetts Bay & $\begin{array}{l}42^{\circ} 25 \mathrm{~N} \\
70^{\circ} 35^{\prime} \mathrm{W}\end{array}$ & $1952-1959$ & 4,008 & 2,440 \\
\hline Cape Henry & $\begin{array}{l}36^{\circ} 56 \mathrm{~N} \\
74^{\circ} 23^{\prime} \mathrm{W}\end{array}$ & $1949-1967$ & 843 & 87 \\
\hline Central Atlantic & $\begin{array}{l}36^{\circ} 20 \mathrm{~N} \\
43^{\circ} 49 \mathrm{~N} \\
45^{\circ} 00^{\prime} \mathrm{W}\end{array}$ & $1959-1960$ & 432 & 480 \\
\hline Subtotal & & & 34,083 & 79,507 \\
\hline \multicolumn{5}{|c|}{ Pacific Ocean } \\
\hline $\begin{array}{l}\text { Farallon Islands } \\
\text { (Subsite A) }\end{array}$ & $\begin{array}{r}37^{\circ} 38 \mathrm{~N} \\
123^{\circ} 08^{\circ} \mathrm{W}\end{array}$ & $1951-1953$ & 3,500 & 1,100 \\
\hline $\begin{array}{l}\text { Farallon Islands } \\
\text { (Subsite B) }\end{array}$ & $\begin{array}{l}37^{\circ} 37^{\prime} \mathrm{N} \\
123^{\circ} 17^{\prime} \mathrm{W}\end{array}$ & $\begin{array}{l}1946-1950 \\
1954-1956\end{array}$ & 44,000 & 13,400 \\
\hline Santa Cruz Basin & $\begin{array}{l}33^{\circ} 40 \mathrm{~N} \\
119^{\circ} 40^{\circ} \mathrm{W}\end{array}$ & $1946-1962$ & 3,114 & 108 \\
\hline Cape Scot & $\begin{array}{l}50^{\circ} 56 \mathrm{~N} \\
136^{\circ} 03 \mathrm{~W} \\
52^{\circ} 25 \mathrm{~N} \\
140^{\circ} 12^{\prime} \mathrm{W}\end{array}$ & $1958-1969$ & 360 & 124 \\
\hline San Diego & $\begin{array}{l}32^{\circ} 00 \mathrm{~N} \\
121^{\circ} 30^{\circ} \mathrm{W}\end{array}$ & $1959-1962$ & 4,415 & 34 \\
\hline Subtotal (oceans) & & & 55,389 & 14,766 \\
\hline \multirow[t]{2}{*}{ Total } & & & 89,472 & 94,273 \\
\hline & \multicolumn{3}{|c|}{ Hydrofracture facility } & \\
\hline \multirow[t]{2}{*}{ ORNL } & \multirow{2}{*}{$\begin{array}{l}\text { Bedded Conasauga } \\
\text { shale underlying the } \\
\text { ORNL site }\end{array}$} & $1959-1965$ & $\begin{array}{l}\text { Small experimental } \\
\text { amounts of grout }\end{array}$ & \\
\hline & & $\begin{array}{l}1966-1980^{d} \\
1982^{\mathrm{e}^{-}} \\
1983^{\mathrm{e}}\end{array}$ & $\begin{array}{l}8.0 \times 10^{3} \mathrm{~m}^{3} \\
3.8 \times 10^{3} \mathrm{~m}^{3} \\
5.5 \times 10^{3} \mathrm{~m}^{3}\end{array}$ & $\begin{array}{l}600,000 \\
200,000 \\
500,000\end{array}$ \\
\hline Total & & & $17.3 \times 10^{3} \mathrm{~m}^{3}$ & $1,300,000$ \\
\hline
\end{tabular}

a Radioactivity is given at time of disposal. Data taken from Table 4.5 of ref. 3 .

Estimated number of containers.

Includes approximately $33,000 \mathrm{Ci}$ of induced activity associated with the U.S.S. Seawolf reactor vessel.

$\mathrm{d}_{\text {Retired after } 18 \text { injections. }}$

eNew facility started up with four injections in 1982 and completed campaign with seven injections in 1983. 
Table 4.16. Significant revisions and changes in the current values for LLW compared to the values in the previous year

\begin{tabular}{|c|c|c|c|c|}
\hline \multirow[t]{2}{*}{ Site } & $\begin{array}{l}\text { DOE/RW-0006, } \\
\text { Rev. } 11 \text { (1995) }\end{array}$ & $\begin{array}{l}\text { DOE/RW-0006, } \\
\text { Rev. } 12(1996)\end{array}$ & \multirow{2}{*}{$\begin{array}{l}\text { Significant revision } \\
\text { or net change }\end{array}$} & \multirow[t]{2}{*}{ Explanation } \\
\hline & Table No. & Table No. & & \\
\hline HANF & 4.7 and 4.12 & 4.7 and 4.12 & $\begin{array}{l}\text { Large decrease in cumulative stored } \\
\text { radioactivity at Hanford (from } \\
205,200 \text { Ci to } 0 \mathrm{Ci} \text { ) }\end{array}$ & $\begin{array}{l}\text { Sources, which accounted for the } \\
205,200 \mathrm{Ci} \text {, were redesignated as MLLW }\end{array}$ \\
\hline INEL & 4.7 and 4.12 & 4.7 and 4.12 & $\begin{array}{l}\text { Large decrease in cumulative stored } \\
\text { radioactivity at INEL (from } \\
1.2 \times 10^{6} \mathrm{Ci} \text { to } 489 \mathrm{Ci} \text { ) }\end{array}$ & $\begin{array}{l}\text { Change was a result of revised reporting } \\
\text { assumptions }\end{array}$ \\
\hline ORNL & $\begin{array}{l}4.7,4.11, \text { and } \\
4.12\end{array}$ & $\begin{array}{l}4.7,4.11, \text { and } \\
4.12\end{array}$ & $\begin{array}{l}\text { Large decrease in cumulative stored } \\
\text { volume and radioactivity at ORNL } \\
\text { (from } 3,538 \mathrm{~m}^{3} \text { to } 1,841 \mathrm{~m}^{3} \text { and from } \\
7.6 \times 10^{5} \mathrm{Ci} \text { to } 4.0 \times 10^{5} \mathrm{Ci} \text { ) }\end{array}$ & $\begin{array}{l}\text { Change was a result of revised reporting } \\
\text { assumptions }\end{array}$ \\
\hline ORNL & $\begin{array}{l}4.2,4.4 \text {, and } \\
4.17\end{array}$ & $\begin{array}{l}4.2,4.4 \text {, and } \\
4.17\end{array}$ & $\begin{array}{l}1994 \text { disposed volume changed } \\
\text { (from } 397 \mathrm{~m}^{3} \text { to } 318 \mathrm{~m}^{3} \text { ) }\end{array}$ & Revision to 1994 reported data \\
\hline
\end{tabular}


Table 4.17. Historical and projected volume, radioactivity, and thermal power characteristics of disposed DOE LLW, except SRS saltstone

\begin{tabular}{|c|c|c|c|c|c|c|}
\hline \multirow{2}{*}{$\begin{array}{l}\text { End of } \\
\text { calendar } \\
\text { year }\end{array}$} & \multicolumn{2}{|c|}{$\begin{array}{l}\text { Volume } e^{2, b} \\
\left(10^{3} \mathrm{~m}^{3}\right)\end{array}$} & \multicolumn{2}{|c|}{$\begin{array}{l}\text { Radioactivity }{ }^{\mathrm{a}, \mathrm{b}} \\
\left(10^{3} \mathrm{Ci}\right)\end{array}$} & \multicolumn{2}{|c|}{$\begin{array}{c}\text { Thermal power } \\
\text { (W) }\end{array}$} \\
\hline & Annual & Cumulative & Annual & Cumulative & Annual & Cumulative \\
\hline 1990 & 60.0 & 2,759 & 545 & 13,516 & 2,013 & 17,844 \\
\hline 1991 & 53.6 & 2,812 & 717 & 13,277 & 2,788 & 18,220 \\
\hline 1992 & 48.3 & 2,860 & 1,078 & 13,401 & 4,947 & 20,741 \\
\hline 1993 & 50.5 & 2,911 & 894 & 13,147 & 3,263 & 20,398 \\
\hline 1994 & 52.1 & 2,963 & 621 & 12,858 & 3,463 & 21,534 \\
\hline 1995 & 48.5 & 3,011 & 422 & 12,550 & 2.378 & 22,195 \\
\hline 1996 & 37.5 & 3,049 & 792 & 12,695 & 4,621 & 25,312 \\
\hline 1997 & 31.5 & 3,080 & 740 & 12,778 & 4,344 & 27,840 \\
\hline 1998 & 31.5 & 3,112 & 740 & 12,850 & 4,344 & 30,092 \\
\hline 1999 & 31.5 & 3,143 & 740 & 12,908 & 4,344 & 32,072 \\
\hline 2000 & 31.5 & 3,175 & 740 & 12,955 & 4,344 & 33,799 \\
\hline 2001 & 31.5 & 3,206 & 740 & 12,992 & 4,344 & 35,297 \\
\hline 2002 & 23.9 & 3,230 & 377 & 12,659 & 2,184 & 34,433 \\
\hline 2003 & 23.9 & 3,254 & 377 & 12,367 & 2,184 & 33,681 \\
\hline 2004 & 23.9 & 3,278 & 377 & 12,106 & 2,184 & 33,002 \\
\hline 2005 & 23.9 & 3,302 & 377 & 11,870 & 2,184 & 32,387 \\
\hline 2006 & 23.9 & 3,326 & 377 & 11,656 & 2,184 & 31,825 \\
\hline 2007 & 23.9 & 3,350 & 377 & 11,462 & 2,184 & 31,312 \\
\hline 2008 & 23.9 & 3,374 & 377 & 11,285 & 2,184 & 30,841 \\
\hline 2009 & 23.9 & 3,398 & 377 & 11,123 & 2,184 & 30,406 \\
\hline 2010 & 23.9 & 3,422 & 377 & 10,975 & 2,184 & 30,005 \\
\hline 2011 & 23.9 & 3,446 & 377 & 10,840 & 2,184 & 29,634 \\
\hline 2012 & 23.9 & 3,470 & 377 & 10,716 & 2,184 & 29,288 \\
\hline 2013 & 23.9 & 3,494 & 377 & 10,602 & 2,184 & 28,966 \\
\hline 2014 & 23.9 & 3,518 & 377 & 10,498 & 2,184 & 28,665 \\
\hline 2015 & 23.9 & 3,542 & 377 & 10,403 & 2,184 & 28,383 \\
\hline 2016 & 23.9 & 3,566 & 377 & 10,316 & 2,184 & 28,118 \\
\hline 2017 & 23.9 & 3,590 & 377 & 10,237 & 2,184 & 27,869 \\
\hline 2018 & 23.9 & 3,613 & 377 & 10,165 & 2,184 & 27,633 \\
\hline 2019 & 23.9 & 3,637 & 377 & 10,100 & 2,184 & 27,410 \\
\hline 2020 & 23.9 & 3,661 & 377 & 10,041 & 2,184 & 27,198 \\
\hline 2021 & 23.9 & 3,685 & 377 & 9,988 & 2,184 & 26,997 \\
\hline 2022 & 23.9 & 3,709 & 377 & 9,940 & 2,184 & 26,805 \\
\hline 2023 & 23.9 & 3,733 & 377 & 9,898 & 2,184 & 26,622 \\
\hline 2024 & 23.9 & 3,757 & 377 & 9,860 & 2,184 & 26,447 \\
\hline 2025 & 23.9 & 3,781 & 377 & 9,827 & 2,184 & 26,280 \\
\hline 2026 & 23.9 & 3,805 & 377 & 9,799 & 2,184 & 26,119 \\
\hline 2027 & 23.9 & 3,829 & 377 & 9,774 & 2,184 & 25,965 \\
\hline 2028 & 23.9 & 3,853 & 377 & 9,754 & 2,184 & 25,817 \\
\hline 2029 & 23.9 & 3,877 & 377 & 9,737 & 2,184 & 25,674 \\
\hline 2030 & 23.9 & 3,901 & 377 & 9,723 & 2,184 & 25,536 \\
\hline
\end{tabular}

aHistorical (beginning of operations through 1994) annual values of volume and radioactivity for each site are from ref. 5. Similar values for 1995 are from ref. 1 . See Tables 4.4, 4.8, 4.13, and 4.14 for more detail. For disposals prior to 1994, radioactivity (by waste type) is decayed from the year of addition using the representative compositions given in Table A.3 of Appendix A. Starting with 1994, representative compositions provided by the sites in the data call for Rev. 11 of this report are used to decay radioactivity.

$b_{\text {Beginning in } 1996, \text { projected disposals are estimated for each active disposal site. Estimated values }}$ reported in this table may not agree with summary data reported in Table 4.8 because projections were reported as either unknown or unavailable by some sites. 
Table 4.18. Projected volume, radioactivity, and thermal power characteristics of DOE LLW saltstone at SRS ${ }^{a}$

\begin{tabular}{|c|c|c|c|c|c|c|}
\hline \multirow{2}{*}{$\begin{array}{l}\text { End of } \\
\text { calendar } \\
\text { year }\end{array}$} & \multicolumn{2}{|c|}{$\begin{array}{l}\text { Volume } \\
\left(10^{3} \mathrm{~m}^{3}\right)\end{array}$} & \multicolumn{2}{|c|}{$\begin{array}{l}\text { Radioactivity } \\
\left(10^{3} \mathrm{Ci}\right)\end{array}$} & \multicolumn{2}{|c|}{$\begin{array}{l}\text { Thermal power } \\
\text { (W) }\end{array}$} \\
\hline & Annual & Cumulative & Annual & Cumulative & Annual & Cumulative \\
\hline 1996 & 45.0 & $72.0^{\mathrm{b}}$ & 4.7 & 4.7 & 43.5 & 43.5 \\
\hline 1997 & 45.0 & 117.0 & 4.3 & 7.8 & 38.8 & 70.8 \\
\hline 1998 & 45.0 & 162.0 & 4.4 & 10.9 & 37.8 & 93.9 \\
\hline 1999 & 45.0 & 207.0 & 4.6 & 14.4 & 36.8 & 114.2 \\
\hline 2000 & 45.0 & 252.0 & 4.9 & 18.3 & 34.5 & 129.3 \\
\hline 2001 & 45.0 & 297.0 & 5.1 & 22.6 & 30.9 & 138.2 \\
\hline 2002 & 45.0 & 342.0 & 5.1 & 27.0 & 27.3 & 143.6 \\
\hline 2003 & 45.0 & 387.0 & 5.2 & 31.5 & 24.6 & 149.9 \\
\hline 2004 & 45.0 & 432.0 & 5.2 & 36.0 & 22.9 & 159.9 \\
\hline 2005 & 45.0 & 477.0 & 5.1 & 40.4 & 22.1 & 174.1 \\
\hline 2006 & 45.0 & 522.0 & 5.1 & 44.8 & 21.7 & 191.6 \\
\hline 2007 & 45.0 & 567.0 & 5.0 & 49.1 & 21.7 & 211.5 \\
\hline 2008 & 45.0 & 612.0 & 5.0 & 53.2 & 21.8 & 232.8 \\
\hline 2009 & 45.0 & 657.0 & 4.9 & 57.3 & 21.9 & 255.2 \\
\hline 2010 & 45.0 & 702.0 & 4.9 & 61.3 & 22.1 & 278.2 \\
\hline 2011 & 45.0 & 747.0 & 4.8 & 65.1 & 22.3 & 301.7 \\
\hline 2012 & 45.0 & 792.0 & 4.8 & 68.9 & 22.5 & 325.5 \\
\hline 2013 & 45.0 & 837.0 & 4.7 & 72.6 & 22.7 & 349.5 \\
\hline 2014 & 45.0 & 882.0 & 4.7 & 76.2 & 22.9 & 373.9 \\
\hline 2015 & 45.0 & 927.0 & 4.6 & 79.7 & 23.1 & 398.5 \\
\hline 2016 & 45.0 & 972.0 & 4.6 & 83.2 & 23.3 & 423.3 \\
\hline 2017 & 45.0 & $1,017.0$ & 4.5 & 86.5 & 23.5 & 448.4 \\
\hline 2018 & 45.0 & $1,062.0$ & 4.5 & 89.8 & 23.6 & 473.7 \\
\hline 2019 & 45.0 & $1,107.0$ & 4.4 & 93.1 & 23.8 & 499.2 \\
\hline 2020 & 45.0 & $1,152.0$ & 4.4 & 96.2 & 24.0 & 525.1 \\
\hline 2021 & 45.0 & $1,197.0$ & .4 .4 & 99.3 & 24.2 & 551.2 \\
\hline 2022 & 45.0 & $1,242.0$ & 4.3 & 102.4 & 24.4 & 577.6 \\
\hline 2023 & 45.0 & $1,287.0$ & 4.3 & 105.4 & 24.6 & 604.2 \\
\hline 2024 & 45.0 & $1,332.0$ & 4.3 & 108.3 & 24.9 & 631.2 \\
\hline 2025 & 45.0 & $1,377.0$ & 4.2 & 111.2 & 25.1 & 658.5 \\
\hline 2026 & 45.0 & $1,422.0$ & 4.2 & 114.0 & 25.3 & 686.1 \\
\hline 2027 & 45.0 & $1,467.0$ & 4.2 & 116.8 & 25.5 & 714.0 \\
\hline 2028 & 45.0 & $1,512.0$ & 4.1 & 119.6 & 25.7 & 742.2 \\
\hline 2029 & 45.0 & $1,557.0$ & 4.1 & 122.3 & 25.9 & 770.7 \\
\hline 2030 & 45.0 & $1,602.0$ & 4.1 & 124.9 & 26.2 & 799.6 \\
\hline
\end{tabular}

asource: ref. 1 of Chapter 2.

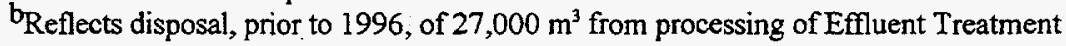
Facility concentrate. 
Table 4.19. Historical annual additions and total volume of LLW at commercial disposal sites ${ }^{\mathrm{a}}$

\begin{tabular}{|c|c|c|c|c|c|c|c|c|}
\hline \multirow{2}{*}{ Year } & \multicolumn{8}{|c|}{ Volume, $\mathrm{m}^{3}$} \\
\hline & Beatty $^{b}$ & $\begin{array}{l}\text { West } \\
\text { Valleyc }^{c}\end{array}$ & $\begin{array}{l}\text { Maxey } \\
\text { Flats }\end{array}$ & Richland & Sheffield & Barnwell & $\begin{array}{c}\text { Annual } \\
\text { total }\end{array}$ & $\begin{array}{l}\text { Cumulative } \\
\text { total }\end{array}$ \\
\hline 1962 & 1,861 & & & & & & 1,861 & 1,861 \\
\hline 1963 & 3,512 & 127 & 2,206 & & & & 5,845 & 7,706 \\
\hline 1964 & 2,836 & 5,940 & 3,872 & & & & 12,648 & 20,354 \\
\hline 1965 & 1,988 & 5,192 & 5,753 & 668 & & & 13,601 & 33,955 \\
\hline 1966 & 3,533 & 3,951 & 5,557 & 2,402 & & & 15,443 & 49,398 \\
\hline 1967 & 3,206 & 7,475 & 7,820 & 773 & 2,527 & & 21,801 & 71,199 \\
\hline 1968 & 3,576 & 3,490 & 8,178 & 1,359 & 2,713 & & 19,316 & 90,515 \\
\hline 1969 & 4,526 & 4,099 & 10,354 & 438 & 2,012 & & 21,429 & 111,944 \\
\hline 1970 & 5,152 & 4,906 & 12,521 & 423 & 2,825 & & 25,827 & 137,771 \\
\hline 1971 & 4,916 & 7,002 & 13,173 & 584 & 4,430 & 1,171 & 31276 & 169,047 \\
\hline 1972 & 4,301 & 9,045 & 15,578 & 654 & 5,956 & 3,757 & 39,291 & 208,338 \\
\hline 1973 & 4,076 & 7,535 & 10,074 & 1,033 & 8,524 & 15,839 & 47,081 & 255,419 \\
\hline 1974 & 4,103 & 8,866 & 8,898 & 1,411 & 12,373 & 18,244 & 53,895 & 309,314 \\
\hline 1975 & 4,943 & 2,243 & 17,098 & 1,500 & 14,116 & 18,072 & 57,972 & 367,286 \\
\hline 1976 & 3,864 & 427 & 13,775 & 2,867 & 13,480 & 40,227 & 74,640 & 441,926 \\
\hline 1977 & 4,742 & 351 & 423 & 2,718 & 17,643 & 45,663 & 71,540 & 513,466 \\
\hline 1978 & 8,874 & 144 & & 7,422 & 1,735 & 61,554 & 79,729 & 593,195 \\
\hline 1979 & 6,491 & 138 & & 12,185 & & 63,861 & 82,675 & 675,870 \\
\hline 1980 & 12,717 & 141 & & 24,819 & & $54,723^{f}$ & 92,400 & 768,270 \\
\hline 1981 & 3,351 & 216 & & 40,732 & & $39,427^{f}$ & 83,726 & 851,996 \\
\hline 1982 & 1,505 & 632 & & 39,606 & & 34,779 & 76,522 & 928,518 \\
\hline 1983 & 1,111 & 1,284 & & 40,458 & & 35,132 & 77,985 & $1,006,503$ \\
\hline 1984 & 2,067 & 966 & & 38,481 & & 34,879 & 76,393 & $1,082,896$ \\
\hline 1985 & 1,388 & 809 & & 40,135 & & 34,389 & 76,721 & $1,159,617$ \\
\hline 1986 & 2,668 & 2,095 & & 18,833 & & 29,612 & 53,208 & $1,212,825$ \\
\hline 1987 & 9,414 & & & 15,765 & & 27,060 & 52,239 & $1,265,064$ \\
\hline 1988 & 2,645 & & & 11,430 & . & 26,391 & 40,466 & $1,305,530$ \\
\hline 1989 & 3,291 & & & 11,562 & & 31,242 & 46,095 & $1,351,625$ \\
\hline 1990 & 1,684 & & & 8,362 & & 22,315 & 32,361 & $1,383,986$ \\
\hline 1991 & 4,539 & & & 11,872 & & 22,368 & 38,779 & $1,422,765$ \\
\hline 1992 & 14,575 & & & 11,271 & & 23,518 & 49,364 & $1,472,129$ \\
\hline 1993 & & & & 5,288 & & 17,145 & 22,433 & $1,494,562$ \\
\hline 1994 & & & & 3,533 & & 20,783 & 24,316 & $1,518,878$ \\
\hline 1995 & & & & 5,804 & & 13,734 & 19,538 & $1,538,416$ \\
\hline Total & 137,455 & 77,074 & 135,280 & 364,388 & 88,334 & 735,885 & & $1,538,416$ \\
\hline
\end{tabular}

aFor a summary of historical additions (1962-1984), see Table 4.6 in ref. 3. For Beatty, Richland, and Barnwell, the additions for 1985-1994 are from Table 4.19 in ref. 5. Information for 1995 is taken from ref. 7.

Beatty ceased accepting LLW Dec. 31, 1992.

CWest Valley includes a commercial state-licensed facility which opened Nov. 18, 1963, and closed Mar. 11, 1975, and an NRC-licensed facility (for on-site fuel reprocessing wastes) which opened in 1966 and continued to receive only onsite-generated LLW associated with water treatment and site cleanup until late 1986 . This license is in abeyance. Disposal operations at the West Valley Demonstration Project (WVDP) have been suspended pending the preparation of an EIS report for the West Valley site closure. The WVDP began in 1980. The LLW volumes reported for 1982 through 1986 are for the WVDP only and are taken from ref. 5. Since the beginning of 1987, LLW generated at the WVDP is stored on-site in engineered facilities pending final disposal (ref. 5)

${ }^{d}$ Closed Dec. 27, 1977. Small perturbations in waste volumes have occurred during site cleanup operations but are not included here since they are inconsequential.

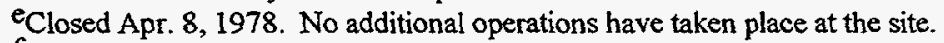

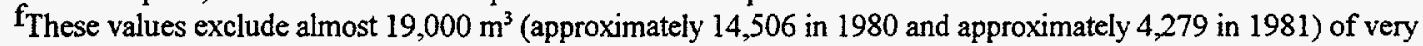
low-level-activity settling pond sludge that was not included in the annual quota. 
Table 4.20. Historical annual additions and total undecayed radioactivity of $L L W$ at commercial disposal sites ${ }^{2}$

\begin{tabular}{|c|c|c|c|c|c|c|c|c|}
\hline \multirow{2}{*}{ Year } & \multicolumn{8}{|c|}{ Radioactivity, $\mathrm{Ci}$} \\
\hline & Beatty $^{b}$ & $\begin{array}{l}\text { West } \\
\text { Valleyc }\end{array}$ & $\begin{array}{l}\text { Maxey } \\
\text { Flats }\end{array}$ & Richland & Sheffield ${ }^{e}$ & Barnwell & $\begin{array}{c}\text { Annual } \\
\text { total }\end{array}$ & $\begin{array}{c}\text { Cumulative } \\
\text { total }\end{array}$ \\
\hline 1962 & $f$ & & & & & & $\mathrm{f}$ & $\mathbf{f}$ \\
\hline 1963 & 5,690 & 100 & 22,556 & & & & 28,346 & 28,346 \\
\hline 1964 & 6,477 & 10,400 & 147,218 & & & & 164,095 & 192,441 \\
\hline 1965 & 6,377 & 22,600 & 63,828 & 144 & & & 92,949 & 285,390 \\
\hline 1966 & 11,974 & 35,400 & 52,737 & 1,606 & & & 101,717 & 387,107 \\
\hline 1967 & 10,894 & 123,100 & 23,273 & 5,378 & 3,850 & & 166,495 & 553,602 \\
\hline 1968 & 6,808 & 10,600 & 45,577 & 64,432 & 2,381 & & 129,798 & 683,400 \\
\hline 1969 & 9,761 & 36,000 & 31,028 & 55,964 & 2,192 & & 134,945 & 818,345 \\
\hline 1970 & 12,304 & 91,900 & 46,969 & 52,820 & 5,427 & & 209,420 & $1,027,765$ \\
\hline 1971 & 4,316 & 436,700 & 720,146 & 23,916 & 7,895 & 4,151 & $1,197,124$ & $2,224,889$ \\
\hline 1972 & 5,228 & 131,300 & 217,351 & 31,809 & 4,857 & 13,575 & 404,120 & $2,629,009$ \\
\hline 1973 & 5,704 & 346,000 & 118,359 & 57,037 & 2,834 & 48,212 & 578,146 & $3,207,155$ \\
\hline 1974 & 23,904 & 6,600 & 143,656 & 12,773 & 3,229 & 13,557 & 203,719 & $3,410,874$ \\
\hline 1975 & 18,388 & 11,600 & 289,570 & 113,341 & 6,103 & 17,428 & 456,430 & $3,867,304$ \\
\hline 1976 & 4,493 & 1,200 & 211,359 & 104,306 & 7,744 & 90,205 & 419,307 & $4,286,611$ \\
\hline 1977 & 23,811 & 900 & 267,063 & 7,465 & 11,147 & 390,121 & 700,507 & $4,987,118$ \\
\hline 1978 & 5,685 & 700 & & 235,548 & 2,547 & 652,061 & 896,541 & $5,883,659$ \\
\hline 1979 & 8,897 & 400 & & 164,787 & & 314,938 & 489,022 & $6,372,681$ \\
\hline 1980 & 148,312 & 300 & & 41,031 & 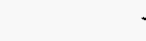 & 143,502 & 333,145 & $6,705,826$ \\
\hline 1981 & 52,214 & 229 & & 43,905 & & 183,744 & 280,092 & $6,985,918$ \\
\hline 1982 & 80,929 & 293 & & 59,007 & & 273,962 & 414,191 & $7,400,109$ \\
\hline 1983 & 1,356 & 255 & & 120,534 & & 383,450 & 505,595 & $7,905,704$ \\
\hline 1984 & 544 & 25 & & 215,286 & & 385,079 & 600,934 & $8,506,638$ \\
\hline 1985 & 453 & 39 & & 287,849 & & 460,571 & 748,912 & $9,255,550$ \\
\hline 1986 & 672 & 13 & & 115,591 & & 116,108 & 232,384 & $9,487,934$ \\
\hline 1987 & 3,353 & 0 & & 42,734 & & 211,026 & 257,113 & $9,745,047$ \\
\hline 1988 & 8,690 & 0 & & 32,067 & & 218,901 & 259,658 & $10,004,705$ \\
\hline 1989 & 42,678 & 0 & & 99,056 & & 725,164 & 866,898 & $10,871,603$ \\
\hline 1990 & 11,323 & 0 & & 92,985 & & 444,277 & 548,585 & $11,420,188$ \\
\hline 1991 & 29,679 & 0 & & 158,784 & & 611,348 & 799,811 & $12,219,999$ \\
\hline 1992 & 90,206 & 0 & & 93,923 & & 815,974 & $1,000,103$ & $13,220,102$ \\
\hline 1993 & & & & 31,422 & & 611,785 & 643,207 & $13,863,309$ \\
\hline 1994 & & & & 6,078 & & 745,301 & 751,379 & $14,614,688$ \\
\hline 1995 & & & & 2,836 & & 168,981 & 171,817 & $14,786,505$ \\
\hline Total & 641,120 & $1,266,654$ & $2,400,690$ & $2,374,414$ & 60,206 & $8,043,421$ & & $14,786,505$ \\
\hline
\end{tabular}

aFor a summary of historical additions (1962-1984), see Table 4.6 in ref. 3 . For Beatty, Richland, and Barnwell, the additions for 1985-1994 are from Table 4.20 in ref. 5 . Information for 1995 is taken from ref. 7.

beatty ceased accepting LLW Dec. 31, 1992.

'West Valley includes a commercial state-licensed facility which opened Nov. 18, 1963, and closed Mar. 11, 1975, and an NRC-licensed facility (for on-site fuel reprocessing wastes) which opened in 1966 and continued to receive only on-sitegenerated LLW associated with water treatment and site cleanup until late 1986 . This license is in abeyance. Disposal operations at the West Valley Demonstration Project (WVDP) have been suspended pending the preparation of an EIS report for the West Valley site closure. The WVDP began in 1980. The LLW radioactivity values reported for 1982 through 1986 are for the WVDP only and are taken from ref. 5 . Since the beginning of $1987, \mathrm{LLW}$ generated at the WVDP is stored on-site in engineered facilities pending final disposal (ref. 5).

Closed Dec. 27, 1977.

elosed Apr. 8, 1978.

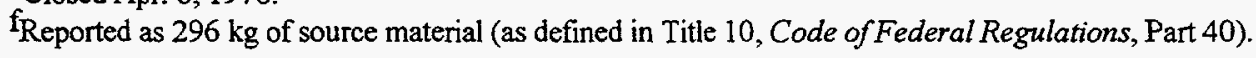


Table 4.21. Distribution of total volume and radioactivity, by state, of LLW shipped to commercial disposal sites in $1995^{a}$

\begin{tabular}{|c|c|c|c|c|c|}
\hline State & $\begin{array}{l}\text { Volume } \\
\left(\mathrm{m}^{3}\right)\end{array}$ & $\begin{array}{l}\text { Radioactivity } \\
\text { (Ci) }\end{array}$ & State & $\begin{array}{l}\text { Volume } \\
\left(\mathrm{m}^{3}\right)\end{array}$ & $\begin{array}{l}\text { Radioactivity } \\
\text { (Ci) }\end{array}$ \\
\hline Alabama & 398 & 78,340 & Nebraska & 68 & 407 \\
\hline Alaska & 0 & 0 & Nevada & 2 & $<1$ \\
\hline Arizona & $<1$ & $<1$ & New Hampshire & $<1$ & $<1$ \\
\hline Arkansas & 55 & 8 & New Jersey & 402 & 996 \\
\hline California & 326 & 409 & New Mexico & 25 & 2 \\
\hline Colorado & 1,954 & 553 & New York & 286 & 1,951 \\
\hline Connecticut & 176 & 841 & North Carolina & 708 & 3,310 \\
\hline Delaware & 2 & $<1$ & North Dakota & 0 & 0 \\
\hline District of Columbia & 4 & $<1$ & Ohio & 250 & 553 \\
\hline Florida & 174 & 2,444 & Oklahoma & 4 & $<1$ \\
\hline Georgia & 519 & 13,798 & Oregon & 2,119 & 1279 \\
\hline Hawaii & 75 & 1,821 & Pennsylvania & 997 & 5,692 \\
\hline Idaho & 41 & $<1$ & Puerto Rico & 0 & 0 \\
\hline IIlinois & 1,636 & 7,044 & Rhode Island & $<1$ & 15 \\
\hline Indiana & 3 & 36 & South Carolina & 2,465 & 4,418 \\
\hline Iowia & 58 & 209 & South Dakota & 7 & $<1$ \\
\hline Kansas & 26 & 398 & Tennessee & 2,232 & 777 \\
\hline Kentucky & 3 & 20 & Texas & 83 & 2,010 \\
\hline Louisiana & 192 & 13,244 & Utah & 127 & 22 \\
\hline Maine & 80 & 78 & Vermont & 3 & 135 \\
\hline Maryland & 125 & 346 & Virgin Islands & 0 & 0 \\
\hline Massachusetts & 311 & 24,903 & Virginia & 1,136 & 951 \\
\hline Michigan & 579 & 1,438 & Washington & 1,461 & 923 \\
\hline Minnesota & 70 & 365 & West Virginia & $<1$ & 5 \\
\hline Mississippi & 284 & 1,543 & Wisconsin & 30 & 348 \\
\hline Missouri & 40 & 183 & Wyoming & 2 & $<1$ \\
\hline \multirow[t]{2}{*}{ Montana } & $<1$ & $<1$ & & - & $\longrightarrow$ \\
\hline & & & Total & 19,538 & 171,817 \\
\hline
\end{tabular}

asource: ref. 7. 
Table 4.22. Historical and projected cumulative volume and radioactivity summary of commercial GTCC LLWa

\begin{tabular}{|c|c|c|c|c|c|c|}
\hline \multirow{3}{*}{ Category } & \multicolumn{3}{|c|}{$1993^{b}$} & \multicolumn{3}{|c|}{$2035^{\mathrm{c}, \mathrm{d}}$} \\
\hline & \multicolumn{2}{|c|}{$\begin{array}{l}\text { Volume } \\
\left(\mathrm{m}^{3}\right)\end{array}$} & \multirow{2}{*}{$\begin{array}{l}\text { Radioactivity } \\
\text { (Ci) }\end{array}$} & \multicolumn{2}{|c|}{$\begin{array}{l}\text { Volume } \\
\left(\mathrm{m}^{3}\right)\end{array}$} & \multirow{2}{*}{$\begin{array}{l}\text { Radioactivity } \\
\text { (Ci) }\end{array}$} \\
\hline & Unpackaged & $\begin{array}{c}\text { ACA } \\
\text { packaged }^{e}\end{array}$ & & Unpackaged & $\begin{array}{c}\text { ACA } \\
\text { packaged }\end{array}$ & \\
\hline \multicolumn{7}{|l|}{ Nuclear utility wastes } \\
\hline - BWR operations & 3.20 & 1.10 & 28,200 & 105.2 & 36.7 & $1,155,517$ \\
\hline - PWR operations & 2.82 & 0.12 & 18,300 & 77.7 & 10.0 & 573,510 \\
\hline LWR operations total & 6.02 & 1.22 & 46,500 & 182.9 & 46.7 & $1,729,027$ \\
\hline - BWR decommissioning & 6.26 & 14.48 & 757,000 & 115.4 & 188.8 & $3,270,412$ \\
\hline - PWR decommissioning & 3.98 & 10.32 & $3,086,500$ & 398.9 & 570.9 & $30,548,517$ \\
\hline LWR decommissioning total & 10.24 & 24.80 & $3,843,500$ & 514.3 & 759.7 & $33,818,929$ \\
\hline Nuclear utility total & 16.26 & 26.02 & $3,890,000$ & 697.2 & 806.4 & $35,547,956$ \\
\hline \multicolumn{7}{|l|}{ Sealed sources } \\
\hline - General license & 0.007 & 0.474 & 1,119 & 0.123 & 8.09 & 18,440 \\
\hline - Specific license & 0.125 & 38.22 & 354,000 & 0.87 & 234 & $1,560,000$ \\
\hline Sealed sources total & 0.13 & 38.69 & 355,119 & 0.99 & 242 & $1,578,440$ \\
\hline DOE-held potential GTCC waste & 0 & 0 & 0 & 0 & 0 & 0 \\
\hline Other generator waste $f$ & 46.9 & 74.2 & 2,738 & 235 & 465 & 12,680 \\
\hline Grand total & 63.3 & 138.9 & $4,247,857$ & 933 & 1,513 & $37,139,076$ \\
\hline
\end{tabular}

a Based on the INEL study of ref. 13. Projected data reported represent base-case scenario projections.

${ }^{\text {Reported cumulative inventory as of December } 31,1993 .}$

cProjected cumulative inventory for end of CY 2035.

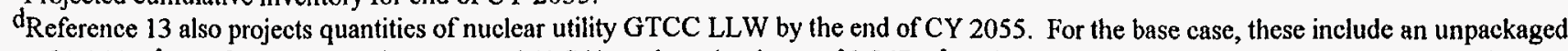
volume of $1,144 \mathrm{~m}^{3}$, an after-concentration-averaged ( $\mathrm{ACA}$ ) packaged volume of $1,347 \mathrm{~m}^{3}$, and an associated radioactivity of $88,400,000 \mathrm{Ci}$.

ACA packaged waste. This is the packaged volume of waste that is classified as GTCC LLW, after all other waste has been classified as Class A, B, or C LLW using concentration-averaging practices.

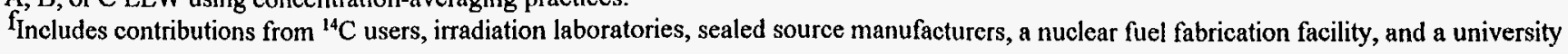
reactor. 
Table 4.23. Breakdown of 1995 low-level radioactive waste by type, volume, and radioactivity received

by commercial disposal sites ${ }^{\mathrm{a}}$

\begin{tabular}{llrr}
\hline $\begin{array}{c}\text { Commercial } \\
\text { site }\end{array}$ & \multicolumn{1}{c}{$\begin{array}{c}\text { Type of } \\
\text { waste }\end{array}$} & $\begin{array}{r}\text { Volume } \\
\left(\mathbf{m}^{3}\right)\end{array}$ & $\begin{array}{r}\text { Radioactivity } \\
(\mathrm{Ci})\end{array}$ \\
\hline Barnwell & Academic & 132 & 39 \\
& Government & 3,171 & 74,834 \\
& Industrial & 3,200 & 24,995 \\
& Medical & 19 & 2 \\
& Utility & 7,212 & 69,111 \\
& & 13,734 & 168,981 \\
& & & 8 \\
Richland & Academic & 81 & 136 \\
& Government & 535 & 4 \\
& Industrial & 1,596 & 2,625 \\
& Medical & 36 & 2,836 \\
\hline
\end{tabular}

aSource: ref. 7. 


\section{URANIUM MILL TAILINGS}

\subsection{INTRODUCTION}

Uranium mill tailings are the residual wastes of milled ore that remain after the uranium has been recovered. They are generated during the process of extracting uranium from the ore fed to the mill. Depending on the ore's chemical characteristics, uranium mills use either an acid leach or an alkaline leach process to recover uranium. Currently, all operable U.S. mills are designed to use the acid leach process. Mill tailings from both processes consist of slurries of sands and clay-like particles called slimes; the tailings slurries are pumped to tailings impoundment ponds for disposal.

\subsection{BY-PRODUCT MATERIAL}

Uranium mill tailings are part of a broad category of radioactive wastes called by-product materials. As defined in DOE Order 5820.2A, by-product material includes two major waste groups:

(1) any radioactive material [except special nuclear material (SNM) such as plutonium or fissile uranium] yielded in, or made radioactive either by exposure to incident radiation or by the process of producing or utilizing SNM; and

(2) the tailings or waste produced by the extraction or concentration of uranium or thorium from any ore processed primarily for its source material (i.e., uranium, thorium, or both) content. This excludes underground ores depleted by uranium solution extraction operations that continue to remain underground.

The basis for the definition of the second group of byproduct materials is Section $11 \mathrm{e}(2)$ of the Atomic Energy Act (AEA) of 1954 (P.L. 83-703, as amended). For this reason, these wastes, which, of course, include uranium mill tailings, are referred to as $11 \mathrm{e}(2)$ by-product materials.
Uranium mill tailings are the only by-product materials considered in this chapter. Additional information and data on 1 le(2) by-product materials from DOE Environmental Restoration Program activities are provided in Chapter 6. The Environmental Restoration Program chapter also reports the volumes of mixed DOE Environmental Restoration 11 e(2) by-product materials, which have both hazardous and radioactive components. For this report, information on thorium mill tailings or other by-product materials is not considered.

\subsection{COMMERCIAL URANIUM MILL TAILINGS}

This section describes the inventories and characteristics of uranium mill tailings generated from uranium ore production at commercially licensed facilities.

\subsubsection{Uranium Ore Production}

U.S. uranium production from conventional milling has declined since 1980; as a consequence, the quantity of mill tailings generated each year has declined (see Table 5.1). During 1995, one conventional mill in the United States was commercially producing uranium concentrates from stockpiled ore mined prior to 1993. This mill accounted for sole generation of $134,000 \mathrm{t}$ of mill tailings (see Table 5.2). At the end of 1995, one of the U.S. mills was operational. Five of the 26 mills were on standby status, and the rest were undergoing various stages of decommissioning. The location and status of each of these mills are indicated on the map shown in Fig. 5.1 (ref. 1). The nonutilization of U.S. uranium mill capacity can be attributed, in large part, to nuclear power plant cancellations and deferments. Since the late 1970s, these have led to lower uranium demand which, in turn, has contributed to lower uranium prices and a steady decline in domestic uranium mining. In addition, cost increases for domestic uranium mining and milling have led to increased reliance on imports of less expensive uranium.

In the history of U.S. uranium production, 1993 and 1994 were the only years with no production from 
conventional milling of ore. Nonconventional concentrate production in 1995 increased to about $2,000 \mathrm{t} \mathrm{U}_{3} \mathrm{O}_{8}$, or $34 \%$ above 1994 production. ${ }^{2,3}$ Nonconventional concentrate production includes by-product processing resulting from the mining of phosphate ore as well as the processing of in situ leach-mining solutions, heap-leach solutions, mine water, and other solutions from reclamation activities. In situ leaching (ISL) technology has been increasingly applied in recent years to mining operations. Of the total $1995 \$ 80 / \mathrm{kg}-\mathrm{U}$ uranium reserves estimated by the Energy Information Administration (EIA), the amount for which ISL is the proposed mining method was about $42 \%$. Because ISL mining generally is successful at lower costs as compared with conventional mining methods, it could gain even wider use in the near future. ISL and byproduct (from phosphate ore) production methods do not generate uranium mill tailings. Residual wastes from nonconventional methods are not considered in this chapter.

\subsubsection{Inventories}

The status of the licensed mills, including their estimated commercial and government-related tailings inventories at the end of 1995, is shown in Table 5.2 (data based on refs. 1-11). For each mill, the amount of tailings generated depends on the amount of ore processed, the ore-feed grade $\left(\mathrm{U}_{3} \mathrm{O}_{8}\right.$ assay), and the percentage of $\mathrm{U}_{3} \mathrm{O}_{8}$ recovered. Table 5.1 lists the annual milling rate, ore grade, and $\mathrm{U}_{3} \mathrm{O}_{8}$ recovery. Through $1995,189.6 \times 10^{6} \mathrm{t}$ $\left(118.7 \times 10^{6} \mathrm{~m}^{3}\right)$ associated mill tailings were generated.

\subsubsection{Waste Characterization}

Because the amount of uranium (by weight) extracted from the ore during milling is relatively small, the dry weight of the tailings produced is nearly equal to the dry weight of the ore processed. Dry tailings typically are composed of 70 to $80 \mathrm{wt} \%$ sand-sized particles and 20 to $30 \mathrm{wt} \%$ finer-sized particles. Acid leaching is preferred for ores with low lime content ( $12 \%$ or less). Those with high lime content require excessive quantities of acid for neutralization and, for economic reasons, are best treated by alkaline leaching. In either leach process, most of the uranium is dissolved, together with the other materials present in the ore (e.g., iron, aluminum, and other impurities). After the ore is leached, the uranium-laden leach liquor is removed from the tailings solids by decantation. After thorough washing, the tailings are pumped as a slurry to a tailings pond. The waste liquid accompanying the tailings solids to the disposal pond is approximately 1 to 1.5 times the weight of the processed ore. Typical characteristics of the tailings solids and liquid are outlined in Table 5.3 (ref. 8).

The tailings pile must have a cover designed to control radiological hazards for a minimum of 200 years and for 1,000 years to the greatest extent reasonably achievable. It must also limit radon $\left({ }^{222} \mathrm{Rn}\right.$ ) releases to $20 \mathrm{pCi} / \mathrm{m}^{2} / \mathrm{s}$ averaged over the disposal area. Radon release limitation requirements apply to any portion of the tailings disposal site unless radium concentrates do not exceed $5 \mathrm{pCi} / \mathrm{g}$ in the first $15 \mathrm{~cm}$ below the surface and $15 \mathrm{pCi} / \mathrm{g}$ in layers more than $15 \mathrm{~cm}$ below the surface. ${ }^{11}$

\subsection{DOE URANTUM MILL TAILINGS}

DOE uranium mill tailings include those resulting from uranium ore milled for defense purposes as well as existing tailings at inactive sites no longer licensed that are administered under the DOE site remedial action projects discussed in Chapter 6.

\subsection{REFERENCES}

1. U.S. Department of Energy, Energy Information Administration, "Comparison of Uranium Mill Tailings Reclamation in the United States and Canada," Uranium Industry Annual 1994, DOE/ELA-0478(94), Washington, D.C. (July 1995).

2. U.S. Department of Energy, Energy Information Administration, "Uranium Industry Annual Survey," Form EIA-858, Washington, D.C. (1995).

3. U.S. Department of Energy, Energy Information Administration, Uranium Industry Annual 1995, DOE/ EIA-0478(95), Washington, D.C. (May 1996).

4. U.S. Department of Energy, Integrated Data Base Report-1994: U.S. Spent Nuclear Fuel and Radioactive Waste Inventories, Projections, and Characteristics, DOE/RW-0006, Rev. 10, Oak Ridge National Laboratory, Oak Ridge, Tennessee (December 1995). 
5. U.S. Department of Energy, Grand Junction Office, and Bendix Field Engineering Corporation, Commingled Uranium Tailings Study, DOE/DP-0011, Vol. 2, Grand Junction, Colorado (June 1982).

6. W. S. White, Directory and Profile of Licensed Uranium Recovery Facilities, NUREG/CR-2869 (ANL/ES-128), Rev. 1, U.S. Nuclear Regulatory Commission, Washington, D.C. (March 1984).

7. U.S. Environmental Protection Agency, "National Emission Standard for Radon-222 Emissions from Licensed Uranium Mill Tailings," Code of Federal Regulations, 40 CFR Part 61, Subpart W (September 1986).

8. U.S. Nuclear Regulatory Commission, Final Generic Environmental Impact Statement on Uranium Milling, Project $M-25$, NUREG-0706, Washington, D.C. (September 1980).

9. U.S. Department of Energy, Grand Junction Office, Statistical Data of the Uranium Industry, GJ0-100(73), Grand Junction, Colorado (Jan. 1, 1973).

10. U.S. Congress, House of Representatives, Committees on Energy and Commerce; Interior and Insular Affairs; Science, Space, and Technology; and Ways and Means, Uranium Revitalization, Tailings Reclamation and Enrichment Act of 1988: Hearing on H.R. 4489, 100th Congress, 2nd sess., pp. 19-21 (Apr. 28, 1988).

11. U.S. Department of Energy, Energy Information Administration, Decommissioning of U.S. Uranium Production Facilities, DOE-EIA-0592, Washington, D.C. (February 1995). 


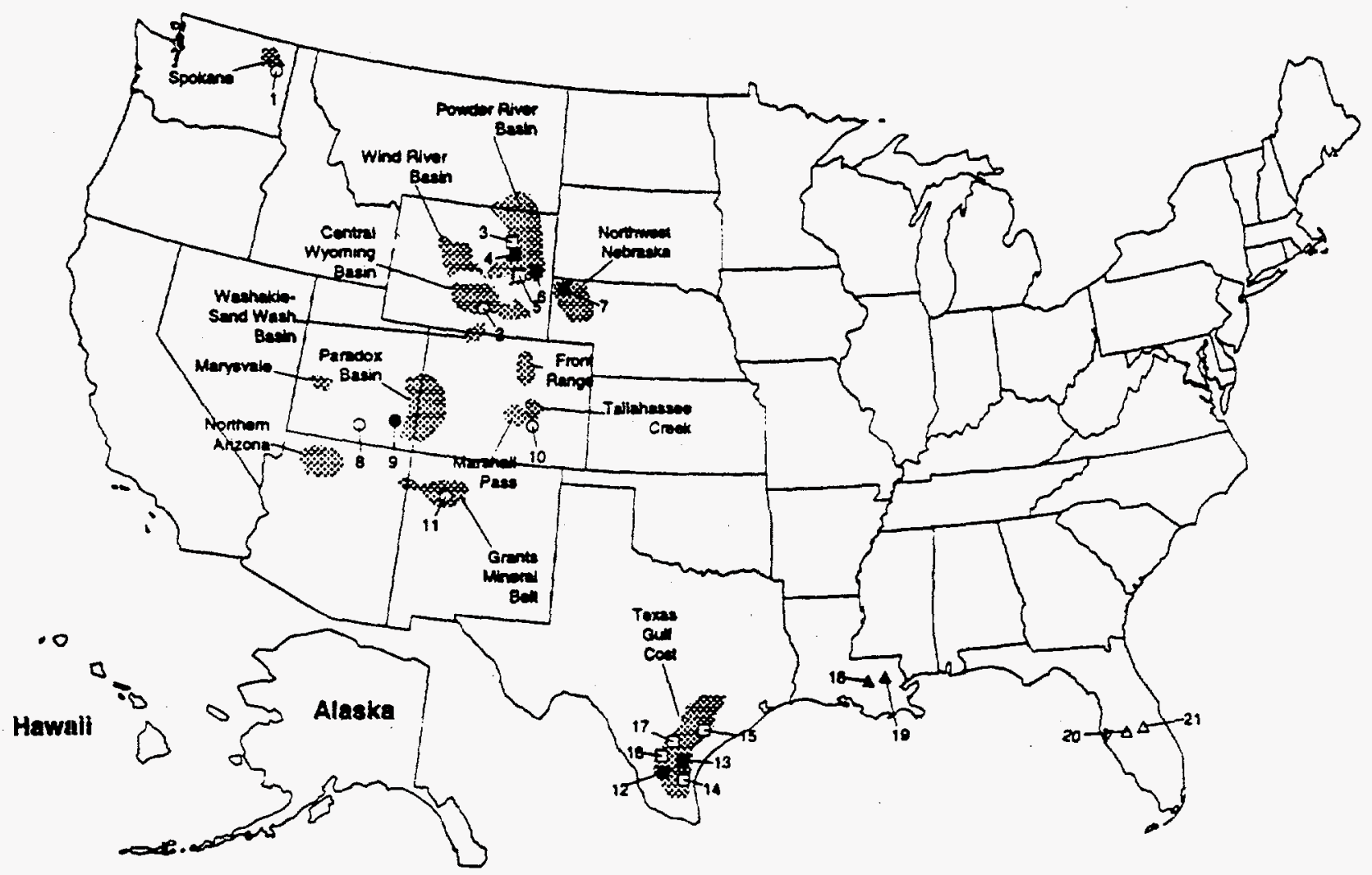

\section{Active at the End of 1995}

4. Malapai Resources. Christensen Ranch

6. Converse County Mining Venture, Highland

7. Crow Butte Resources, Crow Butte

9. Energy Fuels Nuclear, White Mesa

12. Malapai Resources, Holiday-El Mesquite

13. Uranium Resources. Rosita

18. IMC-Agrico, Sunshine Bridge

19. IMC-Agrico. Uncle Sam

\begin{tabular}{|c|c|c|}
\hline Active & $\begin{array}{l}\text { Uranium } \\
\text { Inactive }\end{array}$ & Production Centers \\
\hline & 0 & Conventiona Milis \\
\hline & $\square$ & In Situ Leach Plant \\
\hline$\Delta$ & $\triangle$ & Byproduct From Phosonate Processing \\
\hline 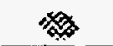 & \multicolumn{2}{|c|}{ Major Uranium Reserve Areas } \\
\hline
\end{tabular}

Inactive at the End of 1995
1. Dawn Mining, Ford
2. Green Mountain Mining Venture. Sweetwater
3. Malapai Resources, Irigaray
5. Rio Algom Mining, Smith Ranch
8. U.S. Energy. Shootaring
10. Cotter Conp., Canon City
11. Rio Algom Mining. Ambrosia
14. Uranium Resources. Kingsville Dome
15. Everest Minerals, Hobson
16. COGEMA Mining. West Cole
17. Malapai Resources, O'Hern
20. IMC-Agrico. Plant City
21. IMC-Agrico, New Wales

Fig. 5.1. Location and status of uranium mills and plants at the end of 1995. Courtesy of U.S. Department of Energy, Energy Information Administration, Washington, D.C. 
Table 5.1. Uranium ore processed, $\mathrm{U}_{3} \mathrm{O}_{8}$ recovery rate, and tailings generated through $1995^{\mathrm{a}} \mathrm{b}$

\begin{tabular}{|c|c|c|c|c|c|c|}
\hline \multirow{2}{*}{$\begin{array}{l}\text { End of } \\
\text { calendar } \\
\text { year }\end{array}$} & \multicolumn{2}{|c|}{ Ore processed } & \multicolumn{2}{|c|}{$\mathrm{U}_{3} \mathrm{O}_{8}$} & \multicolumn{2}{|c|}{ Tailings generated } \\
\hline & $\begin{array}{l}\text { Mass } \\
\left(10^{6} t\right)\end{array}$ & $\begin{array}{c}\text { Grade } \\
\left(\% \mathrm{U}_{3} \mathrm{O}_{8}\right)\end{array}$ & $\begin{array}{l}\text { Recovery } \\
\text { from ore } \\
(\%)\end{array}$ & $\begin{array}{c}\text { Product } \\
\left(10^{3} t\right)\end{array}$ & $\begin{array}{l}\text { Mass }^{e} \\
\left(10^{6} t\right)\end{array}$ & $\begin{array}{l}\text { Volume } \\
\left(10^{6} \mathrm{~m}^{3}\right)\end{array}$ \\
\hline Prior to 1978 & g & $\mathrm{g}$ & $\mathrm{g}$ & g & 108.8 & 68.0 \\
\hline 1978 & 12.5 & 0.134 & 91 & 15.6 & 12.6 & 7.9 \\
\hline 1979 & 14.6 & 0.113 & 91 & 15.3 & 14.5 & 9.1 \\
\hline 1980 & 15.3 & 0.118 & 93 & 17.2 & 15.2 & 9.5 \\
\hline 1981 & 13.2 & 0.115 & 94 & 14.5 & 13.2 & 8.2 \\
\hline 1982 & 7.9 & 0.119 & 96 & 9.9 & 8.1 & 5.0 \\
\hline 1983 & 5.4 & 0.128 & 97 & 7.0 & 5.4 & 3.4 \\
\hline 1984 & 3.9 & 0.112 & 95 & 4.4 & 4.0 & 2.5 \\
\hline 1985 & 1.6 & 0.161 & 96 & 2.8 & 1.6 & 1.0 \\
\hline 1986 & 1.2 & 0.338 & 97 & 4.0 & 1.2 & 0.7 \\
\hline 1987 & 1.3 & 0.284 & 96 & 3.8 & 1.3 & 0.8 \\
\hline 1988 & 1.1 & 0.288 & 95 & 3.2 & 1.1 & 0.7 \\
\hline 1989 & 1.1 & 0.323 & 95 & 3.7 & 1.0 & 0.7 \\
\hline 1990 & 0.7 & 0.293 & 94 & 2.1 & 0.7 & 0.4 \\
\hline 1991 & 0.6 & 0.188 & 92 & 1.2 & 0.6 & 0.4 \\
\hline 1992 & 0.2 & 0.229 & 96 & 0.6 & 0.2 & 0.2 \\
\hline 1993 & 0.0 & 0.000 & 0 & 0.0 & 0.0 & 0.0 \\
\hline 1994 & 0.0 & 0.000 & 0 & 0.0 & 0.0 & 0.0 \\
\hline 1995 & $0.1^{\mathrm{h}}$ & 0.531 & 92 & 0.8 & 0.1 & 0.1 \\
\hline Total $^{i}$ & & & & & 189.6 & 118.7 \\
\hline
\end{tabular}

aSources: Prior to 1984-U.S. Department of Energy, Grand Junction Area Office data files. 1984-1995-Energy Information Administration, "Uranium Industry Annual Survey," Form EIA-858.

bThis table has been revised based on a detailed study of milling data from the Grand Junction

Project Office and EIA files. The values shown include all tailings.

${ }^{c}$ Before in-process inventory adjustments.

${ }^{d}$ Conventional $\mathrm{U}_{3} \mathrm{O}_{8}$ concentrate production.

Includes adjustments to ore-fed amounts for annual mill circuit inventory changes and uranium concentrate production.

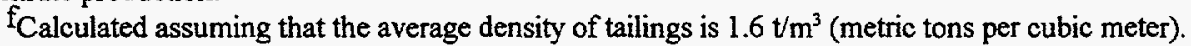

SNot available.

${ }^{\mathrm{h}}$ Stockpiled ore mined prior to 1993.

${ }^{i}$ Because of independent rounding, totals may not equal sum of components. 
Table 5.2. Status of conventional uranium mill sites at the end of $1995^{\text {a }}$

\begin{tabular}{|c|c|c|c|c|c|c|c|c|}
\hline \multirow{3}{*}{ Location } & \multirow{3}{*}{ Operator } & \multirow{3}{*}{$\begin{array}{l}\text { Rated } \\
\text { capacity } b \\
\text { ( } \mathrm{t} / \mathrm{d} \text { ore) }\end{array}$} & \multirow{2}{*}{\multicolumn{2}{|c|}{ Status }} & \multirow{3}{*}{$\begin{array}{c}\text { Tailings } \\
\text { storage } \\
\text { area } \\
\text { (ha) }\end{array}$} & \multicolumn{3}{|c|}{ Total tailings } \\
\hline & & & & & & & & Government \\
\hline & & & Operations ${ }^{b}$ & Tailings $^{c}$ & & $\begin{array}{l}\text { Volume } \\
\left(10^{6} \mathrm{~m}^{3}\right)\end{array}$ & $\begin{array}{l}\text { Mass } \\
\left(10^{6} t\right)\end{array}$ & $\begin{array}{l}\text { portion } \\
\left(10^{6} t\right)\end{array}$ \\
\hline \multicolumn{9}{|l|}{ Colorado } \\
\hline Canon City & Cotter & 1,090 & Shut down, 1987 & & $66 \mathrm{Rg}$ & 1.3 & 2.0 & 0.3 \\
\hline Uravan & Umetco Minerals & $1,180^{h}$ & Decommissioning & Partially stabilized & $34 \mathrm{R}$ & 5.9 & 9.5 & 5.2 \\
\hline Subtotal & & 1,090 & & & $100 \mathrm{R}$ & 7.2 & 11.5 & 5.5 \\
\hline \multicolumn{9}{|l|}{ New Mexico } \\
\hline Ambrosia Lake & Quivira Mining & 6,350 & Shut down, 1985 & Fenced & $131 \mathrm{R}$ & 18.8 & 30.1 & 9.1 \\
\hline Bluewater & Anaconda & $5,440^{h}$ & Decommissioning & Partially stabilized & 199 & 13.6 & 21.7 & 8.0 \\
\hline Church Rock & United Nuclear & $2,720^{\mathrm{h}}$ & Decommissioning & i & $40 \mathrm{R}$ & 2.0 & 3.2 & 0 \\
\hline Grants & Homestake Mining & $3,080^{h}$ & Decommissioning & Unstabilized & $86 \mathrm{R}$ & 12.7 & 20.2 & 10.4 \\
\hline L-Bar & Sohio Western Mining & $1,450^{\mathrm{h}}$ & Decommissioning & $i$ & $46 \mathrm{R}$ & 1.2 & 1.9 & 0 \\
\hline Marquez & Bokum Resources & $1,820^{h} j$ & New (on standby) & Never operated & 0 & 0 & 0 & 0 \\
\hline Subtotal & & 6,350 & & & $502 \mathrm{R}$ & 48.3 & 77.1 & 27.5 \\
\hline \multicolumn{9}{|l|}{ South Dakota } \\
\hline Edgemont & $\begin{array}{l}\text { Tennessee Valley } \\
\text { Authority }\end{array}$ & $680^{h}$ & Decommissioned & Stabilized & 50 & 1.2 & 1.8 & 1.5 \\
\hline & & $\ldots$ & & & 一 & - & - & - \\
\hline Subtotal & & 0 & & & 50 & 1.2 & 1.8 & 1.5 \\
\hline \multicolumn{9}{|l|}{ Texas } \\
\hline Falls City & $\begin{array}{l}\text { Continental Oil/ } \\
\text { Pioneer Nuclear }\end{array}$ & $3,080^{h}$ & Decommissioned & Stabilized & 89 & 6.5 & 10.5 & 0 \\
\hline Panna Maria & Rio Grande Resources & $2,720^{\mathrm{h}}$ & Decommissioned & Stabilized & 101 & 3.9 & 5.9 & 0 \\
\hline $\begin{array}{l}\text { Ray Point } \\
\text { (Felder Facility) }\end{array}$ & Exxon & $1,000^{h}$ & Decommissioned & Stabilized $^{k}$ & 18 & 0.2 & $0.4^{l}$ & 0 \\
\hline Subtotal & & 2,720 & & & 208 & 10.6 & 16.8 & 0 \\
\hline
\end{tabular}


Table 5.2 (continued)

\begin{tabular}{|c|c|c|c|c|c|c|c|c|}
\hline \multirow{3}{*}{ Location } & \multirow{3}{*}{ Operator } & \multirow{3}{*}{$\begin{array}{l}\text { Rated } \\
\text { capacity }{ }^{6} \\
\text { (t/d ore) }\end{array}$} & \multirow{2}{*}{\multicolumn{2}{|c|}{ Status }} & \multirow{3}{*}{$\begin{array}{l}\text { Tailings } \\
\text { storage } \\
\text { area } \\
(\text { ha })^{d}\end{array}$} & \multicolumn{3}{|c|}{ Total tailings } \\
\hline & & & & & & \multirow[b]{2}{*}{$\begin{array}{l}\text { Volume } \\
\left(10^{6} \mathrm{~m}^{3}\right)\end{array}$} & \multirow[b]{2}{*}{$\begin{array}{l}\text { Mass } \\
\left(10^{6} t\right)\end{array}$} & \multirow{2}{*}{$\begin{array}{c}\text { Government } \\
\text { portion } \mathrm{f} \\
\left(10^{6} \mathrm{t}\right)\end{array}$} \\
\hline & & & Operations $^{b}$ & Tailings $^{c}$ & & & & \\
\hline \multicolumn{9}{|l|}{ Utah } \\
\hline Lisbon & Rio Algom & 680 & Decommissioning & i & 14 & 2.2 & 3.5 & 0 \\
\hline Moab & Atlas & $1,270^{h}$ & Decommissioning & Relocation sought & $>80$ & 6.0 & 9.6 & 5.4 \\
\hline Shootering & Plateau Resources & 910 & New (on standby) & Never operated & 28 & 0 & 0 & 0 \\
\hline White Mesa & Energy Fuels & 1,810 & Shut down, 1990; & Partially stabilized & 135 & 2.0 & 3.3 & 0 \\
\hline Subtotal & & 3,400 & & & $>257$ & 10.2 & 16.4 & 5.4 \\
\hline \multicolumn{9}{|l|}{ Washington } \\
\hline Ford & Dawn Mining & 410 & Shut down, 1982 & Wood chip covering & $53 \mathrm{R}$ & 1.8 & 2.8 & 1.1 \\
\hline Sherwood & Western Nuclear & $1,810^{h}$ & Decommissioning & $\mathrm{i}$ & 17 & 1.6 & 2.6 & 0 \\
\hline Subtotal & & 410 & & & $70 \mathrm{R}$ & 3.4 & 5.4 & 1.1 \\
\hline \multicolumn{9}{|l|}{ Wyoming } \\
\hline Bear Creek & Rocky Mountain Energy & $1,810^{\mathrm{h}}$ & Decommissioning & Unstabilized & 61 & 2.7 & 4.3 & 0 \\
\hline Gas Hills & American Nuclear & $860^{h}$ & Decommissioning & Unstabilized & $47 \mathrm{R}$ & 3.3 & 5.4 & 2.0 \\
\hline Gas Hills & Umetco & $1,270^{\mathrm{h}}$ & Decommissioning & Unstabilized & $58 \mathrm{R}$ & 4.6 & 7.3 & 1.9 \\
\hline Highland & Exxon & $2,900^{\mathrm{h}}$ & Decommissioning & Partially stabilized & $116 \mathrm{R}$ & 6.4 & 10.3 & 0 \\
\hline Lucky Mc & Pathfinder & $2,540^{\mathrm{h}}$ & Decommissioning & Unstabilized & $99 \mathrm{R}$ & 6.6 & 10.6 & 2.6 \\
\hline Petrotomics & Petrotomics & $1,360^{h}$ & Decommissioning & Unstabilized & 65 & 3.9 & 6.3 & 0.7 \\
\hline Shirley Basin & Pathfinder & $1,630^{\mathrm{h}}$ & Decommissioning & i & $105 R$ & 4.7 & 7.4 & 0 \\
\hline Split Rock & Western Nuclear & $1,540^{\mathrm{h}}$ & Decommissioning & Interim stabilization & $67 \mathrm{R}$ & 4.4 & 7.0 & 3.0 \\
\hline Sweetwater & $\begin{array}{l}\text { Minerals Exploration/ } \\
\text { Union Energy Mining }\end{array}$ & 2,720 & Shut down, May 1983 & Partially stabilized & 121 & 1.3 & 2.1 & 0 \\
\hline \multicolumn{2}{|l|}{ Subtotal } & 4,350 & & & $739 \mathrm{R}$ & 37.9 & 60.7 & 10.2 \\
\hline \multicolumn{2}{|c|}{1995 total for all sites $b, m, n$} & $18,320^{\circ}$ & & & $>1,926 \mathrm{R}$ & 118.7 & 189.6 & $51.2^{\mathrm{p}}$ \\
\hline
\end{tabular}

(Footnotes on next page.) 


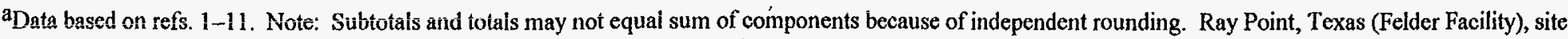
was stabilized during 1987 by Exxon Corporation. Historical data are revised based on detailed study of milling data from the Grand Junction Project Office and EIA files. The values shown include all tailings.

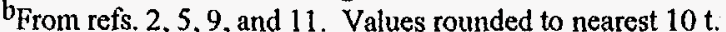

${ }^{c}$ On Aug. 15, 1986, EPA issued its final rules on ${ }^{222}$ Rn emissions from tailings piles. Mill owners have 6 years (subject to certain extensions) to phase out the use of large existing tailings piles. New tailings piles may be contained in small impoundments (less than 16 ha) or disposed of continuously by dewatering and burial (i.e., no more than 4 ha are uncovered at any one time). See ref. 7.

${ }^{\mathrm{d}}$ From refs. 6 and $11 ; 1 \mathrm{ha}=10,000 \mathrm{~m}^{2}$ or approximately 2.5 acres.

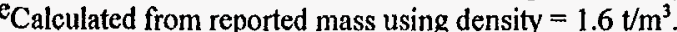

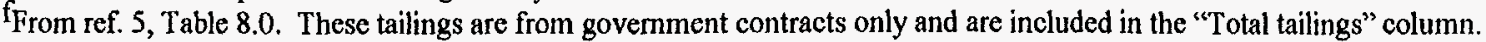

$\mathrm{g}_{\mathrm{R}}=$ revised. From ref. 11 .

hestimates provided are not included in the total. See column labeled "Operations" under "Status" for reason.

$i_{\text {Not available. }}$

jMill construction has not been $100 \%$ complete.

kFrom ref. 11

I From ref. 10

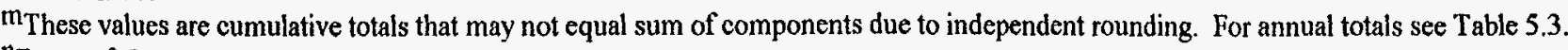

" From ref. 2

From ref. 2 .

pTotal at the end of government-contracted deliveries in 1970 (ref. 5) 
Table 5.3. Typical characteristics of uranium mill tailings ${ }^{\mathrm{a}}$

\begin{tabular}{|c|c|c|c|}
\hline $\begin{array}{l}\text { Tailings } \\
\text { component }\end{array}$ & $\begin{array}{c}\text { Particle size } \\
\text { (um) }\end{array}$ & $\begin{array}{l}\text { Chemical } \\
\text { composition }\end{array}$ & $\begin{array}{l}\text { Radioactivity } \\
\text { characteristics }\end{array}$ \\
\hline Sands & 75 to 500 & $\begin{array}{l}\mathrm{SiO}_{2} \text { with }<1 \% \text { complex silicates } \\
\text { of } \mathrm{Al}, \mathrm{Fe}, \mathrm{Mg}, \mathrm{Ca}, \mathrm{Na}, \mathrm{K}, \mathrm{Se} \text {, } \\
\mathrm{Mn}, \mathrm{Ni}, \mathrm{Mo}, \mathrm{Zn}, \mathrm{U} \text {, and } \mathrm{V} \text {; also } \\
\text { metallic oxides }\end{array}$ & $\begin{array}{l}0.004 \text { to } 0.01 \% \mathrm{U}_{3} \mathrm{O}_{8}{ }^{\mathrm{b}} \\
\text { Acid leaching: } \\
26 \text { to } 100 \mathrm{pCi}{ }^{226} \mathrm{Ra} / \mathrm{g} \\
70 \text { to } 600 \mathrm{pCi}^{230} \mathrm{Th} / \mathrm{g}\end{array}$ \\
\hline Slimes & 45 to 75 & $\begin{array}{l}\text { Small amounts of } \mathrm{SiO}_{2} \text {, but mostly } \\
\text { very complex clay-like silicates } \\
\text { of } \mathrm{Na}, \mathrm{Ca}, \mathrm{Mn}, \mathrm{Mg}, \mathrm{Al} \text {, and } \mathrm{Fe} \text {; } \\
\text { also metallic oxides }\end{array}$ & $\begin{array}{l}\mathrm{U}_{3} \mathrm{O}_{8} \text { and }{ }^{226} \mathrm{Ra} \text { are almos } \\
\text { twice the concentration } \\
\text { present in the sands } \\
\text { Acid leaching: } \\
150 \text { to } 400 \mathrm{pCi}{ }^{226} \mathrm{Ra} / \mathrm{g} \\
70 \text { to } 600 \mathrm{pCi}{ }^{230} \mathrm{Th} / \mathrm{g}\end{array}$ \\
\hline \multirow[t]{2}{*}{ Liquids } & d & $\begin{array}{l}\text { Acid leaching: } \\
\mathrm{pH} 1.2 \text { to } 2.0 ; \mathrm{Na}^{+}, \mathrm{NH}_{4}^{+}, \mathrm{SO}_{4}^{-2} \text {, } \\
\mathrm{Cl}^{-} \text {, and } \mathrm{PO}_{4}^{-3} ; \text { dissolved solids } \\
\text { up to } 1 \%\end{array}$ & $\begin{array}{l}\text { Acid leaching: } \\
0.001 \text { to } 0.01 \% \mathrm{U} \\
20 \text { to } 7,500 \mathrm{pCi}^{226} \mathrm{Ra} / \mathrm{L} \\
2,000 \text { to } 22,000 \mathrm{pCi} \\
{ }^{230} \mathrm{Th} / \mathrm{L}\end{array}$ \\
\hline & & $\begin{array}{l}\text { Alkaline leaching: } \\
\mathrm{pH} 10 \text { to } 10.5 ; \mathrm{CO}_{3}^{-2} \text { and } \mathrm{HCO}_{3}^{-} \text {; } \\
\text { dissolved solids }-10 \% \\
\text {. }\end{array}$ & $\begin{array}{l}\text { Alkaline leaching: } \\
200 \mathrm{pCi}^{226} \mathrm{Ra} / \mathrm{L} \text {; } \\
\text { essentially no }{ }^{230} \mathrm{Th} \\
\text { (insoluble) }\end{array}$ \\
\hline
\end{tabular}

adapted from information in ref. 8.

$\mathrm{b}_{\mathrm{U}_{3}} \mathrm{O}_{8}$ content is higher for acid leaching than for alkaline leaching.

'Separate analyses of sands and slimes from the alkaline leaching process are not available. However, total ${ }^{226} \mathrm{Ra}$ and ${ }^{230} \mathrm{Th}$ contents of up to $600 \mathrm{pCi} / \mathrm{g}$ (of each) have been reported for the combined sands and slimes.

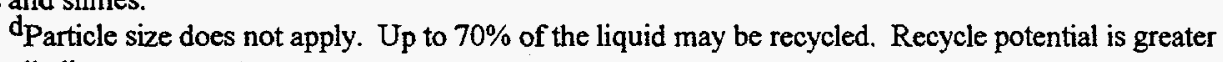
in the alkaline process. 

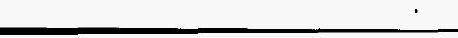


\section{ENVIRONMENTAL RESTORATION PROGRAM}

\subsection{INTRODUCTION}

The overall mission of the DOE Office of Environmental Restoration (EM-40) is to protect human health and the environment from risks posed by inactive, surplus structures and contaminated environmental media. These structures and media contain radioactive and chemically hazardous contaminants as a result of previous activities conducted by DOE and its predecessor agencies. The EM-40 program is accomplishing its mission by remediating sites and facilities in the most cost-efficient and responsible manner possible to provide for future beneficial use of these contaminated areas while complying with applicable environmental regulations.

The environmental restoration program includes a bias for action to expedite actual cleanup wherever and whenever possible. Activities are prioritized based upon several factors, including the need to eliminate risks at sites not controlled by the federal government, the goal of reducing risks at all sites, and compliance with various laws, regulations and agreements. Most actions are designed to either remove or contain contamination in the environment (such as contaminated soil, debris, and ground water) or to decommission contaminated structures (including reactors, chemical processing buildings, and support facilities). Related activities to support remediation actions include treatment of contaminated materials and wastes, transportation of these materials and wastes to storage and disposal facilities, and disposal of wastes in permitted facilities.

Environmental restoration activities include cleanup of buildings and areas that supported defense-related activities (such as nuclear weapon component fabrication) and nondefense, civilian nuclear power activities (such as the development of heat sources for the space program and the operation of small test reactors). Remedial actions are concerned with all aspects of the assessment and cleanup of inactive sites at which releases of radioactive and chemically hazardous substances have occurred. These actions are not limited to the areas directly impacted by the release but also include additional areas to which contaminants may have migrated (such as to ground water). Twenty-two DOE sites are on the U.S. Environmental Protection Agency (EPA) National Priorities List.

Cleanup goals and remedies for each contaminated area are developed through processes established by federal and state laws and other legal agreements. These processes involve decision-makers outside DOE, such as EPA and the impacted state, and include input from other stakeholders such as local citizens and national environmental groups. The principal regulatory requirements for remediation activities are derived from the Comprehensive Environmental Response, Compensation, and Liability Act (CERCLA) and the Resource Conservation and Recovery Act (RCRA). Activities may be subject further to requirements associated with compliance with the National Environmental Policy Act (NEPA) and with regulatory requirements imposed by the states. Other requirements are set forth in various DOE Orders and standards and in other guidance documents.

Decommissioning activities, which occur after facilities have been stabilized and deactivated, address contamination within the structures. The objectives of decommissioning are to eliminate potential risks to human health and safety and the environment and to allow for the reuse of materials, equipment, and buildings to the greatest extent practicable. Most decommissioning activities are concerned with facilities such as reactors, hot cells, processing plants, storage tanks, and other structures from which, in general, few releases to the environment have occurred.

Decommissioning activities are carried out according to requirements set forth in various DOE Orders and standards and other guidance documents. State requirements also apply in certain instances. Based on a joint policy between DOE and EPA, provisions of CERCLA generally govern decommissioning activities, which are conducted as non-time-critical removal actions. Of the 3,500 contaminated facilities that are surplus, or projected to be surplus within the next 10 years, DOE has decommissioned 100 facilities to date. The EM-40 program has placed a priority on minimizing secondary 
waste and has recycled 7,240 metric tons ( $t)(8,000$ tons) of scrap metal from dismantled facilities and equipment (ref. 1). Only those decommissioning activities at facilities currently in the EM-40 program are addressed in this chapter.

The first steps in the remediation process for contamination in environmental media are to identify the contaminants of concern, determine the extent of contarnination, and assess potential threats to human health and the environment. If a significant contamination problem is indicated and if a fast and limited cleanup or containment action could mitigate this problem, DOE may conduct an expedited response action or interim remedial action. To date, DOE has completed over 500 such limited actions (ref. 1).

Upon completion of characterization, a detailed analysis of remedial alternatives is conducted. This analysis is followed by a formal decision-making process, possibly including public meetings and a formal public comment period. If the results of the analysis indicate (a) that a contaminated area does not pose a threat to human health or the environment or (b) that a previously completed limited action adequately addressed the contamination condition, a determination that "no further action" is necessary may be made. Such a determination would be made in conjunction with EPA, the host state, and other stakeholders. However, if a threat is deemed to be present, the appropriate action would be identified and implemented.

A wide range of actions can be implemented to address environmental contamination problems that exist at DOE sites. Current and projected land use is a key component in the decision-making process. For example, in-situ remedies that rely on containment of contaminated materials would be appropriate for the large DOE reservations that are projected to remain under the control of the federal government. In contrast, ex-situ remedies in which contaminated materials are exhumed for treatment and disposal at off-site locations would likely be appropriate for small sites destined to be released for unrestricted or industrial (non-DOE) uses. The most appropriate action to be taken at any given area is sitespecific and depends on the types of contaminants present, the medium in which they are found, and the likelihood of current or future exposures.

To support the planning process, EM-40 has established a database containing key information for the environmental restoration program, such as projected responses for each contaminated area and facility, milestones, projected costs, and anticipated wastes requiring active management. Information in the EM-40 Core Database was provided by the various field offices and was used to support the Baseline Environmental Management Report (BEMR) 1996. The EM-40 Core
Database also serves as the source of information for this chapter (ref. 2).

Waste management information included in the EM-40 Core Database includes data on all waste types that may result from environmental restoration activities including radioactive, sanitary, demolition, and hazardous wastes as regulated by RCRA and the Toxic Substances Control Act (TSCA). This information will assist DOE in planning, constructing, and operating appropriate treatment, storage, and disposal facilities to manage the wastes resulting from implementation of the EM-40 program.

\subsection{OFFICE OF ENVIRONMENTAL RESTORATION}

Environmental restoration activities under the auspices of EM-40 are managed in a decentralized manner. That is, much of the responsibility for program implementation rests with the various Operations and project offices. These field offices have the responsibility for determining the appropriate course of action to take at the various contaminated sites and then directing the remediation activities. The locations of the field offices responsible for directing the DOE environmental restoration program are shown in Fig. 6.1. A listing of the sites in the EM-40 program is given in Table 6.1.

The Headquarters element of the EM-40 program is responsible for establishing policy and providing programmatic guidance to the field offices in implementing this program and determining appropriate priorities. In addition, Headquarters is responsible for formulating and executing the EM-40 budget. Other Headquarters responsibilities include tracking progress on environmental restoration activities and responding to information requests from Congress, other federal agencies, and public-interest groups.

In general, the Operations offices directing the environmental restoration program in the field are the same offices that directed activities at these sites when facilities were operational. For example, the Chicago Operations Office directs energy research and development activities at Ames Laboratory, Argonne National Laboratory, and Brookhaven National Laboratory as well as manages the environmental restoration program at these three laboratories.

Over half of the sites in the EM-40 program are managed under the Uranium Mill Tailings Remedial Action Project (UMTRAP) and the Formerly Utilized Sites Remedial Action Program (FUSRAP). The Albuquerque Operations Office is responsible for implementing UMTRAP, which was authorized in 1978, and involves the stabilization and control of (a) 24 inactive uraniumprocessing sites and associated vicinity properties located 
in 10 states and 2 Indian tribal lands and (b) vicinity properties associated with the Edgemont, South Dakota, inactive uranium mill site, which is owned by the Tennessee Valley Authority (Fig. 6.2). All of the sites are located in the western United States, except for one in Canonsburg, Pennsylvania. Remedial actions have been completed at 20 of the 24 inactive uranium processing sites (ref. 3). The Department is seeking revocation, at the state's request, of the two sites in North Dakota. Remediation of the remaining two UMTRAP sites is expected to be completed in 1997. In addition to the surface contamination present at these sites (mill tailings, soil, and structures), the ground water is contaminated with metals (including uranium and radium) at all sites, except for Lowman, Idaho. Active remediation of contaminated ground water is expected to be necessary at only two of these sites [Monument Valley and Tuba City, Arizona (ref. 2)].

The Oak Ridge Operations Office is responsible for implementing FUSRAP, which is primarily concerned with the cleanup of sites that were formerly used to support the activities of the Manhattan Engineer District, established for the Manhattan Project, and the Atomic Energy Commission (AEC). Private firms and institutions were contracted by the federal government in the 1940s and 1950 s to develop processes and perform research on radioactive materials. The storage and processing of uranium and thorium ores, concentrates, and residues were often involved. Although these sites were cleaned up to formerly acceptable levels, FUSRAP was established in 1974 to identify; reevaluate; and, if necessary, remediate these sites. Most FUSRAP sites are in the eastern half of the country. Currently, 46 sites have been identified in 14 states; 25 of these sites have already been remediated (Fig. 6.3). Remediation of the remaining FUSRAP sites is expected to be completed within the next ten years.

\subsection{ENVIRONMENTAL RESTORATION WASTE CHARACTERISTICS}

The volumes and types of wastes associated with DOE environmental restoration activities are a direct result of the remedy chosen. Waste associated with remediation of contaminated environmental media would occur only when such media are exhumed. For example, no waste would be produced at a site for which an in situ remedy was selected, such as capping an area containing contaminated soil. If a minimal remedial action were selected (e.g., pumping and treating a small pocket of contaminated ground water followed by construction of lateral barriers to minimize future migration), the site would have relatively small waste volumes. However, if large volumes of contaminated environmental media were removed, treated to provide a more suitable waste form for disposal, and then disposed of in an engineered facility, the site would have very large waste volumes.

Environmental restoration wastes are different from those associated with processing operations in that restoration wastes generally have much lower concentrations of radioactive and chemically hazardous substances. Much of the material requiring remediation is a consequence of past activities (e.g., spills, waste disposal, and environmental releases such as liquid discharges to drainage basins). In addition, operations within structures resulted in the contamination of equipment, walls, and floors from routine materialhandling activities and from off-normal incidents such as spills and equipment failure. Decommissioning of these facilities will result in wastes such as wipes, concrete, metal, personal protective clothing, and decontamination solvents that generally have low concentrations of radioactive and chemical contaminants.

Environmental restoration wastes also differ from those resulting from processing operations in that they are generally highly heterogeneous both in physical form and chemical constituency. For example, remediation of an abandoned waste pit could require the exhumation of all materials previously placed into the pit for disposal. This effort could involve any possible combination of objects ranging from small pieces of equipment and drums to entire vehicles such as trucks and forklifts. In addition, a full spectrum of contaminants could be present in these previously disposed materials including those associated with ordnance operations, processing of uranium and thorium ores and concentrates, and the operation of nuclear reactors and associated chemical processing plants. This potential variety is in contrast to waste streams associated with processing activities that have relatively consistent chemical and physical properties.

Because many DOE environmental restoration projects are still in the remedy-selection phase, it is not possible to project definitively the wastes that will result from all of these projects. However, reasonable waste projections can be made based on current site characterization information and planned restoration activities for sites and facilities in the EM-40 program, as reflected in the Core Database (ref. 2). These estimates are presented in Tables 6.2 through 6.6. In addition to waste projections, the volumes of contaminated materials associated with in-situ remedies are also provided in these tables. The information provided in Tables 6.2 through 6.6 is consistent with the base case presented in BEMR 1996 (ref. 1) and reflect proposed site-specific land use assumptions. These estimates do not include contaminated media outside the scope of the current EM-40 program, such as contaminated sediment in the Clinch River near Oak Ridge, Tennessee, and underground materials contaminated by past hydrofracture disposal and nuclear testing at the Oak Ridge Reservation and the Nevada Test Site, respectively. 
Materials in inventory (i.e., those with potential economic value) are also not included in these estimates.

In addition to wastes to be generated, environmental media projected to be left in place have also been assigned a "waste" class in the EM-40 Core Database. This was done to simplify the tracking of all contaminated materials and support response planning, even though these media are technically not wastes unless or until they are removed. Three major radioactive waste classes are associated with environmental restoration activities: LLW, TRUW, and 1 le(2) by-product material. As defined in DOE Order $5820.2 \mathrm{~A}$, LLW is waste that contains radioactivity and is not classified as HLW, TRUW, spent nuclear fuel (SNF), or 1 le(2) by-product material. Environmental restoration activities are not expected to generate any HLW or SNF. TRUW is waste contaminated with alpha-emitting transuranium radionuclides with half-lives greater than 20 years and at concentrations greater than $100 \mathrm{nCi} / \mathrm{g}$ at the time of assay.

As defined in Section 11e(2) of the AEA of 1954 (Pub. L. 83-703, as amended), 11 e(2) by-product material is tailings or waste produced by the extraction or concentration of uranium or thorium from any ore processed primarily for its source material content. Materials being managed under Title 1 of the Uranium Mill Tailings Radiation Control Act of 1978 (Pub. L. 95-604) are defined as residual radioactive material distinct from 11e(2) by-product material. This residual radioactive material is largely uranium mill tailings (UMT), as well as soil and debris contaminated with UMT. Since this material has the same physical and radioactive properties as $11 \mathrm{e}(2)$ by-product material, it is included in this report with $11 \mathrm{e}(2)$ by-product material.

In addition to radioactive contaminants, these materials can also be contaminated with hazardous constituents as regulated by RCRA or TSCA; such wastes are considered mixed wastes. Thus, a total of six waste classes are relevant for radioactively contaminated material resulting from environmental restoration activities: LLW, mixed ILW (MLLW), TRUW, mixed TRUW (MTRUW), 1 le(2) by-product material, and mixed 1 le(2) by-product material.

The estimated volumes of radioactively contaminated materials being managed by the EM- 40 program are given in Tables 6.2 through 6.5 for LLW, MLLW, TRUW, and 11 e(2) by-product material, respectively. The volumes given in Tables 6.4 and 6.5 include the contribution of mixed wastes (the mixed waste volumes are identified in footnotes). The mixed wastes reported in these four tables are limited to RCRA mixed wastes and do not include the contribution of TSCA mixed wastes. TSCA mixed wastes are reported separately in Table 6.6. In addition, radioactive wastes currently in storage at EM-40 facilities are reported in Table 6.7 .
The estimated volumes given in Tables 6.2 through 6.6 are grouped into the following six categories:

1. collection for treatment, storage, and/or disposal by EM-40;

2. collection for treatment, storage, and/or disposal by EM-30;

3. collection for disposition at a commercial facility;

4. in-situ treatment or containment;

5. access/institutional controls or no further action; and

6. not yet determined.

Contaminated materials will be removed and wastes generated under the first three categories (ex-situ responses) with responsibility for final disposition either maintained within the EM-40 program, transferred to the EM-30 program, or targeted for a commercial facility. Wastes will not be generated under the fourth or fifth category, which will involve such measures as capping, monitoring, and retention of land-use controls. The last category addresses materials for which the final disposition is not currently known. Estimates in this group represent the difference between the total volume of a given contaminated material for a specific waste class at a site and the volume projected to be addressed by the indicated responses.

The estimates represent the initial response volumes, that is, the amount collected, not the final waste forms. Thus, changes due to activities such as treatment have not been incorporated. Treatment can result in higher or lower final volumes depending on the specific process used (e.g., stabilization versus incineration). Treatment can also change the waste class (e.g., stabilizing a MLLW material could result in an LLW product). These changes are not reflected in the information provided in Tables 6.2 through 6.7 .

The total volume of solid radioactively contaminated material being address by the EM-40 program is approximately 88 million cubic meters. The two waste classes that constitute the largest volumes of radioactively contaminated material are LLW and $11 \mathrm{e}(2)$ by-product material. Of the material classified as LLW, most (70\%) is projected to be managed in-situ. Los Alamos National Laboratory and the Hanford Site account for most of this volume. The large volume of $11 \mathrm{e}(2)$ by-product material is mainly the result of the large volume of residual radioactive material associated with UMT being remediated under UMTRAP.

The other four waste classes combined contribute about $15 \%$ of the total volume of radioactively contaminated material being addressed by the EM-40 program. Most of this volume is associated with material currently classified as MLLW. In reality, much of this material is probably LLW; characterization of this material is ongoing. The contribution for material classified as 
TRUW is very small, representing about $0.1 \%$ of the total volume of material being addressed by the EM-40 program.

Because environmental restoration information is currently in the initial stages of being compiled at a number of sites, much of the information included in Tables 6.2 through 6.6 is preliminary. In addition, these tables include information only for sites currently in the EM-40 program for which data are available. That is, zero (or very small) volumes may be reported in the EM-40 Core Database for areas of a site at which characterization activities have not been initiated, thus resulting in an underestimate of the total volume of radioactively contaminated media. Conversely, overly conservative assumptions may be incorporated into some estimates to ensure the adequate sizing of treatment and disposal facilities. These uncertainties will continue to be reduced with time as characterization and engineering studies become available to refine these volume estimates.

As described earlier, remedial actions are currently being conducted at a number of sites, especially those associated with the FUSRAP and UMTRAP projects. Many of these are small, interim actions. Wastes resulting from these activities are generally being managed at the site where the remedial action occurred. However, large volumes of UMT are being relocated (consolidated) for a number of UMTRAP sites. In addition, wastes resulting from remedial actions at some sites (such as those being remediated under FUSRAP) are being managed at commercial disposal facilities. One such commercial facility currently being utilized is the Envirocare Facility near Clive, Utah. A summary of radioactive wastes resulting from environmental restoration activities disposed of at this facility through the end of 1995 is given in Table 0.8. More than $44,000 \mathrm{~m}^{3}$ of wastes resulting from $D O E$ environmental restoration activities has been disposed of at that site.

The information contained in this chapter is limited to radioactively contaminated environmental media and wastes, consistent with the scope of this report. The volume estimates given in Tables 6.2 through 6.7 are also limited to solid materials. Liquids, such as contaminated surface water and ground water and liquid wastes currently in storage, are not included. It should not be concluded that sites for which no (or minimal) volumes are indicated in Tables 6.2 through 6.7 have no waste management concerns. Environmental restoration activities at such sites could generate hazardous wastes as regulated by RCRA and TSCA, as well as large volumes of sanitary and demolition wastes. Also, additional characterization activities at these sites may identify areas of radioactive contamination requiring remediation in the future. As it becomes available, such information will be included in future updates of this report.

The volumes of radioactively contaminated materials given in Tables 6.2 through 6.7 are limited to those sites and facilities currently in the EM-40 program. These data are summed across all elements of a site in Tables 6.2 through 6.6 including environmental media, wastes currently in storage, and radioactively contaminated materials that could result from future decommissioning activities. Stored wastes are reported separately in Table 6.7. At a number of sites, wastes resulting from EM-40 activities have been transferred to the Office of Waste Management (EM-30) for treatment, storage, and disposal. These wastes are no longer being managed by EM-40 and are therefore not included in this chapter. Additional information on the MLLW currently in storage at EM-40 sites and that projected to result from future environmental restoration activities are given in Chapter 8 of this report.

The DOE Office of Nuclear Material and Facility Stabilization (EM-60) is responsible for coordinating the transfer of facilities to the Office of Environmental Management (EM-1). As facilities are transferred to EM-1, environmental restoration and waste management information will be developed and included in future updates of this report.

\subsection{REFERENCES}

I. U.S. Department of Energy, Office of Strategic Planning and Analysis, Office of Environmental Management, The 1996 Baseline Environmental Management Report, DOE/EM-0290, Washington, D.C. (June 1996).

2. U.S. Department of Energy, Office of Environmental Restoration, Office of Environmental Management, EM-40 Core Database, electronic database accessed in July 1996, Washington, D.C.

3. U.S. General Accounting Office, Uranium Mill Tailings-Cleanup Continues, but Future Costs Are Uncertain, GAO/RCED-96-37, Washington, D.C. (December 1995). 


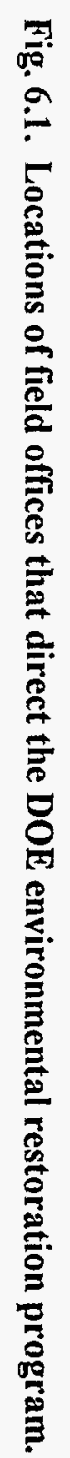

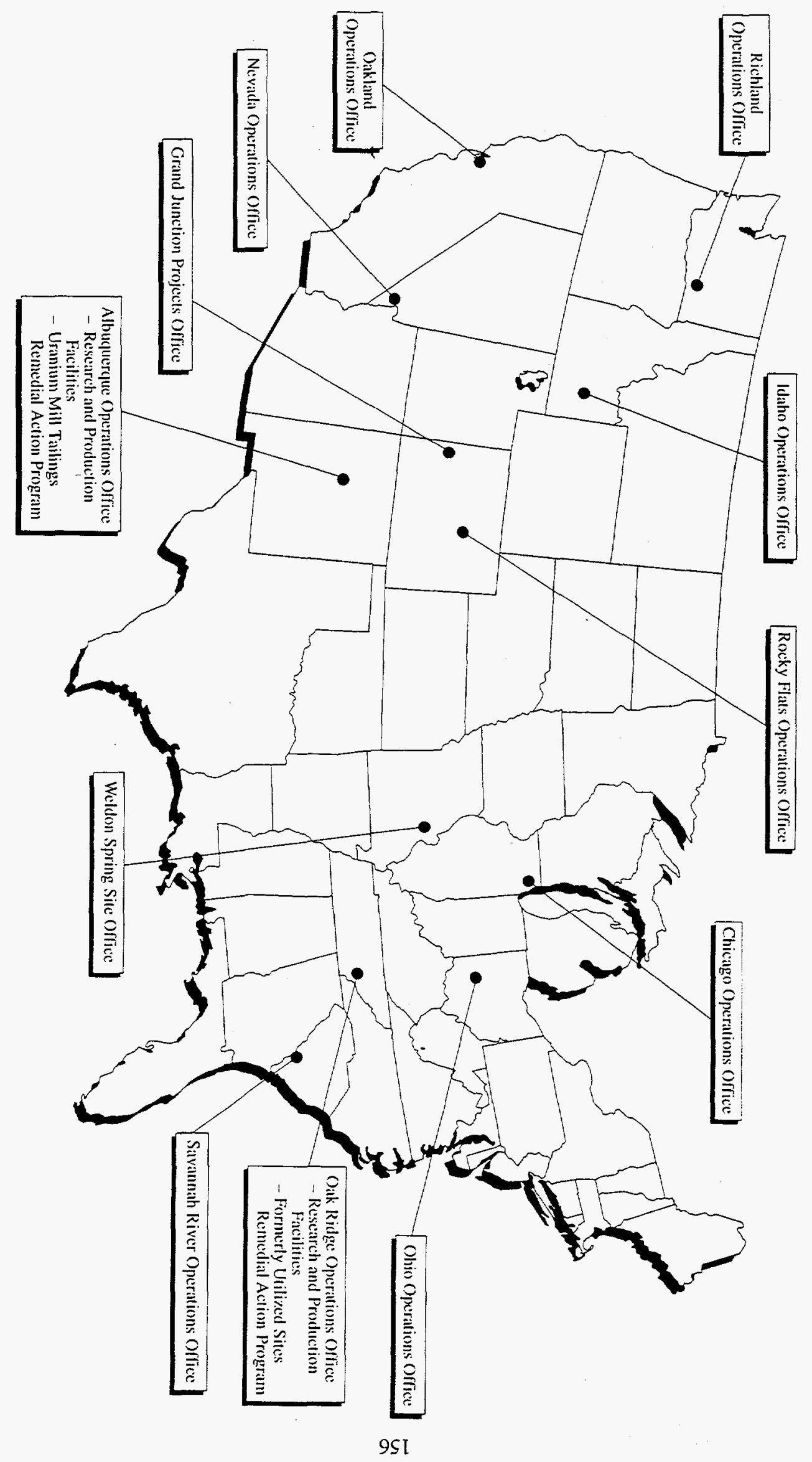

总 


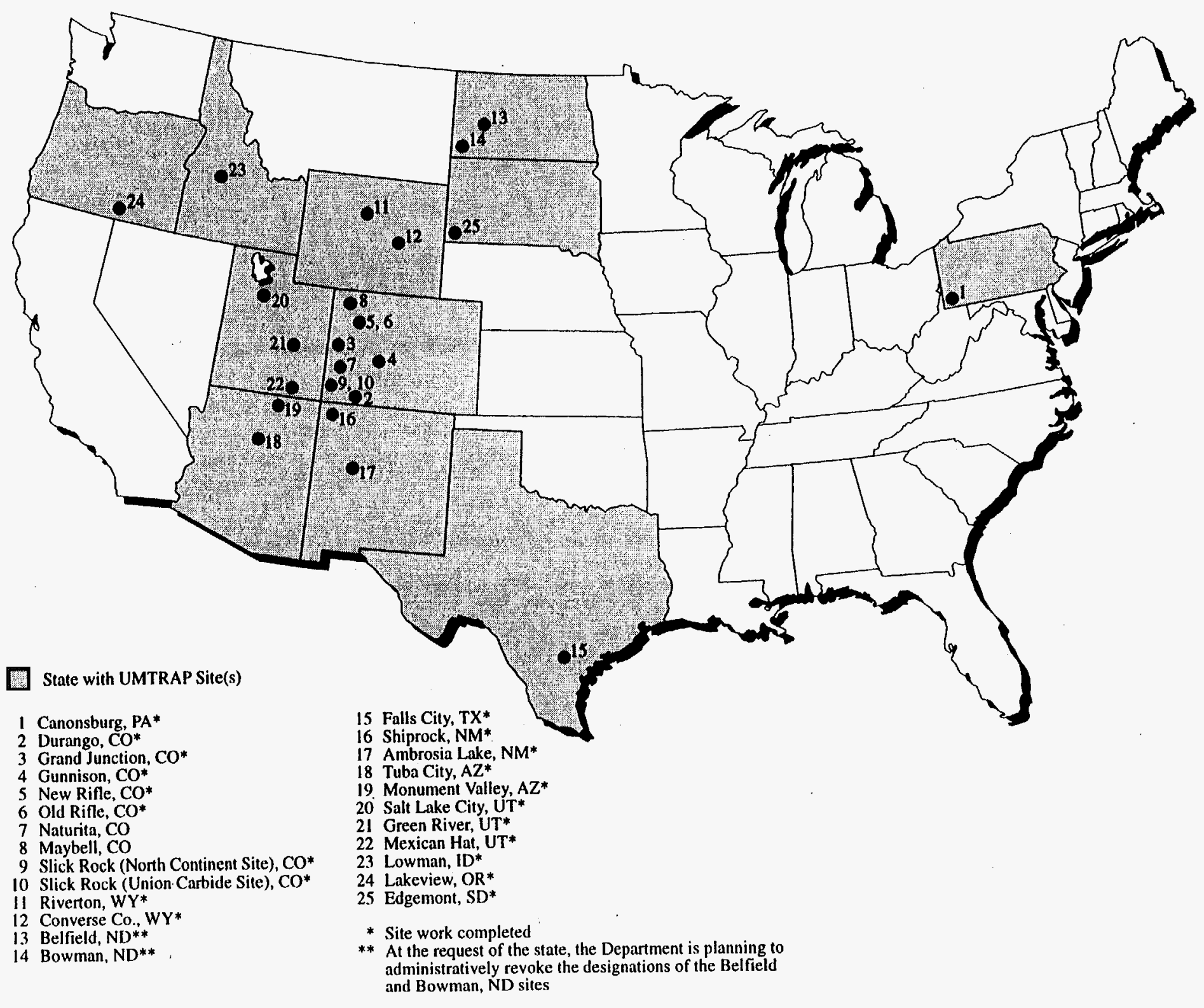

Fig. 6.2. Locations and status of UMTRAP sites. 


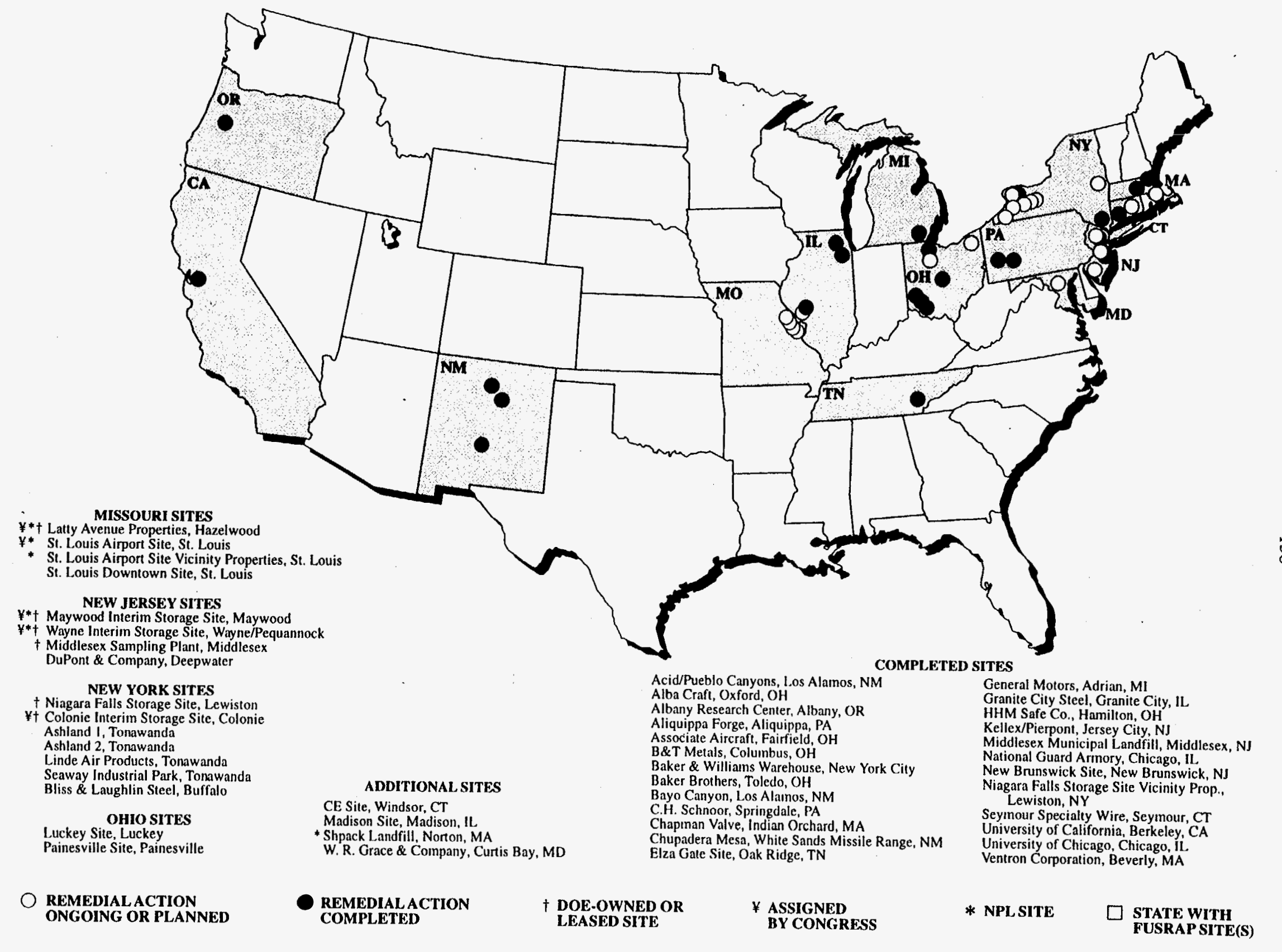

Fig. 6.3. Locations and status of FUSRAP sites. 
Table 6.1. List of sites in the DOE Environmental Restoration Program

\begin{tabular}{|c|c|}
\hline Field office ${ }^{a}$ & Site \\
\hline Albuquerque & $\begin{array}{l}\text { Inhalation Toxicology Research Institute } \\
\text { Kansas City Plant } \\
\text { Los Alamos National Laboratory } \\
\text { Pantex Plant } \\
\text { Pinellas Plant } \\
\text { Sandia National Laboratories/California } \\
\text { Sandia National Laboratories/New Mexico } \\
\text { South Valley Superfund Site } \\
\text { Uranium Mill Tailings Remedial Action Project }\end{array}$ \\
\hline Chicago & $\begin{array}{l}\text { Ames Laboratory } \\
\text { Argonne National Laboratory-East } \\
\text { Argonne National Laboratory-West } \\
\text { Brookhaven National Laboratory } \\
\text { Hallam Site } \\
\text { Piqua Site } \\
\text { Princeton Plasma Physics Laboratory } \\
\text { Site AJPlot M }\end{array}$ \\
\hline Grand Junction & $\begin{array}{l}\text { Grand Junction Projects Office Site } \\
\text { Monticello Remedial Action Project }\end{array}$ \\
\hline Idaho & Idaho National Engineering Laboratory \\
\hline Nevada & $\begin{array}{l}\text { Nevada Test Site } \\
\text { Nevada off-site locations } \mathrm{c}\end{array}$ \\
\hline Oak Ridge & $\begin{array}{l}\text { Formerly Utilized Sites Remedial Action Program } \\
\text { Oak Ridge Reservation } \\
\text { d } \\
\text { Paducah Gaseous Diffusion Plant } \\
\text { Portsmouth Gaseous Diffusion Plant }\end{array}$ \\
\hline Oakland & $\begin{array}{l}\text { Energy Technology Engineering Center } \\
\text { General Atomics Site } \\
\text { General Electric Vallecitos Nuclear Center } \\
\text { Laboratory for Energy-Related Health Research } \\
\text { Lawrence Berkeley Laboratory } \\
\text { Lawrence Livermore National Laboratory } \\
\text { Stanford Linear Accelerator Center }\end{array}$ \\
\hline Ohio & $\begin{array}{l}\text { Battelle Columbus Laboratories } \\
\text { Fernald Environmental Management Project } \\
\text { Mound Plant } \\
\text { Reactive Metals, Inc., Site } \\
\text { Separations Process Research Unit }\end{array}$ \\
\hline Richland & Hanford Site \\
\hline
\end{tabular}


Table 6.1 (continued)

\begin{tabular}{ll}
\hline Field office $^{\mathrm{a}}$ & \multicolumn{1}{c}{ Site } \\
\hline Rocky Flats & Rocky Flats Environmental Technology Site \\
Savannah River & Savannah River Site \\
Weldon Spring & Weldon Spring Site Remedial Action Project \\
\hline
\end{tabular}

aAll of the field offices listed here are Operations offices except for Grand Junction (which reports to Albuquerque Operations) and Weldon Spring (which reports to Oak Ridge Operations). The locations of these field offices is shown in Fig. 6.1.

$\mathrm{b}_{\mathrm{A}}$ listing of sites being addressed under UMTRAP is given in Fig. 6.2.

${ }^{c}$ Consists of Amchitka Island, Alaska; the Rio Blanco and Rulison Test sites in Colorado; the Gnome and Gasbuggy Test sites in New Mexico; the Salmon Test Site in Mississippi; and the Shoal and Central Nevada Test sites in Nevada.

$\mathrm{d}_{\mathrm{A}}$ listing of sites being addressed under FUSRAP is given in Fig. 6.3.

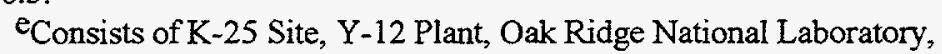
and contaminated areas in the vicinity of Oak Ridge, Tennessee, beyond the boundaries of these three facilities. 
Table 6.2. Projected disposition of radioactively contaminated solid materials classified as $\mathbf{L L W}^{\mathrm{a}}$

\begin{tabular}{|c|c|c|c|c|c|c|c|}
\hline \multirow[b]{2}{*}{ Site } & \multicolumn{7}{|c|}{ Response volume, ${ }^{b} \mathrm{~m}^{3}$} \\
\hline & \multicolumn{3}{|c|}{ Ex-situ } & $\begin{array}{c}\text { In-situ } \\
\text { treatment/ } \\
\text { containment }\end{array}$ & $\begin{array}{l}\text { Access } \\
\text { control or no } \\
\text { further action }\end{array}$ & $\begin{array}{l}\text { Not yet } \\
\text { determined }\end{array}$ & Total \\
\hline Argonne National Laboratory-East & & 8,700 & 23 & & & & 8,800 \\
\hline Argonne National Laboratory-West & 150 & & 6 & & & & 160 \\
\hline Brookhaven National Laboratory & & 400 & 120,000 & & & & 120,000 \\
\hline Energy Technology Engineering Center & & 1,600 & & & & & 1,600 \\
\hline Fernald Environmental Management Project & $1,800,000$ & 180,000 & 480,000 & & & 5,800 & $2,500,000$ \\
\hline \multicolumn{8}{|l|}{ Formerly Utilized Sites Remedial Action Program ${ }^{\mathrm{c}}$} \\
\hline Missouri sites & 160,000 & & & & & & 160,000 \\
\hline New Jersey sites & . 6,300 & & & & & & 6,300 \\
\hline New York sites & 9,400 & & 930 & & & & 10,000 \\
\hline Other sites & 79,000 & & 15,000 & & & & 94,000 \\
\hline General Atomics Site & 1,200 & & & & & & 1,200 \\
\hline Hanford Site & $3,900,000$ & 700 & & $20,000,000$ & & & $24,000,000$ \\
\hline Idaho National Engineering Laboratory & 420,000 & 140,000 & & 79,000 & & & 640,000 \\
\hline Inhalation Toxicology Research Institute & & 9,100 & & & & & 9,100 \\
\hline Laboratory for Energy-Related Health Research & & 3,800 & & & & & 3,800 \\
\hline Lawrence Berkeley Laboratory & & 9,400 & & & & & 9,400 \\
\hline Los Alamos National Laboratory & & 15,000 & & 200,000 & $8,900,000$ & 110,000 & $9,300,000$ \\
\hline Mound Plant & & 3,100 & 120,000 & & & & 120,000 \\
\hline Nevada off-site locations ${ }^{d}$ & & & & 26,000 & & & 26,000 \\
\hline Nevada Test Site & & $1,100,000$ & & 1,200 & & & $1,100,000$ \\
\hline Oak Ridge Reservation & 30,000 & 9,800 & 850,000 & 51,000 & & & 940,000 \\
\hline Paducah Gaseous Diffusion Plant & 770,000 & 150 & & & 56 & & 770,000 \\
\hline Pantex Plant & 54 & & & & & & 54 \\
\hline Portsmouth Gaseous Diffusion Plant & 730,000 & 190 & & & & & 730,000 \\
\hline Reactive Metals, Inc., Site & & 30,000 & & & & & 30,000 \\
\hline Rocky Flats Environmental Technology Site & 61,000 & 36,000 & & & & & 96,000 \\
\hline Sandia National Laboratories/New Mexico & & 36,000 & & & 14,000 & & 50,000 \\
\hline Savannah River Site & & 400,000 & & 310,000 & 1,800 & $1,800,000$ & $2,500,000$ \\
\hline
\end{tabular}


Table 6.2 (continued)

\begin{tabular}{|c|c|c|c|c|c|c|c|}
\hline \multirow[b]{2}{*}{ Site } & \multicolumn{7}{|c|}{ Response volume, ${ }^{b} \mathrm{~m}^{3}$} \\
\hline & \multicolumn{3}{|c|}{ Ex-situ } & $\begin{array}{c}\text { In-situ } \\
\text { treatment } \\
\text { containment }\end{array}$ & $\begin{array}{l}\text { Access } \\
\text { control or no } \\
\text { further action }\end{array}$ & $\begin{array}{l}\text { Not yet } \\
\text { determined }\end{array}$ & Total \\
\hline Separations Process Research Unit & & & 15,000 & & & & 15,000 \\
\hline Site A/Plot M & & & 40 & & & & 40 \\
\hline Total & $8,000,000$ & $1,900,000$ & $1,600,000$ & $21,000,000$ & $8,900,000$ & $1,900,000$ & $43,000,000$ \\
\hline
\end{tabular}

aInformation obtained from the EM-40 Core Database (July 1996). Volume estimates include environmental media such as soil, sediment, sludge, and intermixed rubble/debris; stored wastes; and standing structures and equipment. Sites not listed in this table do not have any radioactively contaminated solid material classified as LLW. The stored waste volumes are also provided separately in Table 6.7 .

bThese volume estimates represent the initial response volumes, not final waste forms. Changes in volumes and waste classes due to treatment are not reflected in this table. All values are preliminary and are being updated as site characterization and engineering studies continue. Values are given to two significant figures unless information was reported to only one significant figure. Some totals may not equal sum of components due to independent rounding.

${ }^{c} A$ listing of the sites being addressed under FUSRAP is given in Fig. 6.3. The radioactively contaminated material classified as LLW in the EM-40 Core Database for those FUSRAP sites (Latty Avenue Properties in Missouri and the Luckey and Painesville sites in Ohio) may be reclassified as 11 e(2) by-product material.

Consists of Amchitka Island, Alaska; the Rio Blanco and Rulison Test sites in Colorado; the Gnome and Gasbuggy Test sites in New Mexico; the Salmon Test Site in Mississippi; and the Shoal and Central Nevada Test sites in Nevada. 
Table 6.3. Projected disposition of radioactively contaminated solid materials classified as MLLWa

\begin{tabular}{|c|c|c|c|c|c|c|c|}
\hline \multirow{3}{*}{ Site } & \multicolumn{7}{|c|}{ Response volume, ${ }^{b} \mathrm{~m}^{3}$} \\
\hline & \multicolumn{3}{|c|}{ Ex-situ } & \multirow{2}{*}{$\begin{array}{c}\text { In-situ } \\
\text { treatment/ } \\
\text { containment }\end{array}$} & \multirow{2}{*}{$\begin{array}{l}\text { Access } \\
\text { control or no } \\
\text { further action }\end{array}$} & \multirow{2}{*}{$\begin{array}{c}\text { Not yet } \\
\text { determined }\end{array}$} & \multirow[b]{2}{*}{ Total } \\
\hline & $\begin{array}{c}\text { Managed by } \\
\text { EM-40 }\end{array}$ & $\begin{array}{l}\text { Transferred } \\
\text { to EM-30 }\end{array}$ & $\begin{array}{c}\text { Commercial } \\
\text { disposal }\end{array}$ & & & & \\
\hline Argonne National Laboratory-East & 160 & 0.4 & & 140,000 & & & 140,000 \\
\hline Battelle Columbus Laboratories & & 12 & 1 & & & & 13 \\
\hline Brookhaven National Laboratory & 3,200 & & 16,000 & & & & 19,000 \\
\hline Fernald Environmental Management Project & & 2,200 & 2,400 & & & & 4,600 \\
\hline \multicolumn{8}{|l|}{ Formerly Utilized Sites Remedial Action Program $\mathrm{c}$} \\
\hline New York sites & & & 12,000 & & & & 12,000 \\
\hline Other sites & 7,200 & & & & & & 7,200 \\
\hline General Atomics Site & 96 & & & & & & 96 \\
\hline Hanford Site & 220 & 100 & & & & & 320 \\
\hline Idaho National Engineering Laboratory & 100,000 & & 65 & 99,000 & & & 200,000 \\
\hline Laboratory for Energy-Related Health Research & & 4,400 & & & & & 4,400 \\
\hline Lawrence Berkeley Laboratory & & & & 42,000 & & & 42,000 \\
\hline Los Alamos National Laboratory & & & 980 & & 500,000 & & 500,000 \\
\hline Nevada off-site locations ${ }^{d}$ & & & & 11,000 & & & 11,000 \\
\hline Nevada Test Site & & 50 & & & & & 50 \\
\hline Oak Ridge Reservation & & 3,900 & & & 450,000 & 1,900 & 460,000 \\
\hline Paducah Gaseous Diffusion Plant & 210,000 & & & & 240,000 & & 450,000 \\
\hline Portsmouth Gaseous Diffusion Plant & 380 & & & & 270,000 & & 270,000 \\
\hline Reactive Metals, Inc., Site & & 11 & 17 & & & & 28 \\
\hline Rocky Flats Environmental Technology Site & 140,000 & 42,000 & & 9,900 & 180,000 & & 380,000 \\
\hline Sandia National Laboratories/New Mexico & & & 1,700 & & 2,600 & & 4,300 \\
\hline Savannah River Site & & 150,000 & & $3,900,000$ & & $6,600,000$ & $11,000,000$ \\
\hline Site A/Plot M & & & 500 & & & & 500 \\
\hline Total & 470,000 & 200,000 & 34,000 & $4,200,000$ & $1,600,000$ & $6,600,000$ & $13,000,000$ \\
\hline
\end{tabular}

a Information obtained from the EM-40 Core Database (July 1996). Volume estimates include environmental media such as soil, sediment, sludge, and intermixed rubble/debris; stored wastes; and standing structures and equipment. Sites not listed in this table do not have any radioactively contaminated solid material classified as MLLW. The stored waste volumes are also provided separately in Table 6.7 .

b These volume estimates represent the initial response volumes, not final waste forms. Changes in volumes and waste classes due to treatment are not reflected in this table. All values are preliminary and are being updated as site characterization and engineering studies continue. Values are given to two significant figures unless information was reported to only one significant figure. Some totals may not equal sum of components due to independent rounding.

$c_{A}$ listing of the sites being addressed under FUSRAP is given in Fig. 6.3

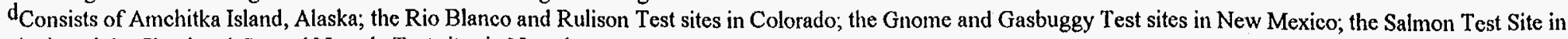
Mississippi; and the Shoal and Central Nevada Test sites in Nevada. 
Table 6.4 Projected disposition of radioactively contaminated solid materials classified as TRUW

\begin{tabular}{|c|c|c|c|c|c|c|c|}
\hline \multirow{3}{*}{ Operations office } & & \multicolumn{6}{|c|}{ Response volume, ${ }^{b} \mathrm{~m}^{3}$} \\
\hline & & \multicolumn{2}{|c|}{ Ex-situ } & \multirow{2}{*}{$\begin{array}{c}\text { In-situ } \\
\text { treatment/ } \\
\text { containment }\end{array}$} & \multirow{2}{*}{$\begin{array}{l}\text { Access } \\
\text { control or no } \\
\text { further action }\end{array}$} & \multirow{2}{*}{$\begin{array}{c}\text { Not yet } \\
\text { determined }\end{array}$} & \multirow[b]{2}{*}{ Total } \\
\hline & & $\begin{array}{c}\text { Managed by } \\
\text { EM-40 }\end{array}$ & $\begin{array}{l}\text { Transferred } \\
\text { to EM-30 }\end{array}$ & & & & \\
\hline Argonne National Laboratory-East & & & 190 & & & & 190 \\
\hline Battelle Columbus Laboratories & & & 95 & & & & 95 \\
\hline General Electric Vallecitos Nuclear Center & & $20^{\mathrm{c}}$ & & & & & 20 \\
\hline Hanford Site & & & 1,800 & & & 84 & 1,900 \\
\hline Idaho National Engineering Laboratory & & & $9,700^{\mathrm{d}}$ & & & & 9,700 \\
\hline Los Alamos National Laboratory & & & & 4,400 & & & 4,400 \\
\hline Oak Ridge Reservation & & $12^{\mathrm{c}}$ & 1,500 & 130 & & & $1,600^{\mathrm{e}}$ \\
\hline Paducah Gaseous Diffusion Plant & & $7^{\mathrm{c}, \mathrm{d}}$ & & & & & 7 \\
\hline \multicolumn{8}{|l|}{ Portsmouth Gaseous Diffusion Plant } \\
\hline Rocky Flats Environmental Technology Site & - & & 1,900 & & & 3,000 & $4,900^{f}$ \\
\hline Sandia National Laboratories/New Mexico & & & & & $4,000^{d}$ & & 4,000 \\
\hline Savannah River Site & & & 65,000 & & & & 65,000 \\
\hline Separations Process Research Unit & & & 36 & & & & 36 \\
\hline Total & & 39 & 80,000 & 4,500 & 4,000 & 3,100 & 91,000 \\
\hline
\end{tabular}

a Information obtained from the EM-40 Core Database (July 1996). Volume estimates include environmental media such as soil, sediment, sludge, and intermixed rubble/debris; stored wastes; and standing structures and equipment and include the contribution of material classified as MTRUW. Sites not listed in this table do not have any radioactively contaminated solid material classified as TRUW. The stored waste volumes are provided separately in Table 6.7.

${ }^{b}$ These volume estimates represent the initial response volumes, not final waste forms. Changes in volumes and waste classes due to treatment are not reflected in this table. All values are preliminary and are being updated as site characterization and engineering studies continue. Values are given to two significant figures unless information was reported to only one significant figure. Some totals may not equal sum of components due to independent rounding.

${ }^{c}$ These wastes are expected to be packaged and eventually transferred to the Waste Isolation Pilot Plant for final disposition.

dMTRUW.

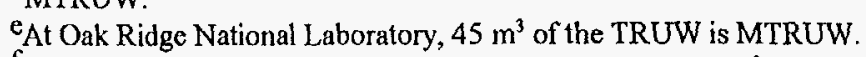

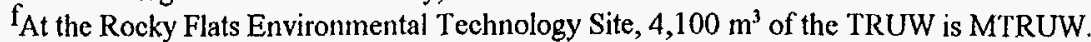




\section{Integrated Data Base Program Report: DOE/RW-0006, Rev. 12}

CHAPTER 6: TABLE 6.5 ERRATA

NOTE: Table 6.5 was revised to correct a couple of erroneous entries and to delete those materials that have been disposed of already in EM-40-managed facilities. These materials are included in Table 6.7. Table 6.5 was revised on January 28, 1997. 
Table 6.5. Projected disposition of radioactively contaminated solid materials classified as $11 \mathrm{e}(2)$ by-product materiala, $\mathrm{b}$

\begin{tabular}{|c|c|c|c|c|c|c|c|}
\hline \multirow{3}{*}{ Operations office } & \multicolumn{7}{|c|}{ Response volume, ${ }^{\mathrm{C}} \mathrm{m}^{3}$} \\
\hline & \multicolumn{3}{|c|}{ Ex-situ } & \multirow{2}{*}{$\begin{array}{c}\text { In-situ } \\
\text { treatment/ } \\
\text { contaminant }\end{array}$} & \multirow{2}{*}{$\begin{array}{l}\text { Access } \\
\text { control or } \\
\text { no further } \\
\text { action }\end{array}$} & \multirow{2}{*}{$\begin{array}{c}\text { Not yet } \\
\text { determined }\end{array}$} & \multirow{2}{*}{ Total } \\
\hline & Managed by EM-40 & $\begin{array}{c}\text { Transferred } \\
\text { to EM-30 }\end{array}$ & $\begin{array}{c}\text { Commercial } \\
\text { disposal }\end{array}$ & & & & \\
\hline Fernald Environmental Management Project & & $11,000^{\mathrm{d}}$ & & & & & 11,000 \\
\hline $\begin{array}{l}\text { Formerly Utilized Sites Remedial Action Program } \\
\end{array}$ & 340000 & & & 10000 & & & Ea \\
\hline New Jersey sites & $\begin{array}{r}340,000 \\
68,000\end{array}$ & & 390.000 & 190,000 & & & $\begin{array}{l}530,000 \\
460000^{f}\end{array}$ \\
\hline New York sites & $280,000 \mathrm{~g}$ & & 460 & & & & 280,000 \\
\hline Other sites & 28,000 & & & & & & 28,000 \\
\hline Grand Junction Projects Office Site & 3,200 & & & & & & 3,200 \\
\hline Monticello Remedial Action Project & $1,900,000$ & & & & 14,000 & & $1,900,000$ \\
\hline Uranium Mill Tailings Remedial Action Program ${ }^{h}$ & $3,200,000$ & & & & & & $3,200,000$ \\
\hline Weldon Spring Site Remedial Action Project & $670,000^{\mathrm{i}}$ & & & & & & 670,000 \\
\hline Total & $6,500,000$ & 11,000 & 390,000 & 190,000 & 14,000 & & $7,100,000$ \\
\hline
\end{tabular}

a Information obtained from the EM-40 Core Database (July 1996). Volume estimates include environmental media such as soil, sediment, sludge, and intermixed rubble/debris; stored wastes; and standing structures and equipment and include the contribution of material classified as mixed 11 e(2) by-product material. Sites not listed in this table do not have any radioactively contaminated solid material classified as $11 \mathrm{e}(2)$ by-product material. The stored waste volumes are provided separately in Table 6.7 .

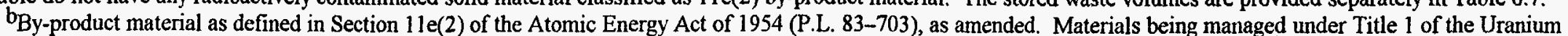
Mill Tailings Radiation Control Act of 1978 (Pub. L. 95-604) are defined as residual radioactive material. Since this material has the same physical and radioactive properties as 11 e(2) by-product material, it is reported here under $11 \mathrm{e}(2)$ by-product material.

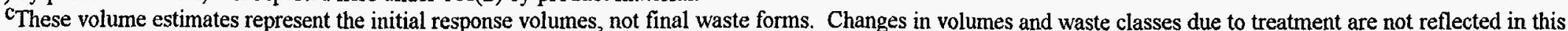
table. All values are preliminary and are being updated as site characterization and engineering studies continue. Values are given to two significant figures unless information was reported to only one significant figure. Some totals may not equal sum of components due to independent rounding.

Residues in storage in four concrete silos.

$\mathrm{e}_{\mathrm{A}}$ listing of the sites being addressed under FUSRAP is given in Fig. 6.3.

Includes $83,000 \mathrm{~m}^{3}$ of $11 \mathrm{e}(2)$ by-product material soil in bulk storage at the Middlesex Sampling Plant and the Wayne and Maywood Interim Storage sites and $24,000 \mathrm{~m}^{3}$ of mixed 1 le(2) by-product soil in bulk storage at the Middlesex Sampling Plant.

gAdditional 190,000 $\mathrm{m}^{3}$ of contaminated soil and residues have been disposed of in a containment cell at the Niagara Falls Storage Site (see Table 6.7).

$\mathrm{h}_{\mathrm{A}}$ listing of the sites being addressed under UMTRAP is given in Fig. 6.2. The volume of mill tailings and debris associated with the 20 sites for which remedial actions have been completed is $27,000,000 \mathrm{~m}^{3}$ (see Table 6.7).

Includes $160,000 \mathrm{~m}^{3}$ of $11 \mathrm{e}(2)$ by-product material soil and debris and $20,000 \mathrm{~m}^{3}$ of mixed 11e(2) by-product material soil and debris in interim storage at the chemical plant area of the Weldon Spring Site. 
Table 6.5. Projected disposition of radioactively contaminated solid materials classified as $11 \mathrm{e}(2)$ by-product material ${ }^{\mathrm{a}, \mathrm{b}}$

\begin{tabular}{|c|c|c|c|c|c|c|c|}
\hline \multirow[b]{2}{*}{ Operations office } & \multicolumn{7}{|c|}{ Response volume, ${ }^{\mathrm{c}} \mathrm{m}^{3}$} \\
\hline & \multicolumn{3}{|c|}{ Ex-situ } & $\begin{array}{c}\text { In-situ } \\
\text { treatment/ } \\
\text { contaminant }\end{array}$ & $\begin{array}{c}\text { Access } \\
\text { control or no } \\
\text { further action }\end{array}$ & $\begin{array}{c}\text { Not yet } \\
\text { determined }\end{array}$ & Total \\
\hline Fernald Environmental Management Project & & 11,000 & & & & & 11,000 \\
\hline \multicolumn{8}{|l|}{ Formerly Utilized Sites Remedial Action Program ${ }^{d}$} \\
\hline New Jersey sites & $68,000^{\mathrm{e}}$ & & 390,000 & & & & 460,000 \\
\hline New York sites & 280,000 & & 460 & & $190,000^{f}$ & & 540,000 \\
\hline Other sites & 28,000 & & & & & & 28,000 \\
\hline Grand Junction Projects Office Site & 3,200 & & & & & & 3,200 \\
\hline Monticello Remedial Action Project & $1,900,000$ & & & & 14,000 & & $1,900,000$ \\
\hline Uranium Mill Tailings Remedial Action Programg & $30,000,000$ & & & & & & $30,000,000$ \\
\hline Weldon Spring Site Remedial Action Project & $670,000^{\mathrm{h}}$ & & & & & & 670,000 \\
\hline
\end{tabular}

a Information obtained from the EM-40 Core Database (July 1996). Volume estimates include environmental media such as soil, sediment, sludge, and intermixed rubble/debris; stored wastes; and standing structures and equipment and include the contribution of material classified as mixed 11 e(2) by-product material. Sites not listed in this table do not have any radioactively contaminated solid material classified as 1 le(2) by-product material. The stored waste volumes are provided separately in Table 6.7 .

${ }^{b}$ By-product material as defined in Section 11e(2) of the Atomic Energy Act of 1954 (P.L. 83-703), as amended. Materials being managed under Title 1 of the Uranium

Mill Tailings Radiation Control Act of 1978 (P.L. 95-604) are defined as residual radioactive material. Since this material has the same physical and radioactive properties as 1 le(2) by-product material, it is reported here under $11 \mathrm{e}(2)$ by-product material.

${ }^{c}$ These volume estimates represent the initial response volumes, not final waste forms. Changes in volumes and waste classes due to treatment are not reflected in this table. All values are preliminary and are being updated as site characterization and engineering studies continue. Values are given to two significant figures unless information was reported to only one significant figure. Some totals may not equal sum of components due to independent rounding.

${ }^{\mathrm{d}} \mathrm{A}$ listing of the sites being addressed under FUSRAP is given in Fig. 6.3.

Includes $24,000 \mathrm{~m}^{3}$ of mixed $1 \mathrm{le}(2)$ by-product material soil in bulk storage at the Middlesex Sampling Plant. The storage pile is covered with a tarp.

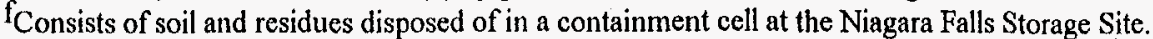

$\mathrm{g}_{\mathrm{A}}$ listing of the sites being addressed under UMTRAP is given in Fig. 6.2. The volume of mill tailings and debris associated with the 20 sites for which remedial actions have been completed is $27,000,000 \mathrm{~m}^{3}$, leaving $3,200,000 \mathrm{~m}^{3}$ of mill tailings and debris for which remedial actions are currently ongoing.

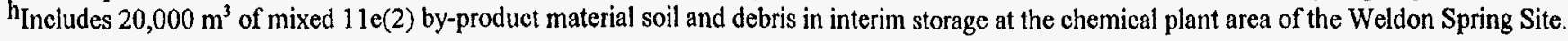


Table 6.6. TSCA mixed waste associated with EM-40 activities ${ }^{\mathrm{a}}$

\begin{tabular}{lcr}
\hline \multicolumn{1}{c}{ Site } & \multicolumn{2}{c}{ Response volume $^{\mathrm{b}}, \mathrm{m}^{3}$} \\
\cline { 2 - 3 } & RASB $^{\mathrm{c}}$ & $\mathrm{RPCB}^{\mathrm{d}}$ \\
\hline Argonne National Laboratory-East & $5^{\mathrm{e}}$ & \\
Grand Junction Projects Office Site & $38^{\mathrm{f}}$ & $46^{\mathrm{f}, \mathrm{g}}$ \\
Mound Plant & $870^{\mathrm{h}}$ & $1^{\mathrm{e}}$ \\
Oak Ridge Reservation & $75^{\mathrm{e}}$ & $3,500^{\mathrm{f}, \mathrm{g}}$ \\
Paducah Gaseous Diffusion Plant & $430^{\mathrm{f}, \mathrm{g}}$ & $3,600^{\mathrm{f}, \mathrm{g}}$ \\
Portsmouth Gaseous Diffusion Plant & & \\
Reactive Metals, Inc., Site & $580^{\mathrm{e}}$ & \\
Separative Process Research Unit & $2^{\mathrm{h}}$ & \\
Weldon Spring Site Remedial Action Project & $7,500^{\mathrm{f} g}$ & \\
\hline
\end{tabular}

anformation obtained from the EM-40 Core Database (July 1996). Volume estimates include environmental media such as soil, sediment, sludge, and intermixed rubble/debris; stored wastes; and standing structures and equipment. Sites not listed in this table do not have any radioactively contaminated solid material classified as mixed TSCA wastes. The stored waste volumes are also provided separately in Table 6.7.

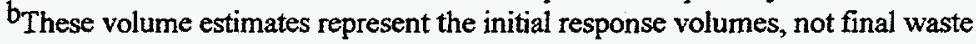
forms. Changes in volumes and waste types due to treatment are not reflected in this table. All values are preliminary and are being updated as site characterization and engineering studies continue. Values are given to two significant figures or the nearest integer (for volumes less than $10 \mathrm{~m}^{3}$ ).

$c_{\text {Radioactive asbestos, i.e., materials contaminated with both radionuclides and }}$ asbestos.

${ }^{\mathrm{d}}$ Radioactive PCBs, i.e., materials contaminated with both radionuclides and polychlorinated biphenyls.

Projected to be transferred to EM-30 for final disposition.

frojected to be managed by EM-40 through final disposition.

gWastes currently in storage.

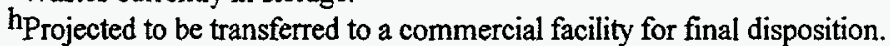


Table 6.7. Volumes $\left(\mathrm{m}^{3}\right)$ of solid radioactive wastes in storage at EM-40 facilities ${ }^{\mathrm{a}}$

\begin{tabular}{|c|c|c|c|c|c|c|c|c|c|}
\hline \multirow[b]{2}{*}{ Site } & \multicolumn{9}{|c|}{ Waste type } \\
\hline & TRUW & MTRUW & LLW & MLLW $^{b}$ & $\begin{array}{c}11 \mathrm{e}(2) \\
\text { by-product } \\
\text { material }\end{array}$ & $\begin{array}{c}\text { Mixed } \\
11 \mathrm{e}(2) \\
\text { by-product } \\
\text { material }\end{array}$ & RASBC $^{\mathrm{c}}$ & $\mathrm{RPCB}^{\mathrm{d}}$ & Total \\
\hline Battelle Columbus Laboratories & 41 & & & & & & & & 41 \\
\hline $\begin{array}{l}\text { Fernald Environmental Management } \\
\text { Project }\end{array}$ & & & 140,000 & 3,500 & $11,000^{\mathrm{e}}$ & & & & 150,000 \\
\hline $\begin{array}{l}\text { Formerly Utilized Sites Remedial } \\
\text { Action Program } \\
\text { Missouri sites } \\
\text { New Jersey sites } \\
\text { New York sites } \\
\text { Other sites }\end{array}$ & & & $\begin{array}{r}24,000^{\mathrm{g}} \\
1,500^{\mathrm{k}}\end{array}$ & & $\begin{array}{c}83,000^{\mathrm{h}} \\
190,000^{\mathrm{j}}\end{array}$ & $24,000^{\mathrm{i}}$ & & & $\begin{array}{r}24,000 \\
110,000 \\
190,000 \\
1,500\end{array}$ \\
\hline General Atomics Site & & & 35 & 5 & & & & & 40 \\
\hline Grand Junction Projects Office Site & & & & & & & & $46^{l}$ & 46 \\
\hline Paducah Gaseous Diffusion Plant & 2 & & 5,200 & 1,000 & & & 430 & 3,500 & 10,000 \\
\hline Portsmouth Gaseous Diffusion Plant & & & 9,000 & 6,800 & & & & 3,600 & 19,000 \\
\hline Reactive Metals, Inc., Site & & & 550 & 28 & & & & & 580 \\
\hline $\begin{array}{l}\text { Uranium Mill Tailings Remedial } \\
\text { Action Program }\end{array}$ & & & & & $27,000,000^{\mathrm{m}}$ & & & & $27,000,000$ \\
\hline $\begin{array}{l}\text { Weldon Spring Site Remedial Action } \\
\text { Project }\end{array}$ & & & & & 160,000 & 20,000 & $7,500^{l}$ & & 190,000 \\
\hline
\end{tabular}

anformation obtained from the EM-40 Core Database (July 1996). Waste volumes are limited to solid wastes and do not include EM-40-generated wastes that are currently in storage facilities managed by EM-30. Volumes are given to two significant figures or the nearest integer (for volumes less than $10 \mathrm{~m}^{3}$ ). Some totals may not equal sum of components, due to independent rounding.

banagement plans for these wastes are provided in site treatment plans developed to meet the requirements of the Federal Facility Compliance Act.

Radioactive asbestos, i.e., materials contaminated with both radionuclides and asbestos.

Radioactive PCBs, i.e., materials contaminated with both radionuclides and polychlorinated biphenyls..

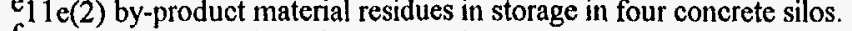

$f_{A}$ listing of sites being addressed under FUSRAP is given in Fig 6.3

gow-level waste soil in bulk storage at the Hazelwood Interim Storage Site. The storage pile is covered with a tarp.

$\mathrm{h}_{1 \mathrm{le}}(2)$ by-product material soil in bulk storage at the Middlesex Sampling Plant and the Wayne and Maywood Interim Storage Sites. All of the storage piles are covered with tarps.

Mixed 1 le(2) by-product material soil in bulk storage at the Middlesex Sampling Plant. The storage pile is covered with a tarp.

$\mathrm{j}_{11 \mathrm{e}(2)}$ by-product material residues and soil disposed of in a containment cell at the Niagara Falls Storage Site.

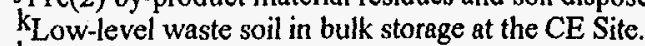

IThe radioactive classification of this waste is $11 \mathrm{e}(2)$ by-product material.

mWaste volume associated with the 20 completed UMTRAP sites (see Fig. 6.2). 



\section{NATURALLY OCCURRING AND ACCELERATOR-PRODUCED RADIOACTIVE MATERIAL}

\subsection{INTRODUCTION}

Naturally occurring and accelerator-produced radioactive material (NARM) is a broad category that includes accelerator-produced radioactive material (ARM) and naturally occurring radioactive material (NORM) and is not source, special nuclear, or by-product material. ARM wastes include materials generated by accelerators used in subatomic particle physics research.

The term NORM refers to materials not covered under the AEA whose radioactivity has been enhanced (i.e., materials whose radionuclide concentrations are either increased or redistributed compared to typical background levels either naturally or as the result of human intervention or processes). Examples are exploration and production wastes from the oil and natural gas industries and phosphate slag piles from the phosphate mining industry. NORM is not used to describe or discuss the natural radioactivity of rocks and soils or background radiation.

NARM wastes are currently not regulated by any federal agency. Responsibility for regulating the disposal of NARM is not addressed in the Atomic Energy Act (AEA). ${ }^{1}$ Regulation of NARM disposal currently rests with the states as part of their authority for ensuring the protection of public health and safety. ${ }^{2,3}$ However, currently a few states do not have regulatory programs for NARM wastes.

\subsection{WASTE CHARACTERIZATION}

The accelerator wastes (ARM) that comprise NARM include accelerator targets, wastes from accelerator maintenance and D\&D, and wastes from radiopharmacentical manufacture. Table 7.1 (based on ref. 4) lists the radionuclides found in ARM wastes. Except for ${ }^{81} \mathrm{Kr},{ }^{145} \mathrm{Pm}$, and ${ }^{22} \mathrm{Na}$, most of these radionuclides have short half-lives that are measured in days, hours, or minutes.

Compared to radioactive wastes associated with most research, industrial, and medical applications, NARM wastes have low radioactivity concentrations. NARM waste with more than $2 \mathrm{nCi} / \mathrm{g}$ of ${ }^{226} \mathrm{Ra}$ or equivalent is commonly referred to as discrete NARM waste; below this threshold, the waste is referred to as diffuse NARM waste.

\subsubsection{Discrete Wastes}

Discrete wastes have a relatively small volume but large radioactivity; these include industrial gauges, old radium watch and industrial dials, radium needles in medical equipment, and resins (filters) that remove radioactive radium and other NORM from ground water.

\subsubsection{Diffuse Wastes}

Diffuse wastes are characterized by a relatively large volume with small radioactivity. ${ }^{5}$ These materials result from industrial processes and include:

- coal ash and slag from utility electrical generation;

- solid wastes from geothermal energy production;

- slag, leachate, and tailings from the mining and processing of metals other than uranium or thorium (e.g., copper);

- sludge from drinking water treatment;

- scale, sludge, produced water, and equipment from oil and natural-gas production containing NORM; and

- wastes (phosphogypsum and slag) from mining phosphate ores for fertilizer (ammonium phosphate) production.

A summary of domestic processes that generate NORM wastes are given in Table 7.2 (based on refs. 6-9). Levels of specific activity for these wastes typically vary from 2-200 pCi $/ \mathrm{g}^{6.10}$ Table 7.3 (adapted from refs. 9 and 11) lists major radionuclides found in diffuse NORM wastes. Estimated radionuclide concentrations reported by the Environmental Protection Agency (EPA) in ref. 9 for each diffuse NORM category are provided in Tables 7.4-7.9.

Descriptions of the specific characteristics of diffuse NORM wastes are given in the following and are based on 
the EPA waste characterization and preliminary risk assessment study of ref. 9 .

\subsubsection{Coal combustion}

Fossil fuels such as coal contain naturally occurring radioactivity from uranium, thorium, radium, and their daughter products. The combustion of coal as a fossil fuel for electric power and industrial applications results in the generation of ash, which is collected at the bottom of power plant boilers and in exhaust stack filters. Consisting mostly of aluminum, iron, calcium, and silicon, coal ash is retained either as bottom ash and boiler slag or as fly ash trapped in exhaust stack filtration devices. Most of the waste is generated as fly ash, which is entrained with the hot flue gases of the combustion process. The remainder of the ash is heavier and settles to the bottom of the boiler to form what is referred to as bottom ash. Liquid boiler slag is formed when some of the bottom ash melts under the intense heat. ${ }^{9}$ Table 7.4 (based on ref. 9) gives a breakdown of the radionuclide concentrations found in NORM wastes from coal combustion. These concentrations, however, can vary widely depending on the mineral content of the coal which, in turn, can vary with mining location and region of the country.

\subsubsection{Geothermal energy production}

Geothermal energy is heat produced and stored in the earth. This energy can be economically extracted from high-temperature crustal rocks, sediments, volcanic deposits, water, steam, and other gases found at accessible depths from the earth's surface. Concentrations of NORM are found in the solid wastes generated by the exploration and development of geothermal systems and the extraction of the earth's geothermal energy for use in either producing electric power or supplying direct heat. These NORM wastes include minerals that precipitate out of solution and form scale or sludge on the inside surfaces of the drilling and production equipment (e.g., steam turbines, heat exchangers, process lines, valves, turbines, and fluidhandling equipment) used to extract geothermal heat. Such wastes contain barium, calcium, and strontium salts (carbonates, sulfates, and silicates) and silica as well as significant concentrations of radium and radium decay products. Radium is slightly soluble and, consequently, can be brought to the surface and coprecipitated with barium and calcium salts onto the inside surfaces of drilling and production equipment. The principal wastes of concern are the scales in piping and production equipment and the filter cake produced from treatment of spent geothermal fluids prior to reinjection. Scales are hard, insoluble sulfate deposits that form on the inside of pipes, tubulars, filters, pumps, well heads, and other water-handling equipment. ${ }^{8}$ Concentrations of NORM in geothermal wastes will vary with the geology and mineralogy of a geothermal resource area along with the physical and chemical changes that occur during energy extraction. ${ }^{9}$ Table 7.5 (based on ref. 9) gives a breakdown of typical radionuclide concentrations found in NORM wastes from geothermal energy production.

\subsubsection{Metal mining and processing}

The mining and processing of metal ores, other than uranium and phosphate, generates large quantities of NORM wastes. These wastes include ore tailings and smelter slag, some of which contain elevated concentrations of uranium, thorium, radium, and their decay products that were originally part of the process feed ore. The extraction process for some ores can yield a waste product (e.g., tailings or slag) that has a higher radionuclide concentration than the original ore. Tailings are the solid materials remaining after physical or chemical benefication (washing, flotation, grinding, and drying) removes the valuable metal constituents from the ore. Slag is the vitreous residue mass left from the smelting (blast furnace melting and conversion) of metal ore for extraction and purification. ${ }^{9}$

The EPA study of ref. 9 describes NORM wastes from the mining and processing of three categories of metals: rare earth metals, special application metals, and metals produced in bulk quantities (i.e., large volumes) by industrial extraction processes. Rare earth (or lanthanide) metals comprise 16 chemical elements, including those with atomic numbers 57 (lanthanum) through 71 (lutetium) as well as yttrium (atomic number 39), which has similar chemical properties. Special application metals are regarded as metals that have unique commercial and industrial uses and include hafnium, tin, titanium, and zirconium. Metals mined and processed in bulk for industrial applications include aluminum, copper, iron, lead, zinc, and precious metals like gold and silver."

The level of NORM found in metal ores depends more on the geologic formation or region than on the particular mineral being mined. Table 7.6 (based on ref. 9) gives a breakdown of typical radionuclide concentrations found in NORM wastes from each of the three categories of mined metals. ${ }^{9}$

\subsubsection{Municipal water treatment}

A small portion of the public water supply systems in the United States treat water containing elevated NORM radionuclide concentrations-most significantly, uranium and radium. Radionuclides are leached into ground or surface water when the water comes in contact with uranium- and thorium-bearing geologic media. The predominant radionuclides found in water include those of uranium, radium, radon, and their decay products. Many 
water treatment technologies typically used for removing solids from water for softening and purification can significantly reduce the level of NORM radioactivity. NORM wastes from municipal water treatment consist of radioactive-contaminated sludges and solids that include filter sludges, spent ion-exchange resins, spent granular activated carbon, and water from filter backwash. Radiumselective ion-exchange resins generate wastes at much higher concentrations than those found in sludge, but in smaller quantities. In fact, some of these wastes fall into the discrete NARM waste category because their concentrations exceed $2 \mathrm{nCi} / \mathrm{g}^{9}$ Table 7.7 (based on ref. 9) gives estimated typical radionuclide concentrations of NORM wastes from municipal water treatment. These concentrations can vary significantly from location to location because of the varying geologic characteristics of different water sources in different regions of the country.

\subsubsection{Oil and natural gas production and processing}

Some oil and natural gas production and processing activities generate NORM wastes. Radium is a major contributor to the radioactivity found in these wastes, which are found in pipe scale and sludge from production and processing operations. Uranium and thorium compounds are mostly insoluble, and as oil and natural gas are brought to the surface, these compounds tend to remain embedded in underground geologic formations. However, some radium and radium daughter products are slightly soluble in water and can become mobilized when ground water (containing dissolved mineral salts) is brought to the surface from production and processing. When this occurs, some radium and its daughters may precipitate out of solution because of geologic chemical changes and reduced temperature and pressure. Radium concentrations from geologic formations can precipitate out in sludges and on the internal surfaces of oil and natural gas piping and production and processing equipment. The solid scale residue typically consists mainly of barium, calcium, and strontium sulfates, silicates, and carbonates along with smaller portions of radium compounds. Sludge deposits, consisting of barium and silica compounds, are generally in the form of oily, loose material. NORM radionuclide concentrations in scales that accumulate in process piping and surface equipment may vary from background soil levels (about $1 \mathrm{pCi} / \mathrm{g}$ ) to several hundred thousand picocuries per gram-with an average activity of about $1000-2000 \mathrm{pCi} / \mathrm{g}^{12}$ Concentrations in sludges range from background levels to several hundred picocuries per gram. Radium and its decay products are also found in elevated concentrations in ground water extracted to the surface from oil drilling. However, these concentrations are much less than those found for the scale or sludge wastes.

NORM wastes from natural gas plant deposits differ from oil production NORM wastes and typically consist of radon decay products plated out on the interior surfaces of pipes, valves, filters, and other gas production and processing equipment. ${ }^{9}$

Table 7.8 (based on ref. 9) gives estimated radionuclide concentrations of NORM wastes from both oil and natural gas production and processing. As with other NORM wastes, the radioactivity concentrations for the scale and sludge wastes are strongly influenced by the natural abundance of radionuclides, the geologic formation conditions where the oil and natural gas are produced, and the characteristics of the production process. ${ }^{9,13}$

\subsubsection{Phosphate mining and fertilizer production}

NORM wastes are generated from the mining and processing of phosphate rock (phosphorite) needed to produce phosphate fertilizers, detergents, animal feed, food products, pesticides, and other phosphorous chemicals. These wastes include ferrophosphorus, phosphogypsum (a hydrated calcium sulfate slurry), piping scale, and slag (calcium silicate). ${ }^{9}$

Phosphogypsum and scale are the principal waste byproducts generated during the production of phosphoric acid and fertilizers. Scale is deposited in small quantities in process piping and in filtration receiving tanks. Phosphate scale wastes are generally regarded as discrete NORM because ${ }^{226} \mathrm{Ra}$ concentrations in small volumes of these materials have been found be as high as $100,000 \mathrm{pCi} / \mathrm{g}^{9,14}$

Ferrophosphorus and phosphate slag are the principal waste by-products from the production of elemental phosphorous, which is produced by the reduction of phosphate rock in large electric furnaces that use carbon and silica as catalysts. Both ferrophosphorus and slag are found in the residual solids that remain from the furnace processing. ${ }^{9}$

Table 7.9 (based on ref. 9) gives estimated radionuclide concentrations in the principal NORM wastes from phosphate mining and fertilizer production. These concentrations include contributions from uranium, thorium, radium, and their radioactive decay products, which are found in mined phosphate ores and have become concentrated in the waste by-products. Actual radionuclide concentrations will vary in location because of varying geological characteristics of phosphate ores in different regions of the country as well as variations in the processes used for phosphate mining and production. ${ }^{9}$

\subsection{GENERATION, INVENTORIES AND PROJECTIONS}

Limited information is available on the generation, inventories, and projections of domestic NARM wastes. Table 7.10 reports the inventories of radium disposed of in recent years by New York State generators. ${ }^{6}$ Currently 
NARM wastes are shipped for disposal to either the Envirocare Facility near Clive, Utah, or the U.S. Ecology, Inc. site near Richland, Washington. As Table 0.8 in Chapter 0 shows, nearly $280,000 \mathrm{~m}^{3}$ of NARM wastes were disposed of at the Envirocare Facility by the end of CY 1995. Most of these wastes were generated from programs sponsored by DOD and EPA. Only a small portion ( $4.5 \%$ ) of the cumulative volume of NARM wastes disposed of at the Envirocare Facility resulted from commercial activities.

Like inventories, projections of NARM (and NORM) are limited and vary according to the region of the country. Table 7.11 (adapted from refs. 9 and 11) reports a summary of estimates of domestic diffuse NORM waste generation, material density, cumulative inventory, and radioactive $\left({ }^{226} \mathrm{Ra}\right)$ concentration. These estimates are reported in refs. 9 and 11 and pertain to wastes from commercial rather than DOE activities. EPA information and data reported from ref. 9 are, in turn, based on refs. 13-26. It should be cautioned that the large quantities reported in Table 7.11 for cumulative NORM inventories are preliminary estimates associated with large ranges of uncertainty. Consequently, caution should be used in trying to extrapolate any of the referenced information and data for purposes such as risk assessment or determining needed disposal facilities. The estimates given in Table 7.11 also do not reflect possible opportunities for the reuse and recycling of NORM materials in commercial and industrial applications. Such applications are further discussed in ref. 9.

The ubiquitous nature of NORM wastes accounts for the apparent enormous inventory of these materials. Even allowing for uncertainties, the estimated cumulative volume of these materials is much larger than the cumulative volume of DOE and commercial radioactive waste and SNF being managed through CY 1995 (see the tables of Chapter 0 and Chapter 6).

The total inventory that should be associated with NORM will depend on what regulatory concentration standards can be applied to these materials on a national basis. Future updates of this document will include additional and updated information and data on both accelerator-produced waste and NORM waste inventories, projections, and characteristics as they become available.

\subsection{REFERENCES}

1. U. S. Congress, Atomic Energy Act of 1954, Pub. L. 83-703, Aug. 15, 1954.

2. Robert E. Berlin and Catherine C. Stanton, Radioactive Waste Management, John Wiley and Sons, New York, 1989.

3. "NRC, States Grapple with BRC, NORM Waste Issues," Nuclear Waste News, Vol. 12, No. 10, p. 85, dated Mar. 5, 1992.

4. Conference of Radiation Control Program Directors, Inc., Guides for Naturally Occurring and Accelerator-Produced Radioactive Materials (NARM), HHS Publication FDA 81-8025, June 1981.

5. The League of Women Voters Education Fund, The Nuclear Waste Primer, Lyons \& Burford, New York, 1993.

6. New York State Low-Level Radioactive Waste Siting Commission, 1994 Source Term Report-Low-Level Radioactive Waste Projections for New York State, Vols. 1 and 2 (Support Document), draft report, second edition, Troy, New York (October 1994).

7. K. P. Smith, An Overview of Naturally Occurring Radioactive Materials (NORM) in the Petroleum Industry, ANL/EAIS-7, Argonne National Laboratory, Argonne, Illinois (December 1992).

8. K. P. Smith and D. L. Blunt, Argonne National Laboratory, Argonne, Illinois, "Scrap Metal Management Issues Associated with Naturally Occurring Radioactive Material," presented at Beneficial Reuse '95, Knoxville, Tennessee, July 31-August 3, 1995.

9. U.S. Environmental Protection Agency, Office of Radiation and Indoor Air, Diffuse NORM Wastes-Waste Characterization and Preliminary Risk Assessment, Draft, RAE-9232/1-2, SC\&A, Inc., and Rogers \& Associates Engineering Corporation, Salt Lake City, Utah (May 1993). 
10. Edward L. Gershey, et al., Low-Level Radioactive Waste-From Cradle to Grave, pp. 34-35, Van Nastrand Reinhold, New York, 1990.

11. U.S. Environmental Protection Agency, Science Advisory Board, Review of the Office of Radiation and Indoor Air Draft Document on Diffuse Naturally-Occurring Radioactive Material (NORM): Waste Characterization and Preliminary Risk Assessment, EPA-SAB-RAC-94-013, Washington, D.C. (May 1994).

12. Kevin J. Grice, Texaco Exploration and Production, Inc., Houston, Texas, correspondence to S. N. Storch, Oak Ridge National Laboratory, Oak Ridge, Tennessee, "Comments on Draft Chapter 7 Naturally Occurring and AcceleratorProduced Radioactive Material," dated Aug. 20, 1996.

13. A. McArthur, "Development and Operation of a NORM Processing and Disposal Facility for the U.S. Oil and Gas Industry," published in CRCPD Publication 88-2, 19th Annual National Conference on Radiation Control, May 18-21, 1987, Boise, Idaho, Conference of Radiation Control Program Directors, Frankfort, Kentucky, 1988.

14. Harlan W. Keaton, "Naturally Occurring Radioactive Materials in the Construction Industry and Current Building Codes," published in Conference of Radiation Control Program Directors, Publication 88-2, 19 th Annual National Conference on Radiation Control, May 18-21, 1987, Boise, Idaho, Conference of Radiation Control Program Directors, Frankfort, Kentucky, 1988.

15. United Nations Scientific Committee on the Effects of Atomic Radiation, Sources and Effects of Ionizing Radiation, 1982 report to the General Assembly, United Nations, New York, 1982.

16. ERC Environmental Energy Services Company, Final Environmental Impact Report for General Plan Amendment, Zone Charge, and Conditional Use Permit, prepared for Planning Department, Imperial County, California (1990).

17. U.S. Environmental Protection Agency, Report to Congress: Wastes from the Extraction and Beneficiation of Metallic Ores, Phosphate Rock, Asbestos, Overburden from Uranium Mining, and Oil Shale, EPA 530-SW-85-033, Office of Solid Waste and Emergency Response, Washington, D.C. (December 1985).

18. J. B. Hedrick and D. A. Templeton, "Rare-Earth Minerals and Metals," Rare-Earth Minerals and Metals Yearbook, U.S. Department of Interior, Washington, D.C., 1985.

19. J. B. Hedrick and D. A. Templeton, "Zirconium and Hafnium," Zirconium and Hafnium Minerals Yearbook, U.S. Department of Interior, Washington, D.C., 1989.

20. U.S. Environmental Protection Agency, Report to Congress on Special Wastes from Mineral Processing, EPA/530-SW-90-070C, Office of Solid Waste and Emergency Response, Washington, D.C. (July 1990).

21. American Water Works Association, 1984 Water Utility Operating Data, Denver, Colorado, 1986.

22. American Water Works Association, 1985 Water Utility Operating Data, Denver, Colorado, 1987.

23. U.S. Department of Energy, Energy Information Administration, Annual Energy Review-1991, DOE/EIA-0384(91), Washington, D.C. (June 1992).

24. U.S. Environmental Protection Agency, Office of Radiation Programs, Radioactivity Distribution in Phosphate Products, By-Products, Effluents, and Wastes, Technical Note ORP/CSD-75-3, Washington, D.C. (August 1975).

25. Conference of Radiation Control Program Directors, Natural Radioactivity Contamination Problems, Report No. 2 , Frankfort, Kentucky, August 1981.

26. American Petroleum Institute, A Naturally Occurring Radioactive Material (NORM) Disposal Cast Study, API Publication 7100, Washington, D.C. (November 1996). 
Table 7.1. Radionuclides found in accelerator-produced radioactive materials ${ }^{a}$

\begin{tabular}{|c|c|c|c|c|c|c|c|}
\hline Nuclide & $\begin{array}{l}\text { Atomic } \\
\text { number }\end{array}$ & Half-life $b$ & $\begin{array}{l}\text { Principal mode(s) } \\
\text { of decayc }\end{array}$ & Nuclide & $\begin{array}{l}\text { Atomic } \\
\text { number }\end{array}$ & Half-life ${ }^{b}$ & $\begin{array}{l}\text { Principal mode(s) } \\
\text { of decayc }\end{array}$ \\
\hline${ }^{11} \mathrm{C}$ & 6 & $20.4 \mathrm{~min}$ & $\beta^{+}$ & ${ }^{81} \mathrm{Rb}$ & 37 & $4.6 \mathrm{~h}$ & $\mathrm{EC}$ \\
\hline${ }^{13} \mathrm{~N}$ & 7 & $10.0 \mathrm{~min}$ & $\beta^{+}$ & ${ }^{82} \mathrm{Rb}$ & 37 & $6.4 \mathrm{~h}$ & $\beta^{+}, \gamma$ \\
\hline${ }^{150} \mathrm{O}$ & 8 & $2.0 \mathrm{~min}$ & $\beta^{+}$ & ${ }^{84} \mathrm{Rb}$ & 37 & $34.0 \mathrm{~d}$ & $\mathrm{EC}$ \\
\hline${ }^{18} \mathrm{~F}$ & 9 & $1.8 \mathrm{~h}$ & $\beta^{+}$ & ${ }^{82} \mathrm{Sr}$ & 38 & $25.0 \mathrm{~d}$ & EC \\
\hline${ }^{22} \mathrm{Na}$ & 11 & $2.6 \mathrm{y}$ & $\beta^{+}, \gamma$ & ${ }^{87 \mathrm{~m}} \mathrm{Sr}$ & 38 & $2.8 \mathrm{~h}$ & IT \\
\hline${ }^{28} \mathrm{Mg}$ & 12 & $21.1 \mathrm{~h}$ & $\beta, \gamma$ & ${ }^{87} Y$ & 39 & $80.3 h$ & $\mathrm{EC}$ \\
\hline${ }^{28} \mathrm{Al}$ & 13 & $2.2 \mathrm{~min}$ & $\beta, \gamma$ & ${ }^{97 \mathrm{~m}} \mathrm{Tc}$ & 43 & $90.0 \mathrm{~d}$ & IT \\
\hline${ }^{33} \mathrm{P}$ & 15 & $25.3 \mathrm{~d}$ & $\beta$ & ${ }^{111} \mathrm{In}$ & 49 & $2.83 \mathrm{~d}$ & $\mathrm{EC}, \gamma$ \\
\hline${ }^{37} \mathrm{Ar}$ & 17 & $34.8 \mathrm{~d}$ & $\mathrm{EC}$ & ${ }^{123} I$ & 53 & $13.1 \mathrm{~h}$ & $\mathrm{EC}, \gamma$ \\
\hline${ }^{43} \mathrm{~K}$ & 19 & $22.2 \mathrm{~h}$ & $\beta, \gamma$ & ${ }^{124} \mathrm{I}$ & 53 & $4.2 \mathrm{~d}$ & $\mathrm{EC}, \gamma$ \\
\hline${ }^{49} \mathrm{Sc}$ & 21 & $57.3 \mathrm{~min}$ & $\beta, \gamma$ & ${ }^{125} \mathrm{I}$ & 53 & $59.7 \mathrm{~d}$ & $E C, y$ \\
\hline${ }^{52} \mathrm{Mn}$ & 25 & $5.7 \mathrm{~d}$ & $\beta^{+}, \gamma$ & ${ }^{126} \mathrm{I}$ & 53 & $13.0 \mathrm{~d}$ & $\mathrm{EC}, \gamma$ \\
\hline${ }^{\mathrm{S} 2} \mathrm{Fe}$ & 26 & $8.3 \mathrm{~h}$ & $\beta^{+}, \gamma$ & ${ }^{127} \mathrm{Xe}$ & 54 & $36.4 \mathrm{~d}$ & $\mathrm{EC}, \gamma$ \\
\hline${ }^{57} \mathrm{Co}$ & 27 & $271 d$ & $\mathrm{EC}$ & ${ }^{131} \mathrm{Cs}$ & 55 & $9.7 \mathrm{~d}$ & $E C$ \\
\hline${ }^{58} \mathrm{Co}$ & 27 & $71 \mathrm{~d}$ & $\mathrm{EC}$ & ${ }^{145} \mathrm{Pm}$ & 61 & $18 \mathrm{y}$ & $\mathrm{EC}$ \\
\hline${ }^{62} \mathrm{Cu}$ & 29 & $9.8 \min$ & $\beta^{+}, \gamma$ & ${ }^{157} \mathrm{Dy}$ & 66 & $8.1 \mathrm{~h}$ & $\mathrm{EC}, \gamma$ \\
\hline${ }^{67} \mathrm{Cu}$ & 29 & $61.7 \mathrm{~h}$ & $\beta, \gamma$ & ${ }^{190} \mathrm{Os}$ & 76 & $9.9 \mathrm{~min}$ & IT \\
\hline${ }^{62} \mathrm{Zn}$ & 30 & $9.2 \mathrm{~h}$ & $\mathrm{EC}, \gamma$ & ${ }^{190} \mathrm{Ir}$ & 77 & $12.2 \mathrm{~d}$ & $\mathrm{EC}, \gamma$ \\
\hline${ }^{66} \mathrm{Gra}$ & 31 & $9.5 \mathrm{~h}$ & $\beta^{+}, \gamma$ & ${ }^{190 \mathrm{~m}} \mathrm{Ir}$ & 77 & $1.2 \mathrm{~h}$ & IT \\
\hline${ }^{68} \mathrm{Gre}$ & 32 & $287 \mathrm{~d}$ & $\mathrm{EC}, \gamma$ & $193 \mathrm{mPt}_{\mathrm{Pt}}$ & 78 & $4.3 \mathrm{~d}$ & IT \\
\hline${ }^{73} \mathrm{As}$ & 33 & $80.3 d$ & $\mathrm{EC}$ & ${ }^{195} \mathrm{Au}$ & 79 & $184.0 \mathrm{~d}$ & $E C, \gamma$ \\
\hline${ }^{73} \mathrm{Se}$ & 34 & $7.1 \mathrm{~h}$ & $\beta^{+}$ & ${ }^{197} \mathrm{Hg}$ & 80 & $64.1 \mathrm{~h}$ & $\mathrm{EC}, \gamma$ \\
\hline${ }^{77} \mathrm{Br}$ & 35 & $56.0 \mathrm{~h}$ & $\beta^{+}, \gamma$ & ${ }^{199} \mathrm{Tl}$ & 81 & $7.4 \mathrm{~h}$ & $\mathrm{EC}, \gamma$ \\
\hline${ }^{77} \mathrm{Kr}$ & 36 & $1.2 \mathrm{~h}$ & $\beta^{+}$ & ${ }^{203} \mathrm{~Pb}$ & 82 & $52.1 \mathrm{~h}$ & $\mathrm{EC}, \gamma$ \\
\hline${ }^{81} \mathrm{Kr}$ & 36 & $2.1 \times 10^{5} y$ & $\mathrm{EC}$ & ${ }^{204} \mathrm{Bi}$ & 83 & $11.3 \mathrm{~h}$ & $E C, \gamma$ \\
\hline
\end{tabular}

a Based on ref. 4.

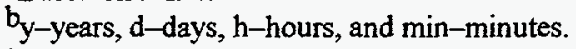

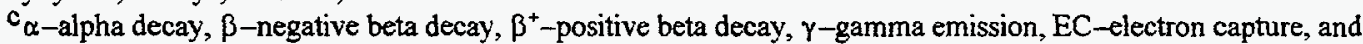
IT-isomeric transition. 
Table 7.2. Domestic processes that generate NORM waste ${ }^{a}$

\begin{tabular}{|c|c|c|c|}
\hline Process & Waste generated & $\begin{array}{l}\text { Classification by } \\
\text { specific activity }\end{array}$ & Major generator locations(s) \\
\hline Coal combustion & $\begin{array}{l}\text { Fly ash } \\
\text { Bottom ash and slag }\end{array}$ & $\begin{array}{l}\text { Diffuse } \\
\text { Diffuse }\end{array}$ & $\begin{array}{l}\text { Midwestern and South } \\
\text { Atlantic states }\end{array}$ \\
\hline Geothermal energy production & Solid wastes & Diffuse & California \\
\hline Manufacturing & $\begin{array}{l}\text { Old/used products: industrial } \\
\text { gauges, radium watches and } \\
\text { industrial dials, and radium } \\
\text { needles in medical equipment }\end{array}$ & Discrete & Various commercial sites \\
\hline \multirow[t]{4}{*}{ Metal mining and processing } & $\begin{array}{l}\text { Slag, leachate, and tailings from } \\
\text { the following: }\end{array}$ & & \\
\hline & Rare earth metals & Diffuse & $\begin{array}{l}\text { California, Florida, and } \\
\text { North Carolina }\end{array}$ \\
\hline & $\begin{array}{l}\text { Special-application metals } \\
\text { (zirconium, hafnium, titanium, } \\
\text { and tin) }\end{array}$ & Diffuse & Ohio, Delaware, and Florida \\
\hline & $\begin{array}{l}\text { Large-volume metal-processing } \\
\text { industries (copper, iron, etc.) }\end{array}$ & Diffuse & $\begin{array}{l}\text { Ohio, Pennsylvania, Indiana, } \\
\text { Mlinois, Michigan, and some } \\
\text { western states }\end{array}$ \\
\hline Municipal water treatment & $\begin{array}{l}\text { Sludge } \\
\text { Radium selective resins }\end{array}$ & $\begin{array}{l}\text { Diffuse } \\
\text { Discrete }\end{array}$ & $\begin{array}{l}\text { North central (e.g., Ilinois) and } \\
\text { coastal plain (e.g., North } \\
\text { Carolina) states and other states }\end{array}$ \\
\hline $\begin{array}{l}\text { Oil and natural gas production } \\
\text { and processing }\end{array}$ & $\begin{array}{l}\text { Scale } \\
\text { Sludge } \\
\text { Contaminated water and } \\
\text { production/processing equipment }\end{array}$ & $\begin{array}{l}\text { Discrete or diffuse } \\
\text { Diffuse } \\
\text { Diffuse }\end{array}$ & $\begin{array}{l}\text { In all petroleum and natural gas } \\
\text { states having production and } \\
\text { processing facilities }\end{array}$ \\
\hline $\begin{array}{l}\text { Phosphate mining and fertilizer } \\
\text { production }\end{array}$ & $\begin{array}{l}\text { Ferrophosphorous } \\
\text { Phosphogypsum }^{\mathrm{d}} \\
\text { Scale } \\
\text { Slag }\end{array}$ & $\begin{array}{l}\text { Diffuse } \\
\text { Diffuse } \\
\text { Discrete } \\
\text { Diffuse }\end{array}$ & $\begin{array}{l}\text { Florida, Idaho, and other states } \\
\text { in the West and Southeast }\end{array}$ \\
\hline
\end{tabular}

abased on ref. 6 (p. 13-9), and refs. 7-8.

bIn fact, many of the wastes generated can be either diffuse or discrete. What is listed in this column is the classification most typically associated with a particular waste generated.

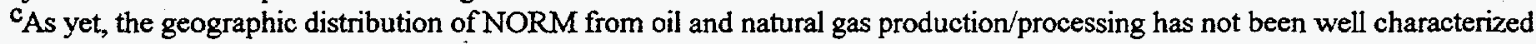
statistically. See refs. 7 and 8 .

dCalcium sulfate. 
Table 7.3. Major radionuclides found in diffuse NORM wastes ${ }^{\mathrm{a}}$

\begin{tabular}{|c|c|c|c|c|c|}
\hline Nuclide & $\begin{array}{l}\text { Atomic } \\
\text { number }\end{array}$ & Half-life ${ }^{b}$ & $\begin{array}{l}\text { Principal } \\
\text { mode(s) of } \\
\text { decayc }\end{array}$ & $\begin{array}{l}\text { Natural } \\
\text { decay } \\
\text { series }\end{array}$ & $\begin{array}{l}\text { Principal } \\
\text { source(s) }\end{array}$ \\
\hline${ }^{238} \mathrm{U}$ & 92 & $4.468 \mathrm{E}+09 \mathrm{y}$ & $\alpha$ & $\mathrm{U}$ & $1,2,4,5,7$ \\
\hline${ }^{235} \mathrm{U}$ & 92 & $7.037 E+08 y$ & $\alpha$ & A & $1,5,7$ \\
\hline${ }^{234} \mathrm{U}$ & 92 & $2.454 \mathrm{E}+05 \mathrm{y}$ & $\alpha$ & $\mathrm{U}$ & $1,2,4,5,7$ \\
\hline${ }^{231} \mathrm{~Pa}$ & 91 & $3.276 \mathrm{E}+04 \mathrm{y}$ & $\alpha$ & A & 1,5 \\
\hline${ }^{232} \mathrm{Th}$ & 90 & $1.405 \mathrm{E}+10 \mathrm{y}$ & $\alpha$ & $\mathrm{T}$ & $1,5,7$ \\
\hline${ }^{230} \mathrm{Th}$ & 90 & $7.54 \mathrm{E}+04 \mathrm{y}$ & $\alpha$ & $\mathrm{U}$ & $1,2,4,5,7$ \\
\hline${ }^{228} \mathrm{Th}$ & 90 & $1.913 \mathrm{y}$ & $\alpha$ & $\mathrm{T}$ & $1,5,6,7$ \\
\hline${ }^{227} \mathrm{AC}$ & 89 & $2.177 E+01 y$ & $\alpha, \beta$ & A & 1,5 \\
\hline${ }^{228} \mathrm{Ra}$ & 88 & $5.75 \mathrm{y}$ & $\beta$ & $\mathrm{T}$ & $1,5,6$ \\
\hline${ }^{226} \mathrm{Ra}$ & 88 & $1.600 E+03 y$ & $\alpha$ & $\mathrm{U}$ & $1,2,3,4,5,6,7$ \\
\hline${ }^{224} \mathrm{Ra}$ & 88 & $3.66 \mathrm{~d}$ & $\alpha$ & $\mathrm{T}$ & $1,5,6,7$ \\
\hline${ }^{222} \mathrm{Rn}$ & 86 & $3.825 \mathrm{~d}$ & $\alpha$ & $\mathrm{U}$ & $1,2,3,4,5,6,7$ \\
\hline${ }^{210} \mathrm{Po}$ & 84 & $1.383 E+02 d$ & $\alpha$ & $\mathrm{U}$ & $1,2,4,5,6,7$ \\
\hline${ }^{210} \mathrm{~Pb}$ & 82 & $1.94 \mathrm{E}+01 \mathrm{y}$ & $\beta$ & $U$ & $1,2,4,5,6,7$ \\
\hline${ }^{40} \mathrm{~K}$ & 19 & $1.277 E+09 y$ & $\beta$ & - & $1,2,4,6,7$ \\
\hline
\end{tabular}

a Based on refs. 9 and 11.

by-years and d-days.

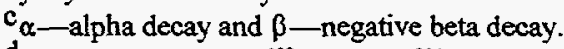

$\mathrm{d}_{\mathrm{U}}$-uranium series ${ }^{238} \mathrm{U}$ parent, ${ }^{206} \mathrm{~Pb}$ stable end daughter); $\mathrm{A}$-actinium series $\left({ }^{235} \mathrm{U}\right.$ parent, ${ }^{207} \mathrm{~Pb}$ stable end daughter); and $\mathrm{T}$-thorium series ( ${ }^{232} \mathrm{Th}$ parent, ${ }^{208} \mathrm{~Pb}$ stable end daughter).

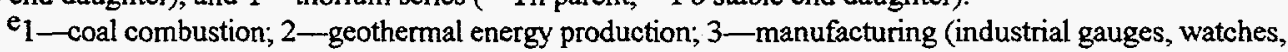
etc., and medical needles); 4-metal mining and processing; 5-municipal water treatment; 6-oil and gas production; and 7-phosphate mining and fertilizer production. 
Table 7.4. Radionuclide concentrations in coal combustion wastes ${ }^{a}$

\begin{tabular}{ccc}
\hline & \multicolumn{2}{c}{$\begin{array}{c}\text { Concentration } \\
\text { (pCi/g) }\end{array}$} \\
\cline { 2 - 3 } Nuclide & Fly ash & $\begin{array}{c}\text { Bottom ash } \\
\text { and slag }\end{array}$ \\
\hline${ }^{238} \mathrm{U}$ & 2.6 & 0.7 \\
${ }^{235} \mathrm{U}$ & 0.13 & 0.03 \\
${ }^{234} \mathrm{U}$ & 2.6 & 0.7 \\
${ }^{231} \mathrm{~Pa}$ & 0.13 & 0.03 \\
${ }^{232} \mathrm{Th}$ & 1.7 & 0.4 \\
${ }^{230} \mathrm{Th}$ & 1.8 & 0.5 \\
${ }^{228} \mathrm{Th}$ & 2.6 & 0.6 \\
${ }^{227} \mathrm{Ac}$ & 0.13 & 0.03 \\
${ }^{228} \mathrm{Ra}$ & 1.4 & 0.4 \\
${ }^{226} \mathrm{Ra}$ & 3.0 & 0.7 \\
${ }^{210} \mathrm{Po}$ & 5.6 & 1.4 \\
${ }^{210} \mathrm{~Pb}$ & 5.4 & 1.4 \\
$\mathrm{Total}$ & 27.1 & 6.9 \\
\hline
\end{tabular}

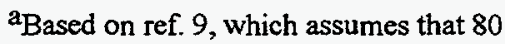
wt $\%$ of the concentration is fly ash.

bxcludes contributions from other (short-lived) radionuclides in decay chains. 
Table 7.5. Radionuclide concentrations in geothermal energy production waste ${ }^{a}$

\begin{tabular}{lc}
\hline Nuclide & $\begin{array}{c}\text { Waste concentration } \\
\text { (pCi/g) }\end{array}$ \\
\hline${ }^{228} \mathrm{Th}$ & 25 \\
${ }^{228} \mathrm{Ra}$ & 93 \\
${ }^{226} \mathrm{Ra}$ & 132 \\
${ }^{210} \mathrm{Po}$ & 96 \\
${ }^{210} \mathrm{~Pb}$ & 96 \\
Total $^{\mathrm{b}}$ & 442 \\
\hline
\end{tabular}

aBased on ref. 9.

Excludes contributions from other (shortlived) radionuclides in decay chains.

Table 7.6. Radionuclide concentrations in metal mining and processing wastes ${ }^{2}$

\begin{tabular}{lccc}
\hline & \multicolumn{3}{c}{ Waste concentration, $\mathrm{pCi} / \mathrm{g}$} \\
\cline { 2 - 4 } Nuclide & $\begin{array}{c}\text { Rare earth } \\
\text { metals }\end{array}$ & $\begin{array}{c}\text { Special- } \\
\text { application } \\
\text { metals }\end{array}$ & $\begin{array}{c}\text { Large-volume } \\
\text { industry } \\
\text { metals }\end{array}$ \\
\hline${ }^{238} \mathrm{U}$ & 900 & 43 & 10.0 \\
${ }^{235} \mathrm{U}$ & 45 & 2.2 & 0.5 \\
${ }^{234} \mathrm{U}$ & 900 & 43 & 10.0 \\
${ }^{231} \mathrm{~Pa}$ & 45 & 2.2 & 0.5 \\
${ }^{232} \mathrm{Th}$ & 2,000 & 22 & 10.0 \\
${ }^{230} \mathrm{Th}$ & 900 & 43 & 10.0 \\
${ }^{228} \mathrm{Th}$ & 2,000 & 22 & 10.0 \\
${ }^{227} \mathrm{Ac}$ & 45 & 2.2 & 0.5 \\
${ }^{228} \mathrm{Ra}$ & & 22 & 10.0 \\
${ }^{226} \mathrm{Ra}$ & 2,000 & 43 & 5.0 \\
${ }^{210} \mathrm{Po}$ & 900 & 30 & 3.5 \\
${ }^{210} \mathrm{~Pb}$ & 630 & 30 & 3.5 \\
Total ${ }^{\mathrm{b}}$ & 10,995 & 305 & 73.5 \\
\hline
\end{tabular}

aBased on ref. 9 .

${ }^{b}$ Excludes contributions from other (short-lived) radionuclides in decay chains. 
Table 7.7. Radionuclide concentrations in municipal water treatment wastes ${ }^{\mathrm{a}}$

\begin{tabular}{llc}
\hline & \multicolumn{2}{c}{$\begin{array}{c}\text { Concentration } \\
\text { (pCi/g) }\end{array}$} \\
\cline { 2 - 3 } Nuclide & Sludges & Radium selective resins \\
\hline${ }^{238} \mathrm{U}$ & 4.0 & $\mathrm{~b}$ \\
${ }^{23} \mathrm{U}$ & 0.03 & $\mathrm{~b}$ \\
${ }^{234} \mathrm{U}$ & 4.0 & $\mathrm{~b}$ \\
${ }^{231} \mathrm{~Pa}$ & 0.03 & $\mathrm{~b}$ \\
${ }^{232} \mathrm{Th}$ & 0.2 & $\mathrm{~b}$ \\
${ }^{230} \mathrm{Th}$ & 0.2 & $\mathrm{~b}$ \\
${ }^{228} \mathrm{Th}$ & $9.0^{\mathrm{c}}$ & $\mathrm{b}$ \\
${ }^{227} \mathrm{Ac}$ & 0.03 & $\mathrm{~b}$ \\
${ }^{228} \mathrm{Ra}$ & $16^{\mathrm{c}}$ & $\mathrm{b}$ \\
${ }^{226} \mathrm{Ra}$ & 16 & $35,000^{\mathrm{d}}$ \\
${ }^{210} \mathrm{Po}$ & 11.0 & $\mathrm{~b}$ \\
${ }^{210} \mathrm{~Pb}$ & 11.0 & $\mathrm{~b}$ \\
& 71.5 & - \\
Total & 71.5 & $\mathrm{~b}$ \\
\hline
\end{tabular}

aBased on ref. 9 .

bunknown.

$c_{\text {Concentration after } 2}$ years of decay and ingrowth.

$\mathrm{d}_{\text {Rough estimate. }}$

'Excludes contributions from other (short-lived) radionuclides in decay chains.

Table 7.8. Radionuclide concentrations in oil and natural gas production and processing wastes $^{a}$

\begin{tabular}{lcc}
\hline & \multicolumn{2}{c}{$\begin{array}{c}\text { Concentration } \\
\text { (pCi/g) }\end{array}$} \\
\cline { 2 - 3 } Nuclide & Scale & Sludge \\
\hline${ }^{228} \mathrm{Th}$ & 120 & 19 \\
${ }^{228} \mathrm{Ra}$ & 120 & 19 \\
${ }^{226} \mathrm{Ra}$ & 360 & 56 \\
${ }^{210} \mathrm{Po}$ & 360 & 56 \\
${ }^{210} \mathrm{~Pb}$ & 360 & 56 \\
Total $^{\mathrm{b}}$ & 1,320 & 206 \\
\hline
\end{tabular}

aBased on ref. 9.

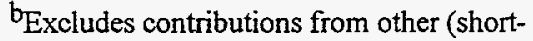
lived) radionuclides in decay chains. 
Table 7.9. Radionuclide concentrations in phosphate wastes ${ }^{2}$

\begin{tabular}{|c|c|c|c|c|}
\hline \multirow{2}{*}{ Nuclide } & \multicolumn{4}{|c|}{$\begin{array}{l}\text { Concentration } \\
(\mathrm{pCi} / \mathrm{g})\end{array}$} \\
\hline & Ferrophosphorous & Phosphogypsum & Scale & Slag \\
\hline${ }^{238} \mathrm{U}$ & b & 6.0 & b & 25 \\
\hline${ }^{235} \mathrm{U}$ & b & 0.3 & b & 1.3 \\
\hline${ }^{234} \mathrm{U}$ & b & 6.2 & b & 24 \\
\hline${ }^{231} \mathrm{~Pa}$ & b & 0.3 & $b$ & 1.3 \\
\hline${ }^{232} \mathrm{Th}$ & $\mathrm{b}$ & 0.27 & b & 0.77 \\
\hline${ }^{230} \mathrm{Th}$ & $\mathrm{b}$ & 13 & $b$ & 32 \\
\hline${ }^{228} \mathrm{Th}$ & b & 1.4 & $b$ & 0.77 \\
\hline${ }^{227} \mathrm{AC}$ & $b$ & 0.3 & $b$ & 1.3 \\
\hline${ }^{228} \mathrm{Ra}$ & $b$ & 0.27 & $\mathrm{~b}$ & 0.77 \\
\hline${ }^{226} \mathrm{Ra}$ & $1.2^{\mathrm{C}}$ & 33 & $1000^{d}$ & 35 \\
\hline${ }^{210} \mathrm{Po}$ & $b$ & 26 & $b$ & 35 \\
\hline${ }^{210} \mathrm{~Pb}$ & $\mathrm{~b}$ & 26 & $\mathrm{~b}$ & 35 \\
\hline Total $^{\mathrm{e}}$ & $b$ & 113 & $\mathrm{~b}$ & 192 \\
\hline
\end{tabular}

a Based on ref. 9 .

Unknown.

$c_{\text {Average for Florida plants. }}$

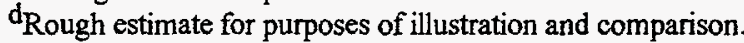

Excludes contributions from other (short-lived) radionuclides in decay chains.

Table 7.10. Quantities of radium disposed of by New York State generators during 1987-91a

\begin{tabular}{lcc}
\hline CY & $\begin{array}{c}\text { Volume } \\
\left(\mathrm{m}^{3}\right)\end{array}$ & $\begin{array}{c}\text { Radioactivity } \\
(\mathrm{Ci})\end{array}$ \\
\hline 1987 & 16.1 & 1.32 \\
1988 & 4.0 & 4.23 \\
1989 & 26.7 & 123 \\
1990 & 6.4 & 0.08 \\
1991 & 6.7 & 1.28 \\
& & \\
Totals & 59.9 & 129.91 \\
\hline
\end{tabular}

abased on ref. 6 (p. 13-9). 
Table 7.11. Estimates of domestic diffuse NORM waste generation, material density, cumulative inventory, and radioactive concentration ${ }^{\mathrm{a}}$

\begin{tabular}{|c|c|c|c|c|c|c|}
\hline \multirow[b]{2}{*}{ Process/waste material } & \multirow{2}{*}{$\begin{array}{l}\text { Current annual } \\
\text { generation } \\
\left(10^{6} \text { t/year }\right)\end{array}$} & \multirow{2}{*}{$\begin{array}{l}\text { Waste } \\
\text { material } \\
\text { density } \\
\left(t / \mathrm{m}^{3}\right)\end{array}$} & \multicolumn{3}{|c|}{ Cumulative inventory } & \multirow{2}{*}{$\begin{array}{l}\text { Average }{ }^{226} \mathrm{Ra} \\
\text { concentration } \\
(\mathrm{pCi} / \mathrm{g})\end{array}$} \\
\hline & & & $\begin{array}{l}\text { Mass } \\
\left(10^{6} t\right)\end{array}$ & $\begin{array}{l}\text { Volume } \\
\left(10^{6} \mathrm{~m}^{3}\right)\end{array}$ & $\begin{array}{l}\text { Radioactivity } \mathbf{c} \\
\text { (Ci) }\end{array}$ & \\
\hline \multicolumn{7}{|l|}{ Coal combustion } \\
\hline Bottom ash and slag & 17 & $(1.50)^{\mathrm{d}}$ & $>337^{\mathrm{e}}$ & $>225$ & 2,300 & $(3.1)$ \\
\hline Fly ash & 44 & 1.20 & $>959^{\mathrm{e}}$ & $>800$ & 26,000 & (3.9) \\
\hline $\begin{array}{l}\text { Geothermal energy production } \\
\text { (scale and filter cake) }\end{array}$ & 0.054 & 1.80 & 0.74 & 0.41 & 330 & 132 \\
\hline \multicolumn{7}{|l|}{$\begin{array}{l}\text { Metal mining and processing-slag, } \\
\text { leachate, and tailings from the } \\
\text { following: }\end{array}$} \\
\hline $\begin{array}{l}\text { Large bulk metal industries } \\
\text { (e.g., aluminum, copper, iron, } \\
\text { and steel) }\end{array}$ & 1,000 & 2.00 & $50,000^{f}$ & 25,000 & $3,700,000$ & (5) \\
\hline - Rare earths & 0.021 & 2.00 & $1^{f}$ & 0.5 & 11,000 & 900 \\
\hline $\begin{array}{l}\text { Special-application metals } \\
\text { (zirconium, hafnium, titanium, } \\
\text { and tin) }\end{array}$ & 0.47 & 2.00 & $20^{\mathrm{f}}$ & 10 & 6,100 & 43 \\
\hline \multicolumn{7}{|l|}{ Municipal water treatment } \\
\hline Radium selective resins & 0.04 & $(1.50)$ & $2 g$ & $>1$ & $>35,000$ & $(35,000)$ \\
\hline Sludges & 0.26 & 1.60 & $10 \mathrm{~g}$ & 6 & 700 & 16 \\
\hline $\begin{array}{l}\text { Oil and gas production waste } \\
\text { (scale and sludge })^{h}\end{array}$ & 0.056 & 1.70 & 4 & 2.3 & 1,210 & 84 \\
\hline \multicolumn{7}{|l|}{ Phosphate production } \\
\hline Ferrophosphorous & $\mathrm{i}$ & $(1.50)$ & $\mathrm{i}$ & $\mathrm{i}$ & i & $1.2 \mathrm{~J}$ \\
\hline Phosphogypsum & 48 & 1.44 & 8,200 & 5,700 & 930,000 & 33 \\
\hline Scale & 0.003 & $(2.00)$ & $\ll 1$ & $<<1$ & $<1,000$ & $(1,000)$ \\
\hline Slag & 1.6 & 1.90 & $324^{\mathrm{k}}$ & 171 & 62,200 & 35 \\
\hline Total & 1,112 & 1.88 & $>59,857$ & $>31,917$ & $>4,775,840$ & 9.6 \\
\hline
\end{tabular}


a Based on refs. 9 and 11. Information and data from ref. 9 are, in turn, based on refs. 13-26.

bstimated from listed waste material density.

Estimated from total radionuclide concentrations listed in Tables 7.4-7.9. Exciudes contributions from ${ }^{40} \mathrm{~K}$.

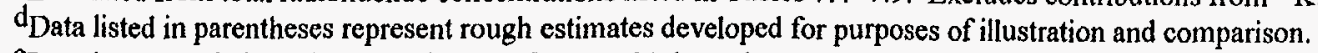

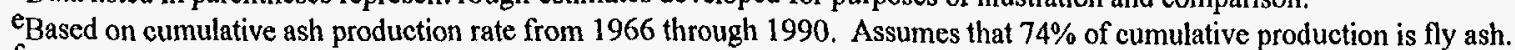

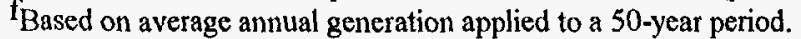

gBased on the average annual generation applied to a 40 -year period.

$h_{\text {Based on ref. } 26 .}$

Unknown.

$\mathrm{j}_{\text {Average for Florida plants. }}$

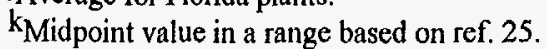




\section{MIXED LOW-LEVEL WASTE}

\subsection{INTRODUCTION}

This chapter reports estimated inventories and generation rates of mixed low-level waste (MLLW) from DOE site and commercial operations. Mixed wastes are radioactive wastes that are also considered hazardous. MLLW are mixed wastes which, radiologically, are low level as defined in Chapter 4.

Other types of radioactive wastes may also be mixed. All high-level wastes ( $H L W s)$ are regarded as mixed and are discussed in Chapter 2. Mixed transuranic wastes are not included in this chapter, but are addressed in the TRUW inventories and projections reported in Chapter 3.

The radioactive components of mixed wastes are subject to the Atomic Energy Act (AEA), as amended, ${ }^{1}$ which, for government sources, is administered by DOE, and, for commercial sources, by NRC (unless a state has obtained agreement-state status). As defined for this report, the hazardous components of mixed wastes are subject to either of two federal statutes that are administered by the U.S. Environmental Protection Agency (EPA) (unless a state has obtained authorization status). These statutes are the Resource Conservation and Recovery Act (RCRA), as amended, ${ }^{2}$ and the Toxic Substances Control Act (TSCA). ${ }^{3}$ Table 8.1 (based on ref. 4) lists those states and territories designated by EPA as having mixed waste authorization. The management of RCRA- and TSCA-regulated mixed wastes are subject to the regulations of EPA ${ }^{5,6}$ and NRC (or the authorized and agreement states), or DOE.

\subsection{SCOPE}

This chapter summarizes the quantities (inventory and generation) and associated characteristics of MLLW from both DOE site and commercial site operations. The DOE MLLW include MLLW for which the hazardous component of the waste is subject to regulation under either RCRA or TSCA (hereafter referred to as either RCRA MLLW or TSCA MLLW, respectively). MLLW which is subject to regulation under both RCRA and TSCA are considered RCRA MLLW.
Relative to the previous issue of this report (DOE/RW-0006, Rev. 11), a key scope change is the exclusion of the Middlesex, Weldon Spring, and Grand Junction sites from this chapter. Radiologically, the mixed waste at these sites is considered 11 e(2) by-product material. Mixed $11 \mathrm{e}(2)$ wastes are addressed exclusively in Chapter 6 (Environmental Restoration Program) of this report. This scope change accounts for most of the difference in the total nationwide inventory of DOE RCRA MLLW from the previous issue of this report (see Sect. 8.4.1).

A significant volume of MLLW is associated with environmental restoration activities. For example, most of the radioactive asbestos results from decommissioning and/or renovation of old structures. In addition, remediation of former landfills used for disposal of radioactively contaminated materials can result in large quantities of RCRA MLLW. MLLW resulting from such activities are provided in Chapter 6 within pertinent tables. The environmental restoration program is a very dynamic program in which wastes are currently being generated by ongoing remedial action activities and certain stored wastes are being treated either on-site or off-site prior to disposal. As such, waste volumes can change significantly in very short periods of time. There may be some discrepancies with MLLW volumes reported in this chapter with those reported in Chapter 6 . These variances occur as a result of differences in (a) the sources of information (e.g., the information in Chapter 6 is limited to environmental restoration activities) and (b) when the information was last updated.

For commercial MLLW, this chapter reports a summary of the cumulative stored inventories and generation documented for a baseline CY (1990) in a national profile study (NUREG/CR-5938, ref. 7) made for the NRC and EPA. The wastes in that study are grouped by facility categories and by major hazardous waste classifications.

Unless otherwise noted, the inventories and projections reported for MLLW in this chapter are separate from those reported for strictly radioactive LLW in Chapter 4. Inventories of MLLW currently stored at DOE 
sites are being thoroughly characterized. As a result, the waste at some sites could require future reclassification, thereby causing significant changes in current inventory data that are currently reported.

\subsection{DOE MLLW DATA SOURCES}

The information and data on DOE RCRA MLLW contained in the previous issue of this report (DOE/RW0006, Rev. 11) was based on the 1995 update to the DOE Mixed. Waste Inventory Report (MWIR) $)^{8}$ data base. In this issue, DOE RCRA MLLW information and data are based on the DOE Site Treatment Plan (STP) Data Base, as updated through December 1996. These data bases differ with respect to certain details of the information and data that are maintained on DOE RCRA MLLW streams. The MWIR data base contains greater detail on the characteristics of the waste streams, whereas the STP data base contains more detail on their planned management. While there was no update to the MWIR data in 1996, the DOE sites have continued to provide updates to the STP data base as part of ongoing efforts to finalize their STPS as required under the Federal Facility Compliance Act (FFCA). ${ }^{10}$

DOE TSCA MLLW information and data reported in this chapter are based on DOE site submittals recently provided to the IDB Program and cited in ref. 11.

\subsection{DOE MLLW}

Figure 8.1 summarizes the estimated MLLW (RCRA and TSCA) volume inventory for major sites in the DOE complex. A corresponding illustration of the estimated annual volume generation is shown in Fig. 8.2. For comparison, Table 8.2 summarizes estimated nationwide volume inventory and annual volume generation of $\mathrm{DOE}$ MLLW (RCRA and TSCA) and commercial MLLW.

\subsubsection{DOE RCRA MLLW}

There is approximately $108,000 \mathrm{~m}^{3}$ of RCRA MLLW in storage throughout the DOE complex with an estimated $28,600 \mathrm{~m}^{3}$ anticipated to be generated over the next 5 years. Table 8.3 summarizes the distribution of the inventory and projected generation across the DOE sites. Most (>90\%) of the inventory is stored at 7 sites (Hanford, INEL, K-25, PORTS, RFETS, SRS, and Y-12).

Table 8.5 summarizes the distribution of RCRA $\mathrm{MLLW}$ inventories and projected generation for the DOE sites according to physical form categories. Definitions of the physical form categories are provided in Table 8.4.
The complex-wide aggregate of the site distributions is provided in Table 8.6. Complex-wide, most ( $~ 90 \%)$ of the inventory consists of inorganic homogeneous solids, debris (inorganic, organic, heterogeneous), and soil/gravel.

The previous edition of this document (DOE/RW-0006, Rev. 11) reported that approximately $138,000 \mathrm{~m}^{3} \mathrm{RCRA} M L L W$ was stored throughout the DOE complex. As discussed above, approximately $108,000 \mathrm{~m}^{3}$ are reported in this edition. The majority of the $30,000 \mathrm{~m}^{3}$ decrease in this edition is due to the exclusion of mixed $11 \mathrm{e}(2)$ waste, particularly that stored at Middlesex (see Sect. 8.2). Table 8.7 summarizes the major contributions to the change in the DOE complexwide inventory.

\subsubsection{DOE TSCA MLLW}

This section highlights the major ground rules and assumptions associated with the DOE site information and data reported in Tables 8.8-8.14. Most of the information and data reported in the subsections that follow describes the TSCA MLLW inventory, generation, and projection ground rules and assumptions documented in the specific DOE site submittals of ref. 11. Corresponding site information associated with RCRA MLLW data are reported in the MWIR (ref. 8) and STP Data Base (ref. 9)

The site inventories and generation rates of $\mathrm{DOE}$ TSCA MLLW are given in this chapter for two major categories of hazardous materials: polychlorinated biphenyls (PCBs) and asbestos. A third category, "Other," has been added to include TSCA MLLWs that contain either mixtures of PCBs and asbestos, other TSCAregulated materials, or both.

Tables 8.8 and 8.9, respectively, report the mass and volume inventory of TSCA MLLW at each DOE site. A volume inventory of about $23,500 \mathrm{~m}^{3}$ is reported for TSCA MLLW throughout the DOE complex. Current annual generation levels for these wastes are reported in mass units in Table 8.10 and in volume units in Table 8.11. Projected TSCA MLLW mass and volume generation levels are given in Tables 8.12 and 8.13, respectively. Significant changes in site reported TSCA MLLW volume inventories from those reported in the previous edition of this report (Rev. 11) are noted in Table 8.14.

The following subsections give some of the significant features of the TSCA MLLW data at each DOE site that are reported in Tables 8.8-8.13.

\subsubsection{Ames Laboratory (Ames)}

Materials containing radioactively-contaminated $\mathrm{PCBs}$ and asbestos comprise the TSCA MLLW inventories and projections for Ames. 


\subsubsection{Argonne National Laboratory-East (ANL-E)}

ANL-E TSCA MLLW inventories are comprised of PCBs. ANL-E projections for TSCA MLLW are not available.

\subsubsection{Argonne National Laboratory-West (ANL-W)}

TSCA MLLW inventories for ANL-W include both PCBs and asbestos. During 1995, TSCA MLLW generation included only asbestos.

\subsubsection{Brookhaven National Laboratory (BNL)}

Both PCBs and solid asbestos materials comprise BNL. TSCA MLLW inventories and generation. Projected wastes reported in Tables 8.12 and 8.13 assume that small quantities will continue to be generated as a result of maintenance activities.

\subsubsection{Energy Technology Engineering Center (ETEC)}

The ETEC site reported no TSCA MLLW inventory for the end of CY 1995, but some asbestos was generated during 1995, and was shipped off site. TSCA MLLW projections anticipated over the next few years include only contributions from asbestos.

\subsubsection{Fernald Environmental Management Project (FEMP)}

Information for this site is not available.

\subsubsection{Fermi National Accelerator Laboratory (FNAL)}

Currently, FNAL has no inventories of TSCA MLLW.

\subsubsection{Hanford Site (Hanford)}

Hanford Site TSCA MLLWs consist of inventories of PCBs. Site projections for PCBs are included in the projections for RCRA MLLW. Hanford inventories and projections for asbestos are not included in this chapter but are included in the site's data for LLW in Chapter 4.

\subsubsection{Idaho National Engineering Laboratory (INEL)}

Inventories of INEL TSCA MLLWs consist solely of PCBs and do not include contributions from ANL-W. It is likely that $\mathrm{PCB}$ wastes will continue to be generated at
INEL; however, estimates of specific future quantities are not yet available. There are also inventories and projections of asbestos wastes at INEL; however, these are not included in this chapter but are included in the site data for LLW in Chapter 4.

\subsubsection{Inhalation Toxicology Research Institute (ITRN)}

Currently, ITRI has no TSCA MLLW.

\subsubsection{K-25 Site}

For the K-25 Site, projections of annual TSCA MLLW generation reported in Tables 8.12 and 8.13 are based on 1995 generation levels. Both the reported TSCA MLLW inventories and projections for this site include only contributions from PCBs.

\subsubsection{Kansas City Plant (KCP)}

Currently, $\mathrm{KCP}$ does not have any inventories of TSCA MLLW, and no quantities of TSCA MLLW are anticipated for the future.

\subsubsection{Lawrence Berkeley Laboratory (LBL)}

LBL TSCA MLLW inventories represent contributions from asbestos during 1995.

\subsubsection{Lawrence Livermore National Laboratory (LLNL)}

Inventories of TSCA-regulated MLLW at LLNL include PCBs and asbestos. No TSCA MLLW is projected from future LLNL activities. Current inventories resulted from either the removal of $\mathrm{PCB}$-containing transformers from service or from renovation or closure of buildings containing asbestos.

\subsubsection{Los Alamos National Laboratory (LANL)}

Inventories of TSCA MLLW at LANL are based on limited records kept of asbestos and PCB wastes during the early operating years. A significant increase occurred during 1993 from the addition of contaminated equipment to the asbestos inventory.

\subsubsection{Mound Plant (Mound)}

TSCA MLLW inventories at Mound include contributions from PCBs and asbestos. After CY 1995, no major generation of $\mathrm{PCB}$ wastes is anticipated. Projected levels for asbestos are based on 1995 generation. 


\subsubsection{Naval Reactor (NR) Sites}

Tables 8.8-8.13 report the total inventories of NR sites. Most of these include contributions from the Bettis Atomic Power Laboratory (BAPL). Projected wastes reported for NR sites in Tables 8.12 and 8.13 include contributions from the INEL Naval Reactors Facility (NRF), BAPL, Knolls Atomic Power Laboratory (KAPL), Kesselring Site, and Windsor Site. These wastes are anticipated to result from a variety of site activities, including facility $\mathrm{D} \& \mathrm{D}$, plant lay-up, ship inactivation, and asbestos removal and replacement.

\subsubsection{Nevada Test Site (NTS)}

Inventories of TSCA MLLW at NTS include only PCB wastes. Future volumes of TSCA MLLW may be generated from NTS D\&D and environmental restoration activities, however no estimates are available at this time.

\subsubsection{Oak Ridge National Laboratory (ORNL)}

PCBs comprise the inventories of TSCA MLLW at ORNL. Site-generated projections reported in Tables 8.12 and 8.13 are based on 1995 generation levels.

\subsubsection{Oak Ridge Institute of Science and Education (ORISE)}

Currently, ORISE does not have any inventories of TSCA MLLW, and no quantities of TSCA MLLW are anticipated for the future.

\subsubsection{Paducah Gaseous Diffusion Plant (PAD)}

PAD site TSCA MLLW inventories include both PCBs and asbestos. Projections for generated TSCA wastes are not currently available.

\subsubsection{Pantex Plant (PANT)}

TSCA MLLW inventories at PANT include contributions from both PCBs and asbestos. The site normally ships these waste materials as soon as a shipping increment is accumulated. TSCA MLLW materials are not stored at PANT for the long term. Current inventories listed in Tables 8.8-8.11 are materials temporarily on hand awaiting shipment. TSCA MLLW projections reported in Tables 8.12 and 8.13 for PANT do not include asbestos materials from future site $D \& D$ activities.

\subsubsection{Pinellas Plant (Pinellas)}

Pinellas currently has no inventories of TSCA MLLW.

\subsubsection{Portsmouth Gaseous Diffusion Plant (PORTS)}

At PORTS, TSCA-regulated MLLW is comprised of both PCBs and asbestos. Reported inventories of these wastes pertain to CY 1994. Projections of generated TSCA wastes from PORTS site activities are currently not available.

\subsubsection{Princeton Plasma Physics Laboratory (PPPL)}

PPPL currently has no inventories of TSCA MLLW.

\subsubsection{Rocky Flats Environmental Technology Site (RFETS)}

Both radioactive PCBs and asbestos are included in the RFETS TSCA MLLW inventories reported in Tables 8.8-8.11. TSCA MLLW projections for RFETS are not currently available.

\subsubsection{Stanford Linear Accelerator Center (SLAC)}

SLAC currently has no inventories of TSCA MLLW.

\subsubsection{Sandia National Laboratory/California (SNL/CA)} MLLW

SNL/CA currently has no inventories of TSCA

\subsubsection{Sandia National Laboratory/New Mexico (SNLNM)}

SNL/NM currently has no inventories of TSCA MLLW.

\subsubsection{Savannah River Site (SRS)}

PCBs comprise the TSCA MLLW inventories reported for SRS. Additional PCB wastes are not anticipated for 1996, and projections are unavailable for 1997 and beyond. No information and data are available on SRS inventories and projections for radioactive asbestos.

\subsubsection{West Valley Demonstration Project (WVDP)}

TSCA MLLW inventories reported for WVDP in Tables 8.8-8.11 include both PCBs and asbestos. Projections reported in Tables 8.12 and 8.13 for TSCA MLLW at WVDP apply only through CY 2001. 


\subsubsection{Y-12 Plant (Y-12)}

Y-12 Plant TSCA MLLW inventories reported in Tables 8.8-8.11 are radioactive PCBs. Contributions from asbestos are not reported because asbestos is considered to be a sanitary waste at the Y-12 Plant. Projections for Y-12 are based on 1995 generation levels for PCB wastes.

\subsection{COMMERCIAL MLLW}

In recent years, the NRC and EPA cosponsored a survey study to compile a national profile of the volumes, characteristics, and treatability of commercially generated MLLW. Such a profile was designed to provide the following:

- states and compacts with information to assist in planning and developing adequate disposal capacity for low-level radioactive waste, including MLLW, as mandated by the Low-Level Radioactive Waste Policy Amendments Act; ${ }^{13}$

- private developers with a clearer idea of the characteristics and volumes of mixed waste and the technical capability and capacity needed to treat this waste; and

- a reliable national data base of the volumes, characteristics, and treatability of commercial mixed waste.

In addition, the data were collected to provide a basis for possible federal actions that would effectively manage and regulate the treatment and disposal of mixed waste. Results from this investigation are documented in ref. 7 and summarized in this report.

The study identified the types and volumes of MLLW generated from five groups of facilities: nuclear utilities, medical facilities, academic institutions, industrial facilities, and NRC-licensed government facilities. The study selected a random sample of 1323 facilities out of a total target population of 2936 facilities. Data from the 1016 completed mixed waste survey questionnaires (77\% response rate) received and the use of appropriate weighting factors indicate that approximately $3950 \mathrm{~m}^{3}$ of MLLW- of which $72 \%$ was liquid scintillation fluids-were generated in the United States in 1990.

The study divided the MLLW into several hazardous stream categories, including the following:
- Liquid scintillation fluids from laboratory counting activities.

- Waste oil from various pumps, equipment, and maintenance activities.

- Chlorinated or fluorinated organics and chlorinated fluorocarbons, including sludges and contaminated filters from dry cleaning, refrigeration, degreasing, and decontamination operations. Chloroform and a number of pesticides are also included.

- Other organics, including miscellaneous solvents, reagents, expired products, and other organic compounds (or materials like rags, wipes, etc., contaminated with such) from research and manufacturing activities, experimental procedures, and laboratory and process equipment cleaning.

- Lead wastes, including lead shielding and lead solutions for research and industrial facilities.

- Mercury wastes, including equipment and debris contaminated with mercury.

- Chromate wastes, including chromium-contaminated solutions for research, maintenance, and waste treatment (ion exchange) operations.

- Cadmium wastes from decontamination activities.

- Aqueous corrosive wastes, including inorganic acids, or, in some instances, bases from cleanup and decontamination activities.

- Other hazardous materials, including materials (a) either not readily assignable to any one of the previous categories or (b) containing a number of different hazardous materials.

Summaries of estimated generation rates, amounts in storage, and amounts treated for each of the five facility categories and each of the hazardous waste stream categories are shown in Tables 8.15 and 8.16, respectively (data from ref. 7). Upper and lower bounds were also set for the volume of mixed waste that is untreatable under current technologies by making the simplifying assumption that liquid scintillation fluids, oil, nonhalogenated organics, and corrosive wastes are treatable. Deducting their total contribution from the estimated total mixed waste generation rate leaves residues of about $524 \mathrm{~m}^{3}$. This upper bound for untreatable mixed waste is about $13 \%$ of the estimated 1990 national generation rate of $3950 \mathrm{~m}^{3}$. However, it was noted that the capacity to treat all of the so-called treatable mixed waste may not be available. 


\subsection{REFERENCES}

1. U.S. Congress, Atomic Energy Act of 1954, Pub. L. 83-703, Aug. 15, 1954.

2. U.S. Congress, Resource Conservation and Recovery Act of 1976, Pub. L. 94-580, Oct. 21, 1976, as amended by the Hazardous and Solid Waste Amendments Acts of 1984, Pub. L. 98-616, Nov. 9, 1984.

3. U.S. Congress, Toxic Substances Control Act of 1976, Pub. L. 94-469, Oct. 11, 1976.

4. Wayne E. Roepe, U.S. Environmental Protection Agency, Arlington, Virginia, correspondence to Steve Storch, $\mathrm{DB}$ Frogram, ORNL, Oak Ridge, Tennessee, dated March 3, 1996, containing the updated respective EPA mixed waste authorization statuses for states and U.S. territories as of February 14, 1996.

5. U.S. Environmental Protection Agency, "Subchapter I-Solid Wastes (continued)," Code of Federal Regulations, 40 CFR Parts 260-299 (July 1, 1996).

6. U.S. Environmental Protection Agency, "Subchapter R-Toxic Substances Control Act," Code of Federal Regulations, 40 CFR Parts 700-789 (July 1, 1996).

7. J. A. Klein et al., National Profile on Commercial Generated Low-Level Radioactive Mixed Waste, prepared by Oak Ridge National Laboratory, Oak Ridge, Tennessee, for U.S. Nuclear Regulatory Commission and U.S. Environmental Protection Agency, NUREG/CR-5938, ORNL-6731 (December 1992).

8. U.S. Department of Energy, Office of Environmental Management, 1995 Mixed Waste Inventory Summary Report, Washington, D.C. (December 1995).

9. U.S. Department of Energy, Office of Environmental Management, Office of Waste Management, Site Treatment Plan Data Base, Washington, D.C., updated through December 1996.

10. U.S. Congress, Federal Facility Compliance Act of 1992, Pub. L. 102-386, Oct. 6, 1992.

11. DOE site TSCA-regulated MLLW data submittal attachments, submitted to the IDB Program during September-November 1996. The following TSCA MLLW submittals were received and reviewed by the IDB Program before analysis and integration. Preceding each submittal is the site (in parentheses) to which it refers.

a. (AMES) Kay Lampe Hannasch, Ames Laboratory, Ames, Iowa, correspondence to Alan Icenhour or Steve Storch, IDB Program, ORNL, Oak Ridge, Tennessee, "Low-Level Waste Data Request for Integrated Data Base Report," dated Sept. 10, 1996.

b. (ANL-E) R. F. Coley, Argonne National Laboratory, Argonne, Illinois, correspondence to Timothy S. Crawford, DOE Argonne Group Manager, Argonne, Illinois, "Low-Level Waste Data Request for the Integrated Data Base Report," dated Sept. 19, 1996.

c. (ANL-W) Rodney B. Allen, Argonne National Laboratory-West, Idaho Falls, Idaho, correspondence to Steve Storch, DB Program, ORNL, Oak Ridge, Tennessee, transmitting updated ANL-W TSCA MLLW information and data, dated Oct. 14, 1996.

d. (BNL) Angela C. Harvey, DOE Brookhaven Area Office, Upton, New York, correspondence to Steven Storch, IDB Program, ORNL, Oak Ridge, Tennessee, "Low-Level Waste Data for Brookhaven National Laboratory," dated Sept. 26, 1996.

e. (ETEC) M. Lee, Rockwell International Corporation, Rocketdyne Division, Canoga Park, California, correspondence to Alan Icenhour or Steve Storch, IDB Program, ORNL, "ETEC/Rocketdyne Low-Level Waste Data for Integrated Data Base (DB) Report," 96ETEC-DRF-0483, dated Sept. 12, 1996. 
f. (FNAL) Andrew E. Mravca, DOE Fermi Group, Batavia, Illinois, correspondence to Alan Icenhour, DB Program, ORNL, Oak Ridge, Tennessee, "Low-Level Waste Data Request for Integrated Data Base Report," dated Sept. 13, 1996.

g. (Hanford) D. E. McKenney, Westinghouse Hanford Company, Richland, Washington, correspondence to T. K. Teynor, DOE Richland Operations Office, Richland, Washington, "Low-Level Waste Data Request for the Integrated Data Base Report," 9602190 R1, dated Sept. 20, 1996.

h. (INEL) Roger R. Piscitella, Idaho National Engineering Laboratory, Idaho Falls, Idaho, correspondence to Steve Storch, IDB Program, ORNL, Oak Ridge, Tennessee, "Idaho National Engineering Laboratory (INEL) Input to Integrated Data Base (IDB) Report-(DOE/RW-0006, Rev. 12)," RRP-08-96, dated Sept. 20, 1996.

i. (TTRD) Stephen R. Rohrer, Lovelace Biomedical and Environmental Research Institute, Inc., Albuquerque, New Mexico, correspondence to Alan Icenhour, IDB Program, ORNL, Oak Ridge, Tennessee, transmitting updated ITRI LLW information and data, dated Aug. 28, 1996.

j. (K-25) A. L. Rivera, Lockheed Martin Energy Systems, Inc., Oak Ridge, Tennessee, correspondence to S. N. Storch, DB Program, ORNL, Oak Ridge, Tennessee, "1996 Low-Level Waste (LLW) Management Data Call," dated Sept. 30, 1996.

k. (KCP) Margaret E. Stockdale, DOE Kansas City Area Office, Kansas City, Missouri, correspondence to Steve Storch, IDB Program, ORNL, Oak Ridge, Tennessee, "LLW Integrated Data Base," dated Nov. 14, 1996.

1. (LANL) I-Li Chen, Los Alamos National Laboratory, Los Alamos, New Mexico, correspondence to Alan Icenhour and Steve Storch, DB Program, ORNL, Oak Ridge, Tennessee, "For 1996 Low-Level Waste," CST-1496-139, dated Sept. 18, 1996.

m. (LBL) Robin Wendt, Lawrence Berkeley Laboratory, Berkeley, California, correspondence to Steve Storch, IDB Program, ORNL, Oak Ridge, Tennessee, "LLW Data Request for DB," WM-96-256, dated Sept. 12, 1996.

n. (LLNL) Richard W. Crawford, Lawrence Livermore National Laboratory, Livermore, California, correspondence to Alan Icenhour or Steve Storch, IDB Program, ORNL, Oak Ridge, Tennessee, transmitting updated LLNL LLW information and data, dated Sept. 20, 1996.

o. (MOUND) James J. Zahora, EG\&G Mound Applied Technologies, Miamisburg, Ohio, correspondence to Robert S. Rothman, DOE Miamisburg Area Office, Miamisburg, Ohio, "Low-level Waste Management Data Call," dated Oct. 16, 1996.

p. (NR sites/BAPL) E. D. Shollenberger, DOE Pittsburgh Naval Reactors Office, West Mifflin, Pennsylvania, correspondence to Alan Icenhour, IDB Program, ORNL, Oak Ridge, Tennessee, "Low-Level Waste Information for the 1996 Integrated Data Base report," dated Oct. 24, 1996.

q. (NR sites/KAPL) A. R. Seepo, DOE Schenectady Naval Reactors Office, Schenectady, New York, correspondence to Alan Icenhour, IDB Program, ORNL, Oak Ridge, Tennessee, "Low-Level Waste Information for the 1996 Integrated Data Base Report,"SBF\#96-38, dated Nov. 5, 1996.

r. (NTS) L. S. Sygitowicz, Bechtel Nevada, Las Vegas, Nevada, correspondence to IDB Program, ORNL, Oak Ridge, Tennessee, "Low-Level Waste Data Request for Integrated Data Base report," El00-WM-96-1550, dated Sept. 20, 1996.

s. (ORISE) No submittal correspondence provided. ORISE data reported is based on personal communication between Charles W. Scott, ORISE, Oak Ridge, Tennessee, and S. N. Storch, IDB Program, ORNL, Oak Ridge, Tennessee, dated Oct. 23, 1996. 
t. (ORNL) A. L. Rivera, Lockheed Martin Energy Systems, Inc., Oak Ridge, Tennessee, correspondence to S. N. Storch, IBB Program, ORNL, Oak Ridge, Tennessee, "1996 Low-Level Waste (LLW) Management Data Call," dated Sept. 30, 1996.

u. (PANT) W. A. Weinreich, Mason \& Hager Corporation, Amarillo, Texas, correspondence to Stephen N. Storch, IDB Program, ORNL, Oak Ridge, Tennessee, "TSCAMLLW Data Requirements for the Integrated Data Base Report," dated Nov. 1, 1996.

v. (Pinellas) Gary C. Schmidtke, DOE Pinellas Area Office, Largo, Florida, personal communication to Stephen N. Storch, IDB Program, ORNL, Oak Ridge, Tennessee, discussing Pinellas Plant TSCA MLLW inventories and projections, dated Nov. 7, 1996.

w. (PPPL) Jerry W. Faul, DOE Princeton Group, correspondence to R. Lang, DOE Chicago Operations Office, Argonne, Mlinois, "PPPL's Low-Level Waste Data for Integrated Data Base Report," TEC. 960927.0002 , dated Sept. 25, 1996.

x. (RFETS) Lisa O'Mary, DOE Rocky Flats Office, Golden, Colorado, correspondence to Steve Storch, IDB Program, ORNL, Oak Ridge National Laboratory, transmitting updated RFETS LLW information and data, dated Sept. 17, 1996

y. (SLAC) Michael Scharfenstein, Stanford University, Stanford, California, correspondence to Alan Icenhour or Steve Storch, IDB Program, ORNL, Oak Ridge, Tennessee, transmitting updated SLAC LLW information and data, dated Sept. 9, 1996.

z. (SNL/CA) Sarah O'Connor, Lockheed Martin Sandia Corporation, Livermore, California, correspondence to Alan Icenhour and Steve Storch, IDB Program, ORNL, Oak Ridge, Tennessee, "Response to Low-Level Waste Data Request for Integrated Data Base Report," dated Sept. 20, 1996.

aa. (SNL/NM) Scott A. Schrader, Sandia National Laboratories/New Mexico, Albuquerque, New Mexico, correspondence to Steve Storch, IDB Program, ORNL, Oak Ridge, Tennessee, "Integrated Data Base Low-Level Waste Data Request," dated Oct. 1, 1996.

ab. (SRS) Nathaniel S. Roddy, Westinghouse Savannah River Company, Aiken, South Carolina, correspondence to Steven Storch, DB Program, ORNL, Oak Ridge, Tennessee, "Low-Level Waste Management Data Call for 1996 (U)," SWE-SWE-96-0411, dated Oct. 4, 1996.

ac. (WVDP) E. B. Martin, West Valley Nuclear Services Company, Inc., West Valley, New York, correspondence to Steven N. Storch, IDB Program, ORNL, Oak Ridge, Tennessee, "Submittal of Low-Level Waste Data Call for the 1995 (sic) Integrated Data Base," WZ:96:0100, dated Sept. 26, 1996.

ad. (Y-12) A. L. Rivera, Lockheed Martin Energy Systems, Inc., Oak Ridge, Tennessee, correspondence to S. N. Storch, IDB Program, ORNL, Oak Ridge, Tennessee, "1996 Low-Level Waste (LLW) Management Data Call," dated Sept. 30, 1996.

12. U.S. Department of Energy, Office of Waste Management, DOE Waste Treatability Group Guidance, Washington, D.C., DOE/LLW-217, Rev. 0 (January 1995).

13. U.S. Congress, Low-Level Radioactive Waste Policy Amendments Act of 1985, Pub. L., 99-240, Jan. 15, 1986. 


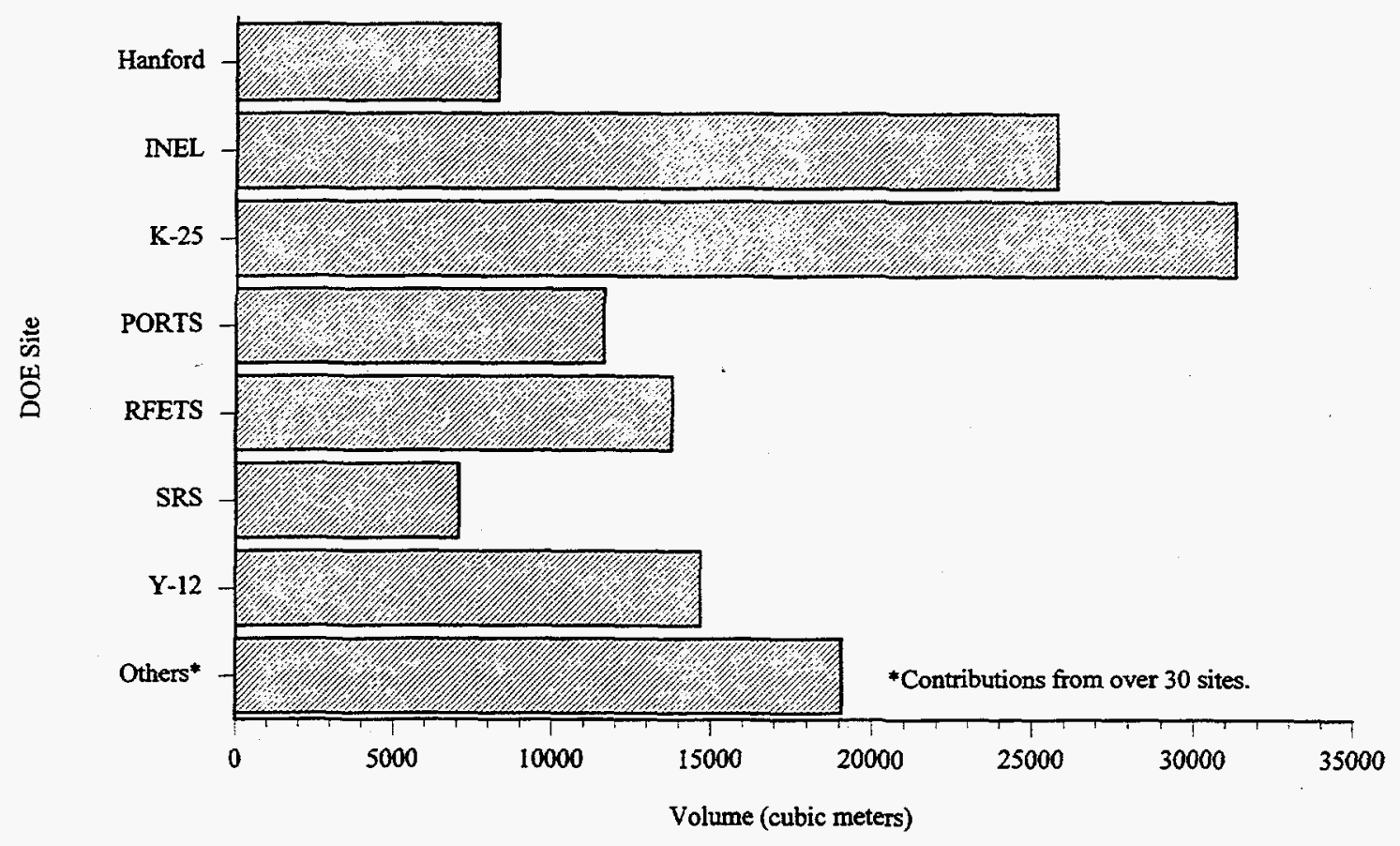

Fig. 8.1. Estimated volume inventory of DOE MLLW (RCRA and TSCA) at the end of 1995.

ORNL DWG 95-8848R

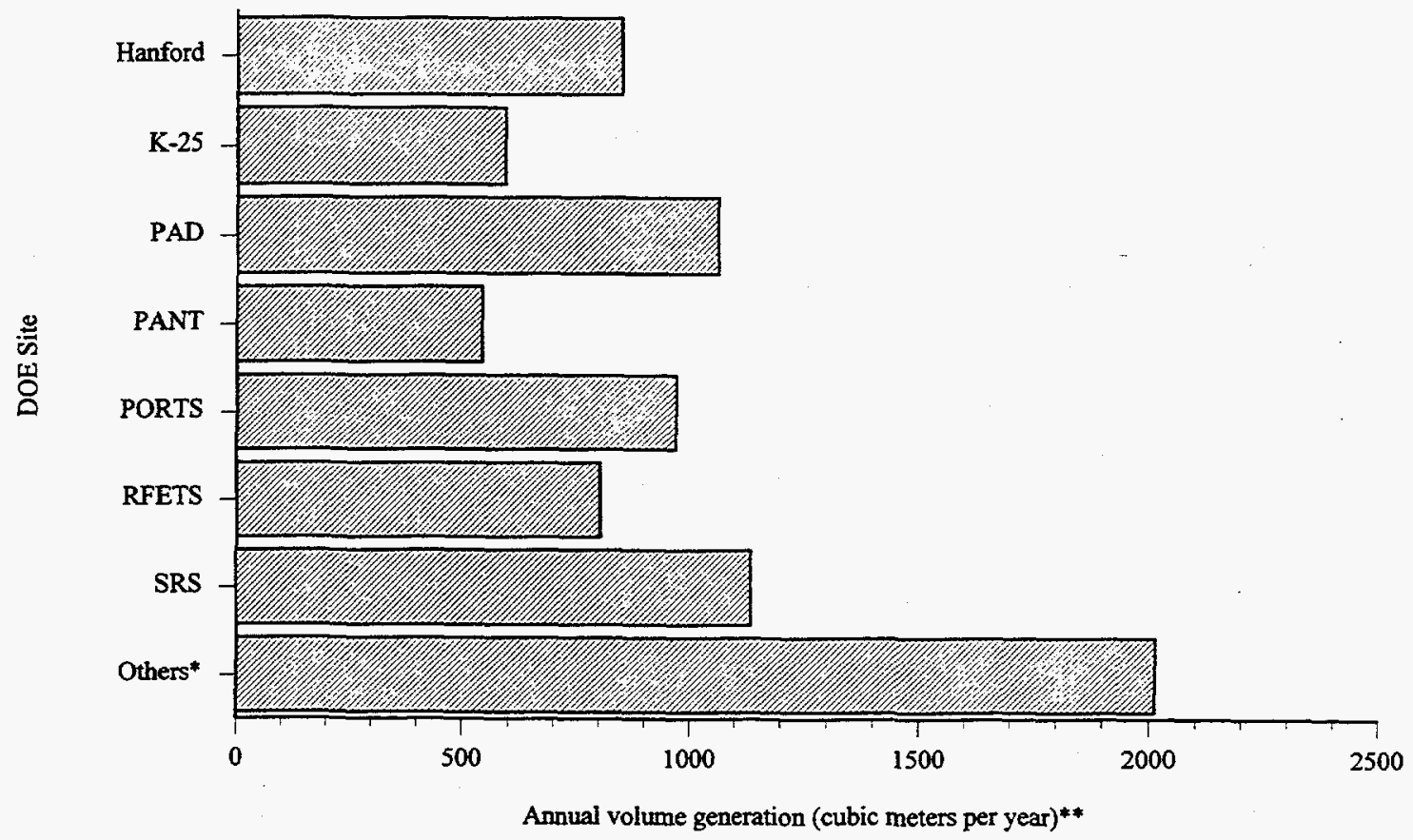

${ }^{*}$ Contributions from over 30 sites.

**Based on an average of 5-year projections.

Fig. 8.2. Estimated volume generation of DOE MLLW (RCRA and TSCA) during 1995. 
Table 8.1. Thirty-nine states and territories with EPA mixed waste authorization as of the end of $1995^{\mathrm{a}}$

\begin{tabular}{lc||lc}
\hline State or territory & Effective date & State or territory & Effective date \\
\hline Alabama & $11 / 23 / 93$ & Nebraska & $12 / 03 / 88$ \\
Arizona & $01 / 22 / 93$ & Nevada & $06 / 29 / 92$ \\
Arkansas & $05 / 29 / 90$ & New Hampshire & $11 / 14 / 94$ \\
California & $08 / 01 / 92$ & New Mexico & $07 / 25 / 90$ \\
Colorado & $11 / 07 / 86$ & New York & $05 / 07 / 90$ \\
Connecticut & $12 / 31 / 90$ & North Carolina & $11 / 21 / 89$ \\
Florida & $02 / 12 / 91$ & North Dakota & $08 / 24 / 90$ \\
Georgia & $09 / 26 / 88$ & Ohio & $06 / 30 / 89$ \\
Guam & $10 / 10 / 89$ & Oklahoma & $11 / 27 / 90$ \\
Idaho & $04 / 09 / 90$ & Oregon & $05 / 29 / 90$ \\
Illinois & $04 / 30 / 90$ & South Carolina & $09 / 13 / 87$ \\
Indiana & $09 / 30 / 91$ & South Dakota & $06 / 17 / 91$ \\
Kansas & $06 / 25 / 90$ & Tennessee & $08 / 11 / 87$ \\
Kentucky & $12 / 19 / 88$ & Texas & $03 / 15 / 90$ \\
Louisiana & $10 / 26 / 91$ & Utah & $03 / 07 / 89$ \\
Michigan & $12 / 26 / 89$ & Vermont & $08 / 06 / 93$ \\
Minnesota & $06 / 23 / 89$ & Washington & $11 / 23 / 87$ \\
Mississippi & $05 / 28 / 91$ & Wisconsin & $04 / 24 / 92$ \\
Missouri & $03 / 12 / 93$ & Wyoming & $10 / 18 / 95$ \\
Montana & $03 / 21 / 94$ & & \\
\hline
\end{tabular}

a Based on ref. 4. Information as of February 14, 1996. 
Table 8.2. Summary of estimated MLLW inventories and annual generation as of 1995

\begin{tabular}{|c|c|c|}
\hline \multirow{2}{*}{ Category } & \multicolumn{2}{|c|}{ Volume, $\mathrm{m}^{3}$} \\
\hline & Inventory & $\begin{array}{l}\text { Annual } \\
\text { generation }\end{array}$ \\
\hline \multicolumn{3}{|l|}{ DOE sites } \\
\hline RCRA/state MLLW & $107,911^{\mathrm{a}}$ & $5,725^{b}$ \\
\hline TSCA MLLWC & 23,468 & 2,234 \\
\hline DOE MLLW total & 131,379 & 7,959 \\
\hline Commercial sites ${ }^{d}$ & 2,116 & 3,949 \\
\hline Envirocare $\mathrm{e}^{\mathrm{e}}$ & 20,143 & 0 \\
\hline
\end{tabular}

aBased on ref. 9. The currentness of these data for the various DOE sites ranges from January 1995 to June 1996.

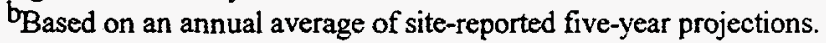

CReported for CY 1995.

${ }^{\mathrm{d}}$ Reported for CY 1990.

'Wastes from commercial- and government-sponsored (DOE, EPA, DOD) activities that are disposed of at the Envirocare Facility near Clive, Utah. DOE wastes include LLW, MLLW, and 1 le(2) by-product material. See Table 0.8 in Chapter 0 , which is based on ref. 19 of Chapter 0. 
Table 8.3. Estimated volume $\left(\mathrm{m}^{3}\right)$ inventories and five-year projections of DOE RCRA MLLW as of 1995 , by site ${ }^{a}$

\begin{tabular}{|c|c|c|c|}
\hline Site & Inventory & $\begin{array}{l}\text { Five-year } \\
\text { projection }\end{array}$ & Total \\
\hline Ames & 0.0 & b & $\mathbf{b}$ \\
\hline ANL-E & 33.7 & 107.5 & 141.2 \\
\hline ANL-W & 411.9 & 368.9 & 780.8 \\
\hline $\mathrm{BNL}$ & 24.0 & 2.8 & 26.8 \\
\hline ETEC & 1.4 & 3.4 & 4.8 \\
\hline FEMP & $2,390.2$ & 153.6 & $2,543.8$ \\
\hline FNAL & b & $b$ & $b$ \\
\hline Hanford & $8,169.5$ & $4,200.4$ & $12,370.0$ \\
\hline $\mathrm{DNEL}^{\mathrm{c}}$ & $25,734.4$ & 899.4 & $26,633.8$ \\
\hline ITRI & 0.0 & $\mathrm{~b}$ & $\mathrm{~b}$ \\
\hline $\mathrm{K}-25$ & $23,076.1$ & $1,345.1$ & $24,421.2$ \\
\hline $\mathrm{KCP}$ & 0.0 & b & b \\
\hline LANL & 608.6 & 107.4 & 716.0 \\
\hline LBL & 6.3 & 7.0 & 13.3 \\
\hline LLNL & 454.0 & $1,765.1$ & $2,219.1$ \\
\hline Mound & 97.1 & 4.5 & 101.6 \\
\hline NR sites ${ }^{d}$ & 77.2 & 157.2 & 234.4 \\
\hline NTS & 306.0 & $2,652.0$ & $2,958.0$ \\
\hline ORISE & b & b & $b$ \\
\hline ORNL & $2,840.5$ & 562.6 & $3,403.1$ \\
\hline PAD & $1,032.2$ & 0.0 & $1,032.2$ \\
\hline PANT & 132.3 & 918.5 & $1,050.8$ \\
\hline Pinellas & 0.0 & b & $b$ \\
\hline PORTS & $8,011.4$ & $3,544.2$ & $11,555.6$ \\
\hline PPPL & 0.0 & b & $b$ \\
\hline RFETS & $13,558.4$ & $4,005.1$ & $17,563.5$ \\
\hline RMI & 21.5 & 7.5 & 29.0 \\
\hline SLAC & $\mathrm{b}$ & b & b \\
\hline SNL/CA & 0.0 & $b$ & b \\
\hline $\mathrm{SNL} / \mathrm{NM}$ & 69.8 & 339.0 & 408.8 \\
\hline SRS & $6,939.5$ & $5,674.7$ & $12,614.2$ \\
\hline WVDP & 147.8 & 39.0 & 186.8 \\
\hline$Y-12$ & $13,729.6$ & $1,732.3$ & $15,461.9$ \\
\hline Others ${ }^{e}$ & 37.9 & 27.0 & 64.9 \\
\hline $\begin{array}{l}\text { Total } \\
\text { (DOE complex) }\end{array}$ & $107,911.3$ & $28,624.2$ & $136,535.5$ \\
\hline
\end{tabular}

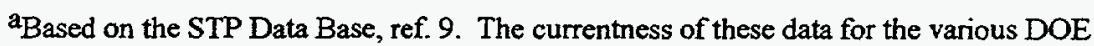
sites ranges from January 1995 to June 1996.

bInformation not available or unknown.

Includes contributions from the Idaho Naval Reactors Facility.

dincludes contributions, if any, from Bettis Atomic Power Laboratory (BAPL), Knolls Atomic Power Laboratory (KAPL), Kesselring Site, and Windsor Site. Also includes contributions, if any, from the Charleston, Mare Island, Norfolk, Pearl Harbor, Portsmouth, and Puget Sound Naval shipyards.

Includes contributions, if any, from Battelle Columbus Laboratory (BCL), Colonie Interim Storage Site (CISS), General Atomics (GA), Laboratory for Energy Related Health Research (LEHR), and Missouri University. 
Table 8.4. Treatability group matrix parameter categories used to characterize DOE RCRA MLLWa

\begin{tabular}{ll}
\hline Matrix parameter category & Description \\
\hline & Liquids \\
\end{tabular}

Aqueous liquids/slurries

Organic liquids

Unknown/other liquids

Inorganic homogeneous solids

Organic homogeneous solids

Unknown/other homogeneous
solids

Soil/gravel

Inorganic debris

Organic debris

Heterogeneous debris

Unknown/other debris

Unknown/other solids
Liquids and slurries containing less than $1 \%$ total organic carbon (TOC)

Liquids and slurries containing $\geq 1 \%$ TOC

Liquids and slurries which can not be categorized as aqueous liquids/slurries or organic liquids because it is not known if the TOC level is less or greater than $1 \%$

\section{Solids ${ }^{\mathrm{c}}$}

Wastes that are at least $50 \mathrm{vol} \%$ inorganic homogeneous solids. Homogeneous solids are defined as solid waste materials, excluding soil/gravel, that do not meet the Environmental Protection Agency (EPA) land disposal restrictions (LDR) criteria for classification as debris. Inorganic homogeneous solids are further defined as those with sufficient inorganic solids content șuch that a minimum of approximately $20 \mathrm{wt} \%$ would remain as residue (i.e., ash/solids) following incineration

Wastes that are at least 50 vol \% organic homogeneous solids. Homogeneous solids are defined as solid waste materials, excluding soil/gravel, that do not meet the EPA LDR criteria for classification as debris. Organic homogeneous solids are further defined as those with a base structure that is primarily organic such that a maximum of approximately $20 \mathrm{wt} \%$ would remain as residue (i.e., ash/solids) following incineration

Wastes that are at least $50 \mathrm{vol} \%$ homogeneous solids, but:

- are insufficiently characterized to enable categorization as either inorganic or organic homogeneous solids, or

- do not meet the criteria for categorization as either inorganic or organic homogeneous solids

Wastes that are at least $50 \mathrm{vol} \%$ soil, including sand and silt or rock and gravel, that do not meet EPA LDR criteria for classification as debris

Wastes that are at least $80 \mathrm{vol} \%$ inorganic materials that meet the EPA LDR criteria for classification as debris. Examples include scrap metal, concrete, glass, and brick

Wastes that are at least 80 vol \% organic materials that meet the EPA LDR criteria for classification as debris. Examples include plastic, rubber, wood, paper, cloth, and biological materials

Wastes that are at least 50 vol \% debris materials that meet the EPA LDR criteria for classification as debris but are not dominant (i.e., at least 80 vol \%) in either inorganic or organic debris materials

Wastes that are at least $50 \mathrm{vol} \%$ materials that meet the EPA LDR criteria for classification as debris but lack adequate characterization information to enable categorizing as inorganic, organic, or heterogeneous debris

Wastes with physically solid matrices for which insufficient characterization information exists to enable categorizing as a homogeneous solid, soil/gravel, or debris 
Table 8.4 (continued)

\begin{tabular}{|c|c|}
\hline Matrix parameter category & Description \\
\hline & Specific waste forms ${ }^{\mathrm{d}}$ \\
\hline Lab packs & $\begin{array}{l}\text { Wastes packaged in lab pack configurations. A lab pack configuration is defined as } \\
\text { two or more waste containers packaged within a larger outer container. Typically, the } \\
\text { inner containers are surrounded by absorbent materials. If present, the absorbents can } \\
\text { be homogeneous solids or debris materials }\end{array}$ \\
\hline Reactive metals & $\begin{array}{l}\text { Reactive metal wastes that meet the criteria for classification as water-reactive or } \\
\text { ignitable-reactive per the Third Third LDR rule ( } 55 \text { FR } 22545 \text { and } 22553 \text { ). Typically, } \\
\text { this waste is sodium metal or sodium metal alloys, but can also include particulate fines } \\
\text { of aluminum, uranium, zirconium, or other pyrophoric materials }\end{array}$ \\
\hline Explosives/propellants & $\begin{array}{l}\text { Wastes consisting of substances which undergo rapid chemical transformations that } \\
\text { produce large amounts of gases and heat. The gases rapidly expand at velocities } \\
\text { exceeding the speed of sound (due to the heat of reaction), which creates a shock wave } \\
\text { and explosion. Waste that meets this definition is categorized as an } \\
\text { explosive/propellant regardless of the specific physical form }\end{array}$ \\
\hline Compressed gases/aerosols & Wastes consisting of pressurized gas cylinders or aerosol cans \\
\hline Elemental mercury & $\begin{array}{l}\text { Wastes that are bulk, pourable liquid mercury. The liquid mercury may be packaged } \\
\text { in a lab pack configuration }\end{array}$ \\
\hline Elemental hazardous metals & $\begin{array}{l}\text { Wastes that are at least } 50 \mathrm{vol} \% \text { solid, bulk elemental hazardous metals that meet the } \\
\text { EPA LDR size criteria for classification as debris. Typical examples of solid elemental } \\
\text { hazardous metals are lead and cadmium }\end{array}$ \\
\hline Beryillium dust & $\begin{array}{l}\text { Wastes that are subject to the metal recovery treatment standard for beryllium dust as } \\
\text { specified in the Third Third LDR rule (55 FR 22545) }\end{array}$ \\
\hline Batteries & $\begin{array}{l}\text { Wastes consisting of lead acid, cadmium, or other batteries. The batteries may be } \\
\text { packaged with absorbent materials }\end{array}$ \\
\hline Unknown/other matrix & $\begin{array}{l}\text { Wastes for which insufficient characterization information is known to enable } \\
\text { categorization as a liquid or solid or as one of the specific waste forms }\end{array}$ \\
\hline & Final waste forms $\mathrm{s}^{\mathrm{e}}$ \\
\hline Immobilized forms & $\begin{array}{l}\text { Wastes that have been immobilized. These include wastes considered to be either } \\
\text { micro- or macro-encapsulated }\end{array}$ \\
\hline Decontaminated solids & Waste that has been decontaminated and is ready for disposal or recycling \\
\hline Other final forms & Final waste forms other than immobilized forms and decontaminated solids \\
\hline \multicolumn{2}{|c|}{$\begin{array}{l}\text { aBased on ref. } 9 \text {. } \\
\text { bThis category addresses wastes that are liquid, including slurries, and are packaged in bulk, free form (i.e., excludes lab } \\
\text { packs). Slurries are defined as liquids with a total suspended/settled solids (TSS) content of } \geq 1 \% \text { and } \leq 30 \% \text {. } \\
\text { "This category addresses waste with physically solid matrices, including sludges. Sludges are defined as having a TSS }>30 \% \text {. } \\
\text { Certain waste with physically solid matrices are excluded from this category (see the "specific waste forms" and "final waste forms" } \\
\text { categories above). } \\
\text { dThis category addresses lab packs and other specific waste forms. The other specific waste forms include waste that (a) is } \\
\text { inherently hazardous (i.e., the bulk material itself is RCRA hazardous), or (b) presents unique treatment or management concerns. } \\
\text { "This category addresses waste that is in final form and meets applicable disposal criteria, including applicable LDR and PCB } \\
\text { treatment standards. }\end{array}$} \\
\hline
\end{tabular}


Table 8.5. Estimated volume $\left(\mathrm{m}^{3}\right)$ inventories and five-year projections of DOE RCRA MLLW as of 1995 , by site and physical form ${ }^{\mathrm{a}}$

\begin{tabular}{|c|c|c|c|c|}
\hline Site & Physical form ${ }^{b}$ & Inventory & $\begin{array}{l}\text { Five-year } \\
\text { projection }\end{array}$ & Total \\
\hline Ames & Not applicable & 0.00 & c & c \\
\hline \multirow[t]{9}{*}{ ANL-E } & Aqueous liquids/slurries & 0.02 & 0.00 & 0.02 \\
\hline & Elemental hazardous metals & 17.53 & 71.74 & 89.27 \\
\hline & Inorganic debris & 0.67 & 5.19 & 5.86 \\
\hline & Inorganic homogeneous solids & 5.12 & 15.00 & 20.12 \\
\hline & Lab packs & 8.59 & 9.65 & 18.24 \\
\hline & Organic debris & 0.79 & 3.97 & 4.76 \\
\hline & Reactive metals & 0.53 & 0.44 & 0.97 \\
\hline & Soil/gravel & 0.40 & 1.50 & 1.90 \\
\hline & ANL-E total & 33.63 & 107.49 & 141.14 \\
\hline \multirow[t]{8}{*}{ ANL-W } & Aqueous liquids/slurries & 0.42 & 0.08 & 0.50 \\
\hline & Elemental hazardous metals & 0.52 & 0.09 & 0.61 \\
\hline & Heterogeneous debris & 2.94 & 1.87 & 4.81 \\
\hline & Inorganic debris & $\mathrm{d}$ & d & 0.01 \\
\hline & Organic debris & 0.16 & 0.08 & 0.24 \\
\hline & Organic homogeneous solids & 20.82 & 23.50 & 44.32 \\
\hline & Reactive metals & 387.03 & 343.31 & 730.33 \\
\hline & ANL-W total & 411.90 & 368.94 & 780.82 \\
\hline \multirow[t]{7}{*}{$\mathrm{BNL}$} & Organic liquids & 17.33 & 0.78 & 18.11 \\
\hline & Elemental hazardous metals & 1.18 & 0.73 & 1.91 \\
\hline & Elemental mercury & 0.65 & 0.10 & 0.75 \\
\hline & Inorganic homogeneous solids & 2.50 & 0.20 & 2.70 \\
\hline & Lab packs & 1.02 & 0.46 & 1.48 \\
\hline & Unknown'other matrix & 1.30 & 0.50 & 1.80 \\
\hline & BNL total & 23.98 & 2.77 & 26.75 \\
\hline \multirow[t]{6}{*}{ ETEC } & Aqueous liquids/slurries & 0.13 & 0.00 & 0.13 \\
\hline & Inorganic debris & 0.10 & 0.84 & 0.94 \\
\hline & Inorganic homogeneous solids & 0.99 & 2.51 & 3.49 \\
\hline & Organic liquids & 0.15 & 0.00 & 0.15 \\
\hline & Reactive metals & $\mathrm{d}$ & 0.00 & $\mathrm{~d}$ \\
\hline & ETEC total & 1.37 & 3.35 & 4.72 \\
\hline \multirow[t]{14}{*}{ FEMP } & Aqueous liquids/slurries & 940.19 & 26.99 & 967.18 \\
\hline & Batteries & 5.00 & 14.09 & 19.09 \\
\hline & Compressed gases/aerosols & 0.59 & 0.30 & 0.89 \\
\hline & Elemental hazardous metals & 2.00 & 5.10 & 7.10 \\
\hline & Elemental mercury & 0.20 & 0.00 & 0.20 \\
\hline & Heterogeneous debris & 62.80 & 10.90 & 73.70 \\
\hline & Inorganic debris & 22.60 & 16.70 & 39.30 \\
\hline & Inorganic homogeneous solids & 612.00 & 15.70 & 627.70 \\
\hline & Lab packs & 0.40 & 0.00 & 0.40 \\
\hline & Organic debris & 12.80 & 0.29 & 13.09 \\
\hline & Organic homogeneous solids & 96.20 & 0.00 & 96.20 \\
\hline & Organic liquids & 460.22 & 63.50 & 523.72 \\
\hline & Reactive metals & 0.40 & 0.00 & 0.40 \\
\hline & Soil/gravel & 170.50 & 0.00 & 170.50 \\
\hline
\end{tabular}


Table 8.5 (continued)

\begin{tabular}{|c|c|c|c|c|}
\hline Site & Physical form ${ }^{b}$ & Inventory & $\begin{array}{l}\text { Five-year } \\
\text { projection }\end{array}$ & Total \\
\hline \multirow{4}{*}{$\begin{array}{l}\text { FEMP } \\
\text { (contd.) }\end{array}$} & Unknown/other homogeneous solids & 3.40 & 0.00 & 3.40 \\
\hline & Unknown/other liquids & 0.10 & 0.00 & 0.10 \\
\hline & Unknown/other solids & 0.80 & 0.00 & 0.80 \\
\hline & FEMP total & $2,390.20$ & 153.57 & $2,543.77$ \\
\hline FNAL & Not applicable & c & c & c \\
\hline \multirow[t]{18}{*}{ FTanford } & Batteries & 1.86 & 2.27 & 4.13 \\
\hline & Elemental hazardous metals & 240.03 & 134.50 & 374.53 \\
\hline & Elemental mercury & 1.45 & 0.66 & 2.11 \\
\hline & Explosives/propellants & 0.00 & 1.14 & 1.14 \\
\hline & Heterogeneous debris & 808.78 & $1,678.04$ & $2,486.82$ \\
\hline & Immobilized forms & 86.47 & 0.00 & 86.47 \\
\hline & Inorganic debris & 535.31 & $1,145.29$ & $1,680.60$ \\
\hline & Inorganic homogeneous solids & $3,779.41$ & 260.65 & $4,040.06$ \\
\hline & Lab packs & 291.35 & 123.67 & 415.02 \\
\hline & Organic debris & $1,690.50$ & 585.92 & $2,276.42$ \\
\hline & Organic liquids & 1.04 & 0.00 & 1.04 \\
\hline & Reactive metals & 5.43 & 0.00 & 5.43 \\
\hline & Soil/gravel & 476.38 & 244.01 & 720.39 \\
\hline & Unknown/other debris & 145.45 & 0.00 & 145.45 \\
\hline & Unknown/other homogeneous solids & 25.32 & 24.30 & 49.61 \\
\hline & Unknown/other matrix & 60.15 & 0.00 & 60.15 \\
\hline & Unknown/other solids & 20.62 & 0.00 & 20.62 \\
\hline & Hanford total & $8,169.54$ & $4,200.44$ & $12,369.98$ \\
\hline \multirow[t]{14}{*}{ INEL } & Aqueous liquids/slurries & 10.73 & 14.50 & 25.24 \\
\hline & Elemental hazardous metals & 433.38 & 119.23 & 552.61 \\
\hline & Elemental mercury & 0.00 & 0.01 & 0.01 \\
\hline & Heterogeneous debris & $19,654.13$ & 80.11 & $19,734.23$ \\
\hline & Immobilized forms & 2.28 & 9.20 & 11.98 \\
\hline & Inorganic debris & .508 .02 & 332.90 & 840.92 \\
\hline & Inorganic homogeneous solids & $3,828.39$ & 86.28 & $3,914.67$ \\
\hline & Lab packs & 3.28 & 2.91 & 6.19 \\
\hline & Organic debris & 709.21 & 189.09 & 898.30 \\
\hline & Organic homogeneous solids & 350.41 & 58.82 & 409.23 \\
\hline & Organic liquids & 10.45 & 6.33 & 16.79 \\
\hline & Reactive metals & 0.93 & 0.00 & 0.93 \\
\hline & Soil/gravel & 222.67 & 0.05 & 222.72 \\
\hline & INEL total & $25,734.38$ & 899.43 & $26,633.82$ \\
\hline ITRI & Not applicable & 0.00 & c & $\mathrm{c}$ \\
\hline \multirow[t]{9}{*}{$K-25$} & Aqueous liquids/slurries & 357.16 & 169.02 & 526.18 \\
\hline & Batteries & 17.56 & 7.09 & 24.65 \\
\hline & Beryllium dust & 0.16 & 0.00 & 0.16 \\
\hline & Compressed gases/aerosols & 4.91 & 1.45 & 6.36 \\
\hline & Elemental hazardous metals & 90.83 & 1.63 & 92.46 \\
\hline & Elemental mercury & 2.80 & 0.25 & 3.05 \\
\hline & Heterogeneous debris & 147.88 & 153.12 & 301.00 \\
\hline & Inorganic debris & 190.38 & 60.82 & 251.19 \\
\hline & Inorganic homogeneous solids & $20,956.75$ & 674.13 & $21,630.88$ \\
\hline
\end{tabular}


Table 8.5 (continued)

\begin{tabular}{|c|c|c|c|c|}
\hline Site & Physical form ${ }^{b}$ & Inventory & $\begin{array}{l}\text { Five-year } \\
\text { projection }\end{array}$ & Total \\
\hline \multirow{8}{*}{$\begin{array}{l}\mathrm{K}-25 \\
\text { (contd.) }\end{array}$} & Lab packs & 31.97 & 4.55 & 36.52 \\
\hline & Organic debris & 162.58 & 103.10 & 265.68 \\
\hline & Organic homogeneous solids & 440.77 & 2.26 & 443.03 \\
\hline & Organic liquids & 352.13 & 117.55 & 469.68 \\
\hline & Reactive metals & 0.08 & 0.00 & 0.08 \\
\hline & Soil/gravel & 278.78 & 49.37 & 328.15 \\
\hline & Unknown solids & 41.42 & 0.77 & 42.19 \\
\hline & $\mathrm{K}-25$ total & $23,076.16$ & $1,345.09$ & $24,421.26$ \\
\hline $\mathrm{KCP}$ & Not applicable & 0.00 & c & c \\
\hline \multirow[t]{12}{*}{ LANL } & Aqueous liquids/slurries & 3.34 & 1.51 & 4.85 \\
\hline & Compressed gases/aerosols & 1.68 & 2.20 & 3.88 \\
\hline & Elemental hazardous metals & 66.91 & 12.70 & 79.61 \\
\hline & Elemental mercury & 0.50 & 0.05 & 0.55 \\
\hline & Inorganic debris & 15.60 & 1.00 & 16.60 \\
\hline & Organic debris & 42.14 & 8.50 & 50.64 \\
\hline & Organic liquids & 55.42 & 23.21 & 78.63 \\
\hline & Reactive metals & 0.20 & 0.05 & 0.25 \\
\hline & Soil/gravel & 49.85 & 2.00 & 51.85 \\
\hline & Unknown/other debris & 19.47 & 11.20 & 30.67 \\
\hline & Unknown/other matrix & 353.49 & 45.00 & 398.49 \\
\hline & LANL total & 608.60 & 107.42 & 716.02 \\
\hline \multirow[t]{7}{*}{ LBL } & Aqueous liquids/slurries & 2.16 & 2.90 & 5.06 \\
\hline & Elemental hazardous metals & 0.43 & 0.00 & 0.43 \\
\hline & Elemental mercury & 0.11 & 0.00 & 0.11 \\
\hline & Heterogeneous debris & 0.11 & 0.15 & 0.26 \\
\hline & Lab packs & 0.20 & 0.12 & 0.32 \\
\hline & Organic liquids & 3.30 & 3.80 & 7.10 \\
\hline & LBL total & 6.31 & 6.97 & 13.28 \\
\hline \multirow[t]{10}{*}{ LLNL } & Elemental mercury & 0.09 & 0.05 & 0.14 \\
\hline & Heterogeneous debris & 62.40 & 72.00 & 134.40 \\
\hline & Inorganic debris & 19.10 & 10.00 & 29.10 \\
\hline & Inorganic homogeneous solids & 250.00 & 240.00 & 490.00 \\
\hline & Lab packs & 6.30 & 6.50 & 12.80 \\
\hline & Organic homogeneous solids & 1.20 & 5.00 & 6.20 \\
\hline & Organic liquids & 92.80 & $1,389.50$ & $1,482.30$ \\
\hline & Soil/gravel & 16.70 & 40.00 & 56.70 \\
\hline & Reactive metals & 5.43 & 2.00 & 7.43 \\
\hline & LLNL total & 454.02 & $1,765.05$ & $2,219.07$ \\
\hline \multirow[t]{5}{*}{ Mound } & Batteries & 0.00 & 0.79 & 0.79 \\
\hline & Elemental hazardous metals & 5.00 & 0.65 & 5.65 \\
\hline & Elemental mercury & 0.02 & d & 0.02 \\
\hline & Lab packs & 43.47 & 3.00 & 46.47 \\
\hline & Organic debris & 0.02 & 0.02 & 0.04 \\
\hline
\end{tabular}


Table 8.5 (continued)

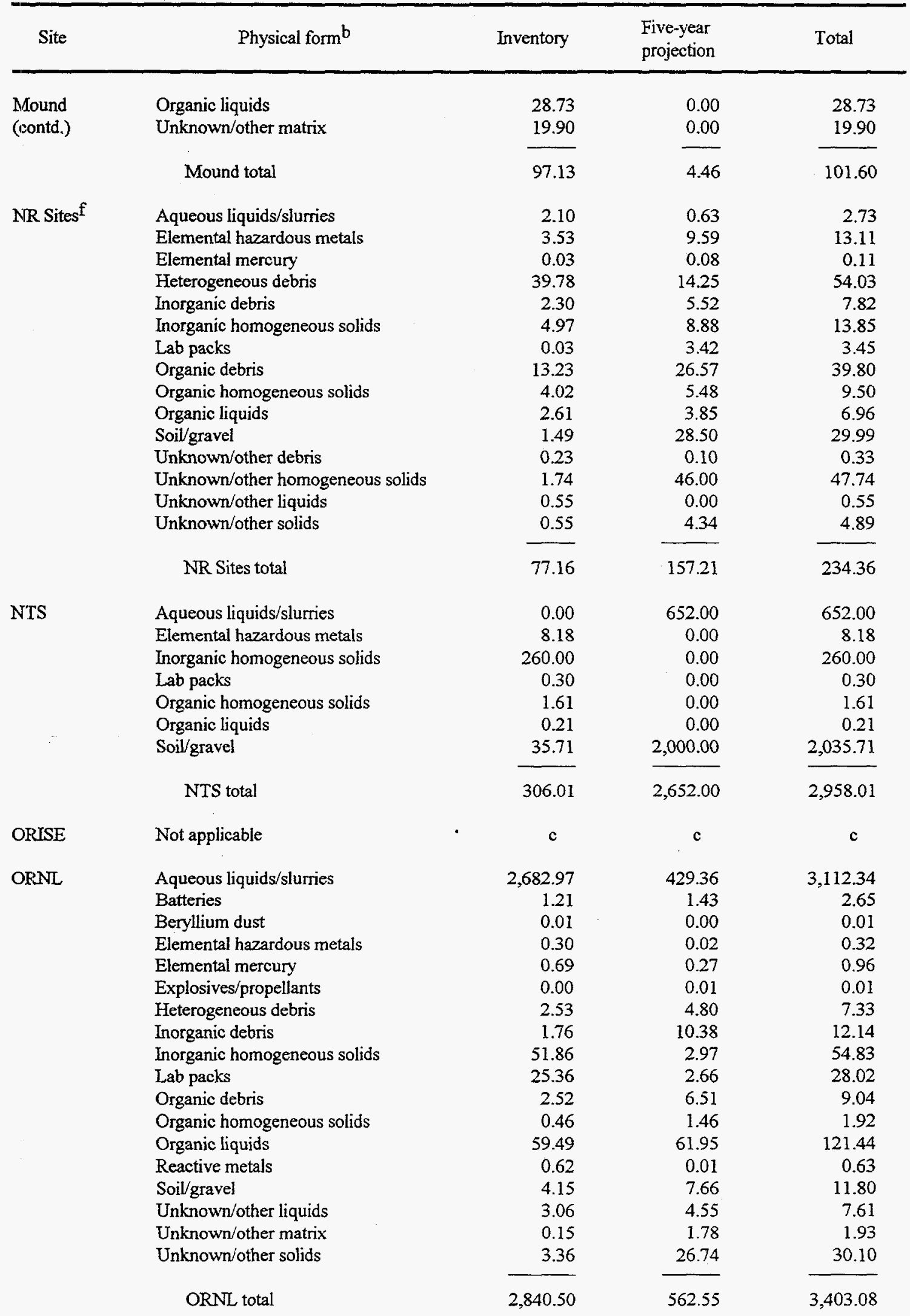


Table 8.5 (continued)

\begin{tabular}{|c|c|c|c|c|}
\hline Site & Physical form ${ }^{b}$ & Inventory & $\begin{array}{l}\text { Five-year } \\
\text { projection }\end{array}$ & Total \\
\hline \multirow[t]{15}{*}{ PANT } & Aqueous liquids/slurries & 1.75 & 24.40 & 26.15 \\
\hline & Batteries & 0.05 & 1.31 & 1.36 \\
\hline & Elemental hazardous metals & 0.06 & 0.15 & 0.21 \\
\hline & Elemental mercury & $d$ & 0.00 & $\mathrm{~d}$ \\
\hline & Heterogeneous debris & 3.81 & 13.85 & 17.66 \\
\hline & Inorganic debris & 12.85 & 569.85 & 582.70 \\
\hline & Inorganic homogeneous solids & 19.26 & 7.86 & 27.12 \\
\hline & Lab packs & 7.25 & 1.82 & 9.07 \\
\hline & Organic debris & 80.06 & 105.94 & 186.00 \\
\hline & Organic homogeneous solids & 0.00 & 1.95 & 1.95 \\
\hline & Organic liquids & 2.20 & 1.40 & 3.60 \\
\hline & Soil/gravel & 0.00 & 190.00 & 190.00 \\
\hline & Unknown/other debris & 0.21 & 0.00 & 0.21 \\
\hline & Unknown/other solids & 4.79 & 0.00 & 4.79 \\
\hline & PANT total & 132.28 & 918.53 & $1,050.82$ \\
\hline \multirow[t]{16}{*}{$\mathrm{PAD}$} & Aqueous liquids/slurries & 61.43 & 0.00 & 61.43 \\
\hline & Batteries & 4.99 & 0.00 & 4.99 \\
\hline & Compressed gases/aerosols & 15.40 & 0.00 & 15.40 \\
\hline & Elemental hazardous metals & 4.25 & 0.00 & 4.25 \\
\hline & Elemental mercury & 0.52 & 0.00 & 0.52 \\
\hline & Explosives/propellants & 0.21 & 0.00 & 0.21 \\
\hline & Heterogeneous debris & 4.63 & 0.00 & 4.63 \\
\hline & Inorganic debris & 541.85 & 0.00 & 541.85 \\
\hline & Inorganic homogeneous solids & 86.95 & 0.00 & 86.95 \\
\hline & Lab packs & 7.44 & 0.00 & 7.44 \\
\hline & Organic debris & 79.11 & 0.00 & 79.11 \\
\hline & Organic homogeneous solids & 20.97 & 0.00 & 20.97 \\
\hline & Organic liquids & 173.21 & 0.00 & 173.21 \\
\hline & Soil/gravel & 5.01 & 0.00 & 5.01 \\
\hline & Unknown/other matrix & 26.25 & 0.00 & 26.25 \\
\hline & PAD total & $1,032.22$ & 0.00 & $1,032.22$ \\
\hline Pinellas & Not applicable & 0.00 & c & c \\
\hline \multirow[t]{15}{*}{ PORTS } & Aqueous liquids/slurries & 119.92 & 83.50 & 203.42 \\
\hline & Batteries & 17.65 & 0.00 & 17.65 \\
\hline & Compressed gases/aerosols & 7.91 & 0.00 & 7.91 \\
\hline & Elemental mercury & 2.66 & 0.00 & 2.66 \\
\hline & Heterogeneous debris & $1,425.46$ & $3,167.20$ & $4,592.66$ \\
\hline & Inorganic debris & 5.64 & 0.00 & 5.64 \\
\hline & Inorganic homogeneous solids & 634.64 & 2.00 & 636.64 \\
\hline & Lab packs & 30.88 & 0.00 & 30.88 \\
\hline & Organic debris & 404.55 & 77.50 & 482.05 \\
\hline & Organic homogeneous solids & 286.56 & 105.00 & 391.56 \\
\hline & Organic liquids & 236.31 & 9.00 & 245.31 \\
\hline & Soil/gravel & $4,837.73$ & 100.00 & $4,937.73$ \\
\hline & Reactive metals & 0.62 & 0.00 & 0.62 \\
\hline & Unknown/other matrix & 0.85 & 0.00 & 0.85 \\
\hline & PORTS total & $8,011.38$ & $3,544.20$ & $11,555.58$ \\
\hline PPPL & Not applicable & 0.00 & $\mathrm{c}$ & c \\
\hline
\end{tabular}


Table 8.5 (continued)

\begin{tabular}{|c|c|c|c|c|}
\hline Site & Physical form ${ }^{b}$ & Inventory & $\begin{array}{l}\text { Five-year } \\
\text { projection }\end{array}$ & Total \\
\hline \multirow[t]{11}{*}{ RFETS } & Aqueous liquids/slurries & 533.26 & 11.17 & 544.43 \\
\hline & Beryllium dust & 3.15 & 0.00 & 3.15 \\
\hline & Elemental hazardous metals & 34.69 & 0.00 & 34.69 \\
\hline & Heterogeneous debris & $1,241.85$ & 822.17 & $2,064.02$ \\
\hline & Inorganic debris & 180.95 & $1,096.38$ & $1,277.33$ \\
\hline & Inorganic homogeneous solids & $10,965.67$ & $1,582.69$ & $12,548.36$ \\
\hline & Lab packs & 47.38 & 40.47 & 87.85 \\
\hline & Organic debris & 14.59 & 38.98 & 53.57 \\
\hline & Organic liquids & 130.80 & 27.23 & 158.03 \\
\hline & Soil/gravel & 406.05 & 386.00 & 792.05 \\
\hline & RFETS total & $13,558.39$ & $4,005.09$ & $17,563.48$ \\
\hline \multirow[t]{7}{*}{ RMI } & Aqueous liquids/slurries & 1.80 & 4.73 & 6.53 \\
\hline & Inorganic debris & 0.00 & 0.23 & 0.23 \\
\hline & Inorganic homogeneous solids & 1.35 & 0.69 & 2.04 \\
\hline & Organic debris & 5.87 & 0.46 & 6.33 \\
\hline & Organic homogeneous solids & 4.93 & 0.69 & 5.62 \\
\hline & Organic liquids & 7.50 & 0.69 & 8.19 \\
\hline & RMI total & 21.45 & 7.49 & 28.94 \\
\hline SLAC & Not applicable & $\mathrm{c}$ & $\mathfrak{c}$ & c \\
\hline $\mathrm{SNL} / \mathrm{CA}$ & Not applicable & 0.00 & c & c \\
\hline \multirow[t]{10}{*}{ SNL/NM } & Aqueous liquids/slurries & 0.03 & 160.00 & 160.03 \\
\hline & Elemental hazardous metals & 0.04 & 1.00 & 1.04 \\
\hline & Elemental mercury & d & 1.00 & 1.00 \\
\hline & Heterogeneous debris & 29.00 & 155.00 & 184.00 \\
\hline & Inorganic debris & 9.74 & 17.00 & 26.74 \\
\hline & Organic debris & 28.06 & 2.00 & 30.06 \\
\hline & Organic liquids & 2.90 & 1.00 & 3.90 \\
\hline & Reactive metals & 0.02 & 1.00 & 1.02 \\
\hline & Unknown/other solids & 0.01 & 1.00 & 1.01 \\
\hline & SNL/NM total & 69.80 & 339.00 & 408.80 \\
\hline \multirow[t]{11}{*}{ SRS } & Aqueous liquids/slurries & 157.60 & $1,556.80$ & $1,714.40$ \\
\hline & Elemental hazardous metals & 155.70 & 45.00 & 200.70 \\
\hline & Elemental mercury & 2.70 & 2.50 & 5.20 \\
\hline & Heterogeneous debris & $2,753.60$ & $1,450.60$ & $4,204.20$ \\
\hline & Inorganic debris & 16.40 & 261.40 & 277.80 \\
\hline & Inorganic homogeneous solids & $2,724.40$ & 154.50 & $2,878.90$ \\
\hline & Organic debris & 13.90 & $1,612.20$ & $1,626.10$ \\
\hline & Organic liquids & 147.40 & 591.70 & 739.10 \\
\hline & Soil/gravel & 16.80 & 0.00 & 16.80 \\
\hline & Unknown/other debris & 951.00 & 0.00 & 951.00 \\
\hline & SRS total & $6,939.50$ & $5,674.70$ & $12,614.20$ \\
\hline \multirow[t]{5}{*}{ WVDP } & Aqueous liquids/slurries & 3.27 & 1.23 & 4.49 \\
\hline & Batteries & 0.20 & 0.14 & 0.34 \\
\hline & Elemental hazardous metals & 1.26 & 0.10 & 1.36 \\
\hline & Elemental mercury & d & 0.00 & d \\
\hline & Heterogeneous debris & 125.54 & 0.07 & 125.61 \\
\hline
\end{tabular}


Table 8.5 (continued)

\begin{tabular}{|c|c|c|c|c|}
\hline Site & Physical form ${ }^{b}$ & Inventory & $\begin{array}{l}\text { Five-year } \\
\text { projection }\end{array}$ & Total \\
\hline WVDP & Inorganic debris & 0.29 & 0.00 & 0.29 \\
\hline \multirow[t]{8}{*}{ (contd.) } & Inorganic homogeneous solids & 10.01 & 32.11 & 42.12 \\
\hline & Lab packs & 0.10 & 0.16 & 0.26 \\
\hline & Organic debris & 1.93 & 2.57 & 4.50 \\
\hline & Organic homogeneous solids & 0.07 & 0.00 & 0.07 \\
\hline & Organic liquids & 2.27 & 2.62 & 4.89 \\
\hline & Unknown/other matrix & 2.86 & 0.00 & 2.86 \\
\hline & Unknown/other solids & 0.02 & 0.02 & 0.04 \\
\hline & WVDP total & 147.82 & 39.01 & 186.83 \\
\hline \multirow[t]{19}{*}{$Y-12$} & Aqueous liquids/slurries & 81.38 & 122.97 & 204.35 \\
\hline & Batteries & 5.20 & 2.54 & 7.74 \\
\hline & Beryllium dust & $\mathrm{d}$ & 0.00 & d \\
\hline & Compressed gases/aerosols & 1.01 & 0.99 & 2.00 \\
\hline & Elemental hazardous metals & 2.26 & 1.30 & 3.56 \\
\hline & Elemental mercury & 1.24 & 0.06 & 1.29 \\
\hline & Heterogeneous debris & 30.73 & 20.17 & 50.90 \\
\hline & Inorganic debris & 13.84 & 37.24 & 51.08 \\
\hline & Inorganic homogeneous solids & $6,620.26$ & $1,293.13$ & $7,913.39$ \\
\hline & Lab packs & 0.05 & 0.00 & 0.05 \\
\hline & Organic debris & 66.22 & 62.68 & 128.90 \\
\hline & Organic homogeneous solids & 51.25 & 7.06 & 58.31 \\
\hline & Organic liquids & 300.92 & 172.88 & 473.80 \\
\hline & Reactive metals & 0.83 & 0.00 & 0.83 \\
\hline & Soil/gravel & $6,476.39$ & 11.24 & $6,487.64$ \\
\hline & Unknown/other liquids & 22.16 & 0.00 & 22.16 \\
\hline & Unknown/other matrix & 0.03 & 0.04 & 0.06 \\
\hline & Unknown/other solids & 55.81 & 0.00 & 55.81 \\
\hline & $Y-12$ total & $13,729.58$ & $1,732.32$ & $15,461.87$ \\
\hline \multirow[t]{10}{*}{ Otherg } & Aqueous liquids/slurries & 12.83 & 0.00 & 12.83 \\
\hline & Elemental hazardous metals & 1.26 & 1.30 & 2.55 \\
\hline & Heterogeneous debris & 8.93 & 10.83 & 19.76 \\
\hline & Inorganic debris & 12.00 & 3.50 & 15.50 \\
\hline & Inorganic homogeneous solids & 2.13 & 0.00 & 2.13 \\
\hline & Lab packs & 0.02 & 0.02 & 0.04 \\
\hline & Organic liquids & 0.01 & 0.00 & 0.01 \\
\hline & Soil/gravel & 0.71 & 11.40 & 12.11 \\
\hline & Other total & 37.89 & 27.05 & 64.93 \\
\hline & Grand total (DOE complex) & $107,911.20$ & $28,624.11$ & $136,535.30$ \\
\hline
\end{tabular}

${ }^{a}$ Based on the STP Data Base, ref. 9. The currentness of these data for the various DOE sites ranges from January 1995 to June 1996.

${ }^{b}$ As described in Table 8.4.

CInformation not available or unknown.

dValue $<0.005 \mathrm{~m}^{3}$.

Includes contributions from the Idaho Naval Reactors Facility.

fIncludes contributions, if any, from Bettis Atomic Power Laboratory (BAPL), Knolls Atomic Power Laboratory (KAPL), Kesselring Site, and Windsor Site. Also includes contributions, if any, from the Charleston, Mare Island, Norfolk, Pearl Harbor, Portsmouth, and Puget Sound Naval Shipyards.

gIncludes contributions, if any, from Battelle Columbus Laboratory (BCL), Colonie Interim Storage Site (CISS), General Atomics (GA), Laboratory for Energy Related Health Research (LEHR), and Missouri University. 
Table 8.6. Estimated volume $\left(\mathrm{m}^{3}\right)$ inventories and five-year projections of DOE RCRA MLLW as of 1995 , by physical form ${ }^{a}$

\begin{tabular}{|c|c|c|c|}
\hline Physical form ${ }^{b}$ & Inventory & $\begin{array}{l}\text { Five-year } \\
\text { projection }\end{array}$ & Total \\
\hline \multicolumn{4}{|l|}{ Liquids } \\
\hline Aqueous liquids/slurries & $4,972.48$ & $3,261.80$ & $8,234.28$ \\
\hline Organic liquids & $2,087.40$ & $2,476.99$ & $4,564.39$ \\
\hline Unknown/other liquids & 25.87 & 4.55 & 30.41 \\
\hline \multicolumn{4}{|l|}{ Solids } \\
\hline Heterogeneous debris & $26,404.90$ & $7,655.13$ & $34,060.03$ \\
\hline Inorganic debris & $2,089.40$ & $3,574.23$ & $5,663.63$ \\
\hline Inorganic homogeneous solids & $50,816.66$ & $4,379.29$ & $55,195.95$ \\
\hline Organic debris & $3,328.24$ & $2,826.39$ & $6,154.63$ \\
\hline Organic homogeneous solids & $1,279.28$ & 211.22 & $1,490.50$ \\
\hline Soil/gravel & $12,999.32$ & $3,071.73$ & $16,071.05$ \\
\hline Unknown/other debris & $1,116.36$ & 11.30 & $1,127.66$ \\
\hline Unknown/other homogeneous solids & 30.46 & 70.30 & 100.75 \\
\hline Unknown/other solids & 127.37 & 32.87 & 160.24 \\
\hline \multicolumn{4}{|l|}{ Specific waste forms } \\
\hline Batteries & 53.72 & 29.66 & 83.39 \\
\hline Beryilium dust & 3.32 & 0.00 & 3.32 \\
\hline Compressed gases/aerosols & 31.49 & 4.94 & 36.43 \\
\hline Elemental hazardous metals & $1,069.32$ & 404.82 & $1,474.14$ \\
\hline Elemental mercury & 13.65 & 5.02 & 18.67 \\
\hline Explosives/propellants & 0.21 & 1.15 & 1.36 \\
\hline Lab packs & 505.36 & 199.41 & 704.77 \\
\hline Reactive metals & 401.12 & 346.81 & 748.92 \\
\hline Final waste forms & 89.25 & 9.20 & 98.45 \\
\hline Unknown/other matrix & 464.96 & 47.32 & 512.28 \\
\hline Total & $107,911.15$ & $28,624.11$ & $136,535.26$ \\
\hline
\end{tabular}

a Based on the STP Data Base, ref. 9.

$\mathrm{b}_{\text {As described in Table } 8.4 .}$ 
Table 8.7. Significant revisions and changes in historical volume $\left(\mathrm{m}^{3}\right)$ inventory data for DOE RCRA MLLW compared to volume $\left(\mathrm{m}^{3}\right)$ inventory data reported previously

\begin{tabular}{|c|c|c|c|}
\hline Site & $\begin{array}{l}\text { Values in current } \\
\text { report }\end{array}$ & $\begin{array}{l}\text { Values in previous } \\
\text { report }\end{array}$ & Explanation \\
\hline Hanford & 8,170 & 6,331 & $\begin{array}{l}\text { The increase in Hanford inventory is } \\
\text { due to on-site generation and receipts of } \\
\text { off-site shipments }\end{array}$ \\
\hline $\mathrm{K}-25$ & 23,076 & 29,473 & $\begin{array}{l}\text { The decrease in } \mathrm{K}-25 \text { inventory is } \\
\text { primarily due to the shipment of } \\
\text { stabilized pond sludge wastes to } \\
\text { Envirocare for disposal }\end{array}$ \\
\hline Middlesex & NA & 24,480 & $\begin{array}{l}\text { The previous report included mixed } \\
11 \text { e(2) wastes at part of the RCRA } \\
\text { MLLW inventories. In this current } \\
\text { report, mixed } 11 \text { er( } 2) \text { wastes are } \\
\text { addressed exclusively in Chapter } 6\end{array}$ \\
\hline WSSRAP & NA & 2,022 & $\begin{array}{l}\text { The previous report included mixed } \\
11 \text { e( } 2 \text { ) wastes at part of the RCRA } \\
\text { MLLW inventories. In this current } \\
\text { report, mixed } 11 \text { e( } 2 \text { ) wastes are } \\
\text { addressed exclusively in Chapter } 6\end{array}$ \\
\hline
\end{tabular}

aDOE/RW-0006, Rev. 12 (December 1996).

bOE/RW-0006, Rev. 11 (September 1995). 
Table 8.8. Mass $(\mathrm{kg})$ inventories through 1995 , by hazard category, of TSCA-regulated MLLW from DOE site activities ${ }^{\mathrm{a}}$

\begin{tabular}{|c|c|c|c|c|}
\hline Site & PCB & Asbestos & Other & Total \\
\hline Ames & 0 & 140 & 0 & 140 \\
\hline ANL-E & 55,636 & 0 & 0 & 55,636 \\
\hline ANL $-\mathrm{W}$ & 333 & 6,114 & 0 & 6,447 \\
\hline BNL & 1,300 & 1,000 & 0 & 2,300 \\
\hline ETEC & 0 & 0 & 0 & 0 \\
\hline FEMP & 0 & 0 & 0 & 0 \\
\hline FNAL & 0 & 0 & 0 & 0 \\
\hline Hanford $b$ & 117,100 & $\mathrm{c}$ & 0 & 117,100 \\
\hline INELd & 850 & 0 & 0 & 850 \\
\hline ITRI & 0 & 0 & 0 & 0 \\
\hline $\mathrm{K}-25$ & $5,998,200$ & 0 & 0 & $5,998,200$ \\
\hline $\mathrm{KCP}$ & 0 & 0 & 0 & 0 \\
\hline LANL & 65,570 & 79,840 & 0 & 145,410 \\
\hline LBL & 0 & 307 & 0 & 307 \\
\hline LLNL & 780 & 58 & 0 & 838 \\
\hline Mound & 2,750 & 20,600 & 0 . & 23,350 \\
\hline NR sites ${ }^{e}$ & 9,240 & 0 & 0 & 9,240 \\
\hline NTS & 165 & 0 & 0 & 165 \\
\hline ORISE & 0 & 0 & 0 & 0 \\
\hline ORNL & 16,534 & 0 & 0 & 16,534 \\
\hline $\mathrm{PAD}$ & $\mathbf{f}$ & $\mathrm{f}$ & 0 & $\mathrm{f}$ \\
\hline PANT & 365 & 1,262 & 0 & 1,627 \\
\hline Pinellas & 0 & 0 & 0 & 0 \\
\hline PORTS & $\mathrm{f}$ & 0 & 0 & f \\
\hline PPPL & 0 & 0 & 0 & 0 \\
\hline RFETS & 192,000 & 101,000 & 0 & 293,000 \\
\hline SLAC & 0 & 0 & 0 & 0 \\
\hline SNL/CA & 0 & 0 & 0 & 0 \\
\hline SNL/NM & 0 & 0 & 0 & 0 \\
\hline SRS & 1,540 & $f$ & $f$ & 1,540 \\
\hline WVDP & 11,086 & 15,727 & 0 & 26,813 \\
\hline$Y-12$ & 460,190 & 0 & 0 & 460,190 \\
\hline Total & $6,933,639$ & 226,048 & 0 & $7,159,687$ \\
\hline
\end{tabular}

${ }^{a}$ Based on the DOE site data submittals of ref. 11. Material may be in interim storage awaiting treatment. Specific site information is provided in Sect. 8.4.2.

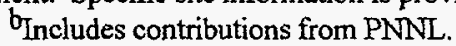

Included in the Hanford LLW inventories reported in Chapter 4.

dExcludes contributions from ANL-W.

'Includes only contributions from Bettis Atomic Power Laboratory (BAPL).

Information not available or unknown. 
Table 8.9. Volume $\left(\mathrm{m}^{3}\right)$ inventories through 1995 , by hazard category, of TSCA-regulated MLLW from DOE site activities ${ }^{a}$

\begin{tabular}{|c|c|c|c|c|}
\hline Site & PCB & Asbestos & Other & Total \\
\hline Ames & 0.0 & 0.7 & 0.0 & 0.7 \\
\hline ANL-E & 57.0 & 0.0 & 0.0 & 57.0 \\
\hline ANL-W & 0.3 & 12.5 & 0.0 & 12.8 \\
\hline $\mathrm{BNL}$ & 1.3 & 2.1 & 0.0 & 3.4 \\
\hline ETEC & 0.0 & 0.0 & 0.0 & 0.0 \\
\hline FEMP & 0.0 & 0.0 & 0.0 & 0.0 \\
\hline FNAL & 0.0 & 0.0 & 0.0 & 0.0 \\
\hline Hanford $b$ & 102.5 & c & 0.0 & 102.5 \\
\hline $\mathrm{INEL}^{\mathrm{d}}$ & 2.2 & 0.0 & 0.0 & 2.2 \\
\hline ITRI & 0.0 & 0.0 & 0.0 & 0.0 \\
\hline$K-25$ & $8,159.3$ & 0.0 & 0.0 & $8,159.3$ \\
\hline $\mathrm{KCP}$ & 0.0 & 0.0 & 0.0 & 0.0 \\
\hline LANL & $2,105.0$ & $4,087.0$ & 0.0 & $6,192.0$ \\
\hline LBL & 0.0 & 0.8 & 0.0 & 0.8 \\
\hline LLNL & 0.5 & $<0.1$ & 0.0 & 0.6 \\
\hline Mound & 3.9 & 41.2 & 0.0 & 45.1 \\
\hline NR sitese & 18.3 & 0.0 & 0.0 & 18.3 \\
\hline NTS & 0.1 & 0.0 & 0.0 & 0.1 \\
\hline ORISE & 0.0 & 0.0 & 0.0 & 0.0 \\
\hline ORNL & 16.3 & 0.0 & 0.0 & 16.3 \\
\hline $\mathrm{PAD}$ & $3,500.0$ & 430.0 & 0.0 & $3,930.0$ \\
\hline PANT & 0.3 & 2.4 & 0.0 & 2.7 \\
\hline Pinellas & 0.0 & 0.0 & 0.0 & 0.0 \\
\hline PORTS & $3,600.0$ & 0.0 & 0.0 & $3,600.0$ \\
\hline PPPL & 0.0 & 0.0 & 0.0 & 0.0 \\
\hline RFETS & 128.0 & 62.9 & 0.0 & 190.9 \\
\hline SLAC & 0.0 & 0.0 & 0.0 & 0.0 \\
\hline $\mathrm{SNL} / \mathrm{CA}$ & 0.0 & 0.0 & 0.0 & 0.0 \\
\hline SNL/NM & 0.0 & 0.0 & 0.0 & 0.0 \\
\hline SRS & 110.0 & $\mathrm{f}$ & f & 110.0 \\
\hline WVDP & 13.0 & 69.2 & 0.0 & 82.2 \\
\hline$Y-12$ & 940.8 & 0.0 & 0.0 & 940.8 \\
\hline Total & $18,758.8$ & $4,708.9$ & 0.0 & $23,467.7$ \\
\hline
\end{tabular}

${ }^{2} B a s e d$ on the DOE site data submittals of ref. 11 . Material may be in interim storage awaiting treatment. Specific site information is provided in Sect. 8.4.2.

Includes contributions from PNNL.

Included in the Hanford LLW inventories reported in Chapter 4.

dExcludes contributions from ANL-W.

Tncludes only contributions from Bettis Atomic Power Laboratory (BAPL).

Information not available or unknown. 
Table 8.10. 1995 mass ( $\mathrm{kg}$ ) generation, by hazard category, of TSCA-regulated MLLW from DOE site activities ${ }^{\mathrm{a}}$

\begin{tabular}{|c|c|c|c|c|}
\hline Site & PCB & Asbestos & Other & Total \\
\hline Ames & 0 & 100 & 0 & 100 \\
\hline ANL-E & 0 & 0 & 0 & 0 \\
\hline ANL_W & 0 & 17 & 0 & 17 \\
\hline $\mathrm{BNL}$ & 230 & 230 & 0 & 460 \\
\hline ETEC & 0 & 18,000 & 0 & 18,000 \\
\hline FEMP & 0 & 0 & 0 & 0 \\
\hline FNAL & 0 & 0 & 0 & 0 \\
\hline Hanford $b$ & 6,900 & $\mathrm{c}$ & 0 & 6,900 \\
\hline NNELd & 54 & 0 & 0 & 54 \\
\hline ITRI & 0 & 0 & 0 & 0 \\
\hline $\mathrm{K}-25$ & 175,500 & 0 & 0 & 175,500 \\
\hline $\mathrm{KCP}$ & 0 & 0 & 0 & 0 \\
\hline LANL & 16 & 18,390 & 0 & 18,406 \\
\hline LBL & 0 & 125 & 0 & 125 \\
\hline LLNL & 53 & 7 & 0 & 60 \\
\hline Mound & 15 & 3,500 & 0 & 3,515 \\
\hline NR sites ${ }^{e}$ & 505 & $28,161^{f}$ & 0 & 28,666 \\
\hline NTS & 0 & 0 & 0 & 0 \\
\hline ORISE & 0 & 0 & 0 & 0 \\
\hline ORNL & 16,492 & 0 & 0 & 16,492 \\
\hline PADg & 224,470 & 187,794 & 0 & 412,264 \\
\hline PANT & 1,017 & 146,288 & 0 & 147,305 \\
\hline Pinellas & 0 & 0 & 0 & 0 \\
\hline PORTSE & 79,990 & 11,500 & 0 & 91,490 \\
\hline PPPL & 0 & 0 & 0 & 0 \\
\hline RFETS & $h$ & $\mathrm{~h}$ & $h$ & $\mathrm{~h}$ \\
\hline SLAC & 0 & 0 & 0 & 0 \\
\hline SNL/CA & 0 & 0 & 0 & 0 \\
\hline SNL/NM & 0 & 0 & 0 & 0 \\
\hline SRS & 0 & h & $\mathrm{h}$ & 0 \\
\hline WVDP & 10,090 & 4,877 & 0 & 14,967 \\
\hline$Y-12$ & 27,227 & 0 & 0 & 27,227 \\
\hline Total & 542,559 & 418,989 & 0 & 961,548 \\
\hline
\end{tabular}

${ }^{a}$ Based on the DOE site data submittals of ref. 11. Material may be in interim storage awaiting treatment. Specific site information is provided in Sect. 8.4.2.

$b_{\text {Includes contributions from PNNL. }}$

Included in the Hanford LLW generation reported in Chapter 4.

dExcludes contributions from ANL-W.

Includes contributions from Bettis Atomic Power Laboratory (BAPL), Knolls Atomic Power Laboratory (KAPL), Kesselring Site, and Windsor Site.

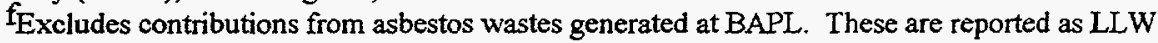
in Chapter 4.

gInformation for CY 1994. Information for CY 1995 not available.

$\mathrm{h}_{\text {Information not available or unknown. }}$ 
Table 8.11. 1995 volume $\left(\mathrm{m}^{3}\right)$ generation, by hazard category, of TSCA-regulated MLLW from DOE site activities ${ }^{\mathrm{a}}$

\begin{tabular}{|c|c|c|c|c|}
\hline Site & $\mathrm{PCB}$ & Asbestos & Other & Total \\
\hline Ames & 0.0 & 0.2 & 0.0 & 0.2 \\
\hline ANL-E & 0.0 & 0.0 & 0.0 & 0.0 \\
\hline ANL-W & 0.0 & $<0.1$ & 0.0 & $<0.1$ \\
\hline BNI & 0.2 & 0.4 & 0.0 & 0.6 \\
\hline ETEC & 0.0 & 18.5 & 0.0 & 18.5 \\
\hline FEMP & 0.0 & 0.0 & 0.0 & 0.0 \\
\hline FNAL & 0.0 & 0.0 & 0.0 & 0.0 \\
\hline Hanford $b$ & 6.9 & c & 0.0 & 6.9 \\
\hline INEL d & 0.3 & 0.0 & 0.0 & 0.3 \\
\hline ITRI & 0.0 & 0.0 & 0.0 & 0.0 \\
\hline $\mathrm{K}-25$ & 321.8 & 0.0 & 0.0 & 321.8 \\
\hline $\mathrm{KCP}$ & 0.0 & 0.0 & 0.0 & 0.0 \\
\hline LANL & 0.3 & 68.6 & 0.0 & 68.9 \\
\hline LBL & 0.0 & 0.3 & 0.0 & 0.3 \\
\hline LLNL & $<0.1$ & $<<0.1$ & 0.0 & $<0.1$ \\
\hline Mound & $<0.1$ & 7.0 & 0.0 & 7.1 \\
\hline NR sites $\mathrm{e}$ & 0.4 & $21.8^{f}$ & 0.0 & 22.2 \\
\hline NTS & 0.0 & 0.0 & 0.0 & 0.0 \\
\hline ORISE & 0.0 & 0.0 & 0.0 & 0.0 \\
\hline ORNL & 20.1 & 0.0 & 0.0 & 20.1 \\
\hline PADg & 286.1 & 774.7 & 0.0 & $1,060.8$ \\
\hline PANT & 0.8 & 357.4 & 0.0 & 358.2 \\
\hline Pinellas & 0.0 & 0.0 & 0.0 & 0.0 \\
\hline PORTSg & 250.0 & 9.9 & 0.0 & 259.9 \\
\hline PPPL & 0.0 & 0.0 & 0.0 & 0.0 \\
\hline RFETS & $\mathrm{h}$ & h & $\mathrm{h}$ & $\mathrm{h}$ \\
\hline SLAC & 0.0 & 0.0 & 0.0 & 0.0 \\
\hline $\mathrm{SNL} / \mathrm{CA}$ & 0.0 & 0.0 & 0.0 & 0.0 \\
\hline SNL/NM & 0.0 & 0.0 & 0.0 & 0.0 \\
\hline SRS & 0.0 & $\mathrm{~h}$ & h & 0.0 \\
\hline WVDP & 10.4 & 25.2 & 0.0 & 35.6 \\
\hline $\mathrm{Y}-12$ & 52.1 & 0.0 & 0.0 & 52.1 \\
\hline Total & 949.6 & $1,284.1$ & 0.0 & $2,233.7$ \\
\hline
\end{tabular}

aBased on the DOE site data submittals of ref. 11. Material may be in interim storage awaiting treatment. Specific site information is provided in Sect. 8.4.2.

bincludes contributions from PNNL.

Included in the Hanford LLW generation reported in Chapter 4.

Excludes contributions from ANL-W.

Includes contributions from Bettis Atomic Power Laboratory (BAPL), Knolls Atomic Power Laboratory (KAPL), Kesselring Site, and Windsor Site.

$\mathrm{f}_{\text {Excludes contributions from asbestos wastes generated at BAPL. These are reported as LLW }}$ in Chapter 4.

gInformation for CY 1994. Information for CY 1995 not available.

$\mathrm{h}_{\text {Information not available or unknown. }}$ 
Table 8.12. Historical and projected annual mass (kg) generation rates for TSCA-regulated MLLW from DOE site activities ${ }^{a}$

\begin{tabular}{|c|c|c|c|c|}
\hline Site & $1995^{b}$ & 1996 & $1997-2001^{\mathrm{c}}$ & $2002-2030^{c}$ \\
\hline Ames & 100 & 100 & 100 & 100 \\
\hline ANL-E & 0 & d & d & d \\
\hline ANL $-W$ & 17 & $d$ & $d$ & d \\
\hline BNL & 460 & 180 & 400 & 400 \\
\hline ETEC & 18,000 & 145,000 & 18,000 & 0 \\
\hline FEMP & 0 & $d$ & d & d \\
\hline FNAL & 0 & 0 & 0 & 0 \\
\hline Hanford ${ }^{e}$ & 6,900 & $\mathrm{f}$ & $\mathrm{f}$ & $f$ \\
\hline INEL $\mathbf{g}$ & 54 & d & d & d \\
\hline ITRI & 0 & 0 & 0 & 0 \\
\hline $\mathrm{K}-25$ & 175,500 & 175,500 & 175,500 & 175,500 \\
\hline $\mathrm{KCP}$ & 0 & 0 & 0 & 0 \\
\hline LANL & 18,406 & 118,400 & 118,400 & 94,750 \\
\hline LB. & 125 & 0 & 125 & 125 \\
\hline LLINL & 60 & 0 & 0 & 0 \\
\hline Mound & 3,515 & 3,500 & 3,500 & 3,500 \\
\hline NR sites $\mathrm{h}, \mathrm{i}$ & 28,666 & 42,919 & 72,656 & 19,500 \\
\hline NTS & 0 & d & $\mathrm{d}$ & d \\
\hline ORISE & 0 & 0 & 0 & $0^{\circ}$ \\
\hline ORNL & 16,492 & 16,492 & 16,492 & 16,492 \\
\hline PAD & 412,264 & $\mathrm{~d}$ & d & $\dot{d}$ \\
\hline PAINT & 147,305 & 82,199 & 43,722 & 4,893 \\
\hline Pinellas & 0 & & & \\
\hline PORTS & 91,490 & d & d & d \\
\hline PPPL & 0 & 0 & 0 & 0 \\
\hline RFETS & d & d & $d$ & $\mathrm{~d}$ \\
\hline SLAC & 0 & 0 & 0 & 0 \\
\hline SNL/CA & 0 & 0 & 0 & 0 \\
\hline SNL/NM & 0 & 0 & 0 & 0 \\
\hline SRS & 0 & 0 & d & d \\
\hline WVDP & 14,967 & 7,500 & d & d \\
\hline$Y-12$ & 27,227 & 27,227 & 27,227 & 27,227 \\
\hline Total & 961,548 & 619,017 & 476,122 & 342,487 \\
\hline
\end{tabular}

a Based on the DOE site data submittals of ref. 11. Specific site information is provided in Sect. 8.4.2.

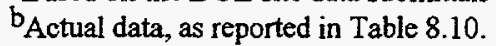

$\mathrm{c}_{\text {Average annual generation rate anticipated for this period. }}$

Information not available or unknown.

Encludes contributions from PNNL.

fHanford projections for PCB's are included in the RCRA/state category of this chapter. Hanford projections for asbestos are included in the Hanford LLW projections of Chapter 4.

gExcludes contribution from ANL-W.

hincludes contributions from INEL Naval Reactors Facility (NRF), Bettis Atomic Power Laboratory (BAPL), Knolls Atomic Power Laboratory (KAPL), Kesselring Site, and Windsor Site.

iExcludes contributions from asbestos wastes generated at BAPL. These are reported as LLW in Chapter 4. 
Table 8.13. Historical and projected annual volume $\left(\mathrm{m}^{3}\right)$ generation rates for TSCA-regulated MLLW from DOE site activities ${ }^{a}$

\begin{tabular}{|c|c|c|c|c|}
\hline Site & $1995^{b}$ & 1996 & $1997-2001^{c}$ & $2002-2030^{c}$ \\
\hline Ames & 0.2 & 0.2 & 0.2 & 0.2 \\
\hline ANL-E & 0.0 & $d$ & d & d \\
\hline ANL-W & $<0.1$ & $d$ & d & $d$ \\
\hline BNL & 0.6 & 0.7 & 0.7 & 0.7 \\
\hline ETEC & 18.5 & 147.0 & 18.4 & 0.0 \\
\hline FEMP & 0.0 & d & d & d \\
\hline FNAL & 0.0 & 0.0 & 0.0 & 0.0 \\
\hline Hanford ${ }^{e}$ & 6.9 & $\mathrm{f}$ & $\mathrm{f}$ & $\mathrm{f}$ \\
\hline INELg & 0.3 & $d$ & d & $d$ \\
\hline ITRI & 0.0 & 0.0 & 0.0 & 0.0 \\
\hline $\mathrm{K}-25$ & 321.8 & 321.8 & 321.8 & 321.8 \\
\hline $\mathrm{KCP}$ & 0.0 & 0.0 & 0.0 & 0.0 \\
\hline LANL & 68.9 & 146.8 & 146.8 & 117.5 \\
\hline LBL & 0.3 & 0.0 & 0.3 & 0.3 \\
\hline LLNL & $<0.1$ & 0.0 & 0.0 & 0.0 \\
\hline Mound & 7.1 & 7.0 & 7.0 & 7.0 \\
\hline NR sitesh,i & 22.2 & 36.7 & 64.4 & 14.9 \\
\hline NTS & 0.0 & d & $d$ & $\mathrm{~d}$ \\
\hline ORISE & 0.0 & 0.0 & 0.0 & 0.0 \\
\hline ORNL & 20.1 & 20.1 & 20.1 & 20.1 \\
\hline PAD & $1,060.8$ & d & d & $\mathrm{d}$ \\
\hline PANT & 358.2 & 200.3 & 106.5 & 44.9 \\
\hline Pinellas & 0.0 & & & \\
\hline PORTS & 259.9 & d & d & d \\
\hline PPPL & 0.0 & 0.0 & 0.0 & 0.0 \\
\hline RFETS & d & d & d & d \\
\hline SLAC & 0.0 & 0.0 & 0.0 & 0.0 \\
\hline SNL/CA & 0.0 & 0.0 & 0.0 & 0.0 \\
\hline SNL/NM & 0.0 & 0.0 & 0.0 & 0.0 \\
\hline SRS & 0.0 & 0.0 & d & d \\
\hline WVDP & 35.6 & 6.6 & $d$ & $d$ \\
\hline $\mathrm{Y}-12$ & 52.1 & .52 .1 & 52.1 & 52.1 \\
\hline Total & $2,233.7$ & 939.3 & 738.3 & 579.5 \\
\hline
\end{tabular}

${ }^{a}$ Based on the DOE site data submittals of ref. 11. Specific site information is provided in Sect. 8.4.2.

${ }_{\text {Actual data, as reported in Table 8.11. }}$.

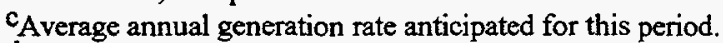

Information not available or unknown.

Includes contributions from PNNL.

fHanford projections for PCB's are included in the RCRA/state category of this chapter. Hanford projections for asbestos are included in the Hanford LLW projections of Chapter 4.

EExcludes contribution from ANL-W.

hincludes contributions from INEL Naval Reactors Facility (NRF), Bettis Atomic Power Laboratory (BAPL), Knolls Atomic Power Laboratory (KAPL), Kesselring Site, and Windsor Site.

iExcludes contributions from asbestos wastes generated at BAPL. These are reported as LLW in Chapter 4. 
Table 8.14. Significant revisions and changes in historical inventory data for $D O E$ TSCA MLLW compared to inventory data reported previously

\begin{tabular}{llcccl}
\hline Site & Waste characteristics & $\begin{array}{c}\text { Values in } \\
\text { current } \\
\text { report }\end{array}$ & $\begin{array}{c}\text { Values in } \\
\text { previous } \\
\text { report }\end{array}$ & $\begin{array}{c}\text { Significant } \\
\text { revisions } \\
\text { and changes }\end{array}$ & Explanation \\
\hline Y-12 & $\begin{array}{l}\text { Inventory of radioactive } \\
\text { PCB wastes }\end{array}$ & $\begin{array}{l}941 \mathrm{~m}^{3} \\
(460,190 \mathrm{~kg})\end{array}$ & $\begin{array}{c}150 \mathrm{~m}^{3} \\
(204,544 \mathrm{~kg})\end{array}$ & $\begin{array}{c}+791 \mathrm{~m}^{3} \\
(+255,646 \mathrm{~kg})\end{array}$ & $\begin{array}{l}\text { Large increase is mainly } \\
\text { due to wastes from site } \\
\text { reroofing activities during } \\
\text { CY 1995 }\end{array}$ \\
\hline
\end{tabular}

aDOE/RW-0006, Rev. 12 (December 1996).

bOE/RW-0006, Rev. 11 (September 1995).

Table 8.15. National commercially generated MLLW profile volume summary, by facility category ${ }^{a}$

\begin{tabular}{lccr}
\hline & \multicolumn{3}{c}{ Waste volume, $\mathrm{m}^{3}$} \\
\cline { 2 - 4 } Facility category & $\begin{array}{c}\text { Generated } \\
\text { in } 1990\end{array}$ & $\begin{array}{c}\text { Stored as of } \\
\text { Dec. } 31,1990^{\mathrm{b}}\end{array}$ & $\begin{array}{r}\text { Treated } \\
\text { in } 1990^{\mathrm{c}}\end{array}$ \\
\hline Academic & 820.7 & 154.2 & $1,581.9$ \\
Government & 750.4 & 78.9 & 612.5 \\
Industrial & $1,428.0$ & $1,197.3$ & $1,115.1$ \\
Medical & 563.6 & 63.1 & 466.3 \\
Nuclear power plants & 385.8 & 622.5 & 216.9 \\
\multicolumn{1}{c}{ Total $^{\mathrm{d}}$} & $3,948.5$ & $2,116.0$ & $3,992.6$ \\
\hline
\end{tabular}

aBased on ref. 7. treatment.

This is not the amount of mixed waste requiring disposal. Some of this waste was being accumulated for

CTreated wastes may include mixed wastes generated in years prior to 1990.

Total reported in this table may not equal the sum of component entries because of round-off and truncation of nurnbers. 
Table 8.16. National commercially generated MLLW profile volume summary, by hazardous waste stream ${ }^{\mathrm{a}}$

\begin{tabular}{|c|c|c|c|}
\hline \multirow[b]{2}{*}{ Hazardous stream } & \multicolumn{3}{|c|}{ Waste volume, $\mathrm{m}^{3}$} \\
\hline & $\begin{array}{l}\text { Generated } \\
\text { in } 1990\end{array}$ & $\begin{array}{l}\text { Stored as of } \\
\text { Dec. } 31,1990^{b}\end{array}$ & $\begin{array}{l}\text { Treated } \\
\text { in } 1990^{\mathrm{c}}\end{array}$ \\
\hline \multicolumn{4}{|l|}{ Organics } \\
\hline Liquid scintillation fluids & $2,837.2$ & 363.4 & $3,371.8$ \\
\hline Waste oil & 148.9 & 178.1 & 139.4 \\
\hline Chlorinated organics & 70.9 & 27.0 & 23.2 \\
\hline Fluorinated organics & 0 & 3.5 & 0 \\
\hline Chlorinated fluorocarbons (CFCs) & 113.2 & 254.7 & 3.7 \\
\hline Other organics & 274.6 & 117.9 & 258.9 \\
\hline Total organics ${ }^{d}$ & $3,444.8$ & 944.6 & $3,797.0$ \\
\hline \multicolumn{4}{|l|}{ Metals } \\
\hline Lead & 81.6 & 138.7 & 6.1 \\
\hline Mercury & 12.5 & 81.1 & 1.5 \\
\hline Chromium & 28.4 & 53.3 & 3.9 \\
\hline Cadmium & 0.3 & 745.2 & 0.1 \\
\hline Total metals ${ }^{d}$ & 122.8 & $1,018.3$ & 11.6 \\
\hline Aqueous corrosives & 80.4 & 12.2 & 2.6 \\
\hline Other hazardous materials & 300.5 & 141.0 & 181.4 \\
\hline Grand totals ${ }^{\mathrm{d}}$ & $3,948.5$ & $2,116.0$ & $3,992.6$ \\
\hline
\end{tabular}

a Based on ref. 7 .

${ }^{b}$ This is not the amount of mixed waste requiring disposal. Some of this waste was being accumulated for treatment.

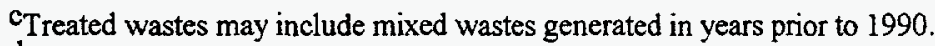

d Totals reported in this table may not equal the sum of component entries because of round-off and truncation of numbers. 

APPENDIX A. RADIOACTIVE WASTE SOURCE TERMS AND CHARACTERISTICS 



\section{APPENDIX A. RADIOACTIVE WASTE SOURCE TERMS AND CHARACTERISTICS}

\section{A.1 DISCUSSION}

In this report, a number of engineering estimates, assumptions, and ground rules are used to determine radioactive waste and spent nuclear fuel projections through the year 2030. Many of these involve parameters that characterize certain types of waste (e.g., see Table A.1). In other instances, estimates were made of the waste volume generated per unit of product throughput for each step in the fuel cycle. This appendix is a compilation of source terms and characteristics used for making radioactive waste projections. Source terms are used to describe quantitative and qualitative characteristics of radioactive wastes. As used in this report, the source term for a particular waste is comprised of two components unique to that waste: (1) the number of curies of radioactivity expressed either per unit of facility production or per unit of waste volume or mass and (2) a listing of the relative radioactivity contributions of component radioisotopes.

The source terms used in the analysis of this report are based on reported historical data, engineering estimates, calculations, and/or experimental data. Documentation of the source terms and key waste-modeling parameters is provided in the following sets of figures and tables (based primarily on refs. 1 through 6). Detailed information on how these source terms and modeling parameters were derived is available, mainly in ref. 1 and its update (ref. 2).

Representative DOE LLW radionuclide compositions are described in Table A.2 (based on ref. 1). Average concentrations for representative radionuclides in LLW disposed of at commercial sites are given in Table A.3 This information was adapted from ref. 3. Table A.4 gives a summary of major sources and estimated characteristics of commercial greater-than-Class-C LLW (data from refs. 4 and 5). Inventories of the LLW incorporated in cement by the West Valley Demonstration Project Radwaste Treatment System are given in Table A.5, which is based on ref. 6 .

\section{A.2 REFERENCES}

1. C. W. Forsberg, W. L. Carter, and A. H. Kibbey, Flowsheets and Source Terms for Radioactive Waste Projections, ORNL/TM-8462, Oak Ridge National Laboratory, Oak Ridge, Tennessee (March 1985).

2. S. L. Loghry, A. H. Kibbey, H. W. Godbee, A. S. Icenhour, and S. M. DePaoli, Low-Level Radioactive Waste Source Terms for the 1992 Integrated Data Base, ORNLTM-11710, Oak Ridge National Laboratory, Oak Ridge, Tennessee (January 1995).

3. G. W. Roles, Characteristics of Low-Level Radioactive Waste Disposed During 1987 Through 1989, NUREG-1418, U.S. Nuclear Regulatory Commission, Washington, D.C. (December 1990).

4. U.S. Department of Energy, Recommendations for Management of Greater-than-Class-C Low-Level Radioactive Waste, DOE/NE-0077, Washington, D.C. (February 1987).

5. O. I. Oztunali, W. D. Pon, R. Eng, and G. W. Roles, Update of Part 61 Impacts Analysis Methodology, Vol. 2 , NUREG/CR-4370, U.S. Nuclear Regulatory Commission, Washington, D.C. (January 1986). 
6. John J. Hollinden, West Valley Nuclear Services Company, Inc., West Valley, New York, correspondence to Steve Storch, DB Program, ORNL, Oak Ridge, Tennessee, "Submittal of Update Information for the 1996 Integrated Data Base Report for the Radwaste Treatment System," WZ:96:0081, dated Aug. 23, 1996. 
Table A.1. Estimated representative unit activity and thermal power characteristics of various types of radioactive materials and wastes

\begin{tabular}{lcc}
\hline $\begin{array}{c}\text { Radioactive material } \\
\text { waste type }\end{array}$ & $\begin{array}{c}\text { Unit activity } \\
\left(\mathrm{Ci} / \mathrm{m}^{3}\right)\end{array}$ & $\begin{array}{c}\text { Unit thermal power } \\
\left(\mathrm{W} / \mathrm{m}^{3}\right)\end{array}$ \\
\hline $\begin{array}{l}\text { Spent nuclear fuel } \\
\text { BWR }\end{array}$ & $1,000,000-10,000,000$ & $3,500-40,000$ \\
PWR & $2,000,000-20,000,000$ & $7,500-65,000$ \\
High-level waste & $1,500-15,000$ & $5-50$ \\
Transuranic waste & & \\
Remote-handled, stored & 1,000 & $1-2$ \\
Contact-handled, stored & $25-50$ & $0.5-1.5$ \\
Buried & $0.25-0.50$ & $0.005-0.010$ \\
& & \\
Low-level waste & & $0.012-0.054$ \\
DOE sites & $9-27$ & $0.30-1.60$ \\
Commercial sites & $\mathrm{c}$ & $0.03-0.10$ \\
Class A & $4.6-6.4$ & $14-15$ \\
Class B & $0.5-0.7$ & $0.003-115^{\mathrm{d}}$ \\
Class C & $55-60$ & $>0.003-\mathrm{No} \mathrm{limit}$ \\
GTCC & $0.1->7,000^{\mathrm{d}}$ & \\
Uranium mill tailings & $>0.1-\mathrm{Nolimit}$ & 0.00020 \\
\hline
\end{tabular}

a Lower-bound levels are based on cumulative spent nuclear fuel discharged; upper-bound levels are based on annual discharges.

based on 1986-1988 Solid Waste Information Management System (SWIMS) and the National Low-Level Waste Management Program (LLWMP) data access system, both of which were maintained by EG\&G, Idaho, Inc., Idaho Falls, Idaho.

Waste classification is defined by the NRC in 10 CFR Part 61.55 on the basis of concentration of certain long- and short-lived radionuclides. The classification system is designed to minimize potential exposures in both the short and long term. The gross $\mathrm{Ci} / \mathrm{m}^{3}$ shown above are representative of typical LLW shipped to commercial disposal sites. Nuclear power plant wastes account for most of the radioactivity $(-96 \%)$ and include Class A, B, and C. Essentially all medical wastes are Class $A$. Industrial wastes are largely Class $A$, but they contain some Class $B$ and $C$.

$\mathrm{d}_{\text {Maximum for }}{ }^{63} \mathrm{Ni}$ in activated metal or ${ }^{90} \mathrm{Sr}$. There is no limit on concentration of ${ }^{3} \mathrm{H},{ }^{60} \mathrm{Co}$, or nuclides with half-lives $<5$ years. The maximum thermal power shown is based on the highest reported gross $\mathrm{Ci} / \mathrm{m}^{3}$ analysis for irradiated core components (1986-1988) and assumes all the activity is due to ${ }^{60} \mathrm{Co}$, which would yield the greatest heat output. If the activity is due to activation products, such as ${ }^{54} \mathrm{Mn},{ }^{58} \mathrm{Co}$, etc., the $\mathrm{Ci} / \mathrm{m}^{3}$ could be much higher for individual shipments, and the total $\mathrm{W} / \mathrm{m}^{3}$ could exceed the value shown.

In temporary storage. The concentration of actinides and ${ }^{129} \mathrm{I}$ determines the lower activity boundary. There is no limit on concentrations of ${ }^{3} \mathrm{H},{ }^{60} \mathrm{Co}$, or nuclides with half-lives $<5$ years. 
Table A.2. Representative DOE LLW radionuclide composition by percent activity ${ }^{a}$

\begin{tabular}{|c|c|c|c|c|c|c|c|c|c|}
\hline \multicolumn{2}{|c|}{ Uranium/thorium } & \multicolumn{2}{|c|}{ Fission product } & \multicolumn{2}{|c|}{ Induced activity } & \multicolumn{2}{|c|}{ Alpha, $<100 \mathrm{nCi} / \mathrm{g}$} & \multicolumn{2}{|c|}{ Other } \\
\hline Nuclide & Composition & Nuclide & Composition & Nuclide & Composition & Nuclide & Composition & Nuclide & Composition \\
\hline${ }^{208} \mathrm{Tl}$ & 0.0017 & ${ }^{60} \mathrm{Co}$ & 0.08 & ${ }^{51} \mathrm{Cr}$ & 4.95 & ${ }^{238} \mathrm{Pu}$ & 2.62 & ${ }^{3} \mathrm{H}$ & 1.22 \\
\hline${ }^{212} \mathrm{~Pb}$ & 0.0045 & ${ }^{90} \mathrm{Sr}$ & 7.77 & ${ }^{54} \mathrm{Mn}$ & 38.10 & ${ }^{239} \mathrm{Pu}$ & 0.20 & ${ }^{14} \mathrm{C}$ & 0.06 \\
\hline${ }^{212} \mathrm{Bi}$ & 0.0045 & ${ }^{90} \mathrm{Y}$ & 7.77 & ${ }^{58} \mathrm{Co}$ & 55.40 & ${ }^{240} \mathrm{Pu}$ & 0.70 & ${ }^{54} \mathrm{Mn}$ & 6.76 \\
\hline${ }^{212} \mathrm{Po}$ & 0.0029 & ${ }^{95} \mathrm{Zr}$ & 1.27 & ${ }^{59} \mathrm{Fe}$ & 0.49 & ${ }^{241} \mathrm{Pu}$ & 96.4 & ${ }^{58} \mathrm{Co}$ & 6.24 \\
\hline${ }^{216} \mathrm{Po}$ & 0.0045 & ${ }^{95} \mathrm{Nb}$ & 2.83 & ${ }^{60} \mathrm{Co}$ & 0.87 & ${ }^{241} \mathrm{Am}$ & 0.004 & ${ }^{60} \mathrm{Co}$ & 18.03 \\
\hline${ }^{224} \mathrm{Ra}$ & 0.0045 & ${ }^{99} \mathrm{Tc}$ & 0,02 & ${ }^{65} \mathrm{Zn}$ & 0.19 & ${ }^{242} \mathrm{Cm}$ & 0.056 & ${ }^{90} \mathrm{Sr}$ & 8.48 \\
\hline${ }^{228} \mathrm{Ra}$ & 0.0269 & ${ }^{125} \mathrm{Sb}$ & 2.93 & & - & ${ }^{244} \mathrm{Cm}$ & 0.020 & ${ }^{90} \mathrm{Y}$ & 8.48 \\
\hline${ }^{228} \mathrm{Ac}$ & 0.0269 & ${ }^{125 \mathrm{~m}} \mathrm{Te}$ & 0.73 & & 100.00 & & ב & ${ }^{99} \mathrm{Te}$ & 0.12 \\
\hline${ }^{228} \mathrm{Th}$ & 0.0045 & ${ }^{106} \mathrm{Ru}$ & 6.39 & & & & 100.000 & ${ }^{134} \mathrm{Cs}$ & 13.98 \\
\hline${ }^{231} \mathrm{Th}$ & 0.0259 & ${ }^{106} \mathrm{Rh}$ & 6.39 & & & & & ${ }^{137} \mathrm{Cs}$ & 18.45 \\
\hline${ }^{232} \mathrm{Th}$ & 0.273 & ${ }^{134} \mathrm{Cs}$ & 0.38 & & & & & ${ }^{137 m} \mathrm{Ba}$ & 17.45 \\
\hline${ }^{234} \mathrm{Th}$ & 33.197 & ${ }^{137} \mathrm{Cs}$ & 17.31 & & & & & ${ }^{238} \mathrm{U}$ & 0.73 \\
\hline${ }^{234} \mathrm{~m} \mathrm{~Pa}$ & 33.197 & ${ }^{137 m} \mathrm{Ba}$ & 16.38 & & & & & & - \\
\hline${ }^{234} \mathrm{~Pa}$ & 0.0034 & ${ }^{144} \mathrm{Ce}$ & 14.67 & & & & & & 100.00 \\
\hline${ }^{235} \mathrm{U}$ & 0.0258 & ${ }^{144} \mathrm{Pr}$ & 14.67 & & & & & & \\
\hline \multirow[t]{6}{*}{${ }^{238} \mathrm{U}$} & 33.197 & ${ }^{147} \mathrm{Pm}$ & 0.06 & & & & & & \\
\hline & - & ${ }^{151} \mathrm{Sm}$ & 0.11 & & & & & & \\
\hline & 100.0000 & ${ }^{152} \mathrm{Eu}$ & 0.09 & & & & & & \\
\hline & & ${ }^{154} \mathrm{Eu}$ & 0.09 & & & & & & \\
\hline & & ${ }^{15 s} \mathrm{Eu}$ & 0.06 & & & & & & \\
\hline & & & 100.00 & & & & & & \\
\hline
\end{tabular}

aBased on ref. 1. 
Table A.3. Average concentrations for representative radionuclides in LLW at commercial disposal sites ${ }^{2}$

\begin{tabular}{|c|c|c|}
\hline Radionuclide & Half-life ${ }^{b}$ & $\begin{array}{l}\text { Concentration } \\
\left(\mathrm{Ci} / \mathrm{m}^{3}\right)\end{array}$ \\
\hline${ }^{3} \mathrm{H}$ & $1.228 \mathrm{E}+01 \mathrm{y}$ & $1.083 E+00$ \\
\hline${ }^{14} \mathrm{C}$ & $5.730 E+03 y$ & $5.079 \mathrm{E}-03$ \\
\hline${ }^{26} \mathrm{Al}$ & $7.300 E+05 y$ & $2.980 \mathrm{E}-10$ \\
\hline${ }^{32} \mathrm{Si}$ & $1.000 E+02 y$ & $3.725 \mathrm{E}-11$ \\
\hline${ }^{32} \mathrm{P}$ & $1.428 \mathrm{E}+01 \mathrm{~d}$ & $9.292 \mathrm{E}-04$ \\
\hline${ }^{35} \mathrm{~S}$ & $8.751 E+01 d$ & $2.208 \mathrm{E}-03$ \\
\hline${ }^{36} \mathrm{Cl}$ & $3.010 E+05 y$ & $6.143 E-06$ \\
\hline${ }^{40} \mathrm{~K}$ & $1.280 E+09 y$ & $1.766 E-07$ \\
\hline${ }^{51} \mathrm{Cr}_{\mathrm{r}}$ & $2.770 E+01 d$ & $7.137 E-02$ \\
\hline${ }^{54} \mathrm{Mn}$ & $3.122 E+02 d$ & $3.895 \mathrm{E}-01$ \\
\hline${ }^{55} \mathrm{Fe}$ & $2.730 \mathrm{E}+00 \mathrm{y}$ & $3.112 \mathrm{E}+00$ \\
\hline${ }^{59} \mathrm{Fe}$ & $4.445 \mathrm{E}+01 \mathrm{~d}$ & $5.081 E-03$ \\
\hline${ }^{58} \mathrm{Co}$ & $7.092 E+01 d$ & $2.047 E-01$ \\
\hline${ }^{60} \mathrm{Co}$ & $5.271 E+00 y$ & $2.242 E+00$ \\
\hline${ }^{59} \mathrm{Ni}$ & $7.500 E+04 y$ & $1.364 \mathrm{E}-03$ \\
\hline${ }^{63} \mathrm{Ni}$ & $1.001 E+02 y$ & $2.692 \mathrm{E}-01$ \\
\hline${ }^{65} \mathrm{Zn}$ & $2.441 E+02 d$ & $1.174 \mathrm{E}-01$ \\
\hline${ }^{85} \mathrm{Kr}$ & $1.072 \mathrm{E}+01 \mathrm{y}$ & $8.147 E-04$ \\
\hline${ }^{89} \mathrm{Sr}$ & $5.055 E+01 d$ & $6.032 E-03$ \\
\hline${ }^{90} \mathrm{Sr}$ & $2.850 E+01 y$ & $6.987 E-02$ \\
\hline${ }^{90} \mathrm{Y}$ & $2.671 E+01 d$ & $6.987 \mathrm{E}-02$ \\
\hline${ }^{91} \mathrm{Y}$ & $5.851 E+01 d$ & $8.859 \mathrm{E}-03$ \\
\hline${ }^{95} \mathrm{Zr}$ & $6.402 E+01 d$ & $1.036 \mathrm{E}-02$ \\
\hline${ }^{94} \mathrm{Nb}$ & $2.030 E+04 y$ & $1.659 E-05$ \\
\hline${ }^{95} \mathrm{Nb}$ & $3.497 E+01 d$ & $1.916 \mathrm{E}-02$ \\
\hline${ }^{93} \mathrm{Mo}$ & $3.500 E+03 y$ & $9.273 E-12$ \\
\hline${ }^{99} \mathrm{Tc}$ & $2.130 E+05 y$ & $1.949 E-04$ \\
\hline${ }^{103} \mathrm{Ru}$ & $3.925 E+01 d$ & $5.900 E-04$ \\
\hline${ }^{108 \mathrm{~m}} \mathrm{Ag}$ & $1.300 E+02 y$ & $5.534 E-06$ \\
\hline${ }^{110 \mathrm{~m}} \mathrm{Ag}$ & $2.498 E+02 d$ & $3.600 E-02$ \\
\hline${ }^{113} \mathrm{Cd}$ & $9.000 E+15 y$ & $4.223 \mathrm{E}-12$ \\
\hline${ }^{124} \mathrm{Sb}$ & $6.020 E+00 d$ & $2.621 E-03$ \\
\hline${ }^{125} \mathrm{Sb}$ & $2.730 E+00 y$ & $1.901 \mathrm{E}-02$ \\
\hline${ }^{123} \mathrm{Te}$ & $1.300 E+13 y$ & $5.710 \mathrm{E}-07$ \\
\hline${ }^{125} \mathrm{I}$ & $6.014 E+00 d$ & $4.570 E-04$ \\
\hline${ }^{129} \mathrm{I}$ & $1.570 E+07 y$ & $2.101 E-05$ \\
\hline${ }^{131} \mathrm{I}$ & $8.040 \mathrm{E}+00 \mathrm{~d}$ & $5.299 \mathrm{E}-03$ \\
\hline${ }^{134} \mathrm{Cs}$ & $2.062 E+00 y$ & $8.661 \mathrm{E}-02$ \\
\hline${ }^{135} \mathrm{Cs}$ & $3.000 E+06 y$ & $1.105 E-05$ \\
\hline${ }^{137} \mathrm{Cs}$ & $3.017 \mathrm{E}+01 \mathrm{y}$ & $2.431 E-01$ \\
\hline${ }^{137} \mathrm{~m} \mathrm{Ba}$ & $2.552 \mathrm{E}+00 \mathrm{~min}$ & $2.300 \mathrm{E}-01$ \\
\hline${ }^{141} \mathrm{Ce}$ & $3.250 E+01 d$ & $1.649 \mathrm{E}-03$ \\
\hline${ }^{144} \mathrm{Ce}$ & $2.849 E+02 d$ & $1.463 E-02$ \\
\hline
\end{tabular}


Table A.3 (continued)

\begin{tabular}{|c|c|c|}
\hline Radionuclide & Half-life $^{b}$ & $\begin{array}{l}\text { Concentration } \\
\left(\mathrm{Ci} / \mathrm{m}^{3}\right)\end{array}$ \\
\hline${ }^{144} \mathrm{Pr}$ & $1.728 \mathrm{E}+01 \mathrm{~min}$ & $1.463 \mathrm{E}-02$ \\
\hline${ }^{144} \mathrm{Nd}$ & $2.100 \mathrm{E}+15 \mathrm{y}$ & $1.689 E-10$ \\
\hline${ }^{147} \mathrm{Pm}$ & $2.623 \mathrm{E}+00 \mathrm{y}$ & $1.317 \mathrm{E}-02$ \\
\hline${ }^{157} \mathrm{~Tb}$ & $1.100 \mathrm{E}+02 \mathrm{y}$ & $1.012 E-10$ \\
\hline${ }^{158} \mathrm{~Tb}$ & $1.800 E+02 y$ & $3.768 E-10$ \\
\hline${ }^{175} \mathrm{Hf}$ & $7.000 E+01 d$ & $1.427 E-03$ \\
\hline${ }^{181} \mathrm{Hf}$ & $4.240 \mathrm{E}+01 \mathrm{~d}$ & $3.235 E-03$ \\
\hline${ }^{187} \mathrm{Re}$ & $4.100 E+10 y$ & $1.772 \mathrm{E}-11$ \\
\hline${ }^{209} \mathrm{Po}$ & $3.253 \mathrm{E}+00 \mathrm{~h}$ & $1.284 \mathrm{E}-10$ \\
\hline${ }^{226} \mathrm{Ra}$ & $1.600 \mathrm{E}+03 \mathrm{y}$ & $2.852 E-04$ \\
\hline${ }^{229} \mathrm{Th}$ & $7.340 \mathrm{E}+03 \mathrm{y}$ & $1.310 \mathrm{E}-10$ \\
\hline${ }^{230} \mathrm{Th}$ & $7.540 \mathrm{E}+04 \mathrm{y}$ & $1.721 \mathrm{E}-08$ \\
\hline${ }^{232} \mathrm{Th}$ & $1.405 \mathrm{E}+10 \mathrm{y}$ & $8.482 \mathrm{E}-03$ \\
\hline${ }^{231} \mathrm{~Pa}$ & $3.276 \mathrm{E}+04 \mathrm{y}$ & $1.016 \mathrm{E}-10$ \\
\hline${ }^{233} \mathrm{U}$ & $1.592 \mathrm{E}+05 \mathrm{y}$ & $2.308 \mathrm{E}-07$ \\
\hline${ }^{234} \mathrm{U}$ & $2.454 \mathrm{E}+05 \mathrm{y}$ & $5.368 E-05$ \\
\hline${ }^{235} \mathrm{U}$ & $7.037 E+08 y$ & $3.179 \mathrm{E}-05$ \\
\hline${ }^{236} \mathrm{U}$ & $2.432 \mathrm{E}+07 \mathrm{y}$ & $7.886 \mathrm{E}-07$ \\
\hline${ }^{238} \mathrm{U}$ & $4.468 \mathrm{E}+09 \mathrm{y}$ & $9.970 \mathrm{E}-03$ \\
\hline${ }^{237} \mathrm{~Np}$ & $2.140 E+06 y$ & $2.210 \mathrm{E}-07$ \\
\hline${ }^{239} \mathrm{Pu}$ & $2.413 E+04 y$ & $1.021 \mathrm{E}-05^{\mathrm{C}}$ \\
\hline${ }^{240} \mathrm{Pu}$ & $6.563 E+03 y$ & $2.504 E-06^{\mathrm{C}}$ \\
\hline${ }^{242} \mathrm{Pu}$ & $3.763 \mathrm{E}+05 \mathrm{y}$ & $6.148 \mathrm{E}-07^{\mathrm{c}}$ \\
\hline${ }^{241} \mathrm{Am}$ & $4.322 E+02 y$ & $4.053 \mathrm{E}-05$ \\
\hline${ }^{243} \mathrm{Am}$ & $7.380 E+03 y$ & $1.398 \mathrm{E}-08$ \\
\hline${ }^{248} \mathrm{Cm}$ & $3.400 E+05 y$ & $6.220 \mathrm{E}-07$ \\
\hline Total & & $8.380 \mathrm{E}+00$ \\
\hline
\end{tabular}

adapted from ref. 3 .

$b_{y}=$ years; $d=$ days; $h=$ hours; $\min =$ minutes; and $s=$ seconds.

Isotopes of plutonium are omitted when this list is applied to waste disposed at Barnwell, South Carolina, because this site has not permitted disposal of plutonium (even though traces of plutonium could have entered with other wastes). 
Table A.4. Estimated sources and characteristics of commercial Greater-Than-Class-C LLWa

\begin{tabular}{|c|c|c|}
\hline Waste source & Physical form & $\begin{array}{l}\text { Primary isotopes of concern } \\
\text { for disposal }\end{array}$ \\
\hline \multicolumn{3}{|r|}{ 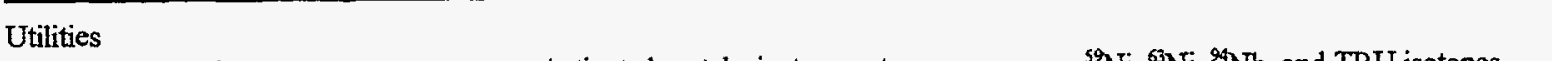 } \\
\hline Reactor operations & $\begin{array}{l}\text { Activated metals, instruments, } \\
\text { filters, ion-exchange resins, sludges }\end{array}$ & ${ }^{59} \mathrm{Ni},{ }^{63} \mathrm{Ni},{ }^{94} \mathrm{Nb}$, and TRU isotopes \\
\hline Reactor decommissioning & Activated metals & ${ }^{59} \mathrm{Ni},{ }^{63} \mathrm{Ni}$, and ${ }^{94} \mathrm{Nb}$ \\
\hline \multicolumn{3}{|l|}{ Fuel testing labs } \\
\hline Bumup lab operation & $\begin{array}{l}\text { Solidified liquids, metal cuttings, } \\
\text { glassware, equipment, ion-exchange } \\
\text { resins }\end{array}$ & ${ }^{90} \mathrm{Sr}$ and TRU isotopes \\
\hline Bumup lab decommissioning & $\begin{array}{l}\text { Solidified liquids, metals, glassware, } \\
\text { equipment }\end{array}$ & ${ }^{90} \mathrm{Sr}$ and IRU isotopes \\
\hline \multicolumn{3}{|l|}{ Sealed sources } \\
\hline Manufacturer operations & Trash, metal, foils & ${ }^{14} \mathrm{C},{ }^{90} \mathrm{Sr},{ }^{137} \mathrm{Cs},{ }^{241} \mathrm{Am}$, and $\mathrm{Pu}$ isotopes \\
\hline Manufacturer decommissioning & Trash, metal, foils & ${ }^{14} \mathrm{C},{ }^{90} \mathrm{Sr},{ }^{137} \mathrm{Cs},{ }^{241} \mathrm{Am}$, and $\mathrm{Pu}$ isotopes \\
\hline Sources designated as waste & Sealed sources & ${ }^{137} \mathrm{Cs},{ }^{238} \mathrm{Pu},{ }^{239} \mathrm{Pu}$, and ${ }^{241} \mathrm{Am}$ \\
\hline Other & $\cdot$ & \\
\hline${ }^{14} \mathrm{C}$ users & Solidified process liquids & ${ }^{14} \mathrm{C}$ \\
\hline Test and research reactors & Activated metals & ${ }^{59} \mathrm{Ni},{ }^{44} \mathrm{Nb}$, and TRU isotopes \\
\hline Other & Soil, trash & ${ }^{241} \mathrm{Am}$ \\
\hline
\end{tabular}

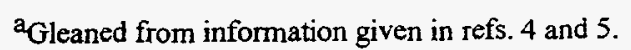


Table A.5. Historical number and volume of drums and classes of LLW incorporated in cement generated in the WVDP Low-Level Radwaste Treatment System ${ }^{a, b, c, d}$

\begin{tabular}{|c|c|c|c|c|}
\hline \multirow{2}{*}{$\begin{array}{l}\text { End of } \\
\text { calendar } \\
\text { year }\end{array}$} & \multicolumn{3}{|c|}{ Number of drums } & \multirow{2}{*}{$\begin{array}{c}\text { Total volume } \\
\text { of drums } \\
\left(\mathrm{m}^{3}\right)\end{array}$} \\
\hline & Class $A^{e}$ & Class.B & Class $\mathrm{C}^{\mathrm{e}}$ & \\
\hline 1987 & $726^{f}$ & $\mathrm{~g}$ & - & 189 \\
\hline 1988 & & $\mathrm{~g}$ & 2,009 & 523 \\
\hline 1989 & & $\mathrm{~g}$ & 4,523 & 1,177 \\
\hline $1990^{\mathrm{h}}$ & & $\mathrm{g}$ & 3,862 & 1,055 \\
\hline 1991 & & $\mathrm{~g}$ & 0 & 0 \\
\hline 1992 & 1,268 & $\mathrm{~g}$ & 268 & 399 \\
\hline 1993 & 2,387 & $\mathrm{~g}$ & 1,298 & 959 \\
\hline 1994. & & g & 2,641 & 687 \\
\hline $1995^{i}$ & & $\mathrm{~g}$ & 1,632 & 425 \\
\hline Total number & 4,381 & $\mathrm{~g}$ & 16,238 & \\
\hline Total volume, $\mathrm{m}^{3}$ & 1,140 & g & 4,225 & 5,414 \\
\hline
\end{tabular}

${ }^{2}$ The so-called square drums used are parallelepipeds of square cross sections $(-0.6 \mathrm{~m} \times 0.6 \mathrm{~m} \times 0.8 \mathrm{~m})$ with a volume of $71 \mathrm{gal}\left(0.27 \mathrm{~m}^{3}\right)$.

${ }^{b}$ The classes are in accordance with the Classes $(A, B$; or $C)$ as set by requirements of the NRC in 10 CFR Part 61.55.

Taken from ref. 6.

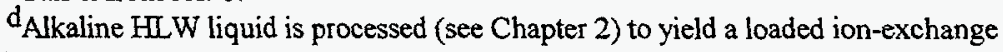
material (zeolite), which is HLW, and an effluent, which is LLW. This effluent is solidified with cement.

EStored in a shielded drum cell.

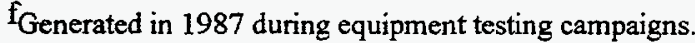

gNo Class-B LLW is expected to be generated with the effluent mentioned in footnote "d."

hProcessing of alkaline supernatant HLW liquid was completed in November 1990.

iProcessing of residual supernatant, sludge waste solutions, and Thorex waste were completed in January 1995. 
APPENDIX B. CHARACTERISTICS OF IMPORTANT RADIONUCLIDES 



\section{APPENDIX B. CHARACTERISTICS OF IMPORTANT RADIONUCLIDES}

\section{B.1 DISCUSSION}

The following Table B.1 lists radionuclides whose characteristics are most often referenced in the variety of studies and evaluations discussed in Chapters 1-7. It includes isotopes for HLW, TRUW, LLW, and UMT as defined by EPA, ${ }^{1}$ $\mathrm{NRC}^{2,3}$ and DOE. ${ }^{4,5}$ The data in Table B.1 were obtained from refs. 6-9.

\section{B.2 REFERENCES}

1. U.S. Environmental Protection Agency, "Environmental Radiation Protection Standards for Management and Disposal of Spent Nuclear Fuel, High-Level and Transuranic Radioactive Wastes," Code of Federal Regulations, 40 CFR Part 191 (July 1, 1996).

2. U.S. Nuclear Regulatory Commission, "Licensing Requirements for Land Disposal of Radioactive Waste," Code of Federal Regulations, 10 CFR Part 61 (Jan. 1, 1996).

3. U.S. Nuclear Regulatory Commission, "Biomedical Waste Disposal," Fed. Regist. 46(47), 16230-16234 (Mar. 11, 1981).

4. U.S. Department of Energy, DOE Order 5820.2A, Radioactive Waste Management, Washington, D.C., Sept. 26, 1988 .

5. U.S. Department of Energy, Energy Information Administration, Domestic Uranium Mining and Milling Industry 1992-Viability Assessment, DOE/EIA-0477(92), Washington, D.C. (December 1993).

6. D. C. Kocher, Radioactive Decay Data Tables, DOE/TIC-1 1026, U.S. Department of Energy, Washington, D.C. (1981).

7. D. C. Kocher, A Radionuclide Decay Data Base—Index and Summary Table, NUREG/CR-1413, ORNL/NUREG-70, Oak Ridge National Laboratory, Oak Ridge, Tennessee (May 1980).

8. E. Browne and R. B. Firestone, Table of Radioactive Isotopes, ed. V. S. Shirley, John Wiley and Sons, Inc., New York (1986).

9. A. G. Croff, ORIGEN2-A Revised and Updated Version of the Oak Ridge Isotope Generation and Depletion Code, ORNL-5621, Oak Ridge National Laboratory, Oak Ridge, Tennessee (July 1980). 
Table B.1. Characteristics of important radionuclides ${ }^{\mathrm{a}}$

\begin{tabular}{|c|c|c|c|c|c|c|c|c|c|c|}
\hline \multirow{2}{*}{ Nuclide } & \multirow{2}{*}{$\begin{array}{l}\text { Atomic } \\
\text { number }\end{array}$} & \multirow{2}{*}{ Half-life $^{b}$} & \multirow{2}{*}{$\begin{array}{l}\text { Principal } \\
\text { mode(s) of } \\
\text { decayc }\end{array}$} & \multicolumn{3}{|c|}{$\begin{array}{c}\text { Major radiation energies }{ }^{d} \\
(\mathrm{MeV} / \mathrm{dis})\end{array}$} & \multicolumn{2}{|c|}{ "Q" value } & \multirow{2}{*}{$\begin{array}{c}\text { Specific } \\
\text { activity } \\
(\mathrm{Ci} / \mathrm{g})\end{array}$} & \multirow{2}{*}{ Daughter(s) } \\
\hline & & & & $\alpha$ & $\epsilon$ & $\gamma$ and $X$ & (MeV/dis) & $(\mathrm{W} / \mathrm{Ci})$ & & \\
\hline${ }^{3} \mathrm{H}$ & 1 & $1.233 \mathrm{E}+01 \mathrm{y}$ & $\beta$ & & 0.00568 & & $5.68 \mathrm{E}-03$ & $3.37 \mathrm{E}-05$ & $9.650 \mathrm{E}+03$ & ${ }^{3} \mathrm{He}$ \\
\hline${ }^{14} \mathrm{C}$ & 6 & $5.730 \mathrm{E}+03 \mathrm{y}$ & $\beta$ & & 0.0495 & & $4.95 \mathrm{E}-02$ & $2.93 E-04$ & 4.457 & ${ }^{14} \mathrm{~N}$ \\
\hline${ }^{26} \mathrm{Al}$ & 13 & $7.2 E+05 y$ & $\mathrm{EC}$ & & 0.4451 & 2.6758 & 3.079 & $1.825 \mathrm{E}-02$ & $1.91 \mathrm{E}-02$ & ${ }^{26} \mathrm{Mg}$ \\
\hline${ }^{32} \mathrm{Si}$ & 14 & $650 y$ & $\beta$ & & 0.0647 & & $2.10 \mathrm{E}-01$ & $1.245 \mathrm{E}-03$ & $1.719 \mathrm{E}+01$ & ${ }^{32} \mathrm{P}$ \\
\hline${ }^{32} \mathrm{P}$ & 15 & $14.282 \mathrm{~d}$ & $\beta$ & & 0.6947 & & $6.95 \mathrm{E}-01$ & $4.12 \mathrm{E}-03$ & $2.853 \mathrm{E}+05$ & ${ }^{32} \mathrm{~S}$ \\
\hline${ }^{35} \mathrm{~S}$ & 16 & $87.51 \mathrm{~d}$ & $\beta$ & & 0.0486 & & $4.86 \mathrm{E}-02$ & $2.88 \mathrm{E}-04$ & $4.263 \mathrm{E}+04$ & ${ }^{35} \mathrm{Cl}$ \\
\hline${ }^{36} \mathrm{Cl}$ & 17 & $3.01 \mathrm{E}+05 \mathrm{y}$ & $\begin{array}{l}\beta(98.1 \%) \\
\operatorname{EC}(1.9 \%)\end{array}$ & & 0.2460 & & $2.460 \mathrm{E}-01$ & $1.458 \mathrm{E}-03$ & $3.299 \mathrm{E}-02$ & ${ }^{36} \mathrm{Ar}$ \\
\hline${ }^{40} \mathrm{~K}$ & 19 & $1.277 \mathrm{E}+09 y$ & $\begin{array}{l}\beta(89.33 \%): \\
\mathrm{EC}(10.67 \%)\end{array}$ & & 0.4545 & 0.1559 & $6.104 \mathrm{E}-01$ & $3.62 \mathrm{E}-03$ & $6.983 \mathrm{E}-06$ & ${ }^{40} \mathrm{Ca}$ \\
\hline${ }^{45} \mathrm{Ca}$ & 20 & $163.8 \mathrm{~d}$ & $\beta$ & & 0.0770 & & $7.70 \mathrm{E}-02$ & $4.56 \mathrm{E}-04$ & $1.780 \mathrm{E}+04$ & ${ }^{45} \mathrm{Sc}$ \\
\hline${ }^{46} \mathrm{Sc}$ & 21 & $83.83 \mathrm{~d}$ & $\beta$ & 0.1120 & 2.0095 & & 2.122 & $1.257 \mathrm{E}-02$ & $3.381 E+04$ & ${ }^{46} \mathrm{Ti}$ \\
\hline${ }^{51} \mathrm{Cr}$ & 24 & $27.704 d$ & $\mathrm{EC}$ & & 0.0031 & 0.0325 & $3.56 \mathrm{E}-02$ & $2.11 E-04$ & $9.240 \mathrm{E}+04$ & ${ }^{\mathrm{st}} \mathrm{V}$ \\
\hline${ }^{54} \mathrm{Mn}$ & 25 & $312.20 \mathrm{~d}$ & $\mathrm{EC}$ & & 0.0034 & 0.8360 & $8.394 \mathrm{E}-01$ & $4.975 \mathrm{E}-03$ & $7.738 E+03$ & ${ }^{54} \mathrm{Cr}$ \\
\hline${ }^{5 s} \mathrm{Fe}$ & 26 & $2.73 \mathrm{y}$ & $\mathrm{EC}$ & & 0.0038 & 0.0016 & $5.4 \mathrm{E}-03$ & $3.2 \mathrm{E}-05$ & $2.500 \mathrm{E}+03$ & ${ }^{55} \mathrm{Mn}$ \\
\hline${ }^{59} \mathrm{Fe}$ & 26 & $44.496 \mathrm{~d}$ & $\beta$ & & 0.1174 & 1.1882 & 1.3056 & $7.741 \mathrm{E}-03$ & $4.918 \mathrm{E}+04$ & ${ }^{59} \mathrm{Co}$ \\
\hline${ }^{57} \mathrm{Co}$ & 27 & $271.77 \mathrm{~d}$ & $\mathrm{EC}$ & & 0.0176 & 0.1252 & $1.428 \mathrm{E}-01$ & $8.464 \mathrm{E}-01$ & $8.456 E+03$ & ${ }^{57} \mathrm{Fe}$ \\
\hline${ }^{58} \mathrm{Co}$ & 27 & $70.92 \mathrm{~d}$ & $\mathrm{EC}$ & & 0.0336 & 0.9758 & 1.0094 & $5.99 \mathrm{E}-03$ & $3.181 \mathrm{E}+04$ & ${ }^{58} \mathrm{Fe}$ \\
\hline${ }^{60} \mathrm{Co}$ & 27 & $5.271 \mathrm{y}$ & $\beta$ & & 0.0958 & 2.5058 & 2.6016 & $1.541 E-02$ & $1.131 E+03$ & ${ }^{60} \mathrm{Ni}$ \\
\hline${ }^{60 m} \mathrm{Co}$ & 27 & $10.47 \mathrm{~min}$ & $\begin{array}{l}\text { IT }(99.75 \%) \\
\beta(0.25 \%)\end{array}$ & & 0.0536 & 0.0066 & $6.02 \mathrm{E}-02$ & $3.57 E-04$ & $2.993 E+08$ & ${ }^{60} \mathrm{Co}$ \\
\hline${ }^{59} \mathrm{Ni}$ & 28 & $7.5 \mathrm{E}+04 \mathrm{y}$ & $\mathrm{EC}$ & & 0.0043 & 0.0024 & $6.72 \mathrm{E}-03$ & $3.98 \mathrm{E}-05$ & $8.079 \mathrm{E}-02$ & ${ }^{59} \mathrm{Co}$ \\
\hline${ }^{63} \mathrm{Ni}$ & 28 & $1.001 \mathrm{E}+02 \mathrm{y}$ & $\beta$ & & 0.0171 & & $1.71 \mathrm{E}-02$ & $1.01 \mathrm{E}-04$ & $6.168 \mathrm{E}+01$ & ${ }^{63} \mathrm{Cu}$ \\
\hline
\end{tabular}


Table B.1 (continued)

\begin{tabular}{|c|c|c|c|c|c|c|c|c|c|c|}
\hline \multirow{2}{*}{ Nuclide } & \multirow{2}{*}{$\begin{array}{l}\text { Atomic } \\
\text { number }\end{array}$} & \multirow{2}{*}{ Half-life ${ }^{b}$} & \multirow{2}{*}{$\begin{array}{l}\text { Principal } \\
\text { mode(s) of } \\
\text { decayc }\end{array}$} & \multicolumn{3}{|c|}{$\begin{array}{l}\text { Major radiation energies } \mathrm{d} \\
\qquad(\mathrm{MeV} / \mathrm{dis})\end{array}$} & \multicolumn{2}{|c|}{ “Q" value } & \multirow{2}{*}{$\begin{array}{c}\text { Specific } \\
\text { activity } \\
(\mathrm{Ci} / \mathrm{g})\end{array}$} & \multirow{2}{*}{ Daughter(s) } \\
\hline & & & & $\alpha$ & $\epsilon$ & $\gamma$ and $X$ & (MeV/dis) & $(\mathrm{W} / \mathrm{Ci})$ & & \\
\hline${ }^{65} \mathrm{Zn}$ & 30 & $244.1 \mathrm{~d}$ & $\mathrm{EC}$ & & 0.0066 & 0.5838 & $5.90 \mathrm{E}-01$ & $3.51 E-03$ & $8.237 \mathrm{E}+03$ & ${ }^{65} \mathrm{Cu}$ \\
\hline${ }^{67} \mathrm{Ga}$ & 31 & $3.261 \mathrm{~d}$ & $\mathrm{EC}$ & & 0.0333 & 0.1549 & $1.882 \mathrm{E}-01$ & $1.115 \mathrm{E}-03$ & $5.975 \mathrm{E}+05$ & ${ }^{67} \mathrm{Zn}$ \\
\hline${ }^{75} \mathrm{Se}$ & 34 & $119.77 \mathrm{~d}$ & $\mathrm{EC}$ & & 0.0134 & 0.3924 & $4.06 \mathrm{E}-01$ & $2.41 \mathrm{E}-03$ & $1.453 \mathrm{E}+04$ & ${ }^{75} \mathrm{As}$ \\
\hline${ }^{79} \mathrm{Se}$ & 34 & $<6.5 \mathrm{E}+04 \mathrm{y}$ & & & 0.0529 & & $5.29 \mathrm{E}-02$ & $3.13 E-04$ & $6.966 \mathrm{E}-02$ & ${ }^{79} \mathrm{Br}$ \\
\hline${ }^{85} \mathrm{Kr}$ & 36 & $1.072 \mathrm{E}+01 \mathrm{y}$ & $\beta$ & & 0.2505 & 0.0022 & $2.53 \mathrm{E}-01$ & $1.50 \mathrm{E}-03$ & $3.923 \mathrm{E}+02$ & ${ }^{85} \mathrm{Rb}$ \\
\hline${ }^{86} \mathrm{Rb}$ & 37 & $18.66 \mathrm{~d}$ & $\beta$ & & 0.6670 & 0.0945 & $7.62 \mathrm{E}-01$ & $4.52 \mathrm{E}-03$ & $8.138 E+04$ & ${ }^{86} \mathrm{Sr}$ \\
\hline${ }^{89} \mathrm{Sr}$ & 38 & $50.55 \mathrm{~d}$ & $\beta$ & & 0.5829 & 0.0001 & $5.83 E-01$ & $3.46 \mathrm{E}-03$ & $2.905 E+04$ & ${ }^{89} \mathrm{Y}$ \\
\hline${ }^{90} \mathrm{Sr}$ & 38 & $2.85 E+01 y$ & $\beta$ & & 0.1958 & & $1.96 \mathrm{E}-01$ & $1.16 \mathrm{E}-03$ & $1.364 \mathrm{E}+02$ & ${ }^{90} \mathrm{Y}$ \\
\hline${ }^{90} \mathrm{Y}$ & 39 & $2.671 \mathrm{~d}$ & $\beta$ & & 0.9332 & & $9.33 \mathrm{E}-01$ & $5.54 \mathrm{E}-03$ & $5.441 E+05$ & $90 \mathrm{Zr}$ \\
\hline${ }^{91} Y$ & 39 & $58.51 \mathrm{~d}$ & $\beta$ & & 0.6039 & 0.0036 & $6.07 \mathrm{E}-01$ & $3.60 \mathrm{E}-03$ & $2.452 \mathrm{E}+04$ & ${ }^{91} \mathrm{Zr}$ \\
\hline${ }^{93} \mathrm{Zr}$ & 40 & $1.53 \mathrm{E}+06 \mathrm{y}$ & $\beta$ & & 0.0471 & 0.0018 & $4.89 \mathrm{E}-02$ & $2.90 \mathrm{E}-04$ & $2.513 \mathrm{E}-03$ & ${ }^{93} \mathrm{Nb}$ \\
\hline${ }^{95} \mathrm{Zr}$ & 40 & $64.02 \mathrm{~d}$ & $\beta$ & & 0.1200 & 0.7337 & $8.54 \mathrm{E}-01$ & $5.06 \mathrm{E}-03$ & $2.148 \mathrm{E}+04$ & ${ }^{95} \mathrm{Nb}$ \\
\hline${ }^{93 m} \mathrm{Nb}$ & 41 & $1.36 \mathrm{E}+01 \mathrm{y}$ & IT & & 0.0281 & 0.0018 & $2.99 \mathrm{E}-02$ & $1.77 \mathrm{E}-04$ & $2.826 \mathrm{E}+02$ & ${ }^{93} \mathrm{Nb}$ \\
\hline${ }^{94} \mathrm{Nb}$ & 41 & $2.03 E+04 y$ & $\beta$ & & 0.1454 & 1.5715 & 1.7169 & $1.018 \mathrm{E}-02$ & $1.873 \mathrm{E}-01$ & ${ }^{94} \mathrm{Mo}$ \\
\hline${ }^{95} \mathrm{Nb}$ & 41 & $34.97 \mathrm{~d}$ & $\beta$ & & 0.0435 & 0.7643 & $8.078 \mathrm{E}-01$ & $4.788 \mathrm{E}-03$ & $3.910 \mathrm{E}+04$ & ${ }^{95} \mathrm{Mo}$ \\
\hline${ }^{93} \mathrm{Mo}$ & 42 & $3500 \mathrm{y}$ & $\mathrm{EC}$ & & 0.0051 & 0.0107 & $1.58 \mathrm{E}-02$ & $9.37 E-05$ & 1.10 & ${ }^{93} \mathrm{Nb}$ \\
\hline${ }^{99} \mathrm{Mo}$ & 42 & $2.748 \mathrm{~d}$ & $\beta$ & & 0.4076 & 0.2723 & $6.799 \mathrm{E}-01$ & $4.028 \mathrm{E}-03$ & $4.796 \mathrm{E}+05$ & ${ }^{99} \mathrm{Tc}$ \\
\hline${ }^{99} \mathrm{Tc}$ & 43 & $2.13 E+05 y$ & $\beta$ & & 0.0846 & & $8.46 \mathrm{E}-02$ & $5.01 \mathrm{E}-04$ & $1.695 \mathrm{E}-02$ & ${ }^{99} \mathrm{Ru}$ \\
\hline${ }^{99 m} \mathrm{Tc}$ & 43 & $6.006 \mathrm{~h}$ & IT & & 0.0142 & 0.1240 & $1.382 \mathrm{E}-01$ & $8.186 \mathrm{E}-04$ & $5.271 \mathrm{E}+06$ & ${ }^{99} \mathrm{Tc}$ \\
\hline${ }^{103} \mathrm{Ru}$ & 44 & $39.254 \mathrm{~d}$ & $\beta$ & & 0.1105 & 0.4851 & $5.96 \mathrm{E}-01$ & $3.53 \mathrm{E}-03$ & $3.227 \mathrm{E}+04$ & ${ }^{103} \mathrm{Rh}$ \\
\hline${ }^{106} \mathrm{Ru}$ & 44 & $1.020 \mathrm{y}$ & $\beta$ & & 0.1004 & & $1.004 \mathrm{E}-01$ & $5.951 \mathrm{E}-04$ & $3.346 \mathrm{E}+03$ & ${ }^{106} \mathrm{Rh}$ \\
\hline${ }^{103 m} \mathrm{Rh}$ & 45 & $56.12 \mathrm{~min}$ & IT & & 0.0375 & 0.0017 & $3.92 \mathrm{E}-02$ & $2.32 E-04$ & $3.253 \mathrm{E}+07$ & ${ }^{103} \mathrm{Rh}$ \\
\hline${ }^{106} \mathrm{Rh}$ & 45 & $2.17 \mathrm{~h}$ & $\beta$ & & 0.3144 & 2.8826 & 3.197 & $1.894 \mathrm{E}-02$ & $3.560 \mathrm{E}+09$ & ${ }^{106} \mathrm{Pd}$ \\
\hline
\end{tabular}


Table B.1 (continued)

\begin{tabular}{|c|c|c|c|c|c|c|c|c|c|c|}
\hline \multirow{2}{*}{ Nuclide } & \multirow{2}{*}{$\begin{array}{l}\text { Atomic } \\
\text { number }\end{array}$} & \multirow{2}{*}{ Half-life ${ }^{b}$} & \multirow{2}{*}{$\begin{array}{l}\text { Principal } \\
\text { mode(s) of } \\
\text { decayc }\end{array}$} & \multicolumn{3}{|c|}{$\begin{array}{l}\text { Major radiation energies } \\
\text { (MeV/dis) }\end{array}$} & \multicolumn{2}{|c|}{ "Q" value e } & \multirow{2}{*}{$\begin{array}{c}\text { Specific } \\
\text { activity } \\
(\mathrm{Ci} / \mathrm{g})\end{array}$} & \multirow{2}{*}{ Daughter(s) } \\
\hline & & & & $\alpha$ & $\epsilon$ & $y$ and $X$ & (MeV/dis) & $(\mathrm{W} / \mathrm{Ci})$ & & \\
\hline${ }^{107} \mathrm{Pd}$ & 46 & $6.5 \mathrm{E}+06 \mathrm{y}$ & $\beta$ & & & 0.0093 & $9.3 \mathrm{E}-03$ & $5.5 \mathrm{E}-05$ & $5.143 E-04$ & ${ }^{107} \mathrm{Ag}$ \\
\hline${ }^{110} \mathrm{Ag}$ & 47 & $24.6 \mathrm{~s}$ & $\begin{array}{l}\beta(99.70 \%) \\
\operatorname{EC~}(0.30 \%)\end{array}$ & & 1.1842 & 0.0316 & 1.216 & $7.208 \mathrm{E}-03$ & $4.169 E+09$ & $\begin{array}{l}{ }^{110} \mathrm{Cd} \text {; } \\
{ }^{110} \mathrm{Pd}\end{array}$ \\
\hline${ }^{110 m} \mathrm{Ag}$ & 47 & $249.76 \mathrm{~d}$ & $\begin{array}{l}\beta(98.64 \%) \\
\text { IT }(1.36 \%)\end{array}$ & & 0.0755 & 2.7392 & 2.815 & $1.669 \mathrm{E}-02$ & $4.750 \mathrm{E}+03$ & $\begin{array}{l}{ }^{110} \mathrm{Cd} ; \\
{ }^{110} \mathrm{Ag}\end{array}$ \\
\hline${ }^{113} \mathrm{Cd}$ & 48 & $9.3 \mathrm{E}+15 \mathrm{y}$ & $\beta$ & & 0.0933 & & $9.13 \mathrm{E}-02$ & $5.412 \mathrm{E}-04$ & $3.402 E-13$ & ${ }^{113}$ In \\
\hline${ }^{113 m} \mathrm{Cd}$ & 48 & $1.37 \mathrm{E}+01 \mathrm{y}$ & $\begin{array}{l}\beta(99.9 \%) \\
\text { IT }(0.1 \%)\end{array}$ & & & 0.1834 & $1.83 E-01$ & $1.08 \mathrm{E}-03$ & $2.168 \mathrm{E}+02$ & $\begin{array}{l}{ }^{113} \mathrm{In} ; \\
{ }^{113} \mathrm{Cd}\end{array}$ \\
\hline${ }^{115 m} \mathrm{Cd}$ & 48 & $44.6 \mathrm{~d}$ & $\beta$ & & 0.6029 & 0.0329 & $6.36 \mathrm{E}-01$ & $3.76 \mathrm{E}-03$ & $2.546 \mathrm{E}+04$ & ${ }^{115} \mathrm{In}$ \\
\hline "111 In & 49 & $2,807 \mathrm{~d}$ & $\mathrm{EC}$ & & 0.0340 & 0.4053 & $4.393 \mathrm{E}-01$ & $2.604 \mathrm{E}-03$ & $4.157 \mathrm{E}+05$ & ${ }^{111} \mathrm{Cd}$ \\
\hline${ }^{113 m} \mathrm{In}$ & 49 & $1.658 \mathrm{~h}$ & IT & & 0.1340 & 0.2555 & $3.89 \mathrm{E}-01$ & $2.31 \mathrm{E}-03$ & $1.673 \mathrm{E}+07$ & ${ }^{113} \mathrm{In}$ \\
\hline${ }^{114 m} I n$ & 49 & $49.51 \mathrm{~d}$ & $\begin{array}{l}\operatorname{IT}(95.7 \%) \\
\operatorname{EC}(4.3 \%)\end{array}$ & & 0.1431 & 0.0943 & $2.37 \mathrm{E}-01$ & $1.40 \mathrm{E}-03$ & $2.313 E+04$ & $\begin{array}{l}{ }^{114} \mathrm{In} ; \\
{ }^{114} \mathrm{Cd}\end{array}$ \\
\hline${ }^{113} \mathrm{Sn}$ & 50 & $115.09 \mathrm{~d}$ & $\mathrm{EC}$ & & 0.1394 & 0.2808 & $4.20 \mathrm{E}-01$ & $2.48 \mathrm{E}-03$ & $1.004 \mathrm{E}+04$ & ${ }^{113}$ In \\
\hline${ }^{117 m} \mathrm{Sn}$ & 50 & $13.61 \mathrm{~d}$ & IT & & 0.1613 & 0.1580 & $3.19 \mathrm{E}-01$ & $1.89 \mathrm{E}-03$ & $7.969 E+04$ & ${ }^{117} \mathrm{Sn}$ \\
\hline${ }^{119 m} \mathrm{Sn}$ & 50 & $293.0 \mathrm{~d}$ & IT & & 0.0783 & 0.0114 & $8.97 E-02$ & $5.32 \mathrm{E}-04$ & $4.478 \mathrm{E}+03$ & ${ }^{119} \mathrm{Sn}$ \\
\hline${ }^{121 m} \mathrm{Sn}$ & 50 & $5.5 \mathrm{E}+01 \mathrm{y}$ & $\begin{array}{l}\text { IT }(77.6 \%) \\
\beta(22.4 \%)\end{array}$ & & 0.0352 & 0.0050 & $4.02 \mathrm{E}-02$ & $2.43 \mathrm{E}-04$ & $5.912 \mathrm{E}+01$ & $\begin{array}{l}{ }^{121} \mathrm{Sn} ; \\
{ }^{121} \mathrm{Sb}\end{array}$ \\
\hline${ }^{123} \mathrm{Sn}$ & 50 & $129.2 \mathrm{~d}$ & $\beta$ & & 0.5222 & 0.0069 & $5.29 \mathrm{E}-01$ & $3.14 \mathrm{E}-03$ & $8.219 \mathrm{E}+03$ & ${ }^{123} \mathrm{Sb}$ \\
\hline${ }^{125} \mathrm{Sn}$ & 50 & $9.64 d$ & $\beta$ & & 0.8110 & 0.3124 & 1.123 & $6.656 \mathrm{E}-03$ & $1.084 \mathrm{E}+05$ & ${ }^{125} \mathrm{Sb}$ \\
\hline${ }^{126} \mathrm{Sn}$ & 50 & $\sim 1 E+05 y$ & $\beta$ & & 0.1249 & 0.0573 & $1.82 \mathrm{E}-01$ & $1.08 \mathrm{E}-03$ & $2.837 \mathrm{E}-02$ & ${ }^{126} \mathrm{Sb}$ \\
\hline${ }^{124} \mathrm{Sb}$ & 51 & $60.20 \mathrm{~d}$ & $\beta$ & & 0.3897 & 1.8523 & 2.242 & $1.329 \mathrm{E}-02$ & $1.749 E+04$ & ${ }^{124} \mathrm{Te}$ \\
\hline${ }^{125} \mathrm{Sb}$ & 51 & $2.73 y$ & $\beta$ & & 0.1257 & 0.4434 & $5.69 \mathrm{E}-01$ & $3.37 \mathrm{E}-03$ & $1.032 \mathrm{E}+03$ & ${ }^{125} \mathrm{Te}$ \\
\hline${ }^{126} \mathrm{Sb}$ & 51 & $12.4 \mathrm{~d}$ & $\beta$ & & 0.3527 & 2.7496 & 3.102 & $1.839 \mathrm{E}-02$ & $8.360 \mathrm{E}+04$ & ${ }^{126} \mathrm{Te}$ \\
\hline${ }^{126 \mathrm{~m}} \mathrm{Sb}$ & 51 & $19.0 \mathrm{~min}$ & $\begin{array}{l}\beta(86 \%) \\
\text { IT }(14 \%)\end{array}$ & & 0.6323 & 1.5484 & 2.181 & $1.292 \mathrm{E}-02$ & $7.854 \mathrm{E}+07$ & $\begin{array}{l}{ }^{126} \mathrm{Te} ; \\
{ }^{126} \mathrm{Sb}\end{array}$ \\
\hline${ }^{123 \mathrm{~m}} \mathrm{Te}$ & 52 & $119.7 \mathrm{~d}$ & IT & & 0.1020 & 0.1482 & $2.502 \mathrm{E}-01$ & $1.482 E-03$ & $8.870 \mathrm{E}+03$ & ${ }^{123} \mathrm{Te}$ \\
\hline${ }^{125 \mathrm{~m}} \mathrm{Te}$ & 52 & $58 \mathrm{~d}$ & IT & & 0.1106 & 0.0361 & $1.467 \mathrm{E}-01$ & $8.690 \mathrm{E}-04$ & $1.801 \mathrm{E}+04$ & ${ }^{125} \mathrm{Te}$ \\
\hline${ }^{127} \mathrm{Te}$ & 52 & $9.35 \mathrm{~h}$ & $\beta$ & & 0.2248 & 0.0048 & $2.30 \mathrm{E}-01$ & $1.36 \mathrm{E}-03$ & $2.639 E+06$ & ${ }^{127} \mathrm{I}$ \\
\hline
\end{tabular}


Table B.1 (continued)

\begin{tabular}{|c|c|c|c|c|c|c|c|c|c|c|}
\hline \multirow{2}{*}{ Nuclide } & \multirow{2}{*}{$\begin{array}{l}\text { Atomic } \\
\text { number }\end{array}$} & \multirow{2}{*}{ Half-life $^{b}$} & \multirow{2}{*}{$\begin{array}{l}\text { Principal } \\
\text { mode(s) of } \\
\text { decayc }\end{array}$} & \multicolumn{3}{|c|}{$\begin{array}{c}\text { Major radiation energies } d \\
\text { (MeV/dis) }\end{array}$} & \multicolumn{2}{|c|}{ "Q" value" } & \multirow{2}{*}{$\begin{array}{c}\text { Specific } \\
\text { activity } \\
(\mathrm{Ci} / \mathrm{g})\end{array}$} & \multirow{2}{*}{ Daughter(s) } \\
\hline & & & & $\alpha$ & $\epsilon$ & $\gamma$ and $X$ & (MeV/dis) & $(\mathrm{W} / \mathrm{Ci})$ & & \\
\hline${ }^{127 \mathrm{~m}} \mathrm{Te}$ & 52 & $109 \mathrm{~d}$ & $\begin{array}{l}\text { IT }(97.6 \%) \\
\beta(2.4 \%)\end{array}$ & & 0.0821 & 0.0111 & $9.32 \mathrm{E}-02$ & $5.52 \mathrm{E}-04$ & $9.432 \mathrm{E}+03$ & ${ }^{127} \mathrm{Te}$ \\
\hline${ }^{129} \mathrm{Te}$ & 52 & $1.160 \mathrm{~h}$ & $\beta$ & & 0.5422 & 0.0624 & $6.05 E-01$ & $3.58 \mathrm{E}-03$ & $2.094 \mathrm{E}+07$ & ${ }^{129} \mathrm{I}$ \\
\hline${ }^{129 m} \mathrm{Te}$ & 52 & $33.6 \mathrm{~d}$ & $\begin{array}{l}\text { IT }(64 \%) \\
\beta(36 \%)\end{array}$ & & 0.2663 & 0.0370 & $3.03 E-01$ & $1.80 \mathrm{E}-03$ & $3.013 E+04$ & $\begin{array}{l}{ }^{129} \mathrm{Te} ; \\
{ }^{129} \mathrm{I}\end{array}$ \\
\hline${ }^{123} I$ & 53 & $13.2 \mathrm{~h}$ & $\mathrm{EC}$ & & 0.0276 & 0.1729 & $2.005 \mathrm{E}-01$ & $1.188 \mathrm{E}-03$ & $1.940 \mathrm{E}+06$ & ${ }^{123} \mathrm{Te}$ \\
\hline${ }^{125} \mathrm{I}$ & 53 & $60.14 \mathrm{~d}$ & $\mathrm{EC}$ & & 0.0179 & 0.0423 & $6.02 \mathrm{E}-02$ & $3.57 \mathrm{E}-04$ & $1.737 \mathrm{E}+04$ & ${ }^{125} \mathrm{Te}$ \\
\hline${ }^{129} \mathrm{I}$ & 53 & $1.57 E+07 y$ & $\beta$ & & 0.0556 & 0.0248 & $8.04 E-02$ & $4.77 \mathrm{E}-04$ & $1.765 \mathrm{E}-04$ & ${ }^{129} \mathrm{Xe}$ \\
\hline${ }^{131} \mathrm{I}$ & 53 & $8.040 \mathrm{~d}$ & $\beta$ & & 0.1913 & 0.3826 & $5.74 \mathrm{E}-01$ & $3.40 \mathrm{E}-03$ & $1.240 \mathrm{E}+05$ & ${ }^{131} \mathrm{Xe}$ \\
\hline${ }^{133} \mathrm{Xe}$ & 54 & $5.245 \mathrm{~d}$ & $\beta$ & & 0.1363 & 0.0459 & $1.82 \mathrm{E}-01$ & $1.08 \mathrm{E}-03$ & $1.872 \mathrm{E}+05$ & ${ }^{133} \mathrm{Cs}$ \\
\hline${ }^{134} \mathrm{Cs}$ & 55 & $2.062 \mathrm{y}$ & $\beta$ & & 0.1639 & 1.5555 & 1.719 & $1.019 \mathrm{E}-02$ & $1.294 \mathrm{E}+03$ & ${ }^{134} \mathrm{Ba}$ \\
\hline${ }^{135} \mathrm{Cs}$ & 55 & $3.0 \mathrm{E}+06 \mathrm{y}$ & $\beta$ & & 0.0563 & & $5.63 \mathrm{E}-02$ & $3.32 \mathrm{E}-04$ & $1.151 \mathrm{E}-03$ & ${ }^{135} \mathrm{Ba}$ \\
\hline${ }^{137} \mathrm{Cs}$ & 55 & $3.017 \mathrm{E}+01 \mathrm{y}$ & $\begin{array}{l}\beta(94.6 \%) \\
\beta(5.4 \%)\end{array}$ & & & 0.1708 & $1.71 \mathrm{E}-01$ & $1.01 \mathrm{E}-03$ & $8.698 \mathrm{E}+01$ & $\begin{array}{l}{ }^{137 m} \mathrm{Ba} \\
{ }^{137} \mathrm{Ba}\end{array}$ \\
\hline${ }^{133} \mathrm{Ba}$ & 56 & $1.054 \mathrm{E}+01 \mathrm{y}$ & $\mathrm{EC}$ & & 0.0547 & 0.4045 & $4.592 \mathrm{E}-01$ & $2.722 \mathrm{E}-03$ & $2.500 \mathrm{E}+02$ & ${ }^{133} \mathrm{Cs}$ \\
\hline${ }^{137} \mathrm{~m} \mathrm{Ba}$ & 56 & $2.552 \mathrm{~min}$ & IT & & 0.0652 & 0.5991 & $6.64 \mathrm{E}-01$ & $3.94 \mathrm{E}-03$ & $5.379 E+08$ & ${ }^{137} \mathrm{Ba}$ \\
\hline${ }^{141} \mathrm{Ce}$ & 58 & $32.50 \mathrm{~d}$ & $\beta$ & & 0.1707 & 0.0770 & $2.48 \mathrm{E}-01$ & $1.47 \mathrm{E}-03$ & $2.848 \mathrm{E}+04$ & ${ }^{141} \mathrm{Pr}$ \\
\hline${ }^{144} \mathrm{Ce}$ & 58 & $284.9 \mathrm{~d}$ & $\beta$ & & 0.0918 & 0.0192 & $1.11 \mathrm{E}-01$ & $6.58 \mathrm{E}-04$ & $3.190 \mathrm{E}+03$ & ${ }^{144} \mathrm{Pr}$ \\
\hline${ }^{143} \mathrm{Pr}$ & 59 & $13.58 \mathrm{~d}$ & $\beta$ & & 0.3156 & & $3.16 \mathrm{E}-01$ & $1.87 E-03$ & $6.731 E+04$ & ${ }^{143} \mathrm{Nd}$ \\
\hline${ }^{144} \mathrm{Pr}$ & 59 & $17.28 \mathrm{~min}$ & $\beta$ & & 1.2091 & 0.0289 & 1.238 & $7.338 \mathrm{E}-03$ & $7.555 \mathrm{E}+07$ & ${ }^{144} \mathrm{Nd}$ \\
\hline${ }^{144 \pi \mathrm{Pr}}$ & 59 & $7.2 \mathrm{~min}$ & $\begin{array}{l}\text { IT }(99.96 \%) \\
\beta(0.04 \%)\end{array}$ & & 0.0464 & 0.0121 & $5.85 \mathrm{E}-02$ & $3.43 \mathrm{E}-04$ & $1.814 \mathrm{E}+08$ & ${ }^{144} \mathrm{Pr}$ \\
\hline${ }^{146} \mathrm{Pm}$ & 61 & $5.53 \mathrm{y}$ & $\begin{array}{l}\text { EC }(66.1 \%) \\
\beta(33.9 \%)\end{array}$ & & 0.0928 & 0.7542 & $8.47 \mathrm{E}-01$ & $5.02 \mathrm{E}-03$ & $4.428 \mathrm{E}+02$ & $\begin{array}{l}{ }^{146} \mathrm{Nd} ; \\
{ }^{146} \mathrm{Sm}\end{array}$ \\
\hline${ }^{147} \mathrm{Pm}$ & 61 & $2.6234 \mathrm{y}$ & $\beta$ & & 0.6196 & & $6.20 \mathrm{E}-02$ & $3.67 E-04$ & $9.270 \mathrm{E}+02$ & ${ }^{147} \mathrm{Sm}$ \\
\hline${ }^{148} \mathrm{Pm}$ & 61 & $5.370 \mathrm{~d}$ & $\beta$ & & 0.7235 & 0.5747 & 1.298 & $7.691 \mathrm{E}-03$ & $1.643 E+05$ & ${ }^{148} \mathrm{Sm}$ \\
\hline${ }^{148 n} \mathrm{Pm}$ & 61 & $41.29 \mathrm{~d}$ & $\begin{array}{l}\beta(95.4 \%) \\
\text { IT }(4.6 \%)\end{array}$ & & 0.1695 & 1.9861 & 2.156 & $1.278 \mathrm{E}-02$ & $2.136 \mathrm{E}+04$ & $\begin{array}{l}{ }^{148} \mathrm{Sm} ; \\
{ }^{148} \mathrm{Pm}\end{array}$ \\
\hline
\end{tabular}


Table B.1 (continued)

\begin{tabular}{|c|c|c|c|c|c|c|c|c|c|c|}
\hline \multirow{2}{*}{ Nuclide } & \multirow{2}{*}{$\begin{array}{l}\text { Atomic } \\
\text { number }\end{array}$} & \multirow{2}{*}{ Half-life ${ }^{b}$} & \multirow{2}{*}{$\begin{array}{l}\text { Principal } \\
\text { mode(s) of } \\
\text { decayc }\end{array}$} & \multicolumn{3}{|c|}{$\begin{array}{l}\text { Major radiation energies } \mathrm{d} \\
\text { (MeV/dis) }\end{array}$} & \multicolumn{2}{|c|}{ "Q" value } & \multirow{2}{*}{$\begin{array}{c}\text { Specific } \\
\text { activity } \\
(\mathrm{Ci} / \mathrm{g})\end{array}$} & \multirow{2}{*}{ Daughter(s) } \\
\hline & & & & $\alpha$ & $\epsilon$ & $\gamma$ and $X$ & (MeV/dis) & $(\mathrm{W} / \mathrm{Ci})$ & & \\
\hline${ }^{151} \mathrm{Sm}$ & 62 & $9.0 \mathrm{E}+01 \mathrm{y}$ & $\beta$ & & 0.1251 & & $1.25 \mathrm{E}-01$ & $7.41 \mathrm{E}-04$ & $2.631 \mathrm{E}+01$ & ${ }^{151} \mathrm{Eu}$ \\
\hline${ }^{152} \mathrm{Eu}$ & 63 & $1.333 E+01 y$ & $\begin{array}{l}\mathrm{EC}(72.08 \%) \\
\beta(27.92 \%)\end{array}$ & & 0.1275 & 1.1628 & 1.290 & $7.646 \mathrm{E}-03$ & $1.729 \mathrm{E}+02$ & $\begin{array}{l}{ }^{152} \mathrm{Sm} ; \\
{ }^{152} \mathrm{Gd}\end{array}$ \\
\hline${ }^{154} \mathrm{Eu}$ & 63 & $8.8 \mathrm{y}$ & $\beta$ & & 0.2794 & 1.2531 & 1.532 & $9.081 \mathrm{E}-03$ & $2.699 \mathrm{E}+02$ & ${ }^{154} \mathrm{Gd}$ \\
\hline${ }^{155} \mathrm{Eu}$ & 63 & $4.96 \mathrm{y}$ & $\beta$ & & 0.0650 & 0.0633 & $1.28 \mathrm{E}-01$ & $7.59 \mathrm{E}-04$ & $4.651 \mathrm{E}+02$ & ${ }^{15 s} \mathrm{Gd}$ \\
\hline${ }^{153} \mathrm{Gd}$ & 64 & $241.6 \mathrm{~d}$ & $\mathrm{EC}$ & & 0.0399 & 0.1015 & $1.414 \mathrm{E}-01$ & $8.381 E-04$ & $3.526 \mathrm{E}+03$ & ${ }^{153} \mathrm{Eu}$ \\
\hline${ }^{157} \mathrm{~Tb}$ & 65 & $150 y$ & $\mathrm{EC}$ & & 0.0031 & 0.0050 & $8.10 \mathrm{E}-03$ & $4.802 \mathrm{E}-05$ & $1.519 E+01$ & ${ }^{157} \mathrm{Gd}$ \\
\hline${ }^{158} \mathrm{~Tb}$ & 65 & $150 y$ & $\begin{array}{l}\mathrm{EC}(82 \%) \\
\beta(18 \%)\end{array}$ & & & & $9.02 \mathrm{E}-01$ & $5.347 \mathrm{E}-03$ & $1.508 \mathrm{E}+01$ & $\begin{array}{l}{ }^{158} \mathrm{Gd} ; \\
{ }^{158} \mathrm{Dy}\end{array}$ \\
\hline${ }^{160} \mathrm{~Tb}$ & 65 & $72.3 \mathrm{~d}$ & $\beta$ & & 0.2535 & 1.1271 & 1.381 & $8.186 \mathrm{E}-03$ & $1.129 \mathrm{E}+04$ & ${ }^{160} \mathrm{Dy}$ \\
\hline${ }^{169} \mathrm{Yb}$ & 70 & $32.02 \mathrm{~d}$ & $\mathrm{EC}$ & & 0.1117 & 0.3121 & $4.238 \mathrm{E}-01$ & $2.512 \mathrm{E}-03$ & $2.414 \mathrm{E}+04$ & ${ }^{169} \mathrm{Tm}$ \\
\hline${ }^{175} \mathrm{Hf}$ & 72 & $70.0 \mathrm{~d}$ & EC & & 0.0439 & 0.3646 & $4.085 \mathrm{E}-01$ & $2.422 \mathrm{E}-03$ & $1.066 \mathrm{E}+04$ & ${ }^{175} \mathrm{Lu}$ \\
\hline${ }^{181} \mathrm{Hf}$ & 72 & $42.39 \mathrm{~d}$ & $\beta$ & & 0.1943 & 0.5441 & $7.54 \mathrm{E}-01$ & $4.47 \mathrm{E}-03$ & $1.702 \mathrm{E}+04$ & ${ }^{181} \mathrm{Ta}$ \\
\hline${ }^{182} \mathrm{Ta}$ & 73 & $115.0 \mathrm{~d}$ & $\beta$ & & 0.2073 & 1.3011 & 1.508 & $8.940 E-03$ & $6.253 E+03$ & ${ }^{182} W$ \\
\hline${ }^{187} \mathrm{Re}$ & 75 & $4.6 E+10 y$ & $\beta$ & & 0.0007 & & 2.59 & $1.535 \mathrm{E}-02$ & $3.823 \mathrm{E}-08$ & ${ }^{187} \mathrm{Os}$ \\
\hline${ }^{192} \mathrm{Ir}$ & 77 & $73.831 \mathrm{~d}$ & $\begin{array}{l}\beta(95.4 \%) \\
\operatorname{EC}(4.6 \%)\end{array}$ & & 0.2162 & 0.8137 & 1.030 & $6.105 E-03$ & $9.211 \mathrm{E}+03$ & $\begin{array}{l}{ }^{192} \mathrm{Pt} \\
{ }^{192} \mathrm{Os}\end{array}$ \\
\hline${ }^{201} \mathrm{Tl}$ & 81 & $3.046 \mathrm{~d}$ & $\mathrm{EC}$ & & 0.0481 & 0.0924 & $1.40 \mathrm{E}-01$ & $8.30 E-04$ & $2.132 \mathrm{E}+05$ & ${ }^{201} \mathrm{Hg}$ \\
\hline${ }^{207} \mathrm{TI}$ & 81 & $4.77 \mathrm{~min}$ & $\beta$ & & 0.4931 & 0.0022 & $4.95 \mathrm{E}-01$ & $2.93 \mathrm{E}-03$ & $1.904 \mathrm{E}+08$ & ${ }^{207} \mathrm{~Pb}$ \\
\hline${ }^{208} \mathrm{TI}$ & 81 & $3.053 \mathrm{~min}$ & $\beta$ & 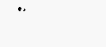 & 0.5979 & 3.3742 & 3.972 & $2.354 \mathrm{E}-02$ & $2.945 E+08$ & ${ }^{208} \mathrm{~Pb}$ \\
\hline${ }^{209} \mathrm{~Pb}$ & 82 & $3.253 \mathrm{~h}$ & $\beta$ & & 0.1980 & & $1.98 \mathrm{E}-01$ & $1.17 \mathrm{E}-03$ & $4.544 \mathrm{E}+06$ & ${ }^{209} \mathrm{Bi}$ \\
\hline${ }^{210} \mathrm{~Pb}$ & 82 & $2.232 \mathrm{E}+01 \mathrm{y}$ & $\beta$ & & 0.0343 & & $3.43 \mathrm{E}-02$ & $2.029 E-04$ & $7.630 \mathrm{E}+01$ & ${ }^{210} \mathrm{Bi}$ \\
\hline${ }^{211} \mathrm{~Pb}$ & 82 & $36.1 \mathrm{~min}$ & $\beta$ & & 0.4523 & 0.0678 & $5.20 E-01$ & $3.083 \mathrm{E}-03$ & $2.468 \mathrm{E}+07$ & ${ }^{211} \mathrm{Bi}$ \\
\hline${ }^{212} \mathrm{~Pb}$ & 82 & $10.64 \mathrm{~h}$ & $\beta$ & & 0.1752 & 0.1453 & $3.20 \mathrm{E}-01$ & $1.90 \mathrm{E}-03$ & $1.389 \mathrm{E}+06$ & ${ }^{212} \mathrm{Bi}$ \\
\hline${ }^{211} \mathrm{Bi}$ & 83 & $2.14 \mathrm{~min}$ & $\begin{array}{l}\alpha(99.727 \%) \\
\beta(0.273 \%)\end{array}$ & 6.5505 & 0.0099 & 0.0467 & 6.607 & $3.916 \mathrm{E}-02$ & $4.184 \mathrm{E}+08$ & $\begin{array}{l}{ }^{207} \mathrm{Tl} \\
{ }^{211} \mathrm{Po}\end{array}$ \\
\hline
\end{tabular}


Table B.1 (continued)

\begin{tabular}{|c|c|c|c|c|c|c|c|c|c|c|}
\hline \multirow{2}{*}{ Nuclide } & \multirow{2}{*}{$\begin{array}{l}\text { Atomic } \\
\text { number }\end{array}$} & \multirow{2}{*}{ Half-life ${ }^{b}$} & \multirow{2}{*}{$\begin{array}{l}\text { Principal } \\
\text { mode(s) of } \\
\text { decay }\end{array}$} & \multicolumn{3}{|c|}{$\begin{array}{c}\text { Major radiation energies }{ }^{d} \\
\text { (MeV/dis) }\end{array}$} & \multicolumn{2}{|c|}{ "Q" value } & \multirow{2}{*}{$\begin{array}{c}\text { Specific } \\
\text { activity } \\
(\mathrm{Ci} / \mathrm{g})\end{array}$} & \multirow{2}{*}{ Daughter(s) } \\
\hline & & & & $\alpha$ & $\epsilon$ & $\gamma$ and $X$ & (MeV/dis) & $(\mathrm{W} / \mathrm{Ci})$ & & \\
\hline${ }^{212} \mathrm{Bi}$ & 83 & $1.0092 \mathrm{~h}$ & $\begin{array}{l}\alpha(35.94 \%) \\
\beta(64.06 \%)\end{array}$ & 2.1740 & 0.5025 & 0.1061 & 2.783 & $1.649 E-02$ & $1.465 \mathrm{E}+07$ & $\begin{array}{l}{ }^{208} \mathrm{Tl} ; \\
{ }^{212} \mathrm{Po}\end{array}$ \\
\hline${ }^{213} \mathrm{Bi}$ & 83 & $45.59 \mathrm{~min}$ & $\begin{array}{l}\alpha(2.16 \%) \\
\beta(97.84 \%)\end{array}$ & 0.1268 & 0.4563 & 0.0825 & $6.66 \mathrm{E}-01$ & $3.95 \mathrm{E}-03$ & $1.934 \mathrm{E}+07$ & $\begin{array}{l}{ }^{209} \mathrm{Tl} \\
{ }^{213} \mathrm{Po}\end{array}$ \\
\hline${ }^{209} \mathrm{Po}$ & 84 & $102 y$ & $\begin{array}{l}\alpha(99.74 \%) \\
\operatorname{EC}(0.26 \%)\end{array}$ & & ' & & 4.9645 & $2.943 \mathrm{E}-01$ & $1.68 \mathrm{E}+01$ & ${ }^{205} \mathrm{~Pb}$ \\
\hline${ }^{210} \mathrm{Po}$ & 84 & $138.4 \mathrm{~d}$ & $\alpha$ & 5.3044 & & & 5.304 & $3.144 \mathrm{E}-02$ & $4.493 E+03$ & ${ }^{206} \mathrm{~Pb}$ \\
\hline${ }^{212} \mathrm{Po}$ & 84 & $2.98 \mathrm{E}-07 \mathrm{~s}$ & $\alpha$ & 8.7844 & & & 8.784 & $5.207 \mathrm{E}-02$ & $1.774 \mathrm{E}+17$ & ${ }^{208} \mathrm{~Pb}$ \\
\hline${ }^{213} \mathrm{Po}$ & 84 & $4.2 \mathrm{E}-06 \mathrm{~s}$ & $\alpha$ & 8.3757 & & & 8.375 & $4.964 \mathrm{E}-02$ & $1.261 \mathrm{E}+16$ & ${ }^{209} \mathrm{~Pb}$ \\
\hline${ }^{215} \mathrm{Po}$ & 84 & $1.780 \mathrm{E}-03 \mathrm{~s}$ & $\alpha$ & 7.3864 & & & 7.386 & $4.378 \mathrm{E}-02$ & $2.948 E+13$ & ${ }^{211} \mathrm{~Pb}$ \\
\hline${ }^{216} \mathrm{Po}$ & 84 & $1.50 \mathrm{E}-02 \mathrm{~s}$ & $\alpha$ & 6.7785 & & & 6.779 & $4.018 \mathrm{E}-02$ & $3.482 E+11$ & ${ }^{212} \mathrm{~Pb}$ \\
\hline${ }^{217} \mathrm{At}$ & 85 & $3.23 E-02 s$ & $\alpha$ & 7.0657 & & 0.0002 & 7.066 & $4.189 E-02$ & $1.610 \mathrm{E}+12$ & ${ }^{213} \mathrm{Bi}$ \\
\hline${ }^{219} \mathrm{Rn}$ & 86 & $3.96 \mathrm{~s}$ & $\alpha$ & 6.8122 & 0.0064 & 0.0560 & 6.875 & $4.076 \mathrm{E}-02$ & $1.301 \mathrm{E}+10$ & ${ }^{215} \mathrm{Po}$ \\
\hline${ }^{220} \mathrm{Rn}$ & 86 & $55.6 \mathrm{~s}$ & $\alpha$ & 6.2878 & & 0.0005 & 6.288 & $3.727 \mathrm{E}-02$ & $9.223 \mathrm{E}+08$ & ${ }^{216} \mathrm{Po}$ \\
\hline${ }^{222} \mathrm{Rn}$ & 86 & $3.825 \mathrm{~d}$ & $\alpha$ & 5.4892 & & 0.0004 & 5.490 & $3.255 \mathrm{E}-02$ & $1.538 \mathrm{E}+05$ & ${ }^{218} \mathrm{Po}$ \\
\hline${ }^{221} \mathrm{Fr}$ & 87 & $4.9 \mathrm{~min}$ & $\alpha$ & 6.3571 & 0.0084 & 0.0277 & 6.393 & $3.789 E-02$ & $1.772 \mathrm{E}+08$ & ${ }^{217} \mathrm{At}$ \\
\hline${ }^{223} \mathrm{Fr}$ & 87 & $21.8 \mathrm{~min}$ & $\beta$ & & 0.3805 & $0.0 \$ 42$ & $4.35 \mathrm{E}-01$ & $2.85 \mathrm{E}-03$ & $3.868 \mathrm{E}+07$ & ${ }^{223} \mathbf{R a}$ \\
\hline${ }^{223} \mathrm{Ra}$ & 88 & $11.43 \mathrm{~d}$ & $\alpha$ & 5.6972 & 0.0731 & 0.1348 & 5.905 & $3.500 \mathrm{E}-02$ & $5.121 E+04$ & ${ }^{219} \mathrm{Rn}$ \\
\hline${ }^{224} \mathrm{Ra}$ & 88 & $3.66 \mathrm{~d}$ & $\alpha$ & 5.6751 & 0.0022 & 0.0103 & 5.688 & $3.372 \mathrm{E}-02$ & $1.593 E+05$ & ${ }^{220} \mathrm{Rn}$ \\
\hline${ }^{225} \mathrm{Ra}$ & 88 & $14.2 \mathrm{~d}$ & $\beta$ & & 0.1057 & 0.0137 & $1.19 \mathrm{E}-01$ & $7.08 E-04$ & $3.920 \mathrm{E}+04$ & ${ }^{225} \mathrm{Ac}$ \\
\hline${ }^{226} \mathrm{Ra}$ & 88 & $1.600 E+03 y$ & $\alpha$ & 4.7741 & 0.0035 & 0.0067 & 4.784 & $2.836 \mathrm{E}-02$ & $9.887 \mathrm{E}-01$ & ${ }^{222} \mathrm{Rn}$ \\
\hline${ }^{228} \mathrm{Ra}$ & 88 & $5.75 y$ & $\beta$ & & 0.0116 & & $1.16 \mathrm{E}-02$ & $6.88 \mathrm{E}-05$ & $2.340 \mathrm{E}+02$ & ${ }^{228} \mathrm{Ac}$ \\
\hline${ }^{225} \mathrm{Ac}$ & 89 & $10.0 \mathrm{~d}$ & $\alpha$ & 5.7501 & 0.0257 & 0.0176 & 5.793 & $3.434 \mathrm{E}-02$ & $5.803 E+04$ & ${ }^{221} \mathrm{Fr}$ \\
\hline${ }^{227} \mathrm{Ac}$ & 89 & $2.177 \mathrm{E}+01$ y & $\begin{array}{l}\beta(98.62 \%) \\
\alpha(1.38 \%)\end{array}$ & 0.0673 & 0.0125 & 0.0002 & $8.00 \mathrm{E}-02$ & $4.74 \mathrm{E}-04$ & $7.233 E+01$ & $\begin{array}{l}{ }^{227} \mathrm{Th} \\
{ }^{223} \mathrm{Fr}\end{array}$ \\
\hline${ }^{228} \mathrm{Ac}$ & 89 & $6.13 \mathrm{~h}$ & $\beta$ & & 0.4292 & 0.9269 & 1.356 & $8.038 \mathrm{E}-03$ & $2.242 \mathrm{E}+06$ & ${ }^{228} \mathrm{Th}$ \\
\hline${ }^{227} \mathrm{Th}$ & 90 & $18.718 d$ & $\alpha$ & 5.9022 & 0.0543 & 0.1113 & 6.068 & $3.597 \mathrm{E}-02$ & $3.073 E+04$ & ${ }^{223} \mathrm{Ra}$ \\
\hline${ }^{228} \mathrm{Th}$ & 90 & $1.913 \mathrm{y}$ & $\alpha$ & 5.3992 & 0.0201 & 0.0034 & 5.423 & $3.214 \mathrm{E}-02$ & $8.196 \mathrm{E}+02$ & ${ }^{224} \mathrm{Ra}$ \\
\hline${ }^{229} \mathrm{Th}$ & 90 & $7.340 \mathrm{E}+03 \mathrm{y}$ & $\alpha$ & 4.8620 & & 0.0343 & 4.896 & $2.902 \mathrm{E}-02$ & $2.127 \mathrm{E}-01$ & ${ }^{223} \mathrm{Ra}$ \\
\hline
\end{tabular}


Table B.1 (continued)

\begin{tabular}{|c|c|c|c|c|c|c|c|c|c|c|}
\hline \multirow{2}{*}{ Nuclide } & \multirow{2}{*}{$\begin{array}{l}\text { Atomic } \\
\text { number }\end{array}$} & \multirow{2}{*}{ Half-life $\mathrm{e}^{\mathrm{b}}$} & \multirow{2}{*}{$\begin{array}{l}\text { Principal } \\
\text { mode(s) of } \\
\text { decayc }^{c}\end{array}$} & \multicolumn{3}{|c|}{$\begin{array}{l}\text { Major radiation energies }{ }^{d} \\
\text { (MeV/dis) }\end{array}$} & \multicolumn{2}{|c|}{ "Q" value } & \multirow{2}{*}{$\begin{array}{c}\text { Specific } \\
\text { activity } \\
(\mathrm{Ci} / \mathrm{g})\end{array}$} & \multirow{2}{*}{ Daughter(s) } \\
\hline & & & & $\alpha$ & $\epsilon$ & $\gamma$ and $X$ & $(\mathrm{MeV} / \mathrm{dis})$ & $(\mathrm{W} / \mathrm{Ci})$ & & \\
\hline${ }^{230} \mathrm{Th}$ & 90 & $7.54 \mathrm{E}+04 \mathrm{y}$ & $\alpha$ & 4.6651 & & 0.0004 & 4.665 & $2.765 E-02$ & $2.109 \mathrm{E}-02$ & ${ }^{226} \mathrm{Ra}$ \\
\hline${ }^{231} \mathrm{Th}$ & 90 & $1.0633 \mathrm{~d}$ & $\beta$ & 0.1732 & & 0.0295 & $2.03 E-01$ & $1.21 \mathrm{E}-03$ & $5.316 \mathrm{E}+05$ & ${ }^{231} \mathrm{~Pa}$ \\
\hline${ }^{232} \mathrm{Th}$ & 90 & $1.405 \mathrm{E}+10 \mathrm{y}$ & $\alpha$ & 4.0056 & & 0.0002 & 4.006 & $2.375 \mathrm{E}-02$ & $1.097 \mathrm{E}-07$ & ${ }^{228} \mathrm{Ra}$ \\
\hline${ }^{234} \mathrm{Th}$ & 90 & $24.10 \mathrm{~d}$ & $\beta$ & & 0.0158 & 0.0094 & $2.52 \mathrm{E}-02$ & $1.49 \mathrm{E}-04$ & $2.316 \mathrm{E}+04$ & ${ }^{234} \mathrm{~Pa}$ \\
\hline${ }^{231} \mathrm{~Pa}$ & 91 & $3.276 \mathrm{E}+04 \mathrm{y}$ & $\alpha$ & 4.9230 & 0.0483 & 0.0399 & 5.011 & $2.970 \mathrm{E}-02$ & $4.723 \mathrm{E}-02$ & ${ }^{227} \mathrm{Ac}$ \\
\hline${ }^{233} \mathrm{~Pa}$ & 91 & $27.0 \mathrm{~d}$ & $\beta$ & & 0.1941 & 0.2042 & $3.98 \mathrm{E}-01$ & $2.36 E-03$ & $2.075 E+04$ & ${ }^{233} \mathrm{U}$ \\
\hline${ }^{234 m} \mathrm{~Pa}$ & 91 & $1.17 \mathrm{~min}$ & $\begin{array}{l}\beta(99.87 \%) \\
\text { IT }(0.13 \%)\end{array}$ & & 0.8227 & 0.0121 & $8.35 E-01$ & $4.95 E-03$ & $6.868 \mathrm{E}+08$ & ${ }^{234} \mathrm{U}$ \\
\hline${ }^{232} \mathrm{U}$ & 92 & $6.89 \mathrm{E}+01 \mathrm{y}$ & $\alpha$ & 5.3065 & & 0.0002 & 5.307 & $3.146 \mathrm{E}-02$ & $2.140 \mathrm{E}+01$ & ${ }^{228} \mathrm{Th}$ \\
\hline${ }^{233} \mathrm{U}$ & 92 & $1.592 E+05 y$ & $\alpha$ & 4.8141 & 0.0055 & 0.0013 & 4.821 & $2.857 \mathrm{E}-02$ & $9.680 \mathrm{E}-03$ & ${ }^{229} \mathrm{Th}$ \\
\hline${ }^{234} \mathrm{U}$ & 92 & $2.454 \mathrm{E}+05 \mathrm{y}$ & $\alpha$ & 4.7732 & & 0.0001 & 4.773 & $2.829 \mathrm{E}-02$ & $6.248 E-03$ & ${ }^{230} \mathrm{Th}$ \\
\hline${ }^{235} \mathrm{U}$ & 92 & $7.037 \mathrm{E}+08 \mathrm{y}$ & $\alpha$ & 4.3785 & 0.0426 & 0.1561 & 4.577 & $2.713 E-02$ & $2.161 \mathrm{E}-06$ & ${ }^{231} \mathrm{Th}$ \\
\hline${ }^{236} \mathrm{U}$ & 92 & $2.342 E+07 y$ & $\alpha$ & 4.4793 & 0.0108 & 0.0015 & 4.492 & $2.662 \mathrm{E}-02$ & $6.469 \mathrm{E}-05$ & ${ }^{232} \mathrm{Th}$ \\
\hline${ }^{238} \mathrm{U}$ & 92 & $4.468 \mathrm{E}+09 \mathrm{y}$ & $\alpha$ & 4.1945 & 0.0095 & 0.0013 & 4.205 & $2.492 \mathrm{E}-02$ & $3.362 \mathrm{E}-07$ & ${ }^{234} \mathrm{Th}$ \\
\hline${ }^{236} \mathrm{~Np}$ & 93 & $1.550 \mathrm{E}+05 \mathrm{y}$ & $\begin{array}{l}\mathrm{EC}(91 \%) \\
\beta(8.9 \%) \\
\alpha(0.20 \%)\end{array}$ & & 0.1967 & 0.1411 & $3.38 \mathrm{E}-01$ & $2.00 \mathrm{E}-03$ & $1.317 \mathrm{E}-02$ & $\begin{array}{l}{ }^{236} \mathrm{U} ; \\
{ }^{236} \mathrm{Pu} \\
{ }^{232} \mathrm{~Pa}\end{array}$ \\
\hline${ }^{237} \mathrm{~Np}$ & 93 & $2.140 \mathrm{E}+06 \mathrm{y}$ & $\alpha$ & 4.7604 & 0.0640 & 0.0327 & 4.857 & $2.879 \mathrm{E}-02$ & $7.049 \mathrm{E}-04$ & ${ }^{233} \mathrm{~Pa}$ \\
\hline${ }^{239} \mathrm{~Np}$ & 93 & $2.355 \mathrm{~d}$ & $\beta$ & & 0.2521 & 0.1740 & $4.26 \mathrm{E}-01$ & $2.53 \mathrm{E}-03$ & $2.320 \mathrm{E}+05$ & ${ }^{239} \mathrm{Pu}$ \\
\hline${ }^{236} \mathrm{Pu}$ & 94 & $2.851 \mathrm{y}$ & $\alpha$ & 5.7521 & 0.0126 & 0.0020 & 5.767 & $3.418 \mathrm{E}-02$ & $5.313 E+02$ & ${ }^{232} \mathrm{U}$ \\
\hline${ }^{238} \mathrm{Pu}$ & 94 & $8.774 \mathrm{E}+01 \mathrm{y}$ & $\alpha$ & 5.4871 & 0.0099 & 0.0018 & 5.499 & $3.2593 \mathrm{E}-02$ & $1.712 \mathrm{E}+01$ & ${ }^{234} \mathrm{U}$ \\
\hline${ }^{239} \mathrm{Pu}$ & 94 & $2.411 \mathrm{E}+04 \mathrm{y}$ & $\alpha$ & 5.1011 & & 0.0001 & 5.101 & $3.024 \mathrm{E}-02$ & $6.216 \mathrm{E}-02$ & ${ }^{235} \mathrm{U}$ \\
\hline${ }^{240} \mathrm{Pu}$ & 94 & $6.563 E+03 y$ & $\alpha$ & 5.1549 & & & 5.155 & $3.056 \mathrm{E}-02$ & $2.279 \mathrm{E}-01$ & ${ }^{236} \mathrm{U}$ \\
\hline${ }^{241} \mathrm{Pu}$ & 94 & $1.44 \mathrm{E}+01 \mathrm{y}$ & $\beta$ & 0.0001 & & 0.0052 & $5.3 E-03$ & $3.2 \mathrm{E}-05$ & $1.030 \mathrm{E}+02$ & ${ }^{241} \mathrm{Am}$ \\
\hline${ }^{242} \mathrm{Pu}$ & 94 & $3.763 E+05 y$ & $\alpha$ & 4.8901 & 0.0081 & 0.0014 & 4.900 & $2.904 \mathrm{E}-02$ & $3.818 \mathrm{E}-03$ & ${ }^{238} \mathrm{U}$ \\
\hline${ }^{244} \mathrm{Pu}$ & 94 & $8.26 \mathrm{E}+07 \mathrm{y}$ & $\begin{array}{l}\alpha(99.875 \%) \\
\operatorname{SPF}(0.125 \%)\end{array}$ & 4.5751 & 0.0007 & 0.0001 & 4.576 & $2.712 \mathrm{E}-02$ & $1.774 \mathrm{E}-05$ & $\begin{array}{l}{ }^{240} \mathrm{U} ; \\
\text { (fission } \\
\text { products) }\end{array}$ \\
\hline${ }^{241} \mathrm{Am}$ & 95 & $4.327 E+02 y$ & $\alpha$ & 5.4801 & 0.0304 & 0.0287 & 5.539 & $3.283 \mathrm{E}-02$ & 3.432 & ${ }^{237} \mathrm{~Np}$ \\
\hline${ }^{242} \mathrm{Am}$ & 95 & $16.01 \mathrm{~h}$ & $\begin{array}{l}\beta(82.7 \%) \\
\operatorname{EC}(17.3 \%)\end{array}$ & & 0.1781 & 0.0180 & $1.96 \mathrm{E}-01$ & $1.16 \mathrm{E}-03$ & $8.084 \mathrm{E}+05$ & ${ }^{242} \mathrm{Cm}$ \\
\hline
\end{tabular}


Table B.1 (continued)

\begin{tabular}{|c|c|c|c|c|c|c|c|c|c|c|}
\hline \multirow{2}{*}{ Nuclide } & \multirow{2}{*}{$\begin{array}{l}\text { Atomic } \\
\text { number }\end{array}$} & \multirow{2}{*}{ Half-life $^{b}$} & \multirow{2}{*}{$\begin{array}{l}\text { Principal } \\
\text { mode(s) of } \\
\text { decayc }\end{array}$} & \multicolumn{3}{|c|}{$\begin{array}{l}\text { Major radiation energies } \\
\text { (MeV/dis) }\end{array}$} & \multicolumn{2}{|c|}{ "Q" value } & \multirow{2}{*}{$\begin{array}{r}\text { Specific } \\
\text { activity } \\
(\mathrm{Ci} / \mathrm{g})\end{array}$} & \multirow{2}{*}{ Daughter(s) } \\
\hline & & & & $\alpha$ & $\epsilon$ & $\gamma$ and $X$ & (MeV/dis) & (W/Ci) & & \\
\hline${ }^{242 \mathrm{~m}} \mathrm{Am}$ & 95 & $1.41 \mathrm{E}+02 \mathrm{y}$ & $\begin{array}{l}\text { IT }(99.55 \%) \\
\alpha(0.45 \%)\end{array}$ & 0.0232 & 0.0403 & 0.0049 & $6.84 \mathrm{E}-02$ & $4.05 \mathrm{E}-04$ & 9.718 & $\begin{array}{l}{ }^{242} \mathrm{Am} \\
{ }^{238} \mathrm{~Np}\end{array}$ \\
\hline${ }^{243} \mathrm{Am}$ & 95 & $7.380 \mathrm{E}+03 \mathrm{y}$ & $\alpha$ & 5.2656 & & 0.0481 & 5.3137 & $3.1496 \mathrm{E}-02$ & $1.993 \mathrm{E}-01$ & ${ }^{239} \mathrm{~Np}$ \\
\hline${ }^{242} \mathrm{Cm}$ & 96 & $162.94 \mathrm{~d}$ & $\alpha$ & 6.0434 & 0.0090 & 0.0018 & 6.0542 & $3.5886 \mathrm{E}-02$ & $3.306 \mathrm{E}+03$ & ${ }^{238} \mathrm{Pu}$ \\
\hline${ }^{243} \mathrm{Cm}$ & 96 & $2.85 \mathrm{E}+01 \mathrm{y}$ & $\begin{array}{l}\alpha(99.76 \%) \\
\operatorname{EC}(0.24 \%)\end{array}$ & 5.8380 & 0.1129 & 0.1316 & 6.083 & $3.605 E-02$ & $5.162 \mathrm{E}+01$ & ${ }^{239} \mathrm{Pu}$ \\
\hline${ }^{244} \mathrm{Cm}$ & 96 & $1.811 E+01 y$ & $\alpha$ & 5.7965 & & 0.0016 & 5.798 & $3.437 E-02$ & $8.090 \mathrm{E}+01$ & ${ }^{240} \mathrm{Pu}$ \\
\hline${ }^{245} \mathrm{Cm}$ & 96 & $8.5 \mathrm{E}+03 \mathrm{y}$ & $\alpha$ & 5.3631 & 0.1342 & 0.1178 & 5.615 & $3.329 \mathrm{E}-02$ & $1.717 \mathrm{E}-01$ & ${ }^{241} \mathrm{Pu}$ \\
\hline${ }^{246} \mathrm{Cm}$ & 96 & $4.73 E+03 y$ & $\alpha$ & 5.3764 & 0.0072 & 0.0014 & 5.385 & $3.192 \mathrm{E}-02$ & $3.072 \mathrm{E}-01$ & ${ }^{242} \mathrm{Pu}$ \\
\hline${ }^{247} \mathrm{Cm}$ & 96 & $1.56 \mathrm{E}+07 \mathrm{y}$ & $\alpha$ & 4.9475 & & 0.3152 & 5.263 & $3.119 E-02$ & $9.278 \mathrm{E}-05$ & ${ }^{243} \mathrm{Pu}$ \\
\hline${ }^{248} \mathrm{Cm}$ & 96 & $3.40 \mathrm{E}+05 \mathrm{y}$ & $\begin{array}{l}\alpha(91.74 \%) \\
\operatorname{SPF}(8.26 \%)\end{array}$ & 4.6524 & & & 4.6524 & $2.7577 \mathrm{E}-02$ & $4.251 \mathrm{E}-03$ & $\begin{array}{l}{ }^{244} \mathrm{Pu} \text {; } \\
\text { (fission } \\
\text { products) }\end{array}$ \\
\hline${ }^{252} \mathrm{Cf}$ & 98 & $2.645 y$ & $\begin{array}{l}\alpha(96.908 \%) \\
\text { SPF }(3.092 \%)\end{array}$ & 5.9308 & 0.0051 & 0.0011 & 5.9370 & $3.5191 \mathrm{E}-02$ & $5.378 \mathrm{E}+02$ & $\begin{array}{l}{ }^{248} \mathrm{Cm} \text {; } \\
\text { (fission } \\
\text { products) }\end{array}$ \\
\hline
\end{tabular}

a Based on refs. 6-9.

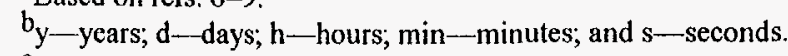

$c_{\alpha}$-alpha decay; $\beta$-negative beta decay; $\mathrm{EC}$ - electron capture; IT -isomeric transition (radioactive transition from one nuclear isomer to another of lower energy); and SPF-spontaneous fission.

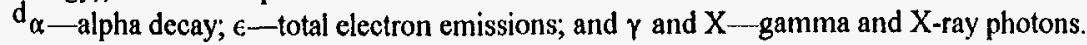

${ }^{2}$ The sum of the average energies for different radiation types in MeV/disintegration or W/Ci (includes alpha and beta particles, discrete electrons, and photons). The "value" indicates the amount of energy (heat) that could be deposited in a radioactive material from each decay event if none of the radiation escaped from the material. Neutrinos are not included. 
APPENDDX C. REFERENCE SITES AND FACILITIES 



\section{APPENDIX C. REFERENCE SITES AND FACIITIES}

This appendix provides information on the major DOE and commercial sites and facilities discussed in this report. The DOE operations and special site offices are identiffed in Table C.1, along with the sites for which they have responsibility. This is followed by Table C.2, which lists DOE Naval Reactors Program (NE-60) offices and sites. Table C.3 lists major DOE sites and facilities referred to in this report, and major commercial radioactive waste disposal sites are given in Table C.4. For each site or facility listed in Tables C.3 and C.4, additional information is provided, including reference symbol or label, location, operations contractor, and, for DOE sites, the supervisory DOE operations and area office. 
Table C.1. DOE operations and special site offices

\begin{tabular}{|c|c|c|c|}
\hline DOE office & Symbol/label & $\begin{array}{l}\text { Generai maiiing address } \\
\text { (Phone number) }^{\mathrm{a}}\end{array}$ & $\begin{array}{c}\text { Radioactive waste sites for which DOE } \\
\text { office has responsibility }\end{array}$ \\
\hline $\begin{array}{l}\text { Albuquerque Operations } \\
\text { Office }{ }^{b}\end{array}$ & DOE/AL & $\begin{array}{l}\text { P.O. Box } 5400 \\
\text { Albuquerque, NM } 87115-5400 \\
(505 / 845-4154)\end{array}$ & $\begin{array}{l}\text { Grand Junction Projects Office sites } \\
\text { Inhalation Toxicology Research Institute } \\
\text { Kansas City Plant } \\
\text { Los Alamos National Laboratory } \\
\text { Pantex Plant } \\
\text { Pinellas Plant } \\
\text { Sandia National Laboratories/California } \\
\text { Sandia National Laboratories/New Mexico } \\
\text { Uranium Mill Tailings Remedial Action Project sites } \\
\text { Waste Isolation Pilot Plant }\end{array}$ \\
\hline Chicago Operations Office ${ }^{c}$ & $\mathrm{DOE} / \mathrm{CH}$ & $\begin{array}{l}\text { Building } 201 \\
9800 \text { South Cass Avenue } \\
\text { Argonne, IL } 60439 \\
(630 / 252-2001)\end{array}$ & $\begin{array}{l}\text { Ames Laboratory } \\
\text { Argonne National Laboratory-East } \\
\text { Argonne National Laboratory-West } \\
\text { Brookhaven National Laboratory } \\
\text { Fermi National Accelerator Laboratory } \\
\text { Princeton Plasma Physics Laboratory }\end{array}$ \\
\hline Idaho Operations Office & DOE/ID & $\begin{array}{l}785 \text { DOE Place } \\
\text { Idaho Falls, ID } 83402 \\
(208 / 526-0111)\end{array}$ & Idaho National Engineering Laboratory \\
\hline Nevada Operations Office & DOE/NV & $\begin{array}{l}\text { P.O. Box } 98518 \\
\text { Las Vegas, Nevada } 89193-8518 \\
(702 / 295-1212)\end{array}$ & Nevada Test Site \\
\hline $\begin{array}{l}\text { Oakland Operations } \\
\text { Office }\end{array}$ & DOE/OAK & $\begin{array}{l}1301 \text { Clay Street } \\
\text { Oakland, CA } 94612-5208 \\
(510 / 273-6383)\end{array}$ & $\begin{array}{l}\text { Energy Technology Engineering Center (Santa Susana Field } \\
\text { Laboratory) } \\
\text { Laboratory for Energy-Related Health Research (Institute of } \\
\text { Toxicology and Environmental Health) } \\
\text { Lawrence Berkeley Laboratory } \\
\text { Lawrence Livermore National Laboratory } \\
\text { Stanford Linear Accelerator Center }\end{array}$ \\
\hline
\end{tabular}


Table C.1 (continued)

\begin{tabular}{|c|c|c|c|}
\hline DOE office & Symbol/label & $\begin{array}{l}\text { General mailing address } \\
\text { (Phone number) }^{\mathrm{a}}\end{array}$ & $\begin{array}{c}\text { Radioactive waste sites for which DOE } \\
\text { office has responsibility }\end{array}$ \\
\hline $\begin{array}{l}\text { Oak Ridge Operations } \\
\text { Office }\end{array}$ & DOE/OR & $\begin{array}{l}\text { P.O. Box } 2001 \\
\text { Oak Ridge, TN } 37831 \\
(423 / 576-5454)\end{array}$ & $\begin{array}{l}\text { Formerly Utilized Sites Remedial Action Program (FUSRAP) sites } \\
\text { Oak Ridge Institute for Science and Education } \\
\text { Oak Ridge K-25 Site } \\
\text { Oak Ridge National Laboratory } \\
\text { Oak Ridge Y-12 Plant } \\
\text { Paducah Gaseous Diffusion Plant } \\
\text { Portsmouth Gaseous Diffusion Plant } \\
\text { Weldon Spring Site Remedial Action Project }\end{array}$ \\
\hline Ohio Field Office & DOE/OH & $\begin{array}{l}\text { P.O. Box } 3020 \\
\text { Miamisburg, OH } 45343-3020 \\
(513 / 865-3977)\end{array}$ & $\begin{array}{l}\text { Battelle Columbus Laboratories Decommissioning Project } \\
\text { Fernald Environmental Management Project } \\
\text { Mound Plant } \\
\text { Reactive Metals, Inc., Extrusion Plant } \\
\text { West Valley Demonstration Project }\end{array}$ \\
\hline Richland Operations Office & DOE/RL & $\begin{array}{l}\text { P.O. Box } 550 \\
\text { 825 Jadwin Avenue } \\
\text { Richland, WA } 99352 \\
(509 / 376-7411)\end{array}$ & $\begin{array}{l}\text { Hanford Site } \\
\text { Pacific Northwest National Laboratory }\end{array}$ \\
\hline Rocky Flats Office & DOE/RF & $\begin{array}{l}\text { P.O. Box } 928 \\
\text { Golden, CO } 80401-0928 \\
(303 / 966-7000)\end{array}$ & Rocky Flats Environmental Technology Site \\
\hline $\begin{array}{l}\text { Savannah River Operations } \\
\text { Office }\end{array}$ & $\mathrm{DOE} / \mathrm{SR}$ & $\begin{array}{l}\text { P.O. Box A } \\
\text { Aiken, SC } 29802 \\
(803 / 725-6211)\end{array}$ & Savannah River Site \\
\hline
\end{tabular}

\footnotetext{
aAccess to main organizations.

b The Albuquerque Operations Office also has the following area offices (monitoring activities of the sites indicated) under its purview: Amarillo (Pantex Plant), Grand Junction (Grand Junction Projects Office), Kansas City (Kansas City Plant), Los Alamos (Los Alamos National Laboratory), and Pinellas (Pinellas Plant).

${ }^{\mathbf{c}}$ The Chicago Operations Office has the following area offices (monitoring activities of the sites indicated) under its purview: Argonne (Argonne National Laboratory-East), Batavia (Fermi National Accelerator Laboratory), Upton (Brookhaven National Laboratory), and Princeton (Princeton Plasma Physics Laboratory).

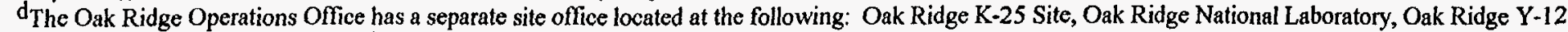

Plant, Paducah Gaseous Diffusion Plant, Portsmouth Gaseous Diffusion Plant, and the Weldon Spring Site Remedial Action Project.
} 


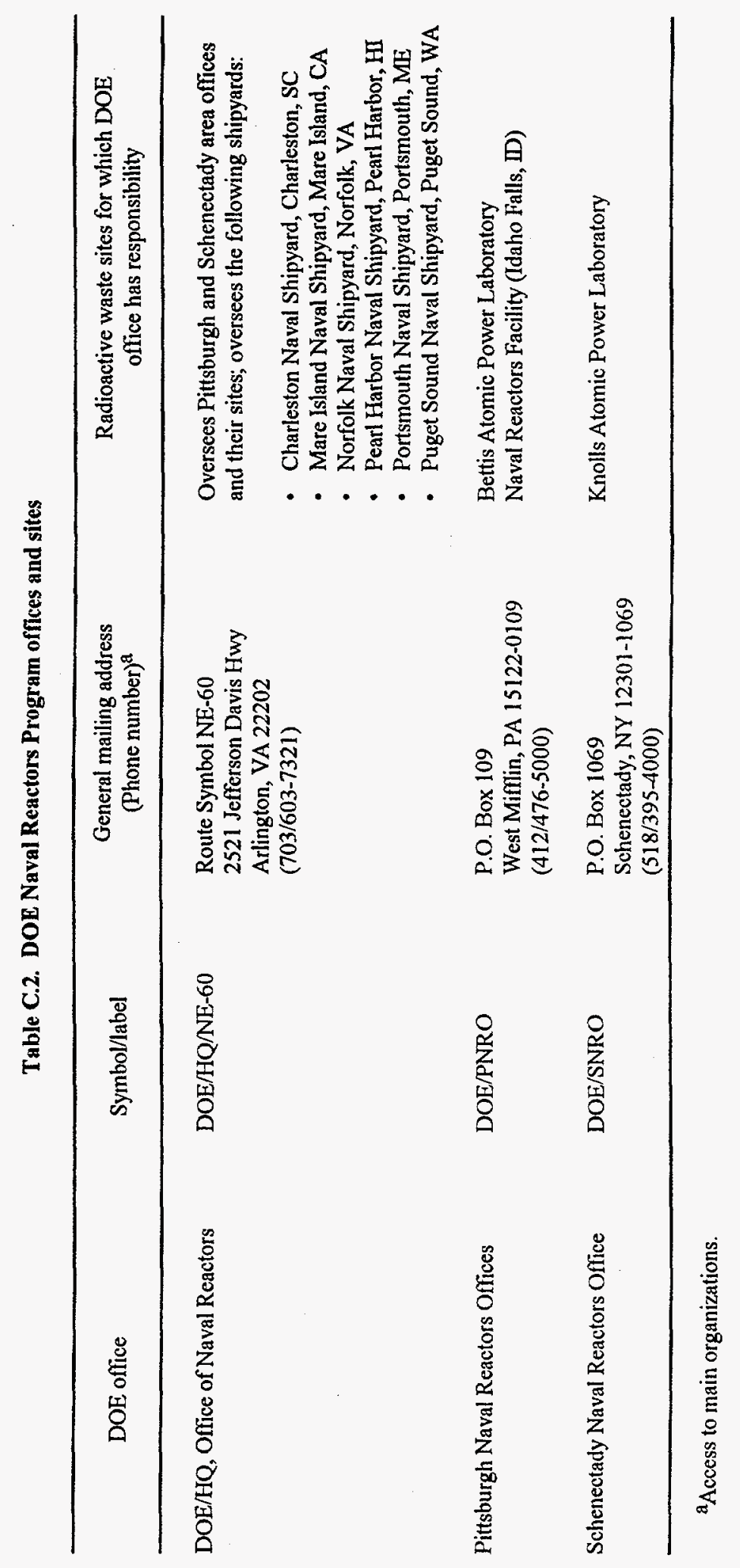


Table C.3. Major DOE sites and facilities referred to in this report

\begin{tabular}{|c|c|c|c|}
\hline Site/facility & Symbol/label & $\begin{array}{l}\text { Principal contractor(s) for site } \\
\text { operations and mailing address } \\
\text { (Phone number) }^{\mathrm{a}}\end{array}$ & $\begin{array}{c}\text { DOE operations/field office } \\
\text { (Phone number) }^{\mathrm{a}}\end{array}$ \\
\hline Ames Laboratory & Ames & $\begin{array}{l}\text { Iowa State University } \\
\text { Technical Administration Services Facility } \\
\text { Ames, IA } 50011-3020 \\
(515 / 294-2680)\end{array}$ & $\begin{array}{l}\text { Chicago } \\
(630 / 252-2001)\end{array}$ \\
\hline Argonne National Laboratory-East & ANL-E & $\begin{array}{l}\text { University of Chicago } \\
9700 \text { South Cass Avenue } \\
\text { Argonne, IL } 60439 \\
(630 / 252-2000)\end{array}$ & $\begin{array}{l}\text { Chicago } \\
\text { Argonne Area Office } \\
(630 / 252-2001)\end{array}$ \\
\hline Argonne National Laboratory-West ${ }^{b}$ & ANL-W & $\begin{array}{l}\text { University of Chicago } \\
\text { Idaho Site } \\
\text { P.O. Box } 2528 \\
\text { Idaho Falls, ID } 83403-2528 \\
(208 / 533-7000)\end{array}$ & $\begin{array}{l}\text { Chicago } \\
(630 / 252-2001)\end{array}$ \\
\hline $\begin{array}{l}\text { Battelle Columbus Laboratories } \\
\text { Decommissioning Project }\end{array}$ & BCLDP & $\begin{array}{l}\text { Battelle Memorial Institute } \\
505 \text { King Avenue } \\
\text { Columbus, OH 43201-2693 } \\
(614 / 424-3989)\end{array}$ & $\begin{array}{l}\text { Ohio Field Office } \\
(513 / 865-3977)\end{array}$ \\
\hline Brookhaven National Laboratory & BNL & $\begin{array}{l}\text { Associated Universities, Inc. } \\
16 \text { South Railroad Street } \\
\text { Upton, NY } 11973-2310 \\
(516 / 282-2123)\end{array}$ & $\begin{array}{l}\text { Chicago } \\
\text { Brookhaven Area Office } \\
(516 / 282-3427)\end{array}$ \\
\hline Colonie Interim Storage Site & CISS & $\begin{array}{l}\text { Bechtel National, Inc. } \\
\text { P.O. Box } 5169 \\
\text { Albany, NY } 12205 \\
(518 / 482-0237)\end{array}$ & $\begin{array}{l}\text { Oak Ridge } \\
(423 / 576-5454)\end{array}$ \\
\hline
\end{tabular}


Table C.3 (continued)

\begin{tabular}{|c|c|c|c|}
\hline Site/facility & Symbol/label & $\begin{array}{l}\text { Principal contractor(s) for site } \\
\text { operations and mailing address } \\
\text { (Phone number) }\end{array}$ & $\begin{array}{l}\text { DOE operations/field office } \\
\text { (Phone number) }^{\mathrm{a}}\end{array}$ \\
\hline $\begin{array}{l}\text { Energy Technology Engineering Center } \\
\text { (Santa Susana Field Laboratory) }\end{array}$ & $\begin{array}{l}\text { ETEC } \\
(\text { SSFL) }\end{array}$ & $\begin{array}{l}\text { Rockwell International } \\
\text { Rocketdyne Division } \\
6633 \text { Canoga Avenue } \\
\text { P.O. Box } 1449 \\
\text { Canoga Park, CA } 91304 \\
(818 / 586-5326)\end{array}$ & $\begin{array}{l}\text { Oakland } \\
(510 / 273-6383)\end{array}$ \\
\hline Fermi National Accelerator Laboratory & FNAL & $\begin{array}{l}\text { University Research Association } \\
\text { P.O. Box } 500 \\
\text { Batavia, I } 60510 \\
(630 / 840-3000)\end{array}$ & $\begin{array}{l}\text { Chicago } \\
\text { Batavia Area Office } \\
(630 / 840-3281)\end{array}$ \\
\hline Fernald Environmental Management Project & FEMP & $\begin{array}{l}\text { Fluor Daniel } \\
\text { Fernald Environmental Restoration } \\
\text { Management Corporation } \\
\text { P.O. Box } 398704 \\
7400 \text { Wiley Road } \\
\text { Cincinnati, OH 45239-8704 } \\
(513 / 738-6200)\end{array}$ & $\begin{array}{l}\text { Ohio Field Office } \\
\text { Fernald Area Office } \\
(513 / 648-3119)\end{array}$ \\
\hline Grand Junction Projects Office & GJPO & $\begin{array}{l}\text { Chem-Nuclear Geotech, Inc. } \\
\text { P.O. Box } 14000 \\
\text { Grand Junction, CO } 81502-5504 \\
(303 / 248-6200)\end{array}$ & $\begin{array}{l}\text { Albuquerque } \\
(505 / 845-4154)\end{array}$ \\
\hline Hanford Site & Hanford & $\begin{array}{l}\text { Fluor Daniel Hanford, Inc. } \\
\text { P.O. Box } 1970 \\
\text { Richland, WA } 99352 \\
(509 / 376-7411)\end{array}$ & $\begin{array}{l}\text { Richland } \\
(509 / 376-7411)\end{array}$ \\
\hline Idaho National Engineering Laboratory & INEL & $\begin{array}{l}\text { Lockheed Martin Idaho Technologies } \\
\text { P.O. Box } 1625 \\
\text { Idaho Falls, ID } 83415-4201 \\
(208 / 526-0111)\end{array}$ & $\begin{array}{l}\text { Idaho } \\
(208 / 526-0111)\end{array}$ \\
\hline
\end{tabular}


Table C.3 (continued)

\begin{tabular}{|c|c|c|c|}
\hline Site/facility & Symbol/label & $\begin{array}{l}\text { Principal contractor(s) for site } \\
\text { operations and mailing address } \\
\qquad \text { (Phone number) }\end{array}$ & $\begin{array}{l}\text { DOE operations/field office } \\
\text { (Phone number) }^{\mathrm{a}}\end{array}$ \\
\hline Inhalation Toxicology Research Institute & ITRI & $\begin{array}{l}\text { Lovelace Biomedical and Environmental } \\
\text { Research Institute, Inc. } \\
\text { P.O. Box } 5890 \\
\text { Albuquerque, NM } 87185 \\
\text { (505/845-1037) }\end{array}$ & $\begin{array}{l}\text { Albuquerque } \\
(505 / 845-4154)\end{array}$ \\
\hline Kansas City Plant & $\mathrm{KCP}$ & $\begin{array}{l}\text { Allied-Signal Aerospace Company } \\
\text { Kansas City Division } \\
\text { Bannister Federal Complex } \\
\text { Kansas City, MO } 64141 \\
(816 / 997-2000)\end{array}$ & $\begin{array}{l}\text { Albuquerque } \\
\text { Kansas City Area Office } \\
(816 / 997-3348)\end{array}$ \\
\hline $\begin{array}{l}\text { Laboratory for Energy-Related Health } \\
\text { Research (Institute of Toxicology and } \\
\text { Environmental Health) }\end{array}$ & $\begin{array}{l}\text { LEHR } \\
\text { (ITEH) }\end{array}$ & $\begin{array}{l}\text { University of California-Davis } \\
\text { Old Davis Road } \\
\text { Davis, CA 95616-8615 } \\
(916 / 752-1340)\end{array}$ & $\begin{array}{l}\text { Oakland } \\
(510 / 273-6383)\end{array}$ \\
\hline Lawrence Berkeley Laboratory & LBL & $\begin{array}{l}\text { University of California } \\
\text { One Cyclotron Road } \\
\text { Berkeley, CA } 94720 \\
(510 / 486-4000)\end{array}$ & $\begin{array}{l}\text { Oakland } \\
\text { Lawrence Berkeley Laboratory } \\
\text { Site Office } \\
(510 / 486-4363)\end{array}$ \\
\hline Lawrence Livermore National Laboratory & LLNL & $\begin{array}{l}\text { University of California } \\
7000 \text { East Avenue } \\
\text { P.O. Box } 808, \text { L-1 } \\
\text { Livermore, CA } 94550 \\
(510 / 422-1100)\end{array}$ & $\begin{array}{l}\text { Oakland } \\
(510 / 273-6383)\end{array}$ \\
\hline Los Alamos National Laboratory & LANL & $\begin{array}{l}\text { University of California } \\
\text { P.O. Box } 1663 \\
\text { Los Alamos, NM } 87545 \\
(505 / 667-5061)\end{array}$ & $\begin{array}{l}\text { Albuquerque } \\
\text { Los Alamos Area Office } \\
(505 / 667-5061)\end{array}$ \\
\hline
\end{tabular}


Table C.3 (continued)

\begin{tabular}{|c|c|c|c|}
\hline Site/facility & Symbol/label & $\begin{array}{l}\text { Principal contractor(s) for site } \\
\text { operations and mailing address } \\
\text { (Phone number) }\end{array}$ & $\begin{array}{l}\text { DOE operations/field office } \\
\text { (Phone number) }^{\mathrm{a}}\end{array}$ \\
\hline Mound Plant & Mound & $\begin{array}{l}\text { EG\&G Mound Applied Technologies } \\
\text { P.O. Box } 3000 \\
\text { Miamisburg, OH } 45343-3000 \\
(513 / 865-4020)\end{array}$ & $\begin{array}{l}\text { Ohio Field Office } \\
\text { Miamisburg Area Office } \\
(513 / 865-3271)\end{array}$ \\
\hline \multicolumn{4}{|l|}{ Naval Reactors Program Facilities } \\
\hline Bettis Atomic Power Laboratory & BAPL & $\begin{array}{l}\text { Westinghouse Electric Corporation } \\
\text { P.O. Box } 79 \\
\text { West Mifflin, PA 15122-0079 } \\
(412 / 476-5000)\end{array}$ & $\begin{array}{l}\text { DOE/HQ Office of Naval } \\
\text { Reactors (NE-60) } \\
\text { Pittsburgh Naval Reactors Office } \\
(412 / 476-5000)\end{array}$ \\
\hline Knolls Atomic Power Laboratory & KAPL & $\begin{array}{l}\text { Lockheed Martin Services Group } \\
\text { P.O. Box } 1072 \\
\text { Schenectady, NY 12301-1072 } \\
(518 / 395-4000)\end{array}$ & $\begin{array}{l}\text { DOE/HQ Office of Naval } \\
\text { Reactors (NE-60) } \\
\text { Schenectady Naval Reactors } \\
\text { Office } \\
(518 / 395-4000)\end{array}$ \\
\hline Naval Reactors Facility (INEL) & NRF & $\begin{array}{l}\text { Lockheed Martin Idaho Technologies } \\
\text { P.O. Box } 2068 \\
\text { Idaho Falls, ID 83403-2068 } \\
\text { (208/526-5526) }\end{array}$ & $\begin{array}{l}\text { DOE/HQ Office of Naval } \\
\text { Reactors (NE-60) } \\
\text { Pittsburgh Naval Reactors Office } \\
(412 / 476-5000)\end{array}$ \\
\hline Nevada Test Site & NTS & $\begin{array}{l}\text { Bechtel Nevada Corporation } \\
\text { P.O. Box } 98521 \\
\text { Mail Stop } 738 \\
\text { Las Vegas, NV } 89193-8521 \\
(702 / 295-9060)\end{array}$ & $\begin{array}{l}\text { Nevada } \\
(702 / 295-1212)\end{array}$ \\
\hline Oak Ridge Institute for Science and Education & ORISE & $\begin{array}{l}\text { Oak Ridge Associated Universities } \\
246 \text { Laboratory Road } \\
\text { P.O. Box } 117 \\
\text { Oak Ridge, TN } 37831-0117 \\
(423 / 576-3000)\end{array}$ & $\begin{array}{l}\text { Oak Ridge } \\
(423 / 576-5454)\end{array}$ \\
\hline
\end{tabular}


Table C.3 (continued)

\begin{tabular}{|c|c|c|c|}
\hline Site/facility & Symbol/label & $\begin{array}{l}\text { Principal contractor(s) for site } \\
\text { operations and mailing address } \\
\text { (Phone number) }\end{array}$ & $\begin{array}{c}\text { DOE operations/field office } \\
\text { (Phone number) }^{\mathrm{a}}\end{array}$ \\
\hline Oak Ridge K-25 Site & $\mathrm{K}-25$ & $\begin{array}{l}\text { Lockheed Martin Energy Systems, Inc. } \\
\text { P.O. Box } 2003 \\
\text { Oak Ridge, TN } 37831-7358 \\
(423 / 576-5454)\end{array}$ & $\begin{array}{l}\text { Oak Ridge } \\
(423 / 576-5454)\end{array}$ \\
\hline Oak Ridge National Laboratory & ORNL & $\begin{array}{l}\text { Lockheed Martin Energy Research } \\
\text { Corporation } \\
\text { P.O. Box 2008 } \\
\text { Oak Ridge, TN } 37831-6235 \\
(423 / 576-5454)\end{array}$ & $\begin{array}{l}\text { Oak Ridge } \\
(423 / 576-5454)\end{array}$ \\
\hline Oak Ridge Y-12 Plant & $\begin{array}{l}\mathrm{Y}-12 \\
.\end{array}$ & $\begin{array}{l}\text { Lockheed Martin Energy Systems, Inc. } \\
\text { P.O. Box } 2009 \\
\text { Oak Ridge, TN } 37831-8010 \\
(423 / 576-5454)\end{array}$ & $\begin{array}{l}\text { Oak Ridge } \\
(423 / 576-5454)\end{array}$ \\
\hline Pacific Northwest National Laboratory & PNNL & $\begin{array}{l}\text { Battelle Memorial Institute } \\
\text { Battelle Boulevard } \\
\text { P.O. Box } 999 \\
\text { Richland, WA } 99352 \\
(509 / 375-2121)\end{array}$ & $\begin{array}{l}\text { Richland } \\
(509 / 376-7411)\end{array}$ \\
\hline Paducah Gaseous Diffusion Plant & PAD & $\begin{array}{l}\text { Lockheed Martin Utility Systems, Inc. } \\
\text { P.O. Box } 1410 \\
\text { Paducah, KY } 42001 \\
(502 / 441-6000)\end{array}$ & $\begin{array}{l}\text { Oak Ridge } \\
\text { Paducah Site Office } \\
(502 / 441-6800)\end{array}$ \\
\hline Pantex Plant & PANT & $\begin{array}{l}\text { Mason \& Hanger-Silas Mason } \\
\text { Company, Inc. } \\
\text { P.O. Box 30020 } \\
\text { Amarillo, TX 79177 } \\
(806 / 477-3000)\end{array}$ & $\begin{array}{l}\text { Albuquerque } \\
\text { Amarillo Area Office } \\
(806 / 477-3000)\end{array}$ \\
\hline
\end{tabular}


Table C.3 (continued)

\begin{tabular}{|c|c|c|c|}
\hline Site/facility & Symbol/label & $\begin{array}{l}\text { Principal contractor(s) for site } \\
\text { operations and mailing address } \\
\text { (Phone number) }^{\mathrm{a}}\end{array}$ & $\begin{array}{l}\text { DOE operations/field office } \\
\text { (Phone number) }\end{array}$ \\
\hline Pinellas Plant & Pinellas & $\begin{array}{l}\text { Lockheed Martin Specialty Components, Inc. } \\
\text { P.O. Box } 2908 \\
\text { Largo, FL 34649-2908 } \\
(813 / 541-8001)\end{array}$ & $\begin{array}{l}\text { Albuquerque } \\
\text { Pinellas Area Office } \\
(813 / 541-8196)\end{array}$ \\
\hline Portsmouth Gaseous Diffusion Plant & PORTS & $\begin{array}{l}\text { Lockheed Martin Utility Systems, Inc. } \\
\text { P.O. Box } 628 \\
\text { Piketon, OH } 45661 \\
(614 / 897-2331)\end{array}$ & $\begin{array}{l}\text { Oak Ridge } \\
\text { Portsmouth Site Office } \\
(614 / 897-2331)\end{array}$ \\
\hline Princeton Plasma Physics Laboratory & PPPL & $\begin{array}{l}\text { Princeton University } \\
\text { P.O. Box } 451 \\
\text { Princeton, NJ 08543 } \\
(609 / 243-2000)\end{array}$ & $\begin{array}{l}\text { Chicago } \\
\text { Princeton Area Office } \\
(609 / 243-3700)\end{array}$ \\
\hline Reactive Metals, Inc., Extrusion Plant & RMI & $\begin{array}{l}\text { RMI Titanium Company } \\
\text { P.O. Box } 579 \\
\text { Ashtabula, OH } 44004 \\
(216 / 992-7442)\end{array}$ & $\begin{array}{l}\text { Ohio Field Office } \\
\text { RMI Decommissioning Project } \\
\text { Office } \\
(216 / 992-7442)\end{array}$ \\
\hline Rocky Flats Environmental Technology Site & RFETS & $\begin{array}{l}\text { EG\&G Rocky Flats, Inc. } \\
\text { P.O. Box } 464 \\
\text { Golden, CO } 80401-0464 \\
(303 / 966-7000)\end{array}$ & $\begin{array}{l}\text { Rocky Flats Office } \\
(303 / 966-7000)\end{array}$ \\
\hline \multicolumn{4}{|l|}{ Sandia National Laboratories } \\
\hline California & $\mathrm{SNL} / \mathrm{CA}$ & $\begin{array}{l}\text { Lockheed Martin Sandia Corporation } \\
\text { P.O. Box } 969 \\
\text { Livermore, CA } 94551-0969 \\
(510 / 294-3000)\end{array}$ & $\begin{array}{l}\text { Albuquerque } \\
(505 / 845-4154)\end{array}$ \\
\hline New Mexico & SNL/NM & $\begin{array}{l}\text { Lockheed Martin Sandia Corporation } \\
\text { P.O. Box } 5800 \\
\text { Albuquerque, NM } 87185-5800 \\
\text { (505/844-5678) }\end{array}$ & $\begin{array}{l}\text { Albuquerque } \\
(505 / 845-4154)\end{array}$ \\
\hline
\end{tabular}


Table C.3 (continued)

\begin{tabular}{|c|c|c|c|}
\hline Site/facility & Symbol/label & $\begin{array}{l}\text { Principal contractor(s) for site } \\
\text { operations and mailing address } \\
\text { (Phone number) }\end{array}$ & $\begin{array}{l}\text { DOE operations/field office } \\
\text { (Phone number) }^{\mathrm{a}}\end{array}$ \\
\hline Savannah River Site & SRS & $\begin{array}{l}\text { Westinghouse Savannah River Company } \\
\text { P.O. Box } 616 \\
\text { Aiken, SC } 29802 \\
(803 / 725-6211)\end{array}$ & $\begin{array}{l}\text { Savannah River } \\
(803 / 725.6211)\end{array}$ \\
\hline Stanford Linear Accelerator Center & SLAC & $\begin{array}{l}\text { Stanford University } \\
\text { P.O. Box } 4349 \\
\text { Palo Alto, CA } 94309 \\
(415 / 926-3300)\end{array}$ & $\begin{array}{l}\text { Oakland } \\
\text { Stanford Site Office } \\
(415 / 926-3208)\end{array}$ \\
\hline Three Mile Island-Unit 2 Reactor & TMI-Unit 2 & $\begin{array}{l}\text { General Public Utilities } \\
\text { P.O. Box } 480 \\
\text { Middletown, PA } 17057 \\
(717 / 944-7621)\end{array}$ & $\begin{array}{l}\text { Idaho } \\
\text { Three Mile Island Site Office } \\
(717 / 944-7621)\end{array}$ \\
\hline Waste Isolation Pilot Plant & WIPP & $\begin{array}{l}\text { Westinghouse Electric Corporation } \\
\text { WIPP Project Office } \\
\text { P.O. Box } 2078 \\
\text { Carlsbad, NM } 88221 \\
(505 / 885-7500)\end{array}$ & $\begin{array}{l}\text { Albuquerque } \\
\text { Carlsbad Area Office } \\
(505 / 234-7327)\end{array}$ \\
\hline Weldon Spring Site Remedial Action Project & WSSRAP & $\begin{array}{l}\text { Jacobs Engineering Group, Inc. } \\
\text { MK-Ferguson Company } \\
7295 \text { Highway } 94 \text { South } \\
\text { St. Charles, MO } 63304 \\
(314 / 441-8978)\end{array}$ & $\begin{array}{l}\text { Oak Ridge } \\
\text { Weldon Spring Site Office } \\
(314 / 441-8978)\end{array}$ \\
\hline West Valley Demonstration Project & WVDP & $\begin{array}{l}\text { Westinghouse Electric Corporation } \\
\text { West Valley Nuclear Services Company, Inc. } \\
10282 \text { Rock Springs Road } \\
\text { P.O. Box 191 } \\
\text { West Valley, NY 14171-0191 } \\
(716 / 942-3235)\end{array}$ & $\begin{array}{l}\text { Ohio Field Office } \\
\text { West Valley Area Office } \\
(716 / 942-4313)\end{array}$ \\
\hline
\end{tabular}

aPhone number for access to main organization.

${ }^{b}$ Part of the Idaho National Engineering Laboratory Reservation.

cPart of the Hanford Site. 
Table C.4. Major commercial radioactive waste disposal sites included in this report ${ }^{a}$

\begin{tabular}{|c|c|c|}
\hline Site & Symbollabel & $\begin{array}{l}\text { Principal contractor(s) for site operations } \\
\text { and mailing address } \\
\text { (Phone number) } \text { b }^{\mathrm{b}}\end{array}$ \\
\hline Barnwell & BARN & $\begin{array}{l}\text { Chem-Nuclear Systems, Inc. } \\
\text { 140 Stoneridge Drive } \\
\text { Columbia, SC 29210 } \\
(803 / 256-0450)\end{array}$ \\
\hline Beatty & BETY & $\begin{array}{l}\text { U.S. Ecology, Nuclear } \\
\text { P.O. Box } 578 \\
\text { Beatty, NV 89003 } \\
(702 / 553-2203)\end{array}$ \\
\hline Envirccare $^{c}$ & ENVR & $\begin{array}{l}\text { Envirocare of Utah, Inc. } \\
46 \text { West Broadway } \\
\text { Suite } 240 \\
\text { Salt Lake City, Utah } 84101 \\
\text { (801/532-1330) }\end{array}$ \\
\hline Maxey Flats & MFKY & $\begin{array}{l}\text { Commonwealth of Kentucky } \\
\text { Department of Environmental Protection/Superfund Branch } \\
\text { Maxey Flats Project } \\
\text { 14 Reilly Road } \\
\text { Frankfort, KY 40601-1190 } \\
\text { (502/564-6716) } \\
\text { Site address: } \\
\text { Maxey Flats Project } \\
\text { Route 2 } \\
\text { P.O. Box 238A } \\
\text { Hillsboro, KY 41049 } \\
(606 / 784-6612)\end{array}$ \\
\hline Richland & RICH & $\begin{array}{l}\text { U.S. Ecology, Nuclear } \\
\text { P.O. Box 638 } \\
\text { Richland, WA } 99352 \\
(509 / 377-2411)\end{array}$ \\
\hline Sheffield & SHEF & $\begin{array}{l}\text { U.S. Ecology, Nuclear } \\
\text { P.O. Box } 158 \\
\text { Sheffield, IL 61361 } \\
(815 / 454-2342)\end{array}$ \\
\hline West Valley & WVNY & $\begin{array}{l}\text { Westinghouse Electric Corporation } \\
\text { West Valley Nuclear Services Company, Inc. } \\
10282 \text { Rock Springs Road } \\
\text { P.O. Box } 191 \\
\text { West Valley, NY 14171-0191 } \\
(716 / 942-3235)\end{array}$ \\
\hline
\end{tabular}


Table C.4 (contrinued)

\begin{tabular}{|c|c|c|}
\hline Site & Symbol/label & $\begin{array}{c}\text { Principal contractor(s) for site operations } \\
\text { and mailing address } \\
\text { (Phone number) }^{b}\end{array}$ \\
\hline
\end{tabular}

\begin{tabular}{|c|c|c|}
\hline $\begin{array}{l}\text { West Valley } \\
\text { (contd.) }\end{array}$ & WVNY & $\begin{array}{l}\text { New York State Energy Research and Development Authority } \\
2 \text { Rockefeller Plaza } \\
\text { Albany, NY } 12223 \\
(518 / 465-6251)\end{array}$ \\
\hline
\end{tabular}

aDoes not include uranium mill tailings sites. See Table 5.2.

b Phone number for access to main organizations.

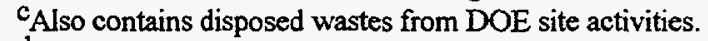

$\mathrm{d}$ The Commonwealth of Kentucky assumed operating contractor responsibilities for the Maxey Flats site in 1992. 

APPENDIX D. INTEGRATED DATA BASE DOCUMENT ACCESS ON THE INTERNET 



\section{APPENDIX D. INTEGRATED DATA BASE DOCUMENT ACCESS ON THE INTERNET}

A copy of this document (IDB Rev. 12) will be accessible via the Internet on the DOE Office of Environmental Management World Wide Web (EM WWW) Server at the Uniform Resource Locator (URL) http://www.em.doe.gov/idb96 .

This document may also be accessed from the EMWWW home page at http://www.em.doe.gov. From the home page menu, select Waste Management. The IDB reports are listed on the Waste Management menu.

The EM WWW Server provides a collection of documents and other information and data relating to DOE/EM's mission. It was established as an EM-wide information resource available to DOE employees, other federal agencies, Congress, state and tribal governments, and the general public. The EM WWW Server is updated and maintained to provide historical and current DOE/EM information. 
APPENDIX E. INTEGRATED DATA BASE READER COMMENT FORM 



\section{APPENDIX E. INTEGRATED DATA BASE READER COMMENT FORM}

To maintain an updated distribution list for this report the Integrated Data Base (IDB) is asking its readership to supply the information requested on the Reader Comment Form provided in the front of this report. When filling out this form, please respond to the questions in Items 1-11 (note that some require two answers). Item 12 requests some personal information (please type or print your complete name and mailing address). To be eligible for future updates of this report, please fold, attach stamp, and mail the completed Reader Comment Form to the Integrated Data Base Program at the mailing address given on the back of the form (and listed below) at your earliest convenience. Also, please notify the IDB Program of any corrections or future changes in your mailing address. Your cooperation and assistance are greatly appreciated.

Integrated Data Base Program

Oak Ridge National Laboratory

Building 4500S, Mail Stop 6111

P.O. Box 2008

Oak Ridge, TN 37831-6111

Phones: 423/574-6823

$423 / 576-7575$

Faxes: $\quad 423 / 576-0327$

423/574-6616

E-mail: sns@ornl.gov

jak@oml.gov 



\section{GLOSSARY OF TERMS}

This glossary gives definitions of some terms commonly used in the main body of this report.

Actinides: Elements with atomic numbers from 89 (actinium) or 90 (thorium) to 103 (lawrencium) inclusive.

Activation product: A radioactive material produced by bombardment with neutrons, protons, or other nuclear particles.

Agreement State: A state that has entered into an agreement with the U.S. Nuclear Regulatory Commission (as specified by the Atomic Energy Act of 1954) and has authority to regulate the disposal of low-level radioactive waste under such an agreement. This term is used in the Low-Level Radioactive Waste Policy Act (Public Law 99-240).

Alpha decay: Radioactive decay in which an alpha particle ( $\left({ }^{4} \mathrm{He}\right.$ nucleus) is emitted.

Beta decay: Radioactive decay in which a beta particle (negative or positive electron) is emitted.

Borosilicate glass: A type of glass containing at least $5 \mathrm{wt} \%$ boric oxide. It is used in glassware that resists heat and is a leading candidate for use in high-level waste immobilization and disposal.

Branching ratio: In branching radioactive decay, the fraction of nuclei that disintegrates in a specific way. (It is usually expressed as a percentage.)

Burnup, specific: The total energy released per initial unit mass of reactor fuel as a result of the fission process occurning. The unit commonly used for specific burnup is megawatt-days per metric ton of initial heavy metal, MWd/MTHHM.

By-product material: (1) Any radioactive material (except special nuclear material) yielded in, or made radioactive by, exposure to the radiation incident to the process of producing or utilizing special nuclear material.
For purposes of determining the applicability of the Resource Conservation and Recovery Act of 1976 to any radioactive waste, the term "any radioactive material" refers only to the actual radionuclides dispersed or suspended in the waste substance. The nonradioactive hazardous waste component of the waste substance will be subject to regulation under the Resource Conservation and Recovery Act; (2) the tailings or waste produced by the extraction or concentration of uranium or thorium from any ore processed primarily for its source material content. Ore bodies depleted by uranium solution extraction operations and which remain underground do not constitute by-product material.

Calcine: A form of high-level waste produced from defense reactor fuel reprocessing waste (at the Idaho Chemical Processing Plant) by heating to a temperature below the melting point to bring about loss of moisture and nonradioactive volatile oxides, thus producing a chemically stable granular powder.

Canister: A metal container used for the storage or disposal of heat-producing, solid, high-level radioactive waste.

Capacity factor, plant: The ratio of the electrical energy actually supplied by a power plant in a given time interval to the electrical energy that could have been produced at continuous full-power operation during the same time period.

Capsules: Encapsulated strontium and cesium high-level wastes produced from defense reactor fuel reprocessing at the Hanford Site. For purposes of this report, inventories of encapsulated strontium and cesium are included in a separate table in the high-level waste section (Chapter 2).

Cladding: A corrosion-resistant tube (commonly aluminum, zirconium alloy, or stainless steel) surrounding the reactor fuel pellets which provides protection from a 
chemically reactive environment and containment of fission products.

Code of Federal Regulations: A documentation of the general rules by the executive departments of the federal government. The code is divided into 50 titles that represent broad areas subject to federal regulation. Each title is divided into chapters that usually bear the name of the issuing agency. Each chapter is further subdivided into parts covering specific regulatory areas.

Control rod: A movable part of the reactor core that is adjusted to regulate the degree of fuel fissioning in the core.

Conversion, fuel: Chemical treatment of yellowcake $\left(\mathrm{U}_{3} \mathrm{O}_{8}\right)$ to uranium hexafluoride $\left(\mathrm{UF}_{6}\right)$ in preparation for enrichment.

Core, nuclear reactor: That part of the reactor which contains the nuclear fuel and in which most or all of the nuclear fissions occur.

Daughter product(s): The nuclide(s) formed by the radioactive decay of the parent radionuclide.

Decay, radioactive: The transition of a nucleus from one energy state to a lower one, usually involving the emission of a photon, electron, neutron, or alpha particle.

Decay chain, radioactive: A series of nuclides in which each member decays to the next member of the chain through radioactive decay until a stable nuclide has been formed.

Decommissioning: Activities taken to reduce the potential health and safety impacts of commercial and DOE-contaminated facilities, including removing a unit from operation, decontamination, entombment, dismantlement, or conversion of the site to another use.

Decommissioning wastes: Wastes (generally low-level) collected or resulting from facility decommissioning activities.

Decontamination: Activities taken to remove unwanted (typically radioactive) material from facilities, soils, or equipment by washing, chemical action, mechanical cleaning, or other (treatment) techniques.

Deep bed plant: A boiling-water reactor facility using a demineralizer vessel for water purification which contains an ion-exchange resin that is $3 \mathrm{ft}$ or more deep.
Disintegration energy (Q-value): The amount of energy released in a particular nuclear disintegration. This is usually expressed in $\mathrm{MeV} /$ disintegration.

DOE waste: Radioactive waste produced from activities supported by the Department of Energy and/or U.S. government defense programs.

Double-shell tank wastes: High-level wastes, generated from defense reactor fuel reprocessing at Hanford, which are stored in double-shelled tanks. These wastes consist of a mixture of liquid and suspended solids referred to as slurry. See also "single-shell tank wastes."

Electron capture: Radioactive decay in which an orbital electron is captured by the nucleus of the radionuclide.

Enrichment, fuel: A nuclear fuel cycle process which increases the concentration of fissionable uranium (i.e., ${ }^{23} \mathrm{U}$ ) in uranium ore above its natural level of $0.71 \%$. (The method currently used in the United States is gaseous diffusion.)

Environmental Impact Statement: A report that documents the information required to evaluate the environmental impact of a project. Such a report informs decision-makers and the public of the reasonable alternatives which would avoid or minimize adverse impacts or enhance the quality of the environment.

Environmental restoration: Cleanup and restoration of sites contaminated with radioactive and/or hazardous substances during past production, accidental releases, or disposal activities.

Equilibrium cycle: An assumed nuclear fuel cycle in which the feed and waste materials of a facility have constant compositions. In a reactor this condition typically results after the third or fourth fuel-loading schedule.

Fabrication, fuel: Conversion of enriched uranium hexafluoride $\left(\mathrm{UF}_{6}\right)$ into pellets of ceramic uranium dioxide $\left(\mathrm{UO}_{2}\right)$. These pellets are then sealed into corrosionresistant tubes of zirconium alloy or stainless steel. The loaded tubes, called fuel elements or rods, are then mounted into special assemblies for loading into the reactor core.

Fertile nuclide: A nuclide capable of being transformed into a fissile nuclide by neutron capture.

Filter/demineralizer plant: A facility that combines filtration and ion-exchange processing using nonregenerable powered resins. 
Fissile nuclide: A nuclide capable of undergoing nuclear fission with thermal neutrons.

Fission, nuclear: The division of a heavy atomic nucleus into two or more isotopes, usually accompanied by the emission of neutrons and gamma radiation.

Fission products: Nuclides produced either by fission or by the subsequent decay of the nuclides thus formed.

Fission, spontaneous: Nuclear fission that occurs without the addition of particles or energy to the nucleus.

Formerly utilized site: A site contaminated with radioactive wastes which was previously used for supporting nuclear activities of the DOE's predecessor agencies, the Manhattan Engineer District (Manhattan Project) and the Atomic Energy Commission.

Fuel assembly: A grouping of nuclear fuel rods that remains integral during the charging and discharging of a reactor core.

Fuel cycle, nuclear: The complete series of steps involved in supplying fuel for nuclear reactors. It includes mining, refining, $\mathrm{UF}_{6}$ conversion, enrichment, fabrication of fuel elements, use in a reactor, and management of radioactive waste. It may also involve chemical processing to recover the fissionable material remaining in the spent nuclear fuel, reenrichment of the fuel material, and/or refabrication of new fuel elements.

Generation (electricity): The process of producing electric energy from other forms of energy; also, the amount of electric energy produced, commonly expressed in kilowatt-hours (kWh) or megawatt-years [MW(e)-years].

Generation (gross): The total amount of electric energy produced by the generating units in a generating station or stations, measured at the generator terminals.

Generation (net): Gross generation less the electric energy consumed at the generating station for station use.

Generation (waste): The origination of new wastes from various facility operations (including production, rework, decontamination and decommissioning, and environmental restoration), including the recovery of pre-1970 transuranic-produced wastes, should their recovery be determined necessary.
Glass frit: A fusible ceramic mixture used to make glass for use in the immobilization and disposal of high-level wastes.

Greater-than-Class-C low-level waste: Waste from commercial sources containing concentrations of radionuclides that exceed U.S. Nuclear Regulatory Commission limits for Class $\mathrm{C}$ low-level radioactive waste, as defined in $10 \mathrm{CFR}$ Part 61.55.

Grout: A mortar or cement mixture used to immobilize radioactive wastes.

Half-life, radioactive: For a single radioactive decay process, the time required for the activity to decrease to one-half of its initial value by that process.

Hazardous waste: Nonradioactive waste that has at least one of the following characteristics: (1) is listed as a hazardous waste in Subpart D of 40 CFR Part 261, (2) exhibits any of the characteristics identified in Subpart C of 40 CFR Part 261, or (3) contains PCBcontaining wastes subject to regulation under the Toxic Substances Control Act and 40 CFR Parts 702-799.

High-level waste: As defined by the Nuclear Waste Policy Act, high-level waste is (1) the highly radioactive material resulting from the reprocessing of spent nuclear fuel, including the liquid waste produced directly in reprocessing and any solid material derived from such liquid waste that contains fission products in sufficient concentrations and (2) other highly radioactive material that the U.S. Nuclear Regulatory Commission, consistent with existing law, determines by rule to require permanent isolation.

Hydrofracture: A process formerly used for permanent disposal of low-level (approximately $0.25 \mathrm{Ci} /$ ) liquid waste at the Oak Ridge National Laboratory. The process involved mixing the waste with a blend of cement and other additives with the resulting grout being injected into shale at a depth of 200 to $300 \mathrm{~m}$. The injected grout hardened into thin, horizontal sheets several hundred meters wide.

Industrial waste: Commercial low-level waste resulting from nonnuclear fuel cycle sources. These include the commercial producers of radiochemicals and radiopharmaceuticals, luminous dial manufacturers, and instruments that incorporate sealed source components (e.g., smoke detectors).

Institutional waste: Commercial low-level waste resulting from bioresearch, medical, and certain 
nonbioresearch sources. Bioresearch wastes include wastes from animal studies at universities. Medical wastes include those generated from diagnostic and therapeutic procedures on humans at hospitals. Nonbioresearch wastes include research reactor wastes; small-volume, sealed radiation sources; and accelerator targets.

Leaching: The process of removal or separation of soluble components from a solid by percolating water or other liquids through the solid.

Low-level waste: As specified in the Low-Level Radioactive Waste Policy Amendments Act of 1985 (Public Law 99-240), radioactive waste not classified as high-level waste, spent nuclear fuel, or by-product material specified as uranium or thorium tailings and waste.

Mill tailings, uranium: Earthen residues that remain after the extraction of uranium from ores. Tailings may also contain other minerals or metals not extracted in the process.

Mixed low-level waste: Waste that satisfies the definition of low-level radioactive waste (LLW) in the Low-Level Radioactive Waste Policy Amendments Act of 1985 and that contains hazardous waste that has at least one of the following characteristics: (1) is listed as a hazardous waste in Subpart D of 40 CFR Part 261, (2) exhibits any of the hazardous waste characteristics identified in Subpart $C$ of 40 CFR Part 261, or (3) contains PCB-containing wastes subject to regulation under the Toxic Substances Control Act and 40 CFR Parts 702-799.

Mixed-oxide fuel: Nuclear reactor fuel composed of plutonium and uranium in oxide form.

Mixed waste: Waste that includes concentrations of both radionuclides and hazardous chemicals.

Moderator: A material used to reduce neutron energy (for fissioning if in a reactor) by elastic scattering.

MRS facility: A proposed facility for the monitored retrievable storage of spent nuclear fuel from commercial power plants. Such a facility would permit continuous monitoring, management, and maintenance of these wastes and provide for their ready retrieval for further processing or disposal.

Naturally occurring and accelerator-produced radioactive materials: Radioactive materials that are considered either naturally occurring and are not source, special nuclear, or by-product material or are produced in a charged particle accelerator.
Neutron activation: The process of irradiating a material with neutrons so that the material itself is transformed into a radioactive nuclide.

Nonfuel components: Nuclear reactor core parts and hardware, excluding the nuclear fuel itself. Such components include shrouds, control rods, fuel channels, in-core chambers, support tubes, and dummy fuel rods.

Parent: A radionuclide that upon decay yields a specified nuclide (the daughter) either directly or as a later member of a radioactive decay series.

Pressure vessel, reactor: A strong-walled container housing the core of most types of power reactors. It usually also contains other core components such as the moderator and control rods.

PUREXTM process: A solvent extraction process that may be used in the reprocessing of uranium/plutoniumbased nuclear fuels.

Radioactivity: The property possessed by certain nuclides of spontaneousiy emitting alpha or beta particles and, sometimes, gamma radiation by the disintegration of atomic nuclei. The units for the rate of decay of radioactive nuclides are the curie, $\mathrm{Ci}\left(3.7 \times 10^{10}\right.$ disintegrations per second exactly), or, in SI units, the becquerel, $\mathrm{Bq}$ (1 disintegration per second).

Reactor, boiling-water: A light-water reactor in which water, used as both coolant and moderator, is allowed to boil in the core. The resulting steam is used directly to drive a turbine.

Reactor, breeder: A reactor that produces more fissionable fuel than it consumes. The new fissionable material is created by a process (breeding) in which fission neutrons are captured in fertile materials.

Reactor, fast flux: A reactor in which fission is induced predominantly by fast neutrons.

Reactor, high-temperature, gas-cooled: A nuclear reactor that uses an inert gas (helinm) as the primary coolant and graphite as the moderator.

Reactor, light-water: A nuclear reactor that uses light water $\left(\mathrm{H}_{2} \mathrm{O}\right)$ as the primary coolant and moderator and slightly enriched uranium as the fuel. There are two types of commercial light-water reactors: boiling-water and pressurized-water. 
Reactor, naval propulsion: A reactor used to power a U.S. Navy vessel.

Reactor, pressurized-water: A light-water reactor in which heat is transferred from the core to a heat exchanger via water kept under high pressure, so that high temperatures can be maintained in the primary coolant system without boiling the water. Steam is generated in a secondary circuit.

Reactor, production: A reactor whose primary purpose is to produce fissile or other materials or to perform irradiations on an industrial scale. Unless otherwise specified, the term usually refers to either a tritium- or plutonium-production facility used to produce materials for nuclear weapons.

Reactor, research: A reactor whose nuclear radiations are used primarily as a tool for basic or applied research. Typically, it has a thermal power of $10 \mathrm{MW}(\mathrm{t})$ or less and may include facilities for testing reactor materials.

Reactor, test: A reactor associated with an engineering-scale test program conducted to develop basic design information or demonstrate safety characteristics of nuclear reactor systems.

Reinserted fuel: Irradiated reactor fuel that is discharged in one cycle and inserted into the same reactor during a subsequent refueling. In a few cases, fuel discharged from one reactor has been used to fuel a different reactor.

Repository, geologic: A facility that has an excavated subsurface system for the permanent disposal of spent nuclear fuel and high-level waste.

Reprocessing, fuel: The chemical/mechanical processing of irradiated nuclear reactor fuel to remove fission products and recover fissile and fertile material.

Salt cake: A salt form of high-level waste stored in tanks, which is produced from neutralizing acidic liquid waste from defense reactor fuel reprocessing with an alkaline agent (caustic soda).

Saltstone: A low-level waste by-product from the solidification of high-level waste at the Savannah River Site. Saltstone is retained in trenches at the Savannah River Site.

Sea-bed disposal: Placement of waste packages in deep ocean sediments.
Sea dumping (disposal): The practice of periodically dumping shiploads of drummed, solidified waste into the ocean at specified locations. (No longer performed.)

Separative work unit: The standard measure of enrichment services. The separative work unit (SWU) is expressed as a unit of mass. For example, 1 kilogram of separative work is expressed as $1 \mathrm{~kg}$ SWU.

Single-shell tank wastes: High-level wastes, generated from defense reactor fuel reprocessing at Hanford, which are stored in single-shelled tanks that contain liquid, sludge, and salt cake. See also "double-shell tank wastes."

Slurry, high-level waste: A watery mixture of highly radioactive, insoluble matter.

Solvent extraction: The separation of materials of different chemical types by exploiting the relative chelating ability of different chemicals which preferentially dissolve in one of the two phases. In spent nuclear fuel reprocessing, liquid-liquid contact of two immiscible solvents (one aqueous, one organic) permits recovery and separation of uranium and plutonium in one phase and fission products in the other phase.

Source material: (1) material containing any combination of uranium or thorium in any physical or chemical form, or (2) ores containing $0.05 \mathrm{wt} \%$ or more of uranium, thorium, or both. Source material excludes special nuclear material (see below).

Source term (IDB Program usage): A set of qualitative and quantitative features used to describe the origin and concentration of radioactive waste. The qualitative features include a flowchart of waste streams generated by a facility or an activity. Quantitative features include (1) the number of curies of radioactivity expressed either per unit of facility production or per unit of waste volume or mass and (2) a listing of the relative concentrations of component radioisotopes per curie of waste activity.

Special nuclear material: Plutonium or fissile uranium (i.e., ${ }^{233} U,{ }^{235} U$ ) enriched to a higher-than-natural assay.

Spent nuclear fuel: Nuclear fuel that has been permanently discharged from a reactor after it has been irradiated. Typically, spent nuclear fuel is measured in terms of either the number of discharged fuel assemblies or the quantity of discharged fuel mass. The latter is measured either in metric tons of heavy metal (i.e., only the heavy-metal content of the spent nuclear fuel is considered) or in metric tons of initial heavy metal (essentially, the initial heavy-metal mass of the fuel before irradiation). 
The difference between these two quantities is the weight of the fission products produced during irradiation.

Standard Contract: A contract between DOE and each commercial owner and/or generator of irradiated nuclear fuel. As indicated in 10 CFR Part 961, the contract specifies the terms and methods of payment, how fees are to be calculated, and how spent nuclear fuel is to be transferred to DOE custody.

Thermal power: A measure of the rate of heat-energy emission that results from the radioactive decay of a material. A unit of thermal power commonly used is the watt (W).

THOREX process: A solvent extraction process developed to reprocess thorium-based nuclear fuels.

Transuranic waste: As defined and used by the U.S. Department of Energy (DOE Order 5820.2A), radioactive waste that, at the time of assay, contains more than $100 \mathrm{nCi} / \mathrm{g}$ of alpha-emitting isotopes with atomic numbers greater than 92 and half-lives greater than 20 years.

Transuranic waste acceptance criteria: A set of requirements/criteria that must be satisfied prior to transport to and emplacement in the Waste Isolation Pilot Plant for disposal.
Transuranic waste certification: The process for verifying that waste meets the applicable requirements/criteria for transport to and emplacement in a repository for disposal.

Transuranic waste, contact-handled: Transuranic waste with a surface dose rate of less than $200 \mathrm{mrem} / \mathrm{h}$.

Transuranic waste nondestructive assay/ nondestructive examination: Nondestructive test procedures performed on suspect transuranic wastes to determine their transuranic isotope concentration. From these tests such wastes can be properly classified (certified) as transuranic or low-level.

Transuranic waste, remote-handled: Transuranic waste with a surface dose rate of greater than $200 \mathrm{mrem} / \mathrm{h}$.

Vitrification: The conversion of high-level waste materials into a glassy or noncrystalline solid for subsequent disposal.

Waste Isolation Pilot Plant: A facility, located near Carlsbad, New Mexico, to be used for demonstrating the safe disposal of transuranic wastes from DOE defenserelated activities.

Yellowcake: A uranium oxide concentrate that results from milling (concentrating) uranium ore. It typically contains 80 to $90 \% \mathrm{U}_{3} \mathrm{O}_{8}$. 


\section{DISTRIBUTION}

\section{DOE Headquarters}

Energy Information Administration, Office of Coal, Nuclear, Electric, and Alternate Fuels, Route Symbol EI-53, 950 L'Enfant Plaza, S.W., Washington, DC 20024

Office of Civilian Radioactive Waste Management, Waste Acceptance Division, Route Symbol RW-44, 1000 Independence Ave., S.W., Washington, DC 20585-0001

Office of Environment, Safety, and Health, Route Symbol EH-20, 1000 Independence Ave., S.W., Washington, DC 20585-0001

Office of Environmental Management, Office of Waste Management, Office of Planning and Analysis, Route Symbol EM-35, Trevion II, 19901 Germantown Road, Germantown, MD 20874-1290

Office of Environmental Management, Office of Environmental Restoration, Office of Program Integration, Route Symbol EM-43, Cloverleaf Building, 20400 Century Blvd., Germantown, MD 20874

Office of Environmental Management, Office of Science and Technology, Route Symbol EM-50, Trevion II, 19901 Germantown Road, Germantown, MD 20874-1290

Office of Environmental Management, Office of Nuclear Material and Facility Stabilization, Route Symbol EM-60, Trevion II, 19901 Germantown Road, Germantown, MD 20874-1290

Office of Naval Reactors, Route Symbol NE-60, 2531 Jefferson Davis Highway, Arlington, VA 22242-5160

\section{DOE Operations and Field Offices}

Albuquerque Operations Office, P.O. Box 5400, Albuquerque, NM 87185-5400

Chicago Operations Office, Building 201, 9800 South Cass Ave., Argonne, Il 60439

Idaho Operations Office, 785 DOE Place, Idaho Falls, ID 83402

Nevada Operations Office, P.O. Box 98518, Las Vegas, NV 89193-8518

Oakland Operations Office, 1301 Clay St., Oakland, CA 94612-5208

Oak Ridge Operations Office, P.O. Box 2001, Oak Ridge, TN 37831-8501

Ohio Field Office, P.O. Box 3020, Miamisburg, OH 45343-3020

Richland Operations Office, P.O. Box 550, 825 Jadwin Ave., Richland, WA 99352

Savannah River Operations Office, P.O. Box A, Aiken, SC 29802 
DOE Sites, Contractors, Special Site/Area Offices, and Special Program Offices

Ames Laboratory, Iowa State University, Technical Administration Services Facility, Ames, IA 5001 1-3020

Argonne National Laboratory, University of Chicago, 9700 South Cass Ave., Argonne, Il 60439

Brookhaven National Laboratory:

A.ssociated Universities, Inc., 16 South Railroad Street, Upton, NY 11973-2310

DOE Brookhaven Area Office, 53 Bell Ave., Upton, NY 11973-2311

DOE Office of Scientific and Technical Information, P.O. Box 62, Oak Ridge, TN 37831

Fermi National Accelerator Laboratory:

DOE Batavia Area Office, P.O. Box 2000, Batavia, IL 60510

University Research Association, P.O. Box 500, Batavia, II 60510

Fernald Environmental Management Project:

DOE Fernald Area Office, P.O. Box 398705, 7400 Wiley Road, Cincinnati, OH 45239-8705

Fluor Daniel, Fernald Environmental Restoration Management Corporation, P.O. Box 398704, 7400 Wiley Road, Cincinnati, OH 45239-8704

Formerly Utilized Sites Remedial Action Program:

Bechtel National, Inc., 151 Lafayette Drive, P.O. Box 350, Oak Ridge, TN 37831-0350

DOE Oak Ridge Operations Office, P.O. Box 2001, Oak Ridge, TN $37831-8723$

Grand Junction Remedial Action Project:

Department of Energy, Grand Junction Projects Office, P.O. Box 2567, Grand Junction, CO 81502

Hanford Site, Fluor Daniel Hanford, Inc., P.O. Box 1970, Richland, WA 99352

Hazardous Waste Remedial Actions Program, Tri-County Mall, P.O. Box 2003, Oak Ridge, TN 37831-7606

Idaho National Engineering Laboratory:

Argonne National Laboratory-West, P.O. Box 2528, Idaho Falls, ID $83401-2528$

Idaho Chemical Processing Plant, Lockheed Martin Idaho Technologies, P.O. Box 4000, Idaho Falls, ID 83404

Lockheed Martin Idaho Technologies, P.O. Box 1625, Idaho Falls, ID 83415-4201

Naval Reactors Facility, P.O. Box 2068, Idaho Falls, ID 83403-2068

Inhalation Toxicology Research Institute:

DOE Kirtland Area Office, P.O. Box 5890, Kirtland Air Force Base, Albuquerque, NM 87185

Lovelace Biomedical and Environmental Research Institute, Inc., P.O. Box 5890, Albuquerque, NM 87185 
Kansas City Plant:

Allied-Signal Inc., Kansas City Division, Bannister Federal Complex, Kansas City, MO 64141

DOE Kansas City Area Office, P.O. Box 410202, Kansas City, MO 64141-0202

Lawrence Berkeley Laboratory, University of California, One Cyclotron Road, Berkeley, CA 94720

Lawrence Livermore National Laboratory, P.O. Box 808, L-1, 7000 East Ave., Livermore, CA 94550

Los Alamos National Laboratory:

DOE Los Alamos Area Office, 528 35th Street, Los Alamos, NM 87544

University of California, P.O. Box 1663, Los Alamos, NM 87545

Mound Plant:

DOE Miamisburg Area Office, P.O. Box 66, Miamisburg, OH 45343-0066

EG\&G Mound Applied Technologies, Inc., P.O. Box 3000, Miamisburg, OH 45343-3000

Naval Reactors Program (DOE/HQ, NE-60) Facilities:

Bettis Atomic Power Laboratory, Westinghouse Electric Corporation, P.O. Box 79, West Mifflin, PA 15122-0079

DOE Pittsburgh Naval Reactors Office, P.O. Box 109, West Mifflin, PA 15122-0109

DOE Schenectady Naval Reactors Office, P.O. Box 1069, Schenectady, NY 12301-1069

Knolls Atomic Power Laboratory, General Electric Company, P.O. Box 1072, Schenectady, NY 12301-1072

Naval Reactors Facility (see Idaho National Engineering Laboratory)

Nevada Test Site:

Bechtel Nevada Corporation, P.O. Box 98521, Mail Stop 738, Las Vegas, NV 89193-8521

Oak Ridge Reservation:

Oak Ridge Institute of Science and Education, Oak Ridge Associated Universities, 246 Laboratory Road, P.O. Box 117, Oak Ridge, TN 37831-0117

Oak Ridge K-25 Site, Lockheed Martin Energy Systems, P.O. Box 2003, Oak Ridge, TN $37831-7358$

Oak Ridge National Laboratory, Lockheed Martin Energy Research Corporation, P.O. Box 2008, Oak Ridge, TN 37831-6235

Oak Ridge Y-12 Plant, Lockheed Martin Energy Systems, P.O. Box 2009, Oak Ridge, TN 37831-8010-

Pacific Northwest National Laboratory, Battelle Memorial Institute, Battelle Boulevard, P.O. Box 999, Richland, WA 99352

Paducah Gaseous Diffusion Plant, Lockheed Martin Utility Systems, P.O. Box 1410, Paducah, KY 42001

Pantex Plant:

DOE Amarillo Area Office, P.O. Box 30030, Amarillo, TX 79120-0030

Mason and Hanger-Silas Mason Co., P.O. Box 30020, Amarillo, TX 79177 
Pinellas Plant:

DOE Pinellas Area Office, P.O. Box 2900, Largo, FL 34649

Lockheed Martin Specialty Components, P.O. Box 2908, Largo, FL 34649-2908

Portsmouth Gaseous Diffusion Plant, Lockheed Martin Utility Systems, P.O. Box 628, Piketon, OH 45661

Princeton Plasma Physics Laboratory, Princeton University, P.O. Box 451, Princeton, NJ 08543

Reactive Materials Incorporated (RM) Extrusion Plant, P.O. Box 579, Ashtabula, OH 44004

Rocky Flats Environmental Technology Site:

DOE Rocky Flats Office, P.O. Box 928, Golden, CO 80401-0928

EG\&G Racky Flats, Inc., P.O. Box 464, Golden, CO 80401-0464

Sandia National Laboratories/California, Lockheed Martin Sandia Corporation, P.O. Box 969, Livermore, CA 94551-0969

Sandia National Laboratories/New Mexico, Lockheed Martin Sandia Corporation, P.O. Box 5800, Albuquerque, NM $87185-5800$

Savannah River Site, Westinghouse Savannah River Company, P.O. Box 616, Aiken, SC 29802

Stanford Linear Accelerator Center, P.O. Box 4349, Palo Alto, CA 94309

Uranium Mill Tailings Remedial Action Program (UMTRAP):

DOE Albuquerque Operations Office, UMTRA. Project Office, P.O. Box 5400, Albuquerque, NM 87115

Jacobs Engineering Group, Inc., Suite 1700, 5301 Central Ave., NE, Albuquerque, NM 87108

Waste Isolation Pilot Plant:

Department of Energy, Waste Isolation Pilot Plant Project Office, P.O. Box 3090, Carlsbad, NM 88221

Westinghouse Electric Corporation, Waste Isolation Pilot Plant Project Office, P.O. Box 2078, Carlsbad, NM 88221

West Valley Demonstration Project:

DOE West Valley Area Office, 10282 Rock Springs Road, P.O. Box 191, West Valley, NY 14171-0191

West Valley Nuclear Services Company, Inc., 10282 Rock Springs Road, P.O. Box 191, West Valley, NY 14171-0191

\section{Federal (non-DOE) Agencies}

Congressional General Accounting Office, P.O. Box 321, Richland, WA 99352

Congressional Office of Technology Assessment, 600 Pennsylvania Ave., S.E., Washington, DC 20510-8025

Environmental Protection Agency, 401 M St., S.W., Washington, DC 20460

Nuclear Regulatory Commission, 1717 H St., N.W., Washington, DC 20555

Extra copies for DOE/OSTI to fill orders (200). 
For additional information regarding the distribution of this report, contact

U.S. Department of Energy Office of Environmental Management

Office of Waste Management

Office of Planning and Analysis Route Symbol EM-35

Trevion II Building 19901 Germantown Road

Germantown, MD 20874-1290

Phone: (301) 903-7126

Fax: (301) 903-9770

E-mail: Mathew.Zenkowich @em.doe.gov
U.S. Department of Energy

Office of Environmental Management

Office of Environmental Restoration

Office of Program Integration

Route Symbol EM-43

Cloverleaf Building 20400 Century Boulevard

Germantown, MD 20874

Phone: (301) 903-8121

Fax: (301) 903-3617

E-mail: Marilyn.Tolbert-

Smith@em.doe.gov
Oak Ridge National Laboratory Integrated Data Base Program

P.O. Box 2008

Building 4500S

Mail Stop 6111

Oak Ridge, TN $37831-6111$

Phones: (423) 574-6823;

(423) $576-7575$

Faxes: (423) 576-0327;

(423) 574-6616

E-mail: jak@ornl.gov sns@ornl.gov 